\title{
Spannglasträger \\ Glasträger mit vorgespannter Bewehrung
}

\author{
Spannglass Beams \\ Glass Beams with Post-Tensioned Reinforcement
}

\begin{abstract}
An der Fakultät Bauingenieurwesen
der Technischen Universität Dresden

zur Erlangung des akademischen Grades

eines Doktor-Ingenieur (Dr.-Ing.) genehmigte
\end{abstract}

\section{Dissertation}

vorgelegt von

Dipl.-Ing. Michael Engelmann

geboren am 08.12. 1983 in Merseburg/Saale

Erster Gutachter

Zweiter Gutachter

Dritter Gutachter

Tag der Verteidigung
Prof. Dr.-Ing. Bernhard Weller

Technische Universität Dresden

Prof. Dr.-Ing. Geralt Siebert

Universität der Bundeswehr München

Prof. Dr. sc. techn. Mike Schlaich

Technische Universität Berlin

24. 08.2017 
Bibliografische Information der Deutschen Bibliothek

Die Deutsche Bibliothek verzeichnet diese Publikation in der Deutschen

Nationalbibliografie; detaillierte bibliografische Daten sind im Internet über http://dnb.ddb.de abrufbar.

Michael Engelmann

Spannglasträger - Glasträger mit vorgespannter Bewehrung

Dissertation. Dresden: Technische Universität, 2017.

(c) 2017 Michael Engelmann

Technische Universität Dresden

Fakultät Bauingenieurwesen

Institut für Baukonstruktion

D-01062 Dresden

Telefon +4935146334845

Telefax +4935146335039

www.bauko.bau.tu-dresden.de 


Karin und meinen Eltern 



\section{Vorwort}

Analoge Forschungsfragen im Konstruktiven Glasbau konnten im Stahlbetonbau bereits beantwortet werden. Die somit verfügbaren Erkenntnisse zu nutzen und den Abstand aufzuholen, motivierte mich zum Schreiben meiner Dissertation. Dieser Ansatz steht gleichwohl für die Grundidee der Arbeit und brachte mir besondere Freude bei dem Verbinden zweier scheinbar so unterschiedlicher Bauarten.

Ein erster Dank gebührt Professor Bernhard Weller, meinem Chef und Doktorvater, für die Unterstützung der Dissertation und der Möglichkeit, eigenständig und eigenverantwortlich zu forschen. Weiterhin möchte ich mich bei Professor Geralt Siebert (Universität der Bundeswehr München) und Professor Maik Schlaich (Technische Universität Berlin) für die Übernahme der weiteren Gutachten bedanken.

Die Hauptuntersuchungen konnte ich im Rahmen des BMWiForschungsvorhabens „Bewehrte Glasträger“ durchführen. Ohne die vertrauensvolle Zusammenarbeit mit den beteiligten Partnern Thiele Glas GmbH und KL megla GmbH wäre eine derarte Forschung nicht möglich - schließlich sind gut 5 Tonnen Glas zu Bruch gegangen und wurden mehr als 200 Meter Seil verwendet. Dabei einen besonderen Dank.

Als besonderen Höhepunkt konnte ich 2014 das Projekt "Spannglasbrücke" verwirklichen, welches mir in wohl einzigartiger Weise ermöglichte, ein kleines Bauvorhaben vom ersten Strich auf einem weißen Blatt Papier bis zur Montage der letzten Teile vor Ort umzusetzen. Die dabei gewonnenen Erfahrungen haben mich nachhaltig geprägt.

Von allen Gutachtern habe ich gelernt, dass ein internationaler Austausch wesentlicher Bestandteil und Quelle weiterführender Forschungsinspiration ist. Daher bin ich glücklich, dass ich die Möglichkeit vieler Reisen nutzen konnte. Der Graduiertenakademie der TU Dresden und dem Deutschen Akademischen Austauschdienst sei für die Bereitstellung zahlreicher Reisestipendien gedankt. 
Letztendlich entsteht eine solche Arbeit nicht ohne die substanzielle Unterstützung durch die Familie, Freunde, Kameraden, Kollegen und beteiligte Studierende. Bei Allen möchte ich mich herzlich bedanken.

Herauszuheben ist die Motivation und Inspiration durch meine Eltern, die wohl immer noch nicht glauben können, dass man das Zerbrechen von Glas zum Beruf machen kann.

Abschließend möchte ich meiner Frau Karin für die geduldige Unterstützung während zahlreicher Wochenenden am Laptop danken - ohne Dich wäre an das Gelingen nicht zu denken gewesen.

Dresden, Oktober 2017

Michael Engelmann 


\section{Kurzfassung}

Glas und Beton sind sich in wesentlichen Materialeigenschaften ähnlich: Beide zeigen gegenüber einer hohen Druckfestigkeit eine vergleichsweise geringe Zugfestigkeit und versagen spröde. Diese Analogie führte zur Entwicklung bewehrter Glasträger, die sich durch eine aufgeklebte Stahllasche an ihrer Biegezugkante auszeichnen. Dadurch wurden die Übertragung von Zugkräften auch im Rissfall möglich, sodass ein duktiles Bauteilverhalten erreicht und der im Konstruktiven Glasbau notwendige Nachweis der Resttragfähigkeit erfüllt wird.

Glasträger mit verbundlos vorgespannter Bewehrung - Spannglasträger - stellen die Fortführung dieses Analogiegedankens dar. Neben einer gezielten Steigerung der Erstrisslast, können die Träger planmäßig überhöht werden. Damit wird einer bisher üblichen Überdimensionierung mit der Anordnung nicht ausgenutzter „Opferscheiben" entgegen gewirkt und sichere sowie materialeffiziente Konstruktionen mit maximaler Transparenz ermöglicht.

Diese Konstruktionsweise wurde bislang ausschließlich für einzelne Sondierungsuntersuchungen in breiter Variantenvielfalt genutzt. Eine Systematik und einheitliche Bezeichnungsweise ist nicht vorhanden. Darüber hinaus beschränken sich verfügbare Ergebnisse auf die Beschreibung der Tragfähigkeit, ohne die Resttragfähigkeit explizit zu belegen oder die Dauerhaftigkeit nachzuweisen.

Mit dieser Arbeit wurde anhand einer Analogiebetrachtung zum Eurocode 2 eine Bezeichnungsweise für bewehrte und vorgespannte Glasträger entwickelt und für vorhandene Konstruktionen erfolgreich angewendet. Darin zeigt sich, dass der Stand der Technik auf diese Weise charakterisierbar ist.

Zusätzlich wird die These aufgestellt, dass sich das Tragverhalten von Spannglasträgern wie im Stahlbeton- und Spannbetonbau beschreiben und die auftretenden Spannkraftverluste analog berechnen lassen. Diese These wird mithilfe experimenteller Studien als Kern dieser Arbeit untersucht und durch eine ergänzende numerische Modellierung bestätigt. 
Zunächst wird das Tragverhalten im Kurzzeit-Biegeversuch an 15 Prüfkörpern unter variierten Bewehrungsgraden und Vorspannkräften untersucht. Dabei zeigen sich gesteigerte Erstrisslasten sowie ein sicheres Verhalten im Anschluss an die Belastung. Durch die Vorspannung wird das Tragverhalten gezielt beeinflusst.

Zusätzlich erbringt eine zerstörungsfreie Untersuchungsreihe an 28 Prüfkörpern unter konstanter Gebrauchslast über 1000 Stunden erstmals eine Beschreibung der auftretenden Spannkraftverluste. Diese sind maßgeblich von der horizontalen Durchbiegung sowie der daraus resultierenden Belastung der Zwischenschicht im Verbund-Sicherheitsglas abhängig. Aus der Größenordnung der Verluste lässt sich schlussfolgern, dass eine Begrenzung dieses Verformungsanteils sowie eine konstruktive Entlastung der Zwischenschicht notwendig sind. Zudem wird die Änderung der Vorspannkraft unter einer Temperaturlast beschrieben. Im Ergebnis zeigt sich, dass dieser Lastfall mittels der linearen Balkentheorie beschreibbar und der damit assoziierte Spannkraftverlust berechenbar ist.

Die Resttragfähigkeit von 24 Spannglasträgern wird mithilfe eines eigens entwickelten Prüfverfahrens bestätigt. Während die Bewehrung einerseits eine Überbrückung von Rissflanken ermöglicht, verursacht die Vorspannkraft andererseits im teilzerstörten Tragsystem bisweilen ein frühzeitiges Versagen. Daher wird empfohlen, die baukonstruktive Detailentwicklung zu intensivieren, um einen größeren Sicherheitsvorteil aus der Konstruktionsweise zu generieren.

Die Arbeit beinhaltet erstmals eine systematische Datensammlung zum Tragverhalten von Spannglasträgern. Es zeigt sich, dass auf eine Anordnung von „Opferscheiben“ zugunsten einer steigenden Materialeffizienz nicht nur verzichtet werden kann, sondern im Sinne eines effektiven Tragverhaltens verzichtet werden muss. Mit der vorgeschlagenen Bezeichnungsweise, den abgeleiteten konstruktiven Maßnahmen sowie den gezeigten Untersuchungsmethoden besteht nunmehr die Möglichkeit, sichere und dauerhafte Spannglasträger zu entwerfen und deren Trageffizienz zu belegen. 


\begin{abstract}
Glass and concrete share essential material characteristics: Their compressive strength exceeds their tensile strength considerably and both of them fail in a brittle manner. This analogy led to the development of reinforced glass beams, which are improved by means of adhesively bonded steel sections in the tensile zone. This improvement allowed for a direct transfer of tensile loads in a post-breakage state and resulted in a ductile structural element, which met the special demand of structural glass for a sufficient residual loadbearing capacity.
\end{abstract}

Glass beams with unbonded, post-tensioned reinforcement - Spannglass Beams - carry this analogy concept on. The members will comprise an increased initial fracture strength and may be uplifted intentionally. This development has rendered the need for over-dimensioning by removing unnecessary sacrificial layers, which will result in a material efficient structure and will maximise transparency.

Solely single exploratory investigations have used this idea in a wide variety of options so far. There is neither a uniform classification nor a consistent nomenclature. Furthermore, available results are limited to the concise description of the short-term load-bearing properties without proving the residual load-bearing capacity explicitly and confirming longterm durability.

This thesis describes the development and the application of a nomenclature for reinforced and pre-compressed glass beams in an analogy study according to Eurocode 2 . The state of technology can be characterised in this manner.

Additionally, the research describes the load-bearing behaviour as well as the calculation of the loss of pre-stress of Spannglass Beams by analogy with concrete structures. As the key section of this thesis, this statement is examined by means of comprehensive experimental studies and completed by a numerical calculation.

Primarily, the load-bearing behaviour of 15 specimens in short-term bending tests and a variety of reinforcement ra- 
tios and pre-stress levels were determined. The results show an increase of initial fracture strength as well as safe behaviour after failure. The pre-stress changes the load-bearing performance significantly.

Furthermore, a non-destructive study including a constant loading for $1000 \mathrm{~h}$ describes the loss of pre-stress in 28 specimens for the first time. The horizontal deflection and the thus resulting shear stresses of the interlayer material of a laminated glass section are the critical parameters. From the magnitude of losses it may be concluded that the deflections need to be limited and the interlayer foils need to be relieved from stress. Moreover, the structural response during a change in temperature is in good agreement with the results obtained from linear beam theory. This allows for an estimation of the associated losses.

Finally, a specifically developed test approach confirms the residual load-bearing capacity of 24 specimens. The reinforcement shows the ability to bridge cracks in the glass. However, it should be noted that pre-stress occasionally causes an early failure of the partially broken Spannglass cross-section. Therefore, intensifying the development of structural details in order to generate an increased advantage concerning safety is recommended.

This contribution contains a systematic acquisition of analytical, experimental and numerical data regarding the loadbearing characteristics of Spannglass Beams for the first time. The use of a sacrificial layers is not necessary. Even more, to reach the most effective load-bearing behaviour, it is necessary to abandon them completely. Implementing the developed nomenclature, realising the recommended structural provisions and using the proposed methods, it is now possible to compose safe and durable Spannglass Beams as well as prove their structural efficiency. 
Inhalt

1 Einleitung 17

1.1 Problemstellung und Motivation 17

1.2 Zielsetzung 21

1.3 Vorgehensweise 23

1.4 Abgrenzung 24

2 Analogiebetrachtung 26

2.1 Zielsetzung 26

2.2 Anwendungsbereich 26

2.3 Begriffe 28

2.3.1 Bewehrte und hybride Glastragwerke 28

2.3.2 Thermische und mechanische Vorspannung 31

2.3.3 Spanngliedkonstruktion und Spannverfahren 33

2.3.4 Lage und Verlauf des Spanngliedes 41

2.3.5 Weitere Begriffe 44

2.4 Grundlagen der Tragwerksplanung 45

2.5 Baustoffe 47

2.5.1 Festigkeit $\quad 47$

2.5.2 Elastische Formänderungseigenschaften $\quad 49$

2.5.3 Kriechen und Schwinden $\quad 51$

2.5.4 Bewehrungsmaterial 59

2.5.5 Komponenten von Spannsystemen 60

2.5.6 Querschnittsgestaltung 61

2.6 Dauerhaftigkeit 62

2.7 Schnittgrößenermittlung 64

2.7.1 Allgemeines 64

2.7.2 Imperfektionen $\quad 65$

$\begin{array}{ll}2.7 .3 \text { Idealisierung } & 67\end{array}$

2.7.4 Lineare Berechnung 68

2.7.5 Nichtlineare Berechnung $\quad 76$

2.7.6 Zeitabhängigkeit der Vorspannkraft $\quad 79$

2.7.7 Vorspannung während der Berechnung 90

2.8 Grenzzustände und Nachweise 90

2.8.1 Grenzzustand der Tragfähigkeit 91

2.8.2 Grenzzustand der Gebrauchstauglichkeit 92

2.8.3 Nachweis der Resttragfähigkeit 93

2.9 Bewehrungs- und Konstruktionsregeln 96

2.10 Zusammenfassung 100 
3 Experimentelle Untersuchungen 103

3.1 Zielsetzung 103

3.2 Prüfkörper - Konstruktion und Materialien 104

3.3 Tragverhalten unter kurzzeitiger Beanspruchung 107

$\begin{array}{ll}\text { 3.3.1 Prüfkörper } & 107\end{array}$

3.3.2 Versuchseinrichtung 107

3.3.3 Untersuchungsverfahren und -bedingungen 109

3.3.4 Analyse- und Auswertungsverfahren 110

3.3.5 Ergebnisse und Ergebnisdiskussion 113

3.3.6 Folgerungen und Zusammenfassung 132

3.4 Tragverhalten unter Dauerlast 134

3.4.1 Prüfkörper 134

3.4.2 Versuchseinrichtung 135

3.4.3 Untersuchungsverfahren und -bedingungen $\quad 136$

3.4.4 Analyse- und Auswertungsverfahren 136

3.4.5 Ergebnisse und Ergebnisdiskussion $\quad 137$

3.4.6 Folgerungen und Zusammenfassung 145

3.5 Resttragfähigkeit 147

3.5.1 Prüfkörper 147

3.5.2 Versuchseinrichtung 148

3.5.3 Untersuchungsverfahren und -bedingungen 149

3.5.4 Analyse- und Auswertungsverfahren 150

3.5.5 Ergebnisse und Ergebnisdiskussion 151

3.5.6 Folgerungen und Zusammenfassung 163

3.6 Tragverhalten unter Temperaturbelastung 165

3.6.1 Prüfkörper 165

3.6.2 Versuchseinrichtung 165

3.6.3 Untersuchungsverfahren und -bedingungen $\quad 166$

3.6.4 Analyse- und Auswertungsverfahren 167

3.6.5 Ergebnisse und Ergebnisdiskussion 170

3.6.6 Folgerungen und Zusammenfassung 176

$\begin{array}{lll}3.7 & \text { Zusammenfassung } & 178\end{array}$

$4 \quad$ Numerische Untersuchungen $\quad 180$

4.1 Zielsetzung 180

4.2 Modellbeschreibung $\quad 181$

4.2.1 Systembeschreibung 181

4.2.2 Einwirkungen 186

4.2.3 Berechnung 186

4.3 Ergebnisse und Ergebnisdiskussion 188

4.3.1 Vergleich mit dem analytischen Modell 188

4.3.2 Modellierung der Umlenkung 188

4.3.3 Einfluss der Zwischenschicht 190 
4.3.4 Auswahl eines Imperfektionswertes $\quad 191$

4.3.5 Seilkraftverlust im Dauerversuch 197

4.4 Zusammenfassung 203

5 Diskussion 206

5.1 Zielsetzung 206

5.2 Tragverhalten unter kurzzeitiger Beanspruchung 207

5.2.1 Tragverhalten unter Vorspannbelastung 207

5.2.2 Trag- und Bruchverhalten unter Biegebelastung 210

5.2.3 Rissverhalten unter Biegebelastung 218

5.2.4 Spannungszuwachs in der Bewehrung 227

5.3 Tragverhalten unter Dauerbelastung 230

5.4 Resttragfähigkeit 234

5.5 Zusammenfassung 238

6 Konstruktive Empfehlungen 242

6.1 Zielsetzung 242

6.2 Teilprojekte 242

6.2.1 Forschungsprojekt „Bewehrte Glasträger“ 242

6.2.2 Spannglasbrücke - glasstec $2014 \quad 243$

6.2.3 Fußgängerbrücke in Nara (Japan) 2015

6.3 Verankerungen 245

6.3.1 Tragfähigkeit der Verankerung 245

6.3.2 Seilkrafteinleitung 246

6.3.3 Toleranzausgleich $\quad 247$

6.3.4 Neigungsausgleich 251

6.4 Vorspannverfahren 253

6.5 Umlenkpunkte 254

6.5.1 Geklotzte Umlenkpunkte 255

6.5.2 Geklebte Umlenkpunkte 257

6.6 Montage 258

6.7 Weiterführende Konstruktionen 261

6.7.1 Spannglasträger mit nachträglichem Verbund 262

6.7.2 Segmentbauweise 263

6.8 Zusammenfassung 264

7 Zusammenfassung und Ausblick 265

7.1 Zusammenfassung 265

$\begin{array}{lll}7.2 & \text { Ausblick } & 270\end{array}$

$8 \quad$ Literatur 273

8.1 Fachbücher und Fachaufsätze 273

8.2 Normen und Richtlinien 284 
Bezeichnungen

Abbildungsverzeichnis und -nachweis

Tabellenverzeichnis

A Analytische Schnittgrößenberechnung

B Kurzzeit-Biegeversuche

C Dauerversuche $1000 \mathrm{~h}$

D Versuche zur Resttragfähigkeit

353

E Biegeversuche unter Temperaturlast

F SOFiSTiK Quelltext 


\subsection{Problemstellung und Motivation}

Glasträger werden als primäre Tragelemente in transparenten Fassaden- und Dachkonstruktionen eingesetzt, um einen hohen Grad an natürlicher Belichtung zu erreichen. ${ }^{1}$ Eine Vielzahl ausgeführter Bauprojekte, wie in Bild 1 beispielhaft ausgewählt, zeigen, dass diese Bauart heute Stand der Technik ist: Die Bemessung erfolgt als Nachweis der Tragfähigkeit und Gebrauchstauglichkeit unter Begrenzung der Hauptzugspannung und Durchbiegung.

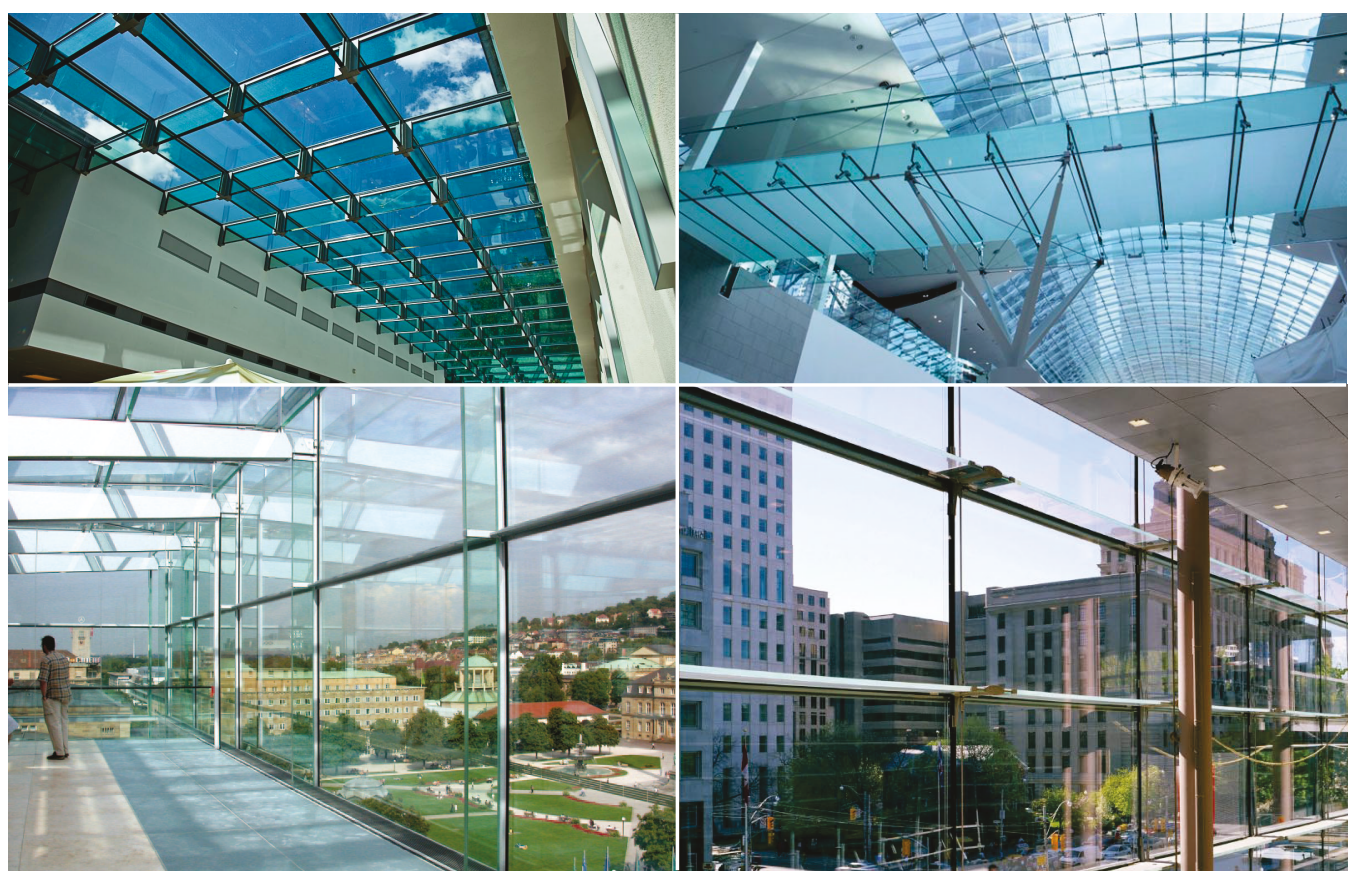

Zusätzlich ist der Nachweis der Resttragfähigkeit zu erbringen. Dieser wird, neben experimentellen Methoden, unter verringerten, außergewöhnlichen Lasten rechnerisch erbracht, indem ein Teil des Glasquerschnitts als nichttragend angenommen wird. Dieser Nachweis wird in der Regel maßgebend, da sich die Querschnittsreduktion größer auswirkt, als die Lastreduktion in der außergewöhnlichen Bemes-
Bild 1

Anwendungen von Glasträgern in Dächern (links oben), als Fußgängerbrücke (rechts oben) oder in Fassaden als horizontale Riegel und vertikale Pfosten (unten).

1 Dabei wird im Zusammenhang mit Glasträgern in Fassaden oft von Glasschwertern geredet, wenn sie eine vorrangig aussteifende Wirkung auf die Verglasungsebene aufweisen. 


\section{Bild 2}

Mit Edelstahlstreifen an Ober- und Unterkante bewehrter Glasträger im Biegeversuch aus einem Forschungsprojekt am Institut für Baukonstruktion der TU Dresden. Die Belastung $M_{\text {res }}$ wird anteilig durch Glas $\left(\mathrm{F}_{\text {res, G}}\right)$ und Bewehrung

$\left(F_{\text {res, }}\right)$ abgetragen.

In Analogie zum Stahlbeton ist ein Lastabtrag nach dem Glasbruch (unten) möglich. sungssituation. Infolgedessen ergibt sich im ungebrochenen Zustand ein geringerer Ausnutzungsgrad, welcher mit einer geringen Materialeffizienz einhergeht. Dieser wurde im Rahmen einer Vielzahl von Forschungsprojekten, welche im Folgenden zusammengefasst werden, durch zusätzliche Bewehrung ausgeglichen - eine Idee, die dem Stahlbetonbau entlehnt ist. Es entstehen bewehrte Glasträger, die einen zusätzlichen Lastabtragspfad über das Bewehrungsmaterial ermöglichen. Der Nachweis der Resttragfähigkeit fällt günstiger aus, da das gebrochene, dennoch drucktragfähige Glas gemeinsam mit der zugtragfähigen Bewehrung als tragfähig beziehungsweise resttragfähig angesetzt wird. ${ }^{2}$

Darüber hinaus kann die Bewehrung im Sinne eines Verbundquerschnittes zum geteilten Lastabtrag zwischen Glas und Bewehrung gezielt ausgenutzt werden. Die Biegeverzerrung des belasteten Trägers erzeugt in Abhängigkeit der Elastizitätsmoduln eine Spannung in den beteiligten Baustoffen. Diese wird zu einer resultierenden Kraft umgerechnet, welche entsprechend ihres inneren Hebelarms im Gleichgewicht mit dem einwirkenden Biegemoment steht (Bild 2).

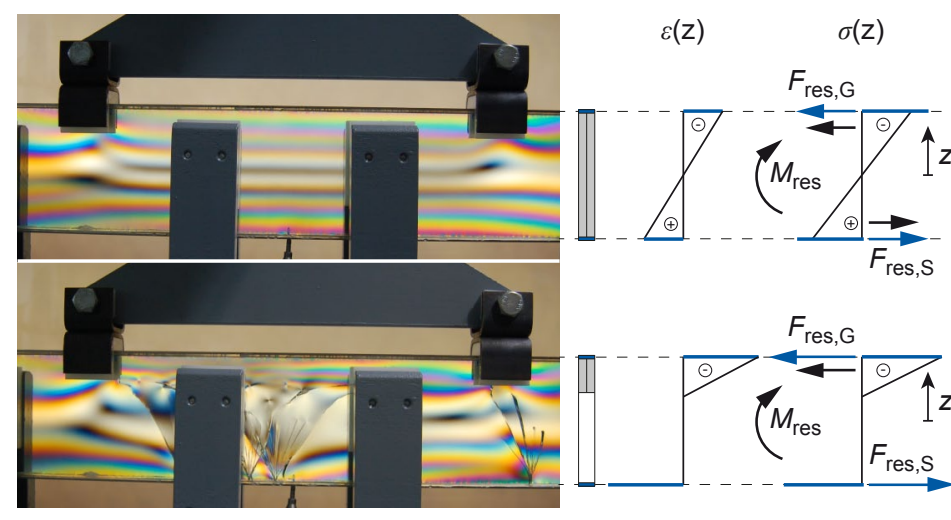

Hierfür sind Forschungsergebnisse verfügbar, deren Gemeinsamkeit darin besteht, dass ein Bewehrungsmaterial wie Stahl oder faserverstärkter Kunststoff stoffschlüssig mit den zugbelasteten Glaskanten verbunden wird. ${ }^{3}$

2 Vergleiche [DIN 18008-1, Kap. 3.1.2]. Auf gering ausgenutzte „Opferscheiben" soll verzichtet werden.

3 Vergleiche [Josef Gartner \& Co 1991], [Bos et al. 2004], [Belis et al. 2009a], [Cagnacci et al. 2009], [Weller et al. 2010b], [Louter 2011], [Louter et al. 2012], [Louter 2013], [Speranzini \& Agnetti 2013]. 
Glasträger zeichnen sich dadurch aus, dass sie aus Verbundglasquerschnitten, das heißt Laminaten mit schubweicher Zwischenschicht, hergestellt werden. Damit ergibt sich die Möglichkeit neben dem Grundmaterial auch die Zwischenschicht voll- oder teilflächig beziehungsweise linear zu bewehren. ${ }^{4}$ In Bild 3 sind einlaminierte Lochbleche und Edelstahlgewebe beispielhaft dargestellt. Neben dem Aufschichten mehrerer Materialien wie Metallbleche oder -gewebe und lonomer- oder PVB-Folien zu einer Zwischenschicht ${ }^{5}$ ist es zudem möglich, gänzlich andere Materialien wie Kunststoffplatten $^{6}$ zu verwenden. Für eine Anwendung als Glasträger bieten sich interne, linear einlaminierte Bänder parallel zur Biegezugkante, wie sie insbesondere in [Louter 2011] beschrieben werden, an.

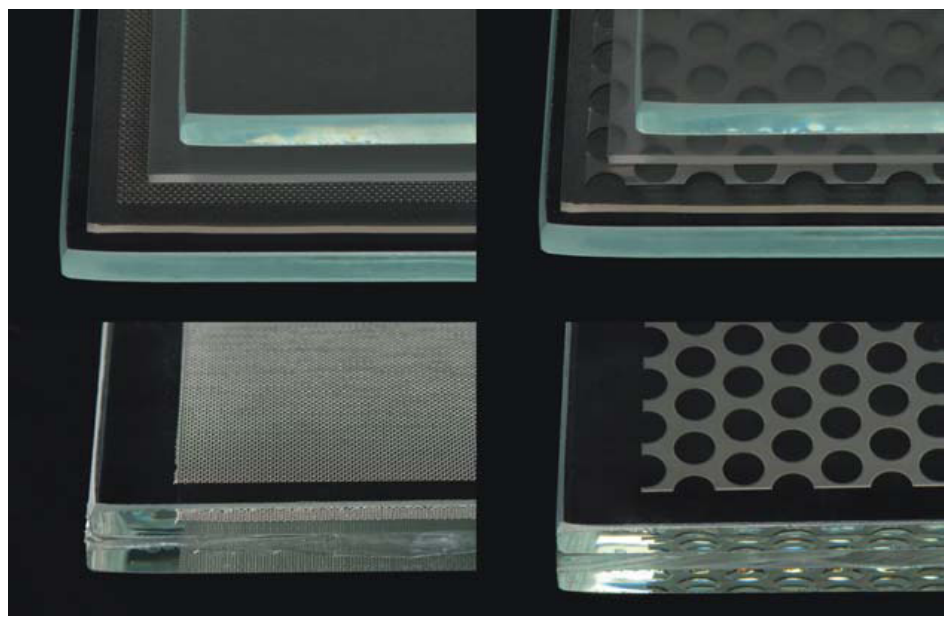

Darüber hinaus sind Verbundquerschnitte mit anderen Baustoffen wie Beton ${ }^{7}$, Holz ${ }^{8}$ oder Kunststoff ${ }^{9}$ möglich (Bild 4). Diese wurden bereits untersucht, haben jedoch noch keinen Weg in die breite Anwendung gefunden. Zum einen zeichnen sich viele der betreffenden Konstruktionen durch einen geringen Glasanteil und somit einer verringerten Transparenz gegenüber unbewehrten, reinen Glasträgern aus. Zum anderen stellte sich die Fügetechnologie zwischen Glas und

4 Vergleiche [Neugebauer 2005], [Feirabend 2010], [Louter 2011], [Cruz \& Valente 2013] und [Härth 2013]

5 Vergleiche [Feirabend 2010].

6 Härth [2013] untersucht Hybridträger aus Glas-Kunststoff-Laminaten mit PMMA, Polyamid und Polycarbonatplatten.

7 Vergleiche [Freytag 2001] und [Freytag et al. 2012].

8 Vergleiche [Cruz \& Pequeno 2008].

9 Vergleiche [Weller et al. 2009a].
Bild 3

Flächige Bewehrung eines Verbundglases mit Lochblechen und Edelstahlgeweben nach [Feirabend 2010].

Oben: Lagen vor dem Herstellen des Verbundes.

Unten: laminiertes Verbundglas.

In diesen Fällen werden Teile der Glasfläche opak und schränken damit den transparenten Gesamteindruck ein. 


\section{Bild 4}

Verbundquerschnitte aus Glas (a), Glas-Beton (b) Glas-Holz (c) und Glas-Stahl (d). Im Vergleich zu Bild 2 und Bild 3 fällt auf, dass der Flächenanteil des Glases im Querschnitt klein ist und damit die Transparenz bei Schrägsicht eingeschränkt bleibt. anderen Baustoffen als problematisch heraus. Oft werden Klebstoffe auf Kunststoffbasis verwendet, welche infolge der natürlichen Alterung und den typischen Belastungen im Bauwesen über die Lebenszeit deutliche Änderungen ihrer mechanischen und optischen Eigenschaften erfahren, die im Ergebnis in der Konstruktion unerwünscht sind. In der Folge wurden Forschungsprojekte motiviert, die lastabtragende Klebverbindungen zwischen Glas und anderen Werkstoffen, die den Anforderungen des Bauwesens gerecht werden, strukturiert untersuchen. (a)

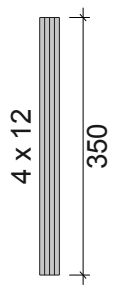

(b)

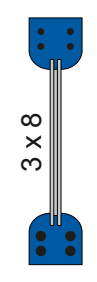

(c)

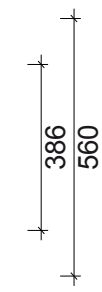

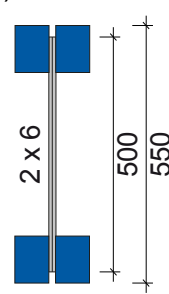

(d)

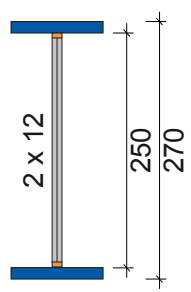

[Cruz \& Pequeno 2008] [Ungermann et al. 2010]

Führt man die Analogie zum Stahlbetonbau für Glasträger mit linearen Zuggliedern weiter, ergibt sich die Idee, die Bewehrung vorzuspannen und an diskreten Punkten mit dem Hauptträger zu verbinden. Die wesentliche Forschungsmotivation dazu ergibt sich aus der Möglichkeit größere Spannweiten bei höheren Lasten zu realisieren und die Durchbiegung gezielt zu beeinflussen. Solche verbundlos vorgespannten Tragwerke - im Weiteren als Spannglasträger bezeichnet - sind bisher nicht konsequent untersucht. ${ }^{10}$ Darauf aufbauend sind Varianten mit nachträglichem Verbund über adhäsive Verbindungen denkbar. ${ }^{11}$

10 Vorversuche an einer geringen Anzahl von Prüfkörpern können [Bos et al. 2004], [Louter et al. 2006], [Louter 2013], [Louter et al. 2013b], [Louter et al. 2013a], [Jordão et al. 2014] und [Cupac \& Louter 2015] entnommen werden. Diese beschränken sich auf einzelne Konstruktionsvarianten mit linear verklebten oder verbundlosen Spanngliedern. In [Weller \& Engelmann 2013] sind Ergebnisse von Vorversuchen für diese Arbeit veröffentlicht. Darüber hinaus wurden Bauprojekte in dieser Konstruktionsart realisiert (Vergleiche [Bünemann 1997], [Schober et al. 2004] und [Waagner Biro 2014]) sowie ein Patent veröffentlicht (Vergleiche [Ludwig \& Seele 1995]).

11 Dabei wird die Verbundfuge (dauerhaft) belastet. Eine solche Belastung wird derzeit in der Anwendung vermieden, birgt jedoch weiteres Potenzial. Vergleiche unter anderem [Louter 2011], [Louter 2013], [Louter et al. 2013b], [Louter et al. 2013a], [Martens et al. 2015a] und [Cupac \& Louter 2015]. 
In vorgespannten Glasträgern (Bild 5) wird eine Druckspannung in der lastabtragenden Glaskante mechanisch eingeleitet. Diese muss zunächst durch eine Dekompressionslast getilgt werden, welche als Traglaststeigerung interpretierbar ist. Darüber hinaus ist eine gezielte Überhöhung des Trägers möglich.
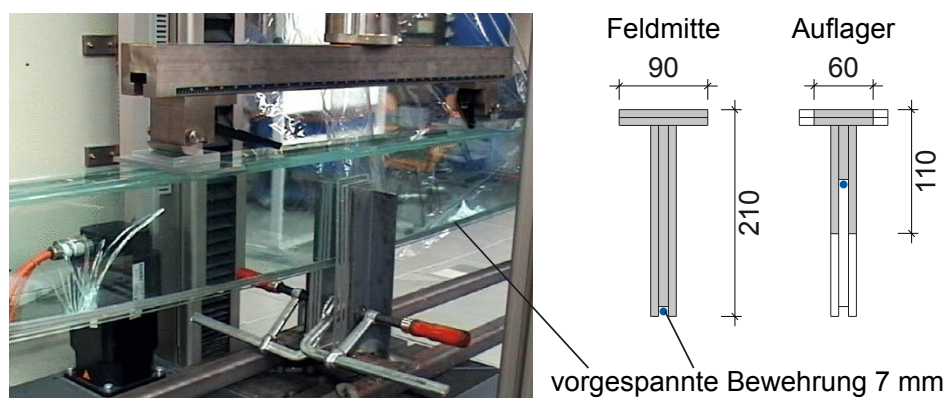

Allerdings führt eine Vorspannung in Form einer zusätzlichen Normalkraftbelastung rechnerisch zu einem früheren Stabilitätsversagen. Dem wird in dieser Arbeit mit der Entwicklung eines „Doppelquerschnitts“ begegnet, welcher mit reduziertem Materialeinsatz eine maximale Tragfähigkeit erreicht und gleichzeitig Raum und nötige Anschlusspunkte für die Bewehrung bietet.

Insgesamt ergibt sich die Fragestellung, wie das Trag- und Resttragverhalten von Spannglasträgern unter Lasten verschiedener Einwirkungsdauer zu beschreiben ist und welche Konstruktionsweisen sich für einen effektiven Einsatz eignen. Durch eine gezielte Untersuchung soll ermöglicht werden, die Materialeffizienz von Glasträgern zu erhöhen und einen Beitrag zur Steigerung der Akzeptanz und Verbreitung derartiger Konstruktionen zu leisten.

\section{$1.2 \quad$ Zielsetzung}

Glasträger mit schlaffer und vorgespannter Bewehrung sind vielfältig, jedoch bisher unsystematisch untersucht. ${ }^{10}$ Neben den konstruktiven Details und dem Tragkonzept wurde eine Fülle von Materialien miteinander kombiniert. Ein einheitliches Bezeichnungskonzept und eine Systematik sind nicht
Bild 5

Glasträger mit vorgespannter Bewehrung entlang der auf Biegezug beanspruchten Unterkante aus [Bos et al. 2004]. Sowohl Flansch als auch Steg des gewählten T-Querschnitts verjüngen sich von Feldmitte zum Auflager. 
vorhanden. Zunächst wird darauf abgezielt, eine solche Systematik zur Kategorisierung der Glasträgerkonstruktionen zu erstellen. Anhand derer wird der Stand des Wissens zusammengefasst, offene Lücken herausgestellt und der Umfang dieser Arbeit eingegrenzt.

Darüber hinaus beschreibt ein analytischer Ansatz das Tragverhalten von Spannglasträgern. Dies betrifft sowohl das Verhalten unter kurzzeitigen und andauernden Lasten, als auch den nötigen Nachweis der Resttragfähigkeit unter Annahme gebrochener Glasschichten.

Im frühen Spannbetonbau wurde zunächst der vollständige Verlust der Vorspannung festgestellt, welcher in weiteren Untersuchungen auf die zeitabhängigen Verformungen von Beton und Bewehrung zurückgeführt wurde. ${ }^{12}$ Daraus resultierten die heute normativ verankerten Betrachtungen zum Spannkraftverlust. Da zeitabhängige Verformungen auch für Verbund-Sicherheitsglas bekannt sind ${ }^{13}$, ist ein Spannkraftverlust in Spannglasträgern ebenso zu erwarten. Daher besteht ein wesentliches Ziel dieser Arbeit darin, das zeitabhängige Verformungsverhalten des belasteten Systems sowie die Spannkraftverluste zu ergründen und damit eine Entwurfsgrundlage zu liefern.

Das Hauptziel dieser Arbeit ist es, die gefundenen analytischen Ansätze experimentell zu belegen, um die Machbarkeit von Spannglasträgern im Rahmen einer Variantenstudie zu zeigen.

Daraus werden Vorschläge für die konstruktive Umsetzung erarbeitet (Bild 6) und die Grundlage für eine analytische Beschreibung gelegt. Mit dieser Arbeit wird angestrebt, eine rechnerische Abschätzung des Tragverhaltens für eine Vorbemessung zu ermöglichen.

12 Vergleiche unter anderem [Zilch \& Weiher 2008].

13 Vergleiche unter anderen [Sobek et al. 2000], [Schuler 2003], [Ensslen 2005], [Kott 2006] und [Sackmann 2008]. 


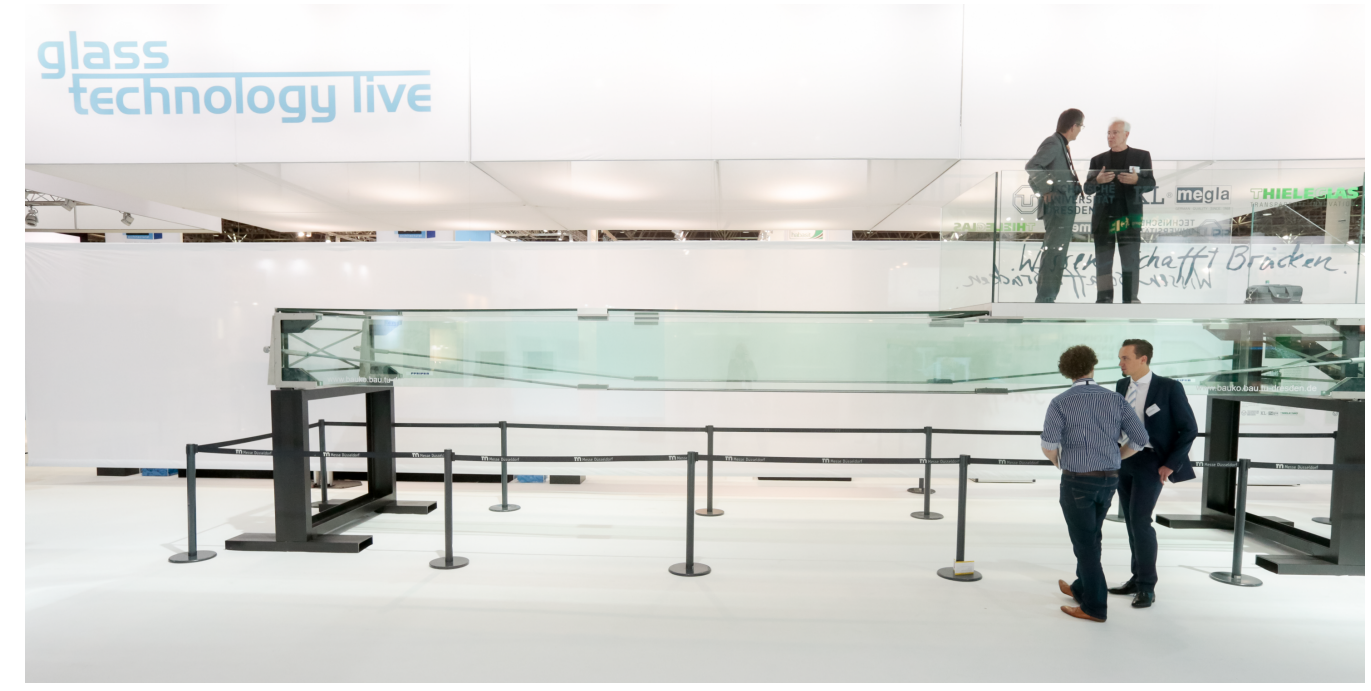

\section{$1.3 \quad$ Vorgehensweise}

Es sind derzeit keine umfassend strukturierten Untersuchungen von Glasträgern mit vorgespannter Bewehrung vorhanden, daher wird der Stand des Wissens aus einer Analogiebetrachtung zur DIN EN 1992 „Eurocode 2: Bemessung und Konstruktion von Stahlbeton- und Spannbetontragwerken“ erschlossen. Dabei werden Konzepte analog auf den Konstruktiven Glasbau übertragen, um den Untersuchungsumfang zu konkretisieren und zu erwartende Ergebnisse, insbesondere analytische Methoden, auszuarbeiten. Gleichzeitig wird darauf aufbauend eine Systematik zur Kategorisierung von Glasträgern erstellt, aus der die Anforderungen an diese Konstruktionen abgeleitet werden.

Hieraus wird ein Arbeitsprogramm entwickelt. Anhand experimenteller Untersuchungen werden die praktische Umsetzbarkeit des Konzeptes "Spannglasträger" gezeigt, Annahmen zur Trag- und Resttragfähigkeit bestätigt sowie die Dauerhaftigkeit überprüft.

Das Arbeitsprogramm beinhaltet experimentelle Studien unter verschiedenen Belastungssituationen und Teilzerstörungszuständen (Bild 7):

\section{Bild 6}

Spannglasträger als Demonstrationsbrücke auf der glasstec 2014. Hier wurde die praktische, konstruktive Umsetzung von Spannglasträgern auf eine Spannweite von $9 \mathrm{~m}$ gezeigt. 
- Vorspannbelastung (kurzzeitig, andauernd)

- Biegebelastung vorgespannter Träger (kurzzeitig, andauernd, wechselnd)

- Temperaturbelastung

Damit wird ein numerisches Modell verifiziert und eine weiterführende Parameterstudie ermöglicht.

Einleitung

Methoden

Ergebnisse

Diskussion

Folgerungen

\section{Bild 7}

Vorgehensweise und Zusammenhang der Kapitel.
Kapitel 1

Wissensgrenzen und resultierende Fragestellung

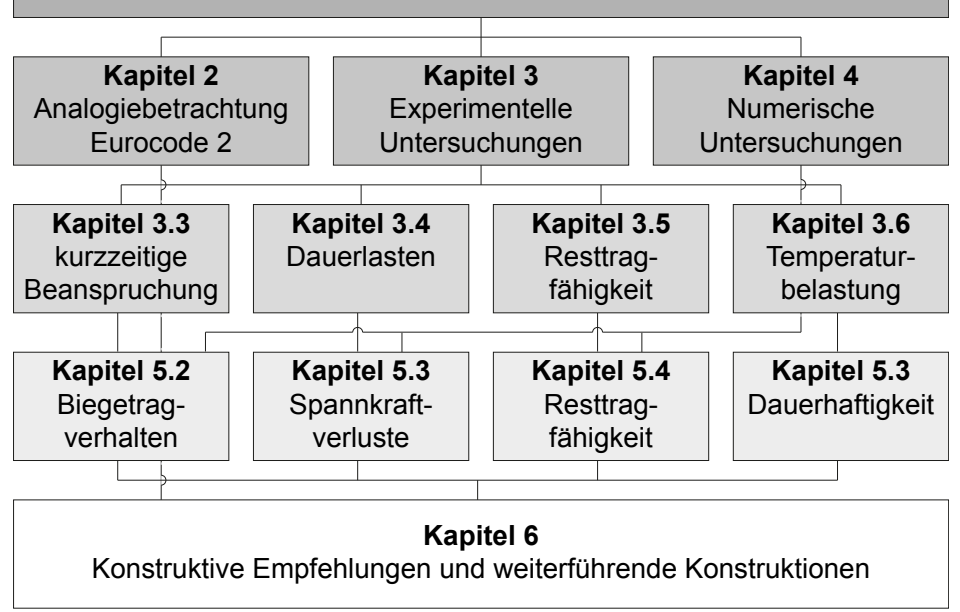

Die Erkenntnisse der Analogiebetrachtung werden den experimentellen und numerischen Ergebnissen gegenübergestellt und diskutiert. Aus den experimentellen Untersuchungen ergeben sich darüber hinaus konstruktive Empfehlungen als Erfahrungssammlung.

\section{$1.4 \quad$ Abgrenzung}

Erstmals werden verbundlos vorgespannte Glasträger umfassend untersucht. Die Ergebnisse dienen als Ausgangsbasis für Konstruktionen im sofortigen oder nachträglichen Verbund, welche jedoch Gegenstand anderer Forschungsaktivitäten bleiben. 
Konstruktive Details wie zum Lastabtrag erforderliche Klebstellen werden nicht vertieft untersucht. Vielmehr werden Anforderungen daran formuliert, die im Rahmen dieser Arbeit beispielhaft, jedoch nicht abschließend, erfüllt wurden.

Die Entwicklung von bewehrten Glasträgern hat gezeigt, dass eine Vielzahl von Parametern wie der Glasaufbau, die Eigenschaften der Zwischenschicht und der Glaskontaktwerkstoffe, Temperatur und Alterung sowie die Konstruktionsweise zu berücksichtigen sind. Die Wirkung der Vorspannung in Interaktion mit diesen Parametern ergibt eine Variantenanzahl, die im Rahmen dieser wissenschaftlichen Arbeit nicht wirtschaftlich experimentell untersucht werden konnte. Daher wird auf eine umfangreiche Geometrievariation zugunsten der verstärkten Nutzung numerischer Methoden verzichtet.

Ein Bemessungskonzept für vorgespannt bewehrte Glasträger bleibt der folgenden Forschung vorbehalten. Dabei müssen weiterführende Problemstellungen wie die Möglichkeit der Austauschbarkeit der vorgespannten Bewehrung oder einzelner Träger nach dem Bruch und der Brandschutz näher erörtert werden. 


\section{$2 \quad$ Analogiebetrachtung}

\section{$2.1 \quad$ Zielsetzung}

Anhand einer Analogiebetrachtung zum Stahlbeton- und Spannbetonbau wird das Themenfeld erschlossen. ${ }^{14}$

Dazu werden die Regelungen der [DIN EN 1992-1-1] analysiert und mit dem Stand des Wissens des konstruktiven Glasbaus verglichen. Es werden Beispiele für bereits umgesetzte Bau- und Forschungsprojekte benannt und nach Konstruktionsarten klassifiziert.

Das Vorgehen des Stahlbeton- und Spannbetonbaus wird analog für den Konstruktiven Glasbau verwendet. Sie bilden die Grundlage für die Entwicklung analytischer Methoden zur Berechnung des Tragverhaltens, insbesondere der Spannkraftverluste in Spannglasträgern. Zudem werden bereits vorab maßgebende Parameter eingegrenzt. Damit öffnet sich das Untersuchungsfeld zum Ableiten notwendiger experimenteller Untersuchungen.

Das folgende Kapitel ist analog der [DIN EN 1992-1-1] gegliedert und bereits um spezifische Themen des Konstruktiven Glasbaus erweitert.

\subsection{Anwendungsbereich}

Der Anwendungsbereich der [DIN EN 1992-1-1] beschränkt sich auf den Entwurf, die Berechnung und die Bemessung von Tragwerken aus Beton, Stahlbeton und Spannbeton und gibt damit ebenso die Grenzen der Analogiebetrachtung vor. Anforderungen an Tragfähigkeit, Gebrauchstauglichkeit und Dauerhaftigkeit nach [DIN EN 1990], einschließlich der Unterscheidung zwischen Prinzipien und Anwendungsregeln, bilden dafür die Basis. Einwirkungen werden für die Bemessung von Glas und Beton gemäß [DIN EN 1991] bestimmt. Beide werden mit Nennung in [DIN 18008] im Konstruktiven

14 Analogien zwischen Beton- und Glasbau werden bereits in [Louter et al. 2005], [Härth 2013] sowie [Weller \& Engelmann 2013] benannt. 
Glasbau übernommen. Auf diese Weise wird das semiprobabilistische Sicherheitskonzept mit einer Begrenzung der Versagenswahrscheinlichkeit zugrunde gelegt. Bild 8 zeigt die Einordnung der [DIN 18008 $]^{15}$ in das Konzept der europäischen Normen.

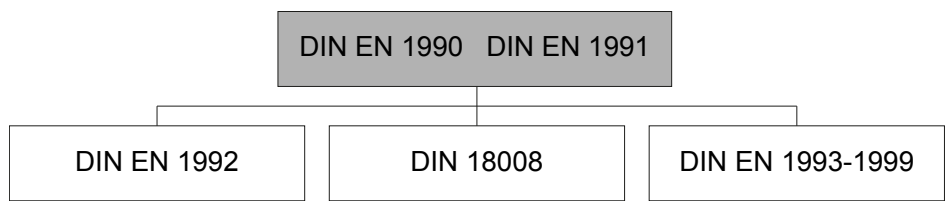

Damit wird eine Bauart normativ erfasst. Zum Nachweis der Tragfähigkeit und Gebrauchstauglichkeit sind zusätzlich Bauprodukte zu benennen. ${ }^{16}$ Dies geschieht entweder auf Basis einer Produktnorm ${ }^{17}$ oder durch zusätzliche Zulassungen ${ }^{18}$.

Weitere Annahmen nach [DIN EN 1992-1-1, Kap. 1.3] sind nicht explizit in [DIN 18008] benannt, können jedoch für den Konstruktiven Glasbau übernommen werden, da es sich um Wiederholungen aus [DIN EN 1990] handelt. Eine Zusammenfassung findet sich in Tafel 1.

Beide Bauarten werden auf analogen Regeln gegründet. Folglich wird das semiprobabilistische Nachweiskonzept der Eurocodes auch auf vorgespannte Glasträger angewendet.

\section{Bild 8}

Einordnung der Glasbaunormung in das Konzept der Eurocodes. Es wird bereits an einem Eurocode for Structural Glass gearbeitet. (Vergleiche [Feldmann et al. 2014]).

15 Anforderungen an den Feuerwiderstand und bauphysikalische Anforderungen (beispielsweise den Wärme- und Schallschutz) sind nicht inbegriffen [DIN EN 1992-1-1, Kap. 1.1] und [DIN 18008-1, Kap. 1].

16 Vergleiche [Musterbauordnung 2002].

17 Für den Stahlbeton- und Spannbetonbau entsprechend der Bauteilkomponenten: Beton nach [DIN EN 206-1] und Bewehrung nach [DIN EN 10080] sowie [DIN EN 10138], letztere unter Vorbehalt ihrer bauaufsichtlichen Einführung [DAfStb 2012, S. 9]. Für den Glasbau: Basisglas nach [DIN EN 572-1] und alle Erzeugnisse, die daraus gefertigt werden, insbesondere thermisch vorgespanntes Einscheiben-Sicherheitsglas nach [DIN EN 12150-1].

18 Im Spannbetonbau in Deutschland ist eine Zulassung von Spannverfahren notwendig [DIN EN 1992-1-1 NA, Kap. 3.4.1 NA.1]. Die Produktnorm [DIN EN 1863] für teilvorgespanntes Glas ist nicht bauaufsichtlich eingeführt [MLTB 2015]. Entsprechend darf TVG in Deutschland nur mit allgemeiner bauaufsichtlicher Zulassung angewendet werden. 


\begin{tabular}{|c|c|}
\hline Stahlbeton- und Spannbetonbau & Konstruktiver Glasbau \\
\hline $\begin{array}{l}\text { Tragwerkplanung durch entsprechend } \\
\text { qualifiziertes Personal. }\end{array}$ & $\begin{array}{l}\text { Kann mit der gleichartigen Nennung in [DIN EN } \\
\text { 1990, Kap. 1.3(2)] übernommen werden. }\end{array}$ \\
\hline $\begin{array}{l}\text { Überwachung und Qualitätskontrolle } \\
\text { in Fabriken, Werken und auf Baustel- } \\
\text { len. }\end{array}$ & $\begin{array}{l}\text { Die Anforderungen an die Produktqualität werden } \\
\text { in Produktnormen, Verwendbarkeits- und Anwend- } \\
\text { barkeitsnachweisen festgelegt. Anforderungen an } \\
\text { die Überwachung auf Baustellen regelt die jeweilige } \\
\text { Bauordnung. }\end{array}$ \\
\hline $\begin{array}{l}\text { Verwendung von Baustoffen entspre- } \\
\text { chend ihrer maßgeblichen Spezifika- } \\
\text { tionen. }\end{array}$ & $\begin{array}{l}\text { In [DIN EN 1990] wird auf die maßgebenden Pro- } \\
\text { duktnormen verwiesen. Dies kann gleichartig für die } \\
\text { einschlägigen Produktnormen für Bauprodukte im } \\
\text { Konstruktiven Glasbau übernommen werden. }\end{array}$ \\
\hline $\begin{array}{l}\text { Angemessene Instandhaltung und } \\
\text { Nutzung entsprechend geplanter } \\
\text { Anforderungen. }\end{array}$ & $\begin{array}{l}\text { Kann mit der gleichartigen Nennung in [DIN EN } \\
\text { 1990, Kap. 1.3(2)] übernommen werden. }\end{array}$ \\
\hline $\begin{array}{l}\text { Erfüllung der Anforderungen an die } \\
\text { Bauausführung nach [DIN EN 13670]. }\end{array}$ & $\begin{array}{l}\text { Eine entsprechende Regelung ist im Konstrukti- } \\
\text { ven Glasbau nicht normativ verankert. Derzeit wird } \\
\text { die Ausführung nach den technischen Regeln des } \\
\text { Glaserhandwerks, Angaben der gültigen bauauf- } \\
\text { sichtlichen Zulassungen und Verglasungsrichtlinien } \\
\text { der Glasveredler geregelt. }^{{ }^{a}}\end{array}$ \\
\hline
\end{tabular}

\section{Tafel 1}

Übersicht zusätzlicher Annahmen zu den allgemeinen Regelungen der [DIN EN 1990] nach [DIN EN 19921-1, Kap. 1.3] im Vergleich mit analogen Regelungen im Konstruktiven Glasbau.

Einzig eine normative Fassung der Anforderungen an die Bauausführung sind noch nicht analog im Glasbau verankert.

\subsection{Begriffe}

Die [DIN EN 1990] wird für alle Bauarten verwendet, sodass ihre Begriffe nicht nur in [DIN EN 1992-1-1, Kap. 1.5.1] übernommen werden, sondern ebenso für den Konstruktiven Glasbau gelten. Ausgesuchte Begriffe bezüglich Bewehrung und Vorspannung werden für eine analoge Klassifikation im Glasbau verwendet und bereits ausgeführte Bauprojekte und Forschungsergebnisse eingruppiert. Damit wird eine Systematik ${ }^{19}$ definiert.

\subsubsection{Bewehrte und hybride Glastragwerke}

Zunächst wird im Stahlbeton- und Spannbetonbau zwischen bewehrten und unbewehrten beziehungsweise gering be-

19 In [Weller \& Engelmann 2013] wurde bereits ein entsprechender Ansatz veröffentlicht. Dieser wird im Weiteren übernommen und fortentwickelt. 
wehrten Bauteilen anhand der Mindestbewehrung unterschieden, welche folgende Aufgaben erfüllt:

- Vermeidung unangekündigten Versagens,

- Vermeidung breiter Risse und

- Aufnahme von Zwangsschnittgrößen.

Zur Querschnittsbemessung wird eine Gleichgewichtsbetrachtung am freigeschnittenen Querschnitt durchgeführt. Im unbewehrten Fall (Bild 9) werden die aus der Verzerrungsannahme resultierenden Spannungen über die Querschnittshöhe getrennt nach Zug- und Druckbeanspruchung zu einer resultierenden Last $\left(F_{G 1}\right.$ und $\left.F_{G 2}\right)$ zusammengefasst. Diese müssen mit den zugehörigen Einwirkungen $\left(\mathrm{M}_{E d}\right.$ und $\left.\mathrm{N}_{E d}\right)$ im Gleichgewicht stehen.

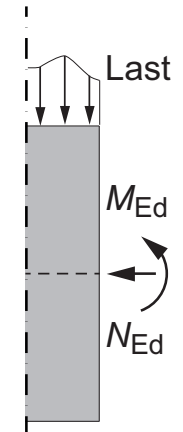

I
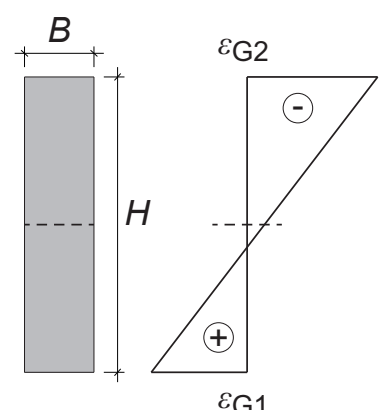

$\varepsilon_{\mathrm{G} 1}$

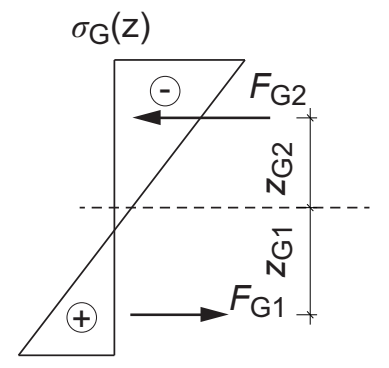

$\sigma_{\mathrm{G} 1}<f_{\mathrm{G}}$
Bild 9

Spannungs- und Verzerrungsverteilung im unbewehrten, ungerissenen Querschnitt.

Für den Fall eines bewehrten Querschnitts (Bild 10) gilt es, die Rechnung um das Bewehrungsmaterial zu erweitern, sodass sich ein zusätzlicher Summand $\left(F_{S 1}\right)$ in der Gleichgewichtsbetrachtung ergibt. Die zugrunde liegende Mechanik ist baustoffunabhängig und wurde bereits erfolgreich im Konstruktiven Glasbau angewendet. ${ }^{14}$ Damit wird das Vorgehen für Berechnungen an bewehrten Referenzträgern dieser Arbeit übernommen.

Neben der Benennung als „bewehrtes“ Bauteil ist es im Glasbau ebenso üblich von „hybriden“ Bauteilen zu sprechen. Dabei werden keine konkreten Definitionen genannt, die eine Entscheidung zwischen beiden Begriffen ermöglichen. Mit beiderlei Bezeichnungen werden Bauteile des Konstruktiven Glasbaus beschrieben, deren Grundmaterial Glas und 
Bild 10

Spannungs- und Verzer-

rungsverteilung im bewehrten Bauteil (exemplarisch).

Ein nicht vorgespanntes Zugglied wird als "schlaffe Bewehrung" bezeichnet.
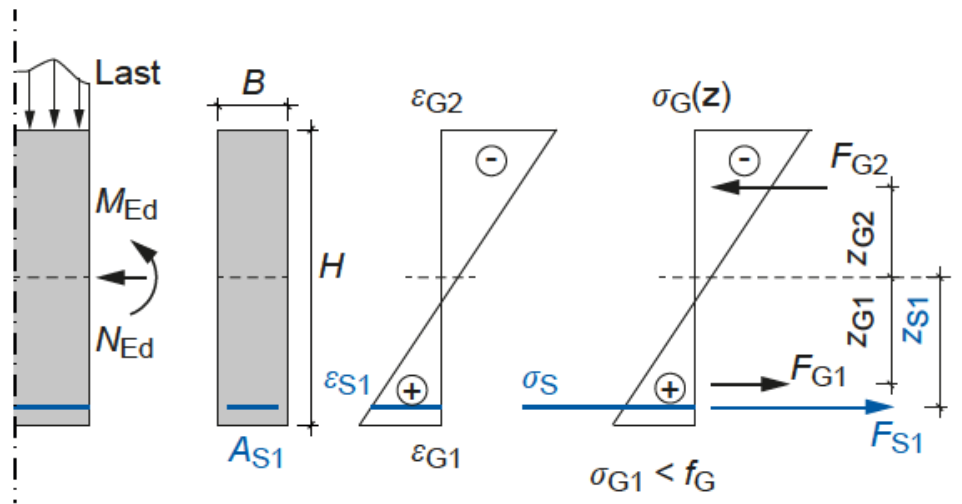

Verbundglas ${ }^{20}$ mit weiteren Materialien kombiniert wird, um günstigere Trageigenschaften im Vergleich zum reinen Grundmaterial zu ermöglichen. Einerseits wird das Zwischenschichtmaterial variiert (Tafel 2), andererseits wird das bestehende Verbund-Sicherheitsglas um weitere konstruktive Bestandteile, die Bewehrung, erweitert.

\begin{tabular}{llll}
\hline & [Feirabend 2010] & [Weimar 2011] & [Härth 2013] \\
\hline $\begin{array}{l}\text { Glas oder Vergla- } \\
\text { sungsmaterial aus } \\
\text { Kunststoff }\end{array}$ & Glas & $\begin{array}{l}\text { Glas oder Kunststoff } \\
\text { (Polycarbonat) }\end{array}$ & $\begin{array}{l}\text { Glas oder Kunststoff } \\
\text { (PMMA, Polyamid } \\
\text { und Polycarbonat) }\end{array}$ \\
\hline Zwischenschicht & $\begin{array}{l}\text { PVB oder SGP mit } \\
\text { Edelstahlgewebe } \\
\text { oder Edelstahl- } \\
\text { Lochblech }\end{array}$ & Polyurethan & $\begin{array}{l}\text { Klebstoffe (Delo } \\
\text { PB® 437 und 4442, } \\
\text { Photowell® 1012) }\end{array}$ \\
\hline
\end{tabular}

Tafel 2

Übersicht alternativer Verbundglasaufbauten.
Unter hybriden Bauteilen versteht man im technischen Sinne eine Zusammensetzung von Einzelteilen, welche für sich genommen bereits eine eigenständig funktionierende Lösung darstellen, zu einem Gesamten, um verbesserte Eigenschaften zu generieren. ${ }^{21}$ Damit kann Verbundglas und VerbundSicherheitsglas bereits als hybrides Bauteil aus Glas und Zwischenschicht bezeichnet werden. Monolithisches Glas wird beispielsweise als Absturzsicherung eingesetzt. Eine Veredelung zu Verbund-Sicherheitsglas ermöglicht darüber hinaus die Splitterbindung im Bruchfall als neuartige

20 Vergleiche [DIN EN ISO 12543-1].

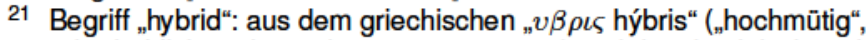
„überheblich“, „übersteigert“, „vermessen“) und dem lateinischen „hybrida“" ("gemischt“, „von zweierlei Herkunft“, „Mischling“, auch „Kreuzung“). [Fremdwörterbuch 2006] 
Sicherheitseigenschaft, die im Anwendungsbereich der [DIN 18008] zudem verpflichtend gefordert ist.

Unter „bewehrt“ im wörtlichen Sinne wird eine Verbesserung oder Verstärkung verstanden. Bezieht man dies auf hybride Bauteile, so kann man unter einem bewehrten Bauteil auch ein hybrides Bauteil verstehen, dessen Eigenschaftsänderungen sich als günstig erwiesen haben, sodass es zu einer deutlichen Verbesserung einer Eigenschaft gekommen ist. Damit stellt sich der Begriff „hybrid“ als Oberbegriff dar und charakterisiert das Auftreten als „Mischform“. Fehlt (zunächst) der umfassende Nachweis günstiger Eigenschaften, ist von einem „hybriden“ Ansatz zu sprechen. Wird eine Eigenschaftsverbesserung, wie beispielsweise eine verbesserte Resttragfähigkeit oder größere Traglasten, nachgewiesen, so ist der Begriff „bewehrt“ zutreffender.

Ein günstiger Effekt konnte sowohl bei der Entwicklung des theoretischen, mechanischen Ansatzes, welcher in Analogie zum Stahlbeton- und Spannbetonbau entwickelt wird (Kapitel 2), als auch bei Entwurf und Prüfung experimentell nachgewiesen werden (Kapitel 3). Dies rechtfertigt die Eingruppierung von Spannglasträgern als bewehrtes Bauteil aus dem großen Pool hybrider Glasbauteile.

\subsubsection{Thermische und mechanische Vorspannung}

Glas wird thermisch ${ }^{22}$ vorgespannt. Dem gegenüber werden unter "Vorspannung“ im Stahlbeton- und Spannbetonbau „allgemein alle dauerhaften Auswirkungen des Vorspannvorgangs“ 23 verstanden. Dabei wird mit „Vorspannen“ ein Verfahren bezeichnet, „bei dem Kräfte in ein Bauteil durch das Spannen von Zuggliedern eingebracht werden“ ${ }^{23}$. Zur Unterscheidung wird dafür im Konstruktiven Glasbau der Oberbegriff "mechanische Vorspannung“ eingeführt und stellt den wesentlichen neuen Parameter für einen Spannglasträger dieser Arbeit dar.

22 Chemisch vorgespanntes Glas wird üblicherweise nicht für Glasträgerkonstruktionen verwendet.

23 Vergleiche [DIN EN 1992-1-1, Kap. 1.5.2.4]. 
Bild 11

Spannung (schematisch) im Glasquerschnitt unter Plattenbiegung aus thermischer und mechanischer Vorspannung sowie äußerer Biegebelastung.

Die Gesamtspannung an der Unterkante zeigt, dass die äußere Belastung allein die Glasfestigkeit $\mathrm{f}_{\mathrm{G}}$ erreicht. Erst durch die thermische und mechanische Vorspannung wird insgesamt ein Nachweis möglich.

Bild 12

Spannungs- und Verzerrungsverteilung im vorgespannt bewehrten Bauteil.

Je nach Konstruktionsart muss die Vordehnung infolge der Vorspannung berücksichtigt werden.

Weitere Summanden aus schlaffer Bewehrung können hinzugefügt werden.
Durch eine äußere Belastung müssen sowohl die thermische als auch die mechanische Vorspannung überwunden werden (Dekompression), bevor die Glasoberfläche eine Zugbeanspruchung erfährt und es bei Überschreitung der Beanspruchbarkeit zum Glasbruch kommen kann (Bild 11).

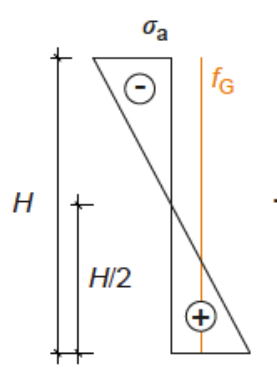

$$
\begin{aligned}
& \text { äußere } \\
& \text { Biegebelastung }
\end{aligned}
$$

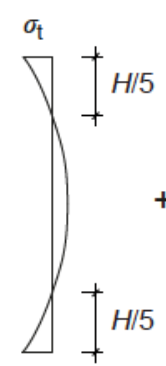

hermische

mechanische Vorspannung

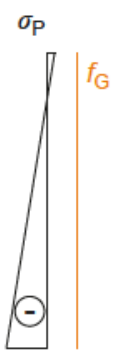

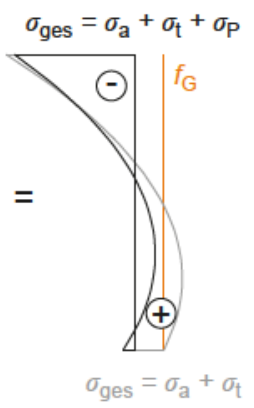

Gesamtspannung
Die Vorspannung wird als Vordehnung beim Aufstellen des Gleichgewichts im Querschnitt berücksichtigt. In Erweiterung der Prinzipien aus Bild 9 sind die Anteile $\left(F_{p i}\right)$ zu berücksichtigen (Bild 12).
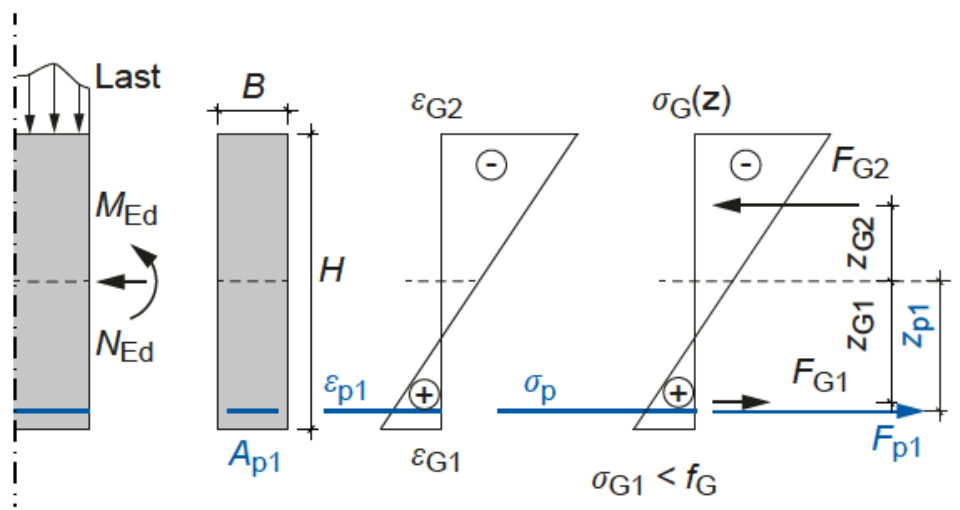

Im Weiteren wird eine Unterscheidung nach der Konstruktion und dem Spannverfahren getroffen. Gleichzeitig werden konstruktive und verfahrenstechnische Anforderungen geklärt. 


\subsubsection{Spanngliedkonstruktion und Spannverfahren}

Es ist eine Lastübertragung zwischen Bewehrung und Grundmaterial nötig, um einen gemeinsamen Lastabtrag zu gewährleisten. Dabei wird im Spannbetonbau nach der Spanngliedkonstruktion und dem Spannverfahren unterschieden:

- Lage der vorgespannten Bewehrung zum Grundquerschnitt (interner und externer Vorspannung).

- Art des Verbundes zwischen Bewehrung und Grundmaterial (Spannglied im sofortigen Verbund, im nachträglichen Verbund und ohne Verbund).

- geometrische Ausdehnung der Bewehrung (lokale, lineare und flächige Bewehrung).

\section{Interne und externe Vorspannung}

Das Spannglied kann zum einen vollständig innerhalb, zum anderen außerhalb der Querschnittsumhüllenden liegen. Im Spannbetonbau wird dazu die interne und die externe ${ }^{24}$ Spanngliedlage unterschieden. ${ }^{25}$

Konstruktionsbeispiele intern und extern geführter Spannglieder im Konstruktiven Glasbau finden sich in Bild 13.

Eine kraftübertragende Verbindung zwischen Spannglied und Grundmaterial ist nur in jenen Bereichen möglich, in denen das Spannglied nahe genug am Grundquerschnitt (intern) liegt. Ist dies nicht der Fall, liegt eine externe Vorspannung vor. Im Gegensatz zu unterspannten Tragwerken (Bild 14) wird die Vorspannkraft nicht in die Unterkonstruktion, sondern in den Hauptquerschnitt übertragen. Am Beispiel des Bild 13 wird deutlich, dass für die externe Spanngliedführung Umlenkkonstruktionen nötig werden, welche deutlich hervortreten und die Transparenz reduzieren. Die-

24 Vergleiche [DIN EN 1992-1-1, Kap. 3.4.2.1]. Zudem ist bei extern vorgespannter Bewehrung zu prüfen, ob diese gegen außergewöhnliche Einwirkungen ausreichend geschützt ist. In diesem Zusammenhang ist im Spannbetonbau der Teilsicherheitsbeiwert zu überdenken. Siehe auch [Fingerloos et al. 2012, S. 210].

25 Vergleiche [DIN EN 1992-1-1, Kap. 1.5.2.3]. 
Bild 13

Derzeit sind mehrere Konstruktionsoptionen von

Glasträgern mit intern und extern geführten Spanngliedern bekannt. Louter [2013] zeigt parallel verlaufende Spannglieder im oberen Bild. Das untere Bild zeigt eine Konstruktionsstudie aus [Firmo et al. 2015] mit extern geführten Spanngliedern.

Darüber hinaus können die Fallbeispiele in Bild 15 sowie Bild 22 ebenso dem Fall interner Spanngliedführung zugeordnet werden.
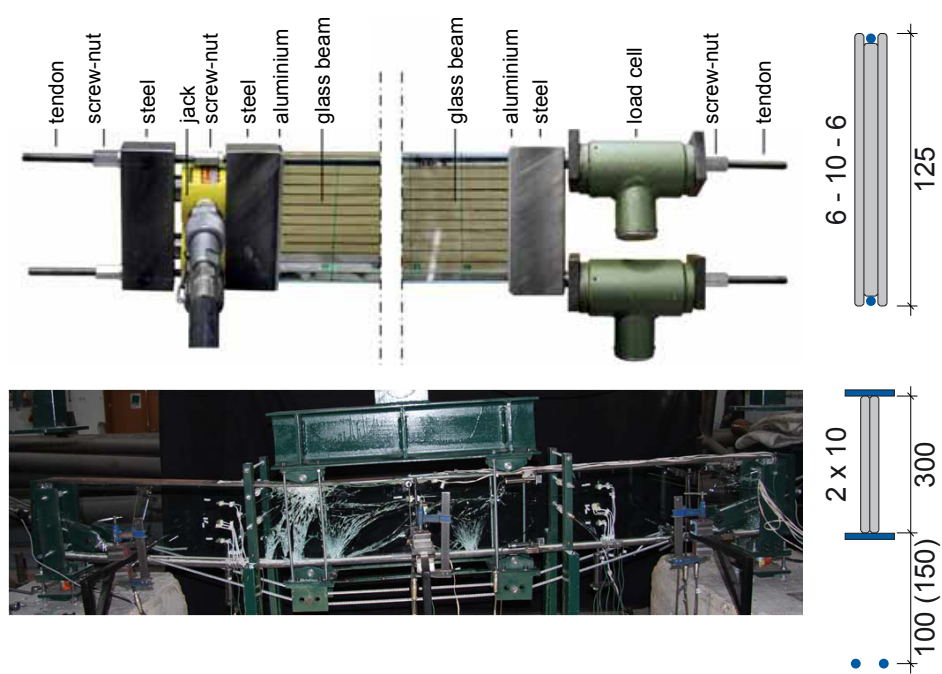

sem intransparenten Gesamteindruck wird durch eine ausschließlich interne Führung der Spannglieder dieser Arbeit entgegengewirkt.

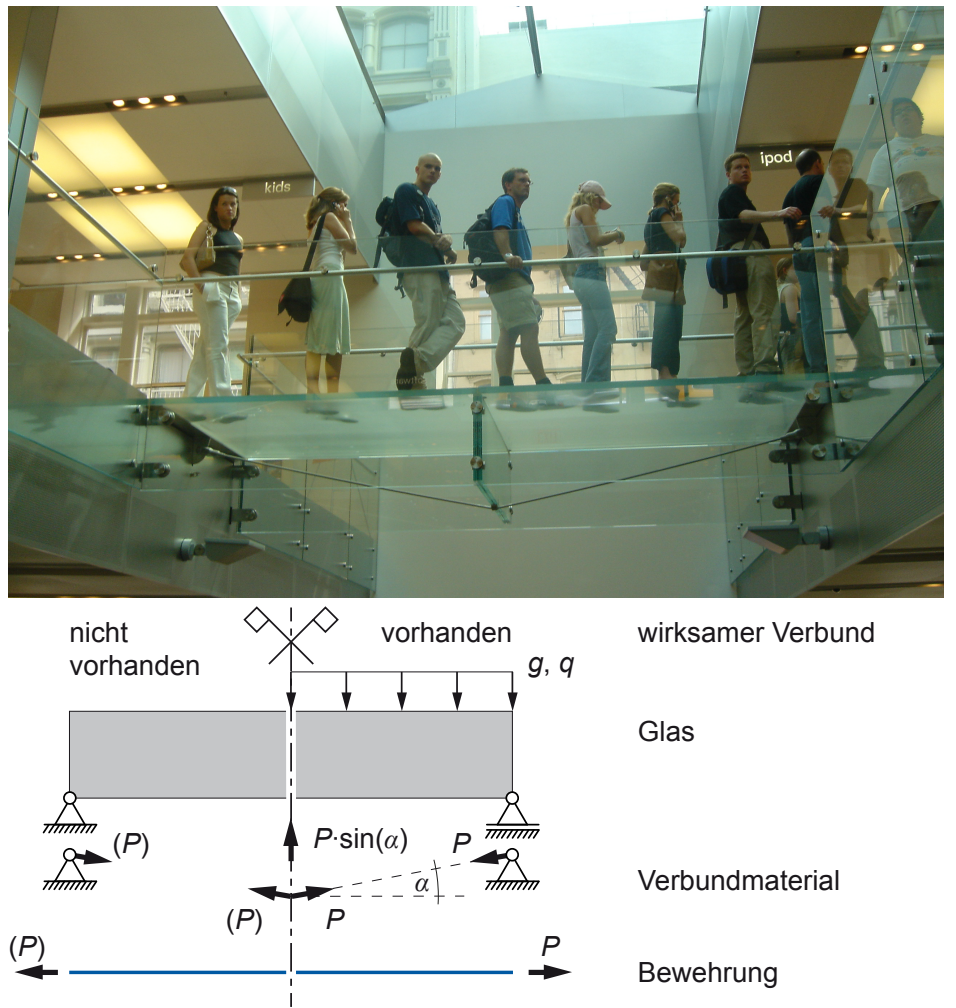




\section{Verbund zwischen Bewehrung und Grundmaterial}

Bei Spannbetonbauteilen mit „sofortigem Verbund“ steht ein wirksamer Verbund bereits mit dem Erhärten des Betons zur Verfügung. ${ }^{26}$ Dabei werden vorgespannte Spannglieder unmittelbar in den frischen Beton (Spannbett) einbetoniert. Ein Lastübertrag wird ausschließlich über die Länge des Spanngliedes erreicht. Eine Verankerungskonstruktion ist nicht vorhanden.

Im Glasbau ist ein derartiger sofortiger Verbund nicht möglich, da Glas nur bei hohen, bewehrungsschädlichen Temperaturen ${ }^{27}$ erweicht. Um einen gleichartigen Lastabtragsmechanismus ohne Verankerungen zu erreichen, muss eine stoffschlüssige Verbindung mittels Klebung zwischen Bewehrung und Glas hergestellt werden. ${ }^{28}$

Cupac \& Louter [2015] stellen einen vorgespannten Glasträger mit sofortigem Verbund vor (Bild 15).

Das Bewehrungsprofil wurde gegen ein steifes Stahlprofil vorgespannt. Darauf wurde ein zweikomponentiger Methacrylat-Klebstoff (Huntsman Araldite® 2047-1) aufgebracht, ein Glasträger aus $24 \mathrm{~mm}$ Verbund-Sicherheitsglas aus Floatglas mit lonomer-Zwischenschicht aufgestellt und binnen 25 Minuten ausgehärtet.

Nach dem Erreichen einer ausreichenden Klebstofffestigkeit, wurde die Bewehrung von der Vorspannkonstruktion entkoppelt, sodass die Vorspannkraft nunmehr nur über die Klebung auf den Glasträger abgetragen wurde. Derzeit liegen jedoch noch keine weiteren Ergebnisse zur Leistungsfähigkeit dieser Konstruktionsart vor. Insbesondere die Dauer-

26 Vergleiche [DIN EN 1992-1-1 NA, Kap. NA 1.5.2.12].

27 Floatglas wird bei circa $1000{ }^{\circ} \mathrm{C}$ hergestellt. Bei diesen Temperaturen verändern sich die Eigenschaften des Gefüges von Stählen signifikant. Insbesondere Spannstähle verlieren ihre durch Kaltumformung gewonnenen Eigenschaften (Spannungsarmglühen) [DIN EN 10052] und [DIN 17022]. Um diese Option dennoch zu ermöglichen, ist eine neue Materialkombination vorzusehen.

28 Linear verklebte Bewehrungen im Glasbau finden sich unter anderem in [Bos et al. 2004], [Bucak et al. 2009b], [Bucak et al. 2009a], [Weller et al. 2009b], [Weller et al. 2010c], [Weller et al. 2010b] und [Louter 2011]. 
Bild 15

Anwendungsbeispiele:

Spannbetonplatte mit Bewehrung im sofortigen Verbund (links) und ein analoger Glasträger nach [Cupac \& Louter 2015] (rechts).

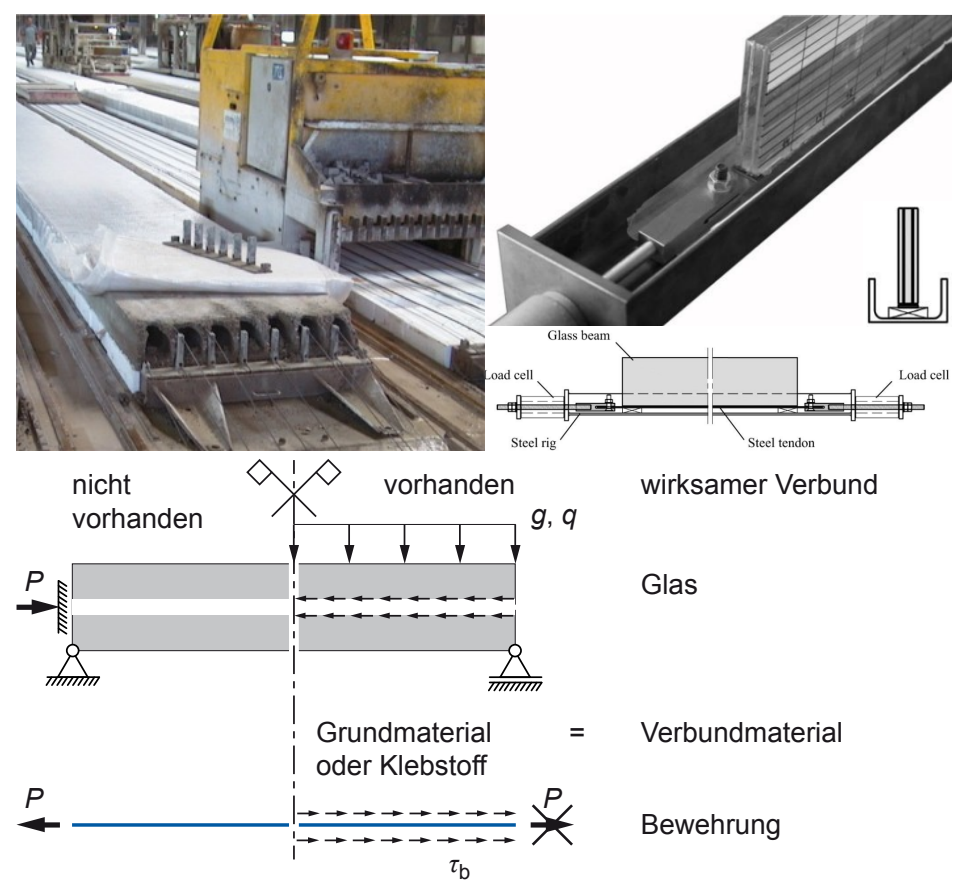

belastung der Klebstoffe infolge der Vorspannung muss als besondere Herausforderung noch untersucht werden.

Des Weiteren kann eine punktuelle Kraftübertragung zwischen Grundmaterial und Bewehrung über Ankerkörper und Umlenkelemente „ohne Verbund“ hergestellt werden. ${ }^{29}$ So wird nach Bild 16 erstmals ein parabolischer Glasträger mit T-Querschnitt mit einem Spannstahlkabel vorgespannt, welches durch ein in der zurückgesetzten Glaskante des Dreifach-Verbund-Sicherheitsglases geklebtes Hohlprofil geführt und an den Trägerenden mit Neoprenkissen und einer stählernen Kopfplatte verankert wird. Aus Bild 16 wird zudem deutlich, dass sich das freiliegende Spannglied mangels Verbund gegenüber dem Glas ungehindert verschieben kann. Der Dehnungszuwachs im Spannglied am höchst belasteten Querschnitt entspricht damit nicht der Dehnung am Grundquerschnitt an gleicher Stelle. Vielmehr ergibt sich die Dehnung der Bewehrung aus der Verzerrung des Glasquerschnitts entlang des Spanngliedes zwischen zwei Ver-

29 Vergleiche [DIN EN 1992-1-1, Kap. NA 1.5.2.15] und [DIN EN 1992-1-1, Kap. 1.5.2.3] sowie [DIN EN 1992-1-1, Kap. 3.4.2.1]. 
ankerungspunkten. Diese Verträglichkeitsbedingung wird mit Gleichung 1 beschrieben.

$$
\begin{array}{ll}
\varepsilon_{p}=\varepsilon_{p, 0}+\Delta \varepsilon_{p}=\sum_{i} \frac{1}{L_{p, i}} \cdot \int_{L_{p, i}} \varepsilon_{G, p}\left(x_{p}, F\right) \mathrm{d} x_{p} \\
\varepsilon_{p} & \text { Dehnung des Spanngliedes } \\
\varepsilon_{p, 0} & \text { Dehnung des Spanngliedes infolge } \\
& \text { initialer Vorspannung } \\
\Delta \varepsilon_{p} & \text { Dehnungsänderung im Spannglied } \\
L_{p, i} & \text { Länge des Spanngliedes zwischen den } \\
\varepsilon_{G, p} & \text { Verankerungspunkten i } \\
& \text { Dehnung des Glases in Höhe des } \\
& \text { Spanngliedes infolge äußerer (Biege-) } \\
& \text { Lasten }
\end{array}
$$
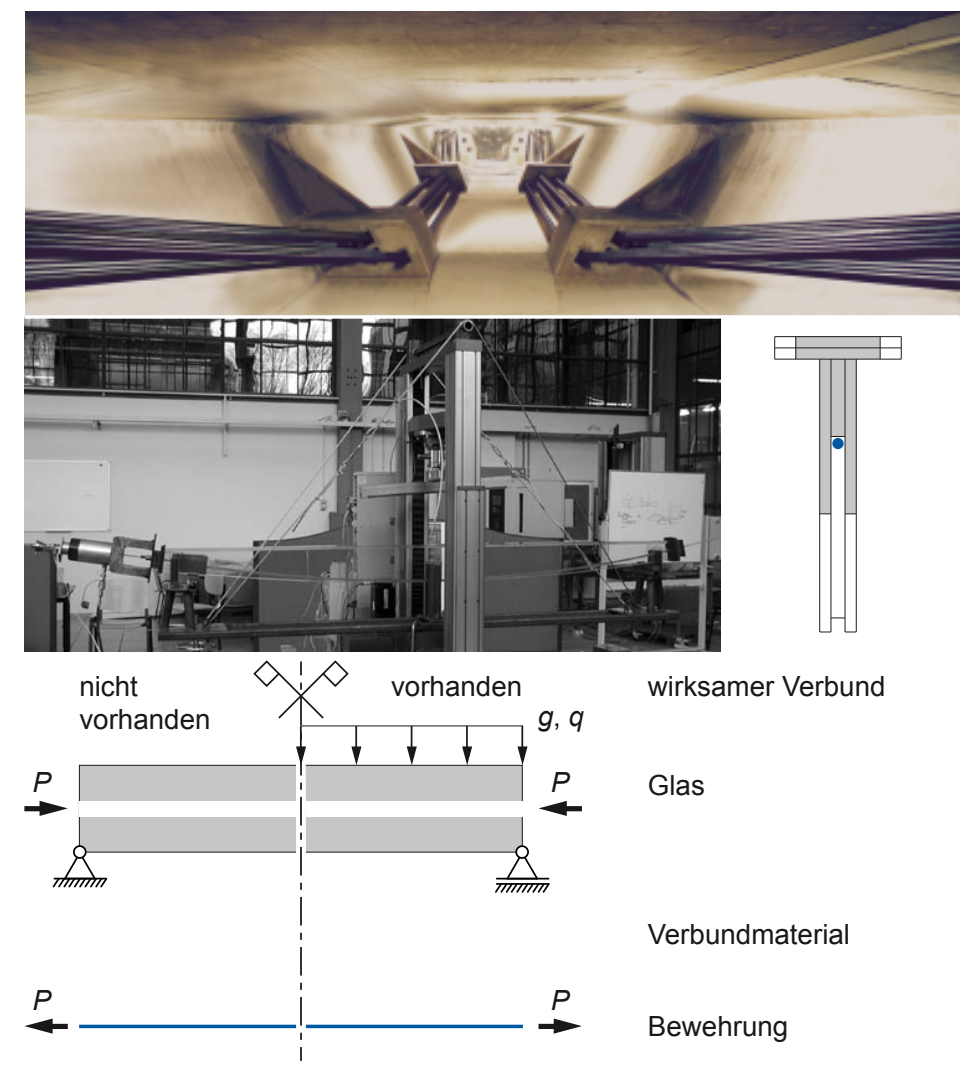

Alternativ wird ein Spannglied im Hüllrohr gegen den bereits erhärteten Beton „nachträglich vorgespannt“ (Bild 17). Dabei wird die Vorspannkraft zunächst unmittelbar punktuell über Ankerkörper in das Grundmaterial eingetragen. Nach dem Verpressen des Hüllrohrs mit einem (betonartigen) Verguß-
Gleichung 1

\section{Bild 16}

Anwendungsbeispiele: Spannbetonträger aus [Abel \& Krautwald 2001] mit Bewehrung ohne Verbund: die Spannglieder liegen innerhalb des Hohlkastquerschnitts frei zwischen den Umlenksätteln (oben).

Glasträger mit vorgespannter Bewehrung aus [Bos et al. 2004] (mittig). Ausschließlich an den Trägerenden und den Umlenkstellen wird wirksam Last übertragen (siehe Kapitel 2.3.4). 
Bild 17

Anwendungsbeispiele: Spannbetonträger mit Bewehrung im nachträglichen Verbund (oben). Die Vorspannung wird zunächst über die Ankerkörper aufgebracht. Anschließend wird der Raum zwischen dem Spannglied und dem Hüllrohr verpresst, um einen wirksamen Verbund zu erstellen.

Ein analog konstruierter Glasträger ist nicht bekannt. mörtel entsteht mit dessen Erhärtung ein tragfähiger Längsverbund. ${ }^{30}$

Es ist zunächst eine Verzerrung des Grundquerschnitts nötig, um weitere Biegelasten über das Verbundmaterial längs der Bewehrung zu übertragen. Eine Dauerbelastung des Verbundstoffes infolge der Vorspannung ist nicht vorhanden und kann damit als vorteilhaft für eine analoge Anwendung im Konstruktiven Glasbau beurteilt werden. Eine derartige Glaskonstruktion ist derzeit nicht bekannt. Sie ist eine Weiterentwicklung des verbundlos vorgespannten Trägers dieser Arbeit um eine stoffschlüssige Verbindung zur unmittelbaren Rissüberbrückung.

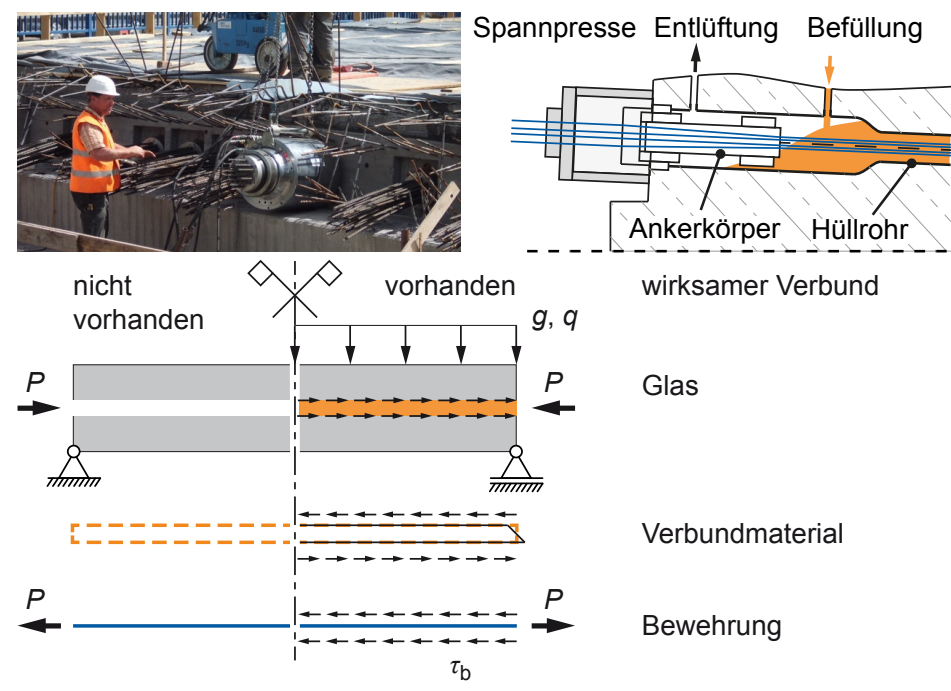

Die obigen Definitionen sind vom verwendeten Grundmaterial unabhängig und können daher analog übernommen werden (Bild 18). Im Konstruktiven Glasbau kommen überwiegend verbundlose Varianten vor, da für die Herstellung eines dauerhaften, linearen Verbundes keine ausreichenden Erfahrungen vorliegen. Die Thematik befindet sich weiterhin im Forschungsstadium. ${ }^{31}$

30 Vergleiche [DIN EN 1992-1-1, NA 1.5.2.13].

31 Vergleiche [Bos et al. 2004], [Louter et al. 2006], [Louter \& Veer 2007b], [Louter 2011], [Louter et al. 2013b], [Louter et al. 2013a], [Weller \& Engelmann 2013], [Weller \& Engelmann 2014a], [Weller \& Engelmann 2014b] und [Engelmann \& Weller 2016]. 


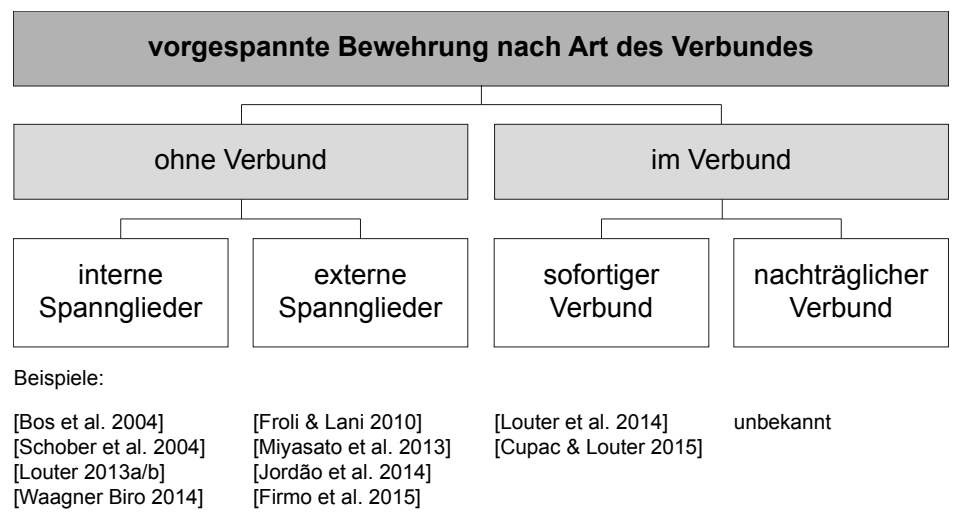

\section{Geometrische Ausdehnung der Bewehrung}

In den oben beschriebenen Fällen „im Verbund“ wird eine lineare Verbindung zwischen Grund- und Bewehrungsmaterial ausgeführt. Vorteilhaft zeigte sich hier, dass über die kontinuierliche Klebung ein Lastübertrag auch unmittelbar zwischen den Rissflanken zweier Glasbruchstücke möglich war. Es wirkt sich günstig auf den Nachweis der Resttragfähigkeit aus und ermöglicht eine höhere Redundanzstufe. ${ }^{32}$

Eine flächige Bewehrung in Anlehnung an mit Matten bewehrte Stahlbetonplatten oder Spannbetonbrücken ist auch im Glasbau denkbar. Die Bewehrung wird auf der Oberfläche auf- beziehungsweise im Zwischenschichtmaterial eingebracht. ${ }^{33}$ Dieses Konzept wird als bewehrtes VerbundSicherheitsglas beschrieben (Bild 19), da eine Verstärkung des Zwischenschichtmaterials (PVB und SGP) durch Gewebe und Lochbleche aus Edelstahl erfolgt, wodurch der gesamte Laminatquerschnitt günstigere (Rest-) Trageigenschaften erreicht. In der Arbeit von Steffen Feirabend zum bewehrten Verbund-Sicherheitsglas ${ }^{34}$ wird ein mechanisches Modell in Analogie zum Stahlbeton- und Spannbetonbau unter Annahme von teilweise auf Zugbeanspruchung ausgefallenen Querschnittsteilen vorgestellt.

32 Vergleiche unter anderem [Bucak et al. 2009b], [Ølgaard et al. 2009], [Correia et al. 2011], [Louter 2011], [Weller et al. 2010c].

33 Einzig Drahtglas nach [DIN EN 572-3] besitzt eine Bewehrungslage im Glasgefüge, um im Bruchfall eine Lastübertragung über die Drahteinlage zwischen den Rissflanken zu erreichen. Das eingelegte Gewebe schädigt das Grundmaterial jedoch derart, dass eine verringerte Festigkeit angesetzt werden muss. Vergleiche auch [Wagner 2002].

34 Vergleiche [Feirabend 2010]. 
Bild 19

Flächige Bewehrung im Stahlbeton- und Spannbetonbau (links) und im Konstruktiven Glasbau nach [Feirabend 2010] (rechts): im Vergleich zum Referenzkörper vorn, trägt die bewehrte Variante hinten mit vollständig gebrochenem Glas weiterhin Lasten ab.

\section{Bild 20}

Lokale, flächige Bewehrung im Stahlbeton- und Spannbetonbau als zusätzliche Durchstanzbewehrung in einem Halbfertigteil aus [Betonbau 11/2015] (links) und im Konstruktiven Glasbau nach [Neugebauer 2005] (rechts)

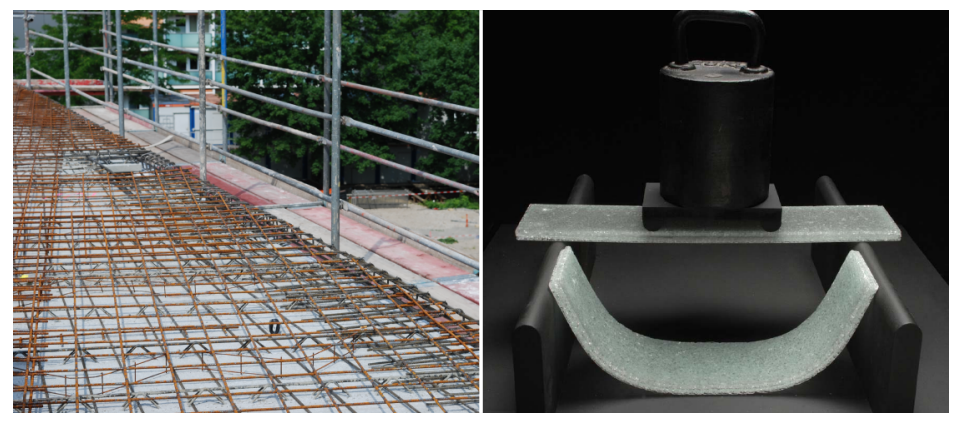

Des Weiteren ist eine flächige Bewehrung zur lokalen Verstärkung hochbeanspruchter Tragwerksteile sinnvoll. Dieses Prinzip ist unabhängig vom Grundmaterial und kann daher im Konstruktiven Glasbau übernommen werden. Insbesondere weil lokale Spannungsspitzen im Glasbau zu vermeiden $\operatorname{sind}^{35}$, kann eine lokale, flächige Bewehrung eine sinnvolle Konstruktionsvariante sein. Darüber hinaus tendieren Verglasungen im Bruchfall dazu, vom Auflager zu rutschen. Daher schlägt Neugebauer [2005] beispielsweise eine lokale Verstärkung vor, um die Resttragfähigkeit günstig zu beeinflussen (Bild 20, rechts).
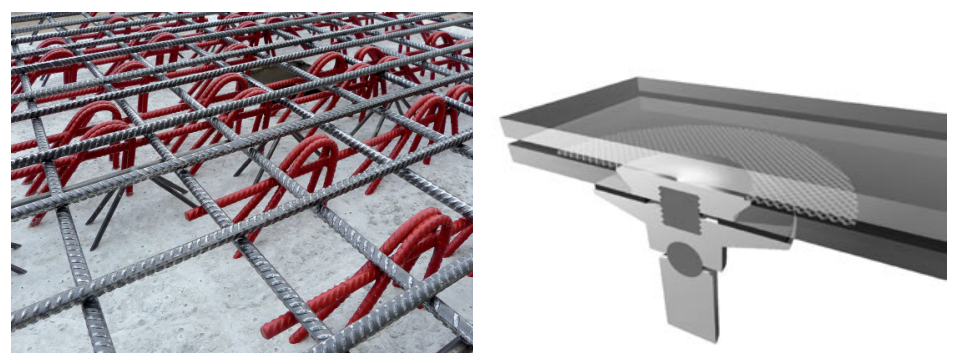

Damit lassen sich Spanngliedkonstruktionen und Spannverfahren für den Konstruktiven Glasbau nach Bild 21 schematisieren.

Bezüglich der Verbundgeometrie wurden bisher ausschließlich lineare Spannglieder in Glasträgern verwendet und zugehörige Ausführungsbeispiele benannt. Eine punktuelle (lokale) oder flächige vorgespannte Bewehrung ist derzeit unbekannt. Hier gilt es noch zu prüfen, inwiefern eine vergleichbare Vorspannung von Verbundglas möglich und sinnvoll ist.

35 Vergleiche [DIN 18008-1, Kap. 10]. 


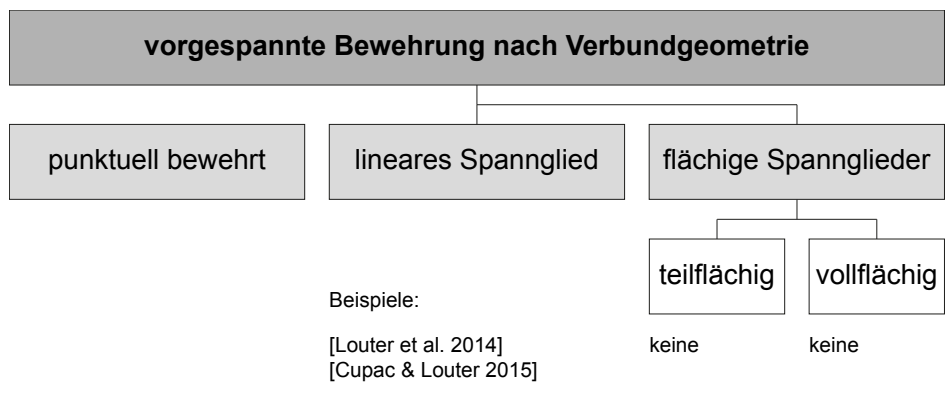

\subsubsection{Lage und Verlauf des Spanngliedes}

Ein Spannglied kann zentrisch oder exzentrisch geführt sein. Bei einer zentrischen Vorspannung fallen die Schwerpunkte der Bewehrung und des Grundquerschnitts zusammen, sodass der Grundquerschnitt ausschließlich durch eine axiale Normalkraft beansprucht wird. Im Beispiel der zentrisch vorgespannten Glasröhren im Tower Place London (Bild 22) liegt das Spannseil im Mittelpunkt eines Kreisringquerschnitts.

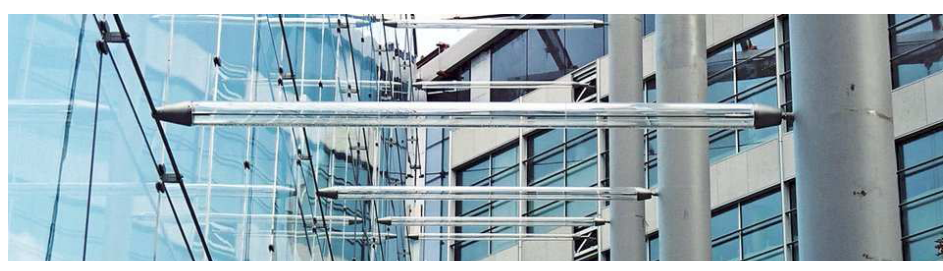

zentrische Vorspannung

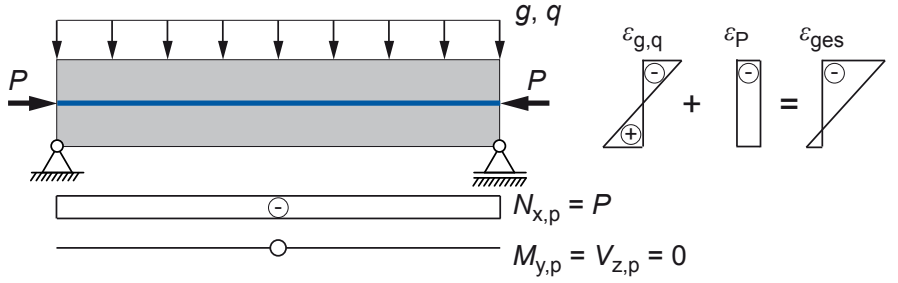

Bild 21

Vorgespannt bewehrte Glaskonstruktionen nach Verbundgeometrie.

Punktuell oder flächig vorgespannte Glaskonstruktionen sind nicht bekannt.

\section{Bild 22}

Die zentrisch vorgespannten Glasstützen der Seilnetzfassade im Tower Place, London (UK) werden in [Wagner 2002], [Kaltenbach 2003] sowie [Sobek et al. 2006] beschrieben.

In [Hightec Glas 2014] werden zentrisch vorgespannte Glasrohre als vertikale Stützen verwendet.

Alternativ können auch mehrere Spannglieder eine zentrische Vorspannung verursachen, wenn deren resultierende Vorspannkraft ebenso durch den Schwerpunkt des Querschnitts verläuft (Bild 23). ${ }^{36}$ Diese Bewehrungsvarianten eignen sich für Glasträger, welche durch eine Wechselbelastung, wie Winddruck und Windsog in Fassadenflächen, belastet werden.

36 Vergleiche [Weller \& Engelmann 2014b]. 
Bild 23

Zentrische Vorspannung durch zwei Bewehrungsseile nach [Weller \& Engelmann 2014b]. Werden beide Seile auf dieselbe Kraft vorgespannt, so verläuft die resultierende Kraft im Schwerpunkt des gläsernen Grundquerschnitts.

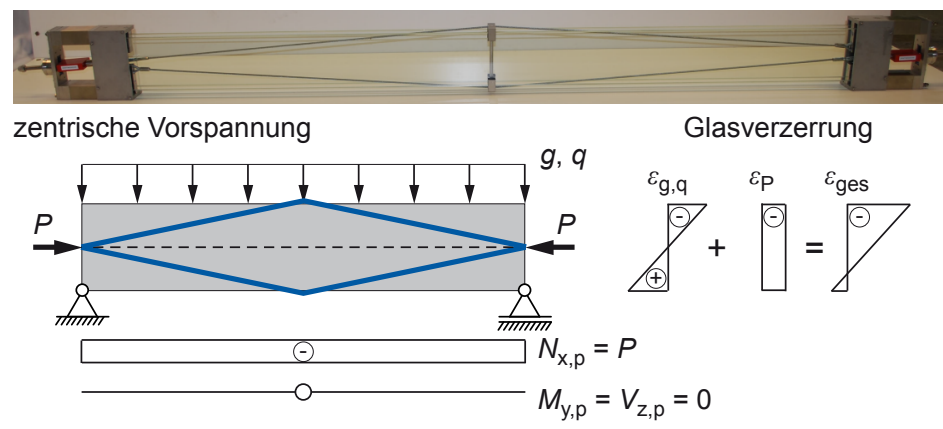

Wird die Bewehrung exzentrisch geführt, so wirken zusätzliche Biegemomente aus der Vorspannung auf den Grundquerschnitt. Diese verursachen eine vertikale Verformung und können zum Einstellen einer Überhöhung konstruktiv ausgenutzt werden. Bild 24, Bild 25 und Bild 26 zeigen dazu gerade, polygonale und parabelförmige Spanngliedverläufe sowie die zugehörigen Umlenkkräfte.

Dieses Konzept ist rein mechanisch begründet und damit unabhängig vom Grundbaustoff des Bauteils. Daher wurde es für den Konstruktiven Glasbau übernommen.

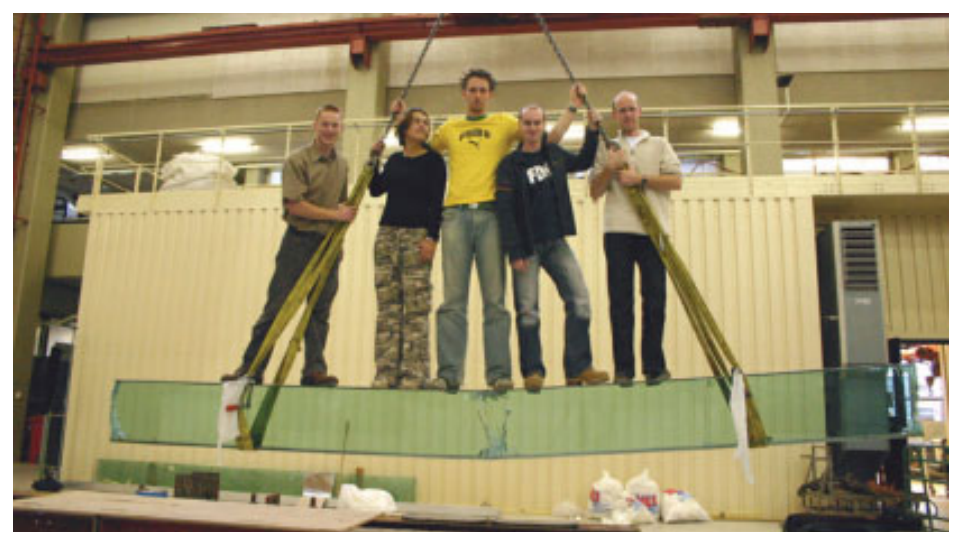

exzentrische Vorspannung

Glasverzerrung

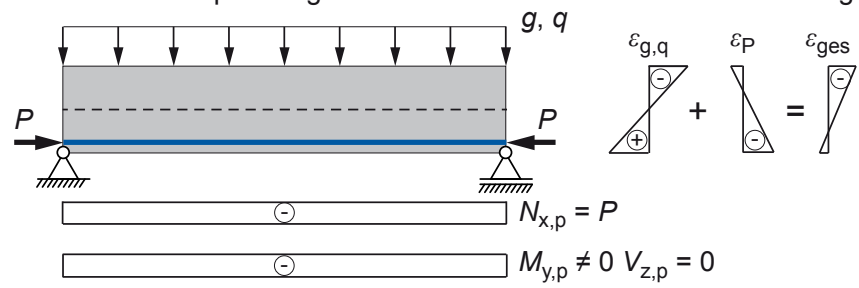

Nach Lage und Verlauf der vorgespannten Bewehrung wurden ausgeführte Beispiele in Forschungs- und Bauprojekten 


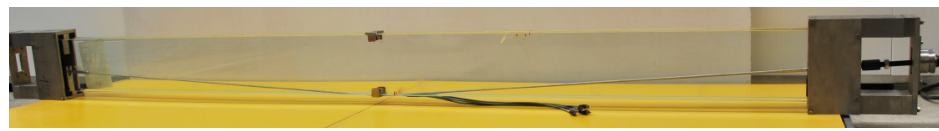

exzentrische Vorspannung
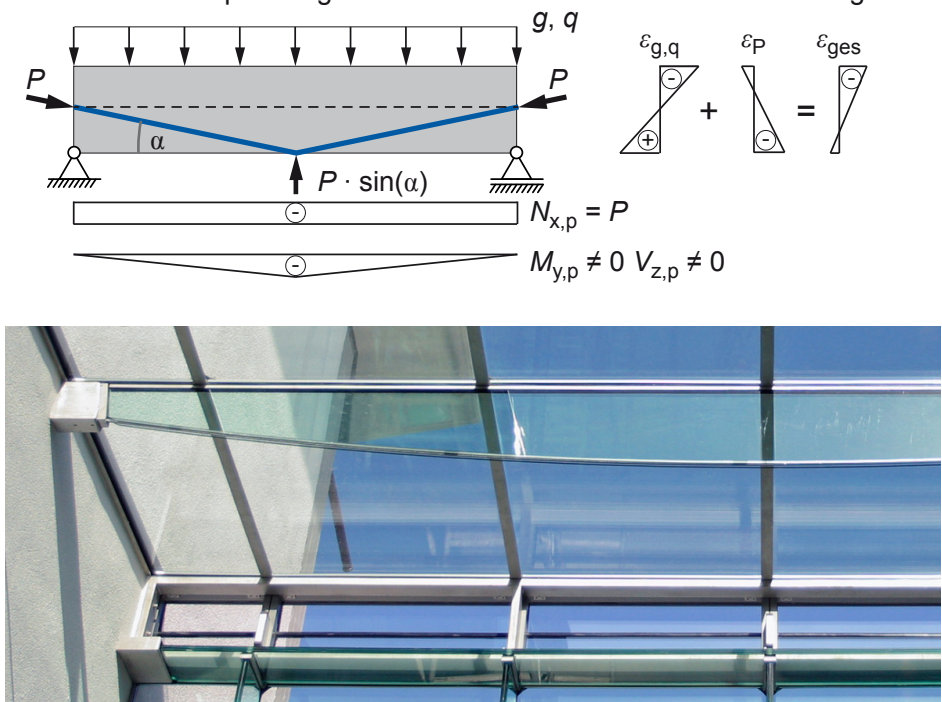

exzentrische Vorspannung

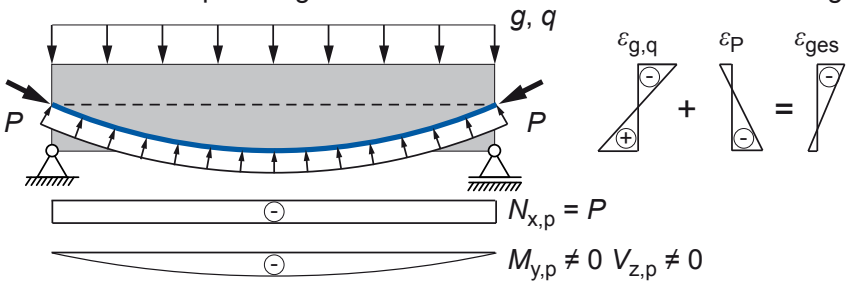

gefunden und eingruppiert. Damit wird die Bezeichnungsweise aus dem Spannbetonbau übernommen (Bild 27).

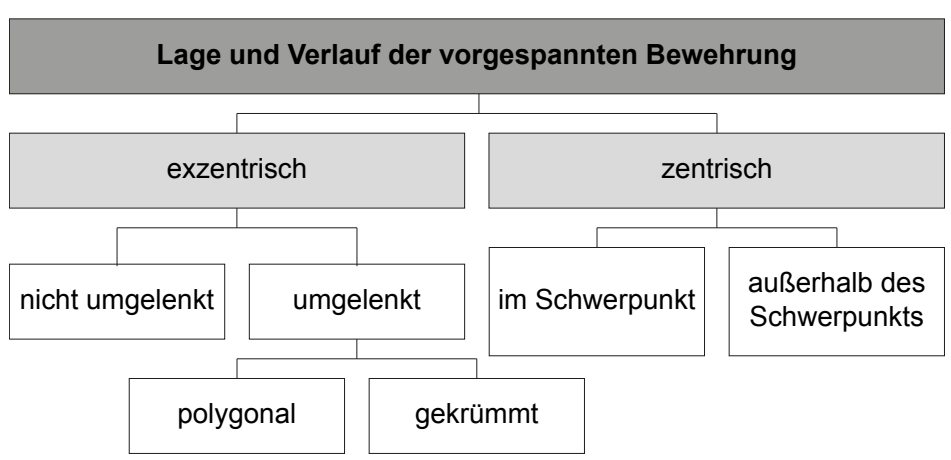

Bild 25

Exzentrisch vorgespannte

Glasträger mit polygonaler Spanngliedführung nach [Weller \& Engelmann 2014b] und [Engelmann \& Weller 2016].

\section{Bild 26}

Exzentrisch vorgespannte Glasträger mit parabelförmiger Spanngliedführung der Therme im Kurort Badenweiler [Schober et al. 2004] und [SBP 2014]. Ein weiteres Objekt von Bünemann [1997] findet sich in der Volksbank Hannover-Linden.

Die Umlenkkräfte wirken über die gesamte Länge des Spanngliedes.

\section{Bild 27}

Übersichtsschema nach Lage und Verlauf der Bewehrung.

Für alle Varianten wurden in Bild 22 bis Bild 26 Beispiele gefunden. 


\subsubsection{Weitere Begriffe: Dekompression und Vorspanngrad}

\section{Dekompression}

Die Dekompression bezeichnet einen „Grenzzustand, bei dem ein Teil des Betonquerschnitts [...] unter Druckspannung steht“ ${ }^{37}$. Es handelt sich im Spannbetonbau um einen Nachweis im Grenzzustand der Gebrauchstauglichkeit zur Begrenzung der Rissbreite, mit dem Ziel, den Korrosionsschutz der Bewehrung sicherzustellen. ${ }^{38}$

Mangels einer Bewehrung innerhalb des Glasquerschnitts werden keine analogen Anforderungen an den Korrosionsschutz gestellt. Eine Ausnahme bildet Drahtglas. ${ }^{33}$

Ein Riss im Glas, insbesondere in fein brechenden, thermisch vorgespannten Glasarten, tritt optisch deutlich in Erscheinung. Durch ein erneutes Überdrücken des gebrochenen Glasquerschnitts verbleibt der Riss sichtbar. Es handelt sich um einen irreversiblen Zustand. Aus Erfahrung wird gerissenem Glas durch den Nutzer keine Tragfähigkeit zugetraut, sodass die Gebrauchstauglichkeit bereits eingeschränkt wird. Das Auftreten eines Risses im unbewehrten Glasträger wird mit dem Nachweis im Grenzzustand der Tragfähigkeit ausgeschlossen. Unter geringeren Gebrauchslasten wird daher kein lastbedingter Riss auftreten, sodass die Definition der Dekompression im Glasbau zunächst obsolet ist.

Dennoch wird in dieser Arbeit der Begriff in Form der Dekompressionslast als Kennzahl für die Leistungsfähigkeit der Vorspannung verwendet.

\footnotetext{
37 Vergleiche [DIN EN 1992-1-1, Kap. NA 1.5.2.25].

38 Vergleiche [DIN EN 1992-1-1, Kap. 7.3].
} 


\section{Vorspanngrad}

Die Definition des Vorspanngrades ist zur Charakterisierung der Vorspannung im Bauteil üblich. ${ }^{39}$ Grundsätzlich bezeichnet er das Verhältnis der Druckspannung infolge der Vorspannung zu einer Zugspannung infolge anderer äußerer Lasten (Gleichung 2).

$$
\begin{array}{ll}
\kappa=\frac{\left|\sigma_{G, p}\right|}{\sigma_{G, g+q}}, \text { hier } & \kappa=\frac{\left|\sigma_{G, p}\right|}{f_{G}} \\
\kappa & \text { Vorspanngrad } \\
\sigma_{G, p} & \text { Druckspannung im Glas infolge Vor- } \\
& \text { spannung } \\
\sigma_{G, g+q} & \text { Glasspannung infolge äußerer Einwir- } \\
f_{G} & \text { kung an der vorgedrückten Zugkante } \\
& \text { Biegezugfestigkeit Glas }
\end{array}
$$

Mit Blick auf Bild 11 wird für den Konstruktiven Glasbau vorgeschlagen, einen Vergleich zur Druckspannung aus der Vorspannung mit der ansetzbaren Glasfestigkeit zu ziehen, um eine Kennzahl zur Wirkung der Vorspannung beim Einsatz von Glasarten unterschiedlicher Festigkeit zu erhalten. So wird Floatglas mit kleinerer Biegezugfestigkeit gegenüber thermisch vorgespanntem Glas und bei sonst gleichen Randbedingungen ( $\sigma_{\mathrm{G}, \mathrm{p}}=$ konstant) einen höheren Vorspanngrad erreichen.

\subsection{Grundlagen der Tragwerksplanung}

Ein Tragwerk muss, unabhängig vom verwendeten Baustoff, Anforderungen an Tragfähigkeit, Gebrauchstauglichkeit und Dauerhaftigkeit erfüllen. ${ }^{40}$ Dazu werden nachzuweisende Grenzzustände, Einwirkungen und Lastkombinationen in [DIN EN 1990] und [DIN EN 1991] definiert. Damit wird die Zuverlässigkeit des Bauteils nach Nutzungsdauer und unter

39 Vergleiche [Rombach 2010]. Dieser Begriff stammt ursprünglich aus der Vorgängernorm [DIN 4227] und wird in [DIN EN 1992] nicht mehr verwendet.

40 Vergleiche [DIN EN 1990]. 
Tafel 3

Arten von untersuchten Einwirkungen und Übersicht über zugehörige Untersuchungsmethoden der Auswirkungen auf Spannglasträger.
Berücksichtigung der Dauerhaftigkeit und Qualitätssicherung festgehalten. ${ }^{41}$

Der Einfluss von Temperaturbelastung, Kriechen und Schwinden ist im Stahlbeton- und Spannbetonbau im Grenzzustand der Gebrauchstauglichkeit zu berücksichtigen. Im Grenzzustand der Tragfähigkeit nur dann, wenn sie wesentlich sind. Glas schwindet nicht, sodass ein Schwindanteil nicht weiter zu berücksichtigen ist. Die weiteren Auswirkungen auf Spannglasträger sind unbekannt und werden im Rahmen dieser Arbeit untersucht. Eine Übersicht bietet Tafel 3.

\begin{tabular}{|c|c|}
\hline $\begin{array}{l}\text { Einwirkung } \\
\text { [DIN EN 1991-1-1] }\end{array}$ & $\begin{array}{l}\text { Untersuchungsmethode der } \\
\text { Auswirkung auf Spannglasträger }\end{array}$ \\
\hline quasi statische Lasten & $\begin{array}{l}\text { Kurzzeit-Biegeversuch } \\
\text { siehe Kapitel 3.3, ab Seite } 107\end{array}$ \\
\hline $\begin{array}{l}\text { Vorspannung } \\
\text { [DIN EN 1992-1-1, } \\
\text { Kap. 2.3.1.4] }\end{array}$ & $\begin{array}{l}\text { Vorspannversuch } \\
\text { siehe Kapitel 3.3, ab Seite } 107\end{array}$ \\
\hline Dauerlasten & $\begin{array}{l}\text { Dauerversuch zur Ermittlung von } \\
\text { Spannkraftverlusten } \\
\text { siehe Kapitel 3.4, ab Seite } 134\end{array}$ \\
\hline $\begin{array}{l}\text { außergewöhnliche } \\
\text { Einwirkungen }\end{array}$ & $\begin{array}{l}\text { Resttragfähigkeitsuntersuchung } \\
\text { siehe Kapitel 3.5, ab Seite } 147\end{array}$ \\
\hline $\begin{array}{l}\text { Temperatureinwirkung } \\
\text { [DIN EN 1992-1-1, } \\
\text { Kap. 2.3.1.2] }\end{array}$ & $\begin{array}{l}\text { Temperaturwechseluntersuchung } \\
\text { siehe Kapitel 3.6, ab Seite } 165\end{array}$ \\
\hline
\end{tabular}

Zusammenfassend kann festgehalten werden, dass für Spannglasträger Nachweise nach [DIN 18008-1] auf Basis des Bemessungskonzeptes nach [DIN EN 1990] und [DIN EN 1991] geführt werden müssen. Die Auswirkung der Einwirkung ist unbekannt und muss untersucht werden. Zugehörige experimentelle und numerische Untersuchungen werden in Kapitel 3 und Kapitel 4 vorgestellt und diskutiert.

41 Entsprechende Regelungen für den Stahlbeton- und Spannbetonbau finden sich in [DIN EN 1991-1-1, Kap. 2]. Analoge Regelungen trifft die [DIN 18008] im Glasbau. 


\section{$2.5 \quad$ Baustoffe}

Die Eigenschaften der Grundbaustoffe Beton und Glas bilden die Basis der Analogiebetrachtung. Im Weiteren werden inre jeweiligen mechanischen und thermischen Eigenschaften dargestellt und verglichen, um analoge Sachverhalte aufzufinden, die eine Übertragung des Vorspannkonzeptes auf den Glasbau ermöglichen. Die Betrachtung wird anschlieBend auf die Bewehrungsbaustoffe und Komponenten ausgeweitet.

\subsubsection{Festigkeit}

Gemäß den Eurocode-Regelungen wird die Festigkeit allgemein als „mechanische Baustoffeigenschaft [beschrieben], welche üblicherweise in Spannungseinheiten ausgedrückt wird." 42 Im Betonbau wird zwischen ein- und mehraxialer Druckfestigkeit (Zylinderdruckfestigkeit oder Würfeldruckfestigkeit) im Betonalter von 28 Tagen und Zugfestigkeit (Spaltzugfestigkeit oder einachsige Zugfestigkeit) unterschieden. ${ }^{43}$ Somit hängen die charakteristischen Festigkeitseigenschaften von Beton vom Prüfverfahren ${ }^{44}$, den Einbaubedingungen $^{45}$ und dem Betonalter ab. Beton wird in Festigkeitsklas$\operatorname{sen}^{46}$ gruppiert, deren Last-Verzerrungs-Modell in Bild 28 dargestellt ist.

Die Festigkeit von Glas ist kein Materialkennwert, sondern ein Kennwert für die Qualität und den Schädigungszustand der zugbeanspruchten Glasoberfläche, welche mithilfe der Bruchmechanik beschreibbar ist. ${ }^{47}$ Die jeweiligen Werte werden jedoch im Rahmen der Materialprüfungen unter Berücksichtigung der bruchmechanischen Einflüsse zu einem cha-

42 Vergleiche [DIN EN 1990, Kap. 1.5.2.16].

43 Vergleiche [DIN EN 1992-1-1, Kap. 3.1].

44 Insbesondere einschließlich der Prüfbedingungen wie Zementtemperatur, Temperatur, Lagerungsbedingungen und Eigenschaften der verwendeten Betonbestandteile wie Zementtyp, -festigkeitsklasse und -temperatur. [DIN EN 1992-1-1, Kap. 3.1.2], [DIN EN 206-1].

45 Wärme- und Nachbehandlung, [DIN EN 206-1]

46 Vergleiche [DIN EN 1992-1-1, Tab. 3.1].

47 Eine prägnante Erläuterung bruchmechanischer Grundkenntnisse im Zusammenhang mit Glasträgern ist in [Härth 2013] zu finden. 


\begin{tabular}{|c|c|c|c|c|c|}
\hline Eigenschaft & Kalk-Natronsilicatgla & & Beton & & Glas / Beton \\
\hline Zugfestigkeit & $\begin{array}{l}\text { Biegezugfestigkeit } \\
45-120 \mathrm{~N} / \mathrm{mm}^{2}\end{array}$ & $a, b, c$ & $\begin{array}{l}\text { Biegezugfestigkeit } \\
1,0-1,6 \mathrm{f}_{\mathrm{ctm}}=1,6- \\
8,3 \mathrm{~N} / \mathrm{mm}^{2}\end{array}$ & $g$ & $5,4-120,0$ \\
\hline Druckfestigkeit & $\begin{array}{l}\text { Druckfestigkeit } \\
500-900 \mathrm{~N} / \mathrm{mm}^{2}\end{array}$ & $d, e$ & $\begin{array}{l}\text { Zylinderdruckfestigkeit } \\
12-100 \mathrm{~N} / \mathrm{mm}^{2}\end{array}$ & $h, k$ & $5,0-75,0$ \\
\hline $\begin{array}{l}\text { Druck-/ } \\
\text { Zugfestigkeit }\end{array}$ & $4,2-20,0$ & & $7,5-12,0$ & & $0,56-1,67$ \\
\hline E-Modul & $70000 \mathrm{~N} / \mathrm{mm}^{2}$ & $a, f$ & $27000-44000 \mathrm{~N} / \mathrm{mm}^{2}$ & $g$ & $1,6-2,6$ \\
\hline Querdehnzahl & $0,20-0,23$ & $a, f$ & $\begin{array}{l}0,20( \pm 0,05) \text { nach } \\
\text { Betonalter und Zusam- } \\
\text { mensetzung }\end{array}$ & $g$ & $1,00-1,15$ \\
\hline Wärmedehnzahl & $9,0 \cdot 10^{-6} \mathrm{~K}^{-1}$ & $f$ & $10,0 \cdot 10^{-6} \mathrm{~K}^{-1}$ & $g$ & 0,90 \\
\hline${ }^{a}$ [DIN EN 572] & ${ }^{d}[\mathrm{DIN} 1249-10]$ & & ${ }^{g}$ [DIN EN 1991-1-1] & & \\
\hline${ }^{b}$ [DIN EN 1863] & ${ }^{e}$ [DIN 51067-1] & & ${ }^{h}$ [DIN EN 206-1] & & \\
\hline${ }^{c}$ [DIN EN 12150-1] & ${ }^{f}$ [DIN 18008] & & ${ }^{k}$ [DIN EN 12390] & & \\
\hline
\end{tabular}

Tafel 4

Charakteristische Festigkeit und elastische Formänderungseigenschaften von Glas und Beton. rakteristischen Festigkeitswert umgerechnet, der als solcher wie für andere Baustoffe verwendet werden kann. ${ }^{48}$

Damit ist im Vergleich von Beton und Glas (Tafel 4), insbesondere thermisch vorgespanntem Glas, eine größere ansetzbare Biegezugfestigkeit festzustellen, welche im Gegensatz zum Stahlbeton- und Spannbetonbau für die Glasbemessung im Grenzzustand der Tragfähigkeit angesetzt werden darf. ${ }^{49}$ Die Druckfestigkeit von normativ erfasstem Beton erreicht nach [DIN EN 1992-1-1] charakteristische Werte von bis zu f $f_{c k}=100 \mathrm{~N} / \mathrm{mm}^{2} .{ }^{50} \mathrm{Im}$ Glasbau dagegen ist für die Druckfestigkeit nach [DIN 18008] kein Wert angegeben. $\mathrm{Er}$ ist in der Regel nicht bemessungsrelevant. Es kann für die Berechnung jedoch von deutlich größeren Werten als im Stahlbeton- und Spannbetonbau ausgegangen werden.

Beide Baustoffe versagen unangekündigt beim Erreichen der Zugfestigkeit - sie sind spröde. Zudem gilt, dass der abso-

48 [DIN 18008] liefert mit Blick auf die zugehörigen Produktnormen ein Konzept zur Ermittlung charakteristischer Zugfestigkeiten unter Berücksichtigung der Lasteinwirkungsdauer, der Belastungsart und der Konstruktion, wodurch die bruchmechanischen Eigenschaften von Glas im Bauwesen adäquat berücksichtigt werden.

49 Vergleiche [DIN EN 1992-1-1, Kap. 6.1].

50 Deutlich höhere Werte für Ultrahochfesten Beton (UHPC) sind derzeit nicht normativ gefasst. 
lute Betrag der Druckfestigkeit ${ }^{51}$ deutlich größer als der entsprechende Wert der Zugfestigkeit ist. Unter reiner Biegebeanspruchung unbewehrter Bauteile sind die absoluten Werte von Druck- und Zugspannung im Bauteil gleich gro ${ }^{52}$, die jeweilige Ausnutzung jedoch nicht, sodass die Zugfestigkeit bemessungsrelevant wird. Eine volle Ausnutzung der Tragpotenziale im Sinne einer gleichmäßigen Ausnutzung auf Druck- und Zugseite wird nicht erreicht. Daher wird Beton zu Stahlbeton bewehrt, ein gemeinsamer Lastabtrag zwischen beiden Baustoffen angestrebt und so gegen ein sprödes Versagen gewirkt. Die Ausnutzung der Zugtragfähigkeit wird im Bewehrungsmaterial, die Drucktragfähigkeit im Grundmaterial $^{53}$ nachgewiesen, sodass eine gleichmäßige Ausnutzung durch die Wahl der Querschnittsflächen möglich wird. Beim analytischen Nachweis bewehrter Glasbauteile wird ein ähnliches Vorgehen gewählt. ${ }^{54}$ Letztlich wird die Zugbeanspruchung im Spannbetonbau durch eine planmäßige Druckbeanspruchung reduziert beziehungsweise vollends egalisiert und damit das Tragvermögen durch eine höhere Gesamtauslastung vergrößert. Ein ähnliches Vorgehen wird durch eine thermische Vorspannung von Floatglas gewählt. Zusätzlich begründen die benannten Festigkeitseigenschaften höhere Tragpotenziale unter Biegebeanspruchung durch die Anordnung einer vorgespannten Bewehrung.

\subsubsection{Elastische Formänderungseigenschaften}

Ebenso wie die Festigkeitsangaben ist die Steifigkeit von Beton von seiner Zusammensetzung und Herstellung ${ }^{55}$ abhängig und verändert sich mit der Erhärtungszeit. ${ }^{56}$ Der E-Modul steigt dabei mit höherer Betonfestigkeitsklasse.

51 Für eine bemessungsrelevante Druckfestigkeit thermisch vorgespannter Gläser und Floatglas im Bauwesen liegen derzeit keine wissenschaftlich ausreichend gesicherten Erkenntnisse vor.

52 Vergleiche $\sigma_{\mathrm{a}}$ in Bild 11, Seite 32.

53 Bei Verwendung einer Druckbewehrung ist die Druckausnutzung ebenso anteilig durch die Bewehrung beeinflusst.

54 Vergleiche [Feirabend 2010], [Louter 2011] und andere.

55 In [DIN EN 1992-1-1, Tab. 3.1] sind Richtwerte für den mittleren E-Modul als Sekantenwert im Betonalter von 28 Tagen angegeben. Eine gesonderte Ermittlung der Werte ist nötig, wenn das Tragwerk empfindlich auf Abweichungen reagiert ([DIN EN 1992-1-1, Kap. 3.1.3(1)])

56 Vergleiche [DIN EN 1992-1-1, Kap. 3.1.3] 
Bild 28

Last-Verzerrungs-Diagramm

für Beton und Glas nach normativ erfassten Materialmodellen in [DIN EN 1992] sowie [DIN 18008].

Positive Verzerrungen werden als (Zug-) Dehnung, negative Werte als (Druck-) Stauchung bezeichnet.

Auf Zugbeanspruchung versagen beide Baustoffe unangekündigt - spröde.

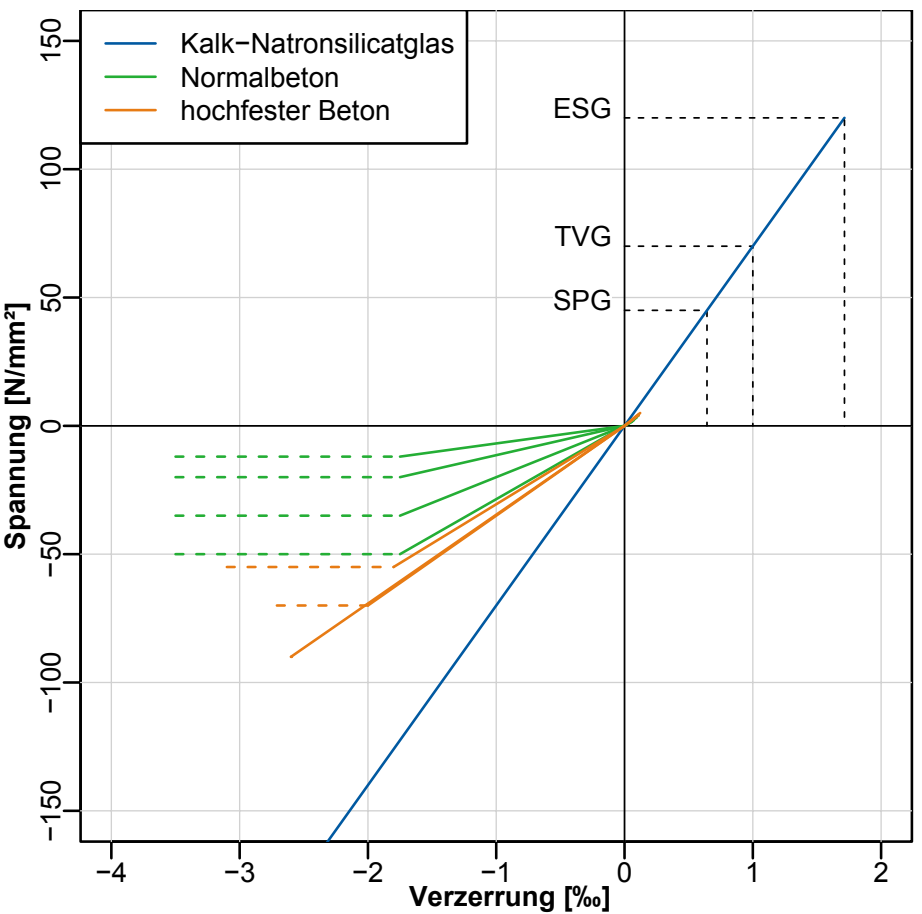

Kalk-Natronsilicatglas im Bauwesen ${ }^{57}$ weist in der Regel einen konstanten, zeitunabhängigen E-Modul von $70000 \mathrm{~N} / \mathrm{mm}^{2}$ auf. Eine Gegenüberstellung ist in Bild 28 dargestellt und gemeinsam mit der Festigkeit in Tafel 4 zusammengefasst.

Die Querdehnzahl von Beton ist ebenso stark von der Zusammensetzung und Feuchte, dem Betonalter sowie dem Belastungsniveau abhängig und wird für den ungerissenen Fall mit $\mu_{B}=0,20$ angesetzt. ${ }^{58}$ Für Berechnungen im Konstruktiven Glasbau wird $\mu_{G}=0,23$ verwendet. ${ }^{59}$

Für Temperatureinwirkungen ist die Kenntnis der Wärmedehnzahl entscheidend, um Zwangsbeanspruchungen und die Interaktion verschiedener Baustoffe in bewehrten Bauteilen fassen zu können. Für Glas und Beton werden Werte von $9,0 \cdot 10^{-6} \mathrm{~K}^{-1}$ beziehungsweise $10,0 \cdot 10^{-6} \mathrm{~K}^{-1}$ verwendet (siehe Tafel 4).

57 Vergleiche [DIN EN 572-1]. Für Borosilicatglas wird ein E-Modul von $60000 \mathrm{~N} / \mathrm{mm}^{2}$ verwendet. [DIN 18008-1].

58 Vergleiche [Fingerloos et al. 2012, S. 218].

59 Die Produktnorm [DIN EN 572-1, Tab. 1] nennt abweichend von [DIN 18008-1] einen geringeren Wert von 0,20 für Kalk-Natronsilicatglas. 
Die Verhältnisse der wesentlichen Festigkeits- und Formänderungseigenschaften von Beton und Glas - insbesondere das Verhältnis von Zug- und Druckfestigkeit - sind ähnlich. Zudem versagen beide Baustoffe spröde. Damit ist eine ähnliche konstruktive Behandlung (Bewehrung und Vorspannung) von Glas wie Beton sinnvoll. Wesentlicher Unterschied ist die fehlende Zeitabhängigkeit der Eigenschaften von Glas ${ }^{60}$, sodass alle Nachweise, für welche zunächst der Zeitpunkt der ungünstigsten Eigenschaftsausprägung aufgefunden werden muss, entfallen. Jedoch wird Flachglas zu Verbund- und Verbundsicherheitsglas veredelt. Zusätzlich kommen Kunststoffe zum Lasteintrag in Glaskonstruktionen zum Einsatz. Deren maßgebende Eigenschaften sind in der Regel zeit- und lastabhängig und müssen daher untersucht werden. Eine Zusammenfassung dazu bietet das nachfolgende Kapitel 2.5.3.

\subsubsection{Kriechen und Schwinden}

Kriechen und Schwinden sind zeitabhängige Materialeigenschaften. ${ }^{61}$

Schwinden ist eine lastunabhängige Verkürzung infolge der Trocknung und Betonerhärtung ${ }^{62}$ und ist für Glas nicht relevant. Entsprechend werden alle Schwindanteile für eine analoge Betrachtung entfernt.

Kriechen ist eine last- und zeitabhängige Verformung unter konstanter Last, welches für Glas in baupraktischen Zeiträumen nicht beobachtbar ist. ${ }^{63}$ Jedoch werden im Glasbau weitere Materialien eingesetzt, welche ein last- und zeitabhängiges Verhalten zeigen. Dies betrifft insbesondere:

60 Lediglich die Belastungsdauer wird mittels des Modifikationsfaktors $k_{\text {mod }}$ bei der Ermittlung der Bemessungsfestigkeit von Glas im Bauwesen nach [DIN 18008] berücksichtigt.

61 Vergleiche [DIN EN 1992-1-1, Kap. 2.3.2.2].

62 Vergleiche [DIN EN 1992-1-1, Kap. 3.1.4(6)].

63 Die dennoch weitverbreitet Ansicht, dass alte Kirchenfenster „kriechen“ wurde widerlegt. Vergleiche [Plumb 1989], [Zanotta 1998], [Stockes 1999]. 
- das verwendete Zwischenschichtmaterial im Verbund- und Verbundsicherheitsglas ${ }^{64}$ sowie

- Kunststoffe ${ }^{65}$ oder andere Materialien, die zum Lasteintrag als Glaskontaktwerkstoff verwendet werden.

Das Kriechen wird mittels der Kriechzahl $\varphi$ als Verhältnis von Kriechverzerrung und elastischer Verzerrung angegeben (Gleichung 3). ${ }^{66} \mathrm{Zu}$ Belastungsbeginn $(t=0)$ liegt noch keine Kriechverzerrung vor, sodass die Kriechzahl stets größer oder gleich Null sein muss.

Gleichung 3

$$
\begin{array}{ll}
\varphi=\frac{\varepsilon_{c}}{\varepsilon_{e l}} & \\
\varphi & \text { Kriechzahl } \\
\varepsilon_{c} & \text { Kriechverzerrung } \\
\varepsilon_{e l} & \text { elastische beziehungsweise soforti- } \\
& \text { ge, zeitunabhängige Verzerrung bei } \\
& \text { Belastungsbeginn }
\end{array}
$$

Der Gesamtprozess des Kriechens von Beton wird als Summe der Teilprozesse Grundkriechen und Trocknungskriechen beschrieben. Analog ergibt sich die zeitabhängige Längenänderung einer Verbundglaskonstruktion aus zwei Einzelscheiben aus den Einzelanteilen von Zwischenschichtmaterial und beidseitiger Klotzung (Gleichung 4 und Bild 29).

Gleichung 4

$$
\Delta L_{V G, c}=2 \cdot \Delta L_{K, c}+\Delta L_{Z, c}=2 \cdot \varepsilon_{K, c} \cdot L_{K, 0}+\varepsilon_{Z, c} \cdot L_{Z, 0}
$$

$$
\begin{array}{ll}
\Delta L_{V G, c} & \text { Längenänderung infolge Kriechens des } \\
\Delta L_{K, c}=L_{K, 0}-L_{K, c} & \text { Verbundglases } \\
\Delta L_{Z, c}=L_{Z, 0}-L_{Z, c} & \text { Längenänderung infolge Klotzungskrie- } \\
& \text { Längen } \\
& \text { Zwischendenschichtmaterials }
\end{array}
$$

Die Längenänderung kann komponentenweise berechnet werden:

\footnotetext{
64 Vergleiche [Sobek et al. 2000], [Schuler 2003], [Sedlacek et al. 2003], [Ensslen 2005], [Kott 2006], [Sackmann 2008].

65 Vergleiche [Ebert 2014].

66 Vergleiche [DIN EN 1992-1-1, Kap. 3.1.4].
} 


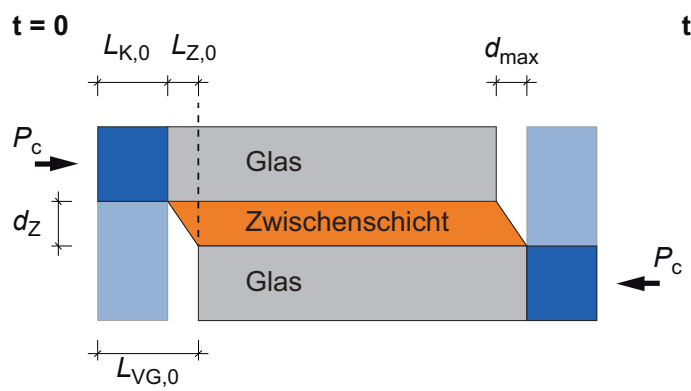

\section{Zwischenschicht}

Für Verbundsicherheitsglas werden verschiedenartige Zwischenschichtmaterialien verwendet. Am häufigsten kommt dabei Polyvinylbutyral (PVB) zum Einsatz. Dessen zeitabhängiges Materialverhalten wurde bereits vielfach untersucht ${ }^{64}$, sodass eine Beschreibung der Verformung mittels des zeitabhängigen Schubmoduls ermöglicht wird.

Dazu schlagen Sobek et al. [2000] eine analytische Fassung des Schubmoduls für PVB vor (Gleichung 5). Diese liegt nach Schuler [2003] auf der sicheren Seite.

a) für $\mathrm{T}>20^{\circ} \mathrm{C}$

$G_{P V B}(T, t)=0,008 \cdot(100-T)-0,0011 \cdot(50+T) \cdot \log (t)$

b) für $\mathrm{T}<10^{\circ} \mathrm{C}$

$G_{P V B}(T, t)=2,0-0,2 \cdot \log (t)$

$G_{P V B}(T, t)$ temperatur- und zeitabhängiger Schubmodul von PVB ( $\mathrm{t}$ in Sekunden)

In [Sedlacek et al. 2003] wird derselbe Zusammenhang für den gesamten Temperaturbereich verwendet.

In baupraktischen Zeiträumen strebt der Schubmodul gegen Null, er ist rechnerisch nicht mehr ansetzbar. Mit steigender Temperatur wird dieser Zustand früher erreicht. ${ }^{67}$ Die Verfor-

67 Analoge Materialgesetze sind auch für andere Zwischenschichtmaterialien zur Anwendung verfügbar. Vergleiche beispielsweise [DuPont SGP 2008].

\section{Bild 29}

Kriechverformung der Glaskonstruktion.

Hier: für ein Verbundglas aus zwei Glasschichten für den Zeitpunkt nach dem Aufbringen der kriecherzeugenden Belastung $\mathrm{P}_{\mathrm{c}}(\mathrm{t}=0)$ und nach dem Auftreten einer zeitabhängigen Verformung $(t>0)$ und einem Kantenversatz von $d_{\max }$. 
mung der Zwischenschicht wird jedoch durch das Tilgen des Kantenversatzes im Verbund-Sicherheitsglas begrenzt, da anschließend keine höheren Lasten in diese Ebene eingetragen werden $\left(\Delta \mathrm{L}_{\mathrm{z}} \leq \mathrm{d}_{\max }\right)^{68}$.

Das Kriechen der Zwischenschicht kann vermieden werden, indem sie frei von einer Schubbelastung bleibt. Dies wird durch die Tilgung des Versatzes mittels dem Planschleifen der Kanten ${ }^{69}$ und/oder ein Ausfüllen des Versatzes mit Injektionsmörtel oder einem Klebstoff erreicht $\left(\tau_{Z}=0\right) .{ }^{70}$ Eine numerische Analyse der Lasteinleitung in eine Verbundglaskante mit Kantenversatz für geklebte und geklotzte Konstruktionen bei unterschiedlichen Temperaturen wurde in [Bukieda 2015] durchgeführt.

Die kriechbedingte Verkürzung der Zwischenschicht bei Schubbelastung ergibt sich aus der Gesamtverformung infolge sofortiger und zeitabhängiger Verformung $\Delta \mathrm{L}_{\mathrm{z}, \mathrm{el}+\mathrm{c}}$ abzüglich der Kriechkomponente $\Delta \mathrm{L}_{\mathrm{z}, \mathrm{c}}$ (Gleichung 6).

Neben den geometrischen Größen muss die Schubspannung in der Zwischenschicht ermittelt werden. Diese wird

68 Maximal zulässiger Kantenversatz $d_{\max }$ im Verbund-Sicherheitsglas nach [DIN EN ISO 12543-5] oder tatsächlicher Kantenversatz, wenn dieser anderweitig nachgewiesen wurde.

69 Einscheiben-Sicherheitsglas nach [DIN EN 12150-1] sowie teilvorgespanntes Glas nach [DIN EN 1863] (beziehungsweise nach Allgemeiner bauaufsichtlicher Zulassung) soll nach dem Vorspannen nicht mehr plangeschliffen werden.

70 Konstruktive Varianten werden in Kapitel 6.3 (ab Seite 245) gegenübergestellt. 
vereinfacht als konstantes Verhältnis der kriecherzeugenden Kraft und der Schubfläche angenommen. ${ }^{71}$

$$
\begin{aligned}
& \Delta L_{Z, c}=\Delta L_{Z, e l+c}-\Delta L_{Z, e l} \\
& =d_{Z} \cdot\left(\tan \left(\gamma_{Z, e l+c}\right)-\tan \left(\gamma_{Z, e l}\right)\right) \\
& =\frac{d_{Z}}{A_{Z}} \cdot\left(\frac{P_{c}\left(t_{c}\right)}{G_{Z}\left(T, t_{c}\right)}-\frac{P_{c}\left(t_{0}\right)}{G_{Z}\left(T, t_{0}\right)}\right) \\
& d_{z} \quad \text { Dicke der Zwischenschicht } \\
& \gamma_{Z, e l+c} \quad \text { Gleitwinkel der Zwischenschicht in- } \\
& \text { folge elastischer und zeitabhängiger } \\
& \text { Verzerrung } \\
& \gamma_{Z, e l} \quad \text { Gleitwinkel der Zwischenschicht infolge } \\
& \text { elastischer Verzerrung } \\
& A_{z}=L_{z} \cdot h_{Z} \quad \text { Schubfläche der Zwischenschicht mit } \\
& P_{c}=\tau_{Z} \cdot A_{Z} \quad \begin{array}{l}
\text { den Abmessungen } L_{z} \text { und } h_{Z} \\
\text { kriecherzeugende Belastung infolge }
\end{array} \\
& \text { der kriecherzeugenden Schubspan- } \\
& \text { nung in der Zwischenschicht zum } \\
& \text { Belastungsbeginn bei } t=t_{0} \text { und zum } \\
& \text { Betrachtungszeitpunkt der Kriechver- } \\
& G_{z}(T, t) \\
& \text { formung } \mathrm{t}=\mathrm{t}_{\mathrm{c}} \text { (Bild 29) } \\
& \text { Schubmodul der Zwischenschicht, z. B. } \\
& \text { nach Gleichung 5, Seite } 53
\end{aligned}
$$

Mit den obigen Angaben wird nun ebenso eine Kriechzahl für die Gleitverformung der Zwischenschicht angegeben:

$$
\begin{aligned}
& \varphi_{Z}\left(T, t_{c}, t_{0}\right)=\frac{\Delta L_{Z, c}}{\Delta L_{Z, e l}}=\frac{G_{Z}\left(T, t_{0}\right)}{G_{Z}\left(T, t_{c}\right)} \cdot \frac{P\left(t_{c}\right)}{P\left(t_{0}\right)}-1 \\
& \varphi_{Z} \quad \text { Kriechzahl Zwischenschicht }
\end{aligned}
$$

In Spannglasträgern nimmt die kriecherzeugende Belastung $P\left(t_{c}\right)$ infolge der Spannkraftverluste ab, sodass eine iterative Ermittlung der Kriechzahl mit einer Abschätzung des Verhältnisses $\mathrm{P}\left(\mathrm{t}_{\mathrm{c}}\right)$ zu $\mathrm{P}\left(\mathrm{t}_{0}\right)$ nötig wird. Auf der sicheren Seite liegend wird angenommen, dass die kriecherzeugende Belastung konstant bleibt, sodass die Kriechzahl der Zwischenschicht in Bild 30 als temperatur- und zeitabhängige Größe dargestellt werden kann.

71 Schuler [2003] zeigt durch einen Vergleich mit einer Finiten-ElementeBerechnung, dass diese Annahme ausreichend genau ist. 
Bild 30

Kriechzahlen einer PVB Zwischenschicht mit dem Materialmodell nach Gleichung 5 für den Temperaturbereich der Dauerlastversuche.

Für den Zeitraum bis $1000 \mathrm{~h}$ werden Kriechzahlen bei variierter Temperatur angegeben und für die Auswertung und Diskussion der Dauerversuche in Kapitel 3 und 5 verwendet.

Der rechnerische Belastungsbeginn wird mit $\mathrm{t}_{0}=1 / 60 \mathrm{~s}$ angenommen. Es resultiert ein Schubmodul von $G_{Z}\left(t_{0}\right)=0,76 \mathrm{~N} / \mathrm{mm}^{2}$.

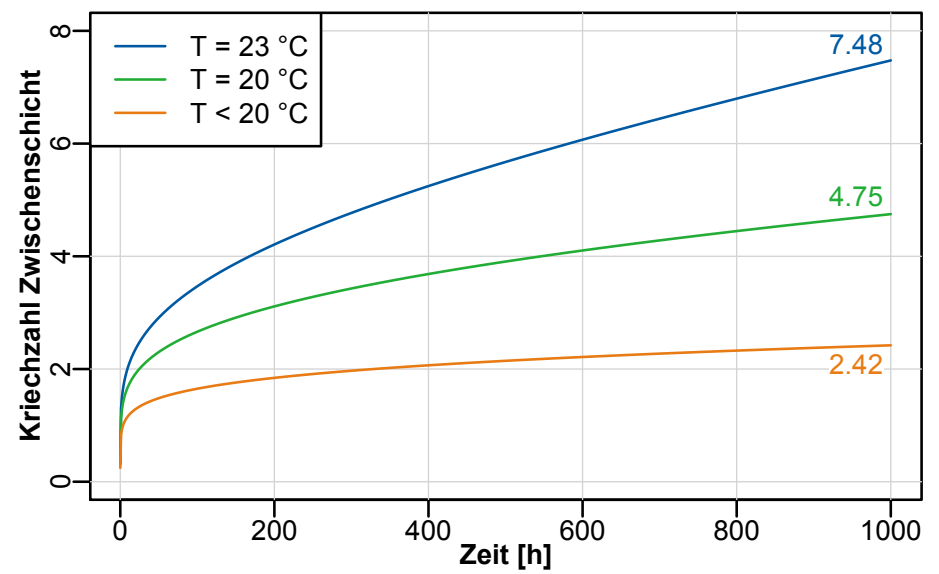

\section{Klotzung}

Für die Beschreibung einer zeitabhängigen Verkürzung einer Kunststoffklotzung wird ebenso eine Kriechzahl ermittelt. Das Gesamtkriechverhalten thermoplastischer Kunststoffe wird in einen zeitabhängigen (viskosen und relaxierenden) sowie einen zeitunabhängigen Anteil getrennt. Ersterer verhält sich bei geringen Lasten linear, bei höheren Lasten jedoch deutlich nichtlinear, was ungünstig große Verformungen hervorruft. Ein nichtlineares Verhalten wird durch die zahlenmäßige Begrenzung der viskosen Stauchung auf $\varepsilon_{\text {vis }} \leq 0,1 \%$ ausgeschlossen. ${ }^{72}$

$$
\Delta L_{K, c}(t)=L_{K, 0} \cdot \varepsilon_{K, c}(t)
$$

$$
\begin{array}{ll}
L_{K, 0} & \text { Ausgangsdicke der Klotzung } \\
\varepsilon_{K, c} & \text { (lineare) Kriechstauchung der Klotzung }
\end{array}
$$

Für die Auswertung der Dauerversuche in dieser Arbeit wird auf die Ergebnisse in Ebert [2014] zurückgegriffen. Darin wird das Verzerrungsverhalten modellhaft einerseits mit einem Findley-Ansatz, andererseits mit einem Burgers-Ansatz analytisch beschrieben. Letzterer wird in [Ebert 2014] für die Beschreibung des Langzeitverhaltens der dort untersuchten Kunststoffe bevorzugt und soll auch in dieser Ar-

72 Vergleiche [Ebert 2014]. 
beit angewendet werden. Es wird nach viskosem und viskoelastischem Anteil getrennt.

$$
\varepsilon_{K, c}(t)=\varepsilon_{K, v i s}(t)+\varepsilon_{K, \text { rel }}(t)
$$

$$
\begin{array}{ll}
\varepsilon_{K, \text { vis }} & \text { viskose Stauchung der Klotzung } \\
\varepsilon_{K, \text { rel }} & \text { relaxierende (visko-elastische) Stau- } \\
& \text { chung der Klotzung }
\end{array}
$$

Für die Beschreibung des Langzeitverhaltens der Klotzungskunststoffe werden beide Summanden nach [Ebert 2014] in Gleichung 10 und Gleichung 11 angegeben.

$$
\varepsilon_{K, v i s}(t)=\frac{\sigma_{0}}{\eta_{0}} \cdot t
$$

Gleichung 10

$\begin{array}{ll}\sigma_{0} & \text { aufgebrachte Anfangsspannung als } \\ & \text { Anfangsbedingung zur Bestimmung } \\ & \text { der Materialkennwerte } \\ \eta_{0} & \text { Viskosität }\end{array}$

$$
\varepsilon_{K, r e l}(t)=\sum_{i} \varepsilon_{K, r e l, i}(t)=\sum_{i} \frac{\sigma_{0}}{E_{r e l, i}} \cdot\left(1-e^{\frac{-t \cdot E_{r e l, i}}{\eta_{r e l, i}}}\right)
$$
Anfangsbedingung zur Bestimmung

Mit diesem Materialmodell werden die Kennwerte $E_{i}$ und $\eta_{i}$ bei einer gewählten Prüflast $\sigma_{0}$ ermittelt, welche sich von der tatsächlich auftretenden kriecherzeugenden Last $\sigma_{K}$ unterscheidet. ${ }^{73}$ Zur Abschätzung der experimentellen Ergebnisse in Kapitel 3 wird angenommen, dass eine lineare Anpassung auf die tatsächliche Last möglich ist, um das Kriechmodell auf dem tatsächlichen Lastniveau anzuwenden. ${ }^{74}$ Setzt man

73 Vergleiche [Ebert 2014].

74 Diese Annahme ist bei einer zukünftigen, vertieften Analyse zu überprüfen und nötigenfalls anzupassen. 
Gleichung 10 und Gleichung 11 in Gleichung 9 ein und multipliziert sie mit $\sigma_{K} / \sigma_{0}$ erhält man Gleichung 12 :

Gleichung 12

$$
\begin{aligned}
& \varepsilon_{K, c}\left(t_{c}\right)=\frac{\sigma_{K}}{\eta_{0}} \cdot t_{c}+\sum_{i} \frac{\sigma_{K}}{E_{r e l, i}} \cdot\left(1-e^{\frac{-t_{c} \cdot E_{r e l, i}}{\eta_{r e l, i}}}\right) \\
& \sigma_{K}=P_{\mathrm{c}} / A_{K} \quad \text { kriecherzeugende Last }
\end{aligned}
$$

Mit diesen Ergebnissen wird die Kriechzahl der Klotzung $\varphi_{K}$ in Abhängigkeit vom Verlustfaktor $\mathrm{P}_{\mathrm{c}}\left(\mathrm{t}_{\mathrm{c}}\right) / \mathrm{P}_{\mathrm{c}}\left(\mathrm{t}_{0}\right)$ ermittelt ${ }^{75}$ und in Bild 31 dargestellt:

$$
\begin{aligned}
\varphi_{K}\left(t_{c}, t_{0}\right) & =\frac{\varepsilon_{K, c}\left(t_{c}\right)}{\varepsilon_{K}\left(t_{0}\right)}=\frac{\frac{\sigma_{K}\left(t_{c}\right)}{\eta_{0}} \cdot t_{c}+\sum_{i} \frac{\sigma_{K}\left(t_{c}\right)}{E_{r e l, i}} \cdot\left(1-\mathrm{e}^{\frac{-t_{c} \cdot E_{r e l, i}}{\eta_{r e l, i}}}\right)}{100 \cdot \frac{\sigma_{K}\left(t_{0}\right)}{E_{K}\left(t_{0}\right)}} \\
& =\frac{E_{K}\left(t_{0}\right)}{100} \cdot \frac{P_{c}\left(t_{c}\right)}{P_{c}\left(t_{0}\right)} \cdot\left[\frac{t_{c}}{\eta_{0}}+\sum_{i} \frac{1}{E_{r e l, i}} \cdot\left(1-\mathrm{e}^{\frac{-t_{c} \cdot E_{r e l, i}}{\eta_{r e l, i}}}\right)\right]
\end{aligned}
$$

Gleichung 13

Damit kann das zeitabhängige Verformungsverhalten von Spannglasbauteilen auf Kriechanteile der in der Konstruktion verwendeten Kunststoffe reduziert werden. Der Einfluss wird bezüglich der Vorspannkraft in Kapitel 2.7.6 untersucht.

75 Die Materialparameter aus [Ebert 2014] resultieren in einem Verzerrungswert in [\%]. Entsprechend ist die Anfangsverzerrung $\varepsilon_{K}\left(\mathrm{t}_{0}\right)$ mit dem Faktor 100 zu multiplizieren. 


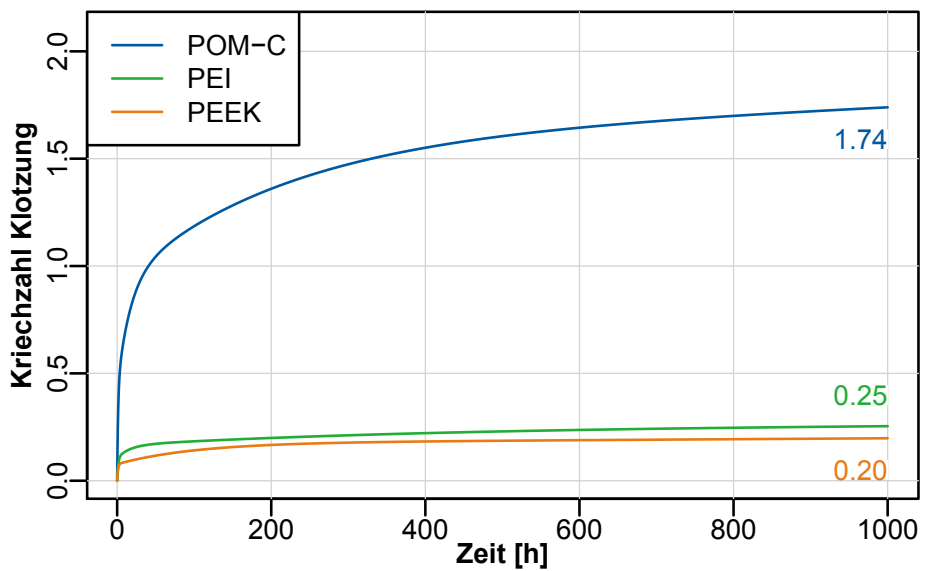

\subsubsection{Bewehrungsmaterial}

Nach [DIN EN 1992] werden sowohl Betonstahl als auch Spannstahl als Bewehrungsmaterialien normativ erfasst. Darüber hinaus wird eine Verstärkung auch mit Fasern und/oder textilen Materialien angewendet.

Im Konstruktiven Glasbau werden folgende Baustoffe als Bewehrungsmaterial im weitesten Sinne verwendet:

- Stahl, ${ }^{76}$

- Spannstahl, ${ }^{77}$

- Glasfaserverstärkter Kunststoff (GFK), ${ }^{78}$

- Holz, ${ }^{79}$

- Stahlbeton ${ }^{80}$ und

- Polycarbonat ${ }^{81}$.

\section{Bild 31}

Kriechzahlen für Klotzungswerkstoffe im Konstruktiven Glasbau mit dem Materialmodell nach Ebert [2014] bei einer angenommenen Temperatur von $23^{\circ} \mathrm{C}$.

Auch hierfür wird auf der sicheren Seite liegend angenommen, dass die kriecherzeugende Last über die Zeit konstant auf dem Niveau der Belastung verbleibt $\mathrm{P}_{\mathrm{c}}\left(\mathrm{t}_{\mathrm{c}}\right)=\mathrm{P}_{\mathrm{c}}\left(\mathrm{t}_{\mathrm{O}}\right)$.

76 Vergleiche [Abeln \& Preckwinkel 2011], [Veer et al. 2003], [Wellershoff \& Sedlacek 2003], [Bos et al. 2004], [Louter \& Veer 2007b], [Louter \& Veer 2007a], [Louter et al. 2007], [Bucak et al. 2009b], [Bucak et al. 2009a], [Ølgaard et al. 2009], [Ungermann et al. 2010], [Weller et al. 2009b], [Weller et al. 2010b], [Weller et al. 2010a], [Louter 2013].

77 Vergleiche [Bos et al. 2004], [Belis et al. 2006], [Louter \& Veer 2007b], [Louter \& Veer 2007a].

78 Vergleiche [Cagnacci et al. 2009], [Louter 2009], [Louter et al. 2010], [Louter 2011].

79 Vergleiche [Cruz \& Pequeno 2008].

80 Vergleiche [Freytag 2001], [Freytag et al. 2012].

81 Vergleiche [Hobbelmann et al. 2001], [Veer et al. 2003], [Weimar 2011], [Härth 2013]. 
Spannstahl hat sich als Bauprodukt für vorgespannte Tragwerke aufgrund seiner hohen Festigkeit, geringer Anfälligkeit gegen Korrosion (Dauerhaftigkeit) sowie seiner vielfältigen Möglichkeiten zum Fügen durchgesetzt. Zusätzlich sind diverse Querschnittsformen verfügbar. Damit kann die Bewehrung so gewählt werden, dass Vorspannkräfte bei Dehnwegen aufgebracht werden, die in akzeptablem Maß während der Lebenszeit des Tragwerks erhalten bleiben. Es sind keine ungünstigen Veränderungen der Materialeigenschaften bei der Anwendung im Glasbau zu erwarten ${ }^{82}$, sodass der Baustoff grundsätzlich ebenso in Spannglasträgern dieser Arbeit verwendet wird. ${ }^{83}$

Es erscheint nicht notwendig, ein gesondertes Bewehrungsmaterial für den Machbarkeitsnachweis von Spannglasträgern zu finden.

\subsubsection{Komponenten von Spannsystemen}

Im Spannbetonbau wird zwischen Verankerungsvorrichtungen oder Ankerkörpern und Spanngliedkopplungen (Kopplungsvorrichtungen oder kurz: Kopplungen) unterschieden. Die zugehörigen Begriffsdefinitionen können sinngemäß übernommen werden. ${ }^{84}$

Diese Komponenten sind keine geregelten Bauprodukte, sodass eine Zulassung nötig ist. ${ }^{85} \mathrm{Im}$ Rahmen der Arbeit werden derartige Konstruktionen für bewehrte Glasträger erstellt, welche ihrerseits einer eigenen Zulassung bedürfen, worin die verwendeten Komponten geregelt sind.

Bild 32 zeigt eine unmittelbare Gegenüberstellung von analogen Konstruktionen im Spannbetonbau und im Konstruk-

82 Tatsächlich werden Spannstähle bereits in Seilnetzfassaden im Zusammenhang mit Glaskonstruktionen angewendet.

83 Es werden die Relaxationsgleichungen nach [DIN EN 1992] angewendet, um die Spannkraftverluste der Dauerversuche in Kapitel 3 zu berechnen.

84 Vergleiche [DIN EN 1992-1-1, Kap. 3.4.1.1].

85 Vergleiche [DIN EN 1992-1-1, Kap. 3.4.1.1], insbesondere [DIN EN 1992-1-1, Kap. 3.4 und 3.4.1 (NA.1)]]. 
tiven Glasbau. Dabei werden Verankerungen im frischen Beton eingegossen. Nach dem Erhärten entsteht ein formund stoffschlüssiger Verbund. Aufgrund der hohen Verarbeitungstemperaturen von Floatglas ist eine analoge Integration der Komponenten nicht möglich, weil die Kaltverformung der Spannstähle bei diesen Temperaturen rückgängig gemacht wird. ${ }^{86}$ Daher kann die Bewehrung von Spannglasträgern nur über eine stoffschlüssige Klebkonstruktion oder einen Formschluss mittels leistungsfähiger Klotzungen verankert werden.
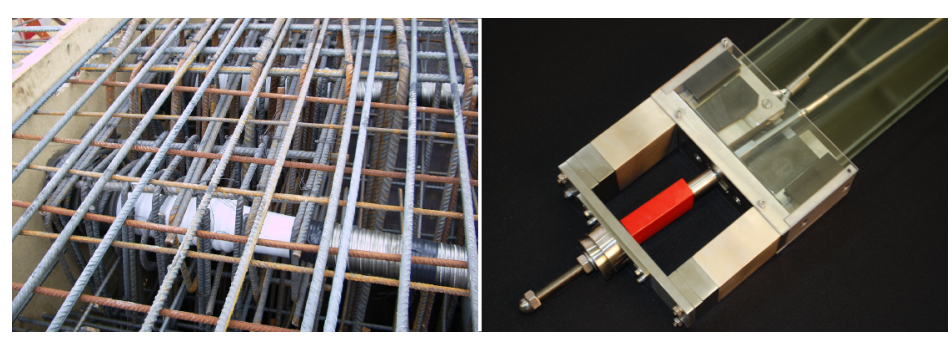

Bild 32

Verankerungen und Kopplungen im Spannbetonbau vor dem Einbau des Betons (links) und im Konstruktiven Glasbau (rechts).

\subsubsection{Querschnittsgestaltung}

Beton wird in der Regel bei Temperaturen oberhalb des Gefrierpunktes auf der Baustelle oder im Fertigteilwerk zum Bauteil verarbeitet, indem das fließfähige Material in eine Schalung gebracht wird. Die Schalung kann variabel hergestellt werden, sodass Betonquerschnitte in nahezu beliebiger Form gegossen werden können. Glas hingegen wird als Flachglas bei Schmelztemperaturen oberhalb von $1000^{\circ} \mathrm{C}$ hergestellt, sodass nur flache Scheiben zu Querschnitten weiterverarbeitet werden. ${ }^{87}$ Für die Herstellung geformter Querschnitte aus Flachglas sind individuell angepasste und damit aufwändige Fügeverfahren nötig. Zusätzlich handelt es sich aufgrund der Plattenform in der Regel um schlanke Querschnitte, bei denen unter Belastung oft ein Stabilitätsversagen auftritt. ${ }^{88}$

86 Vergleiche [DIN EN 10052] und [DIN 17022].

87 Alternativ sind Profilbauglas und Glasrohre verfügbar. Transparente, gegossene Glasbausteine kommen in [Göppert \& Paech 2008] und [Nijsse 2015] im Einzelfall vor. In beiden Fällen wird ein (zeit-) aufwendiges Kühlverfahren zur Herstellung beschrieben.

88 Stabilitätsprobleme von Glasträgern werden analytisch und experimentell in [Luible 2004], [Kasper 2005] und [Langosch et al. 2013] untersucht. 
Bild 33

Doppelquerschnitt.

Links: auf freier Länge Rechts: am Umlenkpunkt.
Hier wird daher das Konzept eines "Doppelquerschnitts" verfolgt (Bild 33). Dabei werden Glasscheiben durch diskrete Verbindungen zu einem abschnittsweisen Hohlkastenquerschnitt kombiniert. Bisherige Versuche, vollständige Hohlkastenquerschnitte herzustellen, beinhalteten lange, linienförmige Klebfugen, deren Herstellverfahren problematisch ist. ${ }^{89}$ Mit der hier gewählten Lösung wird das Fügeproblem minimiert und ein Stabilitätsversagen durch eine gegenseitige Stützung beider Querschnittshälften günstig beeinflusst. Es ergibt sich eine optimierte Materialanwendung, welche durch den offen bleibenden Zwischenraum eine freie Anpassung des Bewehrungsverlaufes ermöglicht.

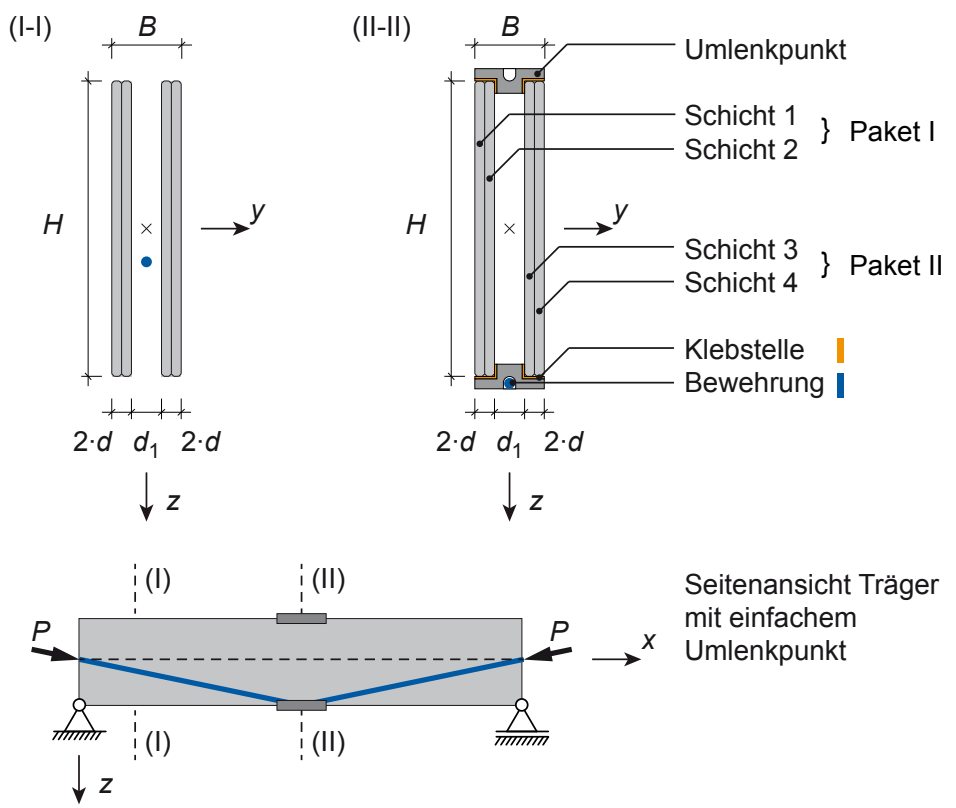

\section{$2.6 \quad$ Dauerhaftigkeit}

Alle Tragwerke sind so zu bemessen, dass zeitliche Eigenschaftsänderungen keinen unvorhergesehenen, ungünstigen Einfluss haben beziehungsweise adäquat berücksichtigt werden. ${ }^{90}$ Es ist zu überprüfen, ob zeitabhängige Effekte vorkommen, ob deren Einfluss günstig oder ungünstig wirkt, wie

\footnotetext{
89 Vergleiche [Bos et al. 2004] und [Louter 2008].

90 Vergleiche [DIN EN 1990, Kap. 2.4].
} 
groß der Einfluss ist und ob er gegebenenfalls vernachlässigbar ist.

Die Eigenschaften von Kalk-Natronsilicatglas sind dabei bereits ausreichend untersucht und in der Produktnorm [DIN EN 572] hinterlegt. Ebenso existieren Untersuchungen zum Dauerhaftigkeitsverhalten von Kunststoffen ${ }^{91}$ und Klebstoffen ${ }^{92}$ im Konstruktiven Glasbau. Damit wird die Dauerhaftigkeit der Komponenten von Spannglasträgern für den Umfang dieser Arbeit als bekannt vorausgesetzt.

In [DIN EN 1992] werden zunächst Anforderungen an die Dauerhaftigkeit ${ }^{93}$ aufgestellt, welche als Grundannahmen für die normativen Regelungen zu sehen sind und bereits in Tafel 1 zusammengestellt wurden. Die werkstoffspezifischen Regelungen im Stahlbeton- und Spannbetonbau beziehen sich auf den Nachweis einer ausreichenden Betondeckung ${ }^{94}$ und damit auf die Vermeidung von Bewehrungskorrosion, eines dauerhaften Verbundes sowie des Brandschutzes $^{95}$. Da die Bewehrung nach dem vorgestellten Doppelquerschnittskonzept nicht vom Grundmaterial umschlossen ist, sind diese Regeln nicht anwendbar. Weiterführend kann untersucht werden, inwiefern eine Korrosionssicherung durch das vollständige Umhüllen mit lastabtragenden Klebungen im Konstruktiven Glasbau möglich ist. So kann es weiterführend sinnvoll sein, die vielfältig auftretenden Umwelteinflüsse, die Änderungen der Materialeigenschaften nach sich ziehen, in Expositionsklassen einzustufen - wie es bereits im Stahlbeton- und Spannbetonbau praktiziert wird. ${ }^{96}$ So ist beispielsweise bekannt, dass es zu einer Delamination von Verbund-Sicherheitsglas kommt, wenn die PVB-Zwischenschicht Feuchte, anderen Stoffen (hier zum Beispiel Klebstoffen), UV-Strahlung sowie wechselnden Temperaturen ausgesetzt ist. ${ }^{97}$ Diese können bereits als Expositionsklassen verstanden werden. Beispielsweise kann es zu einer Feuchtebelastung infolge Luftfeuchte, stehenden Was-

91 Vergleiche [Ebert 2014] und [Kothe 2013].

92 Vergleiche Klebstoffforschung im Konstruktiven Glasbau, beispielsweise in [Härth 2013]

93 Vergleiche [DIN EN 1992-1-1, Kap. 4.3].

94 Vergleiche [DIN EN 1992-1-1, Kap. 4.4]

95 Vergleiche [Fingerloos et al. 2012, S. 234].

96 Vergleiche [DIN EN 1992-1-1, Tabelle 4.1].

97 Vergleiche [Ensslen 2005]. 
sers, offener Beregnung und Tropfenbildung kommen. Einige dieser Lasten können bereits konstruktiv ausgeschlossen beziehungsweise minimiert werden. Dennoch ist ein vollständiger Ausschluss von Feuchtigkeit, zum Beispiel aus dem architektonischen Wunsch nach freien Glaskanten, nie vollkommen Rechnung zu tragen. Es muss ein Kompromiss gefunden werden, der sich in der Definition von Expositionsklassen niederschlagen kann. Ein gleichartiges Vorgehen kann zur einheitlichen Definition dauerhafter Klebungen und Klotzungskonstruktionen führen.

\subsection{Schnittgrößenermittlung}

\subsubsection{Allgemeines}

Für eine zeit- und kostengerechte Schnittgrößenermittlung an Spannglasträgern mit der gewählten Querschnittsform werden ingenieurmäßige Idealisierungen nötig, die in diesem Abschnitt zusammengestellt werden. Sie dienen als Vergleichsgrundlage für die nachfolgenden experimentellen Untersuchungen. Mit dieser Gegenüberstellung wird eine Beurteilung der Idealisierungen und der analytischen Beschreibung des Tragverhaltens möglich.

Man geht in der Regel von homogenen Werkstoffeigenschaften und planmäßiger Geometrie des Tragwerks aus. Abweichungen werden mittels Imperfektionsannahmen und weiteren Idealisierungen (Kapitel 2.7.2 und 2.7.3) berücksichtigt. Eine Unterscheidung der Berechnung mit linearen und nichtlinearen Materialmodellen erfolgt in Kapitel 2.7.4 und 2.7.5.

Im Stahlbeton- und Spannbetonbau wird zwischen globalen und lokalen Schnittgrößen unterschieden. Globale Schnittgrößen ergeben sich an Stellen linearer Verzerrungsverteilung. Lokale Schnittgrößen sind zusätzlich dort zu ermitteln, wo diese Annahme nicht gerechtfertigt ist. Dies betrifft den Bereich lokaler Lasteinleitung, beispielsweise an Auflagern und Verankerungsbereichen oder an Stellen sprunghafter 
Querschnitts- und damit Steifigkeitsänderungen. ${ }^{98}$ Letztere treten auch während der Rissentwicklung im Grundmaterial auf. Daher müssen beim Übergang in den Zustand II besondere Überlegungen angestellt werden. ${ }^{99}$ Die Modellierung der Rissentwicklung für die Schnittgrößenermittlung tragender Glasbauteile ist derzeit ein Forschungsthema und damit noch nicht für Bemessungsaufgaben geeignet. ${ }^{100}$

Zusätzlich ist die Zeitabhängigkeit der Baustoffeigenschaften zu idealisieren, welche insbesondere im Zusammenhang mit der Vorspannung auftreten. Hierfür wurden vielfältige Modelle entwickelt, welche jedoch für eine ingenieurmäßige Anwendung zu umfangreich sind, sodass allgemeingültige Vereinfachungen in Bemessungsnormen zusammengestellt werden. Insbesondere das Kriechen und Schwinden des Betons sowie die geringe Festigkeit der verwendeten Stähle in frühen Spannbetonkonstruktionen hat zu einem Verlust der Vorspannkraft geführt. ${ }^{101}$ Der Vergleich zwischen Stahlbeton- und Spannbetonbau und dem Konstruktiven Glasbau wird in Kapitel 2.7.6 und Kapitel 2.7.7 diskutiert.

\subsubsection{Imperfektionen}

Imperfektionen sind Abweichungen vom planmäßigen Zustand und im Grenzzustand der Tragfähigkeit zu berücksichtigen, wenn sie ungünstige Auswirkungen haben. ${ }^{102}$ Für die Berechnung stabilitätsgefährdeter Tragwerke wie Spannglasträger nach Elastizitätstheorie II. Ordnung, werden geometrische Ersatzimperfektionen berücksichtigt. Die breite Verfügbarkeit ausreichend leistungsfähiger FEM-Programme ermöglichte die Durchsetzung dieser Methode. Sie beinhaltet den Einfluss von:

98 Vergleiche [DIN EN 1992-1-1, Kap. 5.1.1].

99 So verwenden bereits Louter [2011] und Härth [2013] Verzerrungsansätze für gerissene Glasquerschnitte.

100 Einige Forschungsansätze finden sich in [Invernizzi et al. 2011], [Louter 2011] und [Bedon \& Louter 2016].

101 Vergleiche [DIN EN 1992-1-1, Kap. 5.10] sowie [Zilch \& Weiher 2008].

102 Vergleiche [DIN EN 1992-1-1, Kap. 5.2]. 
Bild 34

Infolge der Imperfektion findet eine Biegebelastung $\mathrm{q}_{\mathrm{w}}$ einen Hebelarm $\theta \cdot \mathrm{H} / 2$ um die starke Biegeachse vor, welcher ein Torsionsmoment erzeugt. Auf diese Weise kann das Biegedrillknicken als Spannungsproblem abgebildet werden.

Eine vertikale Imperfektion $\mathrm{w}_{0}$ wird in der Regel nicht angenommen. Sie erzeugt keine zusätzliche ungünstige Verformung unter dem hier behandelten Belastungsszenario.
- geometrischen Imperfektionen aus Vorverdrehung beziehungsweise Vorverschiebung als Abweichungen von der planmäßigen, idealen Geometrie einschließlich Fertigungstoleranzen und Montageungenauigkeiten.

- strukturellen Imperfektionen (Materialimperfektionen) als Abweichung von der planmäßigen Struktureigenschaft wie Eigenspannungen im Sinne von ungewollten verbleibenden Eigenspannungen in thermisch entspanntem Glas, inhomogene Vorspannungszustände thermisch vorgespannter Gläser, Querschnittstoleranzen und Werkstoffinhomogenitäten.

- Tragwerksimperfektionen als Auswirkungen unvermeidbarer Ungenauigkeiten bei der Bauwerkserstellung.

Für Glasträger sind normativ keine Regelungen erfasst. Für eine initiale, seitliche Vorverformung gibt es Untersuchungen in [Luible 2004] und [Belis et al. 2011], die den Einfluss der Glasart und des Laminierprozesses sowie des Zwischenschichtmaterials (PVB und SGP) beinhalten. Dabei wird zusammenfassend ein Wert von L/400 vorgeschlagen (Bild 34).

„perfekt“ „imperfekt" als geometrische

Abweichung von der perfekten Form
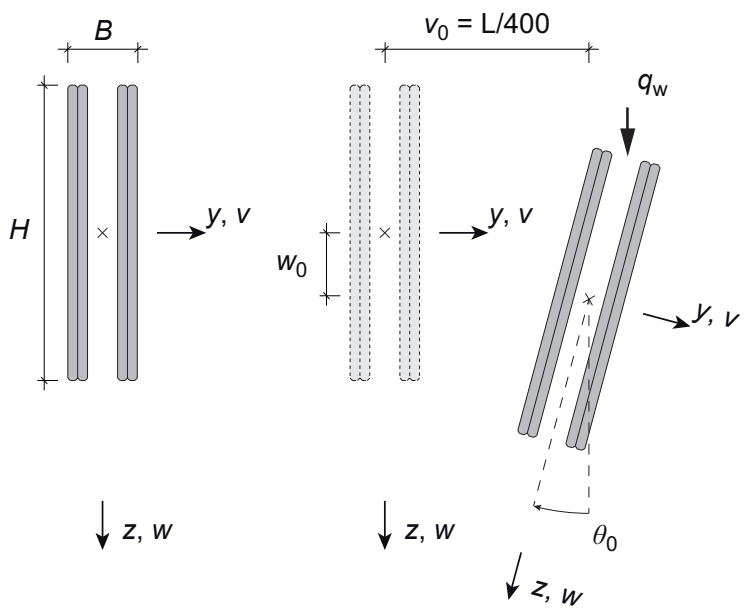


\subsubsection{Idealisierung}

Baukonstruktionen werden in Bauteile zerlegt und im Sinne eines statischen Modells idealisiert, um sie einer ingenieurmäßigen Schnittgrößenberechnung zugänglich zu machen.

In [DIN EN 1991-1-1, Kap. 5.3] werden Zuordnungen zu Tragwerksmodellen entsprechend der Funktion und Geometrie gemacht (Tafel 5). Damit wird sichergestellt, dass der Anwendungsbereich der jeweils anzuwendenden Tragwerksmodelle eingehalten wird.

\begin{tabular}{lll}
\hline Tragwerkmodell & $\begin{array}{l}\text { Stahlbeton- und } \\
\text { Spannbetonbau }\end{array}$ & $\begin{array}{l}\text { Konstruktiver } \\
\text { Glasbau }\end{array}$ \\
\hline Balken & $\mathrm{B} / \mathrm{H}<5 ; \mathrm{L} \geq 3 \cdot \mathrm{H}$ & Glasträger \\
\hline Platte & $\mathrm{B} \geq 5 \cdot \mathrm{H} ; \mathrm{L} \geq 3 \cdot \mathrm{H}$ & $\begin{array}{l}\text { Verglasungen nach } \\
\text { DIN 18008-1 bis }-6\end{array}$ \\
\hline $\begin{array}{l}\text { wandartige } \\
\text { Scheibe }\end{array}$ & $\mathrm{B} / \mathrm{H}<5 ; \mathrm{L}<3 \cdot \mathrm{H}$ & $\begin{array}{l}\text { ausfachende Schei- } \\
\text { be oder Vertikal- } \\
\text { verglasungen unter } \\
\text { Eigenlast }\end{array}$ \\
\hline Stütze & $\mathrm{H} \leq 4 \cdot \mathrm{B}$ & Glasstütze \\
\hline Wand & $\mathrm{B}>4 \cdot \mathrm{H}$ & Glaswand \\
\hline
\end{tabular}

Im Konstruktiven Glasbau ist insbesondere die Abgrenzung zum Glasträger von Belang. Mit der Begrenzung der Schlankheit im Stahlbeton- und Spannbetonbau auf $\mathrm{L} / \mathrm{H} \geq 3$ wird sichergestellt, dass die Idealisierung eines linearen Verzerrungsverlaufes über die Querschnittshöhe unter Biegebelastung ausreichend genau ist. ${ }^{103}$ Mit der Begrenzung auf $\mathrm{B} / \mathrm{H}<5$ wird sichergestellt, dass die Annahme einer unbehinderten Querverzerrung unter Biegung ausreichend genau ist und somit die Anwendung der linearen Balkentheorie rechtfertigt.

Beide Regelungen beziehen sich auf die reine Geometrie, sind materialunabhängig und können damit für den Glasbau übernommen werden. Es bleibt abzuwarten, inwieweit die deutsche und europäische Glasbau-Normung ${ }^{104}$ eine

103 Damit wird die Bernoulli-Hypothese vom Ebenbleiben der Querschnitte eingeführt.

104 Es sind sowohl eine [DIN 18008-7] und ein „Eurocode for Structural Glass" [Feldmann et al. 2014] geplant.
Tafel 5

Übersicht über Idealisierung der Tragwerke nach [DIN

EN 1992-1-1, Kap. 5.3.1] Rippen- oder Kassettendecken sind im Konstruktiven Glasbau unüblich.

B ... Querschnittsbreite H ... Querschnittshöhe

L ....Stützweite 
Abgrenzung zwischen Balken und Platte beziehungsweise Scheibe definiert.

Die geometrischen Angaben in [DIN EN 1991-1-1, Kap. 5.3.2] wie "Mitwirkende Plattenbreite" und „effektive Stützweite" beziehen sich auf konkrete Ausführungsformen im Stahlbetonund Spannbetonbau. Diese Konstruktionsart ist im Glasbau derzeit unüblich. Es bleibt weiterer Untersuchungen vorbehalten, das Potenzial solcher Glaskonstruktionen abzuschätzen und auszunutzen.

\subsubsection{Lineare Berechnung}

Die grundlegende Ingenieuraufgabe liegt in der Wahl eines passenden Verfahrens, um das reale Verhalten eines Tragwerks sicher und wirtschaftlich abbilden zu können. Eine wesentliche Entscheidung besteht in der Wahl eines linearen oder nichtlinearen Ansatzes zur Ermittlung der SchnittgröBen.

Unter einer linearen Berechnung ${ }^{105}$ wird die Idealisierung des Baustoffverhaltens mit einem linearen Baustoffgesetz verstanden. ${ }^{106}$ Für den Stahlbeton- und Spannbetonbau bedeutet dies, dass die Schnittgrößen unter der Annahme ungerissenen Betons und nicht-fließender Bewehrung errechnet werden.

Im Konstruktiven Glasbau werden ebenso Baustoffe, insbesondere Kunststoffe und Metalle, verwendet, die ein nichtlineares Materialverhalten aufweisen. Diese werden jedoch üblicherweise nicht ausgenutzt, um plastische Verformungen zu reduzieren. Daher ist eine lineare Berechnung von Spannungen und Schnittgrößen im Glasbau eine sinnvolle und vielfältig angewandte Methode.

In Analogie zur linearen Berücksichtigung gerissener Betonquerschnitte werden darüber hinaus in Anwendungen

\footnotetext{
105 Hier werden Analogien zu [DIN EN 1992-1-1, Kap. 5.4, 5.5, 5.6] zusammengefasst gesucht.

106 Vergleiche [DIN EN 1990, Kap. 1.5.6].
} 
mit wissenschaftlichem Hintergrund Verfahren angewendet, die die Trageigenschaften des gebrochenen Verbundglases rechnerisch ansetzen. ${ }^{107}$ Mit diesem teilzerstörten Glasquerschnitt ist erneut eine lineare Berechnung möglich. ${ }^{108}$

Grundsätzlich ist sicherzustellen, dass das Kräftegleichgewicht eingehalten wird. Die verbundlos vorgespannten Spannglasträger dieser Arbeit sind innerlich statisch unbestimmt. Es müssen zusätzliche Verformungsrandbedingungen erfüllt werden, um das Kräftegleichgewicht zu finden. Im Folgenden wird das Tragsystem des Spannglasträgers idealisiert und dessen analytische Beschreibung schrittweise hergeleitet. Zunächst wird der Lastfall Vorspannung, dann die äußere Belastung der Bewehrung thematisiert. Damit ist eine Abschätzung des Tragverhaltens möglich.

\section{Vorspannen eines geraden Seils}

Zum mechanischen Vorspannen ist eine äußere Kraft nötig, die das Seil um den Betrag $\Delta \mathrm{L}$ dehnt (Bild 35).

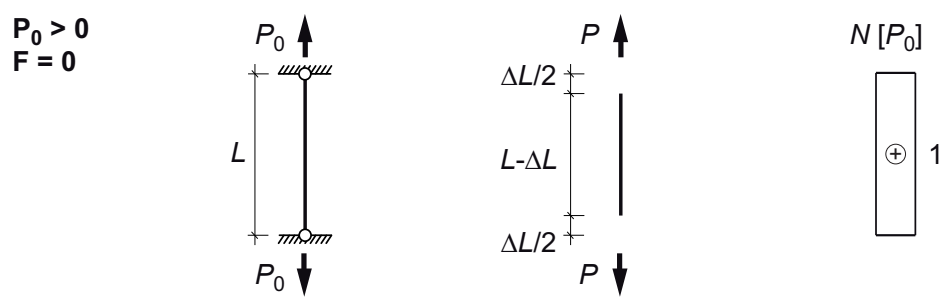

Bild 35

Systembild: ein Seil wird vorgespannt.

Dieser Fall entspricht dem zentrisch, gerade vorgespannten Träger (Bild 22) unter der Annahme, dass die Stauchung der Glaskonstruktion gegenüber der Dehnung des Seils vernachlässigt wird.

107 Vergleiche [Feirabend 2010], [Louter 2011] und [Härth 2013], wobei derzeit noch nicht abschließend geklärt ist, unter welchen Randbedingungen, insbesondere für welche Glasarten und Zwischenschichten, ein sicherer Ansatz gebrochener Schichten möglich ist. Vergleiche auch [Baraldi et al. 2016].

108 [DIN 18008-1, Kap. 9.2.1] gibt an, dass die Resttragfähigkeit einer Glaskonstruktion rechnerisch mit intakten, nicht gebrochenen Glaslagen erfolgen kann. 
Bei bekannten geometrischen Verhältnissen $\left(L, A_{p}\right)$ sowie den Materialeigenschaften $\left(E_{p}\right)$ kann die nötige Vorspannkraft berechnet werden: ${ }^{109}$

Gleichung 14

$$
\begin{array}{ll}
P_{0}=\sigma_{0} \cdot A_{p}=\varepsilon_{p} \cdot E_{p} & A_{p}=\frac{\Delta L}{L} \cdot E_{p} \cdot A_{p} \\
L & \begin{array}{l}
\text { planmäßiger Abstand der Veran- } \\
\text { kerungen, planmäßige Länge des } \\
\text { vorgespannten Seils }
\end{array} \\
\sigma_{p}=P_{0} / A_{p} & \text { Spannung im idealen Seil } \\
A_{p} & \text { Wirksame Querschnittsfläche des Seils } \\
E_{p} & \text { E-Modul der Spannbewehrung }
\end{array}
$$

Das Seil wird an den nun erreichten Auflagerpunkten verankert, sodass die Vorspannkraft nunmehr durch die Lager abgetragen wird. Es findet eine Systemänderung statt. Diese wird in der Regel nicht derart modelliert, vielmehr kann zur Berechnung direkt der letztlich auftretende Normalkraftzustand angenommen werden.

\section{Belastung eines vorgespannten Seils in Seilrichtung}

Ein vorgespanntes Seil wird durch eine äußere Last in Seilrichtung mittig belastet (Bild 36). Die Seilkraft des oberen Tragseils steigt um die äußere Kraft an; die Kraft im unteren Spannseil nimmt um denselben Betrag ab. Sobald die äußere Last die Vorspannkraft erreicht, egalisiert sie die Seilkraft im unteren Seilabschnitt. Dieses kann jedoch keine Drucklasten aufnehmen und trägt nicht mehr zum Lastabtrag bei. Es kommt zu einer Änderung des Tragsystems, da nur der obere Seilabschnitt weiterhin wirkt.

Aus den berechneten Seilkräften ergeben sich deren Längenänderungen und damit wiederrum das Last-Verschiebungsverhalten. In Bild 37 ist ein Vergleich zwischen dem vorgespannten und dem nicht vorgespannten System aus Bild 36 dargestellt. Bis zur Entlastung des Spannseils reagiert das vorgespannte System mit geringeren Verformungen auf eine äußere Belastung.

${ }^{109}$ Einem idealen Seil wird keinerlei Biegesteifigkeit zugeordnet. 
$P_{0}=0$

$\mathrm{F}>0$
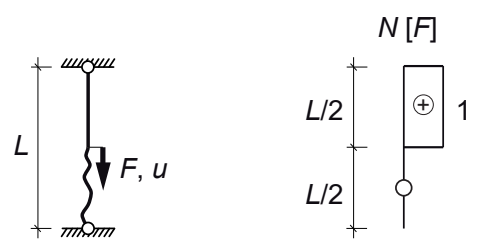

$\mathrm{P}_{0}>0$

$0<F \leq 2 \cdot P_{0}$

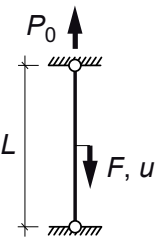

$P_{0} \downarrow$

$P_{0}>0$

$F>2 \cdot P_{0}$
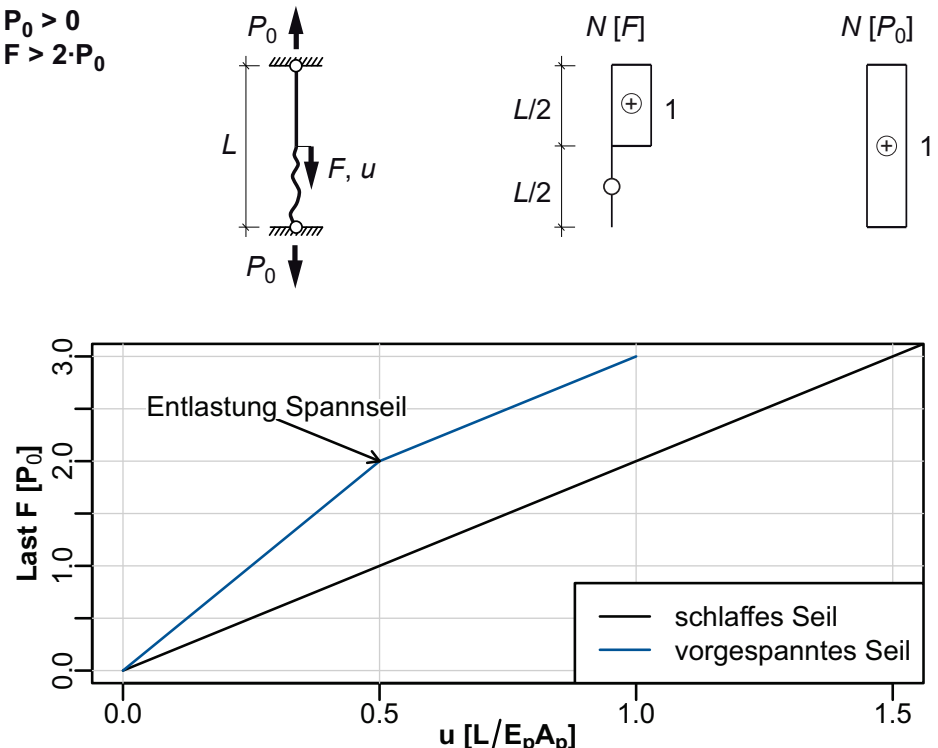

Bild 36

Systembild: ein vorgespanntes Seil wird in Seilrichtung belastet. Die Komponenten der Seilkraft sind getrennt nach Vorspannkraft $P_{0}$ und Auflast $F$ angegeben.

Situation bei einer Belastung unterhalb der Vorspannkraft.

Nach dem Erreichen der Vorspannkraft trägt nur das obere Seil. Es kommt zu einer Systemänderung.

Bild 37

Last-Verformungskurve eines in Seilrichtung belasteten Seils gegenüber einem nicht vorgespannten Seil.

Es zeigt sich, dass das vorgespannte System steifer reagiert. Beim Erreichen der Vorspannkraft kommt es zum Systemwechsel.

Diese Steifigkeitssteigerung durch eine Vorspannung wird gezielt zum Lastabtrag herangezogen. Bei Entlastung eines Seilabschnittes kommt es jedoch zu einer Systemänderung, die in der Berechnung zu berücksichtigen ist. So kann ein Seil auf der Biegedruckseite eines zentrisch vorgespannten Trägers nach Bild 23 vollständig entlastet werden.

\section{Belastung eines Seils quer zur Seilrichtung}

Hier werden Spannglasträger mit gerader, umgelenkter Bewehrung untersucht, wofür eine Umlenkkraft erforderlich ist 
Bild 38

Trennung des gekoppelten Tragsystems in statisch be stimmte Teilsysteme. Über die Verträglichkeitsbedingung findet eine Kopplung der Wirkung statt.
(Bild 25). Damit werden an allen Kontaktpunkten zwischen Hauptträger und Bewehrung Kräfte übertragen. Diese verursachen ihrerseits eine Verformung des Tragwerks, sodass sich die Verbindungspunkte zwischen Bewehrung und Hauptträger verschieben. Daraus resultiert eine erneute Längenänderung des Seiles, welche zu einer Änderung der im Seil wirkenden Kraft führt. Die wirkenden Kräfte sind daher über die Verformung miteinander gekoppelt, das System ist innerlich statisch unbestimmt.

Die Kraftgrößenmethode (Arbeitsgleichung) wird zur Bestimmung der Schnittgrößen angewendet. Dazu wird das unbestimmte System in ein Hauptsystem (,0“ System) und ein System mit Wirkung der statisch Unbestimmten („," System) zerlegt. Es bietet sich an, die Wirkung der Seilkraft als Unbestimmte einzuführen. Sie wird damit direkt als Seilkraftänderung infolge der Biegebelastung interpretiert. Bild 38 zeigt den Ansatz für den Fall der zweifachen Seilumlenkung und einer äußeren Belastung aus Vierpunkt-Biegung.

statisch unbestimmtes System

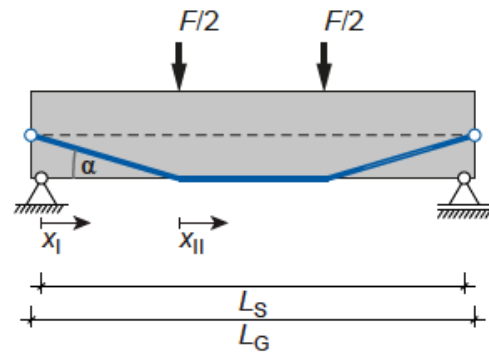

"1" - System

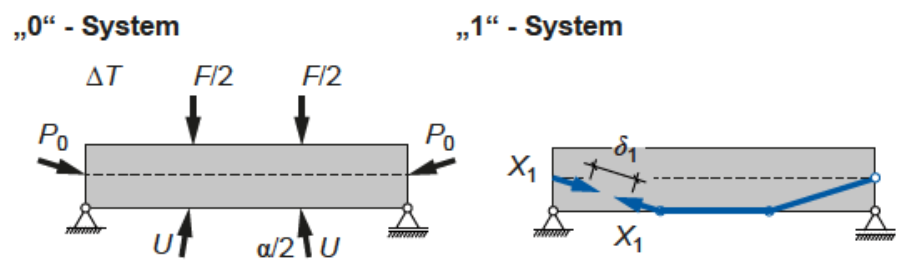

Es ist nun diejenige Seilkraftänderung $X_{1}$ gesucht, die unter der eingetragenen Belastung eine Längung des Seils verur- 
sacht, welche die Lücke $\delta_{1}$ schließt. Dieser Zusammenhang wird als Verträglichkeitsbedingung formuliert:

$$
\delta_{1}=\delta_{10}+X_{1} \cdot \delta_{11}=0
$$

$\begin{array}{ll}\delta_{10} & \begin{array}{l}\text { Längung des Seils infolge äußerer } \\ \text { Lasten } \\ \text { Längung des Seils infolge statisch } \\ \delta_{11}\end{array} \\ X_{1} & \begin{array}{l}\text { unbestimmter Wirkung } \\ \text { statisch Unbestimmte }\end{array}\end{array}$

Die beiden Verschiebungsgrößen $\delta_{10}$ und $\delta_{11}$ ergeben sich mittels der Arbeitsgleichung aus den zugehörigen SchnittgröBenverläufen. Fügt man letztlich deren Ergebnisse zusammen, ergibt sich die statisch Unbestimmte $X_{1}$ als Seilkraftänderung $\Delta P$ in Gleichung 16. Der Berechnungsverlauf ist in Anlage $A$ ausführlich dargestellt.

$$
\begin{aligned}
& x_{1}=-\frac{\delta_{10}}{\delta_{11}}=x_{1 . p} \cdot P_{0}+x_{1 . F} \cdot F+x_{1 . T} \cdot \Delta T \\
& x_{1 . p}=-\frac{E_{p} \cdot A_{p} \cdot\left[27 \cdot I_{y} \cdot\left(2 \cdot \cos ^{2}(\alpha)+1\right)+5 \cdot A_{G} \cdot L_{g e s}^{2} \cdot \sin ^{2}(\alpha)\right]}{E_{p} \cdot A_{p} \cdot\left[27 \cdot I_{y} \cdot\left(2 \cdot \cos ^{2}(\alpha)+1\right)+5 \cdot A_{G} \cdot L_{g e s}^{2} \cdot \sin ^{2}(\alpha)\right]+81 \cdot E_{G} \cdot A_{G} \cdot I_{y}} \\
& x_{1 . F}=\frac{5 \cdot A_{G} \cdot E_{p} \cdot A_{p} \cdot L_{g e s}^{2} \cdot \sin (\alpha)}{162 \cdot I_{y \cdot G} \cdot\left(E_{G} \cdot A_{G}+E_{p} \cdot A_{p}\right)} \\
& x_{1 . T}=\frac{E_{G} \cdot A_{G} \cdot E_{p} \cdot A_{p} \cdot\left(\alpha_{T . p}-\alpha_{T . G}\right)}{E_{G} \cdot A_{G}+E_{p} \cdot A_{p}}
\end{aligned}
$$

Gleichung 16

Damit ist bekannt, welche Kräfte auf den gläsernen Hauptträger wirken. Daraus können alle Schnittgrößen aus der Superposition der Anteile aus dem „0“ und dem „1“ System zur analytischen Beschreibung des Tragverhaltens gewonnen werden. 


\section{Lastumlagerung}

Darüber hinaus können im Stahlbeton- und Spannbetonbau Umlagerungen infolge Rissbildung berücksichtigt werden. ${ }^{110}$ Im Glasbau bedeutet dies die Berücksichtigung eines teilzerstörten, gerissenen Glasquerschnitts bei der Schnittgrößenermittlung. Vereinfachte Verfahren für die Anwendung sind dafür noch nicht bekannt. Das Thema befindet sich weiterhin im Forschungsstadium. So werden ähnliche Methoden beispielsweise in [Feirabend 2010] und [Härth 2013] angewendet. Hierfür sind derzeit noch keine ausreichend gesicherten, allgemeingültigen Verfahren verfügbar, sodass eine gebrochene Glaslage rechnerisch nicht für den Lastabtrag angesetzt wird.

Die Bewehrung des Stahlbetons bringt die Möglichkeit einer plastischen Ausnutzung des Materials, woraus Methoden zur Berücksichtigung nach Plastizitätstheorie entwickelt wurden. ${ }^{111}$ Weiterhin besteht im Glasbau ebenso eine Nachfrage nach einem duktilen Tragverhalten, um dem spröden Bruch des Glasquerschnitts zu begegnen. Ein analoger Ansatz befindet sich für den Glasbau noch im Forschungsstadium. So wenden beispielsweise Martens et al. [2015a] die plastifizierende Stahlbewehrung eines bewehrten gläsernen Durchlaufträgers planmäßig zum Lastabtrag an.

\section{Diskontinuitätsbereiche}

Daneben ist es im Stahlbeton- und Spannbetonbau üblich, in Diskontinuitätsbereichen Stabwerkmodelle zur Bemessung zu verwenden ${ }^{112}$, da aufwendigere numerische Methoden oft unwirtschaftlich sind. Dieses Vorgehen wurde bereits für den Konstruktiven Glasbau übernommen. So beschreibt Fahlbusch [2007] das Resttragverhalten einer gebrochenen Verbundglasplatte mit einem Stabwerkmodell. Ebenso analysiert Puller [2012] den lokalen Lasteintrag einer einlaminierten Stahllasche in die Zwischenschicht einer Verbundglas-

\footnotetext{
110 Vergleiche [DIN EN 1992-1-1, Kap. 5.5].

111 Vergleiche [DIN EN 1992-1-1, Kap. 5.6].

112 Vergleiche [DIN EN 1992-1-1, Kap. 5.6.4].
} 
scheibe mit einem Stabwerkmodell (Bild 39). Ein Übertrag auf normativem Niveau ist dabei noch nicht erfolgt. In Analogie zum Stahlbeton- und Spannbetonbau wird weiterführend vorgeschlagen, die Abbildung von Glaskonstruktionen in wirtschaftlich anzuwendende Stabwerksmodelle fortzuentwickeln.
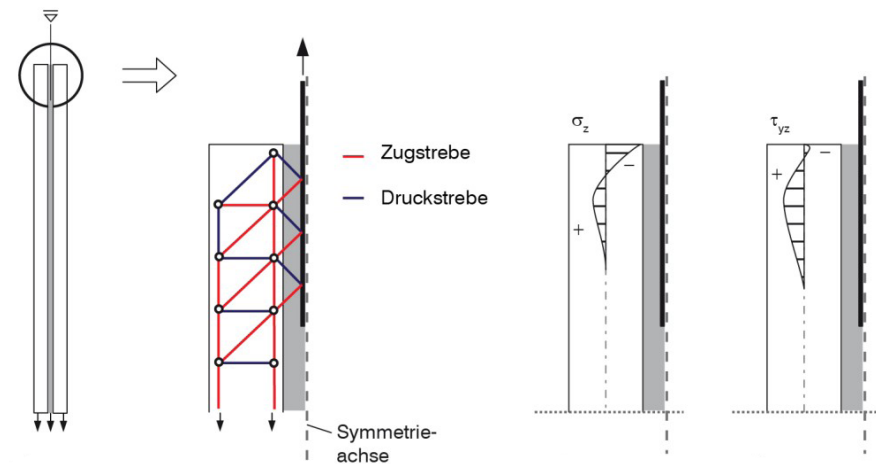

\section{Seitliches Ausweichen schlanker Träger}

Im deutschen nationalen Anhang zum Eurocode 2 werden weitere allgemeine Bestimmungen genannt, die die Anwendung nichtlinearer Methoden betreffen. Demnach muss die Verformung des Tragwerks berücksichtigt werden, wenn sie zu einem wesentlichen Anstieg der Schnittgrößen führen und somit ungünstig wirken. ${ }^{113}$ Dabei ist insbesondere das Biegedrillknicken schlanker Biegeträger zu nennen. Das seitliche Ausweichen schlanker Glasträger ist bereits vielfach beschrieben. ${ }^{114}$ Ebenso wie in Berechnungsmethoden im Stahlbeton- und Spannbetonbau ist der Einfluss zeitabhängiger Verformung zu berücksichtigen. ${ }^{115}$ Im Ergebnis werden alle numerischen Berechnungen an imperfekten Spannglasträgern nach Theorie II. Ordnung geführt.

\footnotetext{
113 Vergleiche [DIN EN 1992-1-1, Kap. 5.1.1.(NA.8-10)].

114 Vergleiche [Luible 2004], [Kasper 2005], [Holberndt 2006]. Darüber hinaus wird Flachglas zu Verbundglas laminiert. Dabei werden maximal 5 bis 7 Glasschichten gestapelt. Durch die Begrenzung der Glasdicken ergibt sich eine Begrenzung der Glasträgerdicken und damit ein herstellungsbedingter Grund, auf den Einsatz größerer Querschnittsbreiten zu verzichten.

115 Vergleiche [DIN EN 1992-1-1, Kap. 5.8.4].
}

\section{Bild 39}

Stabwerkmodell zur Beschreibung des lokalen Lasteintrags in die Zwischenschicht einer Verbundglasscheibe über eine Stahllasche aus [Puller 2012].

Fahlbusch [2007] verwendet ein Modell zur Beschreibung des Resttragverhaltens einer VSG-Scheibe mit vollständig gebrochenem Glas. 
Insbesondere die zusätzliche Normalkraftbeanspruchung durch die Vorspannung sorgt für eine erhöhte Biegedrillknickgefährdung. Die analytischen Grundlagen zur Beschreibung der Stabilitätseigenschaften unter Biege- und Normalkraftbeanspruchung sind gelegt. ${ }^{116}$ Die darin gewählten Randbedingungen müssen mit der konstruktiven Ausführung abgeglichen werden, um ein realitätsnahes Gesamtmodell zu erhalten.

\section{Zusammenfassung zu linearen Berechnungsmethoden}

Damit wird zusammengefasst, dass lineare Berechnungsmethoden unabhängig vom Grundmaterial verwendet werden können.

Des Weiteren ist hiermit ein analytisches Grundgerüst vorhanden, um das Tragverhalten von Spannglasträgern, insbesondere die Beschreibung der auftretenden Seilkräfte, zu beschreiben.

\subsubsection{Nichtlineare Berechnung}

Unter einer nichtlinearen Berechnung wird die modellhafte Berücksichtigung der nichtlinearen Baustoffeigenschaften verstanden. ${ }^{117}$ Dabei wird das Tragverhalten über die elastische Grenze hinaus beschrieben. Im Stahlbeton- und Spannbetonbau werden elastisch-ideal plastisch und elastischplastische Modelle angewandt, um die Materialeigenschaften stufenweise realitätsnah abzubilden. ${ }^{118}$

Nichtlinearitäten können auch für die relevanten Baustoffe im Konstruktiven Glasbau angewandt werden.

\footnotetext{
116 Vergleiche [Langosch 2012].

117 Hier werden Analogien zu [DIN EN 1992-1-1, Kap. 5.7; 5.8 und 5.9] zusammengefasst gesucht.

118 Vergleiche [DIN EN 1990, Kap. 1.5.6 und Kap. 5.7].
} 


\section{Glas}

Glas verhält sich unter Biegebelastung linear elastisch bis zum spröden Bruch. ${ }^{119}$ Damit sind nichtlineare Verfahren für diesen Materialanteil im Querschnitt irrelevant. Gebrochene Querschnittsanteile werden rechnerisch vollständig entfernt, was einen Nachweis auf der sicheren Seite liegend erlaubt. Im Rahmen der experimentellen Studie in Kapitel 3 ist zu klären, inwieweit diese Annahme praktisch erreicht wird. Eine Abweichung zwischen rechnerischem und experimentellem Ergebnis kann in der Tragfähigkeit der gebrochenen Glaslage begründet sein. Insbesondere während der Versuche zur Ermittlung der Resttrageigenschaften von Spannglasträgern in Kapitel 3.5 werden in einigen Fällen alle Glaslagen gebrochen, ohne ein volles Systemversagen zu beobachten, sodass die Mitwirkung des Glases auf Druck notwendigerweise erfolgen muss.

Ein steiferes Tragverhalten als rechnerisch angenommen kann in Analogie zum Stahlbeton- und Spannbetonbau als „tension-stiffening“, als Mitwirkung des intakten Glases zwischen den Rissen oder der Lastumlagerung auf intakte Einzelscheiben im Verbundglas, bezeichnet werden. Auch für diesen Fall liegen jedoch keine vollständigen Untersuchungsergebnisse vor und können ausblickend untersucht werden, wenn zwischen realen Ergebnissen und theoretischem Ansatz ohne Mitwirkung des gebrochenen Glases ausreichend hohe Abweichung festgestellt wird. Dies kann als Indiz für ein auszunutzendes Tragpotenzial gewertet werden.

\section{Zwischenschicht}

Übliche Zwischenschichtmaterialien im Konstruktiven Glasbau sind für ihre nichtlinearen Materialeigenschaften bekannt. Werden Sie rechnerisch angesetzt, können verfügbare Materialmodelle ${ }^{120}$ verwendet werden, um nichtlineare Tragpotenziale zu nutzen. Darüber hinaus bleibt jedoch zu

\footnotetext{
119 Vergleiche Kapitel 2.5, ab Seite 47.

120 Vergleiche [Sobek et al. 2000], [Schuler 2003] sowie [Sedlacek et al. 2003].
} 
berücksichtigen, dass neben den Trageigenschaften auch sicherheitsrelevante Produkteigenschaften, wie eine ausreichende Splitterbindung, zu berücksichtigen sind. Im Zuge der analytischen Ansätze wird die Zwischenschicht durch ein lineares Materialmodell vereinfacht abgebildet.

\section{Glaskontaktwerkstoffe}

Ebenso wie Zwischenschichtmaterialien verhalten sich Glaskontaktwerkstoffe, insbesondere aus der Materialgruppe der Kunststoffe, oft nichtlinear. Nach Ebert [2014] sollte diese Tragreserve nicht herangezogen werden, um bleibende Verformungen an kritischen Konstruktionselementen zu vermeiden.

\section{Bewehrung}

Materialmodelle für Bewehrungsmaterialien, insbesondere Spannstahl, können aus zugehörigen Zulassungen entnommen werden. ${ }^{121}$

Für den Stahlbeton- und Spannbetonbau stehen bereits spezialisierte nichtlineare Berechnungsverfahren auf normativer Grundlage zur Verfügung. ${ }^{122}$ Grundsätzlich wird ein Gleichgewichtsnachweis am verformten System gefordert. Dies ist baustoffunabhängig mechanisch begründet und wird im Konstruktiven Glasbau übernommen. Dafür stehen bereits umfangreiche Untersuchungen ${ }^{123}$ unter der Berücksichtigung glasspezifischer Konstruktionsweisen, wie dem Laminataufbau mit weicher Zwischenschicht, zur Verfügung und können angewendet werden.

${ }^{121}$ Nach [DIN EN 1992-1-1, Kap. 5.1.4] sind Berechnungen nach Theorie I., II. oder III. Ordnung möglich.

122 Vergleiche [DIN EN 1992-1-1, Kap. 5.8 und Kap. 5.9]. Insbesondere die Verwendung von Grenzschlankheiten ermöglicht ein vereinfachtes Berechnungsverfahren.

${ }^{123}$ Vergleiche [Luible 2004], [Kasper 2005], [Holberndt 2006] und [Langosch 2012]. 


\subsubsection{Zeitabhängigkeit der Vorspannkraft}

Das mechanische Vorspannen von Glastragwerken ist der Kern dieser Arbeit. Dabei wird die Vorspannung als Lastfall begriffen, womit er sich direkt auf die Schnittgrößenermittlung auswirkt. Aus dem Spannbetonbau ist bekannt, dass sich die Spannkraft über die Lebenszeit signifikant verändert. Die zugehörigen Betrachtungen können analog für Spannglasträger übernommen werden.

Zur Begrenzung der Spannstahlspannung wird die maximale Vorspannkraft $P_{\max }$ nach dem Absetzen der Pressenkraft $(t=0)$ gemäß der Zulassung der Bewehrung begrenzt. ${ }^{124}$ Damit wird sichergestellt, dass es zu keinen plastischen Verformungen des Spanngliedes kommt. ${ }^{125}$ Diese Begrenzung bezieht sich damit ausschließlich auf die Eigenschaften des Bewehrungsmaterials und kann entsprechend im Glasbau angewendet werden (Gleichung 17).

$$
P_{\max }=A_{p} \cdot \sigma_{p, \max }
$$

$$
\begin{array}{ll}
P_{\max } & \text { maximal auftretende Vorspannkraft } \\
A_{p} & \text { Querschnittsfläche Spannstahl } \\
\sigma_{p, \max } & \text { maximale Spannstahlspannung }
\end{array}
$$

Im Weiteren kann es beim Vorspannen von Spannbetontragwerken zu lokalem Druckversagen hinter der Verankerung kommen. Daher wird die zulässige Betondruckspannung begrenzt. ${ }^{126}$ Eine solche Versagensform kann im Spannglasbau nicht ausgeschlossen werden. In [Ebert 2014] wird die Einleitung von Drucklasten in Glaskanten über Kunststoffklotzungen untersucht, konstruktive Hinweise gegeben sowie Bemessungsvorschläge zu deren Handhabung gemacht. Diese wurden für die Entwicklung der Konstruktionsdetails angewendet. ${ }^{127}$ Darüber hinaus sind tatsächlich verwendete Konstruktionen detaillierter zu untersuchen.

\footnotetext{
${ }^{124}$ Vergleiche [DIN EN 1992-1-1, Kap. 5.10.2.1].

125 Begrenzung auf $0,8 \cdot f_{p k}$ bzw. 0,9 $f_{p 0,1 k}$ gemäß der Zulassung des Spannstahls.

126 Vergleiche [DIN EN 1992-1-1, Kap. 5.10.2.2] sowie spezifische technische Zulassung (abZ, ETA).

${ }^{127}$ Vergleiche Kapitel 3.2, ab Seite 104.
} 
Zumindest ist die Entwicklung der Glasdruckfestigkeit nicht gleichermaßen zeitabhängig wie bei der Aushärtung von Beton, sodass Detailregelungen zu deren Begrenzung bezüglich des Alters entfallen können.

Aus dem Spannbetonbau ist bekannt, dass die eingetragene Spannkraft zeitabhängig abnimmt. ${ }^{128}$ Die Spannkraftverluste werden in sofortige und zeitabhängige Verluste bezüglich des Mittelwerts der Vorspannkraft gegliedert. ${ }^{129}$ Dieser Effekt ist grundsätzlich auch für Spannglasträger zu erwarten und wird daher detailliert betrachtet, um bereits vorab sicherzustellen, dass ein vollständiger Verlust der günstigen Auswirkungen vermieden werden kann.

\section{Zeitunabhängige (sofortige) Verluste}

Sofortige Verluste ergeben sich aus der maximal aufgebrachten Spannkraft $P_{\max }$ abzüglich der Verluste je nach Verbund. Diese sind in Anlehnung an [DIN EN 1992-1-1, Kap. 5.10.3(3)] zusammengestellt:

Gleichung 18

$$
P_{m 0}(x)=P_{\max }-\Delta P_{e l}-\Delta P_{r}-\Delta P_{\mu}-\Delta P_{s l}
$$

$\begin{array}{ll}P_{m 0} & \begin{array}{l}\text { Mittelwert der Vorspannkraft nach dem } \\ \text { Spannvorgang (abzüglich sofortiger } \\ \text { Verluste) } \\ P_{\text {max }}\end{array} \\ \Delta P_{e l} & \begin{array}{l}\text { maximal auftretende Vorspannkraft } \\ \text { Verluste infolge elastischer Verbund- } \\ \text { glasverformung }\end{array} \\ \Delta P_{r} & \begin{array}{l}\text { Verluste infolge Kurzzeitrelaxation des } \\ \text { Spannstahls nach dessen allgemeiner } \\ \text { bauaufsichtlicher Zulassung }\end{array} \\ \Delta P_{\mu} & \begin{array}{l}\text { Reibungsverluste, z. B. an Umlenkun- } \\ \text { gen }\end{array} \\ \Delta P_{s l} & \text { Verluste infolge Verankerungsschlupf }\end{array}$

Die Verluste an Spannkraft im Spannbetonbau sind von der Art des Verbundes abhängig. ${ }^{130}$ Spannkraftverluste bei so-

\footnotetext{
128 Vergleiche [DIN EN 1992-1-1, Kap. 5.10].

${ }^{129}$ Vergleiche [DIN EN 1992-1-1, Kap. 5.10.3].

130 Vergleiche [DIN EN 1992-1-1, Kap. 5.10.4 und Kap. 5.10.5].
} 
fortigem Verbund ${ }^{131}$ werden in dieser Arbeit nicht behandelt, können jedoch konzeptionell für vorgespannte Glasträger zusammengestellt werden:

- Reibungsverluste an Umlenkungen und Verankerungen während des Spannens treten mechanisch bedingt auf und werden experimentell erfasst (Kapitel 3.3).

- Im Zeitraum zwischen dem Vorspannen der Bewehrung und der Herstellung des wirksamen Verbundes zum Grundmaterial treten baustoffbedingt bereits Relaxationsverluste auf, die mit den vorhandenen Kenntnissen über das Bewehrungsmaterial beschreibbar sind.

- Werden Glasträger im Spannbett vorgespannt, kommt es beim Lösen der Bewehrung zur elastischen Verzerrung des Glases sowie des beteiligten Klebstoffes. Dieser Anteil ist unter Berücksichtigung zusätzlich auftretender Mechanismen zu untersuchen.

Die Verluste bei nachträglichem Verbund werden für Glasträger wie folgt ermittelt. Sie werden entsprechend der geltenden Verträglichkeitsbedingung für den Fall „ohne Verbund“ angepasst.

Zunächst wird das Bauteil aus Glas, Klotzungs- und Zwischenschichtmaterial durch die Vorspannkraft elastisch verkürzt:

$$
\Delta P_{e l}=\Delta P_{e l, j}+\Delta P_{K, e l}+\Delta P_{Z, e l}
$$

$\begin{array}{ll}\Delta P_{e l, j} & \text { Spannkraftverluste infolge elastischer } \\ & \text { Glasverzerrung beim Vorspannen } \\ & \text { mehrerer Spannglieder }{ }^{132} \\ & \text { Spannkraftverluste infolge elastischer } \\ & \text { Klotzungsstauchung nach Ebert [2014] } \\ & \text { Spannkraftverluste infolge elastischer } \\ & \text { Verzerrung des Zwischenschichtmate- } \\ & \text { rials. }\end{array}$

Reibungsverluste werden experimentell bestimmt (Kapitel 3.3). Bei externer Vorspannung ohne Verbund wird ein Reibungsverlust nur bei der Berechnung der mittleren Vor-

131 Vergleiche [DIN EN 1992-1-1, Kap. 5.10.4] sowie die Forschungsarbeiten von Cupac \& Louter [2015]. 
spannkraft berücksichtigt. ${ }^{133}$ Im Eurocode 2 werden dazu die mechanischen Gesetzmäßigkeiten der Seilreibung angewendet. Diese sind zunächst vom Grundmaterial unabhängig und werden weiter verwendet.

Der Verlust innerhalb einer Verankerungskonstruktion ist unabhängig von der übrigen Konstruktion und kann daher entsprechend der Angaben der Zulassung übernommen werden, wenn ein derartiges verlustbehaftetes Verankerungsverfahren verwendet wird. Im Rahmen dieser Arbeit wird das Seil über eine Spannmutter am Trägerende verankert. Die experimentellen Untersuchungen eines 9,0 m langen Spannglasträgers haben keine messbaren Verluste aus Verankerungsschlupf gezeigt. ${ }^{134}$

\section{Zeitabhängige Verluste}

Zeitabhängige Spannkraftverluste $\Delta \mathrm{P}_{\mathrm{c}, \mathrm{r}}$ ergeben sich aus zeitabhängigen Verzerrungsanteilen innerhalb der Konstruktion und werden in Anlehnung an [DIN EN 1992-1-1, Kap. 5.10.3(4)] zusammengestellt:

Gleichung 20

$$
P_{m, t}(x)=P_{m 0}(x)-\Delta P_{c, r}(x)
$$

$$
\begin{array}{ll}
P_{m, t} & \text { Mittelwert der Vorspannkraft } \\
P_{m 0} & \text { Mittelwert der Vorspannkraft nach dem } \\
& \text { Spannvorgang (siehe Gleichung 18) } \\
\Delta P_{c, r} & \text { zeitabhängige Vorspannkraftverluste } \\
& \text { infolge Kriechens (siehe Kapitel 2.5.3) } \\
& \text { und Relaxation (siehe Kapitel 2.5.4). }
\end{array}
$$

Der Eurocode 2 gibt einen vereinfachten Zusammenhang zwischen Kriechen, Schwinden und Relaxation und dem Kraftverlust im Spannglied an. ${ }^{135}$ Dieser unterliegt zahlreichen baustoffspezifischen Annahmen und wird daher für den Glasbau neu zusammengestellt. ${ }^{136}$ Als maßgebend für den Gesamtverlust werden die Einzelverluste aus dem Kriechen

\footnotetext{
133 Vergleiche [DIN EN 1992-1-1, Kap. 5.10.5.2(4)].

134 Vergleiche [Engelmann \& Weller 2016].

135 Vergleiche [DIN EN 1992-1-1, Kap. 5.10.6]

136 Vergleiche [Fingerloos et al. 2012].
} 
der Klotzung $\Delta \mathrm{P}_{\mathrm{K}, \mathrm{c}}$ und der Zwischenschicht $\Delta \mathrm{P}_{\mathrm{Z}, \mathrm{c}}$ sowie die Relaxation des Spannstahls $\Delta \mathrm{P}_{\mathrm{p}, \mathrm{r}}$ addiert.

$$
\Delta P_{c, r}=\Delta P_{K, c}+\Delta P_{Z, c}+\Delta P_{p, r}
$$

$\begin{array}{ll}\Delta P_{K, c} & \text { Spannkraftverluste infolge Kriechens } \\ \Delta P_{Z, c} & \text { der Klotzungsmaterialien } \\ & \text { Spannkraftverluste infolge Kriechens } \\ & \text { der Zwischenschicht } \\ & \text { Spannkraftverluste infolge Langzeitre- } \\ & \text { laxation des Spannstahls }\end{array}$

Dabei wird die Verkürzung des Bauteils der Verkürzung des Spanngliedes gleichgesetzt ${ }^{137}$, sodass die folgenden $\mathrm{Zu}$ sammenhänge aus der Wirkung der Klotzung und der Zwischenschicht zusammengestellt werden.

\section{Kriechen der Klotzung}

Die Verkürzung des Spanngliedes $\Delta \mathrm{L}_{p}$ ist äquivalent zur Verkürzung der Klotzung an beiden Enden des Glasträgers infolge einer Kriechstauchung $\left(\Delta \mathrm{L}_{K, \mathrm{c}}\right)$. Der Zusammenhang ist in Gleichung 22 beschrieben.

$$
\begin{aligned}
\Delta P_{K, c} & =A_{p} \cdot \Delta \sigma_{p, c}=A_{p} \cdot E_{p} \cdot \varepsilon_{p, c}=A_{p} \cdot E_{p} \cdot \frac{\Delta L_{p}}{L_{p, 0}} \\
& =E_{p} \cdot A_{p} \cdot \frac{2 \cdot \Delta L_{K, c}}{L_{p, 0}}
\end{aligned}
$$

\begin{tabular}{|c|c|}
\hline$\Delta \sigma_{p, c}$ & $\begin{array}{l}\text { Spannungsänderung der Bewehrung } \\
\text { infolge einer Kriechverformung }\end{array}$ \\
\hline$A_{p}$ & $\begin{array}{l}\text { Wirksame Querschnittsfläche der } \\
\text { Bewehrung }\end{array}$ \\
\hline$E_{p}$ & E-Modul der Bewehrung \\
\hline$\varepsilon_{p, c}$ & $\begin{array}{l}\text { Verzerrung der Bewehrung infolge } \\
\text { Kriechens }\end{array}$ \\
\hline$\Delta L_{p}$ & Verkürzung des Spanngliedes \\
\hline$L_{p, 0}$ & Ausgangslänge des Spanngliedes \\
\hline$\Delta L_{K, c}$ & $\begin{array}{l}\text { Verkürzung der Klotzung infolge Krie- } \\
\text { chens nach Gleichung } 8 \text {, Seite } 56\end{array}$ \\
\hline
\end{tabular}

137 Vergleiche Gleichung 4, Seite 52. 
Mit den in Kapitel 2.5 zusammengestellten Materialeigenschaften der Klotzung kann der Spannkraftverlust in Abhängigkeit der Situation zu Belastungsbeginn mithilfe der Kriechzahl $\varphi_{\mathrm{K}}$ (Gleichung 13) beschrieben werden.

$$
\begin{aligned}
& \Delta P_{K, c}=E_{p} \cdot A_{p} \cdot \frac{2 \cdot L_{K, 0} \cdot \varphi_{K} \cdot \varepsilon_{K}\left(t_{0}\right)}{L_{p, 0}} \\
& \varepsilon_{K}\left(t_{0}\right) \quad \begin{array}{l}
\text { elastische Klotzungsstauchung zu } \\
\text { Belastungsbeginn }
\end{array}
\end{aligned}
$$

Darüber hinaus ist die Lasteinleitung so zu konstruieren, dass es zu einer homogenen Lastverteilung über die Glasträgerhöhe kommt und Spannungsspitzen reduziert werden. Dadurch ist die Annahme einer konstanten Normalstauchung in der Klotzung gerechtfertigt. Der Spannkraftverlust ist damit von der kriecherzeugenden Ausgangsbelastung $P_{c}\left(t_{0}\right)$ im Verhältnis zur Querschnittsfläche der Klotzung $A_{K}$ abhängig.

Gleichung 24

$$
\Delta P_{K, c}=\frac{E_{p} \cdot A_{p}}{E_{K}\left(t_{0}\right) \cdot A_{K}} \cdot \frac{2 \cdot L_{K, 0}}{L_{p, 0}} \cdot \varphi_{K} \cdot P_{K, c}\left(t_{0}\right)
$$

\section{Kriechen der Zwischenschicht}

Ebenso führt eine Verkürzung des Verbundglasträgers durch das Kriechen der Zwischenschicht ${ }^{138}$ zu einer Verkürzung der Bewehrung und damit zu einem Spannkraftverlust:

Gleichung 25

$$
\begin{aligned}
\Delta P_{Z, c}=E_{p} \cdot A_{p} \cdot \frac{\Delta L_{Z, c}}{L_{p, 0}} & \\
\Delta L_{Z, c} \quad & \text { Verkürzung des Verbundglases infolge } \\
& \text { Kriechens der Zwischenschicht nach } \\
& \text { Gleichung 6, Seite 55 }
\end{aligned}
$$

Mittels der vorab bestimmten Kriechzahl $\varphi_{\mathrm{z}}$ (Gleichung 7) wird der Spannkraftverlust in Abhängigkeit der kriecherzeugenden Anfangsbelastung angegeben. Dabei wird vereinfachend davon ausgegangen, dass die Schubspannung in der

138 Vergleiche Bild 29, Seite 53. 
Zwischenschicht aus dem Verhältnis der Anfangskraft und der Fläche der Zwischenschicht ermittelt werden kann.

$$
\Delta P_{Z, c}=\frac{E_{p} \cdot A_{p}}{G_{Z}\left(T, t_{0}\right) \cdot A_{Z}} \cdot \frac{d_{Z}}{L_{p, 0}} \cdot \varphi_{Z} \cdot P_{Z, c}\left(t_{0}\right)
$$

Gleichung 26

\section{Spannstahlrelaxation}

Die Relaxation des Spannstahls tritt in Abhängigkeit der Auslastungen des Spanngliedes auf und kann der Zulassung des Spannstahls entnommen werden. Bei hoher Auslastung und großer Relaxation sind üblicherweise Werte bis $\Delta \sigma_{\mathrm{pr}}=4 \mathrm{~N} / \mathrm{mm}^{2}$ zu erwarten. ${ }^{139}$

$$
\Delta P_{p r}=A_{p} \cdot \Delta \sigma_{p r}
$$

\section{Spannkraftverluste in Analogie zum Eurocode 2}

In [DIN EN 1992-1-1] wird ein vereinfachtes Verfahren zur Ermittlung der Spannkraftverluste infolge von Kriechen und Schwinden des Betons sowie der Relaxation der Bewehrung in Gleichung $5.46^{140}$ angegeben. Diese gilt ebenso für Spannglasträger im sofortigen und nachträglichen Verbund unter der Berücksichtigung der Kriechverformung von Klotzung und Zwischenschicht sowie unter Vernachlässigung einer Schwindverzerrung. ${ }^{141}$ Für verbundlos vorgespannte Spannglasträger muss jedoch zusätzlich die Verträglichkeitsbedingung nach Gleichung 1 (Seite 37 ) erfüllt sein. Umge-

\footnotetext{
139 Vergleiche [DIN EN 1992-1-1, GI. 3.28 bis 3.30].

140 Vergleiche [DIN EN 1992-1-1, Kap. 5.10.6]

141 Vergleiche Gleichung 7, Seite 55 und Gleichung 13, Seite 58.
} 
schrieben zur Ermittlung der zeitabhängigen Verzerrungsänderungen ergibt sich:

Gleichung 28

Gleichung 29

Gleichung 30

$$
\begin{array}{ll}
\varepsilon_{p, c}=\frac{1}{L_{p, i}} \cdot \int_{L_{p, i}} \Delta \varepsilon V G p, c & \left(x_{p}\right) \mathrm{d} x_{p} \\
\varepsilon_{p, c} & \\
& \text { Verzerrungsänderung des Spannglie- } \\
\Delta \varepsilon_{V G p, c} & \text { des infolge eines Kriechvorganges } \\
& \text { Verzerrungsänderung des Verbundgla- } \\
& \text { ses in Höhe der Spanngliedlage infolge } \\
& \text { eines Kriechvorganges } \\
x_{p} & \text { Laufkoordinate entlang der Schwerach- } \\
& \text { se des Spanngliedes. }
\end{array}
$$

Dabei resultiert die Verzerrungsänderung des Spanngliedes aus der zugeordneten Spannungsänderung infolge Kriechens und der Bewehrungsrelaxation.

$$
\begin{aligned}
\Delta \sigma_{p, c+r} & =\Delta \sigma_{p, r}+\Delta \sigma_{p, c}=\Delta \sigma_{p, r}+\Delta \varepsilon_{p, c} \cdot E_{p} \\
\Delta \varepsilon_{p, c} & =\frac{\Delta \sigma_{p, c+r}}{E_{p}}-\frac{\Delta \sigma_{p, r}}{E_{p}}
\end{aligned}
$$

$\begin{array}{ll}\Delta \sigma_{p, c+r} & \text { Spannungsänderung des Spannglie- } \\ & \text { des infolge eines Kriechvorganges und } \\ & \text { Spannstahlrelaxation } \\ & \text { Spannungsänderung des Spannglie- } \\ & \text { des infolge der Spannstahlrelaxation } \\ \Delta \sigma_{p, r} & \text { Spannungsänderung des Spannglie- } \\ & \text { des infolge eines Kriechvorganges }\end{array}$

Die Verzerrungsänderung im Zweifach-Verbundglas ergibt sich aus den Längenänderungen von Klotzung, Zwischenschicht und Glas nach Gleichung 4 (Seite 52).

$$
\Delta \varepsilon_{V G,, c}=\frac{\Delta L_{V G p, c}}{L_{V G, 0}}=\frac{2 \cdot \Delta L_{K p, c}+\Delta L_{Z p, c}+2 \cdot \Delta L_{G p, c}}{L_{V G, 0}}
$$

$\begin{array}{ll}\Delta L_{K p, c} & \text { Längenänderung der Klotzung auf } \\ & \text { Höhe der Spanngliedlage } \\ & \text { Längenänderung der Zwischenschicht } \\ & \text { auf Höhe der Spanngliedlage } \\ & \text { Längenänderung des Glases auf Höhe } \\ & \text { der Spanngliedlage }\end{array}$


Der folgende Abschnitt zeigt die Ermittlung für jeden Summanden. Die Stauchungsänderung der Klotzung auf Höhe der Spanngliedlage $\Delta \varepsilon_{K p, c}$ entspricht damit der Kriechverzerrung nach Gleichung 12 (Seite 58), welche unter Zuhilfenahme der ermittelten Kriechzahl nach Gleichung 13 (Seite 58) wie folgt aufgeschrieben werden kann. Die Belastung resultiert ausschließlich aus der Normalbeanspruchung im Träger.

$$
\begin{aligned}
\Delta L_{K p, c} & =\Delta \varepsilon_{K p, c} \cdot L_{K, 0}=\Delta \varepsilon_{K, c} \cdot L_{K, 0}=\varepsilon_{K}\left(t_{0}\right) \cdot \varphi_{K} \cdot L_{K, 0} \\
& =\frac{\sigma_{K}\left(t_{0}\right)}{E_{K}\left(t_{0}\right)} \cdot \varphi_{K} \cdot L_{K, 0}
\end{aligned}
$$

Im Spannbetonbau wird hierfür zusätzlich die Laständerung über die Zeit sowie die Kriechfunktion als charakteristische Materialeigenschaft des Betons berücksichtigt. Mangels solcher Angaben für Klotzungswerkstoffe wird dieser Summand vernachlässigt und die volle, eingangs wirkende Kraft als kriecherzeugend über den gesamten Zeitraum angesetzt. Damit ergeben sich größere Kriechverzerrungen und folglich rechnerisch größere Spannkraftverluste. Die Annahme liegt demnach auf der sicheren Seite, kann aber ausblickend auf dieser Basis verfeinert werden.

Für das Zwischenschichtmaterial wird unter denselben Annahmen ${ }^{142}$ eine kriechbedingte Längenänderung bestimmt:

$$
\begin{aligned}
\Delta L_{Z p, c} & =\Delta L_{Z, c}=L_{Z, 0} \cdot \varphi_{Z}=d_{Z} \cdot \tan \left(\gamma_{Z}\left(t_{0}\right)\right) \cdot \varphi_{0} \\
& =d_{Z} \cdot \frac{\tau_{Z}\left(t_{0}\right)}{G_{Z}\left(t_{0}\right)} \cdot \varphi_{Z}
\end{aligned}
$$

Die Längenänderung der Glasschichten im Verbundglas ist von der im Glas wirkenden Spannung und damit von den veränderlichen Schnittgrößen entlang der Trägerlängsachse abhängig (Gleichung 33). Der Term entfällt für Träger aus Kalk-Natronsilicatglas $\left(\varphi_{G}=0\right)$, jedoch darf ein Verbundglas nach [DIN EN ISO 12543-1] auch aus einem „Vergla-

\footnotetext{
142 Dies betrifft insbesondere die Annahme, dass das Kriechen der Zwischenschicht konstruktionsbedingt auf eine reine und damit über die Trägerlänge konstante, Normalbeanspruchung zurückzuführen ist.
} 
sungsmaterial aus Kunststoff" bestehen, welches Kriecheigenschaften besitzen kann $\left(\varphi_{G}>0\right) .{ }^{143}$

Gleichung 33

$$
\begin{aligned}
\Delta L_{G p, c} & =\Delta \varepsilon_{G p, c} \cdot L_{G, 0}=\varepsilon_{G p, 0} \cdot \varphi_{G} \cdot L_{G, 0} \\
& =\frac{\sigma_{G p, 0}(x)}{E_{G}} \cdot \varphi_{G} \cdot L_{G, 0}
\end{aligned}
$$

Durch das Einsetzen von Gleichung 29 und Gleichung 30 in Gleichung 28 kann der Spannungsverlust berechnet werden:

$$
\begin{aligned}
\frac{\Delta \sigma_{p, C+r}}{E_{p}}-\frac{\Delta \sigma_{p, r}}{E_{p}} & =\frac{1}{L_{p, i}} \cdot \int_{L_{p, i}} \frac{2 \cdot \Delta L_{K p, c}+\Delta L_{Z p, c}+2 \cdot \Delta L_{G p, c}}{L_{V G, 0}} \mathrm{~d} x_{p} \\
\Delta \sigma_{p, c+r} & =\frac{E_{p}}{L_{p, i}} \cdot \frac{2}{L_{V G, 0}} \cdot \int_{L_{p, i}}\left(\frac{\sigma_{K}\left(t_{0}\right)}{E_{K}\left(t_{0}\right)} \cdot \varphi_{K} \cdot L_{K, 0}+\frac{\sigma_{G p, 0}(x)}{E_{G}} \cdot \varphi_{G} \cdot L_{G, 0}\right) \mathrm{d} x_{p} \\
& +\frac{E_{p}}{L_{p, i}} \cdot \frac{1}{L_{V G, 0}} \cdot \int_{L_{p, i}}\left(\frac{d_{Z} \cdot \tau_{Z}\left(t_{0}\right)}{G_{Z}\left(t_{0}\right)} \cdot \varphi_{Z}\right) \mathrm{d} x_{p}+\Delta \sigma_{p, r}
\end{aligned}
$$

Gleichung 34

Gleichung 35

Damit ist der Spannkraftverlust ${ }^{144}$ von den ständigen Schnittgrößen in Klotzung, Zwischenschicht und Verbundglas, vom Zeitpunkt des Vorspannens $t_{0}$ abhängig. Es wird davon ausgegangen, dass in Klotzung und Zwischenschicht konstruktionsbedingt nur die Vorspannkraft kriecherzeugend wirkt. Diese ist bei flacher Spanngliedneigung näherungsweise konstant über die Trägerlänge, sodass die betreffenden Summanden des bestimmten Integrals direkt angegeben werden.

$$
\sigma_{K}\left(t_{0}\right)=\frac{P_{c}\left(t_{0}\right)}{A_{K}} \quad \text { und } \quad \tau_{Z}\left(t_{0}\right)=\frac{P_{c}\left(t_{0}\right)}{A_{Z}}
$$

Die Glasspannung in Höhe der Spannglieder ergibt sich im Rahmen einer Bemessung aus allen maßgeblichen Einwir-

\footnotetext{
143 So ist denkbar Hybridträger einen zu verwenden. Beispiel: [Härth 2013].

144 Der Spannkraftverlust ist in Gleichung 34 durch die Veränderung der Spannung im Spannglied ausgedrückt.
} 
kungen. ${ }^{145}$ Für die ausschließliche Wirkung der Vorspannkraft ergeben sich die Glasspannungen nach Gleichung 36.

$$
\sigma_{G p}\left(t_{0}\right)=\frac{N_{G, 0}}{A_{G}}+\frac{M_{G, 0}}{I_{y, G}} \cdot z_{p}(x)=-\frac{\delta \cdot P_{0}}{A_{G}} \cdot\left(1+\frac{A_{G}}{l_{y, G}} \cdot z_{p}^{2}(x)\right) \quad \text { Gleichung } 36
$$

Darüber hinaus fallen bei flacher Spanngliedführung die Koordinaten der Bewehrung $x_{p}$ und des Trägers $x$ zusammen.

$$
\begin{aligned}
\Delta \sigma_{p, c+r} & =\frac{P_{0} \cdot E_{p}}{L_{V G, 0}} \cdot\left[\frac{2 \cdot \varphi_{K} \cdot L_{K, 0}}{E_{K}\left(t_{0}\right) \cdot A_{K}}+\frac{\varphi_{Z} \cdot d_{Z}}{G_{Z}\left(t_{0}\right) \cdot A_{Z}}\right. \\
& \left.-\frac{2 \cdot L_{G, 0} \cdot \delta \cdot \varphi_{G}}{E_{G} \cdot A_{G} \cdot L_{p}} \cdot \int_{0}^{L_{p}} 1+\frac{A_{G}}{I_{y, G}} \cdot z_{p}^{2}(x) \mathrm{d} x\right]+\Delta \sigma_{p, r}
\end{aligned}
$$

Gleichung 37

Mit diesem Ansatz sind die zeitabhängigen Verformungskomponenten berücksichtigt. Der Verträglichkeitsbedingung zwischen Bewehrungsseil und dem Grundquerschnitt wird damit gerecht. Die Seilkraft ist somit vom Abstand der Verankerungs- und Umlenkpunkte abhängig. Dieser verändert sich infolge der Durchbiegung des elastischen Trägers und ist bisher nicht berücksichtigt. Es wird befürchtet, dass dieser Effekt für schlanke Spannglasträger zu einer signifikanten Seilkraftänderung führen kann, die durch das bisher abgeleitete Modell nicht abgebildet ist. Daher wird die horizontale und vertikale Durchbiegung im Rahmen der experimentellen Untersuchungen aufgenommen, der Effekt quantifiziert und die Anwendung des vorgestellten Modells diskutiert.

145 Vergleiche [DIN EN 1992-1-1, Kap. 5.10.6]. Demnach wird die Betonspannung unter Ansatz eines Anteils der Eigenlast und der Vorspannung oder aus der quasi-ständigen Kombination ermittelt. 


\subsubsection{Vorspannung während der Berechnung}

Aus den vorab zusammengestellten Ergebnissen wird deutlich, dass die zeitlich veränderliche Vorspannung ebenso Berechnungen an unterschiedlichen Zeitpunkten erfordert:

- Für den Zeitpunkt der maximalen Spannkraft allein, ohne Kombination mit weiteren Lasten $\left(t=0 ; P_{\max }\right)$ für den Nachweis des Spanngliedes und der Teilflächenpressung an Verankerungs- und Umlenkpunkten.

- Für den Zeitpunkt unmittelbar nach dem Aufbringen der Spannkraft $\left(g+P_{m 0}\right)$ für den Nachweis der Bewehrung in Interaktion mit dem Grundwerkstoff (Druckbeanspruchung der vorgedrückten Zugzone und Zugbeanspruchung der Druckzone) ohne zeitlich veränderliche Komponenten $\left(\mathrm{t}=\mathrm{t}_{0}\right)$.

- Für den Zeitpunkt nach dem Aufbringen der äußeren Belastung $\left(g+P_{m t} \oplus Q\right)$ für den Nachweis der Bewehrung unter Berücksichtigung aller sofortigen Verluste $\left(t=t_{1}\right)$.

- Für den Zustand unter Berücksichtigung aller Spannkraftverluste $\left(t \rightarrow \infty: \mathrm{g}+\mathrm{P}_{\mathrm{m} \infty} \oplus \mathrm{Q}\right)$ für den Nachweis der Zugbeanspruchung der vorgedrückten Zugzone sowie der Druckbeanspruchung der Druckzone.

Für die verbundlos vorgespannten Spannglasträger dieser Arbeit wird die Annahme übernommen, dass die Spannglieder zwischen den Umlenkstellen gerade verlaufen. Ein Durchhang aus dem Eigengewicht bleibt unberücksichtigt.

\subsection{Grenzzustände und Nachweise}

Grundsätzlich wird nach dem Grenzzustand der Tragfähigkeit (Kapitel 2.8.1) und dem Grenzzustand der Gebrauchstauglichkeit (Kapitel 2.8.2) unterschieden. Diese sind in jeweils zugeordneten Bemessungssituationen ${ }^{146}$ nachzuweisen und können nötigenfalls auf die geplante Nutzungsdauer bezogen sein, womit Aspekte der zeitlichen Abhängigkeit

146 Vergleiche [DIN EN 1990, Kap. 1.5.2]. 
von Einwirkung und Materialeigenschaften abgedeckt werden. ${ }^{147}$ Im Konstruktiven Glasbau wurde dieses Konzept mit der baurechtlichen Einführung der [DIN 18008] übernommen. Jedoch wird hier zusätzlich ein Nachweis der Resttragfähigkeit ${ }^{148}$ gefordert (Kapitel 2.8.3).

\subsubsection{Grenzzustand der Tragfähigkeit}

Im Grenzzustand der Tragfähigkeit (GZT) wird im Allgemeinen der größte Tragwiderstand eines Bauteils ermittelt, um das Tragwerksversagen beziehungsweise einen Einsturz des Bauwerks zu vermeiden. ${ }^{149}$ Damit wird die Gebrauchstauglichkeit für Personen beziehungsweise die Sicherheit des Tragwerks garantiert. ${ }^{150}$

Im Zuge der Spannbetonplanung wird der Verformungszustand aller Baustoffe berechnet und ein Vergleich der daraus resultierenden Spannungen mit einem Bemessungswert der Festigkeit geführt. ${ }^{151}$ Gleichartig wird die auftretende Hauptzugspannung auf der Glasoberfläche begrenzt. ${ }^{152}$ Für verbundlos vorgespannte Tragwerke ist der Spannungszuwachs im Spannglied aus der Verformung des Gesamttragwerks zu ermitteln. ${ }^{153}$ Daraus folgend ist der Last-Verformungszustand des Glases und des Spanngliedes von Spannglasträgern zu ermitteln, um die Grundlage für einen Machbarkeitsnachweis zu legen.

147 [DIN EN 1990, Kap. 3.1] unterscheidet dabei in ständige (übliche Nutzungsbedingungen), vorrübergehende (zeitlich begrenzte Situationen, wie der Bauzustand) und außergewöhnliche Bemessungssituationen, welche Folgen für Teile des Tragwerks (z. B. Brand oder Anprall) oder das gesamte Tragwerk (z. B. Erdbeben) haben. Diese Regeln werden mit [DIN EN 1992-1-1, Kap. 2.2] unverändert übernommen.

148 Resttragfähigkeit im Sinne der [DIN 18008] beschreibt die „Fähigkeit einer Verglasungskonstruktion im Fall eines festgelegten Zerstörungszustandes unter definierten äußeren Lasten [...] über einen ausreichenden Zeitraum standsicher zu sein" [DIN 18008-1, Kap. 3.1.2].

149 Vergleiche [DIN EN 1990, Kap. 1.5.2.13 und Kap. 1.2.5.15].

150 Dazu ist die Lagesicherheit zu gewährleisten, ein Bruch oder eine übermäßige Verformung sowie zeitabhängige Auswirkungen zu vermeiden. [DIN EN 1990, Kap. 3.3] Die Grundlagen sind in [DIN EN 1990, Kap. 6.4] dargestellt.

151 Vergleiche [DIN EN 1992-1-1, Kap. 6.1 bis Kap. 6.4].

152 Vergleiche [DIN 18008-1, Kap. 8.3.2].

153 Vergleiche [DIN EN 1992-1-1, Kap. 5.10.8]. 
Lokale Belastungen, wie beispielsweise in Lasteinleitungsstellen, können bemessungsrelevant werden. Zu deren Beschreibung und Bemessung wurde im Stahlbeton- und Spannbetonbau ein Modell in Fachwerkanalogie normativ bereitgestellt. ${ }^{154}$ Fachwerkmodelle sind in der Glasbaunormung unbekannt und dienen bisher ausschließlich der Beantwortung von Forschungsfragen. ${ }^{155}$ Derartige Versagensformen werden durch die Anwendung der Empfehlungen zur Auslegung von Klotzungskonstruktionen nach Ebert [2014] konstruktiv ausgeschlossen. ${ }^{156}$

Ein Nachweis der Robustheit, das heißt ein gutmütiges Reagieren auf unplanmäßige Zustände, ist wesentlicher Kern des Machbarkeitsnachweises von Spannglasträgern und wird im Zuge der experimentellen Untersuchungen als Nachweis der Resttragfähigkeit sowie des Verhaltens unter Biegebelastungen mehrfach thematisiert.

\subsubsection{Grenzzustand der Gebrauchstauglichkeit}

Neben dem Tragwerkversagen können auch andere Zustände die Benutzung unter normalen Gebrauchsbedingungen ${ }^{157}$ einschränken. Diese Zustände werden beim Nachweis im Grenzzustand der Gebrauchstauglichkeit (GZG) zusammengefasst. ${ }^{158}$ Dabei wird zwischen umkehrbaren und nicht umkehrbaren Zuständen unterschieden (Tafel 6). ${ }^{159}$

Aus Tafel 6 wird bereits deutlich, dass im Grenzzustand der Gebrauchstauglichkeit von Spannbetonkonstruktionen Spannungen, Rissbreiten und die Durchbiegung begrenzt werden. ${ }^{160}$ Der Nachweis des letzteren ist im Glasbau ebenso üblich und kann mit der Ermittlung des Last-DurchbiegungsVerhaltens von Spannglasträgern beschrieben werden. Dar-

\footnotetext{
154 Vergleiche [DIN EN 1992-1-1, Kap. 6.5].

155 Vergleiche Bild 39, Seite 75.

156 Vergleiche [Ebert 2014]. In Kapitel 6 werden Konstruktionsempfehlung um die Anwendung in Spannglasträgern erweitert.

157 Vergleiche [DIN EN 1990, Kap. 3.4].

158 Vergleiche [DIN EN 1992-1-1, Kap. 1.5.2.14].

159 Die Grundlagen sind in [DIN EN 1990, Kap. 6.5] dargestellt.

160 Vergleiche [DIN EN 1992-1-1, Kap. 7.1 bis Kap. 7.2].
} 


\begin{tabular}{lll}
\hline GZG & $\begin{array}{l}\text { Stahlbeton- und } \\
\text { Spannbetonbau }\end{array}$ & $\begin{array}{l}\text { Konstruktiver } \\
\text { Glasbau }\end{array}$ \\
\hline \multirow{3}{*}{ Umkehrbar } & $\begin{array}{l}\text { Elastische Verfor- } \\
\text { mung, Rissbildung } \\
\text { ohne Plastizierung } \\
\text { der Bewehrung }\end{array}$ & $\begin{array}{l}\text { Elastische Verfor- } \\
\text { mung, Rissheilung }\end{array}$ \\
\hline \multirow{2}{*}{ Nicht umkehrbar } & $\begin{array}{l}\text { Rissbildung mit } \\
\text { Plastizierung der } \\
\text { Bewehrung }\end{array}$ & $\begin{array}{l}\text { Subkritisches und } \\
\text { überkritisches Riss- } \\
\text { wachstum }\end{array}$ \\
\hline
\end{tabular}

Tafel 6

Umkehrbare und nicht umkehrbare Zustände im Grenzzustand der Gebrauchstauglichkeit für den Stahlbeton- und Spannbetonbau nach [DIN EN 1992-1-1, Kap. 7] und den Konstruktiven Glasbau nach [DIN 18008-1, Kap. 8.4].

${ }^{a}$ Vergleiche [Härth 2013]. Darin wird der Prozess der

„Rissheilung“ oder Materialalterung beschrieben, der für eine Vergrößerung der Kantenfestigkeit von Glasträgern verantwortlich gemacht wird.

über hinaus ist ein Nachweis der Rissbreiten im Glas nicht gebräuchlich. Zum einen ist die Bildung eines sichtbaren Risses in einer Glaskonstruktion als Folge eines überkritischen Risswachstums nicht umkehrbar und führt zum Austausch des Bauteils. Zum anderen ist die Bewehrung sichtbar und kann daher visuell auf Korossionsfreiheit geprüft werden. Letztlich ist die Rissfreiheit bereits mit dem Nachweis im Grenzzustand der Tragfähigkeit auf höherem Lastniveau sichergestellt. Weitere Ursachen für das Auftreten von Rissen, wie Nickel-Sulfid-Brüche, Vandalismus, unsachgemäße Verwendung oder unplanmäßige Lasten, können durch die Verwendung als Glasträger nicht beeinflusst werden und unterliegen damit den gleichen Randbedingungen wie andere Glaskonstruktionen. Es ist üblich, eine robuste Konstruktion mittels eines Sicherheitskonzeptes und dem Nachweis der Resttragfähigkeit (Kapitel 2.8.3) zu planen.

Damit wird insgesamt gefolgert, dass zum Nachweis der Machbarkeit von Spannglasträgern einzig die Beschreibung des Durchbiegungsverhaltens nötig ist. ${ }^{161}$

\subsubsection{Nachweis der Resttragfähigkeit}

Für die Bemessung eines Glasbauteils nach [DIN 18008] wird stets ein Nachweis der Resttragfähigkeit gefordert. Ei-

${ }^{161}$ Erste Ergebnisse dazu sind in [Weller \& Engelmann 2014a] sowie [Weller \& Engelmann 2014b] veröffentlicht. 
Bild 40

Resttragfähigkeit nach [Kott 2006] ne allgemeingültige Definition der Resttragfähigkeit ist nicht verfügbar. Vielmehr bietet die Literatur eine Umschreibung dieser Situation. ${ }^{162}$ [DIN 18008] beschreibt sie als Teil des Sicherheitskonzeptes und wird ausdrücklich nicht als Grenzzustand bezeichnet. ${ }^{163}$ Jedoch ist eine Zuordnung des rechnerischen Nachweises der Resttragfähigkeit in den Grenzzustand der Tragfähigkeit unter der außergewöhnlichen Bemessungssituation möglich und wird im internationalen Kontext auch so verstanden. ${ }^{164}$

Nennungen in der Literatur ist gemein, dass ein (Teil-) Schädigungszustand angenommen werden muss, unter dem eine Last (Resttragwiderstand) über einen definierten Zeitraum (Reststandzeit) abgetragen werden muss (Bild 40).

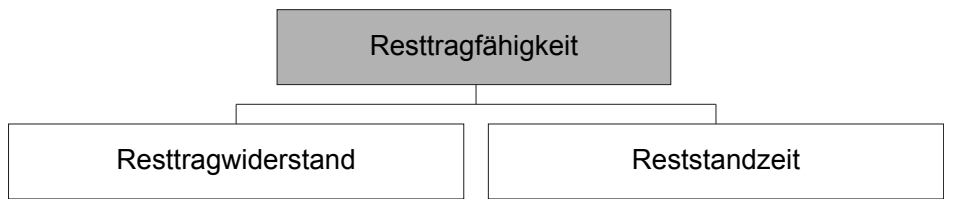

Bereits Hess [2000] unterscheidet zwischen zwei Szenarien. Zum einen muss der Bruch aller Glasscheiben im Verbundglas vermieden werden. Dies geschieht mit ausreichend geringer Bruchwahrscheinlichkeit durch einen Nachweis im Grenzzustand der Tragfähigkeit. Für bewehrte Glasträger kann die Bewehrung zum anderen einen zusätzlich günstigen Effekt bieten, sodass auf „Opferscheiben“ verzichtet werden kann. Jedoch kann es bei unplanmäßigen Einwirkungen bereits unterhalb des Bemessungslastniveaus zum Bruch einzelner Scheiben kommen. Auch in diesem Szenario sind stets die Minderung der Gefährdung von Menschenleben zu berücksichtigen und ein progressives Versagen des Tragwerks ${ }^{165}$ zu vermeiden. Durch einen Nachweis der Resttragfähigkeit wird demnach ein Aspekt der Robustheit der Konstruktion nachgewiesen. ${ }^{166}$

\footnotetext{
162 Vergleiche insbesondere [Kott 2006] und die darin aufgelisteten Verweise nach Bohmann [1999], Wörner et al. [2001], Haas \& Haldimann [2004]. Darüber hinaus auch Hess [2000], Ensslen [2005] und Fahlbusch [2007].

163 Siehe [DIN 18008-1, Kap. 4.1] sowie [DIN 18008-1, Kap. 8 und Kap. 9].

164 Vergleiche [Haldimann et al. 2008].

165 Vergleiche auch [DIN EN 1992-1-1, Kap. 9.10.1].

166 Maßnahmen zum Umgang mit möglichen Schäden sind in [DIN EN 1990, Kap. 2.1] aufgelistet.
} 
Entsprechend ist der Nachweis der Resttragfähigkeit wesentlicher Bestandteil eines Machbarkeitsnachweises von Spannglasträgern. Kern ist die Berechnung der Verformung und der Seilkraft unter einem teilgeschädigten Glasquerschnitt in einer experimentellen Studie (Kapitel 3.5).

Darüber hinaus ist es im Stahlbeton- und Spannbetonbau nötig, den Ausfall eines oder mehrerer Spannglieder zu betrachten, um ein Bauteilversagen ohne Ankündigung auszuschließen. ${ }^{167}$ Dafür werden fünf Verfahren genannt (Tafel 7).

\begin{tabular}{|c|c|}
\hline $\begin{array}{l}\text { Stahlbeton- und } \\
\text { Spannbetonbau }\end{array}$ & Konstruktiver Glasbau \\
\hline $\begin{array}{l}\text { Verfahren A } \\
\text { Mindestbewehrung }^{a}\end{array}$ & $\begin{array}{l}\text { Eine Mindestbewehrung von Glas- } \\
\text { konstruktionen ist nicht definiert }^{b}\end{array}$ \\
\hline $\begin{array}{l}\text { Verfahren B } \\
\text { Spannglieder in soforti- } \\
\text { gem Verbund }^{C}\end{array}$ & $\begin{array}{l}\text { Diese Konstruktionsart wird für } \\
\text { Glasträger in [Cupac \& Louter 2015] } \\
\text { angewendet. Das Verfahren ist } \\
\text { für Spannglasträger dieser Arbeit } \\
\text { mangels Verbund nicht anwendbar. }\end{array}$ \\
\hline $\begin{array}{l}\text { Verfahren C } \\
\text { Zerstörungsfreie Prü- } \\
\text { fung }\end{array}$ & $\begin{array}{l}\text { Die vorgespannte Bewehrung in } \\
\text { Spannglasträgern ist offen zugäng- } \\
\text { lich, sodass eine Inspektion und ein } \\
\text { Austausch von Spanngliedern pro- } \\
\text { blemlos möglich sind. Das Verfahren } \\
\text { kann übernommen werden. }\end{array}$ \\
\hline $\begin{array}{l}\text { Verfahren D } \\
\text { Zuverlässigkeits- } \\
\text { nachweise }^{b}\end{array}$ & $\begin{array}{l}\text { Dieses Verfahren ist sehr interpre- } \\
\text { tationsoffen definiert und wird daher } \\
\text { für den Glasbau zunächst nicht } \\
\text { übernommen. }\end{array}$ \\
\hline $\begin{array}{l}\text { Verfahren E } \\
\text { Nachweis ausreichender } \\
\text { Rissbildung }\end{array}$ & $\begin{array}{l}\text { Eine Rissbildung im Bauteil kündigt } \\
\text { ein Versagen an. Dieser Fall kann } \\
\text { für bewehrte Glasträger übernom- } \\
\text { men werden, wenn sichergestellt } \\
\text { wird, dass ein Riss vor dem Ausfall } \\
\text { des Tragwerks auftritt. Dies ist Be- } \\
\text { standteil der Untersuchungen zur } \\
\text { Resttragfähigkeit. }\end{array}$ \\
\hline \multicolumn{2}{|c|}{$\begin{array}{l}{ }^{a} \text { Fingerloos et al. [2012] verwenden hier abweichend den Begriff } \\
\text { Robustheitsbewehrung. } \\
{ }^{b} \text { Vergleiche Kapitel 2.9, ab Seite } 96 . \\
{ }^{c} \text { In Deutschland nicht zugelassen. Vergleiche } \\
\text { [DIN EN 1992-1-1 NA, NDP zu 5.10.1(6)] } \\
\text { Im Rahmen von abZ oder ZiE ist die Verwendung gegebenenfalls } \\
\text { möglich. [Fingerloos et al. 2012]. }\end{array}$} \\
\hline
\end{tabular}

Tafel 7

Verfahren zum Ausschluss eines unangekündigten Versagens vorgespannter Konstruktionen nach [DIN EN 1992-1-1, Kap. 5.10] 
Mit den Verfahren $C$ und $E$ sind Möglichkeiten verfügbar, die im Konstruktiven Glasbau angewendet werden können.

\subsection{Bewehrungs- und Konstruktionsregeln}

Im Eurocode 2 werden Bewehrungs- und Konstruktionsregeln in Kapitel 8 und Kapitel 9 zusammengestellt. Sie beziehen sich auf konkrete Ausführungsformen im Stahlbetonund Spannbetonbau. Allgemeingültige Konstruktionsregeln im Glasbau können mit dem heutigen Stand der Forschung noch nicht angegeben werden. Daher werden Glaskonstruktionen aus der Literatur in diesem Abschnitt zusammengestellt und um weiterführende Aspekte aus [DIN EN 1992] ergänzt. Ein eigener Erfahrungsbericht findet sich anschlieBend in Kapitel 6.

\section{Materialwahl}

Bereits umgesetzte Bau- und Forschungsprojekte, welche vorgespannte Glasträger beinhalten, sind in Tafel 8 benannt und klassifiziert. Die untersuchten Glasträger bestanden ausschließlich aus thermisch entspanntem Floatglas. Es wird wegen seines günstigen, groben Bruchbildes und der Möglichkeit der nachträglichen Kantenbearbeitung, beispielsweise zum Ausgleich eines VSG-Kantenversatzes, angewendet. Zudem ist es preisgünstiger, da die zusätzlichen Veredelungsschritte des thermischen Vorspannens und Heißlagerns entfallen. Jedoch liegt seine charakteristische Biegezugfestigkeit im Vergleich zu thermisch vorgespanntem Bauglas bei $65 \%$ (TVG) beziehungsweise $38 \%$ (ESG) zuzüglich einer Reduzierung auf $25 \%$ beim Vorhandensein einer ständigen Last, wie der mechanischen Vorspannung, und weiteren $80 \%$ bei Kantenbeanspruchung. ${ }^{168}$ Um die Differenz der Tragfähigkeit aufgrund des Festigkeitsunterschiedes durch die Vorspannung auszugleichen, ist ein Vorspanngrad ${ }^{169}$ von mindestens $135 \%$ nötig. Mit Werten zwischen $30 \%$ und

\footnotetext{
168 Vergleiche [DIN 18008-1].

169 Vergleiche Gleichung 2, Seite 45.
} 
$64 \%$ liegen die Vorspanngrade in Tafel 8 deutlich darunter. Daher ist eine erweiterte Begründung der Materialwahl in anderen Konstruktionen einzubeziehen. Der errechnete Unterschied wird zunächst pauschal der Wirkung der Bewehrung auf die Resttragfähigkeit zugeordnet, da derzeit keine quantifizierenden Methoden verfügbar sind, die den Effekt der Bewehrung auf die Resttragfähigkeit umschreiben.

Die Kanten teilvorgespannter Gläser dürfen nur bedingt nachträglich bearbeitet ${ }^{170}$ werden. Das Bruchbild von TVG eignet sich für sicherheitsrelevante Glasanwendungen mit Anforderungen an die Resttragfähigkeit ${ }^{171}$. Zudem muss die Biegezugfestigkeit für Dauerlasten und bei Kantenbeanspruchung nicht reduziert werden, was eine höhere Vorspannung ermöglich. Daher werden die Spannglasträger dieser Arbeit im Gegensatz zu den bisher verfügbaren Versuchsergebnissen aus TVG konstruiert.

Zur Herstellung des Verbundglases der Träger in anderen Arbeiten (Tafel 8) werden bisher ausschließlich Zwischenschichten verwendet, die aufwendiger zu verarbeiten sind als das üblicherweise eingesetzte PVB. Darüber hinaus wurden alle Glaskanten zur Einleitung der Vorspannkräfte nachgeschliffen, was ebenso einen erhöhten Herstellungsaufwand bedeutet. Diesem Kostennachteil wird in dieser Arbeit mit der Verwendung üblichen Verbund-Sicherheitsglases, ohne weitere Kantenbearbeitung nach dem Laminieren, begegnet. Dieses Grundmaterial ist handelsüblich verfügbar und zu vertretbaren Kosten erhältlich. Die Folgen dieser Entscheidung werden in Kapitel 5 diskutiert und in Kapitel 6 bewertet.

Insgesamt zeigen sich die bekannten Konstruktionen in Tafel 8 als sehr vielfältig, sodass ein unmittelbarer Vergleich nicht möglich ist. Damit ist keine gesicherte Ausgangsbasis vorhanden und wird mit dieser Arbeit erstmals geschaffen.

\footnotetext{
170 Vergleiche [E DIN EN 1863-1/A1]. Darin wird ein zusätzlicher Nachweis im Fall einer nachträglichen Bearbeitung gefordert. Die Thematik wird derzeit aktiv beforscht, sodass der Nachteil zukünftig relativiert werden kann. Vergleiche [Weller et al. 2016].

171 [DIN 18008] ermöglicht beispielsweise die Anwendung von Floatglas und TVG für Horizontalverglasungen.
} 


\begin{tabular}{|c|c|c|c|c|c|}
\hline & $\begin{array}{l}\text { T-Fisch- } \\
\text { bauchträger }\end{array}$ & intern $\mathbf{v}$ & gespannt & $\begin{array}{l}\text { extern vor- } \\
\text { gespannt }\end{array}$ & $\begin{array}{l}\text { Spannbett- } \\
\text { träger }\end{array}$ \\
\hline Verbund & $\begin{array}{l}\text { ohne } \\
\text { Verbund }\end{array}$ & $\begin{array}{l}\text { ohne } \\
\text { Verbund }\end{array}$ & $\begin{array}{l}\text { ohne } \\
\text { Verbund }\end{array}$ & $\begin{array}{l}\text { ohne } \\
\text { Verbund }\end{array}$ & $\begin{array}{l}\text { sofortiger } \\
\text { Verbund }\end{array}$ \\
\hline $\begin{array}{l}\text { Lage und } \\
\text { Verlauf des } \\
\text { Spanngliedes }\end{array}$ & $\begin{array}{l}\text { intern } \\
\text { exzentrisch } \\
\text { parabolisch }\end{array}$ & $\begin{array}{l}\text { intern } \\
\text { zentrisch } \\
\text { gerade }\end{array}$ & $\begin{array}{l}\text { intern } \\
\text { exzentrisch } \\
\text { gerade }\end{array}$ & $\begin{array}{l}\text { extern } \\
\text { exzentrisch } \\
\text { polygonal }\end{array}$ & $\begin{array}{l}\text { intern } \\
\text { exzentrisch } \\
\text { gerade }\end{array}$ \\
\hline $\begin{array}{l}\text { Vorspannkraft } \\
\text { Vorspanngrad }\end{array}$ & 20 kN $30 \%$ & $31 \mathrm{kN} 45 \%$ & $\begin{array}{l}50 \mathrm{kN} ; 66 \mathrm{kN} \\
44 \% ; 58 \%\end{array}$ & 23 kN $54 \%$ & $\begin{array}{l}15 \text { kN; } 25 \text { kN } \\
38 \% ; 64 \%\end{array}$ \\
\hline Anzahl & 2 & 2 & 6 & 1 & 12 \\
\hline $\begin{array}{l}\text { Glasaufbau, } \\
\text { Glasart und } \\
\text { Verbundmaterial }\end{array}$ & $\begin{array}{l}\text { 8-10-8 } \\
\text { Floatglas } \\
\text { Ionomer }\end{array}$ & $\begin{array}{l}\text { 6-10-6 } \\
\text { Floatglas } \\
\text { Acrylat }\end{array}$ & $\begin{array}{l}6-10-6 \\
\text { Floatglas } \\
\text { lonomer }\end{array}$ & $\begin{array}{l}10-10 \\
\text { Floatglas } \\
\text { lonomer }\end{array}$ & $\begin{array}{l}\text { 6-10-6 } \\
\text { Floatglas } \\
\text { lonomer }\end{array}$ \\
\hline Spannweite & $3000 \mathrm{~mm}$ & $800 \mathrm{~mm}$ & $1500 \mathrm{~mm}$ & $3000 \mathrm{~mm}$ & $1500 \mathrm{~mm}$ \\
\hline $\begin{array}{l}\text { Bewehrung } \\
\text { Materialangabe }\end{array}$ & $\begin{array}{l}1 \mathrm{x} ø 7 \mathrm{~mm} \\
\mathrm{f}_{\mathrm{y}, \mathrm{p}}=1670 \\
\mathrm{~N} / \mathrm{mm}^{2}\end{array}$ & $\begin{array}{l}2 \mathrm{x} ø 8 \mathrm{~mm} \\
\text { AISI } 304\end{array}$ & $\begin{array}{l}2 \mathrm{x} ø 8 \mathrm{~mm} \\
2 \mathrm{x} \varnothing 10 \mathrm{~mm} \\
\text { AISI } 304\end{array}$ & $\begin{array}{l}2 \mathrm{x} ø 10 \mathrm{~mm} \\
\mathrm{k} . \mathrm{A} .\end{array}$ & $\begin{array}{l}25 \times 3 \mathrm{~mm}^{2} \\
\text { k.A. }\end{array}$ \\
\hline Quellen & $\begin{array}{l}\text { [Bos et al. } \\
\text { 2004] } \\
\text { [Louter 2011] }\end{array}$ & $\begin{array}{l}\text { [Louter et al. } \\
\text { 2006] }\end{array}$ & $\begin{array}{l}\text { [Louter 2013] } \\
\text { [Louter et al. } \\
2013 a] \\
\text { [Louter et al. } \\
\text { 2013b] }\end{array}$ & $\begin{array}{l}\text { [Jordão et al. } \\
2014 \text { ] } \\
\text { [Firmo et al. } \\
\text { 2015] }\end{array}$ & $\begin{array}{l}\text { [Louter et al. } \\
\text { 2014] } \\
\text { [Cupac \& } \\
\text { Louter 2015] } \\
\text { [Louter \& } \\
\text { Cupac 2015] }\end{array}$ \\
\hline
\end{tabular}

Tafel 8

Übersicht vorgespannter Glasträger mit linearer Bewehrung

\section{Mindestbewehrung}

Die Angaben aus [Louter \& Veer 2007b] reichen nicht fü einen Vergleich.

Mit einer Bewehrung ${ }^{172}$ soll das ungünstige, spröde Materialverhalten des Betons mit unangekündigtem Versagen vermieden werden. Im Versagensfall, beim Erreichen des Rissmomentes $\mathrm{M}_{\mathrm{cr}}$, müssen die zuvor im tragfähigen, ungerissenen Querschnitt abgetragenen Schnittkräfte durch die

172 Gemeint ist hier die Längsbewehrung nach [DIN EN 1992-1-1,

Kap. 9.2.1], da eine analoge Bewehrung für Spannglasträger vorrangig untersucht wird. 
Bewehrung aufgenommen werden. Dafür wird eine Mindestbewehrung $A_{S, \min }$ vorgesehen (Gleichung 38 ).

$$
\begin{array}{lll}
M_{C r}= & f_{G, y} \cdot W_{G}<F_{S} \cdot z=A_{S, \min } \cdot f_{S, y} \cdot z \\
A_{S, \min }=\frac{f_{G, y} \cdot W_{G}}{f_{S, y} \cdot z} & \\
& \multicolumn{1}{c}{\text { Mindestbewehrung }} \\
A_{S, \min } & \text { Biegezugfestigkeit Glas } \\
f_{G, y} & \text { Trägheitsmoment erster Ordnung des } \\
W_{G} & \text { Glasquerschnitts } \\
z & \text { Hebelarm der Bewehrungskraft } \\
f_{S, y} & \text { Streckgrenze der Bewehrung }
\end{array}
$$

Gleichzeitig soll die Bewehrung eines bewehrten Glasträgers ein unangekündigtes, sprödes Versagen des Glases kompensieren ${ }^{173}$, sodass auch hier die Definition einer Mindestbewehrung ${ }^{174}$ sinnvoll ist. Damit ist ein vollständiges Glasversagen ohne vollständiges Versagen des Tragwerks möglich. Die Anordnung von Opfer- oder Verschleißschichten, welche ein vollständiges Glasversagen durch Überbemessung ausschließen sollen, ist nicht nötig und eine effektivere Materialausnutzung bei gewährleisteter Sicherheit wird möglich. Der Einfluss des Bewehrungsgrades wird im Rahmen der folgenden experimentellen Untersuchungen überprüft.

Eine Mindestbewehrung im Stahlbeton- und Spannbetonbau dient somit zusätzlich der Dauerhaftigkeit des Betonbauteils. Zum Korrosionsschutz der Bewehrung müssen breite Risse vermieden werden, durch welche schädigende Medien in den Beton eindringen können. Der Riss des Glases jedoch ist irreversibel ${ }^{175}$ und führt zum Austausch des Bauteils, sodass eine Mindestbewehrung im bewehrten Glasträger keine solche Aufgabe übernehmen muss.

Tragwerke sollen robust konstruiert sein, also auch bei rechnerisch nicht erfassten Situationen gutartig reagieren. Dazu

\footnotetext{
173 Zusätzlich wird beim Erstriss thermisch vorgespannter Gläser Rissenergie frei, welche ebenso abgetragen werden muss, um ein vollständig sprödes Bauteilversagen zu kompensieren.

174 In [Louter 2011] wird der Einfluss der Bewehrungsmenge auf das Tragverhalten bewehrter Glasträger beschrieben.

175 Siehe Tafel 6, Seite 93.
} 
sind konstruktive Regelungen zur Schadensbegrenzung bei außergewöhnlichen Ereignissen normativ erfasst. ${ }^{176}$ Analog wird im Konstruktiven Glasbau ein Nachweis der Resttragfähigkeit gefordert. In beiden Fällen gilt, dass im Fall einer (örtlichen) Schädigung des Tragwerks ein alternativer Lastpfad zur Verfügung stehen muss. So wird ein progressives Versagen vermieden. Daher sind Spannglasträger im Speziellen so zu gestalten, dass der alternative Lastpfad über die Bewehrung auch im Bruchfall zum Lastabtrag genutzt werden kann. Dafür werden experimentelle Untersuchungen in Kapitel 3.5 durchgeführt.

\subsection{Zusammenfassung}

Das Kapitel 2 fasste Inhalte des Eurocode 2 zusammen und übertrug Kerngedanken des Stahlbeton- und Spannbetonbaus auf den Konstruktiven Glasbau, um das Themenfeld zu erschließen, die Zielstellung näher zu definieren und das weitere Vorgehen abzuleiten.

Begriffe zur Beschreibung und Kategorisierung üblicher Konstruktionen, insbesondere die Unterscheidung nach schlaffer und vorgespannter Bewehrung sowie nach Spannverfahren, wurden für den Glasbau als neue Systematik definiert.

Es zeigt sich, dass eine Eingruppierung nach dem vorgeschlagenen Konzept vollständig möglich ist. Sowohl ein sicheres Tragverhalten als auch eine Steigerung der Erstrisslast konnten nachgewiesen werden, sodass dieses vielversprechende Konzept mit dieser Arbeit weiterentwickelt wird.

Vorgespannte Glasträger wurden bisher ausschließlich ohne Verbund oder im sofortigen Verbund untersucht. Aufgrund der geringen Probenanzahl - es sind bisher nur Ergebnisse aus 23 Kurzzeit-Biegeversuchen publiziert ${ }^{177}$ - können noch keine allgemeingültigen Aussagen abgeleitet werden. Daher wird die experimentelle Studie dieser Arbeit ein breiteres

\footnotetext{
176 Vergleiche [DIN EN 1992-1-1, Kap. 9.10].

177 Vergleiche Tafel 8, Seite 98. Davon 11 Prüfkörper ohne Verbund und 12 Prüfkörper mit sofortigem Verbund.
} 
Parameterspektrum bezüglich einer verbundlosen Bewehrungskonstruktion und der Wirkung der Vorspannkraft umfassen. Die Beschreibung des Tragverhaltens unter kurzzeitig wirkenden Lasten ein notwendiges Kriterium zur Gestaltung sicherer Bauteile. Doch nur gemeinsam mit einem Nachweis der Resttragfähigkeit und der Dauerhaftigkeit können hinreichende Argumente gesammelt werden, um nachzuweisen, dass das Konzept einer zusätzlichen mechanischen Vorspannung von Glasträgern sinnvoll ist.

Zum Anknüpfen an die bestehenden Ergebnisse werden zunächst Kurzzeit-Biegeversuche der eigenen Konstruktion durchgeführt und der in der Literatur benannte Einfluss der vorgespannten Bewehrung auf die Trageigenschaften gezeigt. Zusätzlich wird das Tragverhalten mittels der analytischen Abschätzung der Schnittgrößen nach der Kraftgrößenmethode angewandt und dieses Modell hinsichtlich seiner praktischen Anwendbarkeit bewertet. Darüber hinaus wird eine experimentelle Studie zur Resttragfähigkeit zeigen, welchen Einfluss die Vorspannung auf einen teilzerstörten Glasträger hat, um die Grundlagen für den nötigen Nachweis der Resttragfähigkeit zu legen.

Kurzzeit-Biegeversuch

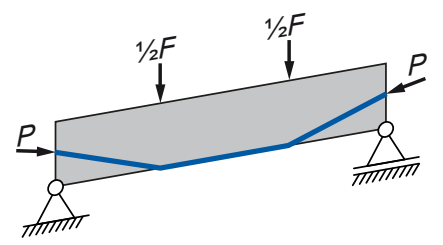

Resttragfähigkeitsversuch

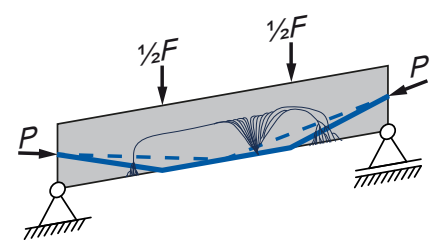

\section{Dauerzeit-Biegeversuch}

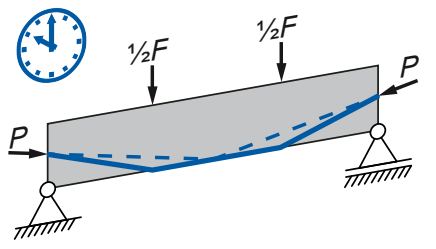

Temperaturlast-Versuch

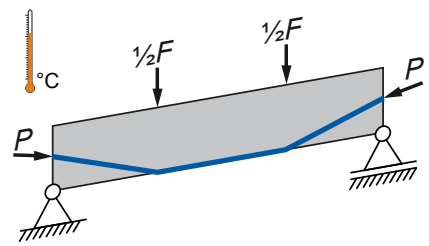

Bild 41

Übersicht notwendiger experimenteller Untersuchungen.

Weiterhin ist die Dauerhaftigkeit nachzuweisen. Es stellt sich die Frage, ob die Spannkraft ausreichend lange erhalten bleibt. Dafür werden die Spannkraftverluste im Biegeversuch unter Dauerlasten sowie unter Temperaturlasten experimentell bestimmt und der analytischen Berechnung gegenüber 
gestellt. Damit wird die These überprüft, wonach die Spannkraftverluste als Folge der zeitabhängigen Verzerrungen der beteiligten Komponten im Lastpfad (Klotzung und Zwischenschicht) sowie der unterschiedlichen Wärmedehnzahlen der beteiligten Baustoffe beschreibbar ist.

Um zusammenzufassen: Die spröden, druckfesten und vergleichsweise zugschwachen Baustoffe Glas und Beton verhalten sind im Grunde ähnlich. Daher ist eine Verwendung in analogen Konstruktionen naheliegend. Nachdem bewehrte Glasträger bereits untersucht sind, wird in dieser Arbeit der nächste Schritt hin zu verbundlos vorgespannten Glasträgern - Spannglasträger - gegangen. Damit werden deren zukünftige Potenziale abgeschätzt, um materialeffiziente transparente Tragwerke zu realisieren und neuartige architektonische Möglichkeiten zu erschließen. 


\section{$3 \quad$ Experimentelle Untersuchungen}

\subsection{Zielsetzung}

Mittels experimenteller Vierpunkt-Biegeversuche soll gezeigt werden, dass die zuvor theoretisch aufgezählten (günstigen) Trageigenschaften praktisch zu erreichen sind. Darüber hinaus soll das Tragverhalten unter verschiedenartigen Einwirkungen aufgezeichnet, beschrieben und letztlich dem analytischen Ansatz aus Kapitel 2 gegenübergestellt werden. Ziel ist, zu bestätigen, dass das Tragverhalten mittels der analytischen Herangehensweise ausreichend gut vorhersagbar ist. Damit soll die Grundlage geschaffen werden, Spannglasträger mit einem einfachen Verfahren vorab zu dimensionieren.

Dieses Kapitel beschreibt zunächst die Konstruktion der Prüfkörper und die verwendeten Materialien in Abschnitt 3.2. Die anschließenden Untersuchungen sind in folgende Zwischenschritte gegliedert:

- Die Kurzzeit-Biegeversuche in Abschnitt 3.3 belegen, dass die Vorspannkraft planmäßig einleitbar ist (Überdrücken der Biegezugkante, Überhöhung des Trägers) sowie das Tragverhalten vor und nach dem Erstriss beeinflusst werden kann.

- Die Untersuchung des Tragverhaltens unter Dauerlast von $1000 \mathrm{~h}$ in Abschnitt 3.4 zeigt die Veränderung der Vorspannkraft über die Lebenszeit des Bauteils.

- Die Untersuchung der Resttragfähigkeit in Abschnitt 3.5 bestätigt ein sicheres Bauteilverhalten unter einem definierten Teilzerstörungszustand im Sinne des im Glasbau üblichen Sicherheitskonzeptes.

- Schließlich bestätigen Untersuchung des Tragverhaltens unter Temperatureinfluss in Abschnitt 3.6, dass das Bauteil im zu erwartenden Einsatztemperaturbereich seine Trageigenschaften erhält. 


\subsection{Prüfkörper - Konstruktion und Materialien}

Für diese Arbeit wurden zwei Prüfkörpertypen mit etwa $2 \mathrm{~m}$ Spannweite verwendet (Bild 42). Wesentliche Variation war die initiale Seilkraft und damit einhergehend der Durchmesser der Bewehrung sowie die Bewehrungsführung mit einfacher Umlenkung in der Feldmitte oder zweifacher Umlenkung in den Drittelspunkten. Die Konstruktion und die verwendeten Materialien waren grundsätzlich gleichartig, sodass die folgenden Angaben für alle Versuche gelten.

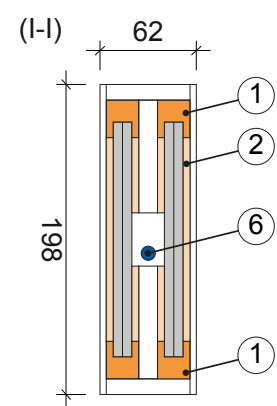

Spannanker

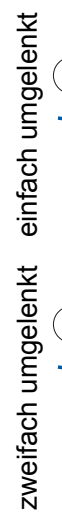$$
\text { i (I) }
$$

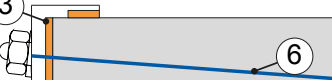

(II-II) $\varlimsup^{54} \nmid$

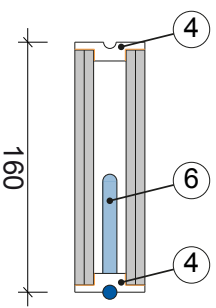

(II)
(III-III) $12,28,12$

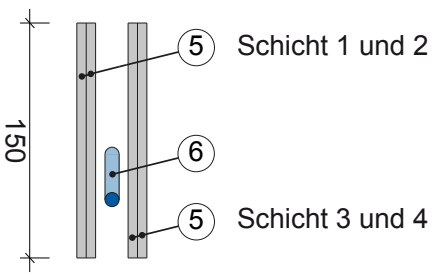

[mm]

(III) Festanker 1000

\section{(4)}

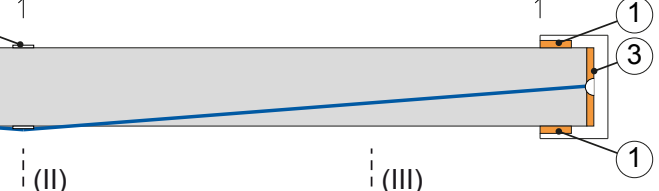

666

667

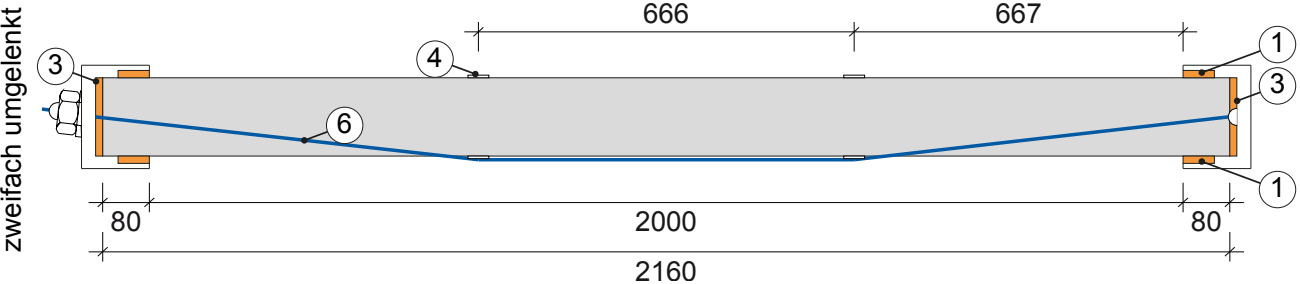

\section{Bild 42}

Querschnitt, Abmessungen und Bezeichnungen innerhalb der Prüfkörper.

(1) Auflagerklotz POM-C $60 \times 24 \times 21 \mathrm{~mm}^{3}$

(2) EPDM Zwischenlage

(3) Vorspannklotz POM-C $150 \times 13 \times 8 \mathrm{~mm}^{3}$

(4) Umlenksattel 1.4571 $54 \times 40 \times 12 \mathrm{~mm}^{3}$

(5) $2 \times 2 \times 6 \mathrm{~mm} \mathrm{VSG}$ aus TVG mit PVB

(6) Bewehrung
Der Trägerquerschnitt bestand aus zwei $150 \mathrm{~mm}$ hohen Paketen Verbund-Sicherheitsglas aus zweimal $6 \mathrm{~mm}$ teilvorgespanntem Glas mit $0,76 \mathrm{~mm}$ Polyvinylbutral-Zwischenschicht. ${ }^{178}$ Die $2160 \mathrm{~mm}$ langen Glaspakete wurden an ihren Enden mit einem Auflagerschuh aus hochlegiertem Stahl ${ }^{179}$ eingefasst, sodass die freie (transparente) Glaslänge $2000 \mathrm{~mm}$ betrug.

178 Damit ergab sich eine reine Glasquerschnittsfläche $A_{G}$ von

$4 \cdot 6 \mathrm{~mm}=150 \mathrm{~mm}=3600 \mathrm{~mm}^{2}$.

179 Chrom-Nickel-Molybdän-Stahl, Werkstoffnummer (Gruppe) 1.44. 
Zwischen den Paketen ergab sich ein offener Spalt von $28 \mathrm{~mm}$, welcher zur verbundlosen Führung der Bewehrung vorgesehen war. Ein Umlenksattel ${ }^{179}$ verband beide Pakete punktuell in der Feldmitte oder den Drittelspunkten über eine Klebfuge $^{180}$ (Bild 43).

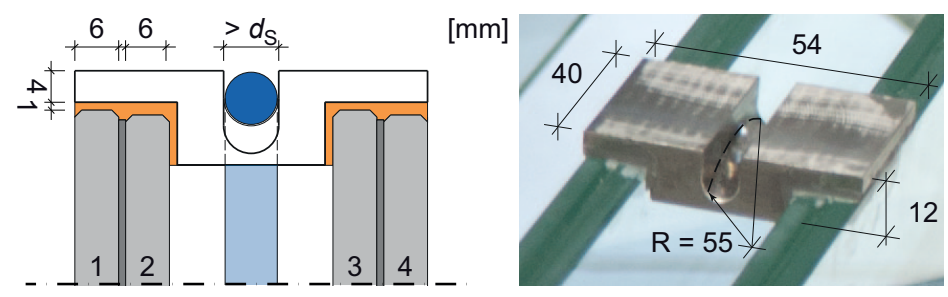

Bild 43

Umlenksattel aus hochlegiertem Stahl (1.4571).

$54 \times 40 \times 12 \mathrm{~mm}^{3}$ mit $1 \mathrm{~mm}$ Klebfuge

Der Glas-Metall-Kontakt wird innerhalb der Auflagerkonstruktion (Bild 44) durch drei Klotzungstypen vermieden. Die jeweils $80 \mathrm{~mm}$ langen Auflagerklotzungen (1) sowie die $150 \mathrm{~mm}$ hohen Vorspannklotzungen (3) zur gezielten Lastüberleitung über Druckkontakt ${ }^{181}$ bestanden aus Polyoxymethylen (POM-C). Die verbleibenden, nicht lastabtragenden Klotzungen (2) waren aus Ethylen-Propylen-Dien-Kautschuk (EPDM) gefertigt.
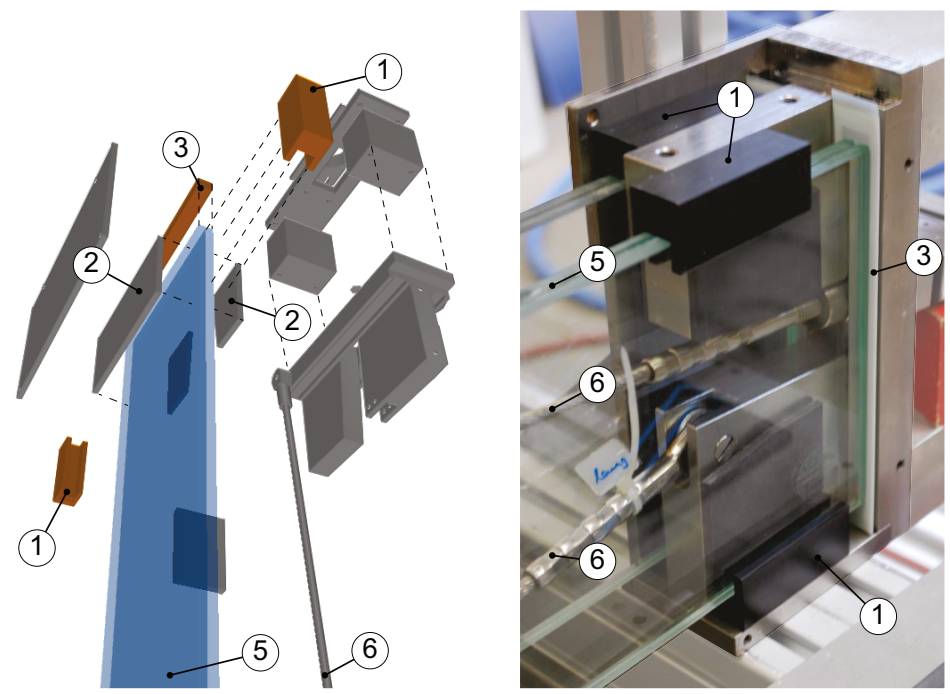

Die Eigenschaften der Bewehrungsseile in Bezug auf den Nenndurchmessern sind in Tafel 9 zu finden. Dabei wiesen

180 Die stoffschlüssige Verbindung wird durch eine Klebfuge aus einem Zwei-Komponenten, schnellhärtenden Acrylat-Klebstoff (SikaFast ${ }^{\circledR 5} 5211$ NT [SikaFast 5211NT]) der Mindestdicke $1 \mathrm{~mm}$ hergestellt.

181 Während die Auflagerklotzungen nach einer Sichtprüfung auf mechanische Beschädigungen wiederverwendet wurden, sind alle Vorspannklotzungen in jedem Versuch erneuert worden.

\section{Bild 44}

Explosionszeichnung der Auflagerkonstruktion. Es wird ein variables Konzept gewählt, mit dem auch zentrisch vorgespannte Konstruktionsarten mit zwei

Bewehrungsseilen nach Bild 23, Seite 42 möglich sind.

(1) Auflagerklotz POM-C $60 \times 24 \times 21 \mathrm{~mm}^{3}$

(2) EPDM Zwischenlage

(3) Vorspannklotz POM-C $150 \times 13 \times 8 \mathrm{~mm}^{3}$

(4) Umlenksattel 1.4571 $54 \times 40 \times 12 \mathrm{~mm}^{3}$ mit $1 \mathrm{~mm}$ Klebfuge

(5) $2 \times 2 \times 6 \mathrm{~mm}$ VSG aus TVG mit PVB

(6) Bewehrung 


\begin{tabular}{|c|c|c|c|c|c|c|}
\hline $\begin{array}{l}d_{p} \\
{[\mathrm{~mm}]}\end{array}$ & $\begin{array}{l}\mathbf{f}_{\mathrm{u}, \mathrm{k}} \\
{\left[\mathrm{N} / \mathrm{mm}^{2}\right]}\end{array}$ & $\begin{array}{l}\mathrm{Z}_{\mathrm{B}, \mathrm{k}} \\
{[\mathrm{kN}]}\end{array}$ & $\begin{array}{l}A_{p} \\
{\left[\mathrm{~mm}^{2}\right]}\end{array}$ & $\begin{array}{l}\text { Konstruktion }{ }^{a} \\
\text { Werksstoffnr. }\end{array}$ & $\begin{array}{l}\alpha_{\mathrm{T}} \\
{\left[\mathrm{K}^{-1}\right]}\end{array}$ & $\begin{array}{l}\mathrm{E}_{\mathrm{Q}} \\
{\left[\mathrm{N} / \mathrm{mm}^{2}\right]^{b}}\end{array}$ \\
\hline 5,0 & $1570^{e}$ & $21^{c, d, k}$ & $15^{k}$ & $\begin{array}{l}1 \times 19^{e} \\
1.4401^{e}\end{array}$ & - & $130000^{b}$ \\
\hline $\begin{array}{l}6,1 \\
\left(\mathrm{PE} 3^{f}\right)\end{array}$ & $1450^{f, g, h}$ & $26^{f, g, h}$ & $22^{f, g, h}$ & $\begin{array}{l}1 \times 19^{f, g, h} \\
1.4401^{f, g, h}\end{array}$ & $16 \cdot 10^{-6 e}$ & $130000^{f, g, h}$ \\
\hline $\begin{array}{l}8,1 \\
\left(\mathrm{PG} 5^{f}\right)\end{array}$ & \multirow[t]{2}{*}{$1770^{f, g, h}$} & $59^{f, g, h}$ & $39^{f, g, h}$ & \multirow{2}{*}{$\begin{array}{l}1 \times 19^{f, g, h} \\
\text { unlegierter } \\
\text { Qualitätsstahl }\end{array}$} & \multirow[t]{2}{*}{$12 \cdot 10^{-6 b}$} & \multirow[t]{2}{*}{$160000^{f, g, h}$} \\
\hline $\begin{array}{l}10,1 \\
\left(\mathrm{PG} 10^{f}\right)\end{array}$ & & $93^{f, g, h}$ & $60^{f, g, h}$ & & & \\
\hline${ }^{a}$ [DIN E & $12385-2]$ & \multirow{3}{*}{$\begin{array}{l}{ }^{d}[ \\
e \\
e \\
{ }^{f}\end{array}$} & V EN ISO 1 & \multicolumn{2}{|c|}{${ }^{g}$ [DIN EN 10264-4] } & \\
\hline${ }_{c}^{b}$ [DIN E & N 1993-1-11] & & rens 2016] & \multirow{2}{*}{\multicolumn{2}{|c|}{$\begin{array}{l}h \text { [DIN EN 12385-1] } \\
k \text { [DIN EN 12385-10] }\end{array}$}} & \\
\hline${ }^{c}[$ Prüfbe & richt 11.07.2013 & & $A-11 / 0160]$ & & & \\
\hline
\end{tabular}

\section{Tafel 9}

Eigenschaften der verwendeten Bewehrung.

$d_{p}$... Seil-Nenndurchmesser

$f_{u, k} \ldots$... Festigkeit

$Z_{\mathrm{B}, \mathrm{k}} \ldots$ Zugtragfähigkeit

$A_{p} \ldots$. . metallischer Querschnitt

$\alpha_{\top} \ldots$ Wärmedehnzahl

$E_{Q} \ldots$ Verformungsmodul

alle Seile eine Nennlänge von $2270 \mathrm{~mm}$ auf. Abzüglich der Länge der verwendeten Gewindefittinge verblieben freie Seillängen von $2038 \mathrm{~mm}, 2064 \mathrm{~mm}, 1990 \mathrm{~mm}$ und $1936 \mathrm{~mm}$.

Unter Verwendung der in Kapitel 2 eingeführten Begriffe wird zusammengefasst, dass im Folgenden ausschließlich intern vorgespannte Glasträger ohne Verbund, mit polygonaler Spanngliedführung und Bewehrungsgraden ${ }^{182}$ von $0,4 \%$ bis $1,7 \%$ untersucht wurden. 


\subsection{Tragverhalten unter kurzzeitiger Beanspruchung}

\subsubsection{Prüfkörper}

Für die Kurzzeit-Biegeversuche wurde die Vorspannkraft und der Seildurchmesser an 15 Prüfkörpern variiert (Tafel 10), sowie die Bewehrung in den Drittelpunkten umgelenkt. Als Referenzen dienten drei unbewehrte (BV 01 bis BV 03) und drei schlaff bewehrte (BV 04 bis BV 06) Träger.

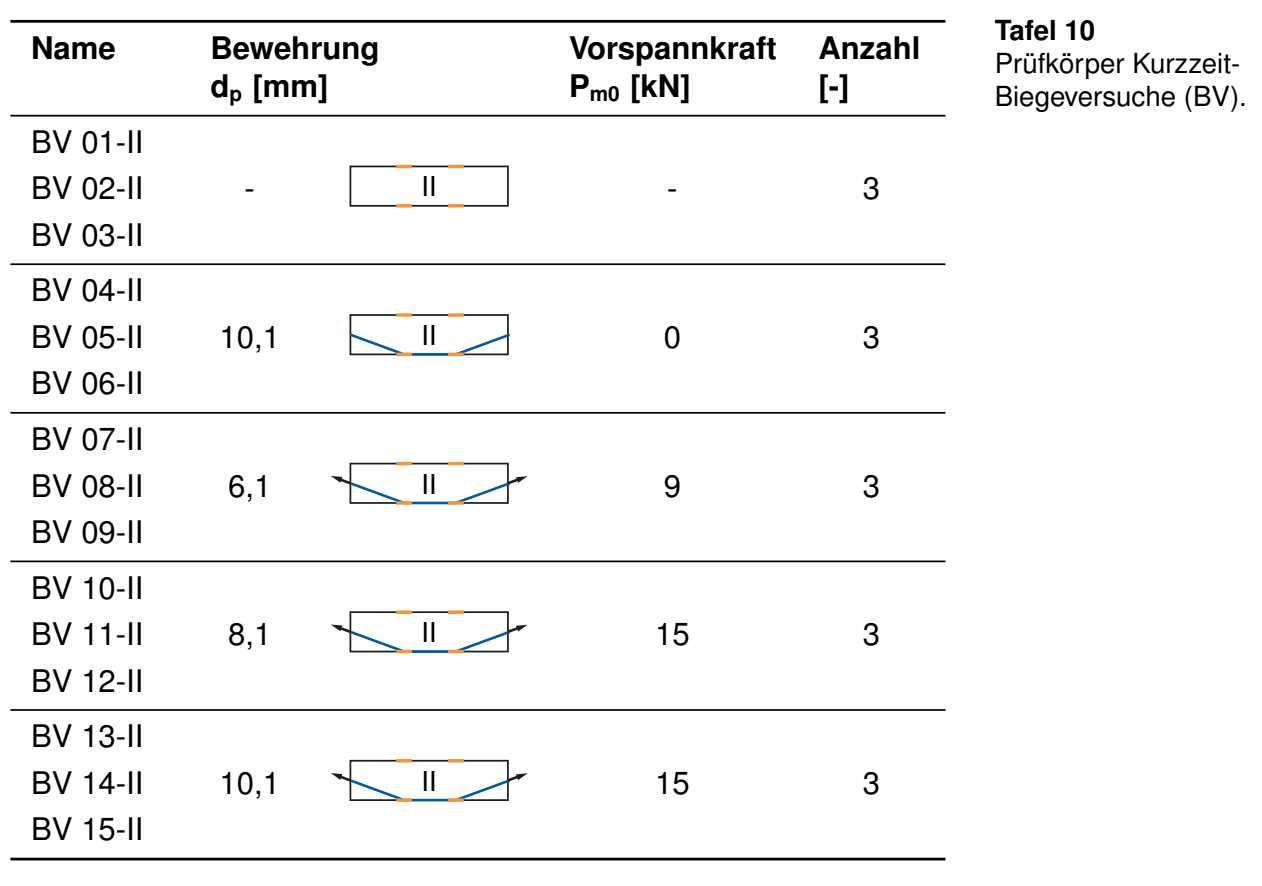

$\Sigma 15$

\subsubsection{Versuchseinrichtung}

Für Biegeversuche unter kurzzeitig wirkenden Lasten wurde ein Vierpunkt-Biegeversuchsstand verwendet (Bild 45). Die Belastung erfolgte weggesteuert über einen einzelnen hydraulischen Prüfzylinder, welcher über eine gelenkig angeschlossene Lasttraverse eine Biegebelastung zweimal F/2 am Prüfkörper erzeugte. Integrierte Sensoren zeichneten sowohl den Traversenweg $\mathrm{w}_{z . T}$, als auch die Kraft $\mathrm{F}$ auf. 
Bild 45

Vierpunkt-

Biegeversuchsstand für kurzzeitige Biegebelastung

(1) Lasttraverse am Prüfzylinder

(2) Kraftmessdose Seilkraft am Spannanker

(3) Wegaufnehmer Spannweg

(4) Wegaufnehmer vertikale Durchbiegung

(5) Wegaufnehmer horizontale Durchbiegung

(6) Festanker

(7) Gabellager
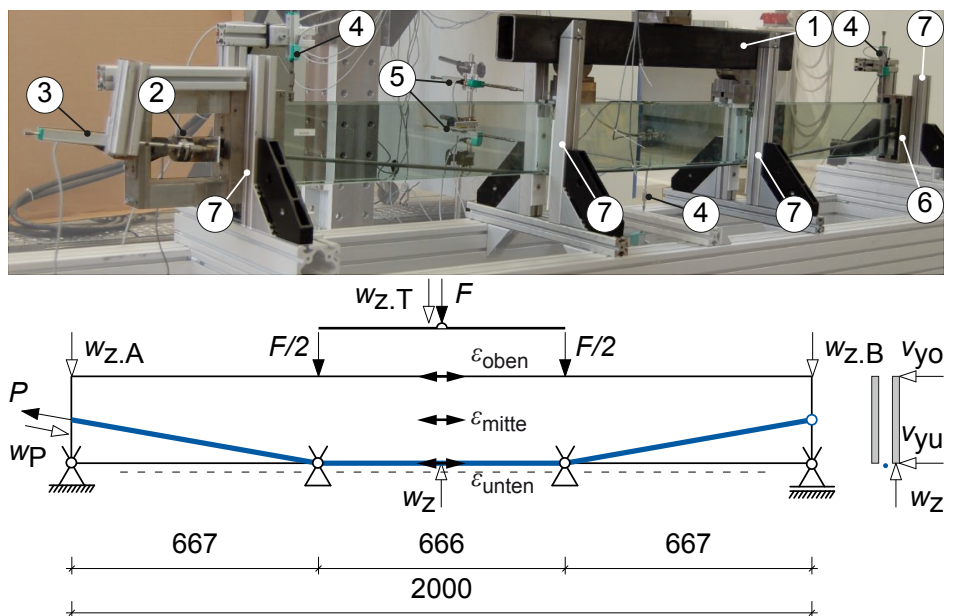

Während des Versuchs wurde die auftretende Seilkraft $N_{p}$ mit einer Kraftmessdose (KMD) sowie der zugehörige Spannweg $w_{P}$ am Spannanker mit einem induktiven Wegaufnehmer gemessen. Die zusätzliche Messung der Seilkraft am Festanker der drei Prüfkörpern BV 08, BV 10 und BV 14 ermöglichte die Quantifizierung der Reibungsverluste an den Umlenkstellen. Darüber hinaus zeichnete ein induktiver Wegaufnehmer die vertikale Durchbiegung der Trägerunterkante $\mathrm{w}_{\mathrm{z}}$ in Feldmitte auf. Während der Biegebelastung wurden die Glasklotzungen innerhalb der Auflagerkonstruktion gestaucht. Dies ist in der Messung der vertikalen Durchbiegung $\mathrm{W}_{\mathrm{z}}$ inbegriffen. Der Einfluss wird rechnerisch unter Berücksichtigung der auflagernahen vertikalen Durchbiegung $w_{z . A}$ und $w_{z . B}$ nach Gleichung 39 entfernt.

$$
\begin{array}{ll}
w_{z . G l a s}=w_{z}-\frac{w_{z . A}+}{2} & w_{z . B} \\
& \\
w_{z . G l a s} & \text { reine vertikale Glasdurchbiegung in } \\
w_{z} & \text { Feldmitte } \\
& \text { gemessene vertikale Durchbiegung } \\
& \text { in Feldmitte inklusive der Verformung } \\
& \text { innerhalb der Auflagerkonstruktion } \\
w_{z . A} \text { und } w_{z . B} & \text { vertikale Durchbiegung der Trägerober- } \\
& \text { kante nahe der Auflager A und B }
\end{array}
$$

Die horizontale Durchbiegung der Trägerober- und Unterkante ( $v_{y \text {.oben }}$ und $\left.v_{\text {y.unten }}\right)$ wurde in Feldmitte am Glaspaket der Scheiben 3 und 4 gemessen. Dieses Scheibenpaket war an dieser Stelle nicht mit dem Paket der Scheiben 1 und 2 
verbunden. Beide Pakete konnten sich unabhängig voneinander horizontal durchbiegen, sodass auf eine Angabe der Querschnittsverdrehung verzichtet wird.

Letztlich nahmen sechs lineare Dehnungsmessstreifen (DMS) die Längsverzerrung der äußeren Scheiben 1 und 4 jeweils an der Oberkante $\left(\varepsilon_{\mathrm{OK}}\right)$, der Unterkante $\left(\varepsilon_{\mathrm{UK}}\right)$ und auf halber Trägerhöhe $\left(\varepsilon_{M}\right)$ in Spannrichtung auf, um das Verzerrungsprofil über die Trägerhöhe zu bestimmen. Alle Versuche wurden mit einem Messverstärker ${ }^{183}$ bei einer Messrate von $5 \mathrm{~s}^{-1}$ aufgenommen.

\subsubsection{Untersuchungsverfahren und -bedingungen}

Die Prüfkörper wurden nach ihrer Herstellung unbelastet für mindestens $24 \mathrm{~h}$ im Prüfraum konditioniert. Nach dem Einbau in den Prüfrahmen fand ein zerstörungsfreier Vorversuch zur Überprüfung der Versuchsbedingungen statt.

Im Vorspannversuch wurde das Seil manuell durch das Anziehen der Spannmutter bis zu einer Vorspannkraft nach Tafel 10 vorgespannt. Anschließend erfolgte ein Biegeversuch mit einer Vorlast von $F=0,5 \mathrm{kN}$, um das Tragverhalten aufzuzeichnen. Letztlich wurde das Versagen im Bruchversuch mit vollständigem Bruch oder schrittweisem Teilversagen charakterisiert und die zugehörigen Risslasten $F_{\text {cr.i }}$ ermittelt. Der erste Riss tritt bei der Erstrisslast $F_{c r .1}$ auf, das Versagen mit der finalen Bruchlast $\mathrm{F}_{\text {Bruch. }}{ }^{184}$

Die Belastung erfolgte weggesteuert mit $0,19 \mathrm{~mm} / \mathrm{s}$, sodass die Biegezugspannung an der Glasunterkante um bis zu $2,3 \mathrm{~N} /\left(\mathrm{mm}^{2} \cdot \mathrm{s}\right)$, unter der einheitlichen Annahme eines unbewehrten Einfeldträgers, anstieg. ${ }^{185}$ Bewehrte und vorgespannte Träger reagieren unter Biegebelastung steifer. ${ }^{186}$ Die analytische Berechnung nach Anlage A zeigt jedoch,

\footnotetext{
183 HBM Spider8®.

184 Versagt der Prüfkörper unmittelbar spröde sind die Erstrisslast und die Bruchlast identisch. $F_{\text {cr.1 }}=F_{\text {Bruch }}$.

185 In [DIN EN 1288-3] wird eine Lastrate von 2,0 $\pm 0,4 \mathrm{~N} /\left(\mathrm{mm}^{2} \cdot \mathrm{s}\right)$ gefordert.

186 Vergleiche Kapitel 2.7, ab Seite 64 und Bild 37, Seite 71.
} 
dass die rechnerische Spannungsrate für diese Fälle einen Wert von $2,25 \mathrm{~N} / \mathrm{mm}^{2}$ nicht unterschreitet.

Die Versuche fanden bei Raumtemperatur $\left(22,7 \pm 0,5^{\circ} \mathrm{C}\right)$ statt.

\subsubsection{Analyse- und Auswertungsverfahren}

\section{Vorspannversuch}

Zunächst wird das Tragverhalten unter der Vorspannbelastung als lineare Regression der Messwerte beschrieben. ${ }^{187}$ Dies ermöglicht eine analytische und kontinuierliche Beschreibung des Belastungsvorganges. Dabei werden stets die Bezeichnungen $a_{i}$ für den Anstieg und $b_{i}$ für den Versatz auf der Ordinate ${ }^{188}$ gewählt. Für den Index i wird die Bezeichnung der Messgröße eingesetzt. Die Güte der Regression wird mittels des Bestimmtheitsmaßes $\mathrm{R}^{2}$ beschrieben und mit folgender Interpretation verwendet: ${ }^{189}$

- $\mathrm{R}_{\mathrm{i}}^{2} \geq 0,95$,sehr gute“,

- $\mathrm{R}_{\mathrm{i}}^{2} \geq 0,85$ "gute“,

- $\mathrm{R}_{\mathrm{i}}^{2} \geq 0,65$ „noch nutzbare" und

- $\mathrm{R}_{\mathrm{i}}{ }^{2}<0,65$ „,nicht nutzbare“ analytische Beschreibung der gemessenen Daten.

Wesentliche Ergebnisse sind die Seilkraftänderung infolge des Spannweges $N_{p}\left(w_{P}\right)$, die Änderung der Glasverzerrung $\varepsilon_{i}(P)$ sowie die vertikale $w_{z}(P)$ und die horizontale Durchbie-

187 Das Vorgehen entspricht dem in [Weller \& Engelmann 2014a].

188 Die Belastung beginnt per Definition bei Null $\left(b_{i}:=0\right)$. Der Parameter wird zu Kontrollzwecken dennoch ermittelt. Eine zu große Abweichung zeigt beispielsweise einen Schlupf im System an.

189 Im Fall einer linearen Regression gibt das Bestimmtheitsmaß als Quadrat des Korrelationswertes $r$ den Grad des linearen Zusammenhangs an. Bei $R_{i}{ }^{2}=1$ beschreibt das Regressionsmodell die (punktuellen) Messdaten vollständig, also ohne Abweichung in jedem Punkt. 
gung $v_{y . i}(P)$ infolge der Vorspannkraft mit den Parametern nach Gleichung 40 bis Gleichung 43.

\begin{tabular}{|c|c|c|c|}
\hline$N_{p}\left(w_{P}\right)$ & $=a_{w P} \cdot w_{P}+b_{w P}$ & mit $\mathrm{R}_{w P}^{2}$ & Gleichung 40 \\
\hline$\varepsilon(P)$ & $=a_{\varepsilon} \cdot P+b_{\varepsilon}(P)$ & mit $\mathrm{R}_{\varepsilon}^{2}$ & Gleichung 41 \\
\hline$w_{z . G l a s}(P)$ & $=a_{w} \cdot P+b_{w}(P)$ & mit $R_{w}^{2}$ & Gleichung 42 \\
\hline$v_{y . i}\left(P_{m 0}\right)$ & $=a_{y} \cdot P+b_{y}$ & mit $\mathrm{R}_{y}^{2}$ & Gleichung 43 \\
\hline
\end{tabular}

\section{Bruchversuch - ungerissener Zustand I}

Das Tragverhalten im weggesteuerten Bruchversuch wird durch die gemessenen Verformungs- und Kraftwerte in Abhängigkeit der Belastung durch die Lasttraverse $w_{z . T}$ beschrieben. Aus Vorversuchen ist bekannt, dass mit einem linearen Tragverhalten zu rechnen ist. ${ }^{190}$ Dieses wird pauschal für alle Versuche bis zu $80 \%$ der Erstrisslast angenommen. Entsprechend werden die gewonnenen Messdaten mittels einer linearen Regressionsfunktion zusammengefasst (Gleichung 44 bis Gleichung 48), um die punktuellen Messwerte analytisch beschreiben zu können.

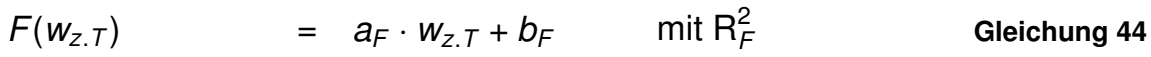

$$
\begin{aligned}
& N_{p}\left(P_{m 0}, w_{z . T}\right) \quad=a_{P} \cdot w_{z . T}+b_{P}\left(P_{m 0}\right) \text { mit } R_{P}^{2} \quad \text { Gleichung } 45 \\
& \varepsilon_{i}\left(P_{m 0}, w_{z . T}\right) \quad=a_{\varepsilon} \cdot w_{z . T}+b_{\varepsilon}\left(P_{m 0}\right) \quad \text { mit } R_{\varepsilon}^{2} \quad \text { Gleichung } 46 \\
& w_{z . G l a s}\left(P_{m 0}, w_{z . T}\right)=a_{w} \cdot w_{z . T}+b_{w}\left(P_{m 0}\right) \text { mit } R_{w}^{2} \quad \text { Gleichung } 47
\end{aligned}
$$


Dabei ist der Traversenweg $w_{z . T}$ im linear elastischen Bereich direkt proportional zur Prüfkraft. Zu Belastungsbeginn wird keine Biegekraft übertragen, sodass der Regressionsparameter $b_{F}$ per Definition Null sein muss. ${ }^{188}$ Damit kann der Traversenweg in Gleichung 44 bis Gleichung 48 durch die Prüfkraft und die zugehörige Steifigkeit $a_{F}$ nach Gleichung 49 ersetzt werden, womit das Tragverhalten auch lastabhängig beschreibbar wird.

$$
w_{z . T}(F)=\frac{F}{a_{F}}
$$

\section{Bruchversuch - Tragverhalten nach Erstriss}

Von bewehrten und vorgespannten Glasträgern wird, neben einer gesteigerten Tragfähigkeit, ein sicheres Tragverhalten nach dem Auftreten des ersten Risses im Glas erwartet. Dieses wird durch einen Vergleich der Bruchlast mit der Erstrisslast angegeben. ${ }^{191}$ Darüber hinaus wird ein Duktilitätskriterium für die Trägerkonstruktion definiert (Gleichung 50). ${ }^{192}$ Dafür wird analog der Traversenweg beim Versagen mit dem Wert beim Auftreten des ersten Glasrisses verglichen.

$$
\begin{array}{ll}
k_{F . d u k}=\frac{F_{c r . i}}{F_{c r .1}} \text { und } & k_{w . d u k}=\frac{W_{z . c r . i}}{W_{z . c r .1}} \\
F_{c r . i} & \text { Risslast } \mathrm{i}(\mathrm{i}>1) \\
F_{c r .1} & \begin{array}{l}
\text { Erstrisslast } \\
\text { vertikale Durchbiegung beim Rissereig- } \\
W_{z . c r . i}
\end{array} \\
\begin{array}{l}
\text { nis } \mathrm{i} \\
\text { vertikale Durchbiegung bei Erstrisslast }
\end{array}
\end{array}
$$

\footnotetext{
191 Martens et al. [2015b] fassen dies als „postfracture performance“ für vorgespannte Glasträger zusammen.

192 [DIN EN 1992-1-1] verwendet analog das Verhältnis der Zugfestigkeit zur Streckgrenze zur Beschreibung der Duktilität der Bewehrung.
} 


\subsubsection{Ergebnisse und Ergebnisdiskussion}

Die Einzelergebnisse der Biegebruchversuche sind in Anlage $B$ zusammengestellt ${ }^{193}$ und werden in diesem Abschnitt getrennt für den Vorspannvorgang, das Biegetragverhalten sowie das Riss- und Bruchverhalten analysiert.

\section{Tragverhalten unter Vorspannbelastung}

Das Verhältnis von Spannweg $w_{P}$ zu Seilkraft $N_{p}$ während des Spannvorgangs ist für drei Beispiele mit Seildurchmessern von $6,1 \mathrm{~mm}, 8,1 \mathrm{~mm}$ und 10,1 mm in Bild 46 (links) dargestellt. Nach einer anfänglichen Krümmung zum Straffen des Seils gehen die Kurven anschließend in einen linearen Verlauf über. Dessen Beginn wird zur Bestimmung der Regressionskoeffizienten ${ }^{194}$ für alle Versuche bei $20 \%$ der angestrebten Vorspannkraft festgelegt. Damit ergeben sich bis zum Erreichen der initialen Vorspannkraft sehr gute lineare Beschreibungen $\left(R_{w P}{ }^{2}>0,98\right)$ der experimentell ermittelten Daten, sodass die analytische Annahme eines linearen Tragverhaltens gerechtfertigt ist.

Daneben (Bild 46, rechts) sind alle Kurvensteigungen $\mathrm{a}_{\mathrm{wP}}$ als Einzelwerte abgedruckt. Sie fallen mit steigendem Seildurchmesser steiler aus. Zum Vergleich ist das analytische Ergebnis nach Gleichung 14 (Seite 70) unter Anwendung der Seilkennwerte aus Tafel 9 (Seite 106) als gestrichelte Linie dargestellt. Die Messergebnisse liegen bei minimal $71 \%$ des rechnerischen Wertes, wobei die Ergebnisse für Seildurchmesser von 6,1 $\mathrm{mm}$ und 8,1 $\mathrm{mm}$ näher am erwarteten Wert rangieren. Die kleineren Steifigkeiten für 10,1 mm dicke Seile ergeben sich daraus, dass eine größere Kraft nötig ist, um die Seile zu straffen, sodass ein Teil der anfänglichen flacheren Kurve $\mathrm{N}_{\mathrm{p}}\left(\mathrm{w}_{\mathrm{P}}\right)$ ebenso in den Regressionsbereich fällt. Darüber hinaus wurde für alle Seile eine Seillänge $L_{p}$ von $2000 \mathrm{~mm}$ angenommen. Mit den tatsächlichen Seillängen einschließlich ihrer Gewindefittinge werden geringe Abweichungen von $81 \%$ bis $112 \%$ errechnet. Das Modell reagiert

\footnotetext{
193 Die Anlagen B, C, D und E wurde mithilfe von [R Core Team 2014] erstellt.

194 Vergleiche Gleichung 40, Seite 111
} 
demnach äußerst sensitiv auf den verwendete Wert der Seillänge. ${ }^{195}$
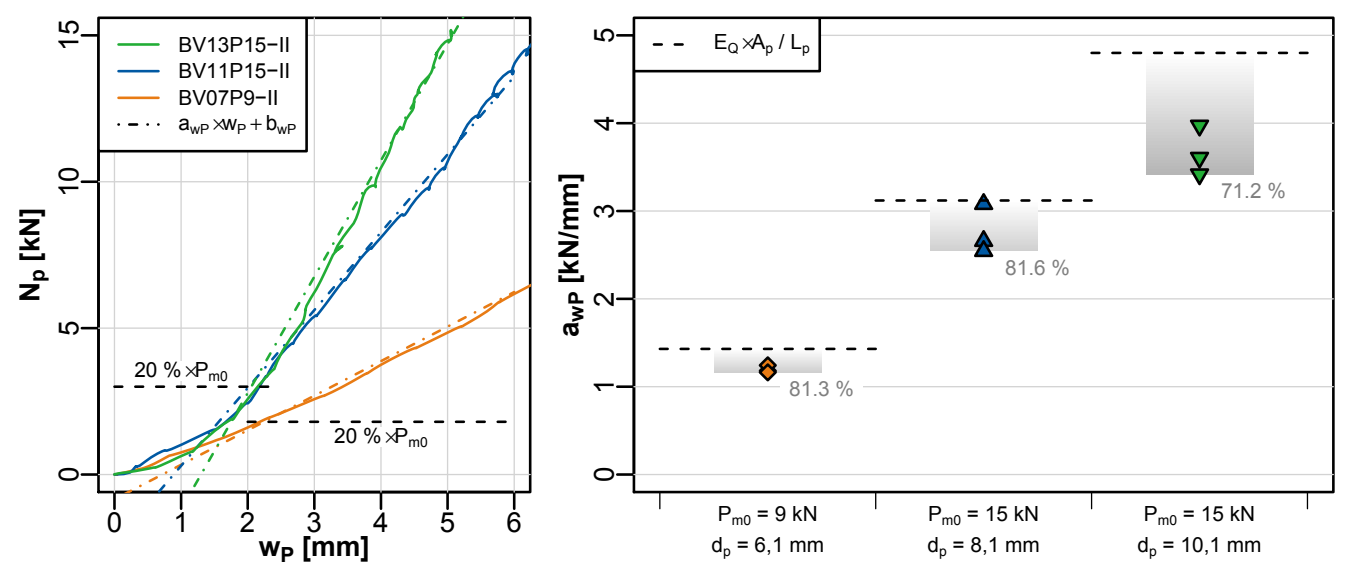

Bild 46

Verhältnis von Spannweg $w_{p}$ zur Seilkraft $N_{p}$ während des Spannvorgangs am Beispiel dreier Prüfkörper (links) und experimentell ermittelte Steifigkeiten $a_{w P}$ für alle Prüfkörper (rechts). Zusätzlich ist das analytische Ergebnis nach Gleichung 111 (Seite 312) vermerkt.

$L_{p}=2000 \mathrm{~mm}$

Außerdem kommt es aufgrund von Imperfektionen ${ }^{196} \mathrm{zu}$ einer seitlichen Durchbiegung und damit zu einer Verkürzung des Trägers infolge der Vorspannung. Im Vergleich zum verwendeten Modell aus Bild 35 (Seite 69) wird das vorgespannte Seil nicht an starren, sondern an nachgiebigen Auflagern verankert, welche zu einer reduzierten Steifigkeit führen ${ }^{197}$ und damit die Abweichungen zwischen analytischem und experimentellem Ergebnis in Bild 46 erklären können. Die Größenordnung der Nachgiebigkeit ist jedoch mangels Wissens über die Verformungsfigur entlang der Trägerlänge unbekannt und wird im Rahmen der numerischen Studie in Kapitel 4 näher untersucht. Damit soll ermittelt werden, ob die horizontale Durchbiegung ein wesentlicher Parameter bei Berechnungen an Spannglasträgern ist.

Bild 47 (links) zeigt die horizontale Durchbiegung beispielhaft für zwei typische Versuche. Der wellenartige Verlauf stimmt mit dem hubweisen, manuellen Anziehen der Spannmutter überein und war daher vermutlich auf ein nicht vollständiges Anliegen der Auflagerkonstruktion am Gabellager des Versuchsstandes zurückzuführen. Dieser Spalt wurde mit jeder

${ }^{195}$ Es sei angemerkt, dass das Verhältnis der Fittinglänge zur freien Seillänge dieser Untersuchung sehr groß ist. Es wurden vergleichsweise "kurze“ Seile verwendet.

196 Vergleiche Kapitel 2.7.2, ab Seite 65.

197 Vergleiche „sofortige Verluste“ in Kapitel 2.7.6, insbesondere Gleichung 18 , Seite 80. 

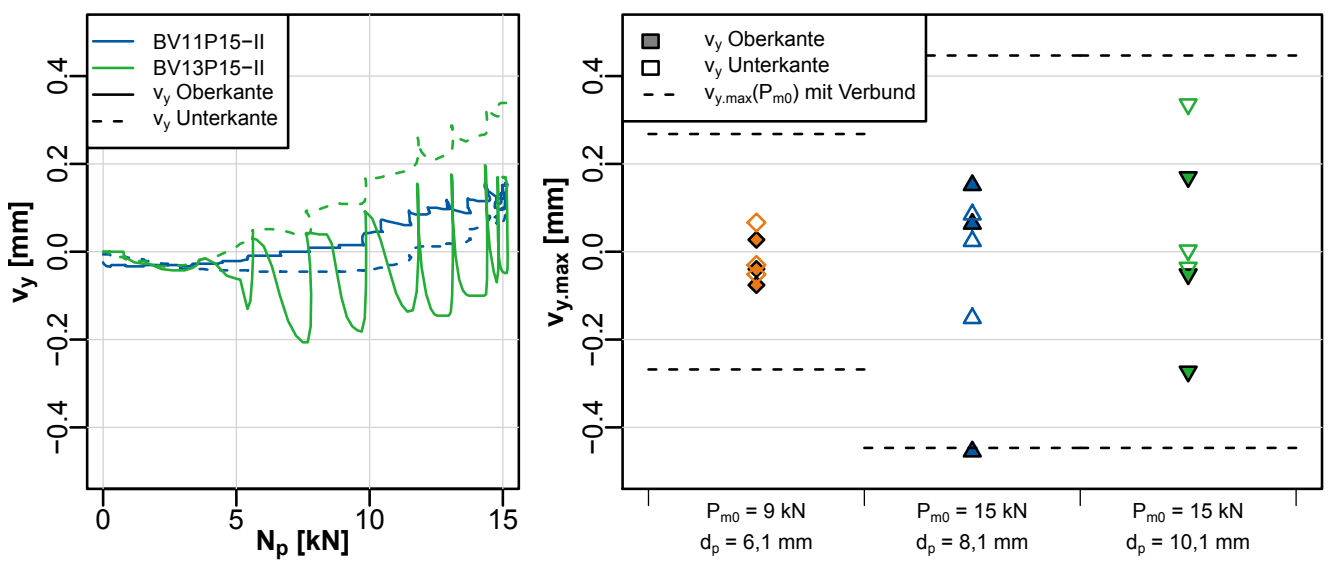

Umdrehung der Spannmutter zunächst geschlossen. Dies verdrehte den Träger am Auflager und resultierte in einer zusätzlichen horizontalen Durchbiegung in Trägermitte. Generell stieg die seitliche Durchbiegung mit steigender Seilkraft, jedoch zeigen die Ergebnisse mit Werten des Bestimmtheitsmaßes $R_{v}{ }^{2}$ zwischen 0,03 und 0,95, im Mittel 0,59 $\pm 0,28$, dass das lineare Regressionsmodell nach Gleichung 43 nicht nutzbar ist.

Infolge der aufgezählten Gründe wird die horizontale Durchbiegung alternativ durch ihre Maximalwerte beim Erreichen der planmäßigen Vorspannkraft ( $v_{\text {y.max }}$ in Bild 47 , rechts) beschrieben. Dieser Wert ist wesentlich, da er das Biegedrillknickversagen der Spannglasträger unter Biegebelastung zusätzlich zur initialen Trägerimperfektion beeinflusst.

Aufgrund der Gabellagerung der Drittelpunkte ist von einer Imperfektion für die Berechnung von 2000 / (3 · 400) $=1,7 \mathrm{~mm}$ im mittleren Drittel auszugehen. ${ }^{198}$ Diese vergröBert sich nach Bild 47 im ungünstigen Fall um einen maximalen Betrag von $0,45 \mathrm{~mm}\left(+26 \%\right.$ für $\left.d_{p}=8,1 \mathrm{~mm}\right)$ und wird als maßgebender Einfluss auf das mögliche Biegedrillknickversagen gewertet.

Darüber hinaus zeigt Bild 47 (links), dass Ober- und Unterkante in unterschiedlichem Maße horizontal durchgebogen

\footnotetext{
198 Belis et al. [2011] giben auf Basis einer umfänglichen Messung eine geometrische Imperfektion von L/400 für unbewehrte Glasträger an.
}

Bild 47

Verhältnis von Seilkraft $\mathrm{N}_{\mathrm{p}}$ zur seitlichen Durchbiegung der Ober- und Unterkante während des Spannvorgangs am Beispiel zweier Prüfkörper (links) und maximale horizontale Durchbiegungen alle Prüfkörper (rechts). 
werden. Es kam demnach zu einer Verdrehung des Glaspaketes um die Trägerlängsachse. Mit den erzielten Ergebnissen ist jedoch keine Angabe einer Verformungsfigur entlang der Trägerlängsachse möglich. ${ }^{199}$ Weitere Angaben zu Vorverdrehungen als geometrische Imperfektion und deren Berücksichtigung fehlen für Glasträger und bilden damit ein offenes Forschungsthema.

Die analytisch errechnete, maximale seitliche Durchbiegung ${ }^{200} v_{y \text {.max }}$ ist zusätzlich in Bild 47 (rechts) als gestrichelte Linie eingezeichnet. Das rechnerische Ergebnis fällt gröBer aus als der experimentelle Wert und liegt demnach auf der sicheren Seite. ${ }^{201}$ Die zitierten Ergebnisse werden damit bestätigt.

Der Anstieg der Kurven im linearen Bereich wird damit als Vorspannsteifigkeit interpretiert, welche maßgeblich durch die Längssteifigkeit des gewählten Seils beeinflusst wird. Mit diesem Ergebnis ist die zweifache Kontrolle des Spannvorganges über Spannkraft und Spannweg möglich, welcher im Spannbetonbau ebenso protokolliert wird.

Zur Beschreibung der Verformungseigenschaften wurden neben der horizontalen auch die vertikale Durchbiegung aufgezeichnet. Die Träger werden mit einer mittleren linearen Steigung von $\mathrm{a}_{\mathrm{wz}}=0,070 \pm 0,004 \mathrm{~mm} / \mathrm{kN}\left(\mathrm{R}_{\mathrm{wz}}{ }^{2}>0,98\right)$ bei Auswertung aller Versuche nach Gleichung 42 vertikal überhöht. Im Experiment fand sich kein deutlicher Unterschied zwischen den Einzelergebnissen bei variierten Nenndurchmessern der Seile. Mit den analytischen Angaben ${ }^{202}$ wird eine Steifigkeit zwischen $0,061 \mathrm{~mm} / \mathrm{kN}$ und $0,067 \mathrm{~mm} / \mathrm{kN}$ ermittelt. Dieser geringe Unterschied (92\%) war experimen-

199 In [Jordão et al. 2014] werden beispielsweise neun Wegaufnehmer zur Bestimmung der Verformungsfigur verwendet.

200 Ein analytisches Vorgehen zur Berechnung der seitlichen Durchbiegung $v_{y . m a x}$ ist in [Weller \& Engelmann 2014a] beschrieben.

201 Unter Annahme des vollen Verbundes im Glaspaket ergeben sich geringere Durchbiegungsergebnisse als ohne eine Berücksichtigung des Schubverbundes im Laminat. Als konservativer Ansatz wird davon ausgegangen, dass beide Glaspakete das gesamte Biegemoment infolge der maximalen Vorspannkraft um die schwache Achse hälftig abtragen und eine Imperfektion im mittleren Felddrittel in der Größenordnung des Erwartungswertes $\mathrm{L} / 1000=2000 /(3 \cdot 1000)=0,67 \mathrm{~mm}$ aus [Belis et al. 2011] vorliegt.

202 Vergleiche Gleichung 107 (Seite 309) und [Weller \& Engelmann 2014a]. 
tell nicht abbildbar. Die vertikale Durchbiegung ist ebenso vorrangig von der wirkenden Seilkraft abhängig. Das analytische Berechnungsmodell (Umlenkkraftmethode) ist nutzbar.

Das Vorspannen zielt darauf ab, ein Spannungsprofil über die Trägerhöhe $\sigma_{P}(z)$ nach Bild 11 (Seite 32) einzustellen. Entsprechend werden die Ergebnisse der Dehnungsmessung an Ober- und Unterkante sowie auf Höhe der Schwerachse des Glasquerschnittes in Tafel 11 zusammengestellt. Experimentell zeigen sich keine erkennbaren Unterschiede in Bezug auf den Seildurchmesser. ${ }^{203}$

Die Verzerrungsverteilung ist vorrangig von der wirkenden Seilkraft abhängig und zeigt eine sehr gute Übereinstimmung $\left(R_{\varepsilon}^{2}>0,98\right)$ zwischen dem linearen Modell nach Gleichung 40 (Seite 111) und den Versuchsergebnissen. Des Weiteren wird deutlich, dass die Oberkante mit steigender Seilkraft auf Zug $\left(a_{\varepsilon . O K}>0\right)$, die Unterkante aufgrund der exzentrischen Spanngliedanordnung in größerem Maße auf Druck $\left(a_{\varepsilon}, U K<0\right)$ beansprucht wird. Die Schwerachse wird aufgrund der Wirkung der Normalkomponente der Seilkraft ebenso überdrückt $\left(a_{\varepsilon . M i t t e}<0\right)$. Die Vorspannkraft wirkt sich somit linear auf die Glasverzerrung aus $\left(\mathrm{R}_{\varepsilon}{ }^{2} \geq 0,98\right)$ und das angestrebte Spannungsprofil, insbesondere ein Vordrücken der Zugkante, stellt sich ein.

\begin{tabular}{lrll}
\hline $\begin{array}{l}\text { Verzerrungs- } \\
\text { parameter }\end{array}$ & $\begin{array}{l}\mathbf{a}_{\varepsilon} \\
{[\mu \mathbf{m} /(\mathbf{m} \cdot \mathbf{k N})]}\end{array}$ & $\begin{array}{l}\mathbf{b}_{\varepsilon} \\
{[\mu \mathbf{m} / \mathbf{m}]}\end{array}$ & \multicolumn{1}{l}{$\begin{array}{l}\mathbf{R}_{\varepsilon} \\
{[-]}\end{array}$} \\
\hline Oberkante $\varepsilon_{\mathrm{OK}}$ & $8,48 \pm 0,52$ & $-0,64 \pm 1,90$ & 0,992 \\
Mitte $\varepsilon_{\mathrm{M}}$ & $-3,90 \pm 0,35$ & $0,13 \pm 2,18$ & 0,982 \\
Unterkante $\varepsilon_{\mathrm{UK}}$ & $-16,25 \pm 0,44$ & $0,89 \pm 2,99$ & 0,997 \\
\hline
\end{tabular}

Im Vergleich zu den Ergebnissen in Tafel 11 werden mittels Gleichung 104 (mit $F=0$ und $\Delta T=0$ ) Stauchungen an der Unterkante zwischen $-14,36$ bis $-15,48 \mu \mathrm{m} /(\mathrm{m} \cdot \mathrm{kN})(88 \%$ bis $95 \%$ ) sowie Zugdehnungen an der Oberkante zwischen 7,14 und $7,70 \mu \mathrm{m} /(\mathrm{m} \cdot \mathrm{kN})(84 \%$ bis $91 \%)$ berechnet. Die Abweichung wird als akzeptabel beurteilt. Die analytische Berechnung kann für eine Abschätzung des realen Tragverhaltens verwendet werden.

203 Einzelwerte in Anlage B, ab Seite 319.
Tafel 11

Regressionsparameter nach Gleichung 41 (Seite 111) zur Beschreibung der Verzerrungsänderung an Oberkante, Mitte und Unterkante als Mittelwerte aus den Einzelmessungen. 
Im Zuge der Versuchsreihe wurden Umlenkkraftverluste ermittelt. Bild 48 (links) zeigt dazu die gemessene Seilkraft am Spannanker (A) und am Festanker (B) während eines gesonderten Vorspannvorgangs. Die Relation der gemessenen Werte sind in Bild 48 (rechts) aufgetragen. Es zeigen sich maximale Unterschiede von $8 \%$ während des Spannvorgangs. Dieses Ergebnis ist auf einen leichten Nachlauf der Messsignale beider Kraftmessdosen zurückzuführen. Während der Haltezeit ergaben sich im Mittel maximal $5 \% \mathrm{Ab}$ weichung. Dieser Betrag wird bei der weiteren Angabe von Seilkräften als Streubereich mit dargestellt.

Der gemessene Unterschied kann sowohl auf Reibungsverluste am Umlenksattel, als auch aus individuellen Unterschieden der beiden verwendeten Kraftmessdosen resultieren. Letzteres wurde ausgeschlossen, indem das Seil zunächst ohne Umlenkung, direkt von Auflager zu Auflager, durch den Spalt im Trägerquerschnitt gespannt wurde. Dabei unterschieden sich beide Messsignale nicht bedeutsam $(<1 \%)$, sodass das oben ermittelte Ergebnis auf die alleinige Wirkung der Umlenkung zurückzuführen war.
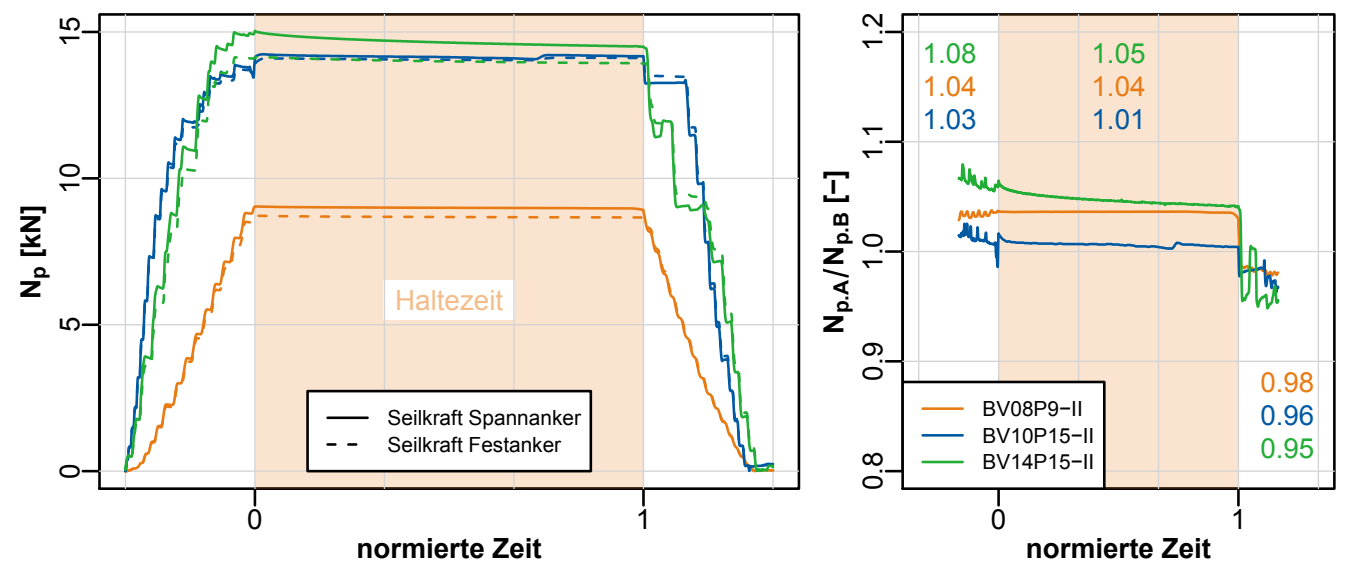

\section{Bild 48}

Umlenkkraftverlust als Verhältnis der Seilkräfte am Spannanker $\mathrm{N}_{\text {p.A }}$ und Festanker $N_{\text {p.B }}$ (links) und die Messwerte während des Vorspannens, Haltens und Entspannens (rechts) jeweils als normierte Zeitdarstellung.

Damit konnten die Ergebnisse aus Voruntersuchungen ${ }^{204}$ bestätigt werden. Die Wirkung der Vorspannkraft auf den Träger wird mit ausreichender Genauigkeit nach linearer Balkentheorie analytisch beschrieben.

${ }^{204}$ Vergleiche [Weller \& Engelmann 2014a]. 


\section{Tragverhalten im ungerissenen Zustand unter Biegebelastung (Zustand I)}

Die Angaben des folgenden Abschnitts beziehen sich auf den Lastbereich bis $80 \%$ der Erstrisslast.

Die Einzelergebnisse in Anlage B zeigen im gewählten Auswertebereich einen linearen Anstieg der Biegesteifigkeit ${ }^{205}$ $\left(R_{F}{ }^{2}>0,99\right)$ als Verhältnis von Biegelast $F$ und Traversenweg $w_{z . T}$. Die unbewehrten Referenzträger zeigten den kleinsten $\left(a_{\mathrm{F}}=1,95 \mathrm{kN} / \mathrm{mm}\right)$, die Gruppe der auf $15 \mathrm{kN}$ vorgespannten Träger mit 10,1 mm Spiralseil den größten $\left(\mathrm{a}_{\mathrm{F}}=\right.$ $2,04 \mathrm{kN} / \mathrm{mm}$ ) Anstieg. Der Unterschied von weniger als $5 \%$ wird als nicht bedeutend eingestuft, sodass das Tragverhalten im weggesteuerten Versuch für alle Träger mit der mittleren Laständerung von $\mathrm{a}_{\mathrm{F}}=1,98 \pm 0,08 \mathrm{kN} / \mathrm{mm}$ beschreibbar ist. ${ }^{206}$

Dem gegenüber fällt das analytische Ergebnis ${ }^{207}$ mit Werten zwischen $3,33 \mathrm{kN} / \mathrm{mm}$ und $3,54 \mathrm{kN} / \mathrm{mm}$ deutlich größer aus. Diese Werte entsprechen vielmehr dem bereinigten Verhältnis aus der Biegelast und der reinen Glasdurchbiegung ${ }^{208}$ mit $3,00 \pm 0,12 \mathrm{kN} / \mathrm{mm}$. Aus dem Unterschied wird der Einfluss der Auflager- und Prüfkonstruktion auf das Ergebnis deutlich. Er wird in den folgenden Ergebnissen mit einem Faktor von 1,98 / 3,00 =0,66 berücksichtigt, um einen Vergleich mit gleichen Randbedingungen zu ermöglichen. Darüber hinaus verbleibt eine Differenz zwischen $0,33 \mathrm{kN} / \mathrm{mm}$ und $0,54 \mathrm{kN} / \mathrm{mm}$ ( $11 \%$ bis $18 \%$ ), welche zusätzlich als Abweichung der weiteren Ergebnisse erwartet wird.

Infolge der Biegebelastung steigt die Seilkraft, wie in Bild 49 (links) beispielhaft gezeigt, linear an $\left(R_{P}^{2}>0,99\right)$. Dabei wird mit steigendem Seildurchmesser eine größer werdende Steifigkeit ap gemessen (Bild 49, rechts). Die Referenzwerte der schlaff bewehrten Träger liegen dabei etwas unterhalb der Ergebnisse der vorgespannt bewehrten Träger bei gleichem Seildurchmesser. Es zeigt sich, dass das Seil zu Belastungs-

\footnotetext{
205 Vergleiche Gleichung 44, Seite 111.

206 Vergleiche Gleichung 49, Seite 112.

207 Einzelwerte in Tafel 37, Seite 311.

208 Siehe Gleichung 39, Seite 108.
} 
beginn $\left(w_{z . T}<5 \mathrm{~mm}\right)$ zunächst durch die Wirkung der Biegelast gestrafft werden muss, was sich in einem Knick der Kurve zeigt. Dies ist für die vorgespannten Konstruktionen bereits mit Blick auf Bild 46 (Seite 114) vorweg genommen und führt im Mittel zu einem kleineren Steifigkeitswert ap. Auf die Güte der linearen Regression wurde jedoch keine wesentliche Auswirkung festgestellt. Zusätzlich ist ein leichtes Abflachen der Seilkraftkurven nahe der Erstrisslast zu beobachten.
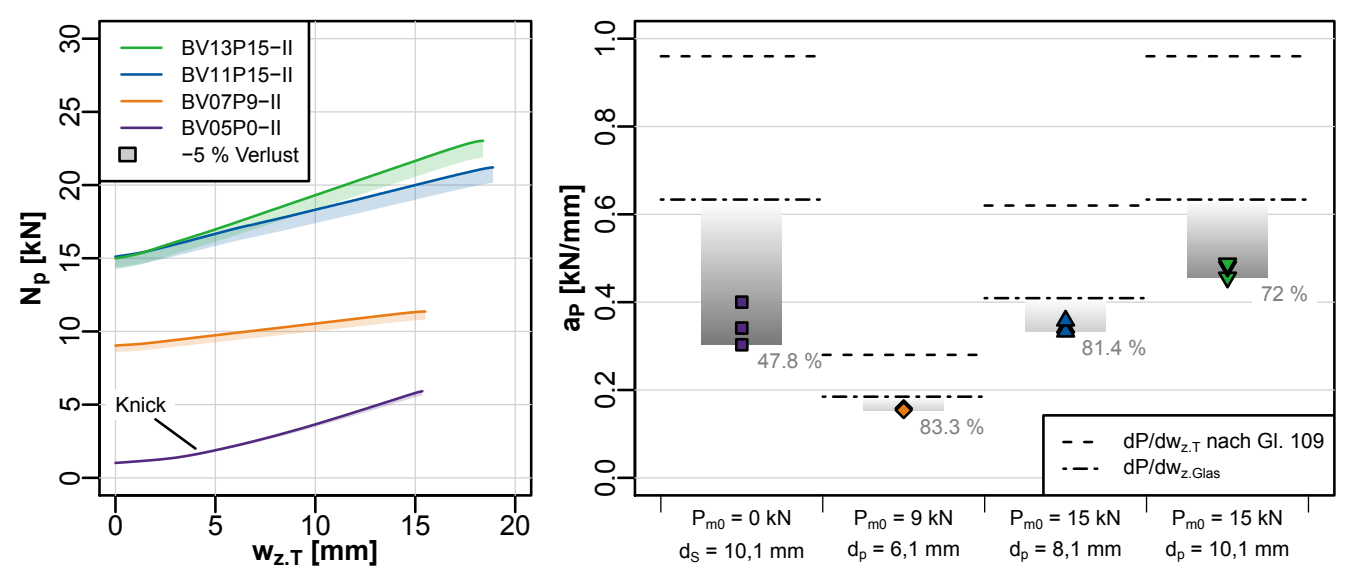

\section{Bild 49}

Beispielhafte Steigerung der Seilkraft während des Spannvorgangs (links) und ermittelte Steifigkeiten $a_{p}$ für alle Prüfkörper (rechts) nach Gleichung 109 (Seite 310 in Anlage A) bis zur Erstrisslast.

Das in Bild 49 (rechts) gezeigte Ergebnis ap ist bezüglich der Traversengeschwindigkiet als auch bezüglich der reinen Glasdurchbiegung ${ }^{209} \mathrm{im}$ rechten Bild 49 eingetragen. Alle experimentellen Werte liegen zwischen $47,8 \%$ und $83,3 \%$ des Erwartungswertes, sodass die Begrenzung der Seilkraft auf der sicheren Seite liegend analytisch erfolgen kann. Es fällt auf, dass alle Prüfkörper mit einem Seildurchmesser von 10,1 mm im Vergleich zum analytischen Ergebnis mit der größten Differenz abschneiden. Daher kann die Seildicke, unabhängig von der Vorspannkraft, als ausschlaggebender Faktor nicht ausgeschlossen werden. Diese Vermutungen werden daher im Rahmen der rechnerischen Abbildung in Kapitel 4 näher untersucht.

Die Biegebelastung wirkt der exzentrischen Vorspannung entgegen und baut die Vorstauchung der Glaskanten line$\operatorname{ar} a b\left(R_{\varepsilon}{ }^{2}>0,99\right)$. Dabei ergab sich kein messbarer Unter-

209 Vergleiche Gleichung 112, Seite 313 in Anlage A. 
schied der Verzerrungsänderung an Ober- und Unterkante die Steifigkeitswerte $a_{\varepsilon}$ in Tafel 12 unterscheiden sich nicht wesentlich. Die darin zusätzlich angegebenen Werte für $b_{\varepsilon}$ spiegeln die anfängliche Verzerrung infolge der Seilkraft aus dem Vorspannversuch wieder. Die Änderung der Verzerrung auf Höhe der Schwerachse ist im Referenzträger ohne Vorspannung planmäßig Null. ${ }^{210}$ Für die bewehrten und vorgespannten Träger wurde ein negativer Anstieg gemessen, dessen Größenordnung erwartungsgemäß deutlich geringer war als die Werte an Ober- und Unterkante. ${ }^{211}$

\begin{tabular}{llrll}
\hline $\begin{array}{l}\text { Verzerrungs- } \\
\text { parameter }\end{array}$ & $\begin{array}{l}\mathbf{a}_{\varepsilon} \\
{[\mu \mathbf{m} /(\mathbf{m} \cdot \mathbf{m m})]}\end{array}$ & $\begin{array}{l}\mathbf{P}_{\mathbf{m 0}} \\
{[\mathbf{k N}]}\end{array}$ & $\begin{array}{l}\mathbf{b}_{\varepsilon} \\
{[\mu \mathbf{m} / \mathbf{m}]}\end{array}$ & $\begin{array}{l}\mathbf{R}_{\varepsilon} \\
{[-]}\end{array}$ \\
\hline Oberkante $\varepsilon_{\mathrm{OK}}$ & $-114,8 \pm 3,2$ & 9,0 & $75,1 \pm 17,6$ & 0,999 \\
& & 15,0 & $133,6 \pm 16,7$ & \\
Unterkante $\varepsilon_{\mathrm{UK}}$ & $112,0 \pm 3,2$ & 9,0 & $-145,8 \pm 10,4$ & 0,998 \\
& & 15,0 & $-249,8 \pm 15,0$ & \\
\hline
\end{tabular}

Aus diesem Ergebnis kann die mittlere Spannungsänderung im Glas mit berechnet werden. ${ }^{212}$ Sie ergibt sich zu $1,5 \mathrm{~N} /\left(\mathrm{mm}^{2} \cdot \mathrm{s}\right)$ und liegt damit bei $66 \%$ des angestrebten Wertes von 2,3 N/(mm $\left.{ }^{2} \cdot \mathrm{s}\right) \cdot{ }^{213}$ Ein Grund dafür kann erneut durch Auswertung der Ergebnisse zur vertikalen Durchbiegung ${ }^{214}$ gefunden werden. Sie gibt die Änderung der vertikalen Glasdurchbiegung abzüglich der Stauchung der Auflagerklotzung an. Im Mittel werden hier $a_{w z}=-0,66 \pm 0,01 \mathrm{~mm} / \mathrm{mm}$ für alle Träger gemessen. Mit einer Traversengeschwindigkeit ${ }^{215}$ von $0,19 \mathrm{~mm} / \mathrm{s}$ biegt sich der reine Glasquerschnitt mit $0,13 \mathrm{~mm} / \mathrm{s}$ durch. Die Idealisierung des Tragsystems mit Vernachlässigung der Stauchung der Klotzung ist demnach nicht ausreichend, sodass die untere Grenze der Lastrate nach [DIN EN 1288-3] mit 1,6 N/(mm².s) verfehlt wird. Auf eine Anpassung der Glasbruchspannungen wird jedoch verzichtet, da die Abweichung einerseits als geringfügig beurteilt wird und andererseits alle Probekörper unter den gleichen Bedingungen geprüft wurden, wodurch weiterhin einen Ergebnisvergleich ermöglicht wird.

\footnotetext{
210 Folglich ist das zugehörige Bestimmtheitsmaß ebenso Null.

211 Die Einzelergebnisse finden sich in Anlage B.

212 Mit der Travensengeschwindigkeit aus Gleichung 109, Seite 310 in Anlage $A$ und $E_{G}=70000 \mathrm{~N} / \mathrm{mm}^{2}$.

213 Vergleiche Tafel 37, Seite 311 in Anlage A.

214 Vergleiche Gleichung 42, Seite 111.

215 Vergleiche Gleichung 109, Seite 310 in Anlage A.
}

Tafel 12

Regressionsparameter nach Gleichung 46 (Seite 111) zur Beschreibung der Verzerrungsänderung an Oberkante, Mitte und Unterkante als Mittelwerte aus den Einzelmessungen. 
Der Einfluss der Stauchung der Klotzung wird für die Versuche unter Dauerlast nach Kapitel 3.4 berücksichtigt, da hier versuchsbedingt keine Messung der Durchbiegung nahe der Auflager erfolgt. Bei einer Ist-Biegelast von $F=10,6 \mathrm{kN}$ wird am Auflager A im Mittel ein Wert von 0,34 $\pm 0,16 \mathrm{~mm}$ und am Auflager $B$ 0,58 $\pm 0,11 \mathrm{~mm}$ bei einer bewehrten Konstruktion sowie $0,52 \pm 0,10 \mathrm{~mm}$ am Auflager $A$ und $0,71 \pm$ $0,10 \mathrm{~mm}$ am Auflager $B$ bei einer unbewehrten Referenzkonstruktion ermittelt.

Letztlich werden die Ergebnisse der horizontale Durchbiegung nach Gleichung 43 (Seite 111) analysiert. Ausgehend von der initialen seitlichen Durchbiegung aus dem Vorspannvorgang wurden sowohl annähernd konstante, als auch ansteigende Durchbiegungen beobachtet. Eine Zuordnung zu einer Konstruktionsart war dabei nicht möglich.

Die lineare Beschreibung war im gewählten Lastbereich nicht durchgehend zutreffend. Im Mittel ergaben sich Bestimmtheitsmaße von $R_{\text {v.unten }}{ }^{2}=0,579 \pm 0,32$ und $R_{v \text {.oben }}{ }^{2}=$ $0,834 \pm 0,16$, die deutlich unter den bisher ermittelten Werten anderer Messsignale liegen sowie eine bedeutend gröBere Standardabweichung aufweisen. Dabei wurden Werte von $\mathrm{R}_{\mathrm{v}}{ }^{2}<0,65$ nur bei geringen Steigungen unterhalb von $0,02 \mathrm{~mm} / \mathrm{mm}$ ermittelt (Bild 50). Darüber ist keine Abhängigkeit der seitlichen Durchbiegung von der Konstruktionsart und der Vorspannkraft zu erkennen.

Bild 50

Veränderung der horizontalen Durchbiegung $a_{v}$ nach Gleichung 43 (Seite 111) gegenüber des zugehörigen Bestimmtheitsmaßes $\mathrm{R}_{\mathrm{v}}{ }^{2}$.

Es sind sowohl die Ergebnisse der Oberkante, als auch der Unterkante dargestellt. Die Wahl der Symbole und des Farbcodes entspricht den voran gegangen Darstellungen.

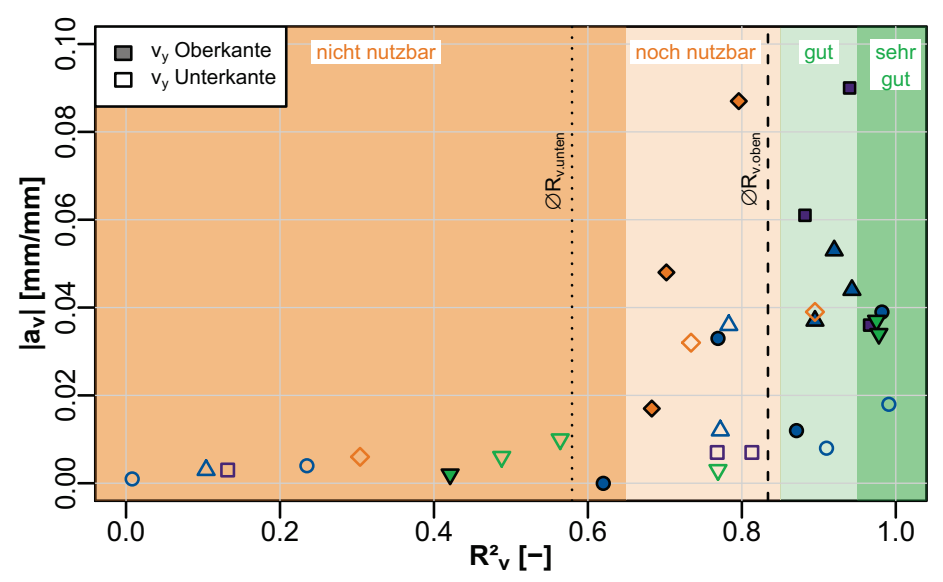

Es kann vermutet werden, dass dieses uneinheitliche Bild auf den zufälligen Charakter der Trägerimperfektion zurück 
zu führen ist, welcher den wesentlichen Einflussfaktor der seitlichen Durchbiegung darstellt. Diese Vermutung wird mittels der numerischen Berechnungen in Kapitel 4 überprüft.

\section{Tragverhalten nach Erstriss (Zustand II)}

Das folgende Bild 51 (links) gibt die Erstrisslast an. Die Ergebnisse liegen im Bereich zwischen 23,7 kN und 36,7 kN, wobei die Mittelwerte jeder Gruppe mit steigender Vorspannkraft ansteigen.

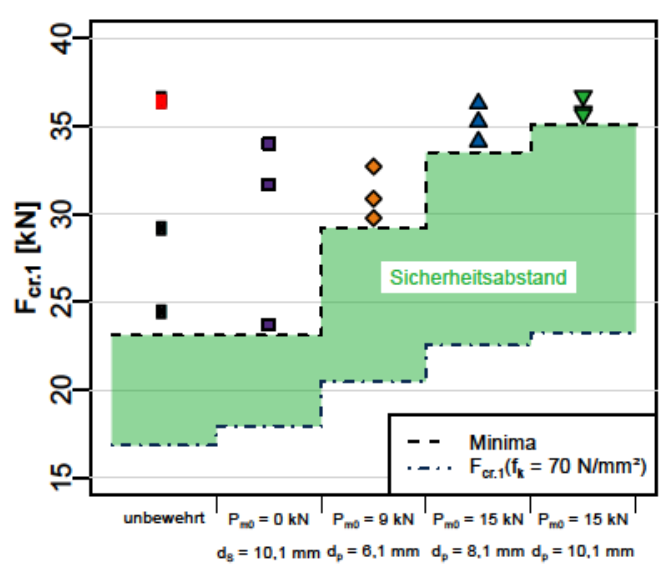

Der unbewehrte Referenzträger BV 03 zeigte mit $36,6 \mathrm{kN}$ unerwartet die zweitgrößte Erstrisslast aller Versuche. Um den Einfluss der Prüfeinrichtung auf diesen Einzelversuch auszuschließen wurde im Anschluss an die Studie eine Wiederholung mit einem einzelnen Prüfkörper durchgeführt. ${ }^{216}$ Dessen Ergebnis ist rot markiert und zeigte erneut eine auffällig hohe Risslast von $36,4 \mathrm{kN}$. Damit wurde ein zufällig hoher Wert aufgrund eines unberücksichtigten Einflusses ausgeschlossen.

Basierend auf den experimentellen Ergebnissen ist die erwartete Erstrisslast $F_{c r .1}\left(f_{k}\right)$ ebenso in Bild 51 (links) einge-

${ }^{216}$ Der Prüfkörper wird mit „BV 03PX-II 2“ bezeichnet. Seine Ergebnisse im Vorspann- und Biegeversuch sind in die obigen Betrachtungen eingeflossen.
Bild 51

Erstrisslast (links) und die zugehörigen Spannungen an der Glasunterkante (rechts).

$\overline{\mathrm{x}} \pm \mathrm{s}=119,7 \pm 16,6 \mathrm{~N} / \mathrm{mm}^{2}$ 
zeichnet. ${ }^{217}$ Dabei zeigt sich ein steigender Trend in Richtung größerer Vorspannkräfte und größerer Seildurchmesser, welcher ebenso durch die Markierung der minimalen Erstrisslasten je Gruppe deutlich wird. Der Unterschied zwischen minimaler Erstrisslast und Biegekraft beim Erreichen der charakteristischen Festigkeit kann als (charakteristischer) Sicherheitsabstand bezeichnet werden.

Bevor jedoch geschlussfolgert wird, dass die Vorspannung die Erstrisslast vergrößert, sind die ermittelten Bruchspannungen ${ }^{218}$ zu überprüfen. Es ist zu befürchten, dass der beobachtete Trend aufgrund zufällig hoher Bruchspannungen zustande kam. Dagegen sprechen die Ergebnisse in Bild 51 (rechts). Hier sind die zugehörigen Glasspannungen sowie der empirische Mittelwertes $\bar{x}$ und die zugehörige Standardabweichung s für alle Versuche dargestellt. Für alle Gruppen liegen Werte in gleichmäßiger Verteilung um den Mittelwert vor. Einzig die Gruppe der mit 9 kN vorgespannten Prüfkörper zeigt eine Tendenz zu geringeren Werten.

In beiden fraglichen Fällen (BV 03 und BV 03-2) beträgt die Bruchspannung $147 \mathrm{~N} / \mathrm{mm}^{2}$ und $142 \mathrm{~N} / \mathrm{mm}^{2}$, welche zweifach über der charakteristischen Festigkeit von teilvorgespanntem Glas liegt (Bild 51, rechts). Bereits Laufs [2000] berichtet von derart hohen Werten für teilvorgespanntes Glas. ${ }^{219}$ Darüber hinaus ist allgemein bekannt, dass die Festigkeitswerte von Glas im Vergleich zu anderen Baustoffen ausgesprochen stark streuen. ${ }^{220}$ Damit werden statistische Ausreißer innerhalb der eigenen Versuchsergebnisse ausgeschlossen.

Insgesamt wird geschlussfolgert, dass die Erstrissspannung den erwartenden Werten üblicher Glasprodukte entsprechen. Damit wird ein Einfluss zufällig hoher Bruchspannungen ausgeschlossen. Für einen finalen Nachweis, im Sinne

217 In Gleichung 44 und Gleichung 45 auf Seite 111, wird die charakteristische Festigkeit von TVG $\left(f_{k}=70 \mathrm{~N} / \mathrm{mm}^{2}\right)$ verwendet. Dies ist möglich, da die ermittelte Regression im hier vorliegenden linear elastischen Bereich (80\% der Bruchlast) gültig ist.

218 Bruchauslösendes Kriterium ist das Erreichen der Festigkeit an der zugbelasteten Glasunterkante.

219 Im Vierpunkt-Biegeversuch zwischen $110 \mathrm{~N} / \mathrm{mm}^{2}$ und $150 \mathrm{~N} / \mathrm{mm}^{2}$.

220 Hier mit einem Variationskoeffizienten von $v=s / \bar{x}=0,14$. In $[B R L A$ 2015/2, Anlage 11.5 Tab. 2] werden für Floatglas Werte zwischen 0,10 und 0,28 angegeben. 
der statistisch fundierten Angabe einer gesteigerten Erstrisslast, ist jedoch eine größere Anzahl an Prüfkörpern ${ }^{221}$ nötig, insbesondere da die Ergebnisse zur Erstrisslast von der vergleichsweise breiten Streuung der Glasfestigkeit überlagert wurden.

Die Versagensart der Prüfkörper wurde in drei Gruppen, den sofortigen sowie den schrittweisen Bruch mit degressiver und progressiver Weiterbelastung (Bild 52), eingeteilt, um anschließend die Rissentwicklung zu analysieren.
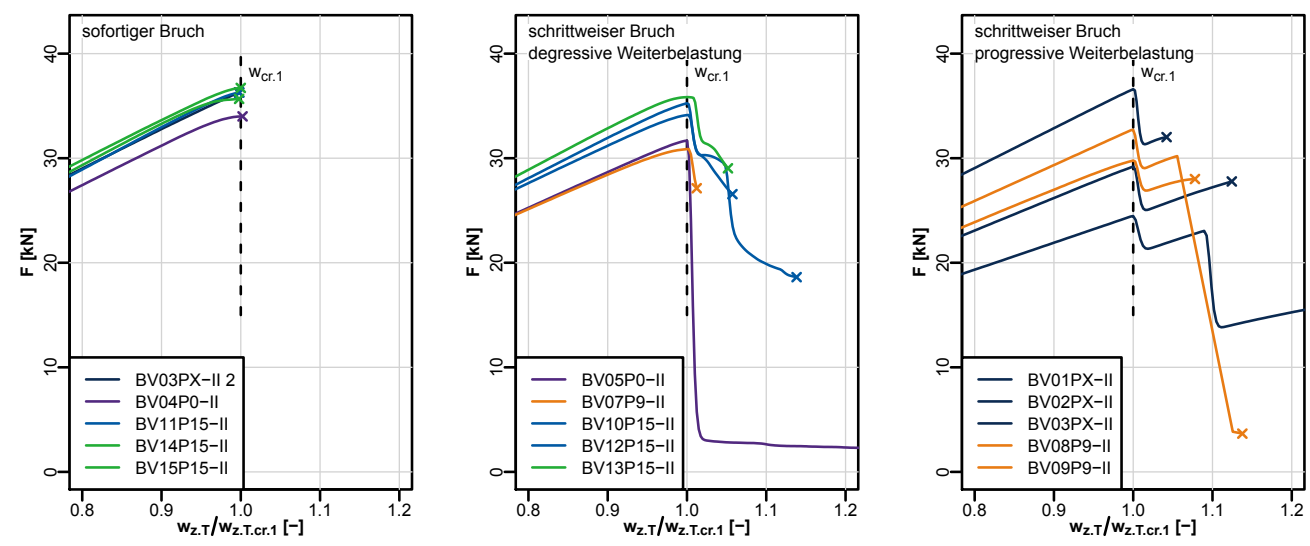

Bild 52

Typisiertes Bruchversagen.

Zum Ersten kam es beim Erreichen der Erstrisslast zum sofortigen „explosionsartigen“ Bruch aller Scheiben und in desDer Sonderfall BV 06 ist nicht dargestellt. sen Folge zum vollständigen Versagen des Trägers (Bild 52, links sowie Bild 53). Dieser Fall trat nur bei bewehrten und vorgespannten Prüfkörpern auf. Die zugehörigen Seilkräfte gingen ebenso unmittelbar mit dem Bruch verloren. Dieses Ergebnis entspricht dem spröden Versagenscharakter üblicher Glaskonstruktionen und wird nicht angestrebt, da keine Sicherheitsreserve nach Bruch gewonnen wurde. Folglich wird kein Duktilitätswert ${ }^{222}$ angegeben.

Zum Zweiten bestand nach dem Riss mindestens einer Scheibe weiterhin die Möglichkeit zum Lastabtrag mit fallender Prüflast bis zum vollständigen Versagen des Trägers. Dieser Fall des schrittweisen Versagens trat für Prüfkörper aller

\footnotetext{
221 Vergleiche beispielsweise [BRL A 2015/2, Anlage 11.5 Tab. 2].

222 Vergleiche Gleichung 50, Seite 112.
} 
Bild 53

Rissentwicklung im Prüfkörper BV 15.

(a) Rissfreier Zustand I

(b) „explosionsartiges" Versagen.

\section{Bild 54}

Rissentwicklung im Prüfkörper BV 10.

(a) Rissfreier Zustand I

(b) Begrenztes Rissbild in Scheibe 4

(c) Erweiterung der Risse im Obergurt (1) sowie einzelnen Rissausläufer (2)

(d) „explosionsartiges“ Versagen.

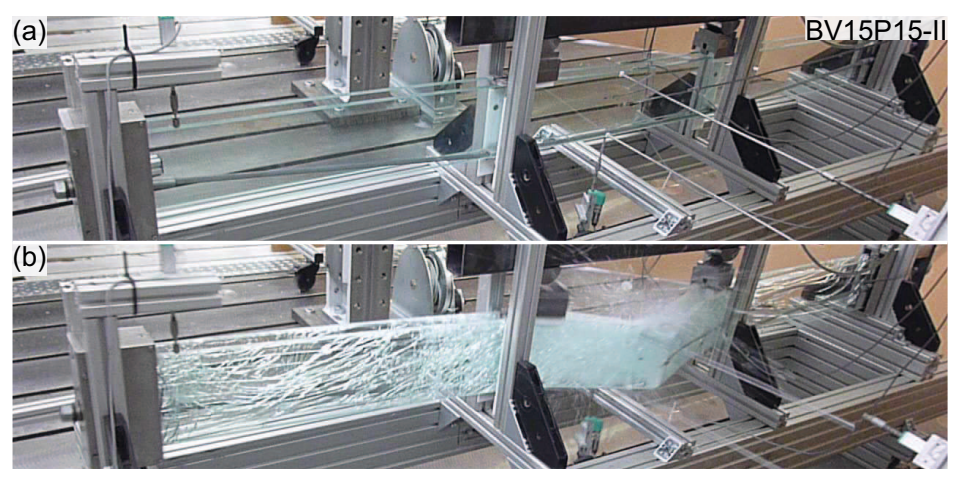

Bewehrungstypen auf. In Bild 54 ist zu erkennen, dass sich zunächst ein begrenztes, dichtes Rissbild in Scheibe 4 des Prüfkörpers BV $10 \mathrm{im}$ mittleren Trägerdrittel einstellt (b). Das Rissbild erweitert sich mit horizontalen Risslinien im Obergurt (c1) sowie einzelnen Rissausläufern in die äußeren Trägerdrittel (c2), um mit dem „explosionsartigen“ Trägerversagen (d) abzuschließen.

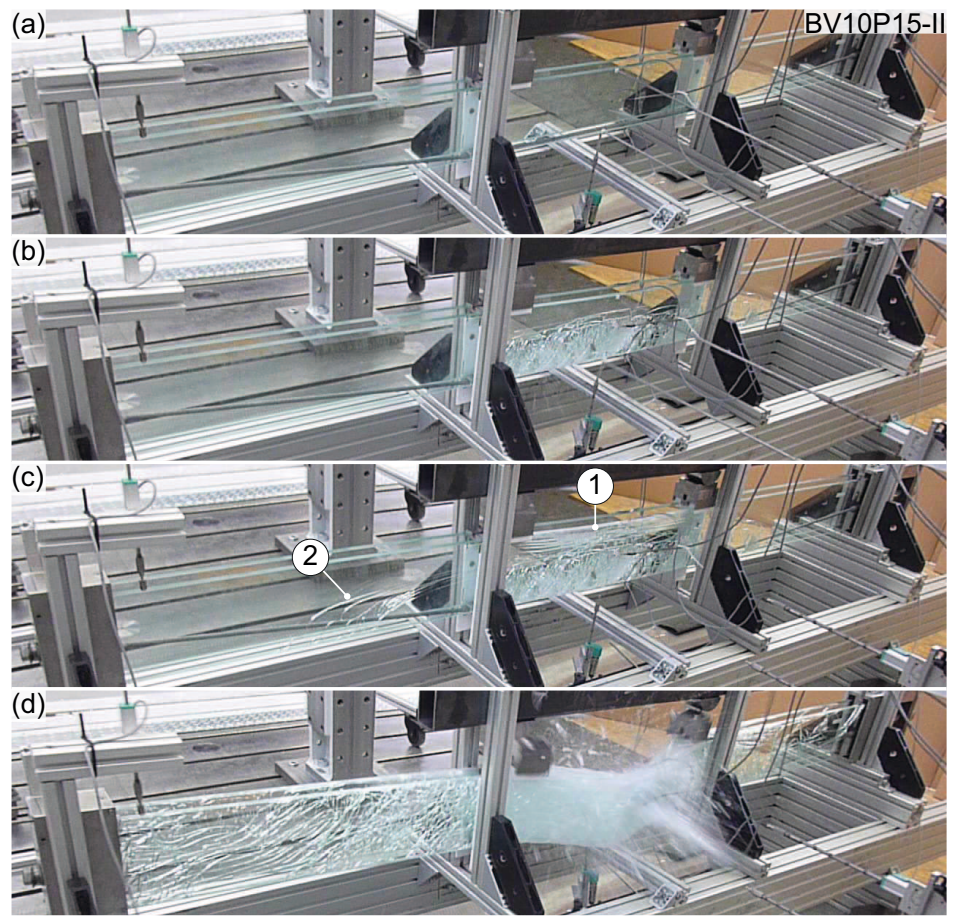

Im dritten Fall verblieb nach dem Erstriss mindestens eine Scheibe intakt, sodass der Verbundglasquerschnitt nach einem kurzzeitigen Lastabfall mit verringerter Steifigkeit progressiv weiter belastet werden konnte. Es handelte sich 
ebenso um ein schrittweises Versagen, welches insbesondere im Fall unbewehrter Referenzträger und bei Prüfkörpern mit $9 \mathrm{kN}$ Vorspannung und 6,1 mm Bewehrungsseil auftrat. Es entstand erneut ein begrenztes, dichtes Rissbild (Bild 55) in Trägermitte beiderseits des Bruchursprungs (1). AnschlieBend erweiterten sich die Risse. Im Fall des hier beispielhaft gezeigten Prüfkörpers BV 08 sind insbesondere lokale Abplatzungen im Obergurtbereich neben der Lasteinleitungsstelle (2) sowie weitergreifende, einzelnen Rissausläufern in das Auflager (3) zu erkennen. Der zufällige Charakter der Rissbildung ist auf die relative Inhomogenität der thermischen Vorspannung zurückzuführen.

Nach dem Erstriss wurde eine weitere Steigerung der Seilkraft in solchen Prüfkörpern vorgefunden, die weiterbelastet werden konnten. Entsprechend traten die bemessungsrelevanten Werte in diesem Tragzustand auf.

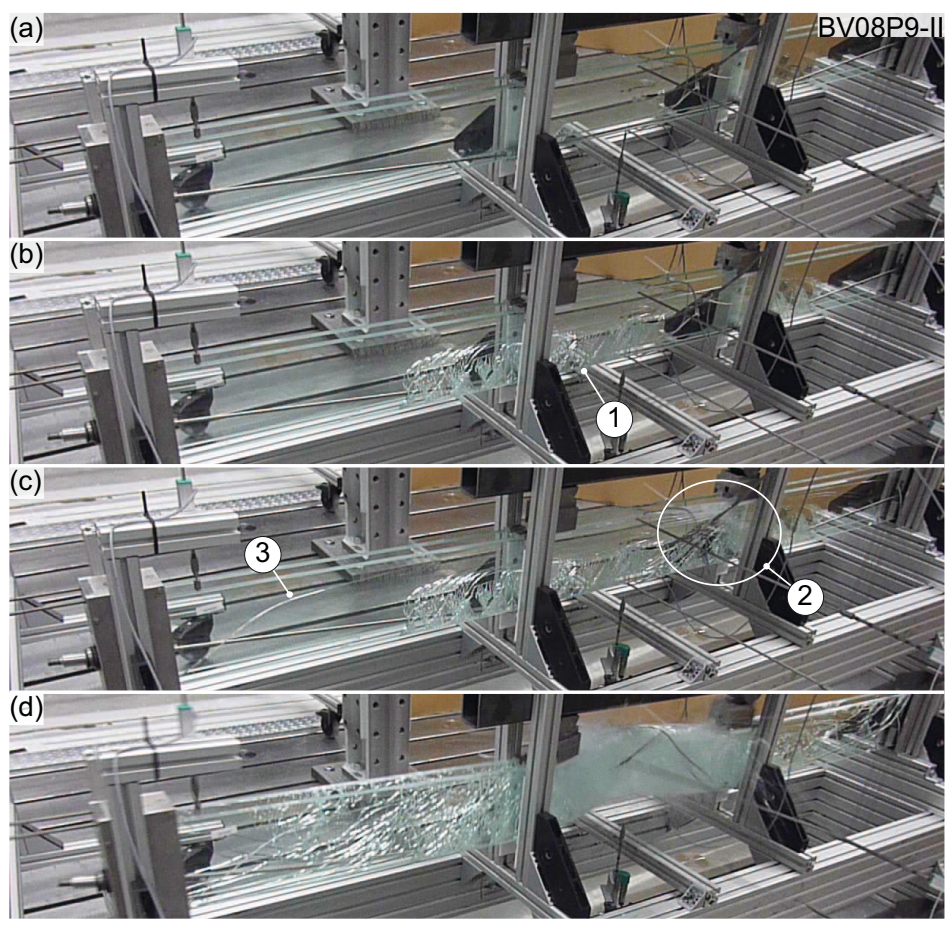

Im Gegensatz zu den bisher veranschaulichten Ergebnissen zeigte der Prüfkörper BV 06 ein anderes Verhalten. Bei jedem Rissereignis wurde jeweils nur eine Schicht im VerbundSicherheitsglas zerstört. Anschließend war eine progressive Weiterbelastung bis zum nächsten Riss möglich. Damit ein-
Bild 55

Rissentwicklung im Prüfkörper BV 08.

(a) Rissfreier Zustand I

(b) Bruchursprung (1) und Ausbreitung des Erstrisses

(c) Erweiterung der Risse unterhalb der Lasteinleitung (2) und in das Auflager (3)

(d) "explosionsartiges" Versagen. 
her ging eine Lastumlagerung vom Glas auf die Bewehrung, welche sich in einer sprunghaft steigenden Seilkraft bei jedem Rissereignis bemerkbar machte. Auch nach dem Riss der letzten Glasschicht war eine Weiterbelastung möglich $\left(\mathrm{k}_{\mathrm{F} \text {.duk }}=6 \%\right)$. Im Gegensatz zu den übrigen bewehrten Prüfkörpern verblieb der Querschnitt vertikal und ohne Falten, sodass das Glas eine ausreichende Druckzone zum gemeinsamen Lastabtrag mit der Bewehrung bereitstellen konnte. Dabei sank sowohl die Seilkraft, als auch die Biegekraft fortlaufend ab. Währenddessen splitterten kontinuierlich Glasteile aus dem Grundquerschnitt ab, bis der Träger bei einem Traversenweg von $95 \mathrm{~mm}$, dem achtfachen Wert im Vergleich zum ersten Glasriss, vollständig versagte. In diesem Fall ist also von einem angekündigten Versagen bei großer Durchbiegung und deutlichem Rissbild zu sprechen, was mit einem sicheren Tragverhalten assoziiert wird. Entsprechend ergibt sich der maximale Kennwert $k_{w . d u k}$ mit $130 \%$. Dabei ist anzumerken, dass es sich um einen Einzelfall handelte und dieser Prüfkörper mit 23,7 kN die geringste Erstrisslast erreichte. Im Vergleich zu den übrigen Versuchen wurde also die geringste elastische Energie beim Erstriss frei, welche ebenso zur geringsten Erschütterung des Prüfkörpers führte und damit vermutlich ein seitliches Versetzen bei jedem Rissereignis vermieden wurde.

Für die Fälle des schrittweisen Versagens sind die Duktilitätskennwerte in Tafel 13 zusammengefasst. Darin zeigt sich, dass das zweite Rissereignis üblicherweise mit verringerten Biegelasten zwischen $72 \%$ und $95 \%$ der Erstrisslast $\mathrm{F}_{\mathrm{cr} .1}$ stattfand. Die zugehörige Durchbiegung stieg um $2 \%$ bis $13 \%$ an. Da ein sprödes Versagen keine weiteren Biegelasten $F_{c r . i>1}$ nach sich zieht und die Verformungsfähigkeit mit $k_{w . d u k}>1$ erhalten bleibt, ist dieses Versagen qualitativ als duktil zu bezeichnen. Eine quantitative Beurteilung kann nicht erfolgen, da keine zulässigen Grenzwerte vorliegen, das Bruchverhalten vielfältig war und zu wenige Prüfkörper zur Verfügung standen, um einen allgemeingültigen Wert neu zu definieren.

Der Bruchursprung konnte, mit Ausnahme des Sonderfalls BV 06, bei allen Prüfkörpern gefunden werden. Es kam so- 
wohl zu Rissen mit Ursprung am aufgeklebten Umlenkpunkt ${ }^{223}$, als auch von der freien Glaskante im Feld. Eine auffällige Häufung oder Tendenz zu einem bevorzugten Ursprung wurde nicht festgestellt. Da gleichzeitig die Bruchspannung im erwarteten Bereich für teilvorgespanntes Glas lag, wird geschlussfolgert, dass die Umlenksättel keinen bestimmenden Einfluss auf das Rissgeschehen hatten. Es wurde ausgeschlossen, dass es zu einem vorzeitigen Versagen, beispielsweise infolge eines Glas-Metall-Kontaktes am Umlenksattel, gekommen ist. ${ }^{224}$ Die Konstruktionsweise ist demnach nutzbar und kann weiterentwickelt werden.

\begin{tabular}{|c|c|c|c|c|c|c|}
\hline \multirow{2}{*}{$\begin{array}{l}\text { Prüf- } \\
\text { körper }\end{array}$} & \multirow{2}{*}{$\begin{array}{l}\mathbf{F}_{\text {cr.i }}[\mathbf{k N}] \\
\mathrm{i}=1\end{array}$} & \multirow{2}{*}{\multicolumn{2}{|c|}{$\mathrm{i}=22^{\left(\mathbf{k}_{\text {F.duk }}[-]\right)} \mathrm{i}=3$}} & \multicolumn{2}{|c|}{$\mathbf{w}_{\text {z.T.cr.i }}[\mathrm{mm}] \quad\left(\mathbf{k}_{\mathrm{w} . \text { duk }}[-]\right)$} & \multirow{2}{*}{$i=3$} \\
\hline & & & & $\mathrm{i}=1$ & $\mathrm{i}=2$ & \\
\hline BV 06 & 31,68 & $2,01 \quad(6 \%)$ & - & 15,39 & $19,95(130 \%)$ & - \\
\hline BV 07 & 30,87 & $22,15(72 \%)$ & - & 15,54 & $15,81(102 \%)$ & - \\
\hline BV 10 & 35,25 & $26,21(74 \%)$ & - & 17,90 & $18,96(106 \%)$ & - \\
\hline BV 12 & 34,12 & $29,31(86 \%)$ & $16,20(47 \%)$ & 16,49 & $17,29(105 \%)$ & $18,81(114 \%)$ \\
\hline BV 13 & 35,84 & $28,68(80 \%)$ & - & 18,43 & $19,38(105 \%)$ & - \\
\hline BV 01 & 24,46 & $23,04(94 \%)$ & $15,75(64 \%)$ & 12,31 & $13,41(109 \%)$ & $15,20(123 \%)$ \\
\hline BV 02 & 29,20 & $27,83(95 \%)$ & - & 14,97 & $16,87(113 \%)$ & - \\
\hline BV 03 & 36,60 & 32,03 (87\%) & - & 18,05 & $18,85(104 \%)$ & - \\
\hline BV 08 & 29,78 & $28,01(94 \%)$ & - & 15,12 & $16,34(108 \%)$ & - \\
\hline BV 09 & 32,71 & $30,20(92 \%)$ & $3,51(11 \%)$ & 15,73 & $16,61(106 \%)$ & $17,94(114 \%)$ \\
\hline
\end{tabular}

Alle unbewehrten Träger sackten nach dem Versagen aller Glaslagen herab, bis sie auf der Prüfeinrichtung zum Liegen kamen. Demgegenüber wurden alle bewehrten und vorgespannten Träger weiterhin durch das Seil gehalten (Bild 56). Damit stellt die Bewehrung ein nutzbares Mittel dar, um einen zusätzlichen Lastpfad bereitzustellen. Die Redundanz wird vergrößert und die Resttragfähigkeit in diesem experimentellen Last- und Versagensszenario gesteigert. Nichtsdestotrotz wird davon ausgegangen, dass ein solches Versagen mit ausreichender Sicherheit durch eine Bemessung ausgeschlossen wird. Entsprechend sei an dieser Stelle auf die Untersuchungen zur Resttragfähigkeit in

${ }^{223}$ Nichtsdestotrotz bildet der Umlenksattel einen Steifigkeitssprung im Trägerquerschnitt, welcher eine konstruktive Spannungsspitze nach sich zieht.

224 Demgegenüber wurden bereits in [Weller \& Engelmann 2014b] Konstruktionsarten ausgeschlossen, bei denen es zu einem vorzeitigen Versagen als Folge einer konstruktiven Spannungsspitze kam.
Tafel 13

Biegelast und Traversenweg beim ersten, zweiten und dritten Rissereignis sowie Verhältnisse nach Gleichung 50, Seite 112. 

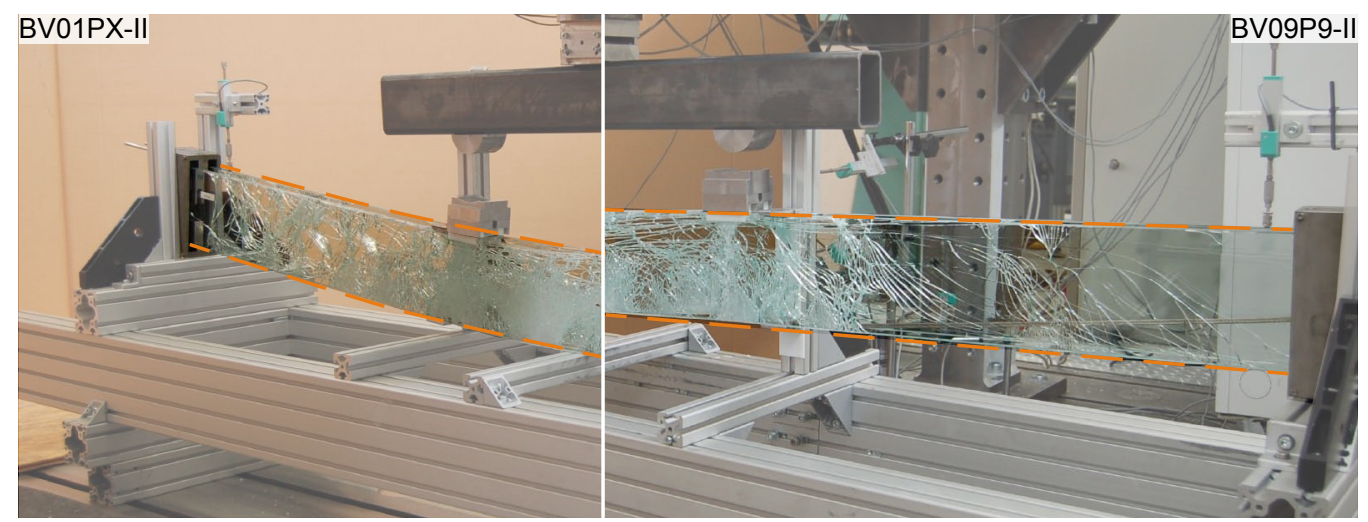

Bild 56

Der unbewehrte Referenzträger BV 01 (links) sackte nach dem Versuch bis auf die Prüfeinrichtung, während der Spannglasträger BV 09 (rechts) auch nach dem vollständigen Glasbruch vom Seil gehalten wurde.
Kapitel 3.5 verwiesen. Es bietet sich damit die Möglichkeit auf „Opferscheiben“ im Glasquerschnitt zu verzichten und deren Aufgabe der Bewehrung zuzuordnen.

Nach dem Versagen war ein Verlust der Seilkraft in allen Biegeversuchen zu verzeichnen, welcher mit der Bruchform in Verbindung gebracht wurden. Unbewehrte Referenzträger verblieben nach dem Versuchsende nahezu vertikal (Bild 57, links), während die bewehrten Prüfkörper ein deutliches seitliches Ausweichen zeigten. Infolge des Glasbruches und dem damit einhergehenden Steifigkeitsverlust kam es zu vertikalen Bruchkanten und einer polygonalen Bruchform im Grundriss (Bild 57, rechts). Dadurch verkürzt sich die Trägerlänge über die Seillängung aus der Vorspannkraft und Biegebelastung hinaus, was sich im Verlust der Seilkraft bemerkbar machte. Angesichts dieses Faltenwurfs war der Träger nicht mehr zum planmäßigen Lastabtrag befähigt. Die Bewehrung eines Spannglasträgers führt demnach zu einer veränderten Versagensform.

Ein weiteres charakterisierendes Merkmal zur Beschreibung der Konstruktionseigenschaften ist das Bruchbild nach dem Versagen. Je kleiner die Einzelbruchstücke, desto geringer die Tragfähigkeit des Verbundglases. Alle Probekörper zeigten ein feines Bruchbild im mittleren Drittel und größere Bruchstücke in den äußeren Dritteln der Trägerlänge. ${ }^{225}$ Dies war auf die höhere Energiefreisetzung beim Bruch im höchstbeanspruchten Mittelteil des Trägers zurückzuführen.

225 Siehe auch Bild 56 (rechts), Seite 130. 

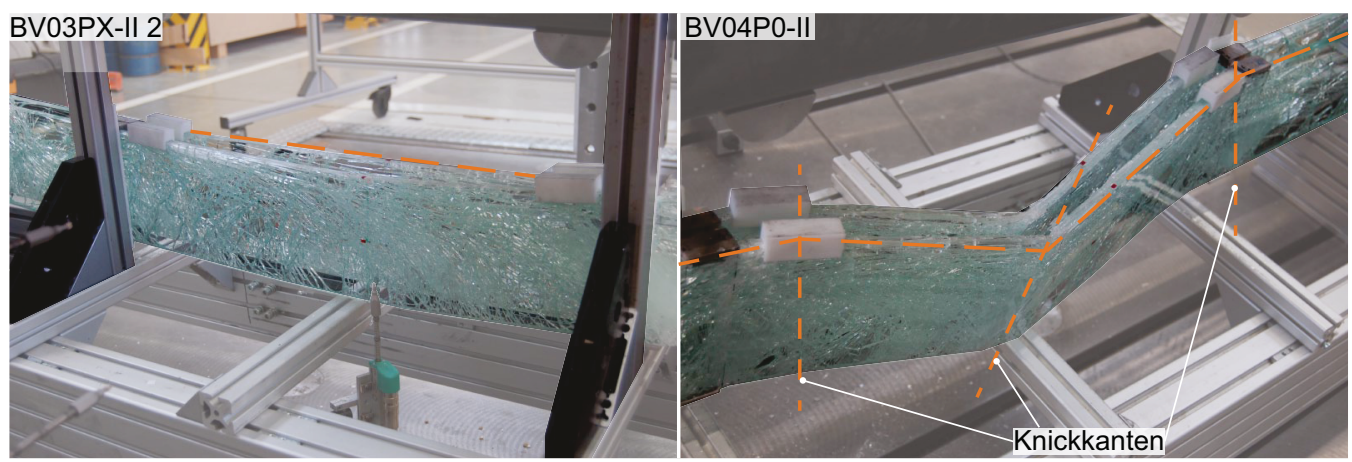

Bild 57

Die Ungleichverteilung zeigte, dass das gelieferte Glas keine

Der unbewehrte Referenzträger BV 03 (links) verblieb Brucheigenschaften von Einscheiben-Sicherheitsglas hatte. Der Rissfortschritt bis zum Rand, oft mit einer deltaförmigen Verzweigung an den Kanten („Rissdelta“), schließt auch Floatglas aus. Das Bruchbild zeigte daher die zu erwarteten Eigenschaften teilvorgespannten Glases. ${ }^{226}$

nach dem Versagen vertika stehen, während der bewehrte Träger BV 04 (rechts) mit einem Knick zur Seite ausweicht. Dieses Verhalten war typisch für bewehrte und vorgespannte Prüfkörper.

Das Laminieren von Einzelscheiben zu Verbund-Sicherheitsglas soll die Splitterbindung im Bruchfall gewährleisten. In allen Fällen wurden unterhalb der Prüfkörper ausschließlich feine, als ungefährlich beurteilte Glassplitter aufgefunden. Es war jedoch auffällig, dass die Oberkante des Glases in großem Maße zerstört war: vielfach kam es zum Abgang von Glas in der Nähe der Verbundfolie, auf der nur noch sehr flache Glasmuscheln haften blieben (Bild 58).

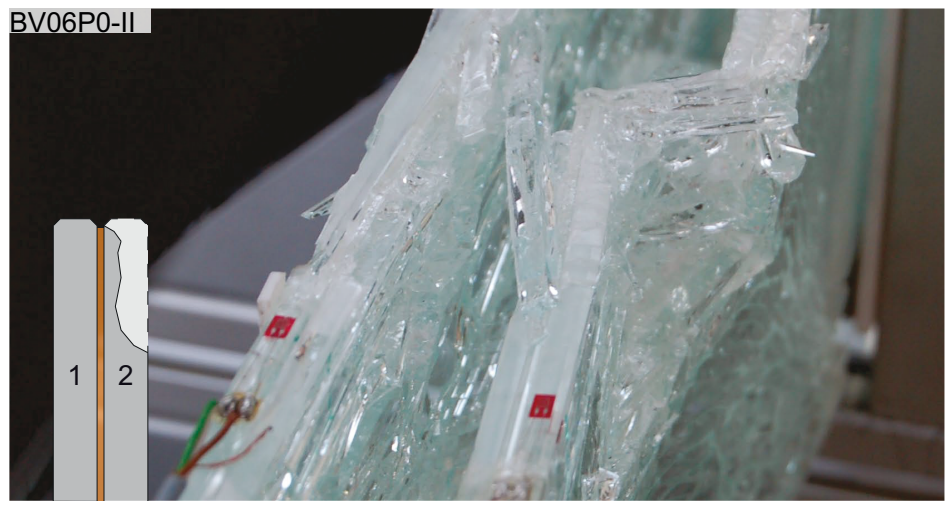

Bild 58

Obere Glaskante des Prüfkörpers BV 06 nach dem Versagen.

Das Glas ist großflächig abgeplatzt, sodass in der Druckzone ausschließlich die Verbundfolie mit aufhaftenden, flachen Glasmuscheln verblieb.

${ }^{226}$ Ein Bruchbildvergleich im Sinne der zugehörigen Produktnormen beziehungsweise -zulassungen ist nicht möglich, da darin ein Versuch im unbelasteten Zustand durchgeführt wird. Vielmehr werden hier allgemein bekannte, charakterisierende Eigenschaften verglichen. 
Die Splitterbindung durch die Folie war weiterhin gewährleistet, vielmehr versagte der Träger innerhalb des Glasvolumens. Auf diese Weise entstanden Lücken in der Druckzone, welche, gemeinsam mit der ebenso verlorenen Biegezugsteifigkeit, zu einem Versagen in diesem Querschnitt führte. Soll also ein duktiles Verhalten oder eine höhere Traglast erreicht werden, muss diese Versagensform vermieden werden. Es ist demnach empfehlenswert den Nachweis einer verbleibenden Druckzone nach dem Glasriss zu führen.

\subsubsection{Folgerungen und Zusammenfassung}

In diesem Kapitel wurden die Ergebnisse einer experimentellen Biegestudie bis zur Bruchlast dargestellt. Darin ist erstmals der nötige Spannweg zum Aufbringen der initialen Seilkraft für eine größere Anzahl an Prüfkörpern beschrieben. ${ }^{227}$ Nach dem Straffen der Seile stellte sich ein lineares Verhältnis zwischen Spannweg und Seilkraft ein, welches mit der Längssteifigkeit des Seils gut beschreibbar ist. Aus diesem Untersuchungsteil resultiert zudem ein Wert zu Umlenkkraftverlusten aus Reibung von $5 \%$.

Darüber hinaus wurden die Ergebnisse einer Vorstudie ${ }^{228}$ bestätigt, wonach sich die Glasverzerrung und vertikale Durchbiegung linear, die horizontale Durchbiegung jedoch nichtlinear, zur Vorspannkraft verhält. Damit ist die Vorspannung planmäßig in das Glas einleitbar und das Tragverhalten unter diesem Belastungsszenario beschrieben.

Aus den anschließenden weggesteuerten Biegeversuchen wurde für alle Träger eine Lastrate $a_{F}$ angegeben, die es ermöglicht, die Versuchsergebnisse im linearen Bereich bis zu $80 \%$ der Erstrisslast kraftbezogen anzugeben. Dabei steigerte sich die Seilkraft entsprechend des analytischen Ergebnisses, womit ein geteilter Lastabtrag zwischen Glas und Spannbewehrung nachgewiesen wurde.

\footnotetext{
227 In [Engelmann \& Weller 2016] wurde bereits der Spannweg für drei $9 \mathrm{~m}$ lange Spannglasträger angegeben.

228 Vergleiche [Weller \& Engelmann 2014a].
} 
Es wurde tendenziell eine Steigerung der Erstrisslast beobachtet, welche jedoch unter Berücksichtigung der generell stark streuenden Glasfestigkeit erst mit einer größeren Prüfkörperanzahl allgemeingültig nachweisbar wird. Das finale Versagen kann aufgrund der hohen, im System gespeicherten Energie, als „explosionsartig“ beschrieben werden, wobei keine Steigerung der Traglasten beobachtet wurde.

Das sicherheitsrelevante Rissverhalten wurde in drei Gruppen eingeteilt: neben dem sofortigen Bruch war eine degressive oder eine progressive Weiterbelastung möglich. Dabei entstand ein Rissfächer ausgehend vom Bruchursprung, welcher sich in den verbleibenden Träger unterhalb der Druckzone verzweigte. Für alle Prüfkörper, die nach dem ersten Glasriss weiter belastet werden konnten, wurde die Duktilität quantifiziert. Dafür ist sowohl eine Angabe der Last, als auch der Durchbiegung nötig. Derzeit fehlen jedoch allgemein anerkannte Grenzwerte, wodurch eine weiterführende Forschung gerechtfertigt wird.

Die Entwicklung der Seilkraft ist mit analytischen Mitteln gut beschreibbar, wobei die bemessungsrelevanten Maximalwerte nach dem Erstriss auftraten.

Es zeigte sich, dass die Versagensform durch die Bewehrung und das Vorspannen beeinflussbar ist. Im Gegensatz zu unbewehrten Referenzträgern wurde der vollständig gerissene Querschnitt bewehrter Träger weiterhin vom Bewehrungsseil getragen. Jedoch kam es infolge des Auffaltens des Glasquerschnitts entlang seiner Bruchlinien zu einem vollständigen Seilkraftverlust, sodass ein Überdrücken der Einzelrisse nicht möglich war.

Letztlich hatte die gewählte Querschnittsform mit aufgeklebten Umlenkungen keinen negativen Einfluss auf die Träger, sodass geschlussfolgert wird, dass das Querschnittskonzept konstruktiv nutzbar ist.

Zusammenfassend bleibt festzuhalten, dass die eingangs gestellten Ziele, das Tragverhalten zu beschreiben und das Bruchverhalten durch Bewehrung und Vorspannung gezielt zu beeinflussen, erreicht wurden. 


\subsection{Tragverhalten unter Dauerlast}

\subsubsection{Prüfkörper}

Für die Versuche unter Dauerlast wurden der Seildurchmesser sowie die Bewehrungsführung von 28 Prüfkörpern (Tafel 14) variiert. Zum einen wurde das Seil einfach in Trägermitte (,I"), zum zweiten zweifach in den Drittelspunkten (,II“) umgelenkt. Als Referenzen dienten vier unbewehrte (DV01 bis DV04) und vier schlaff bewehrte Träger (DV05 bis DV08).

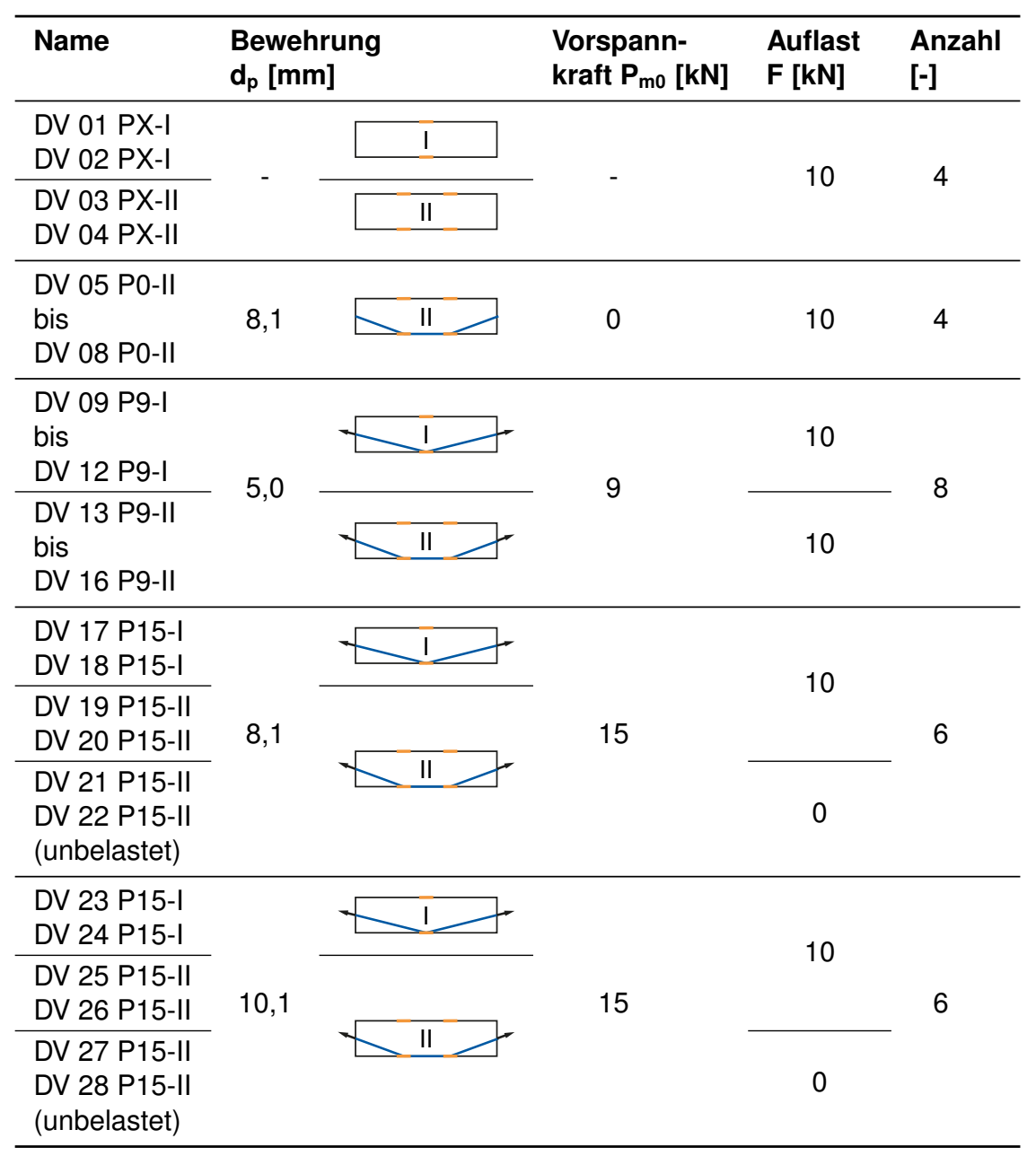

Tafel 14

Prüfkörper Dauerversuche (DV). 


\subsubsection{Versuchseinrichtung}

Für Dauerlastversuche wurde ein Vierpunkt-Biegeversuchsstand für eine gleichzeitige Untersuchung von vier Prüfkörpern verwendet (Bild 59). Die Belastung erfolgte über eine Traverse und einen Hebelarm, welcher mit Betonsteinen eine Biegekraft von $\mathrm{F}=10 \mathrm{kN}$ am Prüfkörper erzeugte. ${ }^{229}$ Dieses gewählte Belastungsniveau entspricht näherungsweise einem Drittel der mittleren Erstrisslast nach Kapitel 3.3 und wird als mögliches Gebrauchslastniveau interpretiert.

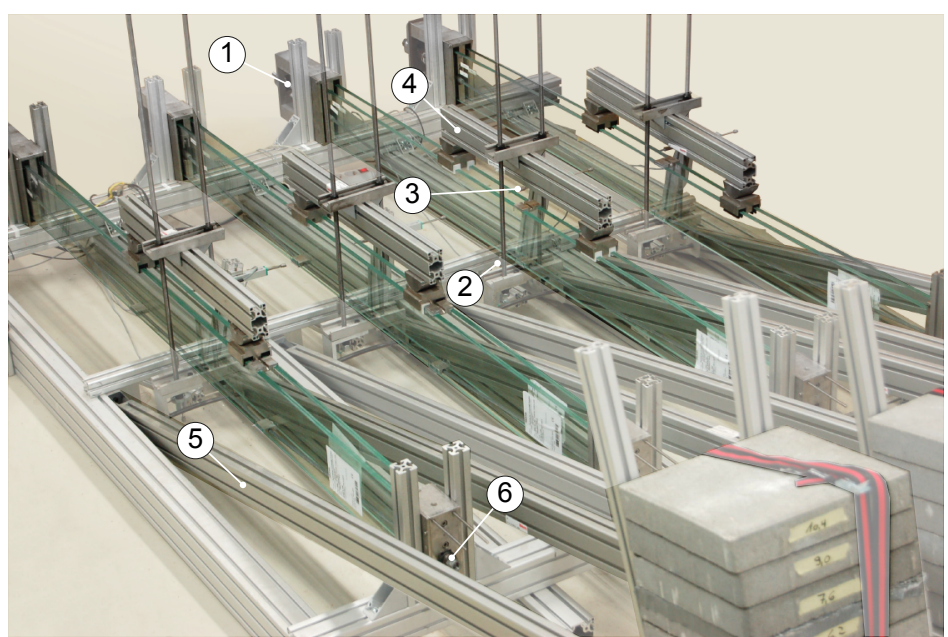

\section{Bild 59}

Vierpunkt-

Biegeversuchsstand für dauerhafte und gleichzeitige

Biegebelastung von vier Prüfkörpern

(1) Kraftmessdose Seilkraft am Spannanker

(2) Wegaufnehmer vertikale Durchbiegung

(3) Wegaufnehmer horizontale Durchbiegung (Obergurt)

(4) Lasttraverse

(5) Hebelarm mit Betongewichten

(6) Festanker

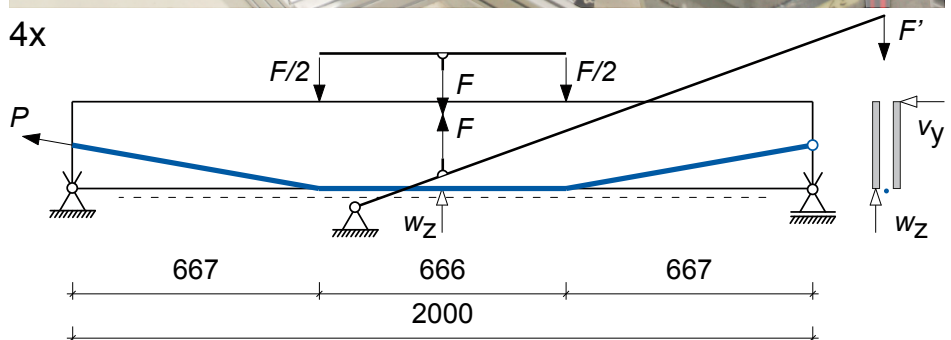

Während der Versuchsdauer von $1000 \mathrm{~h}$ wurde die auftretende Seilkraft $N_{p}(t)$ mit einer Kraftmessdose auf der Spannseite, die vertikale Durchbiegung der Trägerunterkante in Feldmitte $w_{z}(t)$ sowie die horizontale Durchbiegung der Trägeroberkante in Feldmitte $v_{y}(t)$ gemessen. Die Aufzeichnung erfolgte mit einem zwölf-kanaligen Datenloggersystem ${ }^{230}$ im Netzbetrieb mit Notfallstromversorgung und einer Soll-

229 Hier wird der Sollwert der Biegelast angegeben. Im Vorfeld der Versuche wurde die auftretende Biegelast individuell gemessen und in Anlage $\mathrm{C}$ dokumentiert $\left(\mathrm{F}_{\text {ist }}=10,2 \pm 0,14 \mathrm{kN}\right)$.

230 Drei ALMEMO®2590-4AS Universalmessgeräte. 
Messrate von $10 \mathrm{~min}^{-1}$. Während des Aufbringens der Belastung und während der ersten $72 \mathrm{~h}$ wurde eine erhöhte Messrate von $1 \mathrm{~s}^{-1}$ und $1 \mathrm{~min}^{-1}$ verwendet. Ein separates System zeichnete zusätzlich die Raumtemperatur auf.

\subsubsection{Untersuchungsverfahren und -bedingungen}

Die Prüfkörper wurden nach ihrer Herstellung unbelastet für mindestens $24 \mathrm{~h}$ im Prüfraum konditioniert. Nach dem Einstellen der angestrebten Vorspannkraft $P_{\mathrm{mo}}$ (Tafel 14), wurde die Belastungseinrichtung montiert und jeder Träger nacheinander mit der vorgesehenen Biegebelastung beaufschlagt. Nachdem alle vier Prüfkörper eines Durchlaufes belastet waren, begann die Versuchslaufzeit. Nach mindestens $1000 \mathrm{~h}$ erfolgten die vollständige Entlastung der Prüfkörper sowie das Aufzeichnen des Entlastungsverhaltens für weitere $24 \mathrm{~h}$ zu Kontrollzwecken.

\subsubsection{Analyse- und Auswertungsverfahren}

Es wurde erwartet, dass die Seilkraft über die Versuchsdauer abnimmt. Entsprechend wurde die maximale Seilkraft $\mathrm{N}_{\mathrm{p} \text {.max }}$ zu Versuchsbeginn sowie die Seilkraft nach $1000 \mathrm{~h}$ $\left(N_{p .1000 h}\right)$ bestimmt. Die Differenz beider Werte ergibt den Seilkraftverlust $\triangle \mathrm{P}$. Er resultiert maßgebend aus den Kriechverformungen der Zwischenschicht und der Klotzung. ${ }^{231} \mathrm{De}-$ ren Last-Zeit-Abhängigkeit ergibt sich analytisch als logarithmischer Zusammenhang. Entsprechend wird der experimentell bestimmte Seilkraftverlauf über die Zeit $t$ in Stunden ebenso als logarithmische Regression beschrieben (Gleichung 51). Die Güte der Regression ergibt sich aus dem Be-

231 Vergleiche Gleichung 5, Seite 53 und Gleichung 12, Seite 58. 
stimmtheitsmaß $R^{2}$ mit ihrer verbalen Beschreibung nach Kapitel 3.3.4.

$$
\begin{array}{ll}
\begin{array}{ll}
N_{p}(t)=a_{\log } \cdot \ln \left(\frac{t}{h}\right)+ & b_{\log } \quad \text { mit } \mathrm{R}^{2} \\
a_{\log } \text { und } b_{\log } & \text { Regressionsparameter } \\
\mathrm{R}^{2} & \text { Bestimmtheitsmaß der Regression }
\end{array}
\end{array}
$$

Gleichung 51

Darüber hinaus wird der relative Seilkraftverlust als Verhältnis des absoluten Seilkraftverlustes und der anfänglichen Seilkraft nach Gleichung 52 angegeben.

$$
\Delta P_{\text {rel }}=\frac{\Delta P}{N_{\text {p.max }}}=\frac{N_{\text {p.1000h }}-N_{p . \max }}{N_{\text {p.max }}}
$$

$\begin{array}{ll}\Delta P_{\text {rel }} & \text { relativer Seilkraftverlust } \\ \Delta P & \text { absoluter Seilkraftverlust über 1000 h } \\ N_{p .1000 \mathrm{~h}} & \text { Seilkraft am Versuchsende } \\ N_{p . \max } & \text { Seilkraft zu Versuchsbeginn, entspricht } \\ & \mathrm{N}_{\mathrm{p}}(\mathrm{t}=0)\end{array}$

\subsubsection{Ergebnisse und Ergebnisdiskussion}

Die Einzelergebnisse der Dauerlastversuche sind in Anlage $\mathrm{C}$ zusammengestellt.

Alle Prüfkörper in Bild 60 zeigten erwartungsgemäß einen Verlust an Seilkraft im Prüfzeitraum. Im Vergleich dazu trat bei schlaff bewehrten Prüfkörpern kein messbarer Kraftverlust auf. In keinem Fall wurde eine nennenswerte Auswirkung der schwankenden Raumtemperatur (im Mittel $18,5^{\circ} \mathrm{C}$ bis $24,9^{\circ} \mathrm{C}$ ) auf den Verlauf beobachtet.

Die Seilkraftänderung über die Zeit wird mit einer logarithmischen Regression nach Gleichung 51 beschrieben. Die zugehörigen Bestimmtheitsmaße in Tafel 15 zeigen, dass die Messdaten gut und sehr gut mit dieser Methode beschreibbar sind. Einzig das Mindestbestimmtheitsmaß der Träger mit 5,0 mm Bewehrung zeigt Werte deutlich unterhalb von 


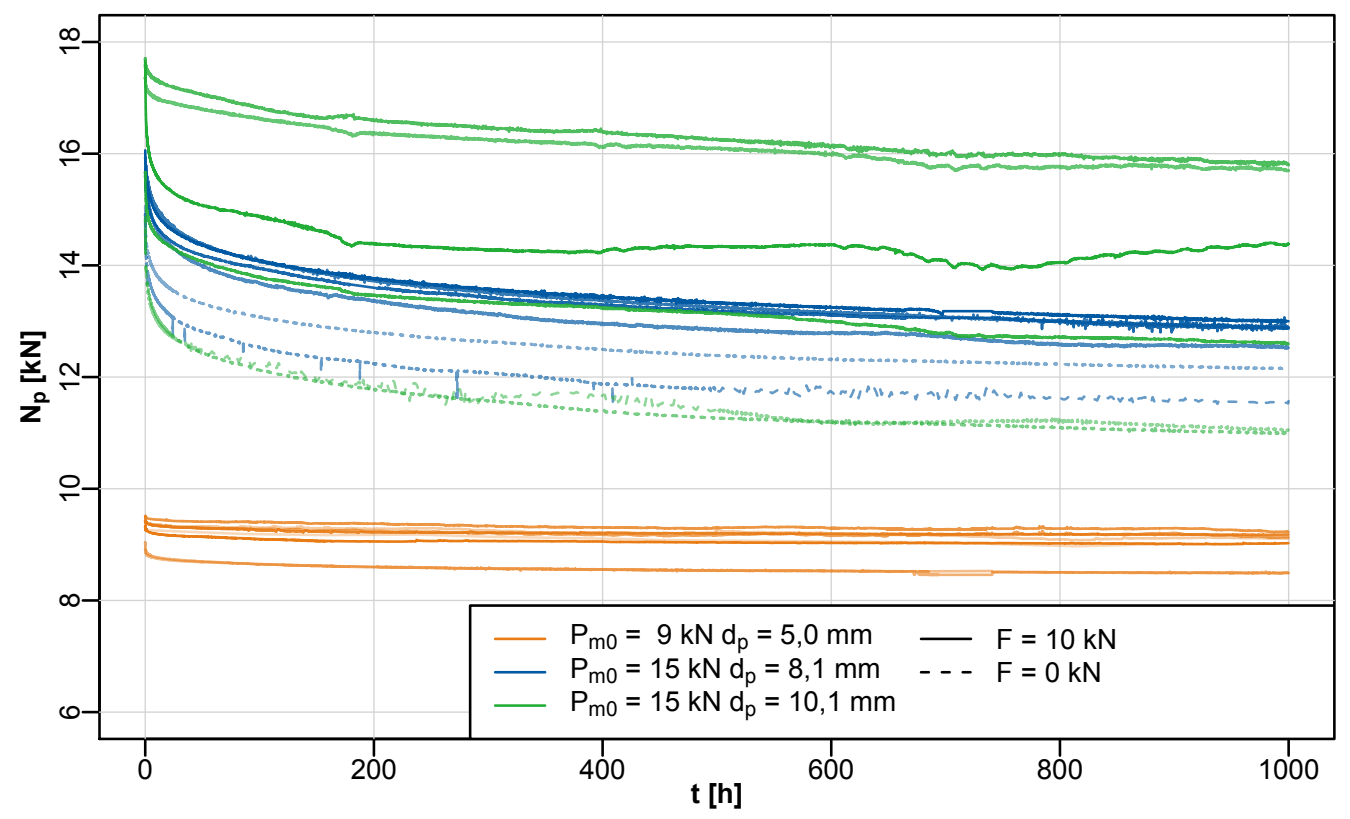

Bild 60

Verlauf der Seilkraft aller vorgespannt bewehrten Spannglasträger über die gesamte Versuchszeit.

Die schlaff bewehrten Referenzprüfkörper zeigten keinen messbaren Seilkraftverlust und sind daher hier nicht dargestellt.

0,85. Dies liegt darin begründet, dass die kontinuierliche Datenaufzeichnung des Prüfkörpers DV 16 im Versuchsverlauf ausgefallen ist und die Regressionsanalyse mit den per Hand aufgezeichneten Messwerten erfolgte. ${ }^{232}$

Der Seilkraftverlauf der schlaff bewehrten Referenzprüfkörper kann mit der gewählten Regression nicht beschrieben werden. Die Bestimmtheitsmaße liegen mit Werten zwischen 0,26 und 0,64 deutlich unterhalb der Ergebnisse der vorgespannten Träger. Als Grund ist die nahezu konstante Seilkraft über die Versuchsdauer zu benennen, welche nicht die Eigenschaft einer logarithmischen Funktion zeigt.

Die Güte der Regression wird im folgenden Abschnitt durch das Fehlermaß als Abweichung zwischen dem gemessenen Wert (Bild 60) und dem errechneten Wert ${ }^{233}$ analysiert.

Im linken Bild 61 zeigt sich ein Fehlermaß für vorgespannte Träger mit 9,0 kN Vorspannung nahe Null über den gesamten

\footnotetext{
232 Während des Versuchsverlaufes über $1000 \mathrm{~h}$ wurden 15 Werte per Hand aufgezeichnet, was einer verringerten mittleren Messrate von $2,5 \cdot 10^{-4} \mathrm{~min}^{-1}$ entspricht.

233 Vergleiche Gleichung 51, Seite 137.
} 


\begin{tabular}{llll}
\hline Gruppe & $\begin{array}{l}\mathbf{a}_{\text {log }} \\
{[\mathbf{k N}]}\end{array}$ & $\begin{array}{l}\mathbf{b}_{\text {log }} \\
{[\mathbf{k N}]}\end{array}$ & $\begin{array}{l}\mathbf{R}^{2} \\
{[-]}\end{array}$ \\
\hline $\begin{array}{l}\text { Referenz } \\
\text { bewehrt } \\
d_{\mathrm{S}}=8,1 \mathrm{~mm}\end{array}$ & $-0,008 \pm 0,02$ & $1,36 \pm 0,22$ & $\geq 0,26$ \\
\hline $\begin{array}{l}P_{\mathrm{m} 0}=9 \mathrm{kN} \\
d_{\mathrm{p}}=5,0 \mathrm{~mm}\end{array}$ & $-0,036 \pm 0,01$ & $9,24 \pm 0,26$ & $\geq 0,65$ \\
\hline $\begin{array}{l}P_{\mathrm{m} 0}=15 \mathrm{kN} \\
d_{\mathrm{p}}=8,1 \mathrm{~mm}\end{array}$ & $-0,377 \pm 0,04$ & $15,53 \pm 0,24$ & $\geq 0,93$ \\
\hline $\begin{array}{l}P_{\mathrm{m} 0}=15 \mathrm{kN} \\
d_{p}=8,1 \mathrm{~mm} \\
\text { (unbelastet) }\end{array}$ & $-0,308 \pm 0,02$ & $14,12 \pm 0,25$ & $\geq 0,97$ \\
\hline $\begin{array}{l}P_{\mathrm{m} 0}=15 \mathrm{kN} \\
d_{\mathrm{p}}=10,1 \mathrm{~mm}\end{array}$ & $-0,274 \pm 0,06$ & $16,61 \pm 1,20$ & $\geq 0,83$ \\
\hline $\begin{array}{l}P_{\mathrm{m} 0}=15 \mathrm{kN} \\
d_{p}=10,1 \mathrm{~mm} \\
\text { (unbelastet) }\end{array}$ & $-0,374 \pm 0,01$ & $13,70 \pm 0,06$ & $\geq 0,98$ \\
\hline
\end{tabular}

\section{Tafel 15}

Regressionsparameter des Seilkraftverlaufes über die Zeit im DauerlastBiegeversuche (DV) als Mittelwerte \pm Standardabweichung je Gruppe nach Gleichung 51, Seite 137.

In dieser Gruppe verfehlte ein einzelner Prüfkörper das Bestimmtheitsmaß von 0,85 .
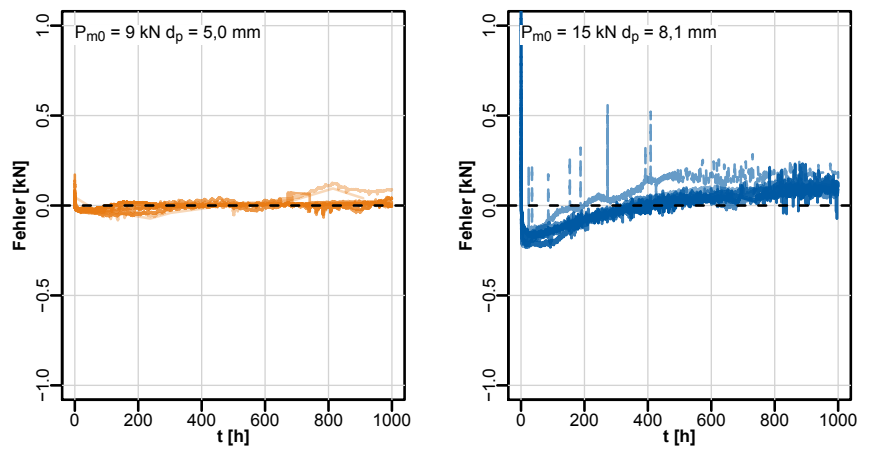

Verlauf. Dieses Ergebnis liegt aufgrund der kleinsten gemessenen Seilkraftverluste vor, welche die kleinsten absoluten Fehler nach sich ziehen.

Die Fehleranalyse zeigt zudem, dass die Abweichung auch im Extremfall unterhalb von $0,5 \mathrm{kN}$ liegt und beträgt damit weniger als $4 \%$ der Sollkraft zu Beginn. Dieser Zustand wird als akzeptabel bewertet. Unter zusätzlicher Berücksichtigung der Bestimmtheitsmaße (Tafel 15) wird geschlussfolgert, dass die gewählte Regression ein zweckmäßiges Mittel zur analytischen Beschreibung der Ergebnisse darstellt.

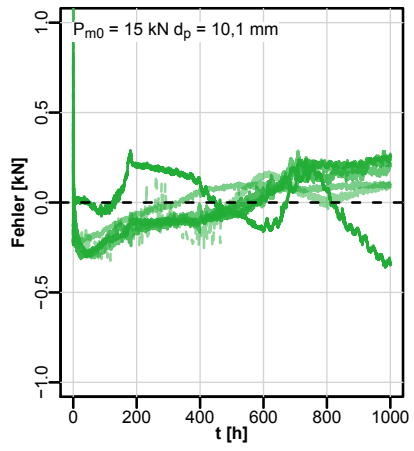

Bild 61

Abweichung zwischen gemessener Seilkraft und Regression nach Gleichung 51 als Fehlermaß über die Versuchszeit. 
Im Speziellen zeigt sich für die Probekörper mit $15 \mathrm{kN}$ Vorspannung (Bild 61, mittig und rechts) während der ersten Versuchshälfte bis $500 \mathrm{~h}$ ein Fehler im negativen Bereich: Die Regression ergibt kleinere Seilkräfte als die Messung. Im weiteren Verlauf kehrt sich dieser Trend um und die Regression prognostiziert größere Seilkräfte als die Messung. Das systematische Auftreten dieses Verhaltens lässt schlussfolgern, dass der analytische Zusammenhang den Seilkraftverlust bei Extrapolation der Ergebnisse unterschätzt. Daher wird die stets abnehmende Seilkraft für Prüfkörper mit $15 \mathrm{kN}$ Vorspannung alternativ durch eine dreiparametrige Potenzfunktion nach Gleichung 53 beschrieben.

Gleichung 53

$$
\begin{array}{ll}
N_{p}(t)=a_{p o t} \cdot\left(\frac{t}{h}\right)^{b_{p o t}} & +c_{p o t} \text { mit } \mathrm{R}^{2} \\
a_{p o t}, b_{p o t} \text { und } c_{p o t} & \text { Regressionsparameter } \\
\mathrm{R}^{2} & \text { Bestimmtheitsmaß der Regression }
\end{array}
$$

Die hierfür ermittelten Regressionsparameter sind in Tafel 16 wiedergegeben. Darin zeigt sich, dass das experimentelle Ergebnis ebenso „sehr gut" abgebildet werden kann $\left(R^{2}>0,99\right)$. Die Begutachtung des zugehörigen Fehlers in Bild 62 zeigt im Gegensatz zur Näherung mit einer logarithmischen Funktion (Bild 61) geringere absolute Werte. Darüber hinaus verlaufen die Kurven in allen Fällen vergleichsweise horizontal um Null. Ein systematischer Fehler ist nicht mehr erkennbar, woraus gefolgert wird, dass die Regressionsfunktion nach Gleichung 53 für diese Fälle besser nutzbar ist. Das Zusammenspiel der experimentellen Resultate und der analytischen Ergebnisse aus Kapitel 2.7 wird umfassend in Kapitel 5.3 diskutiert.

Die absoluten Ergebnisse des Seilkraftverlustes in Tafel 17 gruppieren sich nach dem Bewehrungsdurchmesser. Spannglasträger mit Bewehrungsdurchmessern von 10,1 mm übernahmen den größten Anteil der Biegelast und starteten mit der größten mittleren Vorspannkraft von $17,2 \mathrm{kN}$ und einem anschließenden Abfall von 2,52 kN (-14,7\%). Die zugehörigen Referenzprüfkörper ohne Auflast zeigten einen größeren Seilkraftverlust von $-26,8 \%$. Dem gegenüber verloren die ebenso auf eine Sollkraft von $P_{m 0}=15 \mathrm{kN}$ vorgespannten 


\begin{tabular}{|c|c|c|c|c|}
\hline Gruppe & $\begin{array}{l}a_{\text {pot }} \\
{[k N]}\end{array}$ & $\begin{array}{l}b_{\text {pot }} \\
{[-]}\end{array}$ & $\begin{array}{l}\text { Cot } \\
{[\mathrm{kN}]}\end{array}$ & $\begin{array}{l}R^{2} \\
{[-]}\end{array}$ \\
\hline $\begin{array}{l}P_{\mathrm{m} 0}=15 \mathrm{kN} \\
\mathrm{d}_{\mathrm{p}}=8,1 \mathrm{~mm}\end{array}$ & $\begin{array}{l}-1,649 \\
\pm 0,34 \\
\end{array}$ & $\begin{array}{l}0,140 \\
\pm 0,014\end{array}$ & $\begin{array}{l}17,06 \\
\pm 0,43 \\
\end{array}$ & $>0,99$ \\
\hline $\begin{array}{l}P_{\mathrm{m} 0}=15 \mathrm{kN} \\
\mathrm{d}_{\mathrm{p}}=8,1 \mathrm{~mm} \\
\text { (unbelastet) }\end{array}$ & $\begin{array}{l}-2,102 \\
\pm 0,41\end{array}$ & $\begin{array}{l}0,112 \\
\pm 0,008\end{array}$ & $\begin{array}{l}16,32 \\
\pm 0,18\end{array}$ & $>0,99$ \\
\hline $\begin{array}{l}P_{m 0}=15 \mathrm{kN} \\
d_{p}=10,1 \mathrm{~mm}\end{array}$ & $\begin{array}{l}-0,430 \\
\pm 0,47\end{array}$ & $\begin{array}{l}0,293 \\
\pm 0,096\end{array}$ & $\begin{array}{l}17,03 \\
\pm 0,87\end{array}$ & $>0,99$ \\
\hline $\begin{array}{l}P_{\mathrm{m} 0}=15 \mathrm{kN} \\
d_{p}=10,1 \mathrm{~mm} \\
\text { (unbelastet) }\end{array}$ & $\begin{array}{l}-3,145 \\
\pm 0,08\end{array}$ & $\begin{array}{l}0,092 \\
\pm 0,005\end{array}$ & $\begin{array}{l}16,94 \\
\pm 0,03\end{array}$ & $>0,99$ \\
\hline
\end{tabular}

Tafel 16

Alternative Regressionsparameter der Seilkraftentwicklung über die Zeit im Dauerlast-Biegeversuche (DV) als Mittelwerte \pm Standardabweichung je Gruppe nach Gleichung 53, Seite 137.

Die Ergebnisse des Prüfkörpers DV23 (AusreiBer) wurden hierfür nicht berücksichtigt.
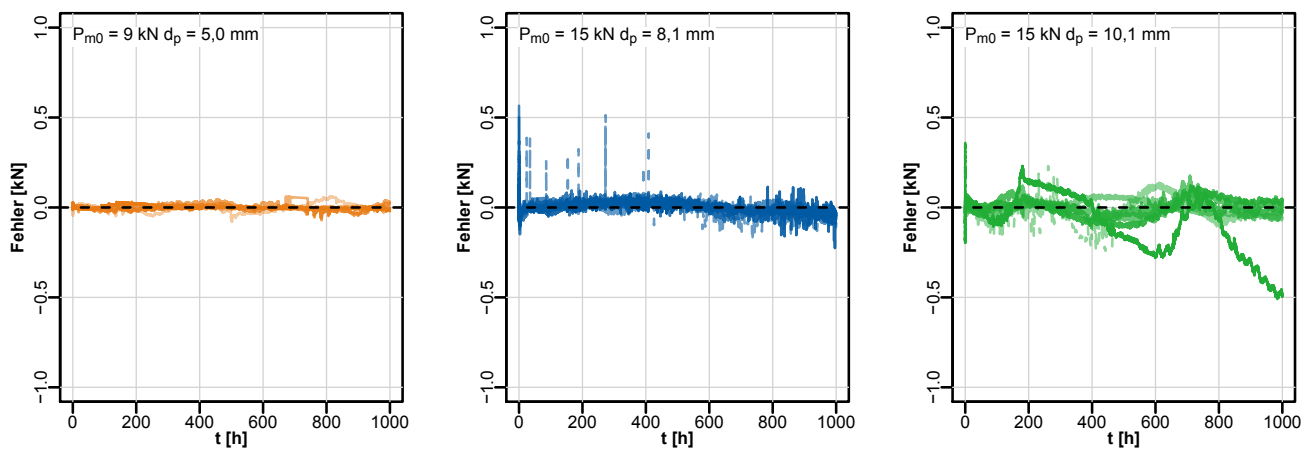

Bild 62

Abweichung zwischen gemessener Seilkraft und Regression nach Glei-

Träger mit Bewehrungsdurchmessern von 8,1 mm im Mittel 3,02 kN (-19,0\%). Die zugehörigen Referenzprüfkörper ohne Auflast verhielten sich vergleichbar (-21,9\%). Den kleinsten chung 53 (Fehler) über die Versuchszeit. mittleren Verlust von $0,35 \mathrm{kN}(-3,8 \%)$ verzeichneten die Träger der Vorspannkraft $9 \mathrm{kN}$ bei einem Seildurchmesser von $5,0 \mathrm{~mm}$. Im Vergleich dazu wurde bei den schlaff bewehrten Referenzprüfkörpern kein maßgebender Seilkraftverlust verzeichnet. Diese Bewertung stützt sich auf die Auswertung der Kurvenverläufe in Bild 60. Darin wird deutlich, dass der größte Verlustanteil unmittelbar nach Belastungsbeginn eintrat. Im weiteren Versuchsverlauf wurde ein kleiner werdender Verlust erfasst.

Des Weiteren sind in Tafel 17 die analytisch ermittelten Seilkraftverluste entsprechend Gleichung 37 eingetragen. Die so ermittelten Ergebnisse liegen deutlich unter den experimentellen Werten. Daher ist das analytische Modell nicht ausrei- 
Tafel 17

Ergebnisse DauerlastBiegeversuche (DV) gegenüber dem analytischen Modell nach Gleichung 37

Mittelwerte \pm Standardabweichung.

Die Eingangswerte der Seilkraft können unter Berücksichtigung der Auflast nach Gleichung 103 analytisch ermittelt werden. chend, um den Effekt zu beschreiben. Neben dem Kriechen von Klotzung und Zwischenschicht aufgrund einer Normalbelastung der Komponenten müssen weitere, bisher unberücksichtigte Anteile die Seilkraftreduzierung hervorrufen. Nur für die schlaff bewehrten Prüfkörper können mit Gleichung 37 realitätsnahe Ergebnisse berechnet werden. Jedoch liegen diese nahe Null, sodass eine quantitative Aussage zur Güte des analytischen Ansatzes nicht erfolgt.

\begin{tabular}{lcll}
\hline Gruppe & $\mathbf{N}_{\text {p.max }}[\mathbf{k N}]$ & $\Delta \mathbf{P}[\mathbf{k N}]$ & $\Delta \mathbf{P}_{\text {Gl. 37 }}[\mathbf{k N}]$ \\
& $\mathbf{N}_{\text {p.1000h }}[\mathbf{k N}]$ & $\Delta \mathbf{P}_{\text {rel }}[-]$ & $\Delta \mathbf{P}_{\text {rel }}[-]$ \\
\hline Referenz & $1,34 \pm 0,19$ & $-0,02 \pm 0,11$ & $-0,07$ \\
bewehrt & $1,32 \pm 0,21$ & $-1,5 \%$ & $-2,2 \%$ \\
$d_{\mathrm{S}}=8,1 \mathrm{~mm}$ & & & \\
\hline$P_{\mathrm{m} 0}=9 \mathrm{kN}$ & $9,31 \pm 0,22$ & $-0,35 \pm 0,10$ & $-0,12$ \\
$d_{p}=5,0 \mathrm{~mm}$ & $8,96 \pm 0,30$ & $-3,8 \%$ & $-0,6 \%$ \\
\hline$P_{\mathrm{m} 0}=15 \mathrm{kN}$ & $15,85 \pm 0,19$ & $-3,02 \pm 0,16$ & $-0,66$ \\
$d_{p}=8,1 \mathrm{~mm}$ & $12,83 \pm 0,21$ & $-19,0 \%$ & $-2,0 \%$ \\
\hline$P_{\mathrm{m} 0}=15 \mathrm{kN}$ & $15,19 \pm 0,14$ & $-3,33 \pm 0,27$ & $-0,59$ \\
$d_{p}=8,1 \mathrm{~mm}$ & $11,86 \pm 0,41$ & $-21,9 \%$ & $-1,9 \%$ \\
(unbelastet) & & & \\
\hline$P_{\text {m0 }}=15 \mathrm{kN}$ & $17,15 \pm 1,00$ & $-2,52 \pm 0,86$ & $-1,06$ \\
$d_{p}=10,1 \mathrm{~mm}$ & $14,63 \pm 1,50$ & $-14,7 \%$ & $-3,0 \%$ \\
\hline$P_{\mathrm{m} 0}=15 \mathrm{kN}$ & $15,06 \pm 0,01$ & $-4,04 \pm 0,04$ & $-0,90$ \\
$d_{p}=10,1 \mathrm{~mm}$ & $11,02 \pm 0,04$ & $-26,8 \%$ & $-3,0 \%$ \\
(unbelastet) & & & \\
\hline
\end{tabular}

Betrachtet man darüber hinaus die Seilkraftverluste mit Blick auf die Anzahl der Seilumlenkungen zeigen sich bei Trägern mit 5,0 mm und 8,1 mm Bewehrungsdurchmesser keine nennenswerten Unterschiede. Bei Prüfkörpern mit 10,1 mm Seilen zeigten die Träger mit einfacher Umlenkung einen deutlich größeren Seilkraftverlust (Tafel 18).

\begin{tabular}{lll}
\hline Gruppe & $\begin{array}{l}\text { Einfache } \\
\text { Umlenkung }\end{array}$ & $\begin{array}{l}\text { Zweifache } \\
\text { Umlenkung }\end{array}$ \\
\hline$P_{\mathrm{m} 0}=9 \mathrm{kN}$ & $-0,31 \pm 0,05$ & $-0,40 \pm 0,12$ \\
$d_{p}=5,0 \mathrm{~mm}$ & (DV 09 bis DV 12) & (DV 13 bis DV 16) \\
\hline$P_{\mathrm{m} 0}=15 \mathrm{kN}$ & $-3,08($ DV 17) & $-3,06$ (DV 19) \\
$d_{p}=8,1 \mathrm{~mm}$ & $-2,79($ DV 18) & $-3,16$ (DV 20) \\
\hline$P_{\mathrm{m} 0}=15 \mathrm{kN}$ & $-3,41($ DV 23) & $-1,91($ DV 25) \\
$d_{p}=10,1 \mathrm{~mm}$ & $-3,10$ (DV 24) & $-1,66$ (DV 26) \\
\hline
\end{tabular}


Die Seilkraft ist an die Verschiebung der Umlenkpunkte gekoppelt ${ }^{234}$, welche durch die konstruktive Ausbildung beeinflusst wird. Daher werden im Folgenden die Ergebnisse der horizontalen und vertikalen Durchbiegungsänderung nach Gleichung 54 in Bild 63 während des Versuchsverlaufes dargelegt und zur Erklärung der beiden letztgenannten Ergebnisse herangezogen. Die Durchbiegungen zeigen sich als kontinuierlich zunehmend. Dabei finden sich die kleinsten Änderungen bei den unbewehrten Referenzkörpern und den vorgespannten Trägern ohne Auflast.

$$
\Delta w_{z}=w_{z .1000 \mathrm{~h}}-w_{z .0} \text { und } \Delta v_{y}=v_{y .1000 \mathrm{~h}}-v_{y .0}
$$

$\Delta w_{z}$ und $\Delta v_{y} \quad$ Durchbiegungsänderungen über die Versuchsdauer von $1000 \mathrm{~h}$

Die vertikale Durchbiegung nimmt sowohl bei schlaff bewehrten, als auch bei vorgespannten Trägern in der Regel um bis zu $2 \mathrm{~mm}$ zu. Im extremen Einzelfall werden 6,1 mm erreicht. Dies kann beispielsweise an der Lasteinleitung der Biegelast begründet liegen, wobei eine Klotzung beide Scheiben eines VSG-Paketes übergreift und beim Vorliegen eines individuellen Kantenversatzes nur eine Schicht direkt, die zweite über die Zwischenschicht, belastet. Damit ergeben sich zwei Durchbiegungsergebnisse, von denen nur eine tatsächlich gemessen wird. Dabei wird die Zwischenschicht belastet und beginnt zu Kriechen. ${ }^{235}$

Ein ähnliches Ergebnis ergibt sich für die horizontale Durchbiegung mit kleinsten absoluten Beträgen bei unbewehrten Referenzprüfkörpern und unbelasteten, vorgespannten Trägern. Insgesamt zeigt sich jedoch ein uneinheitliches Bild, ohne weitere Zuordnung der Seilkraft zum Durchbiegungsergebnis. Verantwortlich hierfür kann erneut die zufällig verteilte Trägerimperfektion gemacht werden. Sie ist maßgeblicher Faktor für die horizontale Durchbiegung, da sie der Belastung einen zusätzlichen Hebelarm zur Verfügung stellt. Hieraus resultiert ein Biegemoment um die schwache Querschnittsachse, welches eine Schubbelastung in der Zwi-

\footnotetext{
${ }^{234}$ Vergleiche Kapitel 2.7.4, ab Seite 68.

235 Vergleiche Kapitel 2.5.3, ab Seite 51. Über dieses Phänomen wurde bereits in [Härth 2013] berichtet.
} 
Bild 63

Durchbiegungsānderungen in horizontaler und vertikaler Richtung.

Gleichung 55 schenschicht erzeugt. Diese Last wirkt dauerhaft ${ }^{236}$ und verursacht eine Kriechverformung.

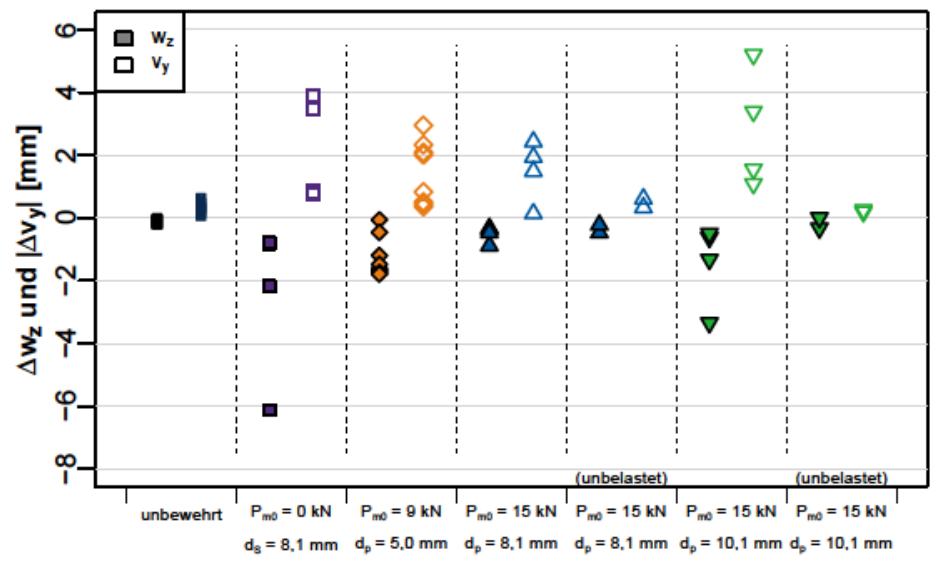

Da beide Durchbiegungskomponenten die Länge des Seiles und damit die Seilkraft beeinflussen, wird in Bild 63 die absolute Verschiebung $\delta_{\mathrm{yz}}$ infolge der horizontalen und vertikalen Durchbiegung nach Gleichung 54, getrennt nach der Anzahl der Umlenkpunkte, dargestellt.

$$
\delta_{y z}=\sqrt{\Delta w_{z}^{2}+\Delta v_{y}^{2}}
$$

Die Ergebnisse in Bild 64 verdeutlichen, dass Träger mit zweifacher Umlenkung eine deutlich geringere absolute Verschiebung gegenüber der einfach umgelenkten Variante aufweisen. Der Unterschied zeigt sich bei vorgespannten Trägern mit 10,1 mm deutlicher, als bei den verbleibenden Varianten, was als Grund für die Ergebnisse aus Tafel 18 benannt werden kann. Einschränkend ist jedoch zu sagen, dass die Seillänge durch die Durchbiegung am Umlenkpunkt bestimmt wird. Mit der hier angewandten Prüfmethode wird jedoch ausschließlich die Durchbiegung in Feldmitte gemessen, sodass für eine detailliertere Untersuchung ein aufwendigeres Messverfahren mit detaillierter Bestimmung der Verformungsfigur über die Prüfzeit nötig ist. Als weitere Möglichkeit wird eine numerische Fassung des Phänomens in Kapitel 4 erfolgen und eine Gegenüberstellung mit dem

\footnotetext{
${ }^{236}$ Neben der ständig wirkenden Eigenlast und Vorspannkraft findet sich zusätzlich eine konstante und im Versuch dauerhaft Biegelastung.
} 
analytischen Ansatz nach Kapitel 2.7.6 und den experimentellen Ergebnissen im Rahmen der Diskussion in Kapitel 5 behandelt.

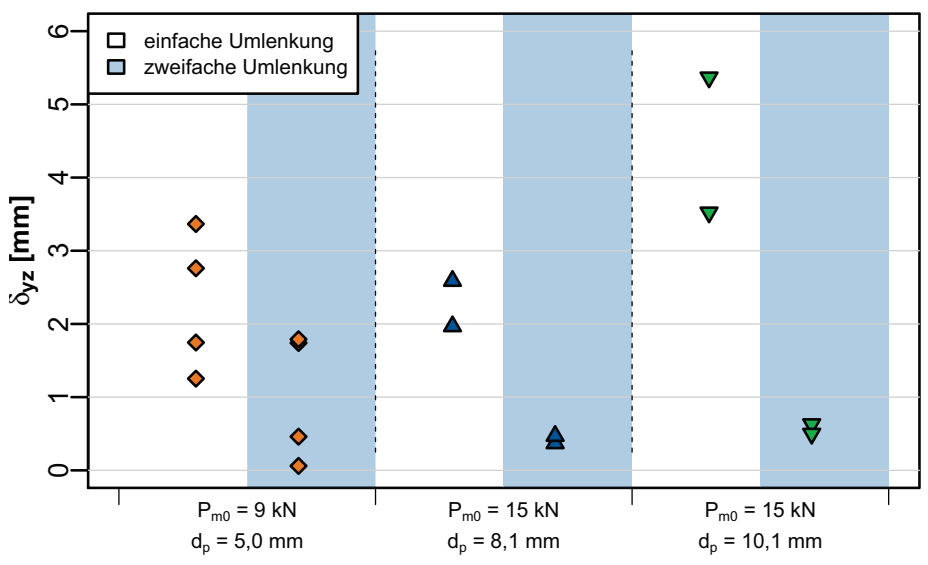

\subsubsection{Folgerungen und Zusammenfassung}

In diesem Kapitel wurde die Auswirkung einer dauerhaften Belastung auf Spannglasträger experimentell ermittelt. Dabei zeigte sich ein Spannkraftverlust von $19 \%$ bis zu $27 \%$ entsprechend der gewählten Konstruktion und der Vorspannkraft. Unbelastete Referenzträger, mit folglich geringerer Seilkraft, zeigten den größeren Seilkraftverlust. Daher wird geschlussfolgert, dass die lastabhängigen nichtlinearen Verformungsparameter ${ }^{237}$ einen geringeren Einfluss haben als die Verformungsfigur infolge der Lastanordnung. So führt die unten am Träger angreifende Umlenkkraft aus den vorgespannten Seilen zu einer anderen Verformungsfigur, als die oben angreifende Biegelast, da dort infolge der Vorverdrehung des Querschnitts andere Hebelverhältnisse vorliegen. Darüber hinaus wurde die Lasttraverse in Versuchen ohne Auflast nicht verwendet, sodass ein Einfluss des Prüfrahmens auf die Ergebnisse nicht vollständig ausgeschlossen werden kann.

237 Beispielsweise das Kriechen von Klotzung und Zwischenschicht sowie die Relaxation des Bewehrungsmaterials. Vergleiche Kapitel 2.5.3, ab Seite 51.
Bild 64

Absolute Verschiebung $\delta_{y z}$ des Trägerquerschnitts in Feldmitte aus horizontaler und vertikaler Durchbiegung. 
Letztlich wurde das Tragverhalten unter Dauerlast erstmals und zielgemäß sowohl als Logarithmus- als auch als Potenzfunktion beschrieben. Dem gegenüber verfehlte die analytische Abschätzung das reale Ergebnis jedoch deutlich - es ist hier nur bedingt anwendbar. Entsprechend wird das analytische Modell mittels einer numerischen Studie in Kapitel 4 nachgerechnet und der bisher unberücksichtigte Einfluss der Durchbiegung überprüft. Darüber hinaus folgt in Kapitel 5 eine umfassende Diskussion der Parameter, welche einen Spannkraftverlust verursachen. 


\subsection{Resttragfähigkeit}

\subsubsection{Prüfkörper}

Für die Versuche zur Bestimmung der Resttragfähigkeit wurden 24 Prüfkörper benutzt (Tafel 19). Es handelte sich um Spannglasträger, welche nach dem zerstörungsfreien Dauerversuch nach Kapitel 3.4 vorbelastet und nun weiterverwendet wurden. ${ }^{238}$ Analog variierten die Vorspannkraft, der Seildurchmesser sowie die Bewehrungsführung. Als Referenz dienten vier unbewehrte (RT 01 bis RT 04) und vier schlaff bewehrte Träger (RT 05 bis RT 08).

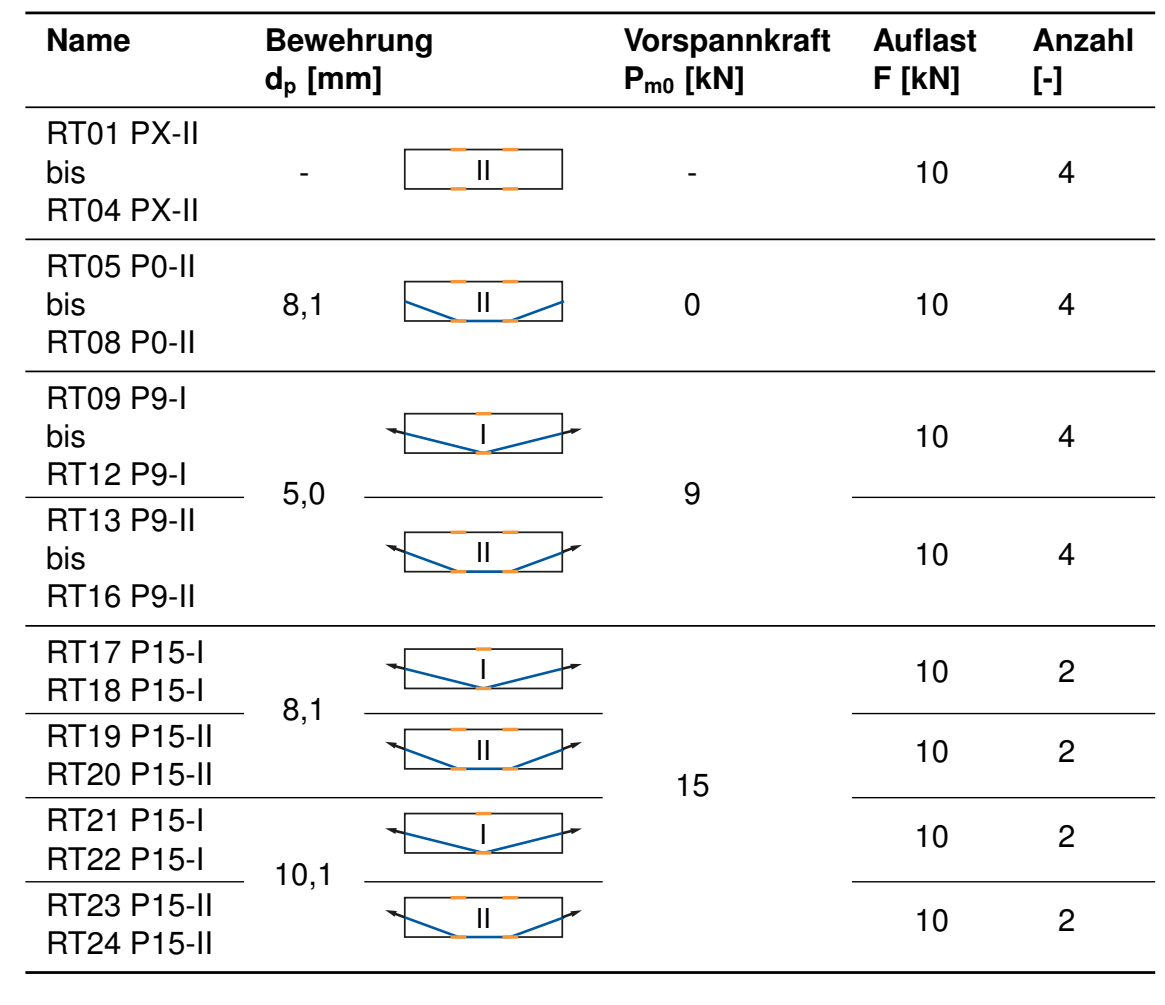

Tafel 19

Prüfkörper

Resttragfähigkeits-Versuche (RT).

238 Entsprechend entfallen die unbelasteten Prüfkörper DV 21/22 und DV 27/28 für die Versuche im Kapitel 3.5. 


\subsubsection{Versuchseinrichtung}

Für Versuche zur Bestimmung der Resttragfähigkeit wurde ein eigener Vierpunkt-Biegeversuchsstand konzipiert (Bild 65), der eine stetige Belastung bei konstanter Kraft ermöglichte. Die Krafteinleitung erfolgte über eine Traverse und einen Hebelarm, welcher mit drei Betonsteinen eine Biegebelastung von etwa $10 \mathrm{kN}$ am Prüfkörper erzeugte. ${ }^{239}$

\section{Bild 65}

Vierpunkt-

Biegeversuchsstand zur Ermittlung der Resttragfähigkeit. Die Prüfkraft wird über einen Hebelarm mittels Betongewichten aufgebracht.

(1) Kraftmessdose Seilkraft am Spannanker

(2) Wegaufnehmer vertikale Durchbiegung

(3) Wegaufnehmer horizontale Durchbiegung (Obergurt)

(4) Lasttraverse

(5) Hebelarm

(6) Betongewichte

(7) Festanker

(8) Dehnungsmessung

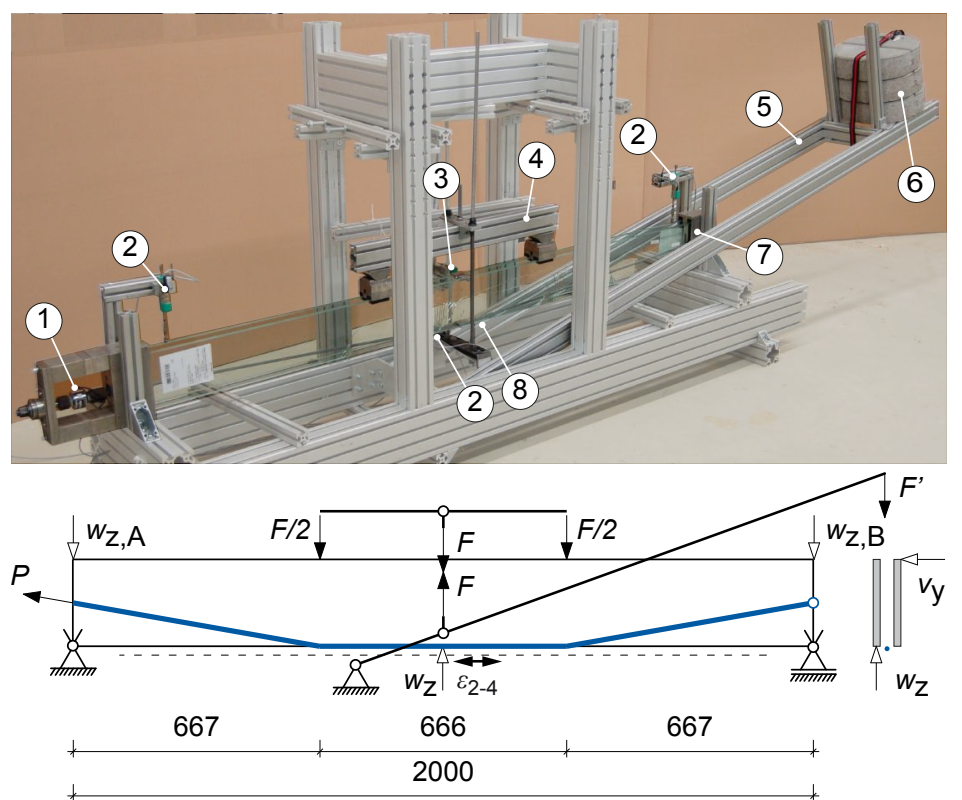

Während des Versuchs wurde die Seilkraft $N_{p}(t)$ mit einer Kraftmessdose auf der Spannseite, die vertikale Durchbiegung der Unterkante in Feldmitte $\mathrm{w}_{\mathrm{z}}(\mathrm{t})$ sowie die horizontale Durchbiegung der Oberkante in Feldmitte $v_{y}(t)$ mit Wegsensoren gemessen. Darüber hinaus wurde die Durchbiegung des Glases am Auflager $w_{z, A}$ und $w_{z, B}$ gemessen, um später eine Deformation der Klotzung innerhalb der Auflagerkonstruktion rechnerisch aus dem Ergebnis entfernen zu können. ${ }^{240}$ Einachsige Folien-Dehnungsmessstreifen ermittelten die Verzerrung der intakten Glasschichten in Feldmitte auf der unteren Kantenfläche des Glases.

\footnotetext{
239 Hier wird der Sollwert der Biegelast angegeben. Im Vorfeld der Versuche wurde die auftretende Biegelast $\left(F_{\text {ist }}\right)$ individuell gemessen und in Anlage D dokumentiert.

240 Siehe Gleichung 39, Seite 108.
} 
Die Datenaufzeichnung erfolgte mit einem acht-kanaligen Messverstärker bei einer Messrate von 0,02 $\mathrm{s}^{-1}$ während der Standzeit und $5 \mathrm{~s}^{-1}$ während dem Aufbringen der Biegebelastung und dem Anschlagen der Glasschichten. ${ }^{241}$ Zusätzlich wurde die Raumtemperatur mit einem separaten System aufgezeichnet.

\subsubsection{Untersuchungsverfahren und -bedingungen}

Für Glasträger existiert kein allgemeingültiges Verfahren zur Bestimmung der Reststandzeit und des Resttragwiderstandes. Daher wurde ein eigenes, geeignetes Verfahren entwickelt. Hier stehen die vier Glasschichten im Trägerquerschnitt bei Raumtemperatur unter konstanter Biegebelastung $^{242}$ auf Dauerlastniveau und werden durch Anschlagen schrittweise zerstört.

Nach dem Einstellen der Vorspannkraft $P_{\mathrm{mo}}$ (Tafel 19), wurde die Belastungseinrichtung montiert und der Prüfkörper mit der vollen Biegekraft belastet. Anschließend wurde die Glasschicht 1 mittels eines stählernen Meißels und eines Hammers im mittleren Drittel, im Bereich des maximalen Biegemomentes, an der Glasunterkante angeschlagen bis es zum Glasbruch kam. ${ }^{243}$ Der Träger verblieb anschließend für $24 \mathrm{~h}$ im Prüfrahmen (Standzeit). Dabei handelt es im um eine im Konstruktiven Glasbau übliche Zeitspanne für den Nachweis gebrochener Überkopfverglasungen. ${ }^{244}$ War der Träger weiterhin intakt, wurde das gleiche Vorgehen anschließend analog an den Glasschichten 4, 2 und 3 fortgesetzt (Bild 66).

${ }^{241}$ HBM Spider8® an einem PC mit der Steuersoftware catman Easy. Dabei entspricht die gewählte Messfrequenz von $0,02 \mathrm{~s}^{-1}$ der kleinsten möglichen Messrate.

242 Entspricht dem Resttragwiderstand. Vergleiche auch Tafel 14.

${ }^{243}$ Alle Bruchursprünge sollten den gleichen Abstand $( \pm 5 \mathrm{~cm})$ zum Auflager haben.

244 Derartige Regelungen werden derzeit von den Obersten Bauaufsichtsbehörden der Länder, beispielsweise in Hessen und Bayern, vorgegeben. Vergleiche [Glaserlass Hessen 2004] und [Merkblatt ZiE Bayern 2015]. Künftig soll eine solche Regelung auch in [DIN 18008-1 (NA 1419)] zu finden sein. 


\section{Bild 66}

Schrittweises Zerstören der Glasschichten unter Vorspann- und Biegebelastung. Zwischen jedem Schritt wurde der Prüfkörper für $24 \mathrm{~h}$ belassen.

Nach $96 \mathrm{~h}$ endet der Versuch, wenn in der Zwischenzeit kein Versagen stattgefunden hat.

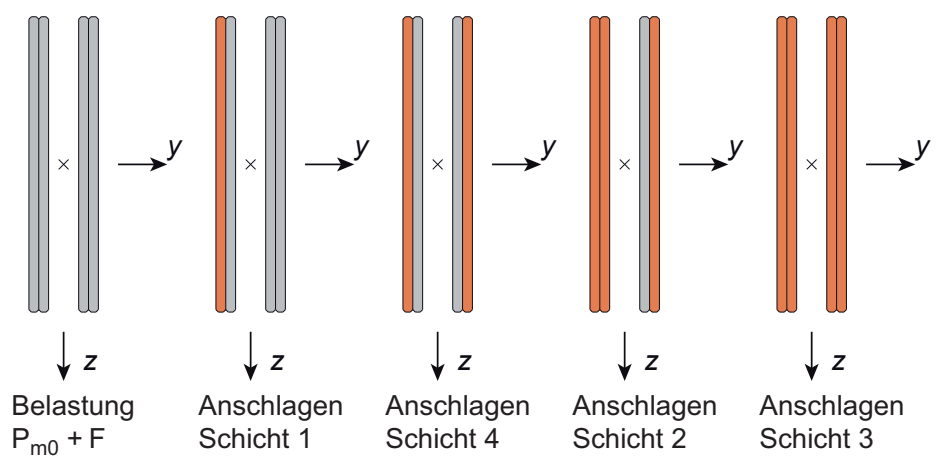

Versuchsdauer $\quad t_{0}=0 \quad t_{24 \mathrm{~h}}=24 \mathrm{~h} \quad t_{48 \mathrm{~h}}=48 \mathrm{~h} \quad t_{72 \mathrm{~h}}=72 \mathrm{~h}$

Bei vier Glasschichten ergab sich eine maximale Versuchsdauer von $96 \mathrm{~h}$. Der Zeitpunkt des früheren Versagens markierte die Reststandzeit. Die Reihenfolge ergab sich aus der Annahme, dass es zum maximalen Steifigkeitsverlust um die schwache Achse kommt, welches dem maßgebenden Parameter für ein Biegedrillknickversagen nach Kapitel 3.3 entspricht.

\subsubsection{Analyse- und Auswertungsverfahren}

Nach dem Versuch existiert für jeden Träger ein Datensatz, welcher die vertikale und horizontale Durchbiegung, die Verzerrung der intakten Glaskanten sowie die Seilkraft im Verlauf der Prüfzeit enthält. Als einheitliches Vergleichskriterium wurde festgelegt, dass die Messwerte jeweils $10 \mathrm{~s}$ vor und nach dem Anschlagen zur Auswertung herangezogen werden, da zu erwarten war, dass sie zum Ersten mit steigendem Zerstörungszustand und zum Zweiten über die Versuchszeit ${ }^{245}$ veränderlich sind.

Damit wird der Traganteil aller gebrochenen Glasschichten ermittelt, indem das Messergebnis dem idealisierten analytischen Berechnungsergebnis, in dem der Traganteil der gebrochenen Glasschichten vollständig vernachlässigt ist, gegenübergestellt wird.

${ }^{245}$ Vergleiche Kapitel 3.4.5, ab Seite 137. 
Die Trageigenschaften sind von der Anzahl und dem Verlauf der Risslinien abhängig. Daher wurde eine Fotodokumentation zur Vermessung der Linien erstellt. Ein entsprechendes Datenblatt in Anlage $D$ dokumentiert dieses Ergebnis für jeden Einzelversuch. Hierin wurden alle Fotos mit einer Grafiksoftware ${ }^{246}$ entzerrt ${ }^{247}$, woraus eine frontale, schematisch Abbildung des Bruchbildes resultierte. Als Bildmaßstab fungierte die Höhe des Glasquerschnitts zwischen zwei Umlenkpunkten. Daraus wurden sowohl die Höhe des Bruchfächers $h_{z}$ (Zugzonenhöhe), die Höhe der verbleibenden, ungebrochenen Druckzone $\mathrm{H}-\mathrm{h}_{\mathrm{z}}$ als auch die Form des Bruchverlaufes bestimmt und für einen Vergleich auf einheitlicher, quantifizierter Grundlage heran gezogen. Jedoch erfolgt das Entzerren des Fotos und die Ermittlung der Messstellen nach dem Eindruck des Betrachters, sodass ein subjektiver Charakter im Ergebnis enthalten bleibt.

Schließlich ergab sich der Versagenszeitpunkt beim vollständigen Verlust der Tragfähigkeit (Reststandzeit). Zusätzlich wird der Versagenscharakter verbal beschrieben, um eine Gruppierung der Ergebnisse zu ermöglichen und potentielle Versagensmodi für den zukünftigen Aufbau eines Bemessungsmodells oder der Ableitung konstruktiver Regeln herauszustellen.

\subsubsection{Ergebnisse und Ergebnisdiskussion}

Die Einzelergebnisse der Versuche zur Ermittlung der Resttragfähigkeit sind in Anlage $\mathrm{D}$ zusammengestellt.

Mit dem Anschlagen jeder Glasschicht bildete sich ein charakteristisches Rissbild heraus (Bild 67). Ausgehend vom Bruchursprung (1) verzweigten sich eine Vielzahl von Risslinien nach oben (2) und wurden im weiteren Verlauf in Richtung der Auflager abgelenkt. Auf Höhe der Druckzone (3) des Querschnitts verliefen die anfänglichen Risse horizontal (4) und vereinigten sich in der Regel. In der weiteren Fol-

\footnotetext{
246 Adobe® Illustrator® CS5 Version 15.0.0.

247 Die örtlichen Bedingungen im Prüfraum erlaubten nur Bilder in Schrägsicht.
} 
Bild 67

Charakteristisches Rissbild im Resttragfähigkeits-

Versuch am Beispiels des des Prüfkörpers RT 23.

Vom Bruchursprung (1) verzweigt der Bruchfächer (2), bis sie im Druckbereich (3) des Glasquerschnitts horizontal ablegenkt wird (4), wobei sich Einzelrisse vereinen und als Rissdelta an der Unterkante (5) auslaufen. Vereinzelt laufen Risslinien innerhalb der Druckzone (6) horizontal zurück. ge verzweigte sich dieser Riss im Bereich der Drittelspunkte, unterhalb der Lasteinleitungsstellen, in Richtung der Trägerunterkante. Dort zeigte sich die für teilvorgespanntes Glas typische Auffächerung zu einem Rissdelta (5).

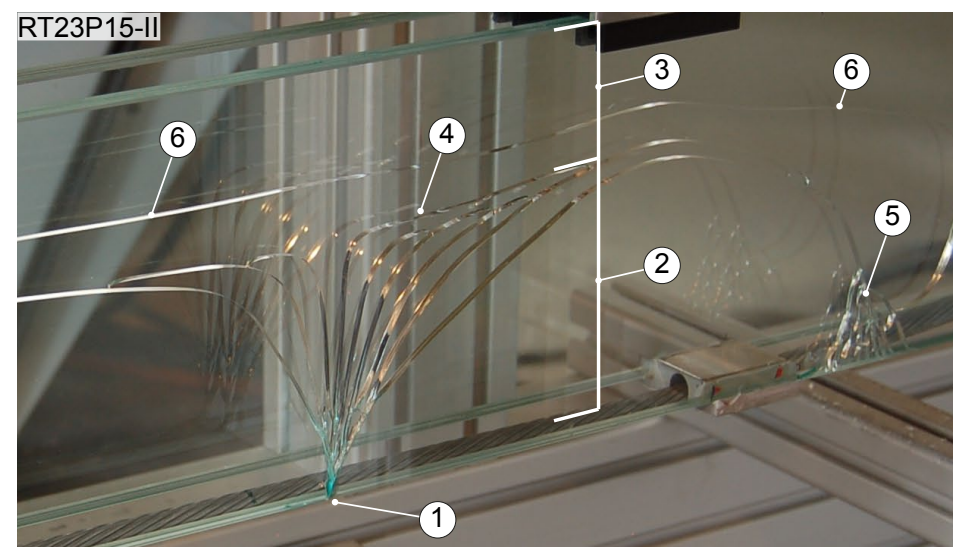

\section{Durchbiegung und Seilkraft}

Bild 68 zeigt den Verlauf eines Resttragfähigkeitsversuches beispielhaft für den Prüfkörper RT 19. Darin vergrößert sich die vertikale Durchbiegung $w_{z}$ ausgehend von einem Wert von $-3,59 \mathrm{~mm}$ um $0,22 \mathrm{~mm}$ infolge des ersten Anschlagens, um $0,60 \mathrm{~mm}$ infolge des zweiten Anschlagens und schließlich um 1,55 mm nach dem dritten Anschlagen sprunghaft. Der Träger sackt erwartungsgemäß kontinuierlich durch, bis der Wegsensor nach $72 \mathrm{~h}$ und einer zusätzlichen Durchbiegung von 5,24 mm den Kontakt zur Glaskante verliert, sodass keine folgenden Messwerte dargestellt werden. Dieses Ergebnis wurde qualitativ für alle Prüfkörper festgestellt und bestätigt die Annahmen für die analytische Berechnung. ${ }^{248}$

Zudem kommt es zu einer veränderlichen horizontalen Durchbiegung. Erwartungsgemäß vergrößert sich dieser Wert innerhalb der ersten $24 \mathrm{~h}$, analog zum Versuch unter Dauerlasten nach Kapitel 3.4.5, deutlich. Mit dem Bruch der zweiten Scheibe kommt es jedoch zu einer Rückstellung. AnschlieBend, bis zur Versuchsdauer von $72 \mathrm{~h}$, verbleibt die Durchbiegung praktisch unverändert, bis es mit dem Anschlagen der letzten Scheibe zu einer kontinuierlich zunehmenden

248 Vergleiche Kapitel 2.7 sowie Anlage A, ab Seite 317. 
Neigung kommt, welcher nach 88,6 h zum Kollaps des Prüfkörpers führt. Im Gegensatz zur vertikalen Durchbiegung konnte hier jedoch keine Systematik im Vergleich mit anderen Prüfkörpern festgestellt werden.

Schließlich stellt Bild 68 (rechts) die Verzerrung der intakten Glaskanten dar. Analog zur horizontalen Durchbiegung kommt es in allen 24-stündigen Abschnitten zu einem kontinuierlichen Anstieg sowie zu einem Sprung beim Anschlagen jeder Einzelscheibe. Hierin zeigt sich eine Lastumlagerung auf das intakte Glas. Auch hierbei handelt es sich um ein typisches Verhalten, welches repräsentativ für alle Prüfkörper steht.

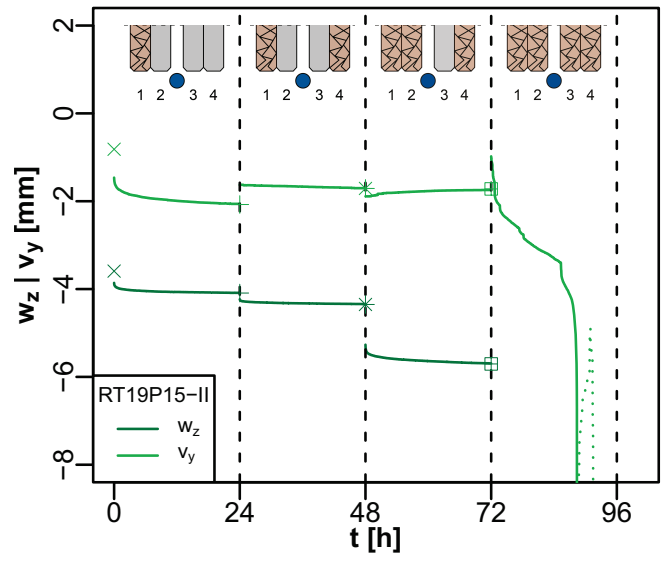

Betrachtet man die auftretenden Seilkräfte für alle Prüfkörper mit steigendem Teilzerstörungsgrad, welcher über die Standzeit in Schritten von je $24 \mathrm{~h}$ in Bild 69 vereinfachend linear dargestellt ist, so fällt zunächst auf, dass sich mit jedem Anschlagen ein Sprung im Verlauf ergibt. Die maximale Seilkraft fiel mit dem maximalen (Teil-) Zerstörungszustand zusammen. Demnach ist das Bewehrungsseil für den Fall des Teilzerstörungszustandes des Glases auszulegen. Dies bestätigt den analytischen Berechnungsansatz ${ }^{249}$, da die Glassteifigkeit mit jedem Anschlagen verringert wird.

Darüber hinaus war keine wesentliche Änderung der Seilkraft binnen jeder der 24-stündigen Abschnitte zu verzeich-

\section{Bild 68}

Verlauf von Durchbiegung und Verzerrung während des exemplarischen Resttragfähigkeitsversuchs des Prüfkörpers RT19 P15-II.

249 Vergleiche Tafel 41, Seite 317 in Anlage A. 
nen. Eine Ausnahme bildeten die beiden Prüfkörper RT 17 und RT 18 mit einem auf $15 \mathrm{kN}$ vorgespannten Seil und einer einzelnen Umlenkung. Für diese war ein deutliches Absinken der Seilkraft innerhalb von $48 \mathrm{~h}$ zu verzeichnen. Mit diesem Ergebnis zeigt sich eine Tendenz zu unterschiedlichen Ergebnissen nach der Anzahl der Umlenkungen.
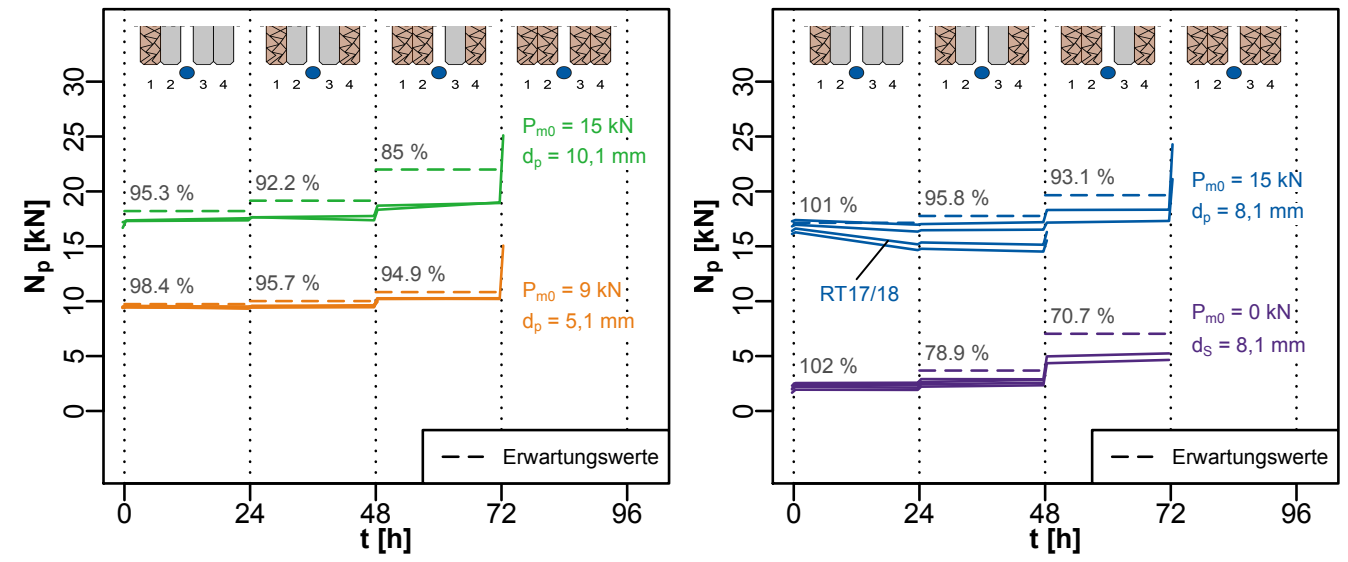

\section{Bild 69}

Absolute Änderung der Seilkraft vor und nach dem Anschlagen einer Glasschicht getrennt nach farblich gekennzeichneten Gruppen.

Erwartungswerte nach Tafel 41 (Seite 317) bereinigt um die Verformung am Auflager nach Gleichung 39, Seite 108.

Zusätzlich sind die Seilkräfte nach der analytischen Rechnung ${ }^{250}$ als gestrichelte horizontale Linien in Bild 69 markiert und im Vergleich dazu die größte gemessene Seilkraft als Verhältniswert festgehalten. Mit dem Anschlagen der ersten Scheibe ist die rechnerische Annahme, wonach der Traganteil der gebrochenen Scheibe nahezu vollständig verloren geht, praktisch sehr realitätsnah (95,3\% bis $102 \%)$. In der weiteren Folge verringert sich das Verhältnis auf $78,9 \%$ bis $95,8 \%$ und $70,7 \%$ bis $94,9 \%$. Daher kann angenommen werden, dass auch eine gebrochene Glasschicht im VSG teilweise zum Lastabtrag beiträgt. Zusätzlich werden die tatsächlich vorhandenen Tragreserven des Seils mit steigendem Zerstörungszustand in zunehmend geringerem Maße ausgenutzt. Es fällt besonders auf, dass die schlaff bewehrten Träger eine vergleichsweise größere Tragreserve hinterlassen. Die Anwendung der analytischen Berechnungsmethode bleibt daher für die Auslegung des Seils auf der sicheren Seite und bildet das Tragverhalten für die begrenzten Ergebnisse dieser Studie (qualitativ) realitätsnah ab.

250 Vergleiche Tafel 41, Seite 317 in Anlage A. 
Im Vergleich dazu verhielten sich die Prüfkörper RT 21 und RT 22 andersartig. Nach dem Anschlagen der Schicht 1 (beispielhaft für RT 21 in Bild 70, links) zeigte sich eine stetig abfallende Seilkraft. Gleichzeitig nahm die Glasdehnung an der Unterkante des Trägers in allen Schichten sukzessive zu. Dies war ebenso für beide Durchbiegungskomponenten zu beobachten (Bild 70, rechts). Die ansteigende seitliche Durchbiegung bedeutete eine Biegebelastung um die schwache Achse und bewirkte dabei eine Schubbelastung der Zwischenschicht. Diese entzog sich der Last durch Verformung, sodass es zu einer zunehmenden Vergrößerung der Durchbiegung und Krümmung des Glases bis zum Erreichen einer kritischen Spannung und folglich dem Glasbruch in den verbleibenden Glasschichten kam. Einerseits war das Bruchbild dabei deutlich sichtbar - das Tragversagen trat mit entsprechendem zeitlichen Vorlauf ein, was einem günstigen Tragverhalten mit angekündigtem Versagen entspricht. Andererseits trat dieser Fall bereits mit dem Bruch der ersten Glasschicht auf, sodass das Tragsystem als nicht redundant zu beschreiben ist und dieses Verhalten als nicht ausreichend resttragfähig beurteilt wird.
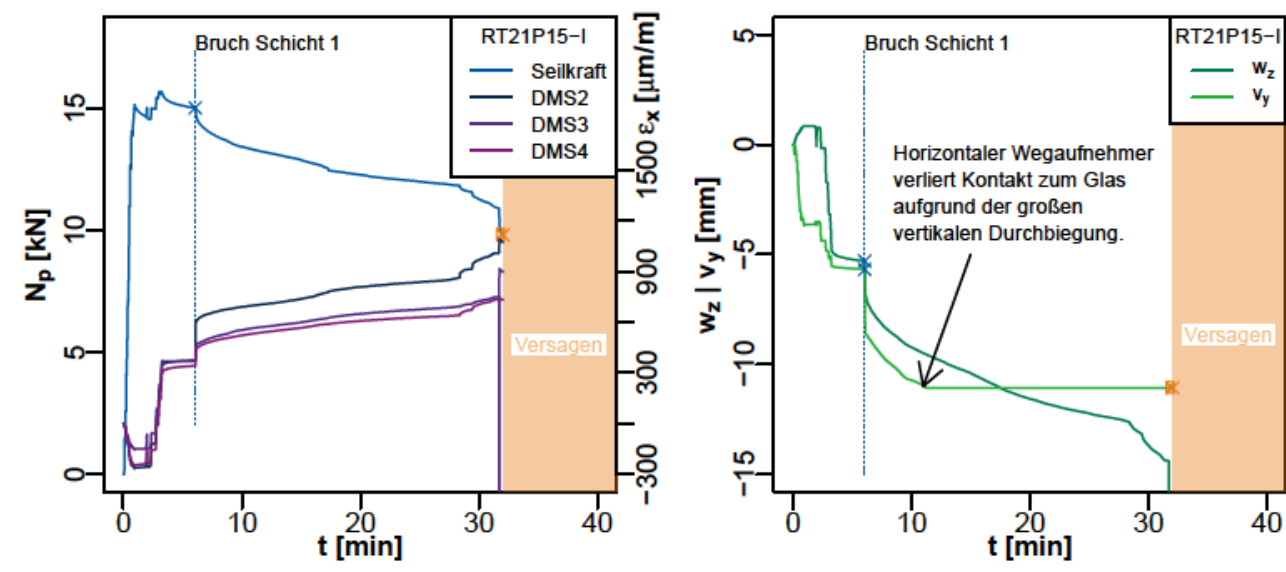

Bild 70

Seilkraft, Glasverzerrung und Durchbiegung beispielhaft für den Prüfkörper RT 21. 


\section{Reststandzeit}

In Bild 71 wird der Zeitpunkt des kompletten Versagens im Resttragfähigkeitsversuch (Reststandzeit), getrennt nach Konstruktions- und Bewehrungsart, dargestellt. Fast alle unbewehrten Referenzprüfkörper verloren ihre Tragfähigkeit mit dem Anschlagen der Schicht 2 nach $48 \mathrm{~h}$. Einzig ein Träger der Gruppe hielt der Biegebelastung für weitere $5 \mathrm{~h}$ stand. Nach dem Verlust der Tragfähigkeit sackten die Prüfkörper infolge der Rissaufweitung im Glas bis auf den Prüfrahmen herab. $^{251}$

Bild 71

Reststandzeit aller Prüfkörper in Gruppen nach der Konstruktionsweise.

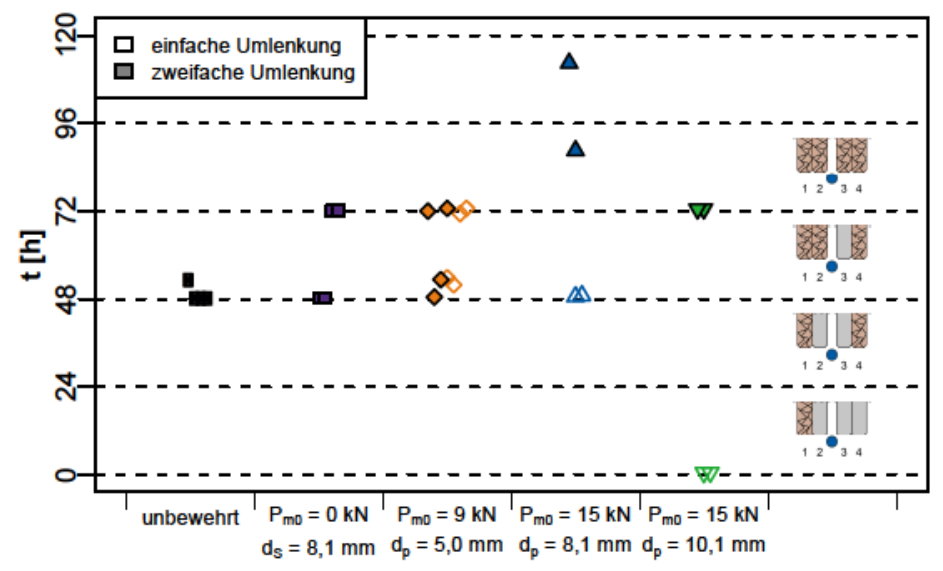

Zwei der schlaff bewehrten Glasträger versagten ebenso bereits unmittelbar nach dem Anschlagen der Scheibe 2 nach $48 \mathrm{~h}$. Die zwei verbleibenden Prüfkörper dieser Konfiguration waren mit einer einzigen intakten Glasschicht für weitere $24 \mathrm{~h}$ tragfähig. Nach dem Versagen (72 h) blieb das Bewehrungsseil weiterhin intakt und hielt den Träger oberhalb des Prüfrahmens. ${ }^{252}$ Diese Auswirkung der Bewehrung wurde bereits unter kurzzeitiger Biegebelastung beobachtet ${ }^{253}$ und im Vergleich zu unbewehrten Trägern als günstig beurteilt.

Ein ähnliches Ergebnis wurde bei den vorgespannten Prüfkörpern mit 5,0 mm Seildurchmesser erzielt. Eine Hälfte der Prüfkörper versagte unmittelbar beim Anschlagen der dritten Scheibe oder innerhalb weniger Stunden danach. Die

\footnotetext{
251 Vergleiche auch Bild 56 (links), Seite 130.

252 Vergleiche auch Bild 56 (rechts), Seite 130.

${ }^{253}$ Vergleiche Kapitel 3.4.5, ab Seite 137.
} 
zweite Hälfte der Prüfkörper konnte mit der verbleibenden Scheibe 3 bis $72 \mathrm{~h}$ der Belastung widerstehen. Dabei wurde kein deutlicher Unterschied zwischen Trägern mit einem oder zwei Umlenkpunkten beobachtet. Hier wird vermutet, dass der Bewehrungsgrad und die Vorspannkraft nicht ausreichend groß war, um das Resttragverhalten zu beeinflussen.

In der Gruppe der mit $15 \mathrm{kN}$ vorgespannten Spannglasträger zeigte sich ein auffällig breites Ergebnisspektrum. Zunächst versagten jene Prüfkörper mit 8,1 mm Seildurchmesser und einfacher Umlenkung nach $48 \mathrm{~h}$ gleichzeitig mit dem Anschlagen der Glasscheibe 2. Im Unterschied dazu konnten sich bei den äquivalenten Prüfkörpern mit zweifacher Umlenkung auch nach dem Anschlagen der letzten Glasschicht ein Gleichgewicht zwischen dem Glas in der Druckzone und dem Seil in der Zugzone ausbilden. Darüber hinaus kann nicht ausgeschlossen werden, dass ein Teil der Zugbelastung über das verbleibende Glas in der Zugzone beziehungsweise durch den gemeinsamen Lastabtrag und die Lastübertragung zwischen einzelnen Schichten im Verbund-Sicherheitsglas erfolgte. Diese Träger versagten erst nach 88,6 h und 112,5 h. Im Gegensatz zu den Trägern mit 5,0 mm Bewehrung wurde hierfür ein deutlich günstigerer Einfluss der vorgespannten Bewehrung auf das Tragverhalten beobachtet.

Der Einfluss der Umlenkungen und damit der Konstruktionsart wird im Vergleich der Träger mit dem größten Bewehrungsgrad bei einem Seildurchmesser von $10,1 \mathrm{~mm}$ offenbar: Während die Prüfkörper mit zweifacher Umlenkung (RT 23 und RT 24) eine gesteigerte Reststandzeit von $72 \mathrm{~h}$ zeigten, brachen die verbleibenden zwei Prüfkörper RT 21 und RT 22 mit einfacher Umlenkung innerhalb einer halben Stunde nach dem Anschlagen der ersten Glasschicht. Hierbei bildete sich ein dichtes, vollflächiges Rissnetz über die gesamte betreffende Glasschicht zusätzlich zum charakteristischen Bruchfächer aus (Bild 72).

Ein irregulärer Vorspannungszustand des Glases als Grund für dieses Verhalten wird ausgeschlossen, da alle Prüfkörper aus der selben Produktionscharge stammten. Darüber 
hinaus entsprach das Ergebnis nicht dem Rissbild, welches von einem Träger aus Einscheiben-Sicherheitsglas erwartet wurde. ${ }^{254}$ Vielmehr waren im Bereich der Oberkanten auffällig fein verzweigte Risslinien zu erkennen ((2) in Bild 72), welche typischerweise bei einem hohen Lastnivau auftreten. Darüber hinaus zeigten die betroffenen Prüfkörper eine deutlich sichtbare seitliche Neigung in Richtung der gebrochenen Außenscheibe 1. Daher wird eine überdurchschnittlich große Vorverformung, aus geometrischer Imperfektion ${ }^{255}$ und konstruktiven Gegebenheiten ${ }^{256}$, als Grund für diese Ergebnisse vermutet. Hierin zeigt sich ein ungünstiger Effekt der Vorspannung: Infolge des künstlich erzeugten Bruchs der ersten Glasschicht lehnten sich die Prüfkörper deutlich sichtbar zur Seite, was im Gegensatz zum typischen Bild 67 ein abweichendes, flächenhaftes Bruchbild erzeugte (Bild 72).
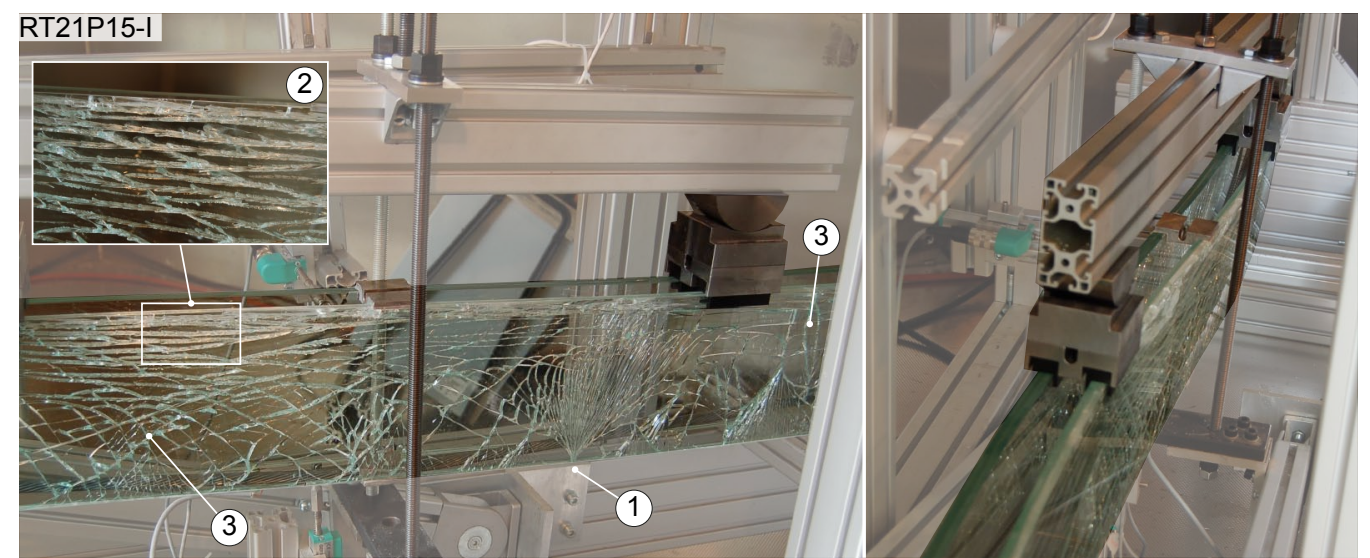

Bild 72

Abweichendes Rissbild (links) und deutliche seitliche Durchbiegung (rechts).

(1) Bruchursprung und charakteristischer Bruchfächer

(2) auffällige fein, verzweigte Risslinien parallel zur Trägeroberkante

(3) vollflächige Risse
Insgesamt zeigt sich, dass die Ausbildung des Rissbildes einen deutlichen Einfluss auf das Tragverhalten im teilzerstörten Zustand hat.

\footnotetext{
254 Während der Studie wurde eine Fehllieferung mit ESG-Scheiben untersucht. Erst nach dem Anschlagen der ersten Schicht zeigte sich der Fehler in Form eines sehr feinen Bruchbilds mit kleinen Bruchstücken, welches für diese Glasart typisch sind. Die Ergebnisse dieser Untersuchung wurden für diese Arbeit nicht berücksichtigt.

${ }^{255}$ Vergleiche Kapitel 2.7.2, Seite 65.

256 Der Einfluss der Konstruktion auf die seitliche Durchbiegung wird in Kapitel 6, ab Seite 242 dargelegt.
} 


\section{Rissbilder in Teilzerstörungszuständen}

Mit steigendem (Teil-) Zerstörungszustand wurden die Bruchfächer höher und breiter (Beispiel in Bild 73). Es wurde eine höhere Bruchenergie frei, sodass mehr Risslinien gebildet wurden, was sich in einem feineren Rissbild äußerte. Darüber hinaus entstanden vereinzelt Rissfortschritte bis ins Auflager oder bis in die Oberkante des Glases. Während der Standzeit von jeweils $24 \mathrm{~h}$ erweiterten sich einige Risse, jedoch ohne erkennbare Systematik. Dies betraf sowohl die zuletzt angeschlagene Schicht, sowie alle benachbarten Glaslagen.

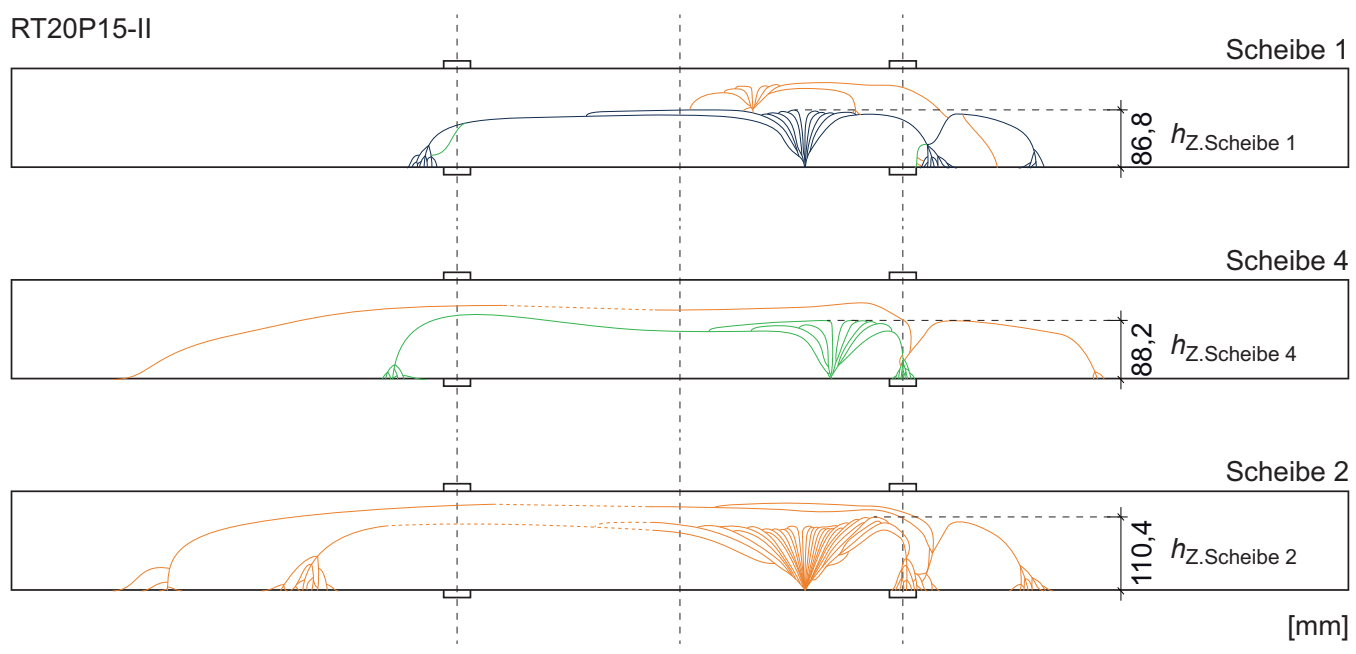

Bild 73

Aus allen Einzelrissbildern ${ }^{257}$ wurde die Zugzonenhöhe abgeschätzt. Bild 74 zeigt dazu eine Zusammenfassung nach Gruppen ohne Markierung der Anzahl der Umlenkungen, da hier kein auffälliger Unterschied beobachtet wurde. Zwischen den unbewehrten und den schlaff bewehrten Trägern zeigte sich kein wesentlicher Unterschied beim Anschlagen der Scheiben 1 und 4. Alle Messergebnisse liegen hier dicht beieinander. Mit steigender Vorspannkraft verringerte sich die Höhe des Bruchfächers, wobei die Werte für Prüfkörper mit 5,0 mm Seildurchmesser auffällig streuten. Im weiteren Verlauf der blauen und grünen Linie ist eine steigende Tendenz zu erkennen. Hierbei scheint eine Vergrößerung des
Rissbilder des Prüfkörpers RT 20 P15-II.

\section{Risslinien}

— zu Versuchsbeginn

- nach $24 \mathrm{~h}$

nach $48 \mathrm{~h}$

257 siehe Anlage D, ab Seite 353. 
Bild 74

Mittelwerte der Bruchfächerhöhe nach Bewehrungsgruppen und Vorspannung.

Die ungebrochene Glashöhe oberhalb des Bruchfächers ergibt sich aus der Differenz zu $150 \mathrm{~mm}$.
Bewehrungsgrades keinen spürbaren Vorteil, möglicherweise gar einen Nachteil, zu erbringen, sodass weiterführende Analysen anzuraten sind. Für das Anschlagen der Scheibe 2 wird die fallende Tendenz deutlicher: Die Bruchfächerhöhe fällt von $118 \mathrm{~mm}$ auf $107 \mathrm{~mm}$ und $105 \mathrm{~mm}$ sowie letztlich auf $100 \mathrm{~mm}$. Der dementsprechend verbleibende, ungebrochene Glasquerschnitt in der Druckzone wird mit steigendem Vorspanngrad und initialer Vorspannkraft größer.

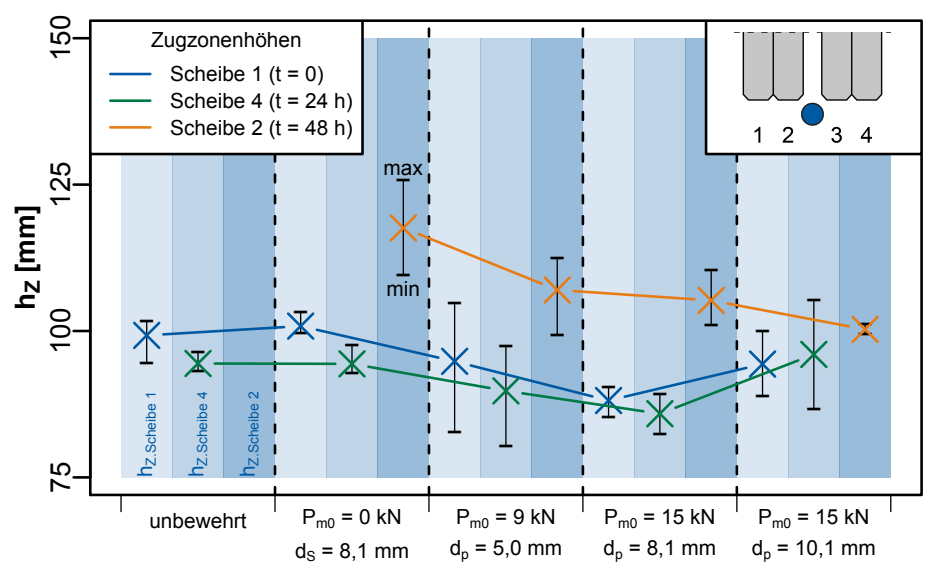

Es ergibt sich also der Eindruck, dass die Vorspannung zu einer größeren Tragsicherheit führen kann und damit das Konzept „Spannglasträger" sein Potenzial hin zu sichereren Glaskonstruktionen im Ansatz gezeigt hat. Einschränkend ist jedoch festzuhalten, dass die quantitativen Werte vergleichsweise nah beieinander liegen. Unter zusätzlicher Berücksichtigung der geringen Prüfkörperanzahl wird die Beobachtung nur qualitativ bewertet. Daher ist das Ergebnis, dass mit steigender Vorspannung einer größerer Glasquerschnitt intakt und damit tragfähig bleibt, ausschließlich als Tendenz zu bewerten. Fortführende Untersuchungen, insbesondere das Auffinden einer günstigen Kombination von Vorspannkraft und Steifigkeit des Glasquerschnitts sowie der Bewehrung, müssen dieses Trend bestätigen.

\section{Charakter des finalen Versagens}

Das Versagen eines Prüfkörpers nach dem Anschlagen der vierten Glasschicht fand in dieser Studie nur im Einzelfall 
statt. Es kam in der Regel zu einem Versagen vor dem Ablauf der maximalen Standzeit von $96 \mathrm{~h}$. Daher wird im Weiteren das aufgetretene Versagen typisiert, um die beobachteten Versagensmodi zu charakterisieren und zu systematisieren.

Nach dem vollständigen Versagen sackten die unbewehrten Referenzprüfkörper bis auf den Prüfrahmen ab (Bild 75, links). Die Rissflanken wurden durch die Zwischenfolie überbrückt, welche infolge der auftretende Zugbelastung eine große Dehnung zeigte. Dem gegenüber neigten sich bewehrte und vorgespannte Träger in der Regel zur Seite, bis es zu einem Auffalten des gebrochenen Glases kam (Bild 75, rechts). Das Seil vermochte den zerstörten Träger oberhalb des Lastrahmens zu halten. Ein äquivalentes Verhalten zeigten die bewehrten Prüfkörper BV 04 bis BV $15 \mathrm{im}$ Kurzzeit-Biegeversuch ${ }^{258}$. Demnach erbrachte das gewählte Vorgehen im Resttragfähigkeitsversuch eine vergleichbare Zerstörungssituation. Das neu entwickelte Prüfverfahren wird daher als zweckmäßig beurteilt.

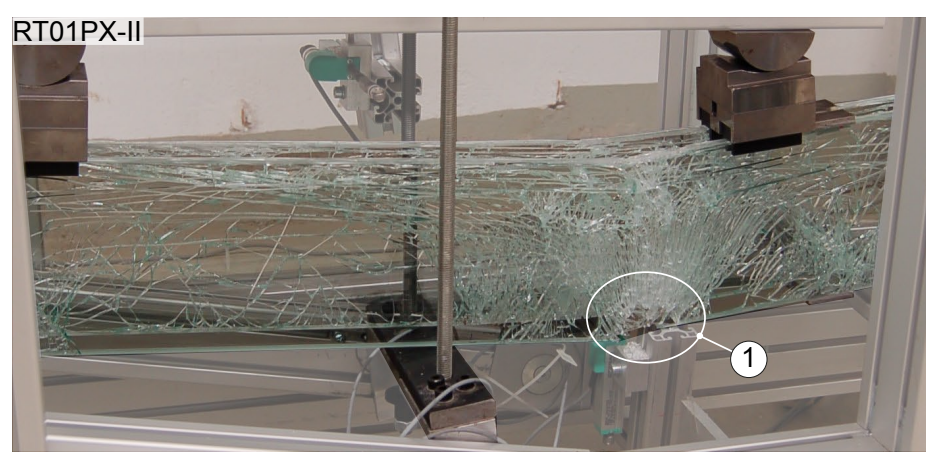

Darüber hinaus wurden im Einzelfall weitere Sonderformen des Versagens, die auf zusätzliche Versagensmechanismen hindeuten, beobachtet. Deren Anaylse ist notwendig, um perspektivisch sicherere Spannglasträger konstruieren zu können.

Die Prüfkörper RT 10, RT 14 und RT 17 versagten in einer zweiwelligen Biegeform (Bild 76, links). Offenbar wurde in diesen Fällen eine höhere Bruchenergie frei, welche sich zu-

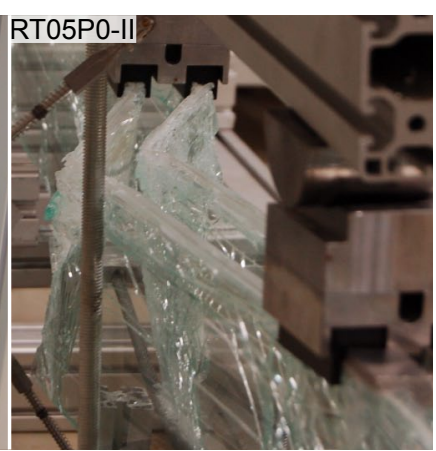

Bild 75

Unbewehrte Träger am Beispiel des Prüfkörpers RT01 (links) kamen nach dem Versagen auf dem Prüfrahmen zum Liegen. Die Zwischenschicht überbrückte den Rissursprung mit großer Dehnung (1). Dem gegenüber versagten bewehrte und vorgespannte Träger (rechts) in der Regel einwellig durch ein Auffalten. Das Seil verhinderte ein Absacken des Glases. 
sätzlich durch ein flächenhaftes Rissbild über die gesamte Trägerlänge und einer auffällig großen seitlichen Durchbiegung äußerte. Hier fand sich ein zu den Prüfkörpern RT 21 und RT 22 ähnliches Ergebnis bei höherem Teilzerstörungszustand. Zusätzlich kam es zum Abreißen je eines Klebpunktes. Dabei blieben Glaslanzen am Umlenkpunkt zurück, sodass das Loslösen durch eine Auftrennung im Verbundglas begründet lag. ${ }^{259}$ Diese Versagensform ist zu vermeiden, da das Glas ohne einen tragfähigen Umlenkpunkt nicht mehr durch das Seil gestützt wird und es infolge eines flächenhaften Risses zu einem maximalen Steifigkeitsverlust kommt, welcher sich in einer minimalen verbleibenden Tragfähigkeit äußert. Zu deren Vermeidung ist daher eine baukonstruktive Weiterentwicklung der Fügestelle im Detail notwendig.

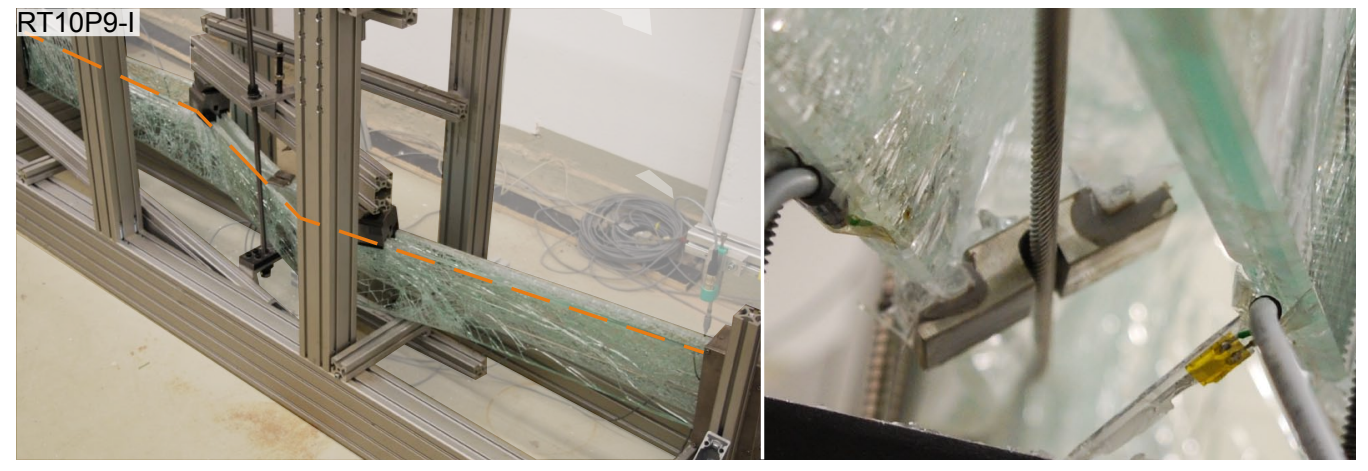

\section{Bild 76}

Mehrwellige Versagensfom mit flächigem Rissbild.

Hierbei ist es vereinzelt zum Abreißen der Umlenkpunkte kommen.
Prüfkörper RT 15 zeigte neben dem üblichen Auffalten der Trägeroberkante zusätzlich ein Abscheren in der Nähe eines Umlenkpunktes (Bild 77). In diesem Bereich, zwischen den Lasteinleitungen der Umlenk- und Biegekraft, entstanden eine Vielzahl von Risslinien auf kleinem Raum, sodass das Versagen dem eines Fließgelenkes ähnelte. Ein analoges Abscherverhalten wurde für die Prüfkörper RT 11 und RT 16 auf freier Kantenlänge, abseits der geklebten Umlenkpunkte, beobachtet. Dabei zeigte sich allgemein im vollständig gebrochenen Glaspaket ein Versatz in der Unterkante (1). Trotz des gebrochenen Glases war in diesen Bereichen ein Lastübertrag möglich.

259 Entsprechend wurde weder ein adhäsives oder kohäsives Versagen der Klebfuge beobachtet. 


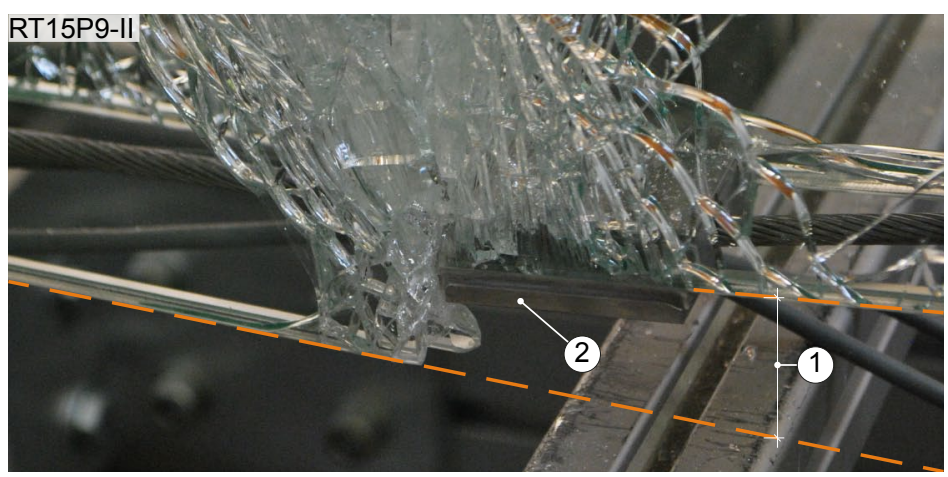

Bild 77

Das gebrochene Glas im

Prüfkörper RT 15 schert

neben dem Umlenkpunkt ab.

Es kommt zu einem deutlich

sichtbaren Versatz (1) der

Glasunterkante beiderseits

der Umlenkung (2).

\subsubsection{Folgerungen und Zusammenfassung}

In diesem Kapitel wurden die Ergebnisse der Resttragfähigkeitsuntersuchung zusammengestellt. Es wurde eine Methode vorgeschlagen und erfolgreich angewendet, um sowohl die Reststandzeit, als auch die Resttraglast zu ermitteln.

Unter der gewählten Lastsituation zeigte sich die Schädigungssituation nach $48 \mathrm{~h}$ als kritisch. Hier wird die dritte Schicht im VSG-Paket angeschlagen, sodass die verbleibende Glasschicht im bewehrten Fall weiterhin durch das Seil gestützt wird. Eine effektive Tragstruktur wird dann erreicht, wenn unter Gebrauchslasten alle Schichten gebrochen sein können und eine verbleibende Standzeit erreicht wurde. Mit diesem Nachweis können alle Schichten im Grenzzustand der Tragfähigkeit angesetzt werden, sodass das Prinzip einer „Opferscheibe“ entfallen kann. Dieses Ziel wurde nur teilweise erreicht, sollte aber ausblickend anvisiert werden.

Für eine derartige Untersuchung liegen nach Recherche des Autors keine vergleichbaren Ergebnisse vor. Daher wurden die beobachteten Trageffekte, Bruchbilder und Versagensmodi erstmals umfassend beschrieben. Der Versagenszeitpunkt konnte durch die Bewehrung und eine Vorspannung beeinflusst werden. Insgesamt wurden sowohl ungünstige, als auch günstige Eigenschaften beobachtet:

Infolge einer deutlichen seitlichen Durchbiegung beim Glasbruch verringert sich die Standzeit. Diese Verformungsrichtung wird durch die Vorspannung begünstigt, sodass diese 
Konstruktionsart bei exzentrischer Belastung um die schwache Achse ungünstig wirkt. Diese Eigenschaft ist weiter zu analysieren, um sie perspektivisch vermeiden zu können.

Das Bewehren und Vorspannen von Glasträgern soll im Ziel zu günstigen Effekten führen. Dazu erbrachten bewehrte und vorgespannte Träger einerseits die gleiche Standzeit, wie die unbewehrte Referenz, andererseits wurde eine vergröBerte Reststandzeit und ein Aufrechterhalten der Konstruktion durch die Bewehrung im Sinne der Bereitstellung eines weiteren Lastabtragspfades, insbesondere für Prüfkörper mit zweifacher Umlenkung, beobachtet. Die Wahl der Querschnittskonstruktion und der Anzahl der Stützstellen steuerte die mögliche Biegeform der Glaspakete war demnach bedeutend für die Trageigenschaften während einer Situation mit teilweise zerstörten Glasschichten.

Zusätzlich ist eine mögliche Reduzierung der Bruchfächerhöhe zu nennen. Diese günstigen Effekte sind weiter zu analysieren und die Konstruktion zu verfeinern. Eine Abgrenzung zwischen einem sicheren günstigen und ungünstigen Verhalten bleibt einer weiterführenden Untersuchung sowie einer weiteren baukonstruktiven Detailentwicklung mit einer größeren Anzahl an Prüfkörpern vorbehalten.

Insgesamt wurden die aufgetretenden Phänomene erstmals beschrieben und gruppiert, sodass eine erste Datengrundlage zur weiteren Verfügung bereit steht. Dabei hat sich der Spannglasträger bei Wahl einer günstigen Variante als resttragfähig gezeigt. 


\subsection{Tragverhalten unter Temperaturbelastung}

\subsubsection{Prüfkörper}

Für die zerstörungsfreien Temperaturlastversuche wurden zwei Prüfkörper mit zweifacher Seilumlenkung nach Tafel 20 mit einem 8,1 mm Spannseil ${ }^{260}$ verwendet.

\begin{tabular}{|c|c|c|c|c|}
\hline Name & $\begin{array}{l}\text { Bewehrung } \\
d_{p}[\mathrm{~mm}]\end{array}$ & $\begin{array}{l}\text { Vorspannkraft } \\
\mathrm{P}_{\mathrm{m} 0}[\mathrm{kN}]\end{array}$ & $\begin{array}{l}\text { Auflast } \\
F[k N]\end{array}$ & $\begin{array}{l}\text { Anzahl } \\
{[-]}\end{array}$ \\
\hline TV01-II & 8,1 & $0 ; 3 ; 6 ; 9$ & $0 ; 5 ; 10$ & 1 \\
\hline TV02-II & & $0 ; 3 ; 6 ; 9$ & $0 ; 5 ; 10$ & 1 \\
\hline
\end{tabular}

Tafel 20

Prüfkörper Temperatur-

Biegeversuche (TV).

Die Vorspannkraft und die Auflast werden gemäß der Kombinationsmatrix in Tafel 21 variiert. Als Referenzen dienen die unbewehrte (PX) und die schlaff bewehrte (P0) Variante.

\begin{tabular}{llllll}
\hline $\mathbf{F}$ & \multicolumn{1}{c}{$\mathbf{P}_{\mathbf{m} \mathbf{0}}$ ohne } & $\mathbf{0 k N}$ & $\mathbf{3 k N}$ & $\mathbf{6 k N}$ & $\mathbf{9 k N}$ \\
\hline $\mathbf{0 k N}$ & PX F0 & P0 F0 & P3 F0 & P6 F0 & P9 F0 \\
$\mathbf{5 k N}$ & PX F5 & P0 F5 & P3 F5 & P6 F5 & P9 F5 \\
$\mathbf{1 0 k N}$ & PX F10 & P0 F10 & P3 F10 & P6 F10 & P9 F10 \\
\hline
\end{tabular}

Tafel 21

Versuchsmatrix TemperaturBiegeversuche (TV).

Es ergeben sich 30 zerstörungsfreie Prüfungen. Die Prüfkörper werden nach jedem Versuch wiederverwendet.

\subsubsection{Versuchseinrichtung}

Die Versuchseinrichtung, welche für die Bestimmung der Resttragfähigkeit zum Einsatz kam, ${ }^{261}$ wurde in einer Temperierkammer aufgebaut. Darin heizte ein Elektroofen die Raumluft auf eine Soll-Temperatur von $60^{\circ} \mathrm{C}$. Ein Ventilator vermischte die Luft innerhalb der Prüfkammer, um eine gleichmäßige Temperaturverteilung zu erreichen. Die Ofenregelung erfolgte über ein internes System.

260 siehe Tafel 9, Seite 106.

261 Vergleiche Bild 65, Seite 148. 
Bild 78

Referenzglaskörper aus Temperaturversuch.

Die Verzerrung in der Mitte der rechteckigen Oberfläche $\left(75 \times 75 \mathrm{~mm}^{2}\right)$ wurde mit einem biaxialen Dehnungsmessstreifen aufgezeichnet.
Während des Versuchs wurden die auftretende Seilkraft $N_{p}(T, t)$ sowie die Verzerrung aller vier Glaskanten $\varepsilon_{\text {Signal }} 1$ bis 4 aufgezeichnet. Die Messung der vertikalen Durchbiegung $w_{z}$ und horizontaler Durchbiegung an der Glasoberkante $v_{y}$ in Feldmitte erfolgte zur Überwachung des Versuchsverlaufes. Ein Fe-Cu/Ni Thermoelemente ${ }^{262}$ nahm die Raumtemperatur in der Nähe des Prüfkörpers auf. Darüber hinaus wurden die einachsigen Verzerrung parallel zu den Kanten einer rechteckigen unbelasteten Referenzglasscheibe der Dicke $8 \mathrm{~mm}$ gemessen (Bild 78).

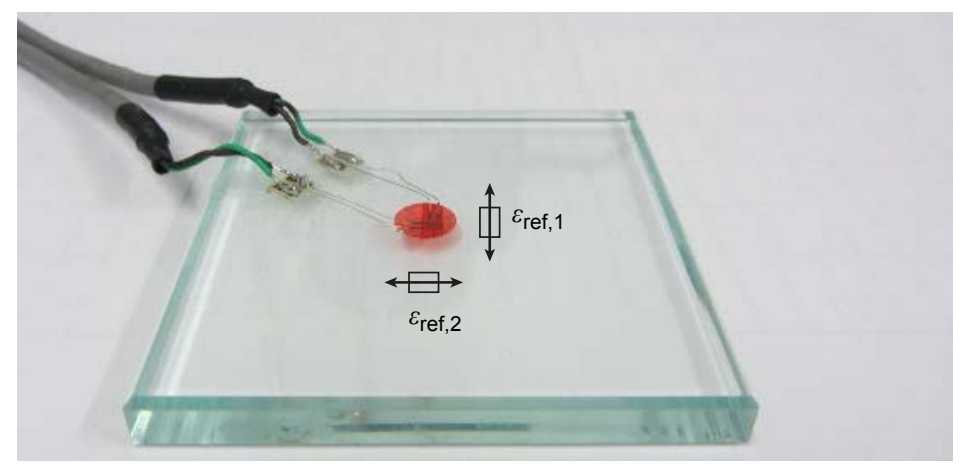

Die Datenaufzeichnung erfolgte mit je einem sechs- und acht-kanaligen Messverstärker ${ }^{263}$ bei einer Messrate von $0,02 \mathrm{~s}^{-1}$.

\subsubsection{Untersuchungsverfahren und -bedingungen}

Für die Ermittlung des Tragverhaltens von Glasbauteilen unter Temperaturlast ist kein allgemeingültiges Prüfverfahren verfügbar. Daher wurde eine vierstufige Methode neu entwickelt. ${ }^{264}$ Zunächst wurde die Vorspann- und Biegebelastung analog zum Ablauf in Kapitel 3.5 aufgebracht. Im Rahmen von Vorversuchen ${ }^{265}$ wurden die anschließend nötigen Heizund Haltezeiten bestimmt. Demnach dauert es 90 bis 100

262 GEFRAN T4P-1-J-5-AU. Zur Kontrolle wurde die Lufttemperatur zusätzlich unmittelbar unterhalb der Kammerdecke, circa $1 \mathrm{~m}$ oberhalb des Prüfkörpers, aufgezeichnet.

263 HBM Spider8®.

264 Zu Beginn der Ergebnisauswertung wurde der Nachweis geführt, dass alle Versuche unabhängig voneinander unter gleichartigen Bedingungen durchgeführt wurden. 
Minuten bis die Lufttemperatur in der Klimakammer erstmals den Zielwert von $60^{\circ} \mathrm{C}$ überschreitet und der Ofen seinen Regelzyklus beginnt. Dabei wurde die Oberflächentemperatur des Glases kontinuierlich mit einem Kontaktthermometer gemessen. Im Anschluss kühlten der Prüfkörper, die Belastungseinrichtung und die geöffnete Klimakammer auf Raumtemperatur herunter, sodass der nächstfolgende Durchlauf unter den gleichen Anfangsbedingungen gestartet werden konnte.

\subsubsection{Analyse- und Auswertungsverfahren}

Die Versuchsergebnisse werden aufbauend auf früheren Arbeiten ${ }^{265}$ hinsichtlich der Änderung der Seilkraft und der Verformung ausgewertet. Im Fokus dieser Untersuchung stand die erwartete Seilkraftänderung sowie ein möglicher vollständiger Seilkraftverlust bei einer Temperatursteigerung.

Aus Vorversuchen ${ }^{265}$ ist bekannt, dass es im Zeitraum zwischen 100 und 240 Minuten zu keiner wesentlichen Änderung der Glasoberflächentemperatur und der Durchbiegung mehr kommt. Daher werden die Mittelwerte über diesen Zeitbereich für den Vergleich mit den Ausgangswerten verwendet. Gleichung 56 beschreibt den Zusammenhang beispielhaft für die Seilkraft.

$$
\Delta N_{p}=N_{p}(t=0)-\varnothing N_{p}(t=100 \text { bis } 240 \min )
$$

$$
\begin{array}{ll}
\Delta N_{\mathrm{p}} & \text { Seilkraftänderung } \\
N_{\mathrm{p}}(t=0) & \text { Seilkraft zu Versuchbeginn (einschließ- } \\
& \text { lich der Wirkung von Biegelast } \mathrm{F} \text { und } \\
\varnothing N_{\mathrm{p}} & \text { Vorspannung } \mathrm{P}_{\mathrm{m} 0} \text { ) } \\
& \text { Mittlere Seilkraft, hier im Zeitbereich } \\
& \text { von } 100 \text { bis } 240 \text { Minuten }
\end{array}
$$

Ebenso wurde der Einfluss der Temperatur auf die Messmittel in Vorversuchen ${ }^{265}$ untersucht. Demnach verbleiben die Signale der Wegaufnehmer und der Kraftmessdose im gewählten Einsatzbereich von der Umgebungstemperatur

265 Vergleiche [Bukieda 2016]. 
unabhängig und werden direkt verwendet. Im Unterschied dazu wird das Signal der Dehnungsmessung nach der Aufzeichnung angepasst. ${ }^{266}$ Demnach wird die scheinbare Verzerrung $\varepsilon_{\text {app }}$ und die thermische Verzerrung des Dehnungsmessstreifens ${ }^{267}$ aus dem Signal $\varepsilon_{\text {Signal }}$ entfernt, sodass sich die kompensierte Verzerrung $\varepsilon_{\text {komp }}$ ergibt (Gleichung 57 ).

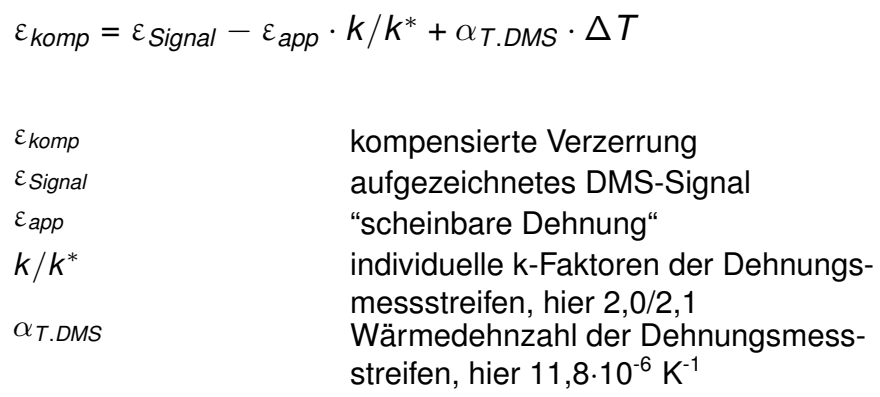

Diese kompensierte Gesamtverzerrung beinhaltet Anteile aus der thermischen Verzerrung des Glases und der mechanischen Verzerrung der Glasunterkante in Feldmitte aus Vorspannung und Biegebelastung (Gleichung 58).

Die mechanische Verzerrung aus der Biegebelastung ist temperaturunabhängig. Die Dehnung aus der Vorspannung kann mit dem Messwert der Seilkraft errechnet werden. ${ }^{268}$ Damit wird der mechanische Anteil aus dem kompensier-

\footnotetext{
266 Das Verfahren ist ausführlich in [Engelmann et al. 2016] beschrieben und wird hier zusammenfassend dargestellt.

267 Zum Einen ist der spezifische Widerstand des DMS-Gittermatierials temperaturabhängig, zum Anderen unterscheiden sich die Wärmedehnzahlen des (Glas-) Bauteils und des DMS-Gitters. Der daraus resultierende Effekt ist ausschließlich durch eine Temperaturänderung induziert und wird als "scheinbare Dehnung" oder "thermisches Ausgangssignal“ bezeichnet. Eine prägnante zusammenfassung findet sich in [MM TN-504-1 2010]. Der Hersteller der Dehnungsmessstreifen stellt die dafür nötigen Parameter zur Verfügung. Die „scheinbare Dehnung“ der verwendeten Geräte liegt im Einsatztemperaturbereich der Versuchsreihe von $20^{\circ} \mathrm{C}$ bis $60^{\circ} \mathrm{C}$ zwischen 8,0 und $-24,0 \mu \mathrm{m} / \mathrm{m}$.

268 Damit ist die statisch unbestimmte Wirkung inbegriffen.
} 
ten Dehnungssignal entfernt ${ }^{269}$, sodass die reine thermische Verzerrung verbleibt. Mit diesem Wert wird eine Wärmedehnzahl der Konstruktion nach Gleichung 59 berechnet.

$$
\begin{aligned}
& \alpha_{T . \text { Träger }}=\frac{\varepsilon_{\text {therm }}}{\Delta T}=\frac{\varepsilon_{\text {komp }}-\varepsilon_{P}-\varepsilon_{F}}{\Delta T} \\
& \alpha_{T . \text { Träger }} \quad \text { Wärmedehnzahl der Konstruktion }
\end{aligned}
$$

Der Vergleich der Wärmedehnzahlen erlaubt eine Aussage zum thermischen Verzerrungsverhalten innerhalb der Trägerkonstruktion. Die auftretenden Effekte werden nach der zweidimensionalen Übersicht in Bild 79 schrittweise bestimmt:

1. Einfluss der Konstruktion durch den Vergleich des Referenzglaskörpers mit dem unbewehrten und unbelasteten Glasträger (PX F0).

2. Einfluss der Bewehrung durch den Vergleich des unbewehrten mit dem schlaff bewehrten Träger (PO F0).

3. Einfluss der Vorspannung durch den Vergleich der schlaff bewehrten (P0 F0) mit den vorgespannt bewehrten Trägern (P3|P6|P9 F0).

4. Einfluss der Auflast durch den gruppenweisen Vergleich der unbelasteten (F0) mit den belasteten Trägern (F5|F10).

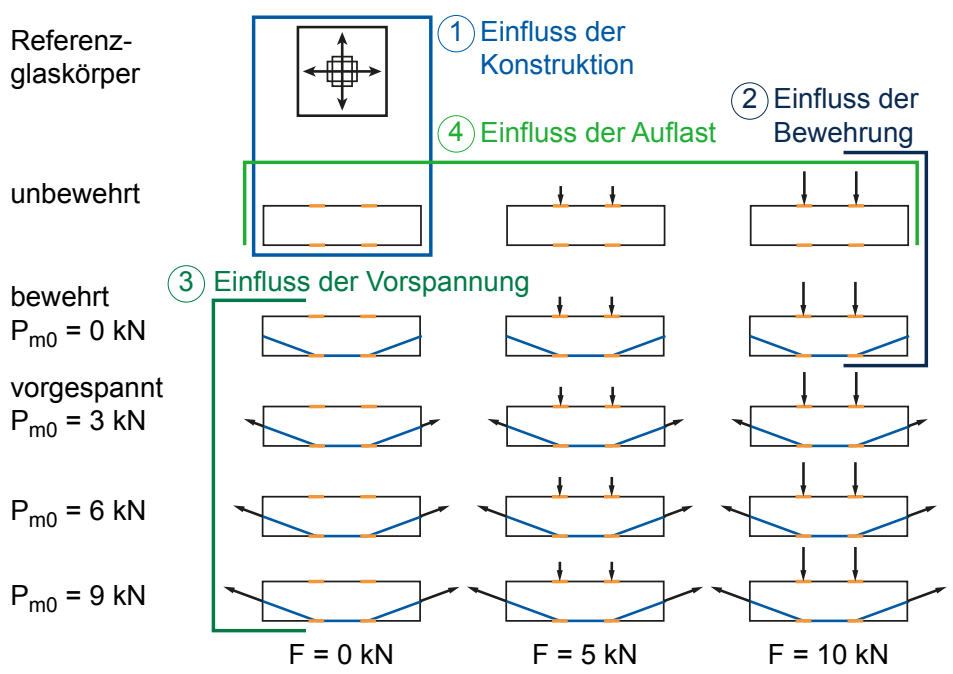

269 Dies ist mittels einer Berechnung nach linearer Balkentheorie auf Basis der Ergebnisse aus Kapitel 3.3 möglich.
Gleichung 59

Bild 79

Vorgehen bei der Auswertung der Biegeversuche unter Temperaturlast. 


\subsubsection{Ergebnisse und Ergebnisdiskussion}

Die Einzelergebnisse der 30 zerstörungsfreien Temperaturlastversuche sind in Anlage E zusammengestellt. Ausgehend von einer Raumtemperatur von $25,7 \pm 2,4^{\circ} \mathrm{C}$ wurden der Prüfraum auf $63,1 \pm 2,4^{\circ} \mathrm{C}$ aufgeheizt. Die Prüfkörper erfuhren weder einen Glasbruch, noch eine andere Art der Beschädigung während der Versuche.

\section{Seilkraft und Verformung}

Zuerst werden die Versuche anhand der repräsentativen Einzelergebnisse des Durchlaufes mit dem Prüfkörper 1 bei einer Biegelast von $F=5 \mathrm{kN}$ und einer Vorspannung $P_{\mathrm{m} 0}=6 \mathrm{kN}$ nachempfunden (TV01-II P6F5, Bild 80).

Zunächst zeigt Bild 80 (links) den Verlauf der Lufttemperatur in der Prüfkammer. Ausgehend von $22,5^{\circ} \mathrm{C}$ steigt sie innerhalb von 100 Minuten auf die Zieltemperatur von $60^{\circ} \mathrm{C}$ (Heizzeit). Zwischen 120 und 240 Minuten ist eine periodische Schwankung als Regelzyklus der Klimakammer zu erkennen (Haltezeit). In diesem Zeitraum wird die mittlere Temperatur ${ }^{270}$ von $66,0 \pm 2,5^{\circ} \mathrm{C}$ bestimmt, sodass eine relevante Temperaturänderung von $43,5 \mathrm{~K}$ resultiert. Im Prüfzeitraum ändert sich ebenso die Seilkraft. Sie beginnt infolge der Vorspannung und der Seilkraftzunahme aus der Biegelast bei $6,54 \mathrm{kN}^{271}$ und fällt bis zum Erreichen der Zieltemperatur kontinuierlich ab. Im anschließenden Haltezeitraum wird der wellige Temperaturverlauf durch einen Mittelwert der Seilkraft von 6,04 kN (Gleichung 56) abgebildet. Damit ergibt sich eine Seilkraftreduzierung von $0,5 \mathrm{kN}$.

Im rechten Bild 80 ist die Verformung des Prüfkörpers unter Temperaturlast dokumentiert. Die in grün markierte Durchbiegung in horizontaler und vertikaler Richtung ändert sich nicht maßgebend. Des Weiteren dehnt sich der Referenzglaskörper auf einen Mittelwert von 423,8 $\mu \mathrm{m} / \mathrm{m}$. Daraus re-

$270 \varnothing T$ analog Gleichung 56.

271 Im Vergleich dazu resultiert das analytische Ergebnis nach Gleichung 102 mit der Vorspannung nach Tafel 36 zu 6,87 kN. Die Abweichung von $5 \%$ wird als zulässig bewertet. 


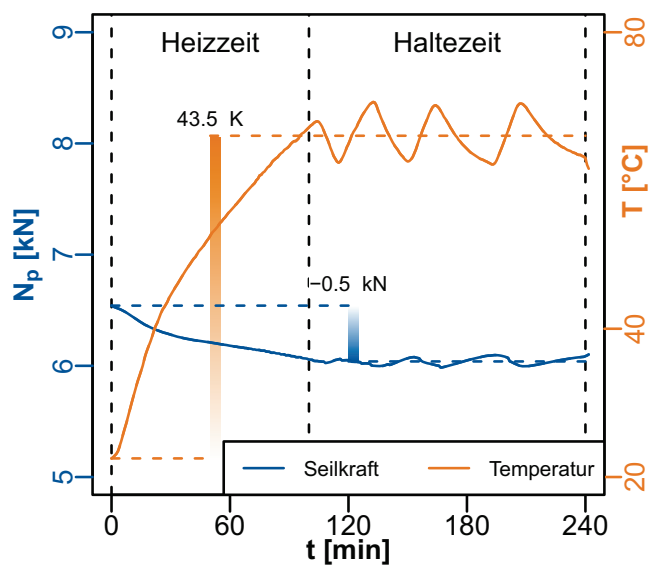

sultiert eine Wärmedehnzahl unter der gemessenen Temperaturänderung von $9,74 \cdot 10^{-6} \mathrm{~K}^{-1}$. Dem gegenüber beginnt der Verlauf der Verzerrungsänderung des Trägers bei $164,4 \mu \mathrm{m} / \mathrm{m}$ infolge der Glaskantenbeanspruchung aus der Vorspannung und der Biegelast, gefolgt von einer Änderung um 430,8 $\mu \mathrm{m} / \mathrm{m}$. Aus diesem Ergebnis folgt ein Wärmedehnzahl des Spannglasträgers nach Gleichung 59 von 9,90 . $10^{-6} \mathrm{~K}^{-1}$.

Erwartungsgemäß wurde eine Seilkraftreduzierung und eine Verzerrungsänderung gemessen. Dem gegenüber verblieb die Durchbiegung infolge der Temperaturlast im nicht messbaren Bereich. Die entspricht den Ergebnissen im analytischen Berechnungsmodell.

Für alle gemessenen Ergebnisse gilt, dass während der Haltezeit ein horizontaler Verlauf zu erkennen ist. Daher kann davon ausgegangen werden, dass der Prüfkörper ausreichend und konstant erwärmt wurde und somit das Belastungsverfahren anwendbar ist.

\section{Freie Glasverzerrung unter Temperaturlast}

In Tafel 22 werden die Wärmedehnzahlen des Referenzglaskörpers aus jedem Versuch nach Gruppen zugehöriger Glasträgerversuche tabelliert. Darin zeigt sich für alle Versuche
Bild 80

Versuchsergebnisse unter Temperaturlast zum Prüfkörper 1 unter $5 \mathrm{kN}$ Biegelast und $6 \mathrm{kN}$ Vorspannkraft. 
Tafel 22

Wärmedehnzahl des Referenzglaskörpers.

Gruppenweise Zusammenfassung der Einzelergebnisse aus Anlage $\mathrm{E}$ als Mittelwerte \pm Standardabweichung. ein rechnerisches Ergebnis von 9,46 $\pm 0,16 \cdot 10^{-6} \mathrm{~K}^{-1}$, welcher $5 \%( \pm 1,8 \%)$ oberhalb der Angabe aus [DIN EN 572-1]

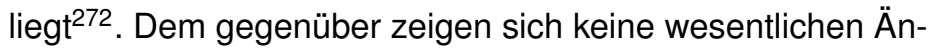
derungen nach Versuchsgruppen, sodass geschlussfolgert wird, dass alle Versuche unabhängig voneinander und unter vergleichbaren Bedingungen stattfanden. Damit ist ein Vergleich der Ergebnisse auf einheitlicher Basis möglich, obwohl alle Einzelversuche nacheinander und mit variierenden Temperaturprofilen stattfanden. Die Temperaturlastaufbringung ist damit wiederhol- und reproduzierbar.

\begin{tabular}{llll}
\hline Gruppen & & $\alpha_{\text {T.Ref }}\left[\mathbf{1 0}^{-6} \mathbf{K}^{-1}\right]$ & Anzahl \\
\hline Prüfkörper & TV01 & $9,51 \pm 0,14$ & 15 \\
& TV02 & $9,42 \pm 0,16$ & 15 \\
\hline Bewehrung & unbewehrt & $9,43 \pm 0,12$ & 3 \\
& $P_{m 0}=0$ & $9,45 \pm 0,10$ & 3 \\
& $P_{m 0}=3 \mathrm{kN}$ & $9,51 \pm 0,16$ & 3 \\
& $P_{m 0}=6 \mathrm{kN}$ & $9,49 \pm 0,23$ & 3 \\
& $P_{m 0}=9 \mathrm{kN}$ & $9,42 \pm 0,17$ & 3 \\
\hline Biegelast & $\mathrm{F}=0 \mathrm{kN}$ & $9,46 \pm 0,17$ & 5 \\
& $\mathrm{~F}=5 \mathrm{kN}$ & $9,51 \pm 0,18$ & 5 \\
& $\mathrm{~F}=10 \mathrm{kN}$ & $9,42 \pm 0,11$ & 5 \\
\hline alle & & $9,46 \pm 0,16$ & 30 \\
\hline
\end{tabular}

\section{Einfluss der Konstruktion - unbewehrte Träger unter Temperaturlast}

Im Vergleich zum Referenzglaskörper zeigte die unbelastete und unbewehrte Trägerkonstruktion (PX F0) mit 8,72 . $10^{-6} \mathrm{~K}^{-1}$ und $8,17 \cdot 10^{-6} \mathrm{~K}^{-1}$ deutlich geringere Wärmedehnzahlen, welche auf die Zusammenstellung der Glaspakete zu einem Spannglasträger einschließlich seiner geklebten Umlenkpunkte zurückzuführen ist. Daher muss zwischen der freien Temperaturverzerrung und der Verzerrung der Trägerkonstruktion unterschieden werden. In dieser Konstellation liegt neben der Temperaturlast eine Biegung unter Eigenlast vor. Diese Lastkombination resultiert jedoch in keiner merklichen vertikalen Durchbiegung $(<1 / 100 \mathrm{~mm} / \mathrm{K})$ und verdeutlicht damit den geringen Beitrag der Temperaturänderung auf die Durchbiegung im Vergleich zu den Anteilen aus der

272 Vergleiche auch Tafel 4 (Seite 48). 
Vorspannung und Biegebelastung nach Gleichung 107 (Seite 309 in Anlage A).

Zusätzlich wurde der Effekt der Temperaturlast anhand des unbewehrten Referenzträgers unter variierter Auflast bestimmt. Hier ergaben sich schrittweise höhere Wärmedehnzahlen der Trägerkonstruktion von 9,28 $10^{-6} \mathrm{~K}^{-1}$ und 9,20 . $10^{-6} \mathrm{~K}^{-1}$ unter $5 \mathrm{kN}$ Auflast (PX F5) sowie $9,43 \cdot 10^{-6} \mathrm{~K}^{-1}$ und $9,62 \cdot 10^{-6} \mathrm{~K}^{-1}$ unter $10 \mathrm{kN}$ Auflast (PX F10). Demnach kann die Biegelast in dieser Untersuchung als bestimmender Faktor auf die Ergebnisse gewertet werden.

\section{Einfluss der Bewehrung - schlaff bewehrte Träger unter Temperaturlast}

Im nächsten Schritt wird der Einfluss der Bewehrung auf das Tragsystem unter Temperaturlast im Vergleich zum unbewehrten Träger untersucht. Dabei wurden mit 8,77 $\cdot 10^{-6} \mathrm{~K}^{-1}$ und $8,35 \cdot 10^{-6} \mathrm{~K}^{-1}$ vergleichbar große Wärmedehnzahlen ermittelt (P0 F0). Unter steigender Temperatur ist demnach nicht von einer Zwängung infolge der hinzugefügten Bewehrung auszugehen. Mit Blick auf die größeren Wärmedehnzahlen der Bewehrung ${ }^{273}$ gegenüber dem Glas war dies zu erwarten und ist nunmehr experimentell bestätigt.

In allen Versuchen mit schlaff bewehrten Trägern wird eine Verringerung der anfangs eingestellten Seilkraft mit Werten zwischen 1,09 N/K und 3,29 N/K festgestellt. Dieses Ergebnis wird jedoch nur qualitativ bewertet, da die gemessenen Seilkräfte mit Auslastungen von maximal einem Prozent ${ }^{274}$ weit unterhalb der Auslastungsgrenzen des Bewehrungsmaterials liegen.

Unter steigender Auflast (P0 F5 und P0 F10) werden ebenso steigende Wärmedehnzahlen gemessen. Diese Zunahme ist im Vergleich zum Ergebnis der unbewehrten Träger unter jeweils gleicher Biegelast in derselben Größenordnung dokumentiert.

273 Vergleiche Tafel 9, Seite 106.

${ }^{274}$ Hier wird die Seilkraft infolge der Auflast mit dem charakteristischen Wert nach Tafel 9 (Seite 106) verglichen. 


\section{Einfluss der Vorspannung - vorgespannte Träger unter Temperaturlast}

Im letzten Schritt wird die Frage geklärt, inwiefern das Vorspannen der Bewehrung im Vergleich zum schlaff bewehrten Tragsystem einen Einfluss auf das Tragverhalten zeigt. Dabei resultiert eine Wärmedehnzahl der Konstruktion von $8,89 \pm 0,21 \cdot 10^{-6} \mathrm{~K}^{-1}$ ohne bedeutsame Unterschiede nach der Größe der Vorspannkraft (P3 F0; P6 F0; P9 F0). Die Einzelwerte liegen geringfügig oberhalb der vorab bestimmten Ergebnisse für unbewehrte und schlaff bewehrte Prüfkörper.

Neben der Vorspannkraft wurde zudem die Auflast variiert, sodass die Prüfkörper der Reihen P3 bis P9 sowie F0 bis F10 durch insgesamt drei unterschiedlichen, jedoch voneinander abhängigen Belastungen ausgesetzt waren. Hier zeigten sich ebenso steigende Wärmedehnungen mit größer werdender Biegelast. Eine zusätzliche Abhängigkeit von der gewählten initialen Vorspannkraft ist aufgrund der geringen Versuchsanzahl nur scheinbar vorhanden (Bild 81).

Bild 81

Nach mechanischer Belastung gruppierte Wärmedehnzahlen der Glasträger gegenüber der unbehinderten Wärmedehnung des Referenzglaskörpers.

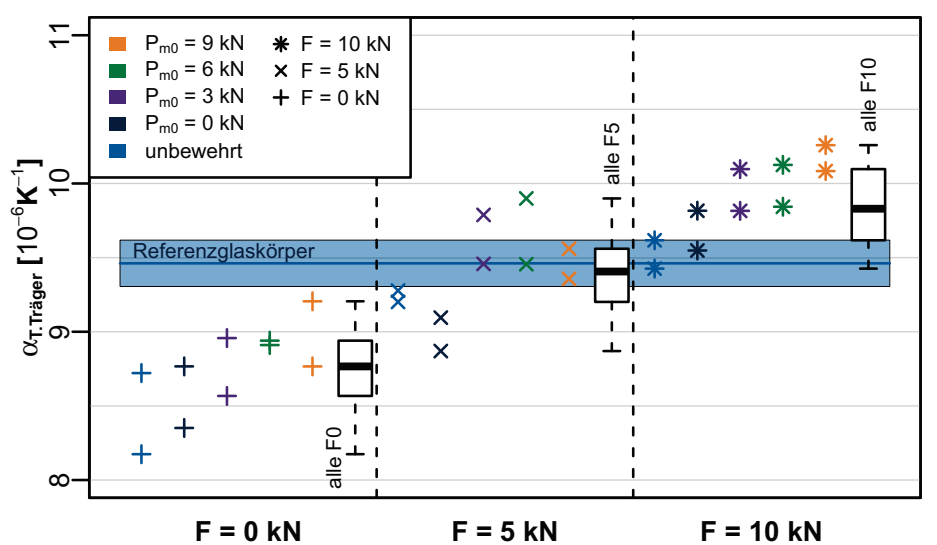

Zunächst wurde festgestellt, dass die Glasverzerrung der Träger unter Biege- und Vorspannkraft sehr gut mit dem analytischen Modell nach Kapitel 2.7 berechenbar ist. Ebenso konnte die Wärmedehnzahl des unbelasteten Referenzglaskörpers mit ausreichend großer Nähe zum Erwartungswert bestimmt werden. Jedoch scheint die thermische Verzerrung der Träger von der Biegebelastung abzuhängen, was gegen 
das analytische Berechnungsmodell spricht. ${ }^{275}$ Dies kann durch den Einfluss der Belastungseinrichtung bedingt sein. Aufgrund der Ergebnisse der Vorversuche ${ }^{265}$ wird dieses Risiko als gering bewertet.

Darüber hinaus wird die seitliche Durchbiegung, welche zu einer zusätzlichen mechanischen Verzerrung führt, in der analytischen Berechnung vernachlässigt und kann als Grund für den beobachteten Effekt vermutet werden. Bild 82 zeigt daher die ermittelten Wärmedehnzahlen gegenüber den auf die Temperaturänderung normierten horizontalen Durchbiegungen. Darin ist zu erkennen, dass vor allem solche Versuche mit großer Biegebelastung $(F=10 \mathrm{kN})$ zu den größten seitlichen Durchbiegungen führen. Damit ist mit einem größeren, vernachlässigten Verzerrungsanteil aus seitlicher Durchbiegung mit steigender Biegelast $F$ zu rechnen. Dies erzeugt nach Gleichung 58 scheinbar größere Wärmedehnzahlen. Es wird geschlussfolgert, dass die seitliche Durchbiegung auch unter Temperaturlasten für die Verzerrungsberechnung nicht zu vernachlässigen ist. Darüber hinaus ist aufgrund dieses nicht erfassten Effektes eine Beurteilung eines konstruktiven Temperaturzwanges nicht möglich.

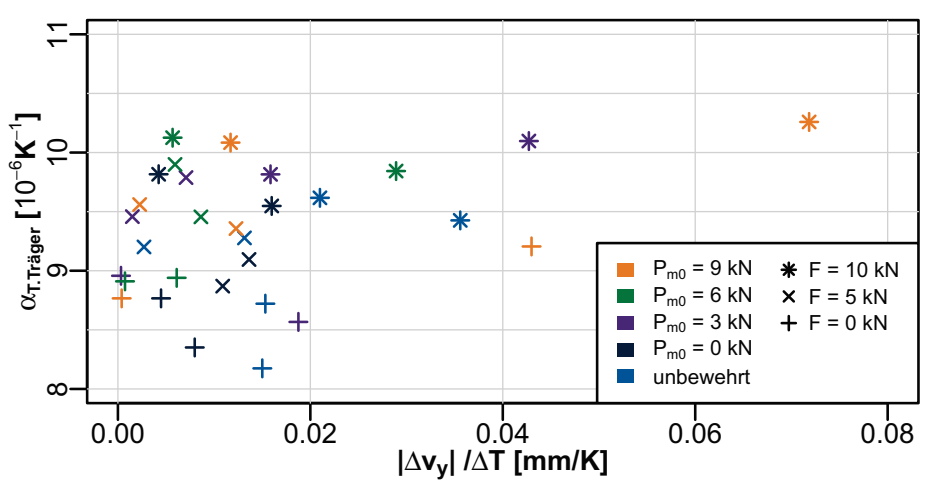

Letztlich sind in Bild 83 die Folgen einer Temperaturänderung auf die Seilkraft dargestellt. Die Linien zeigen dabei die rechnerische Seilkraft nach Gleichung 102 (Seite 307 in Anlage A). Die gewählten Farben spiegeln die eingangs eingestellte Vorspannkraft $P_{m 0}$ wieder. Dem gegenüber sind die

275 Zur Berechnung der Verzerrungsänderung unter einer Temperaturlast unter Zuhilfenahme der Gleichung 105 (Seite 308 in Anlage A) entfällt der Summand, welche die Biegelast enthält. Demnach verbleibt der Verzerrungsanteil aus der Biegebelastung unverändert.
Bild 82

Wärmedehnzahlen der Glasträger gegenüber der normierten seitlichen Durchbiegung. 
zugehörigen experimentellen Ergebnisse als Kreuze eingetragen.

Bild 83

Analytisch berechnte (Gleichung 102, Seite 307) und experimentell ermittelte Seilkräfte unter Temperaturlast.

Das rechnerische Ergebnis (Linien) beschränkt sich auf $0 \leq \Delta \mathrm{T} \leq 45 \mathrm{~K}$

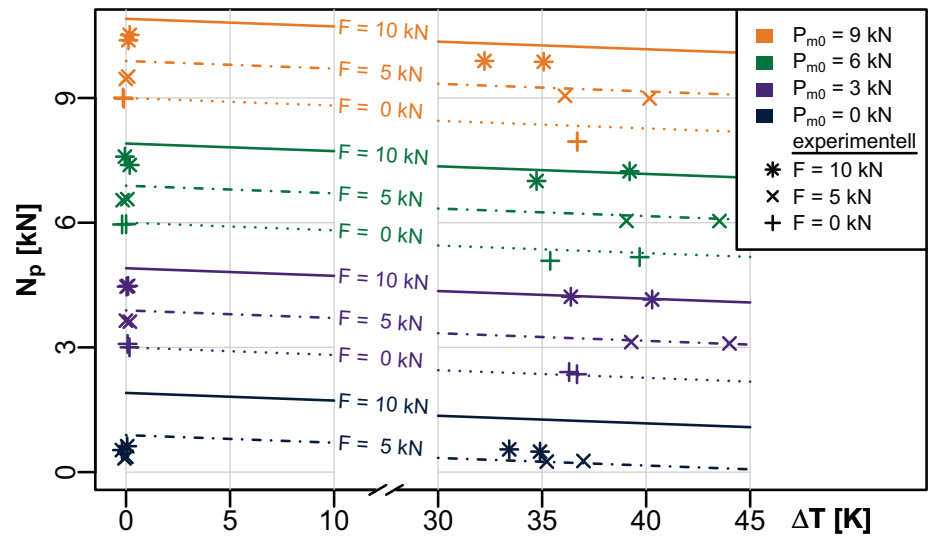

Das experimentelle Ergebnis der schlaff bewehrten Spannglasträger zeigt sowohl zu Versuchsbeginn, als auch unter erhöhter Temperatur deutliche Abweichungen vom analytischen Ergebnis (28\% bis $126 \%$ ). Aufgrund des geringen Lastniveaus wird dies jedoch als unproblematisch erachtet.

Für die vorgespannten Träger wird ein Verhältnis zwischen rechnerischem und experimentellem Ergebnis von $91 \%$ bis $103 \%$ zu Versuchsbeginn (bei $\Delta T=0$ ) und $95 \%$ bis $103 \%$ bei erhöhten Temperaturen während der Haltezeit festgestellt. Die Ergebnisse werden gruppenübergreifend ermittelt, da keine Abhängigkeit zwischen der Abweichung von analytischem Ergebnis und experimentellem Messwert der Seilkraft nach der Größe der Auflast oder Vorspannkraft festgestellt wurde. Daraus wird geschlussfolgert, dass Gleichung 102 (Seite 307 in Anlage A) zur Berechnung der Seilkräfte unter Temperaturlasten verwendbar ist.

\subsubsection{Folgerungen und Zusammenfassung}

Das Ziel der Ergebnisdiskussion bestand darin, den Anwendungsbereich der analytischen Abschätzung nach Kapitel 2 einzugrenzen. ${ }^{276}$ In diesem rechnerischen Modell werden

276 Dabei wird auf Ergebnisse aus [Engelmann et al. 2016] aufgebaut. 
folgende Eigenschaften der Konstruktion vernachlässigt, obwohl ein temperaturabhängiger Einfluss auf das Berechnungsergebnis nicht auszuschließen ist:

- Temperaturabhängigkeit der Zwischenschicht- und der Klotzungseigenschaften ${ }^{277}$

- Lasteinleitung in eine Kante des Verbundglases aufgrund des Kantenversatzes und der damit einher gehenden Exzentrizität im Sinne zusätzlicher Biegung um die schwache Trägerachse sowie die damit einher gehende zusätzliche Schubbelastung der Zwischenschicht ${ }^{278}$

- konstruktiver Temperaturzwang im Bereich der Klebverbindung zwischen zwei Glaspaketen im Querschnitt im Fall einer ungleichmäßigen Temperaturänderung ${ }^{279}$

- horizontale Durchbiegung aufgrund von Imperfektionen und die damit einhergehende Schubbelastung der Zwischenschicht $^{280}$

In diesem Abschnitt wurde das Tragverhalten der Spannglasträger unter einer Temperaturlast sowie einer vielfältigen Kombination von Biege- und Vorspannkraft untersucht. Dazu wurde ein Prüfverfahren entwickelt und erprobt. Im Ergebnis zeigt sich, dass die horizontale Durchbiegung der Träger zur Ermittlung der Glasverzerrung berücksichtigt werden muss. Darüber hinaus zeigte sich die erwartete Seilkraftabnahme unter steigender Temperatur. Diese kann mit dem linearen Modell aus Kapitel 2.7 realitätsnah berechnet werden, sodass die Wirkung einer thermischen Belastung auf die Vorspannung der Glasträger vorab abgeschätzt werden kann. Die erzielten Ergebnisse beziehen sich jedoch zunächst ausschließlich auf den vorgestellten Parameterbereich. Darüber hinaus lässt die geringe Anzahl der Versuche keine allgemeingültige Aussage zu. Jedoch kann auf dieser Basis mit weiterführenden Untersuchungen eine Bemessung von Spannglasträgern anvisiert werden. Grundsätzlich wird es mit den Ergebnissen möglich einen vollständigen Seilkraftverlust aus einer Temperaturlast zu vermeiden, wodurch das Konzept „Spannglasträger“ aufrechterhalten werden kann.

\footnotetext{
277 Vergleiche Kapitel 2.5, ab Seite 47.

278 Vergleiche Kapitel 2.5.3, ab Seite 51 und Bild 29, Seite 53.

279 Vergleiche Kapitel 2.5.6, ab Seite 61.

280 Vergleiche Kapitel 2.7, ab Seite 64.
} 


\subsection{Zusammenfassung}

In diesem Kapitel wurde die praktische Umsetzbarkeit des Konzeptes "Spannglasträger" gezeigt und die getroffenen Annahmen aus der Analogiebetrachtung mit vier Versuchsserien überprüft.

Das Tragverhalten beim Aufbringen der Vorspannkraft auf den Glasträger zeigte sich entsprechend dem analytischen Modell. Insbesondere die ermittelten Umlenkkraftverluste waren auf vernachlässigbar kleinem Niveau. Darüber hinaus wurde eine Biegebelastung anteilig durch das Glas und die Bewehrung aufgenommen. Mit steigender Vorspannung wurde tendenziell eine Steigerung der Erstrisslast beobachtet und eine Mehrzahl verschiedener Versagensformen als Datengrundlage für weiterführende Detailuntersuchungen charakterisiert. Das Querschnittskonzept mit geklebten Umlenkpunkten zeigte sich dabei als unkritisch. Nach dem Bruch aller Scheiben kam es in der Regel zu einem seitlichen Versagen und in dessen Folge zu einem Verlust der Vorspannkraft. Dennoch wurde der gebrochene Träger durch das Seil an Ort und Stelle gehalten und damit die Resttragsicherheit gewährleistet.

Unter einer Dauerlast über $1000 \mathrm{~h}$ wurden maximale Vorspannkraftverluste zwischen $19 \%$ und $27 \%$ ermittelt, welche konstruktionsabhängig analytisch beschrieben wurden. Damit ist eine Datengrundlage verfügbar, um das analytische Modell aus der Analogiebetrachtung zum Spannbetonbau anzuwenden und eine Vorhersage zur verbleibenden Vorspannkraft am Ende der Lebenszeit des Bauteils zu treffen.

Mit einem teilzerstörten Glasquerschnitt ergaben sich sowohl günstige als auch ungünstige Trageigenschaften. Dabei zeigte sich insbesondere die seitliche Durchbiegung als bestimmender Faktor. Tendenziell resultierte die Vorspannung der Glasträger in einer vergrößerten Reststandzeit, was den günstigen Einfluss der Konstruktionsweise auf die Sicherheit der Bauteile unterstreicht.

Auch unter einer Temperatursteigerung wurde eine Verringerung der Seilkraft entsprechend des analytischen Berech- 
nungsmodells ermittelt. Damit wird eine Abschätzung der zu erwartenden Veränderungen möglich.

Eine Reproduktion der theoretisch anvisierten Eigenschaften wurde auch praktisch unter Laborbedingungen erreicht. Dazu wurden individuelle Prüfmethoden neu entwickelt, erprobt und anhand von 98 Versuchsdurchläufen mit 46 Prüfkörpern angewendet. Damit ergab sich erstmals eine breite Datengrundlage zur Beschreibung der Trag- und Versagenseigenschaften von Spannglasträgern auf deren Basis angesprochene, umfangreichere Untersuchungen zielgerichtet eingeschränkt werden können. Die beobachteten, vielfältigen Effekte wurden charakterisiert und kategorisiert, um als Ausgangspunkt der vertieften Detailentwicklung und -untersuchung zu dienen.

Damit sind Spannglasträger mit den angegebenen Grenzen beschreibbar. Das vorgestellte Konzept ist praktisch umsetzbar.

Im Rahmen der experimentellen Versuche dieses Kapitels wurden mit unter Vermutungen über die Begründung der Ergebnisse geäußert, deren Nachweis außerhalb der Grenzen der Studie liegen. Zur Ergänzung werden daher numerische Simulationen durchgeführt und im folgenden Kapitel 4 zusammengefasst. 


\section{$4 \quad$ Numerische Untersuchungen}

\subsection{Zielsetzung}

Ein numerisches Modell verbindet die analytische Berechnung aus Kapitel 2 mit den in Kapitel 3 experimentell ermittelten Trageigenschaften und soll dazu beitragen, bisher offen gebliebene Fragestellungen zu klären.

Eingangs getroffene analytische Idealisierungen werden dazu schrittweise abgebaut, um das experimentell beobachtete, reale Tragverhalten mit minimaler Abweichung numerisch zu ermitteln. Dies betrifft insbesondere:

- die Wirkung der aufgeklebten Umlenkpunkte und der Materialeigenschaften der Zwischenschicht auf das Gesamttragverhalten,

- die räumliche Verformung des Tragwerks in Abhängigkeit von der tatsächlich vorliegenden Imperfektion sowie

- das Zusammenspiel der Einzeleffekte bei der numerischen Berechnung der Spannkraftverluste.

Dazu wird das numerische Modell entsprechend der Annahmen für die analytische Rechnung (lineare Balkentheorie I. Ordnung, ohne Imperfektionen) aufgestellt und ein Ergebnisvergleich der resultierenden Seilkraft und der vertikalen Durchbiegung durchgeführt. Für dieses verifizierte System wird eine Eigenwertanalyse als Parameterstudie mit variierten Eigenschaften - der Schubsteifigkeit der Zwischenschicht und der Drehsteifigkeit der aufgeklebten Umlenkpunkte - durchgeführt, um den möglichen Wertebereich dieser Parameter zu reduzieren. Damit wird im nächsten Schritt ein Imperfektionswert ausgewählt, welcher das reale Tragverhalten unter Vorspannbelastung (Spannweg) und Biegebelastung (vertikale und horizontale Durchbiegung sowie Seilkraftänderung) realitätsnah abbildet.

Mit dem beschriebenen Vorgehen wird das numerische Modell validiert und beweist damit seine Tauglichkeit zur Klärung offener Fragestellungen, die sonst nur mit erhöhtem ex- 
perimentellen Aufwand ${ }^{281}$ zu beantworten sind. Dies betrifft insbesondere:

- die Abweichung zwischen dem experimentellen Ergebnis und der analytischen Berechnung des Spannweges, ${ }^{282}$

- die Beurteilung des Einflusses der Steifigkeit der Zwischenschicht und der geklebten Umlenkpunkte sowie der Imperfektion im Biegeversuch ${ }^{283}$ sowie im Dauerlast-Versuch ${ }^{284}$ und

- die Beurteilung des Einflusses der räumlichen Durchbiegung auf den Seilkraftverlust im Vergleich zur analytischen Abschätzung. ${ }^{285}$

Als zweites Ziel soll der Einfluss einzelner Idealisierungsschritte gewichtet werden. Er mündet in Empfehlungen für die Modellierung von Spannglasträgern zur Abschätzung der Trageigenschaften einer konkreten Konstruktion sowie der Beurteilung der Ergebnisstreuung im Experiment.

\subsection{Modellbeschreibung}

\subsubsection{Systembeschreibung}

Der Spannglasträger wird als räumliches Stabwerk modelliert (Bild 84). Dabei ist jedes VSG-Paket (A) des Doppelquerschnitts als Biegeträger im Abstand ihrer Schwerachsen zwischen Gabellagern abgebildet.

Die aufgeklebten Seilumlenkungen der realen Konstruktion definieren den Abstand beider Glaspakete und leiten die auftretenden Seilkräfte um. Sie werden daher als unverformbare Stäbe idealisiert und mittels Drehfedern der Steifigkeit $c_{m y}$ und $\mathrm{C}_{\mathrm{mz}}$ mit den Hauptträgern verbunden (B). Sie bestimmen damit die Querschnittssteifigkeit in ihrem Wirkungsbereich.

\footnotetext{
281 Hieraus leiten sich Aufgaben für die weiterführende Forschung ab. 282 Vergleiche Bild 46, Seite 114.

283 Vergleiche Kapitel 3.3.5, ab Seite 119.

284 Vergleiche Kapitel 3.4.5, ab Seite 137.

285 Vergleiche Kapitel 3.4.5, ab Seite 137.
} 


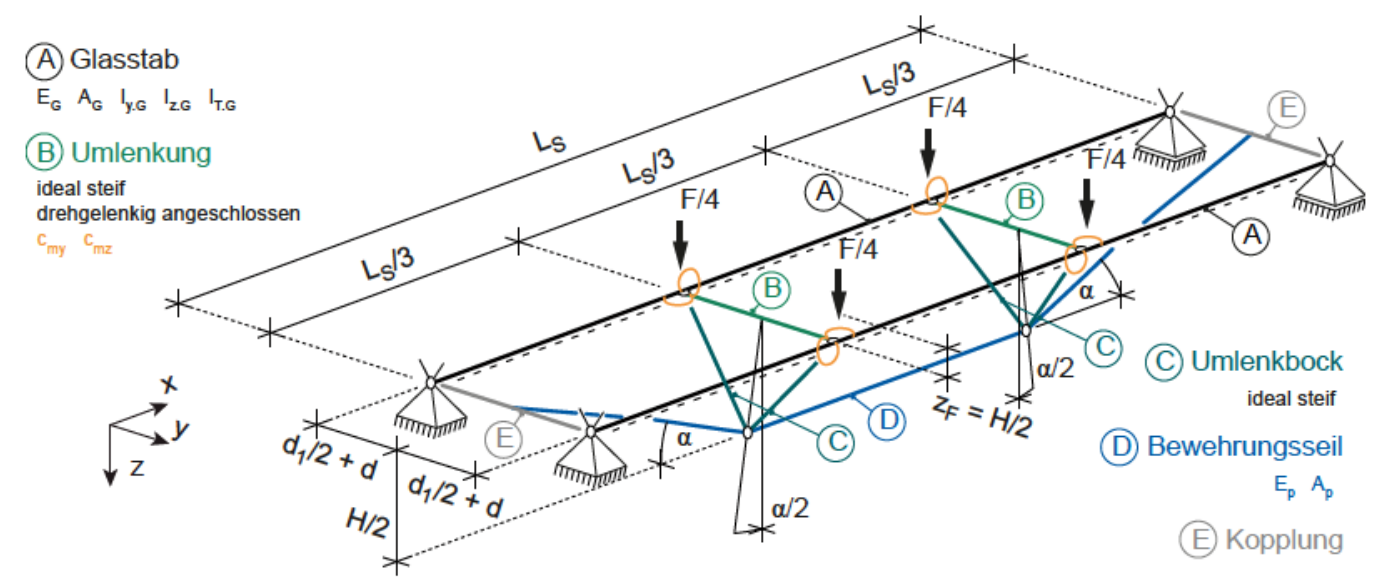

\section{Bild 84}

Räumliches Stabwerkmodell zur numerischen Abbildung des Tragverhaltens von Spannglasträgern mit „Doppelquerschnitt".

Ls...Spannweite

F ....Biegelast

d ....Glasschichtdicke

$\mathrm{d}_{1}$....Abstand Glaspakete

H ... Querschnittshöhe

$\alpha$.... Seilneigung

$\mathrm{z}_{\mathrm{F}}$... Exzentrizität Biegelast

Unter Annahme einer ideal drehsteifen Verbindung (bei maximaler Federsteifigkeit $\mathrm{c}_{\mathrm{m}}$ ) verbinden die Umlenkungen die Glaspakete lokal zu einem Hohlkasten, während bei rechnerisch fehlender Drehsteifigkeit ${ }^{286}$ ausschließlich der Glaspaketabstand konstant bleibt (Bild 85).

Unter der Umlenkung wird je ein ideal steifer Umlenkbock aus Pendelstäben (C) angeschlossen, um die Wirkung der Umlenkrolle abzubilden. Sie sind um den Winkel $\alpha / 2$ nach außen geneigt und stehen somit in der Wirkungslinie der Umlenkkraft. Damit ergibt sich in jedem Abschnitt die gleiche Seilkraft, wodurch man der realen Konstruktion gerecht wird, in der das Seil (D) frei über den Umlenkpunkt gleiten

\section{Bild 85}

Idealisierung des

realen „Doppelquer-

schnitts“ mit aufgeklebten

Umlenkpunkten.

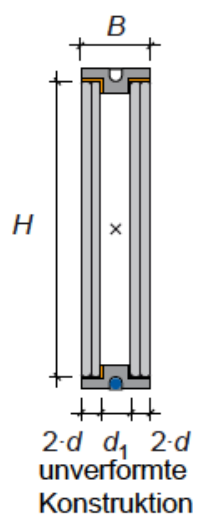

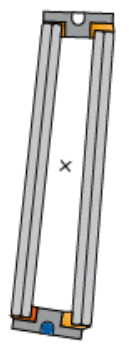

verformte Konstruktion (überhöht)

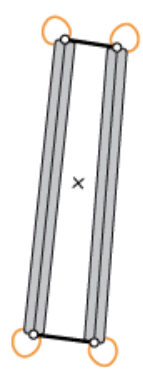

Idealisierung von Idealisierung der Klebung und VSG-Pakete zu Umlenkpunkt Biegestäben

${ }^{286}$ Hierfür werden die verwendeten Drehfedern durch ideale Gelenke ersetzt. 
kann. ${ }^{287}$ Die Seile sind zudem zwischen den Glasauflagern an den Trägerenden fixiert. Dabei erfahren die Glasauflagerknoten die gleiche Längsverschiebung in x-Richtung wie die Seilenden (E).

Die Querschnittswerte der Glasstäbe werden getrennt nach Freiheitsgraden angegeben. Bei einer Biegebelastung um die starke Achse wird immer der vollständige Glasquerschnitt belastet. Somit ergibt sich das zugehörige Flächenträgheitsmoment aus der Glashöhe H und der Summe der Dicken aller Einzelscheiben. Gleichung 60 gibt den Zusammenhang für zwei Glasscheiben im VSG-Paket an.

$$
I_{y \cdot G}=\frac{2 \cdot d \cdot H^{3}}{12}
$$

Gleichung 60

Demgegenüber wird die Zwischenschicht bei einer Biegung um die schwache Achse belastet. Entsprechend fällt das Flächenträgheitsmoment $I_{z . G} z$ wischen die Extremfälle „mit vollem Verbund“ und „ohne Verbund“ nach Gleichung 61 unter Annahme gleich dicker Einzelschichten des Glasaufbaus.

$$
\begin{aligned}
& I_{z . G . v o l l e r ~ V e r b u n d ~}=\frac{H \cdot(2 \cdot d)^{3}}{12}=\frac{2 \cdot H \cdot d^{3}}{3} \\
& I_{z . G . \text { ohne Verbund }}=\frac{H \cdot d_{\text {ohne Verbund }}^{3}}{12}=\frac{H \cdot d^{3}}{6} \\
& \text { mit } d_{\text {ohne Verbund }}=\sqrt[3]{d^{3}+d^{3}}=\sqrt[3]{2} \cdot d
\end{aligned}
$$

Zwischenwerte sind von der Schubsteifigkeit der Zwischenschicht abhängig. Dieser Teilverbund wird mit einer effektiven Dicke $d_{\text {eff }}$ modelliert. Ein solcher effektiver Querschnitt soll unter Vierpunkt-Biegung dieselbe Durchbiegung $\delta_{\text {eff }}$ aufweisen, wie der tatsächliche Laminatquerschnitt $\delta_{\text {Laminat }}$ (Bild 86 und Gleichung 62).

287 Alternativ bieten einige FEM-Programme eine Funktion an, die einem Seilstrang die gleiche Normalkraft aufzwingt. In diesem Fall ist der Umlenkbock jedoch steif an die Hauptträger anzuschließen. Diese alternative Modellierung kann in SOFiSTiK mit der slip Anweisung erstellt werden. Vergleiche [Sofistik AG 2014]. 
Bild 86

Die Durchbiegung des Laminates (links) entspricht der Durchbiegung eines monolithischen Trägers effektiver Dicke (rechts).

Gleichung 62

Gleichung 63

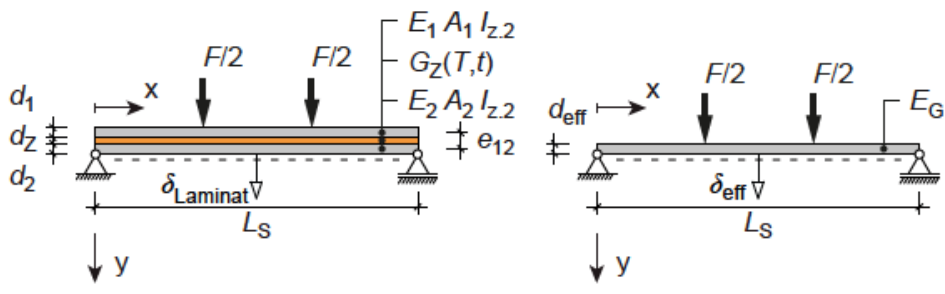

$$
\begin{array}{ll}
\delta_{\text {Laminat }} & =\delta_{\text {eff }}=\frac{23 \cdot F \cdot L_{S}^{3}}{1296 \cdot E_{G} \cdot I_{\text {z.eff.G }}} \\
\text { mit } I_{\text {Z.eff.G }}= & \frac{B \cdot d_{\text {eff }}^{3}}{12} \\
\delta_{\text {Laminat }} & \begin{array}{l}
\text { Durchbiegung eines Laminatquer- } \\
\text { schnitts } \\
\text { Durchbiegung des effektiven Quer- } \\
\text { schnitts }
\end{array}
\end{array}
$$

Durch das Umstellen nach der effektiven Glasdicke ergibt sich der gesuchte Zusammenhang (Gleichung 63) in Abhängigkeit der Durchbiegung des Laminatquerschnitts ${ }^{288}$, wodurch das zeit- und temperaturabhängige Tragverhalten über das verwendete Materialgesetz der Zwischenschicht ${ }^{289}$ eingebunden wird. Eine detaillierte Herleitung ist in Anlage $A$ ab Seite 314 zusammengestellt.

$$
\begin{gathered}
d_{\text {eff }}(T, t)=\sqrt[3]{\frac{23}{108 \cdot H} \cdot \frac{F \cdot L_{S}^{3}}{E_{G} \cdot \delta_{\text {Laminat }}\left(G_{Z}(T, t)\right)}} \\
\delta_{\text {Laminat }}\left(G_{Z}(T, t)\right) \\
\begin{array}{l}
\text { Durchbiegung eines Laminatquer- } \\
\text { schnitts nach Gleichung } 115 \text { in Anla- } \\
\text { ge A, Seite } 315
\end{array}
\end{gathered}
$$

Die Bewehrung wird als Seilelement mit einem Vollkreisquerschnitt modelliert. ${ }^{290}$ Die zeitabhängigen Trageigenschaften der Konstruktion werden über die Reduzierung des E-Moduls $\mathrm{E}_{\mathrm{p}}$ mittels des Faktors $\eta_{\mathrm{p}}$ berücksichtigt (Gleichung 64). Dieser beeinhaltet das Verhältnis der Seilkraft zum Betrachtungszeitpunkt zur initialen Seilkraft. Entspre-

288 Eine Herleitung für die Anwendung auf ein Verbundglas auf Basis von [Wölfel 1987] ist in [Schuler 2003] und [Ensslen 2005] zu finden.

289 Vergleiche Gleichung 5, Seite 53.

290 Der Flächeninhalt ergibt sich aus dem metallischen Querschnitt der Seile nach Tafel 9, Seite 106. 
chend der Verträglichkeitsbedingung verkürzt sich die Glaskonstruktion in gleicher Weise wie die Bewehrung. Entsprechend kann der Seilkraftverlust aus dem Kriechen der Klotzung und der Zwischenschicht ebenso über eine Reduzierung der Seilsteifigkeit berücksichtigt werden.

$$
\begin{aligned}
& E_{p . e f f}=\frac{P_{c}-\Delta P_{K, c}-\Delta P_{Z, c}-\Delta P_{p r}}{P_{c}} \cdot E_{p}=\eta_{p} \cdot E_{p} \\
& E_{p . e f f} \quad \text { effektive Seilsteifigkeit } \\
& \triangle P_{K, C} \quad \text { Seilkraftverlust aus dem Kriechen der } \\
& \text { Klotzung (Gleichung 24, Seite 84) } \\
& \Delta P_{Z, c} \quad \begin{array}{l}
\text { Seilkraftverlust aus dem Kriechen } \\
\text { der Zwischenschicht (Gleichung } 26
\end{array} \\
& \text { Seite 85) } \\
& \Delta P_{p r} \quad \text { Seilkraftverlust aus der Relaxation der } \\
& \begin{array}{ll}
\eta_{p} & \begin{array}{l}
\text { Bewehrung (Gleichung 27, Seite 85) } \\
\text { rechnerischer Verlustfaktor }
\end{array}
\end{array}
\end{aligned}
$$

Damit können die Einflüsse aller beteiligter Materialien in der Konstruktion rechnerisch getrennt betrachtet werden:

- $\eta_{p}(1)=1$ beschreibt die Situation nach dem Ende des Vorspannens $(t=0)$ ohne Verluste.

- $\eta_{p}(r)$ beschreibt ausschließlich den Seilkraftverlust infolge der Relaxation der Bewehrung. Diese ist im Dauerversuch nach $1000 \mathrm{~h}$ nahezu Null und wird daher rechnerisch hier nicht berücksichtigt.

- $\eta_{p}(K)$ beschreibt ausschließlich den Seilkraftverlust infolge des Kriechens der Klotzung.

- $\eta_{p}(Z)$ beschreibt ausschließlich den Seilkraftverlust infolge des Kriechens der Zwischenschicht.

- $\eta_{p}(K Z)$ beschreibt den Seilkraftverlust infolge des Kriechens der Klotzung und der Zwischenschicht.

Eine Werteübersicht ist in Tafel 40 in Anlage A auf Seite 317 zu finden.

Für alle beteiligten Materialien wird ein linear elastisches Verhalten ohne Festigkeits- oder Plastizierungsgrenze unterstellt, deren Einhaltung im Ergebnis überprüft wird. 


\subsubsection{Einwirkungen}

Die Berechnung erfolgt unter Berücksichtigung der gleichzeitigen Wirkung zweier Lastfälle. ${ }^{291}$ Zunächst wird die Vorspannung als Vordehnung der Seilelemente $\varepsilon_{0}$ einbezogen ${ }^{292}$ und schrittweise von $0 \%$ bis $150 \%$ der zugehörigen initialen Seilkraft $P_{m 0}$ erhöht. Daneben wird die Biegelast in globaler z-Richtung (richtungstreu) wirkend entsprechend eines Vierpunkt-Biegeversuchs in den Drittelspunkten am Obergurt $\left(\mathrm{Z}_{\mathrm{F}}=\mathrm{H} / 2\right)$ als Einzellasten auf jedes VSG-Paket, ausgehend vom unbelasteten Zustand $(F=0)$, schrittweise um je $5 \mathrm{kN}$ gesteigert.

\subsubsection{Berechnung}

Zur Berechnung wird die FEM-Software SoFiSTiK $2014^{293}$ einschließlich ihrer Terminologie ${ }^{294}$ verwendet.

Zunächst werden alle Einzellastfälle zum Vergleich mit der analytischen Berechnung (Anlage A) linear und ohne Imperfektion nach Theorie I. Ordnung gelöst. Im nächsten Schritt erfolgt die Ermittlung der ersten drei positiven Eigenwerte unter einer Biegelast von $\mathrm{F}=10 \mathrm{kN}$. Der Eigenwert ist als Lastfaktor zum Erreichen der Bruchlast interpretierbar. ${ }^{295}$ Die daraus resultierenden Eigenformen werden mit einem Imperfektionswert skaliert, um einen spannungsfreien Primärlastfall zu erstellen. Es wird zunächst der perfekte Träger $(\mathrm{L} / \infty)$ analysiert. Anschließend wird die Vorverformung schrittweise von L/2000, L/1000 (Mittelwert nach [Belis et al. 2004]), L/400 (5\%-Fraktilwert nach [Belis et al. 2004]) und

291 Die Eigenlast bleibt unberücksichtigt, da ihre Auswirkungen in der experimentellen Versuchsserie nicht gemessen wurden. Die Temperaturlast kann analog zu den Ergebnissen in Kapitel 3.6 ergänzt werden.

292 Vergleiche Gleichung 14, Seite 70.

293 Im Detail kommen die Module AQUA 16.53, Sofimsha 16.23, Sofiload 16.03 und ASE 14.04 zum Einsatz.

294 Siehe [Sofistik AG 2014].

295 Der Eigenwert markiert das Versagen im „perfekten“ System (Verzweigungsproblem), während das Bruchlastergebnis einem Versagen des „imperfekten“ Systems (Spannungsproblem) entspricht. Die tatsächliche Bruchlast liegt damit unterhalb der idealen Verzweigungslast. Dennoch kann der Eigenwert als Vergleichsindikator für die Beschreibung des Modellverhaltens verwendet werden. Vergleiche [Petersen 1982]. 
L/300 gesteigert. ${ }^{296}$ Mit dem so erstellten Träger erfolgt die Berechnung des Stabilitätsverhaltens (Spannungsproblem) nach Theorie III. Ordnung ${ }^{297}$. Im folgenden Schritt wird der „imperfekte“ Träger mit dem Vorspannungslastfall belastet, um die Schnittgrößen und Durchbiegungen als Ausgangspunkt der Biegelast numerisch zu ermitteln. Anschließend erfolgt eine schrittweise Biegebelastung analog der Durchführung der Biegeversuche in Kapitel 3.3. Als Ergebnis resultiert das numerisch ermittelte Last-Verformungsverhalten des Spannglasträgers für einen Teilverbundzustand mit einem Schubmodul bei $23^{\circ} \mathrm{C}$ und einer Lastzeit von $60 \mathrm{~s}$ analog dem experimentellen Kurzzeit-Biegeversuch (Kapitel 3.3, ab Seite 107).

Im letzten Schritt wird das zeitabhängige Tragverhalten modelliert. Dabei wird das „imperfekte“ Grundsystem vorgespannt und das Gleichungssystem für jeden gewählten Zeitschritt mit angepassten Glas- und Seilsteifigkeiten ${ }^{298}$ erneut gelöst. Durch die Imperfektion kommt es zur Biegung um die schwache Achse und damit zur Schubbelastung der Zwischenschicht, welche sich, neben der Schubbelastung aus der Normalkraft ${ }^{299}$, als weitere zeitabhängige Lastkomponente manifestiert. Als Zeitschritte werden, ausgehend vom System "mit vollem Verbund“ ( $\mathrm{t}=0)$, die Zeitpunkte nach $1 \mathrm{~s}$, $1 \mathrm{~min}, 1 \mathrm{~h}$ sowie $50 \mathrm{~h}, 100 \mathrm{~h}, 250 \mathrm{~h}, 500 \mathrm{~h}, 750 \mathrm{~h}$ und $1000 \mathrm{~h}$ gewählt. Die Ergebnisse aus diesem Intervall werden mit den Messwerten aus dem Dauerlastversuch (Kapitel 3.4) verglichen.

Der resultierende Quelltext ist in Anlage F hinterlegt.

296 Dabei ergibt sich die rechnerische Spannweite L ohne seitliche Lagerung aus der Spannweite $L_{S}=2000 \mathrm{~mm}$, während mit seitlicher Lagerung in den Drittelspunkten deren Abstand mit $L=L_{S} / 3$ maßgebend wird.

297 Hier werden die Bezeichnungen aus SoFiSTiK 2014 verwendet. Es handelt sich um eine geometrisch nichtlineare Berechnung einschließlich der Effekte aus einer Systemänderung für die Bewehrungsseile. Dabei erfolgt ein Update der tangentialen Steifigkeit nach jedem Iterationsschritt (Linesearchverfahren). Die Seile verbleiben ideal gestreckt. Ein innerer Seildurchhang wird rechnerisch nicht berücksichtigt. Vergleiche [Sofistik AG 2014].

298 Siehe Gleichung 63 und Tafel 39 in Anlage A, Seite 316.

299 Vergleiche $\eta_{p}(Z)$ in Gleichung 64, Seite 185. 


\subsection{Ergebnisse und Ergebnisdiskussion}

\subsubsection{Vergleich mit dem analytischen Modell}

Die Kurzzeit-Biegeversuche für Prüfkörper nach Tafel 10 (Seite 107) werden nach Biegetheorie I. Ordnung nachgerechnet. In Tafel 23 sind die Berechnungsergebnisse sowie ein Vergleich der vertikalen Durchbiegung und der Seilkraftänderung mit der analytischen Lösung abgedruckt.

Tafel 23

Berechnungsergebnisse des Stabwerkmodells und Vergleich mit der analytischen Lösung nach Gleichung 102 (Seite 307) für die Seilkraft und Gleichung 106 (Seite 106) für die vertikale Durchbiegung.

\begin{tabular}{clll}
\hline $\begin{array}{l}\text { Bewehrung } \\
\mathbf{d}_{\mathbf{p}}[\mathbf{m m}]\end{array}$ & $\mathbf{P}_{\mathbf{m} \mathbf{0}}[\mathbf{k N ]}$ & $\begin{array}{l}\mathbf{N}_{\mathbf{p} . \mathrm{FEM}} \\
{[\mathbf{k N} / \mathbf{k N}]}\end{array}$ & $\begin{array}{l}\mathbf{W}_{\mathbf{z . F E M}} \\
{[\mathbf{m m} / \mathbf{m m}]}\end{array}$ \\
\hline unbewehrt & & - & $0,3000(100,0 \%)$ \\
10,1 & 0 & $0,2544(94,2 \%)$ & $0,2832(100,4 \%)$ \\
6,1 & 9,0 & $0,0808(97,3 \%)$ & $0,2951(100,0 \%)$ \\
8,1 & 15,0 & $0,1708(96,0 \%)$ & $0,2895(100,0 \%)$ \\
10,1 & 15,0 & $0,2548(94,4 \%)$ & $0,2836(100,6 \%)$ \\
\hline
\end{tabular}

Die ermittelten Abweichungen von $94,2 \%$ bis $97,3 \%$ und $100,0 \%$ bis 100,6\% in Bezug auf die analytische Lösung werden als ausreichend klein beurteilt. Die größere Abweichung der Seilkraft resultiert aus der getroffenen Idealisierung am Umlenkpunkt und liegt im Bereich der ermittelten Umlenkkraftverluste ${ }^{300}$. Die Modellierung ist somit validiert und nutzbar, um das lineare Tragverhalten analog zur analytischen Lösung abzubilden.

\subsubsection{Modellierung der Umlenkung}

Die tatsächliche Steifigkeit der Klebkonstruktion am Umlenkpunkt ist unbekannt. Für diese Arbeit wird die Wirkung der geklebten Umlenksättel durch eine Studie des Parameters $\mathrm{c}_{\mathrm{m}}=\mathrm{c}_{\mathrm{my}}=\mathrm{c}_{\mathrm{mz}}$ beschrieben .

Dafür gibt Bild 87 das Verhältnis zwischen der Drehsteifigkeit der Verbindungsfedern zu den ersten drei positiven Eigenwerten des Systems unter Biegebelastung $(F=10 \mathrm{kN})$ an. Es wird zwischen der Lagerung eines freien Einfeldträgers ohne seitliche Lagerung (links) analog dem statischen

300 Vergleiche Kapitel 3.3.5, ab Seite 113. Insbesondere Bild 48, Seite 118. 
System im Dauerlastversuch ${ }^{301}$ und einer Situation mit seitlicher Lagerung (rechts) analog dem statischen System im Kurzzeit-Biegeversuch ${ }^{302}$ unterschieden.

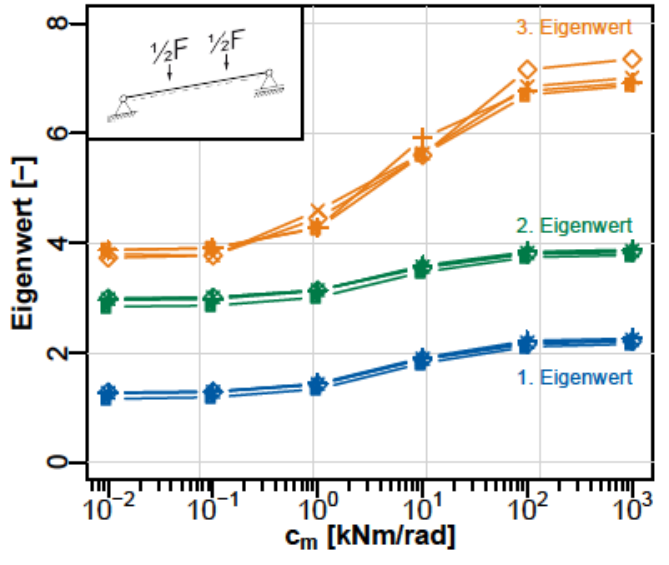

Mit steigender Drehsteifigkeit vergrößern sich die zugehörigen Eigenwerte (Bild 87). Dabei wurden keine wesentlichen Unterschiede zwischen verschieden Seildurchmessern festgestellt. Beim frei spannenden Einfeldträger (Bild 87, links) ergibt sich erwartungsgemäßig eine einwellige, zweiwellige und dreiwellige Eigenform zu den korrespondierenden ersten $(1,16$ bis 2,27$)$, zweiten $(2,84$ bis 3,88$)$ und dritten $(3,72$ bis $7,35)$ Eigenwerten als parallele Verschiebung beider VSGPakete in dieselbe Richtung. Darin entspricht die dritte Eigenform qualitativ den ersten beiden Eigenformen ( 3,72 bis 6,89 ) bei seitlicher Lagerung in Bild 87 (rechts). Diese überlagern sich im gesamten Bereich der Drehsteifigkeit. Beim Erreichen des ersten Eigenwertes verschiebt sich einer der Hauptträger seitlich, während beim zahlenmäßig naheliegenden zweiten Eigenwert der zweite Hauptträger versagt. Diese Eigenwerte werden für einen Vergleich mit den Bruchlasten der experimentellen Studie herangezogen. ${ }^{303}$ Es ist zu erwarten, dass die tatsächlichen Bruchlasten unterhalb der zugehörigen Eigenwerte liegen. ${ }^{295}$ Hier wird angenommen, dass dies bei seitlicher Lagerung für Drehsteifigkeiten bis $c_{m}=10 \mathrm{kNm} / \mathrm{rad}$ für die ersten beiden Eigenwerte der Fall ist.

\footnotetext{
301 Vergleiche Bild 59, Seite 135.

302 Vergleiche Bild 45, Seite 108.

${ }^{303}$ Vergleiche Kapitel 3.3.5, ab Seite 113.
}

Bild 87

Einfluss der Drehsteifigkeit der Umlenkungen auf den Eigenwert unter VierpunktBiegebelastung $(\mathrm{F}=10 \mathrm{kN})$ bei einem gewählten Schubmodul $\mathrm{G}_{\mathrm{Z}}\left(1 \mathrm{~min}, 23^{\circ} \mathrm{C}\right)$ $\left(d_{\text {eff }}=10,46 \mathrm{~mm}\right)$.

Links: ohne seitliche Lagerung.

Rechts: mit seitlicher Lagerung. Zudem sind die zu erwartenden Eigenwerte aus der Bruchlast aus Kapitel 3.3.5 in rot markiert. ${ }^{295}$ 
Für die weitere numerische Berechnung wird ein Wertebereich zwischen $\mathrm{c}_{\mathrm{m}}=0$ (konservativ) und $\mathrm{c}_{\mathrm{m}}=10 \mathrm{kNm} / \mathrm{rad}$ für die Analyse im Biegeversuch und ein Wert von $c_{m}=10 \mathrm{kNm} / \mathrm{rad}$ unter Dauerlast gewählt.

Insgesamt zeigt sich, dass die Steifigkeit der geklebten Umlenkkonstruktion eine deutliche Änderung der Verzweigungslast zur Folge hat. Ebenso kann die seitliche Lagerungsbedingung des Spannglasträgers, beispielsweise durch den Anschluss des Obergurtes an die Verglasung, ein Versagen bei höherem Eigenwert und damit ebenso bei höheren Bruchlasten verursachen. Hier ergibt sich eine konstruktive Möglichkeit die Traglast der Konstruktion günstig zu beeinflussen, sodass eine weiterführende Erforschung der Eigenschaften des Konstruktionsdetails als zielführend beurteilt wird.

\subsubsection{Einfluss der Zwischenschicht}

Im nächsten Schritt wird der Einfluss durch die Schubsteifigkeit der Zwischenschicht studiert. Dazu sind die Eigenwerte in Abhängigkeit des Schubmoduls $G_{Z}$ beziehungsweise der zugehörigen effektiven Glasdicke $d_{\text {eff }}{ }^{304} z$ wischen den Grenzwerten „voller Verbund“ und „ohne Verbund“ getrennt nach Lagerungsbedingungen (ohne und mit seitlicher Lagerung) in Bild 88 dargestellt. Der Einfluss des Seils auf den Eigenwert ist als Ergebnis einer nicht gesondert dargestellten Vorstudie erneut vernachlässigbar.

Bild 88 zeigt in allen Fällen einen fallenden Eigenwert bei verringerter effektiver Glasdicke. Daher ist eine höhere Traglast beim Einsatz von Zwischenschichten mit größerer Steifigkeit zu erreichen. Darüber hinaus werden bei fehlender seitlicher Lagerung geringere Eigenwerte ermittelt, als mit zusätzlicher Stützung. Daher ist die Lagerungssituation weiterhin ein nicht zu vernachlässigender Faktor bei der Ermittlung der Trageigenschaften.

${ }^{304}$ Vergleiche Gleichung 63, Seite 184. 


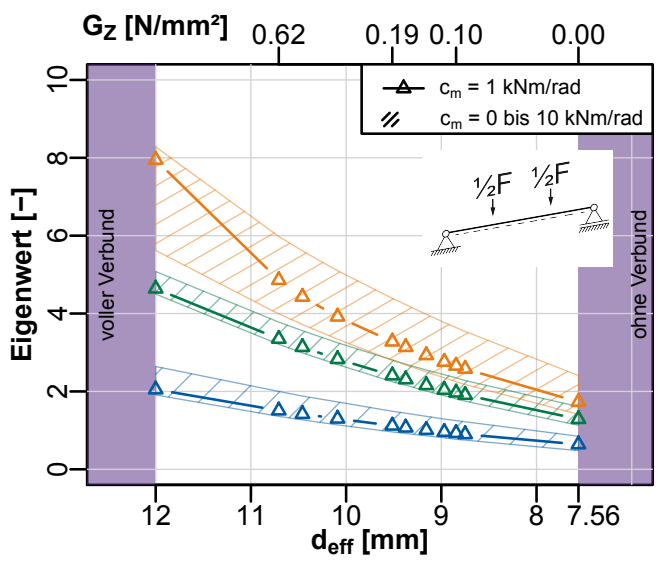

Mit den Ergebnissen in Bild 88 (rechts) wird erneut ein Vergleich mit den korrespondierenden Bruchlasten aus Kapitel 3.3.5 vorgenommen. Dabei durchstreifen die ersten beiden Eigenwerte den Wertebereich zwischen einer effektiven Dicke von $10,0 \mathrm{~mm}\left(\mathrm{G}_{Z}=0,33 \mathrm{~N} / \mathrm{mm}^{2}\right)$ und $8,75 \mathrm{~mm}$ $\left(G_{Z}=0,10 \mathrm{~N} / \mathrm{mm}^{2}\right)$. Diese Werte ergeben sich nach dem Materialmodell für eine PVB-Zwischenschicht ${ }^{305}$ im KurzzeitBiegeversuch ab einer Belastungszeit von $60 \mathrm{~s}$ bei $23^{\circ} \mathrm{C}$ als untere Grenze. Entsprechend der Dauer der KurzzeitBiegeversuche wird der Schubmodul für eine Belastungszeit von 1 min gewählt.

\subsubsection{Auswahl eines Imperfektionswertes}

\section{Imperfektion im Vorspannversuch}

Das Seil im numerischen Modell wird mittels einer Vordehnung belastet, welche multipliziert mit der rechnerischen Seillänge ${ }^{306}$ den Spannweg $w_{p}$ ergibt. Dabei wird der imperfekte Träger im Rahmen einer Parameterstudie verformt, sodass die Seilkraft $P_{m 0}$ unter Einhaltung der Verträglichkeitsbedingung ${ }^{307}$ resultiert. Damit wird die Idealisierung ei-

\footnotetext{
305 Vergleiche Gleichung 5, Seite 53.

306 Die rechnerische Seillänge im numerischen Modell beträgt $2009 \mathrm{~mm}$.

307 Vergleiche Gleichung 1, Seite 37.
}

\section{Bild 88}

Einfluss der Schubsteifigkeit auf den Eigenwert unter Biegelast von $\mathrm{F}=10 \mathrm{kN}$ zwischen den Grenzwerten „voller Verbund“ ( $\mathrm{d}_{\text {eff }}$ $=12,0 \mathrm{~mm}$ ) und „ohne Verbund" ( $\left.d_{\text {eff }}=7,56 \mathrm{~mm}\right)$.

Der Einfluss der geklebten Umlenkung wird im Wertebereich zwischen $\mathrm{C}_{\mathrm{m}}=0$ und $10 \mathrm{kNm} / \mathrm{rad}$ als gestreifte Fläche abgebildet.

Link: ohne seitliche Lagerung.

Rechts: mit seitlicher Lagerung. Zudem sind die zu erwartenden Eigenwerte aus der Bruchlast aus Kapitel 3.3.5 rot markiert. ${ }^{295}$ 
ner ebenen Biegung im analytischen Modell zugunsten der Berücksichtigung der räumlichen Durchbiegung eingestellt.

Die numerischen Ergebnisse im Lastbereich bis $150 \%$ der angestrebten Vordehnung liegen mit $\mathrm{a}_{\mathrm{wP}}=1,37 \mathrm{kN} / \mathrm{mm}$, $2,89 \mathrm{kN} / \mathrm{mm}$ und $4,29 \mathrm{kN} / \mathrm{mm}$ für Seildurchmesser von $6,1 \mathrm{~mm}$, $8,1 \mathrm{~mm}$ und $10,1 \mathrm{~mm}$ zwischen $96 \%$ und $90 \%$ des analytischen Ergebnisses ${ }^{308}$ und mit einem Unterschied von $105 \%$ bis $117 \%$ näher am experimentellen Ergebnis, als die analytische Rechnung ${ }^{309}$. Damit bewirkt die Berücksichtigung der räumlichen Durchbiegung ein realitätsnäheres Ergebnis und ist in einer abschätzenden Berechnung einzubeziehen. Es wird kein wesentlicher Einfluss der Imperfektionsannahme auf die Seilkraftänderung, sowohl mit als auch ohne Modellierung einer zusätzlichen seitlichen Lagerung im Drittelspunkt, festgestellt.

Tafel 24 gibt die Durchbiegung in horizontaler y-Richtung je Bewehrungstyp bei $100 \%$ Vordehnung an. Dabei wird der Imperfektionswert ausgehend vom ,perfekten“ Träger $(\mathrm{L} / \infty)$ bis auf $\mathrm{L} / 300$ gesteigert. Zudem werden die beiden Lagerungssituationen „ohne seitliche Lagerung“ in der jeweils ersten Zeile und "mit seitlicher Lagerung" in der jeweils zweiten Zeile angegeben.

Tafel 24

Horizontale Durchbiegung bei $100 \%$ Vordehnung sowie variierten Imperfektionsannahmen und Lagerungsbedingungen im numerischen Modell.

\begin{tabular}{|c|c|c|c|c|c|c|}
\hline \multicolumn{2}{|c|}{ Bewehrung } & \multicolumn{5}{|c|}{$v_{y}\left(100 \% \varepsilon_{0}\right)[\mathrm{mm}]$ ohne/mit seitl. Lagerung } \\
\hline$d_{p}[\mathrm{~mm}]$ & $P_{\mathrm{m} 0}[\mathrm{kN}]$ & $\mathrm{L} / \infty$ & $\mathrm{L} / 2000$ & L/1000 & $\mathrm{L} / 400$ & $\mathrm{~L} / 300$ \\
\hline \multirow[t]{3}{*}{6,1} & 9,0 & 0 & 1,19 & 2,38 & 5,95 & 7,93 \\
\hline & & 0 & 0,38 & 0,77 & 1,92 & 2,55 \\
\hline & erimentell: & $\leftarrow 0$ & $\mathrm{~mm} \rightarrow$ & & & \\
\hline \multirow[t]{2}{*}{8,1} & 15,0 & 0 & 1,53 & 3,06 & 7,65 & 10,2 \\
\hline & & 0 & 0,43 & 0,87 & 2,17 & 2,89 \\
\hline \multirow[t]{3}{*}{10,1} & 15,0 & 0 & 1,54 & 3,09 & 7,71 & 10,3 \\
\hline & & 0 & 0,44 & 0,87 & 2,17 & 2,90 \\
\hline & experimentell: & \multicolumn{5}{|c|}{$\leftarrow 0,45 \mathrm{~mm} \rightarrow$} \\
\hline
\end{tabular}

Eine seitliche Durchbiegung des „perfekten“ Trägers tritt erwartungsgemäß nicht auf, steigt dann aber mit größer werdender Imperfektion überproportional an. Bei allen Imperfektionswerten ergeben sich im numerischen Ergebnis ohne seitliche Lagerung drei- bis fünffach höhere horizontale

\footnotetext{
308 Vergleiche Gleichung 14, Seite 70 und Gleichung 40, Seite 111.

309 In Bild 46 (Seite 114) ist ein Abstand von $113 \%$ bis $131 \%$ dargestellt.
} 
Durchbiegungen als mit seitlicher Lagerung. Im experimentellen Ergebnis ${ }^{310}$ wurden maximal 0,27 $\mathrm{mm}\left(P_{\mathrm{m} 0}=9,0 \mathrm{kN}\right)$ beziehungsweise $0,45 \mathrm{~mm}\left(P_{\mathrm{m} 0}=15,0 \mathrm{kN}\right)$ ermittelt. Diese Werte werden in der numerischen Rechnung mit seitlicher Lagerung und einer Imperfektion von L/2000 knapp (102\%) berechnet. Größere Imperfektionswerte als L/1000 liefern mit mehr als $285 \%$ und $193 \%$ deutlich zu große Durchbiegungen für eine numerische Abschätzung des experimentellen Ergebnisses.

Eine Imperfektion bewirkt im numerischen Modell eine auf bis zu $126 \%$ vergrößerte vertikale Durchbiegung (Tafel 25 ). Im Vergleich zwischen den Situationen mit und ohne seitlicher Lagerung zeigen sich nur bei Bewehrungsdurchmessern von $8,1 \mathrm{~mm}$ und 10,1 mm sowie einer Imperfektionsannahme von mehr als L/1000 deutliche Unterschiede von mehr als 5\%. Mit größerer Imperfektion reagiert das System weicher, da die Biegung um eine neue Hauptachse im verdrehten Querschnitt erfolgt, was in einer Biegesteifigkeit unterhalb des maximal möglichen Wertes ${ }^{311}$ resultiert. Eine Abschätzung dieser Eigenschaft im ,perfekten“ System ist nach linearer Balkentheorie nicht mehr möglich. Im Experiment wurde für alle Bewehrungsdurchmesser ein Ergebnis von $\mathrm{a}_{\mathrm{wz}}=0,070 \pm 0,004 \mathrm{~mm} / \mathrm{kN}$ angegeben. ${ }^{312}$ Daraus resultieren vertikale Durchbiegungen von $0,63 \pm 0,036 \mathrm{~mm}$ für Seildurchmesser von 6,1 $\mathrm{mm}$ und $1,05 \pm 0,060 \mathrm{~mm}$ bei einem Seildurchmesser von 10,1 mm für einen Vergleich mit den Ergebnissen in Tafel 25.

\begin{tabular}{|c|c|c|c|c|c|c|}
\hline \multicolumn{2}{|c|}{ Bewehrung } & \multicolumn{5}{|c|}{$\mathbf{w}_{\mathbf{z}}\left(\mathbf{1 0 0} \% \varepsilon_{0}\right)[\mathrm{mm}]$ ohne/mit seitl. Lagerung } \\
\hline$d_{p}[m m]$ & $\mathrm{P}_{\mathrm{mo}}[\mathrm{kN}]$ & $\mathrm{L} / \infty$ & $\mathrm{L} / 2000$ & L/1000 & $\mathrm{L} / 400$ & $\mathrm{~L} / 300$ \\
\hline \multirow[t]{3}{*}{6,1} & 9,0 & 0,61 & 0,63 & 0,66 & 0,73 & 0,77 \\
\hline & & 0,61 & 0,63 & 0,66 & 0,73 & 0,77 \\
\hline & erimentell: & $\leftarrow 0$ & $\pm 0,036$ & $\mathrm{~mm} \rightarrow$ & & \\
\hline \multirow[t]{2}{*}{8,1} & 15,0 & 1,01 & 1,04 & 1,08 & 1,17 & 1,22 \\
\hline & & 1,01 & 1,03 & 1,05 & 1,11 & 1,17 \\
\hline \multirow[t]{3}{*}{10,1} & 15,0 & 1,00 & 1,04 & 1,07 & 1,17 & 1,22 \\
\hline & & 1,00 & 1,03 & 1,05 & 1,10 & 1,13 \\
\hline & experimentell: & &, $05 \pm 0$ & $060 \mathrm{~mm}$ & & \\
\hline
\end{tabular}

Tafel 25

Vertikale Durchbiegung im numerischen Vorspannversuch bei $100 \%$ Vordehnung.

Es wurden variierte Imperfektionsannahmen und Lagerungsbedingungen berücksichtigt.

\footnotetext{
${ }^{310}$ Vergleiche Bild 47, Seite 115.

311 Vergleiche Gleichung 61, Seite 183.

312 Vergleiche Kapitel 3.3.5, ab Seite 116.
} 
Für einen Seildurchmesser von 6,1 mm betrifft dies einen Imperfektionsbereich bis L/1000 (mit seitlicher Lagerung), während bei größeren Durchmessern praktisch eine Imperfektion bis $L / 400$ zutreffend ist. Damit werden die Angaben in [Belis et al. 2004] mit einem Imperfektionsmittelwert von L/1000 und einem Fraktilwert von L/400 indirekt bestätigt. Für die weitere Berechnung wird ein Imperfektionswert von L/1000 als realistisch betrachtet.

\section{Imperfektion im Kurzzeit-Biegeversuch}

Schließlich wird überprüft, ob das Modell das Tragverhalten unter Biegebelastung ausreichend realitätsnah wiedergibt, um zu bestätigen, dass die bisher getroffenen Annahmen verwendet werden können.

Bis zu einer Biegelast von $30 \mathrm{kN}$ steigt die numerisch ermittelte, vertikale Durchbiegung linear an. Dies entspricht der experimentellen Beobachtung, wonach bis zu $80 \%$ der Bruchlast ein lineares Tragverhalten vorlag. Damit wird, durch die analoge Anwendung der Gleichung 44 (Seite 111), eine Biegesteifigkeit $a_{F}$ für das numerische Ergebnis ermittelt und in Tafel 26 zusammengefasst. Sie entspricht für das „perfekte" System der analytischen Lösung. ${ }^{313}$ Mit steigender Imperfektion verringert sich die Steifigkeit $\mathrm{a}_{\mathrm{F}}$ auf bis zu $67 \%$ des Ausgangswertes. Für den Erwartungswert der Imperfektion von $\mathrm{L} / 1000$ ergeben sich $3 \%$ bis $6 \%$ geringere Werte.

Ansonsten ist die Biegesteifigkeit durch den Bewehrungsdurchmesser bestimmt. Mit steigender metallischer Querschnittsfläche vergrößert sich $\mathrm{a}_{\mathrm{F}}$, wobei kein merklicher Unterschied zwischen vorgespannten und schlaff bewehrten Optionen erkennbar ist.

Bild 89 zeigt die Änderung der Seilkraft während des numerischen Biegeversuchs getrennt nach Bewehrungstypen. Dabei zeigen sich fallende Steifigkeiten $a_{p}$ mit größer werdender Imperfektion. Im kritischen Lastbereich ( $\mathrm{F}>30 \mathrm{kN})$ wird

\footnotetext{
313 Vergleiche Tafel 37 in Anlage A, Seite 311. Die Abweichungen im Bereich von $1 \%$ resultieren aus den Eigenschaften der numerischen $\mathrm{Be}$ rechnung als Näherungslösung.
} 


\begin{tabular}{|c|c|c|c|c|c|c|}
\hline \multicolumn{2}{|c|}{ Bewehrung } & \multicolumn{3}{|c|}{$a_{F}(F \leq 30 k N)[k N / m m]$} & \multirow[b]{2}{*}{$\sqcup 400$} & \multirow[b]{2}{*}{ ᄂ/300 } \\
\hline $\mathrm{d}_{\mathrm{p}}[\mathrm{mm}]$ & $\mathrm{P}_{\mathrm{mo}}[\mathrm{kN}]$ & $\mathrm{U}_{\infty}$ & L/2000 & レ1000 & & \\
\hline \multirow{2}{*}{\multicolumn{2}{|c|}{ unbewehrt }} & 3,33 & 3,29 & 3,22 & 2,96 & 2,71 \\
\hline & & $100,0 \%$ & $\% 98,8 \%$ & $96,8 \%$ & $88,8 \%$ & $81,7 \%$ \\
\hline \multirow[t]{2}{*}{10,1} & 0 & 3,53 & 3,48 & 3,40 & 3,00 & 2,73 \\
\hline & & $100,0 \%$ & $\% 98,6 \%$ & $96,2 \%$ & $85,0 \%$ & $77,2 \%$ \\
\hline \multirow[t]{2}{*}{6,1} & 9,0 & 3,38 & 3,34 & 3,25 & 2,84 & 2,59 \\
\hline & & $100,0 \%$ & $\% 98,5 \%$ & $96,0 \%$ & $84,0 \%$ & $76,4 \%$ \\
\hline \multirow[t]{2}{*}{8,1} & 15,0 & 3,45 & 3,39 & 3,28 & 2,74 & 2,41 \\
\hline & & $100,0 \%$ & $\% 98,4 \%$ & $95,0 \%$ & $79,4 \%$ & $70,0 \%$ \\
\hline \multirow[t]{2}{*}{10,1} & 15,0 & 3,52 & 3,45 & 3,31 & 2,70 & 2,36 \\
\hline & & $100,0 \%$ & $\% 98,0 \%$ & $94,0 \%$ & $76,5 \%$ & $66,9 \%$ \\
\hline
\end{tabular}

Tafel 26

Numerisch ermittelte Biege-

steifigkeit $\mathrm{a}_{\mathrm{F}}$ in Abhängigkeit von der Imperfektionsannahme im linearen Lastbereich für $\mathrm{F} \leq 30 \mathrm{kN}$ sowie Verhältnis zur Steifigkeit des „perfekten“ Trägers.

Analoge experimentelle Ergebnisse sind in Kapitel 3.3.5 (Seite 119) sowie Anlage B abgedruckt.

ein auffälliges Abflachen, gar ein Abfallen, der Kurven deutlich. Dies geht mit einer merklichen Verdrehung im Vorfeld des Stabilitätsversagens der Träger einher. Die Abgrenzung zum linearen Tragbereich muss daher bereits bei kleineren Biegelasten erfolgen. Für die Ermittung der zugehörigen Seilsteifigkeit ap wird ein Wert von $\mathrm{F} \leq 20 \mathrm{kN}$ gewählt und in Tafel 27 zusammengestellt. Es zeigen sich erneut verringerte Steifigkeiten mit steigendem Imperfektionswert. Die experimentell bestimmten Mittelwerte von $a_{p}=0,237 \mathrm{kN} / \mathrm{mm}$, $0,522 \mathrm{kN} / \mathrm{mm}$ und $0,720 \mathrm{kN} / \mathrm{mm}$ für vorgespannte Prüfkörper der Gruppen mit Seildurchmessern von 6,1 mm, 8,1 mm und $10,1 \mathrm{~mm}$ sind zwischen die Imperfektionsgruppen bis $\mathrm{L} 400$ nach Tafel 27 einzuordnen.

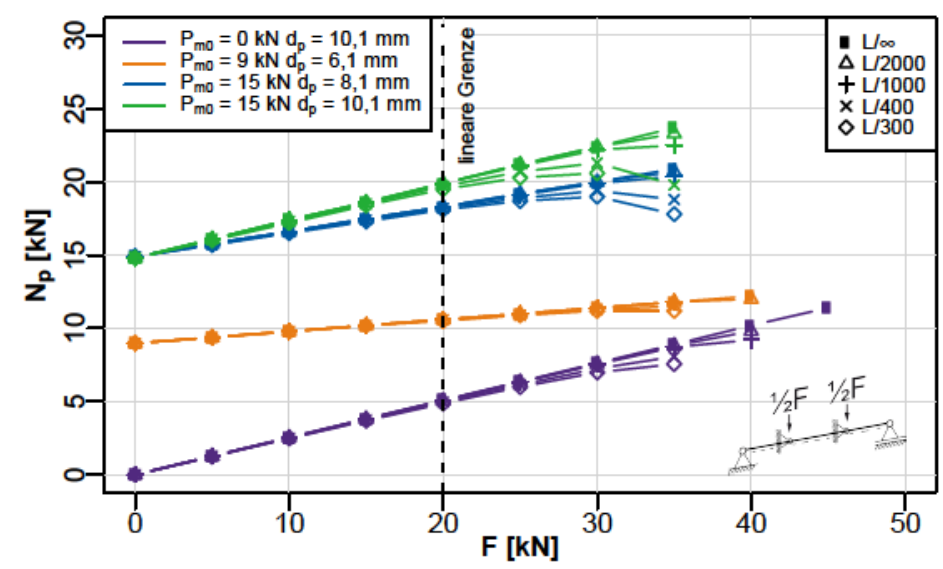

Bild 89

Verhältnis zwischen Biegelast und Seilkraft.

Es ist sinnvoll, den Imperfektionswert zur größeren Steifigkeit $a_{p}$, hier $\sqcup 1000$, für die folgenden Betrachtungen zu wählen, da daraus rechnerisch eine höhere Seilkraft resultiert. Dies wirkt für die Auslegung des Seils ungünstig. Darüber hinaus 
Tafel 27

Seilkraftänderung $a_{p}$ im numerischen Modell in Abhängigkeit von der Imperfektionsannahme sowie Verhältnis zur Steifigkeit bei Annahme eines „perfekten“ Trägers.

\begin{tabular}{|c|c|c|c|c|c|}
\hline \multicolumn{2}{|c|}{ Bewehrung } & \multicolumn{4}{|c|}{$a_{P}(F \leq 20 k N)[k N / m m]$} \\
\hline$d_{p}[\mathrm{~mm}]$ & $P_{m 0}[k N]$ & $\mathrm{L} / \infty \quad \mathrm{L} / 2000$ & $\mathrm{~L} / 1000$ & $\mathrm{~L} / 400$ & $\mathrm{~L} / 300$ \\
\hline \multirow[t]{3}{*}{10,1} & 0 & 0,899 & 0,859 & 0,745 & 0,665 \\
\hline & & $100,0 \% 98,2 \%$ & $95,6 \%$ & $82,9 \%$ & $73,9 \%$ \\
\hline & erimentell: & $\leftarrow 0$ &, $720 \mathrm{kN}$ & $\mathrm{mm} \rightarrow$ & \\
\hline \multirow[t]{3}{*}{6,1} & 9,0 & 0,270 & 0,263 & 0,231 & 0,200 \\
\hline & & $100,0 \% 98,6 \%$ & $96,0 \%$ & $84,3 \%$ & $73,0 \%$ \\
\hline & erimentell: & $\leftarrow 0,237 \mathrm{kN} / \mathrm{r}$ & $\mathrm{mm} \rightarrow$ & & \\
\hline \multirow[t]{3}{*}{8,1} & 15,0 & $0,586 \quad 0,576$ & 0,557 & 0,454 & 0,386 \\
\hline & & $100,0 \% 98,4 \%$ & $95,1 \%$ & $77,5 \%$ & $65,9 \%$ \\
\hline & erimentell: & $\leftarrow 0,522 \mathrm{kN} / \mathrm{s}$ & $\mathrm{mm} \rightarrow$ & & \\
\hline \multirow[t]{3}{*}{10,1} & 15,0 & $0,894 \quad 0,876$ & 0,841 & 0,658 & 0,556 \\
\hline & & $100,0 \% 98,0 \%$ & $94,0 \%$ & $73,5 \%$ & $62,1 \%$ \\
\hline & erimentell: & $\leftarrow 0,720 \mathrm{kN} / \mathrm{l}$ & $\mathrm{mm} \rightarrow$ & & \\
\hline
\end{tabular}

ergeben sich größere Normalkräfte im Glas, welche ungünstig für das beobachtete Stabilitätsversagen sind, wodurch die Annahme insgesamt auf der sicheren Seite liegt.

Zuletzt ist die Frage zu klären, ob die zufällige Trägerimperfektion Grund für die vergleichsweise große Bandbreite seitlicher Durchbiegungsergebnisse und die schlechte Passung der zugehörigen linearen Regression sein kann. ${ }^{314} \mathrm{Da}$ zu zeigt Tafel 28 die Änderung der seitlichen Durchbiegung während der Steigerung der Biegebelastung auf $F=30 \mathrm{kN}$. Gegenüber dem Erwartungswert der Imperfektion (L/1000) sind Unterschiede der seitlichen Durchbiegungsänderung zwischen den Faktoren 0,5 und 3,3 möglich. Zudem ergaben sich auch numerisch deutlich nichtlineare Beziehungen zwischen der Biegelast und der Durchbiegung, welche für die rechnerische Abbildung des Spannungsproblems so zu erwarten waren. Daher wird die zufällig verteilte Trägerimperfektion als maßgeblicher Parameter für die seitliche Durchbiegung von Spannglasträgern beurteilt.

Es bleibt zu prüfen inwieweit die Imperfektionsannahmen für unbewehrte VSG-Träger nach [Belis et al. 2004] auf den Doppelquerschnitt der Träger dieser Arbeit vorbehaltlos übertragbar sind. Es besteht die Vermutung, dass durch die lokale Verbindung beider Glasträgerpakete andere Vorverformungsfiguren, beispielsweise eine gegenläufige Vorverfor-

${ }^{314}$ Vergleiche Kapitel 3.3.5, ab Seite 119. 


\begin{tabular}{|c|c|c|c|c|c|c|}
\hline \multicolumn{2}{|c|}{ Bewehrung } & \multicolumn{5}{|c|}{$v_{y}(F=20 k N)-v_{y}(F=0 k N)[m m]$} \\
\hline$d_{p}[\mathrm{~mm}]$ & $P_{\mathrm{m} 0}[\mathrm{kN}]$ & $\mathrm{L} / \infty$ & $\mathrm{L} / 2000$ & $\mathrm{~L} / 1000$ & $\mathrm{~L} / 400$ & $\mathrm{~L} / 300$ \\
\hline \multicolumn{2}{|l|}{ unbewehrt } & 0 & 0,77 & 1,50 & 3,68 & 4,91 \\
\hline & & - & $51 \%$ & $100 \%$ & $245 \%$ & $327 \%$ \\
\hline \multirow[t]{2}{*}{10,1} & 0 & 0 & 0,94 & 1,86 & 4,61 & 6,09 \\
\hline & & - & $50 \%$ & $100 \%$ & $247 \%$ & $327 \%$ \\
\hline \multirow[t]{2}{*}{6,1} & 9,0 & 0 & 1,01 & 2,01 & 5,03 & 6,69 \\
\hline & & - & $50 \%$ & $100 \%$ & $249 \%$ & $332 \%$ \\
\hline \multirow[t]{2}{*}{8,1} & 15,0 & 0 & 1,36 & 2,73 & 6,68 & 8,80 \\
\hline & & - & $50 \%$ & $100 \%$ & $244 \%$ & $322 \%$ \\
\hline \multirow[t]{2}{*}{10,1} & 15,0 & 0 & 1,56 & 3,11 & 7,36 & 9,49 \\
\hline & & - & $50 \%$ & $100 \%$ & $237 \%$ & $305 \%$ \\
\hline
\end{tabular}

Tafel 28

Änderung der seitlichen Durchbiegung während der Steigerung der Biegebelastung im numerischen Modell sowie Verhältnis zum Ergebnis bei einer Imperfektion von L/1000.

mung beider Pakete, möglich sind. Hier besteht ausblickend weiterer Forschungsbedarf.

Aus der Gesamtbetrachtung aller Analysen wird zusammenfassend die weitere Verwendung eines Imperfektionswertes von L/1000 abgeleitet.

\subsubsection{Seilkraftverlust im Dauerversuch}

\section{Dauerlastversuch ohne Auflast}

Bild 90 zeigt die numerisch errechnete Seilkraft im Dauerlastversuch für Prüfkörper ohne Auflast. ${ }^{315}$ Die zugehörigen Zahlenwerte sind in Tafel 29 zusammengestellt. Ausgehend von initialen Seilkräften von knapp $15 \mathrm{kN}$ resultieren für Seildurchmesser von $8,1 \mathrm{~mm}$ und $10,1 \mathrm{~mm}$ nach $1000 \mathrm{~h}$ Verluste von $0,54 \mathrm{kN}$ und $0,82 \mathrm{kN}$. Dabei stellte sich die größte Abnahme innerhalb der ersten $100 \mathrm{~h}$ ein. Anschließend flacht die Kurve zunehmend ab. Der qualitative Kurvenverlauf wird im Vergleich zum experimentellen Ergebnis daher gut abgebildet. Darüber hinaus werden mittels der analytischen Herangehensweise mit $0,60 \mathrm{kN}$ und $0,90 \mathrm{kN}$ nahezu die gleichen Werte errechnet. Die Berechnung verfehlt die experimentellen Ergebnisse jedoch deutlich. Je nach Regressionsfunktion ergeben sich zwischen 2,23 kN und 4,66 kN Verlust. 

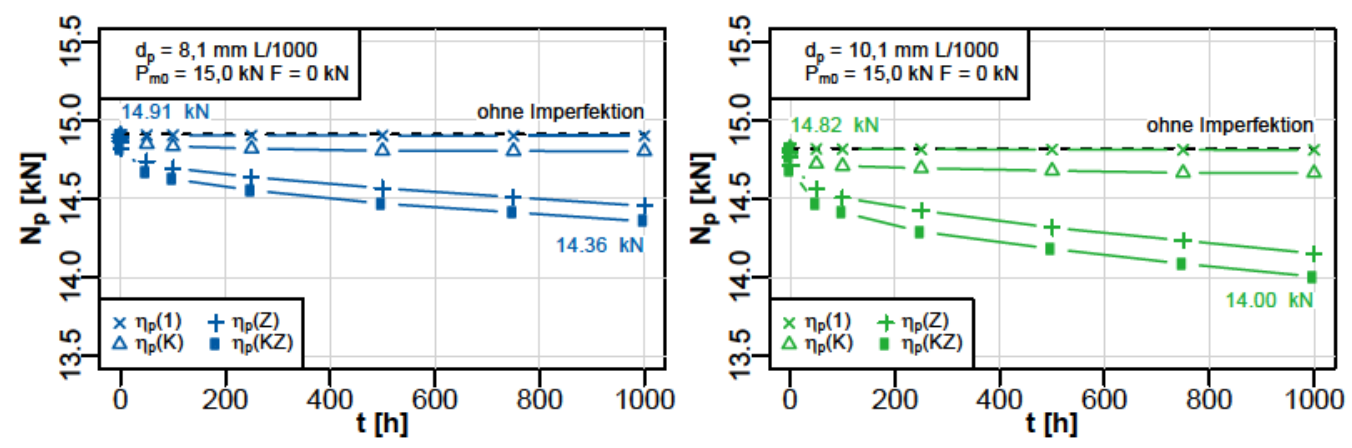

Bild 90

Numerisch berechnete Seilkraftveränderungen ohne Auflast $(F=0)$ analog der Prüfkörper im DauerlastVersuch (Tafel 14, Seite 134) sowie Trennung der Wirkanteile mit dem Faktor $\eta_{p}$ nach Gleichung 64, Seite 185.

Die prägnanten Unterschiede lassen vermuten, dass bisher nicht berücksichtigte Verformungsanteile eine zusätzliche Rolle spielen. Diese können darin begründet sein, dass die Eigenform unter Biege- und nicht unter Vorspannlasten ermittelt wurde.

Entsprechend wird in einem weiteren numerischen Rechenschritt die erste Eigenform unter Vorspannbelastung bestimmt und eine erneute Berechnung der Seilkraft nach $1000 \mathrm{~h}$ durchgeführt. Mit einer Imperfektion von $\sqcup 1000$ reduzierten sich die Seilkräfte für beide Seildurchmesser auf $99 \%$ des Ergebnisses mit der Eigenform unter Biegelast. Eine weitere Berechnung mit $\sqcup 400$ ergab eine Verringerung auf $94 \%$ bis $96 \%{ }^{316}$ Damit ist es gerechtfertigt der Eigenformannahme einen Einfluss auf das Ergebnis zuzuschreiben. Jedoch werden erst mit verhältnismäßig großen Ausmitten quantitativ deutliche Veränderungen ermittelt. Daher liegt die Vermutung nahe, dass die experimentellen Ergebnisse mit Prüfkörpern vergleichsweise großer initialer Vorverformung ermittelt wurden. Es wird für zukünttige Untersuchungen daher vorgeschlagen, die tatsächliche Trägerimperfektion vorab zu vermessen und in die Analyse einzubeziehen. Zusätzlich kann der Versuchsapparat selbst einen Einfluss gehabt haben. Im Unterschied zum Dauerlastversuch mit Auflast wurde die Lasttraverse und der Hebelarm im Versuch ohne Auflast nicht verwendet. Letztlich können konstruktive

\footnotetext{
${ }^{316}$ Unter der Vorspannbelastung ergibt sich eine dreiwellige Eigenform. Zur besseren Vergleichbarkeit wurde diese so skaliert, dass der absolute Betrag der initialen seitlichen Vorverformung nahezu gleich dem Ausgangswert bei der Eigenformermittlung unter der Biegelast ist.
} 
Besonderheiten eine Rolle spielen. Sie werden in Kapitel 6 besprochen.

\section{Dauerlastversuch mit Auflast}

Bild 91 zeigt die errechneten Seilkraftveränderungen unter der Wirkung der Biegelast von $10 \mathrm{kN}$. Für die schlaff bewehrte Variante $\left(d_{p}=8,1 \mathrm{~mm}\right)$ und vorgespannte Träger mit $5,0 \mathrm{~mm}$ Seildurchmesser wird qualitativ erneut ein stetig kleiner werdender Verlust errechnet, wobei nach $1000 \mathrm{~h}$ im numerischen Rechenversuch noch $96 \%$ bis $98 \%$ der initialen Kraft verbleiben. Auch dieses Ergebnis deckt sich gut mit der analytischen Berechnung (Tafel 30).
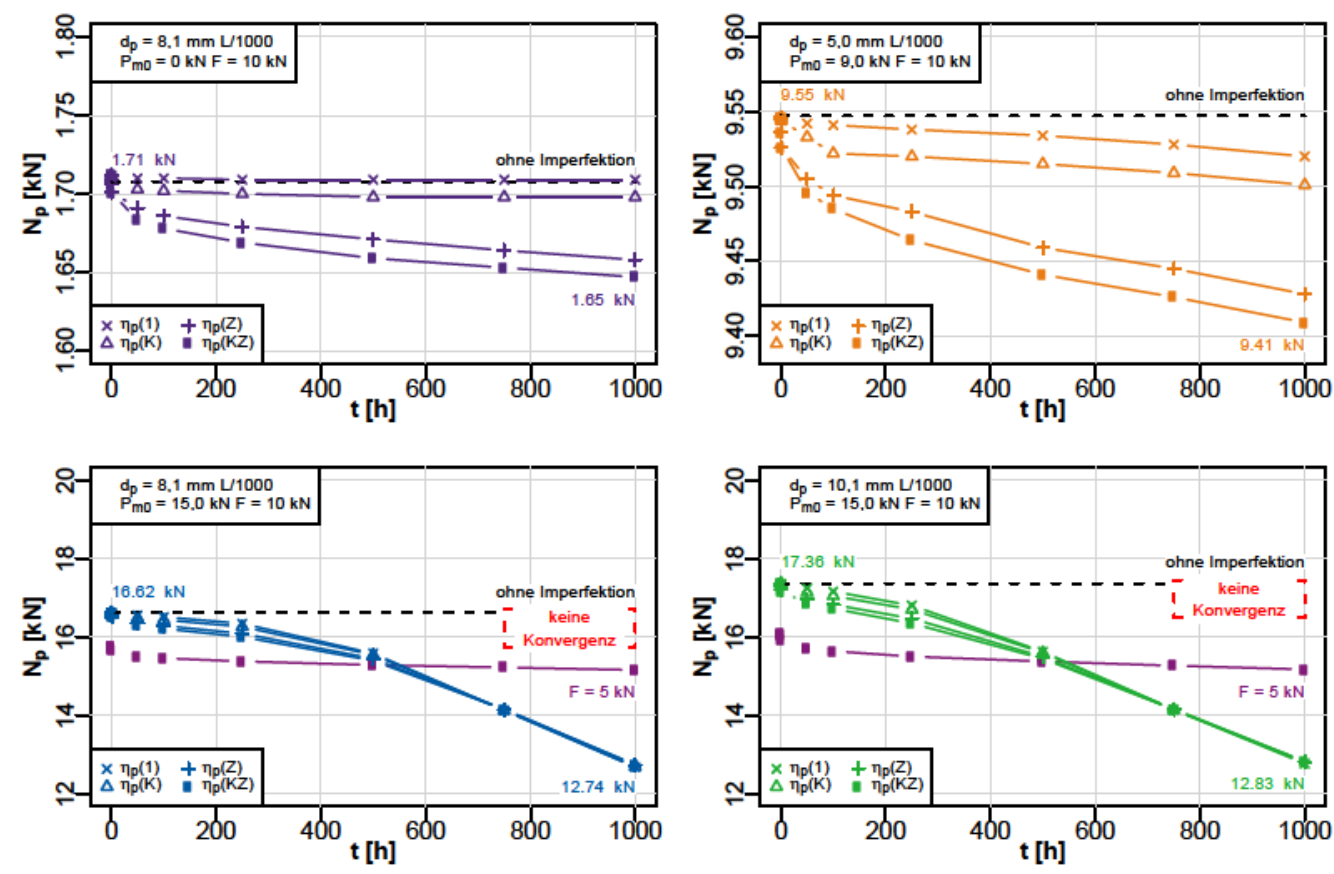

Im Gegensatz dazu zeigen Spannglasträger mit $15 \mathrm{kN}$ Vorspannung einen qualitativ andersartigen Kurvenverlauf mit progressiv zunehmendem Seilkraftverlust. Betrachtet man den Zeitpunkt $t=1000 \mathrm{~h}$, so stellt man fest, dass die analytische Rechnung deutlich geringe Verluste ausweist, während das numerische Ergebnis sehr nah am experimentellen Er-

Bild 91

Numerisch berechnete Seilkraftveränderungen mit Auflast $(\mathrm{F}=10 \mathrm{kN})$ analog zu den Prüfkörpern im Dauerlast-Versuch (Tafel 14, Seite 134) sowie Trennung der Wirkanteile mit dem Faktor $\eta_{p}$ nach Gleichung 64, Seite 185. 
Tafel 29

Als Ausgangspunkt der Berechnung dient in allen dargestellten Ergebnissen die Seilkraft zum Zeitpunkt $\mathrm{t}=0$ aus der jeweiligen numerischen Berechnung $\left(\mathrm{N}_{\text {p.max }}\right.$ in Tafel 30). gebnis liegt. Aufgrund des andersartigen Verlaufes wird dies jedoch als zufällig bewertet.

Als Grund kann eine mögliche Überlastung des Systems mit kleiner werdenden Querschnittssteifigkeiten benannt werden. So zeigt eine Vergleichsrechnung mit halbierter Biegelast ( $F=5 \mathrm{kN}$ in Bild 91) den erwarteten Verlauf der Seilkraft mit flach auslaufendem Kurvenende und der Tendenz zu einem stabilen Endwert. Für diese These spricht zudem die fehlende Konvergenz im Modell bei einer Betrachtung des Systems „ohne Imperfektion“ - die Verzweigungslast wurde bereits überschritten. ${ }^{317}$ In diesem Zusammenhang ist die Imperfektionsannahme kritisch zu hinterfragen, da, im Gegensatz zum System „ohne Imperfektion“, unter Annahme einer initialen Vorverformung bei $t=1000 \mathrm{~h}$ ein Gleichgewicht im Tragsystem numerisch gefunden wurde. Daher wird im nächsten Schritt der Einfluss der Imperfektionsannahme im Dauerlastversuch näher analysiert.

\begin{tabular}{|c|c|c|c|c|c|c|}
\hline \multicolumn{3}{|c|}{ Bewehrung } & \multicolumn{4}{|c|}{$N_{p}(t=1000 h)[k N](L / 1000)$} \\
\hline & $\mathbf{P}_{\mathrm{m} 0}$ & & analytisch & experim & tell & numerisch \\
\hline$[\mathrm{mm}]$ & {$[\mathbf{k N}]$} & {$[\mathrm{kN}]$} & Gl. 37 & Tafel 15 & Tafel 16 & $\eta_{p}(K Z)$ \\
\hline 8,1 & 15,0 & 0 & 14,31 & 12,77 & 10,34 & 14,36 \\
\hline 10,1 & 15,0 & 0 & 13,92 & 12,24 & 11,57 & 14,01 \\
\hline 8,1 & 0 & 10 & 1,64 & 1,30 & - & 1,65 \\
\hline 5,0 & 9,0 & 10 & 9,43 & 9,30 & - & 9,41 \\
\hline 8,1 & 15,0 & 10 & 15,96 & 14,01 & 12,28 & 12,74 \\
\hline 10,1 & 15,0 & 10 & 16,30 & 15,47 & 14,11 & 12,83 \\
\hline
\end{tabular}

Wirkung der Imperfektion im Dauerlast-Versuch

Das folgende Bild 92 zeigt einen Vergleich der numerisch berechneten Seilkraftverluste für zwei Trägervarianten mit veränderten Imperfektionsannahmen. Mit Seilkraftverlusten zwischen $0,68 \mathrm{kN}$ und $4,98 \mathrm{kN}$ in Abhängigkeit der gewählten Imperfektionen wird deutlich, dass deren Wert einen bedeutenden Einfluss auf die errechnete Seilkraft hat. Eine veränderte Imperfektion führt zu qualitativ unterschiedlichen Kurvenkrümmungen. Dabei zeigten sich bei Werten von $\mathrm{L} / 2000$ und $\mathrm{L} / 1000$, progressiv zunehmende Verläufe,

317 Der zugehörige Eigenwert in Bild 88 (Seite 191, links) nähert sich ab $500 \mathrm{~h}$ dem Wert Eins. 
während sowohl ohne eine Imperfektion als auch bei Werten von $\mathrm{L} 400$ und $\mathrm{L} 300$ flacher werdende Kurven zu erkennen sind. Dies ist auf unterschiedliche Verformungsfiguren während der nichtlinearen Berechnung zurückzuführen. Darüber hinaus wird deutlich, dass sowohl bei großen Imperfektionswerten, als auch bei „perfektem“ Träger stellenweise keine Konvergenz gefunden wurde, was eine Lastsituation nahe der Überlast vermuten lässt. Des Weiteren legen die Berechnungsergebnisse den Schluss nahe, dass die Stabilität unter Dauerlasten für die ungünstigste Kombination aus Seilkraft und Auflast, folglich der resultierenden Normalspannung im Glas, zu untersuchen ist. Diese kann während der gesamten Lastgeschichte in der hier gezeigten Untersuchung sowohl zu Beginn $(t=0)$ als auch am Versuchsende $(t=1000 h)$ auftreten, da die Biegesteifigkeit um die schwache Achse bezüglich des Stabilitätsnachweises schneller abnimmt als die Seilkraft als einzig zeitlich veränderliche Lastgröße.

Das Modell zeigt damit, dass die vergleichsweise breite Ergebnisstreuung auf die Imperfektion und der daraus resultierenden Belastung der Zwischenschicht erklärt werden kann. Daher erfolgt im nächsten Abschnitt eine Trennung der Materialeinflüsse mit dem Faktor $\eta_{p}$, um diese in zukünftigen Untersuchungen gezielt beeinflussen zu können.

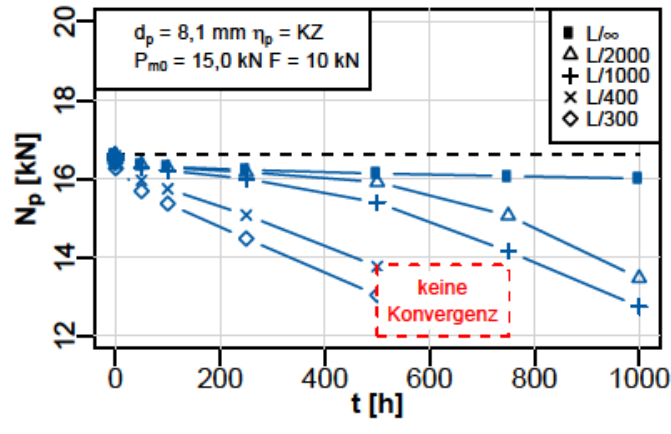

In den Bildern 90, 91 und 92 sind bereits die Seilkraftverläufe unter Annahme der alleinigen Wirkung einzelner Materi-

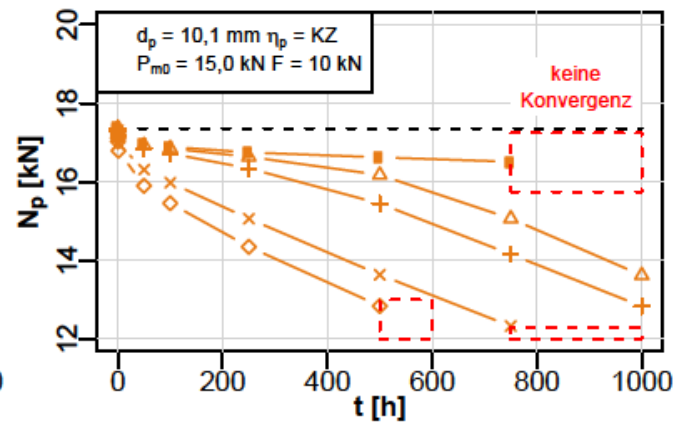

Bild 92

Numerisch berechnete Seilkraftveränderungen mit Auflast ( $F=10 \mathrm{kN}$ und $P_{m 0}=15 \mathrm{kN}$ ) für variierte Imperfektionswerte sowie Trennung der Wirkanteile mit dem Faktor $\eta_{p}$ nach Gleichung 64, Seite 185. 
alparameter eingetragen und als Ergebnis nach $1000 \mathrm{~h}$ in Tafel 30 quantitativ zusammengestellt.

Unter Vernachlässigung der Eigenlast verbleibt ein unbelasteter Spannglasträger unverformt. Unterstellt man dem „perfekten“ System (ohne Imperfektion) keine zeitabhängigen Trageigenschaften, oder betrachtet ausschließlich den Zeitpunkt der ersten Belastung $(t=0)$, so wird die wirkende Seilkraft $N_{p}$ konstant bleiben. Dieses Ergebnis ist als gestrichelte, horizontale Linie über die Zeit in den Bildern dargestellt.

Unterstellt man im nächsten Schritt eine Vorverformung, so kommt es zur Biegung um die schwache Achse. Daraufhin wird ein Seilkraftverlust $\left(\eta_{p}(1)\right)$ berechnet, da sich die Biegesteifigkeit $I_{\text {z.eff.G }}$ infolge der Zeitabhängigkeit der Zwischenschichtsteifigkeit reduziert. ${ }^{318}$ Mit weniger als $2 \%$ Anteil am Gesamtverlust nach $1000 \mathrm{~h}$ sind diese jedoch für Trägerkonfigurationen, welche ausschließlich durch einen Lastfall belastet wurden, als nicht maßgebend einzustufen.

Beim vorgespannten und biegebelasteten Träger mit 5,0 mm Seildurchmesser kommt es bereits zu einem deutlichen Beitrag von $20 \%$ zum Seilkraftverlust als Folge der initialen Schiefstellung. Darüber hinaus resultiert der gesamte Seilkraftverlust von Trägern unter Biegelast und mit $15 \mathrm{kN}$ Vorspannung aus dieser geometrischen Situation. In diesen Fällen ist daher einerseits eine dauerhaft steifere Zwischenschicht zu wählen. Andererseits sollte die Belastung der Zwischenschicht durch konstruktive Maßnahmen reduziert werden. ${ }^{319}$ Insgesamt zeigt sich, dass die Imperfektion auch bei der Berechnung der Spannkraftverluste ein wesentlicher und zu berücksichtigender Parameter ist.

Neben dem Biegeanteil kommt es zu einer Stauchung der Materialien längs der Trägerachse. ${ }^{320}$ Daraus resultiert eine Stauchung der Klotzung und der Zwischenschicht $\left(\eta_{p}(K Z)\right)$, welche einen Gesamtverlust der Seilkraft von $0,07 \mathrm{kN}$ bis $0,82 \mathrm{kN}$ nach sich zieht. Betrachtet man die Beiträge ge-

\footnotetext{
318 Vergleiche Gleichung 61, Seite 183.

319 Vergleiche Kapitel 6, ab Seite 242.

320 Vergleiche Bild 29, Seite 53.
} 


\begin{tabular}{|c|c|c|c|c|c|c|c|}
\hline \multicolumn{3}{|c|}{ Bewehrung } & \multicolumn{5}{|c|}{$\Delta P(t=1000 h)[k N](L / 1000)$} \\
\hline $\begin{array}{l}d_{p} \\
{[\mathrm{~mm}]}\end{array}$ & $\begin{array}{l}P_{m 0} \\
{[k N]}\end{array}$ & $\begin{array}{l}\mathbf{F} \\
{[\mathrm{kN}]}\end{array}$ & $\begin{array}{l}N_{\text {p.max }} \\
{[k N]}\end{array}$ & $\eta_{p}(K Z)$ & $\eta_{\mathrm{p}}(\mathrm{K})$ & $\eta_{p}(Z)$ & $\eta_{p}(1)$ \\
\hline 8,1 & 15,0 & 0 & 14,91 & $\begin{array}{r}-0,551 \\
100 \%\end{array}$ & $\begin{array}{c}-0,106 \\
19,2 \%\end{array}$ & $\begin{array}{l}-0,455 \\
82,6 \%\end{array}$ & $\begin{array}{r}-0,008 \\
1,4 \%\end{array}$ \\
\hline 10,1 & 15,0 & 0 & 14,82 & $\begin{array}{r}-0,817 \\
100 \%\end{array}$ & $\begin{array}{l}-0,159 \\
19,5 \%\end{array}$ & $\begin{array}{l}-0,671 \\
82,1 \%\end{array}$ & $\begin{array}{r}-0,012 \\
1,5 \%\end{array}$ \\
\hline 8,1 & 0 & 10 & 1,71 & $\begin{array}{r}-0,065 \\
100 \%\end{array}$ & $\begin{array}{l}-0,010 \\
15,4 \%\end{array}$ & $\begin{array}{l}-0,050 \\
76,9 \%\end{array}$ & $\begin{array}{r}-0,001 \\
1,5 \%\end{array}$ \\
\hline 5,0 & 9,0 & 10 & 9,55 & $\begin{array}{r}-0,137 \\
100 \%\end{array}$ & $\begin{array}{l}-0,046 \\
33,6 \%\end{array}$ & $\begin{array}{l}-0,119 \\
86,9 \%\end{array}$ & $\begin{array}{c}-0,027 \\
19,7 \%\end{array}$ \\
\hline 8,1 & 15,0 & 10 & 16,62 & $\begin{array}{r}-3,866 \\
100 \%\end{array}$ & $\begin{array}{c}-3,921 \\
101 \%\end{array}$ & $\begin{array}{r}-3,883 \\
100 \%\end{array}$ & $\begin{array}{r}-3,932 \\
102 \%\end{array}$ \\
\hline 10,1 & 15,0 & 10 & 17,36 & $\begin{array}{r}-4,527 \\
100 \%\end{array}$ & $\begin{array}{l}\text { n.k. } \\
-\end{array}$ & $\begin{array}{r}-4,546 \\
100 \%\end{array}$ & $\begin{array}{r}-4,590 \\
101 \%\end{array}$ \\
\hline
\end{tabular}

trennt, so zeigt sich die Zwischenschicht mit mehr als $80 \%$, gegenüber der Klotzung mit 19\% Anteil am Gesamtverlust, als maßgebender Beitrag und spiegelt damit die eingangs erstelle Definition in Gleichung 64 (Tafel 29) wieder.

Will man die Seilkraftverluste in Spannglasträgern begrenzen ist daher primär die Konstruktion und die Materialwahl der Zwischenschicht maßgebend. Erst im zweiten Schritt kann ein alternatives Klotzungsmaterial zu einem günstigeren Dauerlastverhalten führen.

\subsection{Zusammenfassung}

In diesem Kapitel wurde ein Stabwerkmodell aufgebaut und angewendet, um das experimentell ermittelte Tragverhalten auch numerisch abzubilden. Zunächst konnte gezeigt werden, dass das numerische Modell die analytisch ermittelten Ergebnisse errechnet, sodass die folgenden Betrachtungen auf einer gemeinsamen Basis erfolgen konnten.

Es zeigte sich, dass die Annahmen zur Steifigkeit der geklebten Umlenkpunkte einen wesentlichen Einfluss auf das Berechnungsergebnis hat. In dessen Folge wurde ein Werte-
Tafel 30

Seilkraftänderungen nach $1000 \mathrm{~h}$ unter Annahme der gemeinsamen Kriechwirkung von Klotung und Zwischenschicht $\left(\eta_{p}(K Z)\right)$ sowie der ausschließlichen Wirkung der Klotzung $\left(\eta_{\mathrm{p}}(\mathrm{K})\right)$, der Zwischenschicht $\left(\eta_{p}(Z)\right)$ und ohne Längskriechanteile, bei einziger Wirkung der initialen seitlichen Durchbiegung $\left(\eta_{p}(1)\right)$.

Aufgrund der nichtlinearen Berechnung kann keine lineare Superposition unterstellt werden, sodass Verhältnisse > $100 \%$ auftreten. 
bereich der Umlenksteifigkeit für die weiteren Erörterungen gewählt.

Die Eigenschaften der Zwischenschicht zeigten sich ebenso als maßgebend auf das Berechnungsergebnis. Darin wurde festgelegt, dass das temperatur- und zeitabhängige Materialmodell aus der Literatur weiterverwendet wird. Entsprechend wurde eine Belastungszeit von $60 \mathrm{~s}$ und eine Raumtemperatur von $23^{\circ} \mathrm{C}$ für die Kurzzeit-Biegeversuche gewählt. Für die Nachrechnung der Dauerlast-Versuche verblieb der Zeitparameter veränderlich bei einem ebenso gewählten, konstanten Temperaturwert.

Die räumliche Durchbiegung als Folge der Systemimperfektion ist bereits unter einer Vorspannbelastung zu berücksichtigen. Gemeinsam mit einer Parameterstudie unter Biegebelastung wurde ein Imperfektionswert von L/1000 zur Nachrechnung der Versuche bestimmt.

Damit zeigten sich diese Modellannahmen innerhalb eines physikalisch möglichen Wertebereichs der Umlenksteifigkeit, Zwischenschichtsteifigkeit und initialer Imperfektion als tauglich für die Nachrechnung der Biegeversuche unter Dauerlasten. Das vorgeschlagene numerische Modell ermittelt qualitative und quantitative Spannkraftverluste, die in wesentlichen Fällen mit den experimentellen Ergebnissen übereinstimmen. Abweichende Ergebnisse wurden diskutiert und weiterer Forschungsbedarf festgestellt, welche die tatsächliche Verformungsfigur über die Trägerlänge detaillierter beschreiben und so die zu wählende Imperfektionsannahme konkretisieren. Darüber hinaus ist eine konstruktive Entlastung der Zwischenschicht zu berücksichtigen und die Steifigkeit der Umlenkkonstruktion in einer Detailuntersuchung zu präzisieren. Daher wird das aufgestellte Stabwerkmodell vorläufig nicht abgelehnt. Es ist zu empfehlen Dauerlasten, auf Spannglasträger soweit zu reduzieren, dass der Einfluss der Imperfektion vernachlässigbar wird. Darüber hinaus ist ein Lastmodell zu entwickeln, welches die Veränderlichkeit der realen Bedingungen berücksichtigt, da, im Gegensatz zu den gezeigten numerischen Berechnungen und experimentellen Studien, in der Realität keine konstanten Dauerlasten auf dem gewählten Niveau auftreten. 
Die eingangs gestellten Fragen wurden beantwortet. Dabei wurden sowohl die Imperfektionsannahme als auch die räumliche Durchbiegung des Trägers als bestimmender $\mathrm{Pa}$ rameter auf das Tragverhalten beurteilt. Darüber hinaus kann der Spannkraftverlust über den Einbezug der Zwischenschichteigenschaften beeinflusst werden. Erst danach sind die Eigenschaften der Umlenkung und der Klotzungskonstruktion wichtig. Daher wird empfohlen, alle benannten $\mathrm{Pa}-$ rameter in einer weiterführenden Modellierung zu berücksichtigen. 


\section{$5 \quad$ Diskussion}

\subsection{Zielsetzung}

In diesem Kapitel werden Hauptaussagen und neue Erkenntnisse der Arbeit rekapituliert und in den Stand der Forschung im Konstruktiven Glasbau eingeordnet. Im Mittelpunkt stehen vorzugsweise Beobachtungen aus Untersuchungen mechanisch vorgespannter Glasträger. Liegen stellenweise keine Ergebnisse vor, kommen Erkenntnisse schlaff bewehrter oder unbewehrter Glaskonstruktionen zur Anwendung. Dabei werden analytische, experimentelle und numerische Ergebnisse gemeinsam betrachtet. Studien aus dem Stahlbeton- und Spannbetonbau ergänzen den Vergleich.

Die Besprechung gliedert sich in drei Teile: Nachdem das Tragverhalten unter kurzzeitiger Beanspruchung, einschließlich dem Rissverhalten und dem Spannungszuwachs in der Bewehrung, evaluiert wurde, erfolgt anschließend die Diskussion der Spannkraftverluste unter Dauerbelastung und der Resttragfähigkeit, um die gewonnenen Ergebnisse zu begründen und zu bewerten.

Folgende Leitfragen sollen beantwortet werden:

- Sind höhere Erstriss- und Traglasten durch eine Bewehrung und Vorspannung möglich?

- Ist eine Überdimensionierung von Glasträgern im Sinne des Sicherheitskonzeptes im Bauwesen weiterhin nötig?

- Sind Spannglasträger sichere und dauerhafte Bauteile?

- Welche Konstruktionen sind zu empfehlen?

Darüber hinaus erfolgt eine kritische Betrachtung der eigenen Untersuchungen. Daraus werden Empfehlungen für den weiterführenden Forschungsbedarf abgeleitet. 


\subsection{Tragverhalten unter kurzzeitiger Beanspruchung}

\subsubsection{Tragverhalten unter Vorspannbelastung}

\section{Spannglasträger}

Die verbundlose Vorspannung ist die wesentliche Neuerung der Spannglasträger dieser Arbeit. Es erfolgte eine Beschreibung des nötige Spannweges zum Aufbringen der Vorspannkraft sowie der daraus resultierenden Verformungen. ${ }^{321}$ Eine numerische Studie bestätigte, dass die initiale Vorverformung auch bei der Beschreibung des Tragverhaltens unter Vorspannung zu berücksichtigen ist. ${ }^{322}$ Schließlich ist die Vorspannkraft mit der gewählten Konstruktion planmäßig in den Glasquerschnitt einleitbar: Es kommt infolgedessen zur Kompression der Biegezugkante und zur Überhöhung des Spannglasträgers.

\section{Glasträger mit vorgespannter Bewehrung}

Analoge Ergebnisse vorgespannter Glasträger sind in der Literatur in der Regel nicht explizit benannt. Dabei beschränken sich erste Sondierungsversuche ${ }^{323}$ auf die Analyse des Tragverhaltens unter Biegebelastung im Anschluss an den Vorspannvorgang sowie auf das Ausarbeiten einer nutzbaren Konstruktion zum Einleiten der Vorspannkraft. In Ergebnissen der jüngeren Zeit wird die Überwachung des Vorspannvorgangs angesprochen ${ }^{324}$ beziehungsweise beispielhafte Einzelergebnisse ${ }^{325}$ für einen im Spannbett vorgespannten Glasträger gezeigt. Darin wird jedoch betont, dass der Einfluss der horizontalen Durchbiegung während des Vorspannvorgangs nicht zu vernachlässigen ist. ${ }^{326}$

321 Vergleiche Kapitel 3.3, ab Seite 107.

322 Vergleiche Kapitel 4.3.5, ab Seite 199.

${ }^{323}$ Vergleiche [Bos et al. 2004], [Louter et al. 2006], [Belis et al. 2006], [Louter 2013] und [Louter et al. 2013b].

324 Vergleiche [Jordão et al. 2014] und [Firmo et al. 2015].

325 Vergleiche [Louter et al. 2013a], [Louter et al. 2014], [Cupac \& Louter 2015] und [Louter \& Cupac 2015].

${ }^{326}$ Auch Belis et al. [2006] stellen als Ergebnis einer numerischen Studie fest, dass geometrische Vereinfachungen zu vermeiden sind und Imperfektionen einen wesentlichen Einfluss auf das Ergebnis haben. 


\section{Spannbetonträger}

Der Spannvorgang im Spannbetonbau ist ein aufwändiges Verfahren, welches sorgfältig vorbereitet und dokumentiert wird, sowie durch Fachpersonal durchzuführen und zu überwachen ist. ${ }^{327}$ Obwohl Betonträgerquerschnitte vergleichsweise gedrungen sind, sollen bei einer Vorspannung externer Spannglieder Biegemomente nach Theorie II. Ordnung berücksichtigt werden. ${ }^{328}$

Das Tragverhalten verbundlos vorgespannter Betonträger wurde in den 1980er Jahren ausführlich untersucht und in [Trost et al. 1984] für Einfeldträger sowie in [Zimmermann 1988] und [Weller 1988] für Durchlaufträger dokumentiert. Die Hauptaussagen dieser Grundlagenuntersuchungen werden zum Vergleich verwendet. Der Spannvorgang wird darin ebenso überwacht, jedoch werden keine detaillierten Angaben über die daraus resultierenden Verformungen gemacht.

\section{Vergleichende Beurteilung}

Vergleicht man alle verfügbaren Ergebnisse, dann zeigen sie, dass eine Vorspannkraft planmäßig in einen Glasquerschnitt einleitbar ist. Damit sind die gewählten Konstruktionen grundsätzlich nutzbar, um das analytisch vorab beschriebene Tragverhalten praktisch umzusetzen. Die eigenen Ergebnisse zeigen, dass eine detaillierte Beschreibung des Verformungsverhaltens, ergänzt um den Vorspannungslastfall, erforderlich ist, um eine sichere und kontrollierbare Anwendung auf hohem Qualitätsniveau, analog dem Spannbetonbau, sicherzustellen. Die hier beschriebenen Erkenntnisse können als Grundlage für eine weitere Entwicklung verwendet werden.

\footnotetext{
327 Vergleiche [DIN EN 1992-1-1, Kap. 5.10.2.3] und [DIN EN 13670 Kap. 7]

328 Vergleiche [DIN EN 1992-1-1, Kap. 5.10.7(1)].
} 


\section{Kritik}

Es bleibt jedoch zu berücksichtigen, dass die verwendeten Querschnittskonzepte ${ }^{329}$ sowie die verfügbaren Konstruktionen zur Einleitung der Vorspannkraft in die Glaskante sehr vielfältig sind und die Vergleichbarkeit der Ergebnisse einschränken. Für diese Arbeit wurden geklebte und geklotzte Varianten konstruiert und die schmale sowie die lange Glaskante als Ort der Lasteinleitung gewählt. Darüber hinaus hat sich gezeigt, dass die Methode der Vorspannung Einfluss auf das Ergebnis haben kann.

\section{Weiterer Forschungsbedarf}

Die Imperfektionsfigur als Vorverdrehung und Vorverschiebung in Abhängigkeit von der Einbaurichtung und der Bewehrungskonstruktion ist zu ermitteln, um eine sichere Berechnung der auftretenden Schnittgrößen, insbesondere der zusätzlichen Verformungen durch die Vorspannung, zu ermöglichen.

Zudem sind Empfehlungen für tolerierbare Verformungen beim Vorspannvorgang abzuleiten und die Folgen des Vorspannvorgangs im Rahmen eines Qualitätssicherungs- und Überwachungskonzepts zu quantifizieren und einzugrenzen.

Die Weiterentwicklung der Lasteinleitung in die Verbundglaskante trägt weiterführend dazu, bei übermäßige Schiefstellungen und nicht tolerierbare Verformungen zu vermeiden. ${ }^{330}$

\footnotetext{
${ }^{329}$ In der Literatur werden einfache Laminatquerschnitte mit unterschiedlichen Zwischenschichtmaterialien und Kantenbearbeitungen untersucht. 330 Dazu kann beispielsweise auf die Ergebnisse in [Ebert 2014] und eine Vorstudie in [Bukieda 2015] aufgebaut werden.
} 


\subsubsection{Trag- und Bruchverhalten unter Biegebelastung}

\section{Spannglasträger}

Spannglasträger verhalten sich unter Biegebelastung zunächst linear elastisch (Bild 93). Analog zum Stahlbetonund Spannbetonbau wird diese Situation ohne sichtbare Risse als „Zustand I" bezeichnet. Eine ergänzende numerische Untersuchung zeigte, dass die Ausprägung der Imperfektionsfigur sowie der vergleichsweise große Wertebereich der Materialkennzahlen der Zwischenschicht maßgebliche Einflussfaktoren sind. ${ }^{331}$

Schließlich kam es zum Erstriss im Glas und somit zum Übergang in den „Zustand II“, wobei eine Steigerung der Erstrisslast mit steigender Vorspannung ermittelt wurde. Insbesondere die für Glas übliche breite Streuung der Bruchspannungswerte ${ }^{332}$ überlagerte das Ergebnis und ist daher in der Auslegung solcher Konstruktionen zu berücksichtigen. Entweder versagten die Träger unmittelbar oder es konnte eine Weiterbelastung nach einem Sprung in der Traglastkurve erfolgen. Der Lastabtrag endete mit einem vollständigen Versagen unterhalb der Erstrisslast. In keinem Fall kam es zum Fließen oder Versagen der Bewehrung. ${ }^{333}$ Somit wurden keine höheren Traglasten nachgewiesen. Das zugehörige Versagen kann als „explosionsartig“ beschrieben werden. Dabei wurden zudem Teile des Glases vom Querschnitt abgesprengt. Anschließend trug das Bewehrungsseil den gebrochenen Träger.

\section{Glasträger mit vorgespannter Bewehrung}

Vorgespannte Glasträgerkonstruktionen aus der Literatur zeigten ein linear elastisches Tragverhalten bis zum Erstriss. Anschließend war eine Weiterbelastung möglich. Zu deren Kategorisierung werden die Definitionen nach Nielsen

\footnotetext{
331 Vergleiche Kapitel 4.3, ab Seite 188.

332 Vergleiche Bild 51, Seite 123. $v=s / \bar{x}=0,14$.

333 Eine Bezeichnung als „Zustand III“ oder „yield stage“ ist beispielsweise in [Nielsen 2009] und [Louter 2011] üblich.
} 
[2009], welche dem Stahlbetonbau entlehnt sind, verwendet und in Bild 93 (A und B) dargestellt.

Im Vergleich zur Erstrisslast erfolgte die Weiterbelastung „unterbewehrter“ Träger bei geringeren Werten ${ }^{334}$, etwa auf dem Niveau der Erstrisslast ${ }^{335}$ oder in „normal bewehrten“ Konstruktionen mit einer deutlichen Traglaststeigerung über die Erstrisslast hinaus, sodass es mitunter zum Fließen der Bewehrung ${ }^{336}$ kam. Louter [2011] beschreibt die so klassifizierten Träger als „duktil“ und definiert einen Zusammenhang mit dem Bewehrungsgrad für den Glasbau. Demnach führt ein steigender Bewehrungsgrad zu einer Vergrößerung, ein fallender Bewehrungsgrad zu einer Verringerung des in Kapitel 3.3.4 (Seite 112) definierten Duktilitätskennwertes $k_{\text {duk.F. }}$

Die Erstrisslast mechanisch vorgespannter Glasträger konnte im Vergleich zu unbewehrten ${ }^{337}$ und schlaff bewehrten ${ }^{338}$ Trägern gesteigert werden. Das finale Versagen war in der Regel ebenso „explosionsartig“.

\section{Spannbetonträger}

Betonträger mit Vorspannung ohne Verbund zeigten in vorangegangenen Untersuchungen bis zum ersten Riss, und damit bis zum Übergang in den Zustand II, ein linear elastisches Tragverhalten. ${ }^{339}$ Anschließend riss die Betonzugzone sukzessive ohne ausgeprägten Sprung in der Traglastkurve, sodass sich die Geschwindigkeit des Lastanstiegs im weggesteuerten Biegeversuch verringerte (Bild 93, C). Dieser Übergang wurde jedoch, anders als bei Trägern mit Spann-

${ }^{334}$ Im Fall der unbewehrten Referenzprüfkörper in [Louter 2013], [Louter et al. 2013b], [Louter et al. 2013a], [Louter et al. 2014] und [Cupac \& Louter 2015].

335 Im Fall von schlaff bewehrten Prüfkörpern in [Nielsen 2009] und [Louter 2013].

336 Vergleiche [Bos et al. 2004], [Louter et al. 2006], [Louter 2013], [Louter et al. 2013b], [Louter et al. 2013a], [Jordão et al. 2014], [Cupac \& Louter 2015] (für schlaffe und vorgespannte Bewehrung) und [Louter \& Cupac 2015]. Für einen hybriden Querschnitt mit ausgeprägten Stahlflaschen auch in [Firmo et al. 2015].

337 Vergleiche [Cupac \& Louter 2015], [Louter et al. 2014], [Louter 2013], [Louter et al. 2013b] und [Louter et al. 2013a].

338 Vergleiche [Cupac \& Louter 2015] und [Louter \& Cupac 2015].

339 Vergleiche [Trost et al. 1984], [Zimmermann 1988] und [Weller 1988]. 


\section{Bild 93}

Prinzipbilder der Versagenstypen "normal bewehrt", „unterbewehrt" und „semispröde" auf Basis der Definition in [Nielsen 2009] und [Louter 2011] im Vergleich zum typischen Verhalten von Spannbetonbauteilen.

„Überbewehrte“ Träger versagen unmittelbar mit dem Erreichen der Erstrisslast $\left(\mathrm{F}_{\mathrm{cr} .1}=\mathrm{F}_{\text {Bruch }}\right)$.

Im Gegensatz zum Tragverhalten bewehrter Glasträger ist für Spannbetonträger kein ausgeprägter Sprung in der Traglastkurve üblich. (Vergleiche [Geßner et al. 2017, Bild 1].)

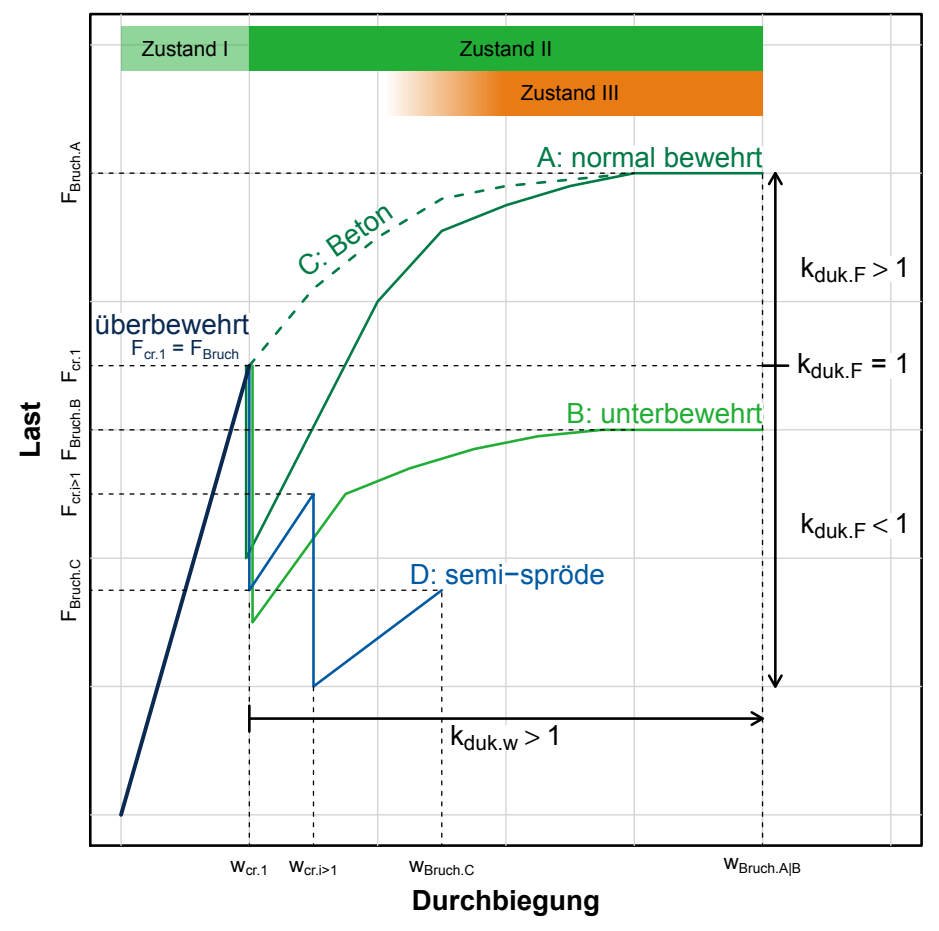

bewehrung im Verbund, als vergleichsweise abrupt beschrieben. Man ging von einer nahezu abgeschlossenen Rissbildung unmittelbar nach dem Erstriss aus. Letztlich war ein Herausplatzen der Betondruckzone für Träger mit Vorspannung ohne Verbund typisch. Als Grund dafür wurde insbesondere die mit steigender Last vergrößerte Einschnürung der Betondruckzone und die daraus resultierende Querverzerrung genannt. Diese führte bei einer lokalen Überschreitung der Betonzugfestigkeit zur Bildung von Längsrissen, welche ursächlich für ein Herausbrechen des Betons waren. ${ }^{340}$

\section{Vergleichende Beurteilung}

Nur ein Teil der eigenen Spannglasträger zeigte ein vergleichbares Verhalten. Damit erreichen andere Glasträgerkonstruktionen stabilere Werte. Einschränkend ist festzustellen, dass nur die Ergebnisse in [Louter 2013], [Louter et al.

340 Vergleiche [Trost et al. 1984]. 
2013b], [Louter et al. 2013a] sowie [Louter et al. 2014] eine ähnliche, verbundlos vorgespannte Konstruktion mit rein mechanisch verankerten, jedoch nicht umgelenkten, Spanngliedern und ohne weitere (schlaffe) Bewehrung enthielten. Zudem handelte es sich um Floatglasquerschnitte mit steiferer lonomer-Zwischenschicht und kürzeren Spannweiten. Die vorliegende Konstruktionsvielfalt zeigt, dass ein unmittelbarer Ergebnisvergleich nicht auf einheitlicher Basis möglich ist. Daher können mit dem heutigen Stand des Wissens keine allgemeingültigen Aussagen für vorgespannte Glasträger beliebiger Material- und Geometriewahl getroffen werden.

Im Weiteren beschreibt Nielsen [2009] „überbewehrte“ Träger, welche im Anschluss an die linear elastische Entwicklung unmittelbar und vollständig ohne Plastifizierung der Bewehrung versagen $\left(k_{F \text {.duk }}=1\right.$ und $\left.k_{w \text {.duk }}=1\right)$. Die bisher noch nicht eingruppierten eigenen Spannglasträger zeigten derartige Eigenschaften. Dafür spricht insbesondere die vergleichsweise geringe Ausnutzung der metallischen Querschnitte bei Seildurchmessern von $8,1 \mathrm{~mm}$ und $10,1 \mathrm{~mm} \cdot{ }^{341}$ Die Träger dieser Arbeit sind als schlank zu charakterisieren und zeigten daher eine deutlich sichtbare seitliche Durchbiegung, welche ein Versagen ankündigte. Es bleibt jedoch offen, inwiefern diese Eigenschaft genutzt werden kann, um ein sicheres Bauteilverhalten zu ermöglichen.

Darüber hinaus existiert nach Nielsen [2009] eine weitere Kategorie, bei der nach dem Steifigkeitsverlust infolge des Erstrisses mindestens ein zusätzlicher linear elastischer Belastungspfad folgt $\left(\mathrm{k}_{\mathrm{w} \text {.duk }} \geq 1\right.$ mit beliebigem $\left.\mathrm{k}_{\mathrm{F} \text {.duk }}\right)$. Dieser wurde mit einem Versagen der Verankerung in Verbindung gebracht. ${ }^{342}$ Ein solches schrittweises Versagen trat in dieser Arbeit sowohl für unbewehrte als auch für schlaff bewehrte Träger auf und kann somit nicht auf die Wirkung der Bewehrung, ihr Verbundversagen oder die Vorspannung zurück geführt werden. Es wird daher in Erweiterung zu Nielsen [2009] die Bezeichnung "semi-spröde“ vorgeschlagen, da es einerseits durch ein plötzliches Versagen ohne Plastifizierung gekennzeichnet ist, aber andererseits ein vollständiges

\footnotetext{
341 Vergleiche auch Tafel 32, Seite 217.

342 Bezeichnung als ",anchorage failure" in [Nielsen 2009] mit Bezug auf einen rein linear verbundenen Bewehrungsquerschnitt.
} 
Versagen durch einen sich vorab einstellenden Teilzerstörungszustand anzeigt (Bild 93, D). In weiterführenden Untersuchungen ist eine detaillierte Trennung der als ursächlich herausgestellten Einzeleffekte mit einer größeren Anzahl von Prüfkörpern vorzunehmen.

Ein „explosionsartiges“ Versagen wurde sowohl bei Versuchen aus der Literatur für Beton- und Glasträger, als auch bei den eigenen Untersuchungen festgestellt (Bild 94). Somit wird ein ähnlicher Versagensmechanismus unterstellt. Entsprechend wird im Stahlbeton- und Spannbetonbau eine querdehnungsbehindernde Bewehrung vorgesehen. Diese kann als lokale konstruktive Verstärkung auch im Glasbau geplant werden. So können die druckbeanspruchten Glaskanten von Spannglasträgern mit zusätzlichen Umlenkpunkten beklebt werden, welche eine Querdehnungsbehinderung ermöglichen.
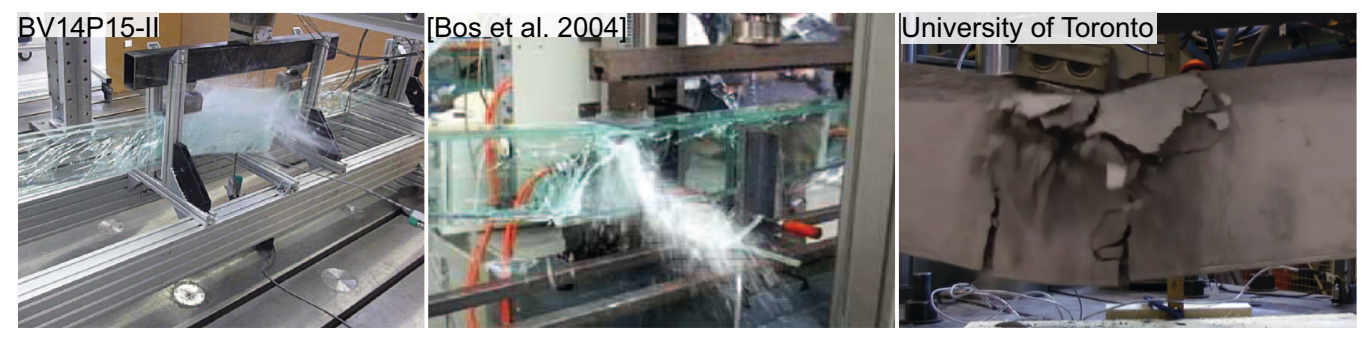

Bild 94

Momentaufnahmen: Versagen des Prüfkörpers BV 14 im Vergleich zu einem vorgespannten Glasträger aus [Bos et al. 2004] sowie einem Spannbetonträger aus einem Biegeversuch an der University of Toronto, Kanada.
Bos et al. [2004] führt das „explosionsartige“ Verhalten auf die durch die Vorspannung zusätzlich eingebrachte elastische Energie zurück. Entsprechend muss vorgeschlagen werden, die Vorspannkraft zu reduzieren, um einen Erstriss bei geringeren Lasten zu ermöglichen, sodass die Entwicklung eines Rissbildes allmählich erfolgt.

Darüber hinaus ist zu berücksichtigen, dass Glasträger, insbesondere nach dem vollständigen Bruch oder einem Teilversagen, schlanke Querschnittsanteile beinhalten und somit unter einer Biegebelastung zum Stabilitätsverlust neigen. Zu deren Berücksichtigung kann die Druckzonenhöhe berechnet und eine relevante Druckzonenlänge ermittelt werden, womit ein rechnerischer Knicknachweis als Ersatzstab ermöglicht wird (Bild 95). 


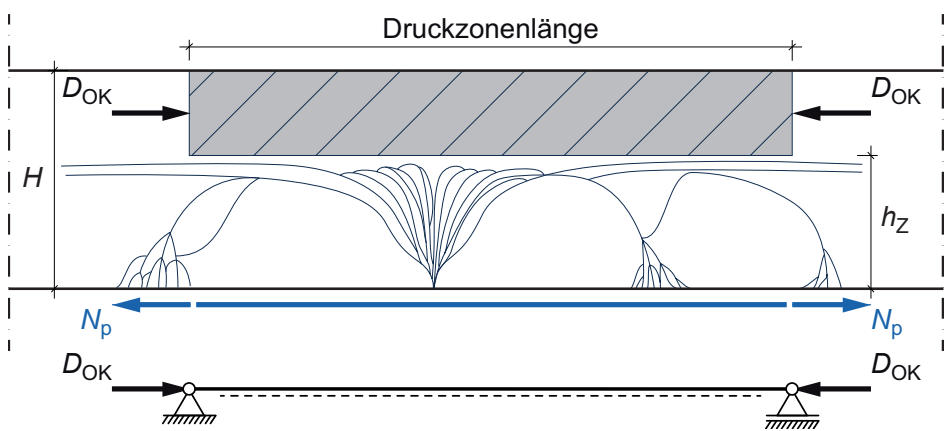

Zwar wurden in den hier vorgestellten Versuchen derarte Versagensmechanismen vermutet, jedoch fehlen heute noch belastbare Daten zur Ermittlung einer relevanten Druckzonenlänge für den Konstruktiven Glasbau, woraus sich eine weiterführende Forschungsaufgabe ergibt.

Alternativ muss eine Konstruktionsweise zur Verfügung gestellt werden, die in der Lage ist, die frei werdende elastische Energie sicher aufzunehmen. Damit können auch „semispröde" und überbewehrte Glasträger ein sicheres Tragverhalten erlangen. Dies kann durch eine Lastumlagerung erreicht werden, bei der sich das Tragverhalten eines Hängewerks einstellt - der verbleibende Verbundglasquerschnitt sackt auf das Bewehrungsseil herab ohne herunter zu falIen. ${ }^{343}$ Einer Gefährdung eines Nutzers unterhalb der Verglasung wird damit begegnet. ${ }^{344}$

Um nach dem Erstriss weiterhin eine ausreichende Tragfähigkeit zu gewährleisten, müssen, entsprechend dem Verhältnis der Teilsicherheitsbeiwerte ${ }^{345}$, im Grenzzustand der Tragfähigkeit bis zu 50\% höhere Lasten abgetragen werden. Dies kann für „,normal bewehrte“ Glasträger analog der Betonbemessung erfolgen. Die hier gezeigten Spannglasträger zeigen jedoch zunächst einen Abfall der Lastkurve nach dem Erstriss $\left(k_{\text {duk.F }}<1\right)$. Dieses Tragverhalten kann nur dann als

\footnotetext{
343 Luible [2004] bezeichnet ein solches Bauteil als „reststandsicher“.

344 So wurde bespielsweise in Fahlbusch [2007] eine Überkopfverglasung aus einem Verbundsicherheitsglas aus Einscheiben-Sicherheitsglas durch Stahlseile unterspannt, welche das herabfallende Glaspaket im Bruchfall auffingen. Damit war den Anforderungen eines sicheren Nachbruchzustandes genüge getan, obwohl diese Überkopfverglasung allein nach [DIN 18008-2, Kap. 5.1 und 5.9] als nicht ausreichend sicher angesehen wird.

345 Vergleiche $\gamma_{\text {Q.GZT }}=1,5$ und $\gamma_{\text {Q.GZG }}=1,0$ in [DIN EN 1990].
}

Bild 95

Schematische Ermittlung einer Druckzonenlänge in der Oberkante (OK) im gebrochenen Glasträger. (ohne Maßstab) 
günstig angesetzt werden, wenn eine bedeutende Lastreduzierung durch eine Lastumlagerung auf benachbarte Bauteile, beispielsweise in einem Glasdachsystem mit mehreren Einzelträgern, möglich ist, sodass die auf dem Einzelbauteil verbleibende Bemessungslast mindestens auf das Niveau der Gebrauchslast ohne Lastumlagerung zurück fällt. Ein entsprechendes Glasträgersystem muss daher einen Nachweis der Systemredundanz erfüllen. Daraus ergibt sich die Aufgabe, einen Nachweis des sicheren Systemversagenverhaltens zu entwickeln.

Nichtsdestotrotz verbleibt das primäre Ziel, auch ein Einzelbauteil sicher zu gestalten und ein duktiles Verhalten zu erreichen. Jedoch sind derzeit keine Mindestwerte der Duktilität von bewehrten Glasträgern definiert. Daher werden die wenigen, heute zur Verfügung stehenden Vergleichswerte in Tafel 31 zusammengestellt. Darin zeigt sich, dass Spannglasträger im Vergleich zu anderen mechanisch vorgespannten Glasträgern deutlich kleinere Duktilitätskennwerte zeigen. Diese wiederum erreichen jedoch ihrerseits das Niveau aus dem Spannbetonbau nicht. Insbesondere der mögliche, weitere Verformungsweg nach dem Erstriss $k_{w . d u k}$ erwies sich im Vergleich zu den Minimalwerten im Spannbetonbau bisher als deutlich größer. Daher ist zum einen zu prüfen, ob beim Bau schlanker Glasträger auch die horizontale Durchbiegung als Duktilitätskriterium aufgenommen wird. Zum zweiten obliegt es der weiterführenden Forschung, klare Grenzwerte im Sinne eines integralen Sicherheitskonzeptes zu definieren. ${ }^{346}$ Es wird vorgeschlagen, bis auf weiteres das Erreichen der Mindestwerte aus dem Spannbetonbau anzustreben.

\section{Kritik}

Insgesamt stellt sich die Frage, warum in dieser Arbeit vielfach ein überbewehrter Träger konstruiert und untersucht wurde. Das beobachtete Tragverhalten ist maßgeblich darauf zurückzuführen. Zunächst erfolgte die Auswahl der Seil-

346 Diese Fragen werden auf europäischer Ebene derzeit mit der Entwicklung eines „Eurocode for Structural Glass“ beraten. Vergleiche [Feldmann et al. 2014]. 


\begin{tabular}{|c|c|c|c|c|}
\hline & \multirow{2}{*}{$\begin{array}{l}\text { Spannglas } \\
\text { Tafel } 13 \text {, } \\
\text { Seite } 129\end{array}$} & \multicolumn{2}{|c|}{ Glasträger } & \multirow{2}{*}{$\begin{array}{l}\text { Spannbeton } \\
\text { ohne } \\
\text { Verbund }^{d}\end{array}$} \\
\hline & & $\begin{array}{l}\text { ohne } \\
\text { Verbund }^{a}\end{array}$ & $\begin{array}{l}\text { sofortiger } \\
\text { Verbund }^{b}\end{array}$ & \\
\hline \multirow{2}{*}{$\begin{array}{r}\mathrm{k}_{\mathrm{F} \text {.duk }} \min \\
\max \end{array}$} & 0,72 & 1,17 & 1,26 & 1,36 \\
\hline & 0,95 & 4,21 & 1,71 & 3,51 \\
\hline \multirow{2}{*}{$\begin{array}{r}\mathrm{k}_{\mathrm{w} . \text { duk }} \min \\
\max \end{array}$} & 1,02 & $1,50^{c}$ & $1,70^{c}$ & 8,01 \\
\hline & 1,30 & $16,0^{c}$ & $30,0^{c}$ & 42,0 \\
\hline Anzahl & 10 Prüfkörper & \multicolumn{2}{|c|}{23 Prüfkörper } & 5 Prüfkörper \\
\hline \multicolumn{5}{|c|}{$\begin{array}{l}\text { a } \text { [Bos et al. 2004], [Louter et al. 2006], [Louter 2013], [Louter et al. 2013k } \\
b \text { [Louter et al. 2014], [Louter \& Cupac 2015], [Cupac \& Louter 2015]. } \\
{ }^{c} \text { Aus Ablesewert im Last-Durchbiegungs-Diagramm. } \\
{ }^{d} \text { [Trost et al. 1984]. }\end{array}$} \\
\hline
\end{tabular}

Tafel 31

Vergleich minimaler und maximaler Duktilitätskennwerte aus dem Konstruktiven Glasbau und dem Spannbetonbau. durchmesser zur Untersuchung des Einflusses des Bewehrungsgrades. Entsprechend wurden die Vorspannkräfte am Seil des geringsten Durchmessers orientiert. Zudem war die Abschätzung der zu erwartenden maximalen Seilkräften unsicher, da hohe Glasfestigkeiten im Einzelfall möglich waren, wodurch auf der sicheren Seite liegend große Sicherheitsreserven bei der Auslegung der Seile angesetzt wurden. Erwartungsgemäß resultierten die größten Seilkräfte aus der Untersuchung der Resttragfähigkeit (Tafel 32). Dabei sollte ein Versagen des Seils in allen Tragsituationen vermieden werden. Mit den hier vorliegenden Ergebnissen kann nun im nächsten Schritt eine Auslegung des Seils mit höheren Auslastungen vorgenommen werden.

\begin{tabular}{rccc}
\hline \multicolumn{2}{c}{ Bewehrung } & \multicolumn{2}{c}{ maximale Seilkräfte [kN] $\left(\mathbf{N}_{\mathbf{p . m a x}} / \mathbf{Z}_{\mathbf{B . k}}\right)$} \\
$\mathbf{d}_{\mathbf{p}}$ [mm] & $\mathbf{P}_{\mathbf{m} 0}[\mathbf{k N}]$ & Kurzzeit-Biegelast & Resttragfähigkeit \\
\hline 8,1 & 0 & - & $5,2(9 \%)$ \\
10,1 & 0 & $13,8(15 \%)$ & - \\
\hline 5,0 & 9,0 & - & $15,1(72 \%)$ \\
6,1 & 9,0 & $11,8(45 \%)$ & - \\
8,1 & 15,0 & $21,2(36 \%)$ & $24,3(41 \%)$ \\
10,1 & 15,0 & $23,4(25 \%)$ & $25,1(27 \%)$ \\
\hline
\end{tabular}

Zudem wurden die Kurzzeit-Biegeversuche dieser Arbeit sowie die in der Literatur mit zusätzlichen seitlichen Lagerungen durchgeführt, um ein frühzeitiges seitliches Ausweichen der Träger zu verhindern. Diese Versuchsanordnung bildet jedoch keine reale Baukonstruktion ab. Daher ist zu prüfen, inwieweit die beobachteten Effekte auf eine reale Konstruktion übertragen werden können. Es bleibt zu befürchten, dass die günstigen Effekte einer mechanischen Vorspannung von

\section{Tafel 32}

Maximale Seilkräfte unter kurzzeitiger Biegelast (Kapitel 3.3, ab Seite 107) und während der Untersuchung der Resttragfähigkeit (Kapitel 3.5, ab Seite 147) sowie deren Auslastung gegenüber der charakteristischen Werte aus Tafel 9 , Seite 106. 
Glasträgern überschätzt werden, da in der Realität zusätzliche Trageffekte wirken. Zur Eingrenzung des Ergebnisumfangs ist dieses Vorgehen jedoch sinnvoll, sodass sich aus dem Vergleich zwischen dem realen Bauteilverhalten und den vorgestellten Laboruntersuchungen eine Aufgabe für die weiterführende Forschung ergibt.

\section{Weiterer Forschungsbedarf}

Es besteht somit weiterer Forschungsbedarf in der Trennung der Einzeleffekte, die den Tragverlust bewirken innerhalb einer umfangreicheren experimentellen Studie. Weiterhin ist das Tragverhalten der Druckstrebe im gebrochenen Glasquerschnitt zu beschreiben und der Nachweis der Systemredundanz für eine Bemessung zu untersuchen.

Zudem ist die horizontale Durchbiegung bei der Beschreibung der Duktilität von Glasträgern zu berücksichtigen, sowie Duktilitätskennzahlen für den Glasbau festzulegen oder die Mindestwerte des Spannbetonbaus zu übernehmen.

Schließlich sind die Laborergebnisse auf reale Baukonstruktionen zu übertragen.

\subsubsection{Rissverhalten unter Biegebelastung}

\section{Spannglasträger}

Eine Biegelast auf einen Spannglasträger dekomprimiert die vorgedrückte Glaskante bis zum Erreichen der kritischen Zugspannung (Bild 96, oben). An der Stelle des ungünstigsten Verhältnisses von Zugspannung und Zustand der Glasoberfläche kam es zum Glasbruch und zur abrupten Ausbildung eines Bruchfächers (Bild 96, (1)) sowie der Bildung von weiter verzweigten Rissausläufern.

Die Rissausbreitungsgeschwindigkeit ist so groß, dass praktisch sofort ein abgeschlossenes Rissbild vorlag, welches 
nahezu unverändert verblieb, bis es infolge der fortschreitenden Belastung zu einem weiteren Bruchfächer kam. Darüber hinaus überlagerte ein Flächenriss infolge der thermischen Vorspannung des Glases dieses Geschehen.

Dabei wurde die druckbeanspruchte Oberkante gestaucht, sodass es fallweise zum örtlichen Versagen kam (Bild 96, (2)).

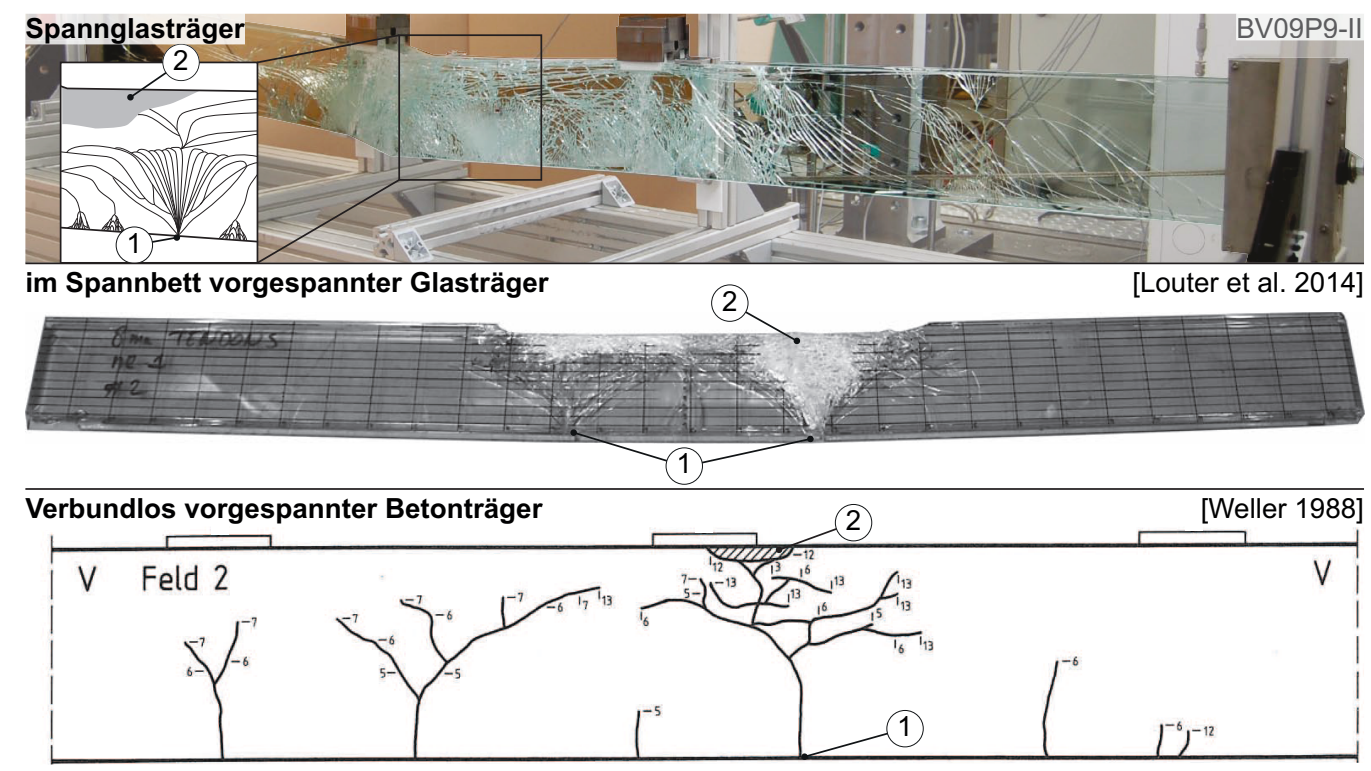

\section{Bewehrte und unbewehrte Glasträger}

Nur vereinzelt wurde die Rissbildung in Glasträgern detailliert angesprochen. Hess [2000] berichtet nach Biegeversuchen mit weit variierten, jedoch unbewehrten Glasträgern von Rissfächern, welche vom Bruchursprung an der Zugkante ausgingen. Diese blieben für Floatglasträger auf den Bereich beiderseits des Ursprungs begrenzt, wobei die Risslinien nahe des Obergurtes vertikal in die Oberkante ausliefen. ${ }^{347}$ Für Träger aus teilvorgespanntem Glas ergaben sich zusätzlich zum Fächer Risse über den gesamten Trä-
Bild 96

Rissverhalten von Spannglasträgern (oben), vorgespannten Glasträgern mit sofortigem Verbund (mittig) und einem verbundlos vorgespannten Betonträger (unten).

(1) Rissursprung

(2) lokal zerstörte Druck-

${ }^{347}$ Eine typische, vergleichbare Abbildung findet sich in Bild 99, links. 
Bild 97

Beispiel für Horizontalrisse in der Druckzone unterhalb des Umlenkungspunktes aus dem Projekt „Spannglasbrücke" in [Engelmann \& Weller 2016].

Während das Rissbild beiderseits der Klebung sehr fein erscheint, bilden sich im Bereich der Klebfläche weniger und längs ausgerichtete Risslinien. ger mit zum Teil unerwartet kleinen Bruchstücken. ${ }^{348}$ Erst als Verbundglaskonstruktion aus zwei TVG-Schichten und einer Scheibe Einscheiben-Sicherheitsglas mit PVB-Zwischenschicht ergab sich ein Rissbild mit „Druckgewölbe“, welches durch auslaufende Rissenden in die Unterkante und einem nahezu rissfreien Obergurt gekennzeichnet war. Dabei verblieb die Schicht aus Einscheiben-Sicherheitsglas ungebrochen. Lediglich horizontale Risse wurden im Druckbereich aufgefunden und die Vermutung eines Versagens infolge einer Querzugwirkung beschrieben (Bild 97).

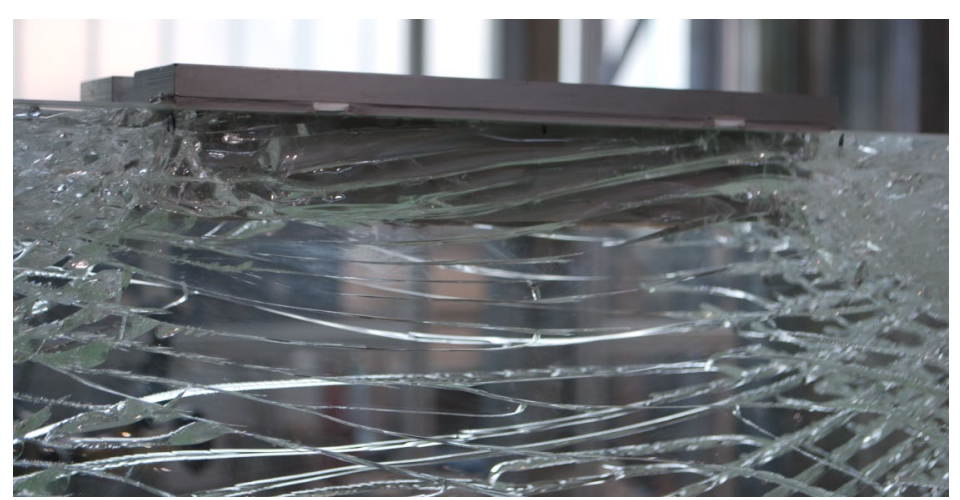

In [Belis et al. 2009b] sind vergleichende Biegeversuche an unbewehrten Glasträgern mit PVB- sowie mit steiferer lonomer-Zwischenschicht dokumentiert. Das Rissbild in den Floatglasträgern blieb durch die geänderte Folie unbeeinflusst. Jedoch zeigte sich ein anderes Tragverhalten zwischen den aufklaffenden Rissursprüngen. Mit einer PVBZwischenschicht wurden größere Rissöffnungen möglich (Bild 98), welche mit einer Delamination der Folie vom Glas endeten. Demgegenüber überbrückte die verwendete steifere Folie nur vergleichsweise kleinere Risslücken und es kam zum Versagen durch den Anriss der Folie. Eine signifikante Übertragung von Zuglasten über die Zwischenschicht und damit eine tragfähige Rissüberbrückung wurde nicht beobachtet.

Im Gegensatz zum üblichen „Bruchfächer“ in Verbundglasträgern (Bild 99, links) bildete sich in Hybridträgern mit einer

\footnotetext{
348 Dies wurde mit einem vergleichsweise hohen thermischen Vorspanngrad begründet. Insofern kann ein Vergleich der Rissbilder mit den Ergebnissen aus dieser Arbeit nur eingeschränkt erfolgen. Vergleiche [Hess 2000].
} 


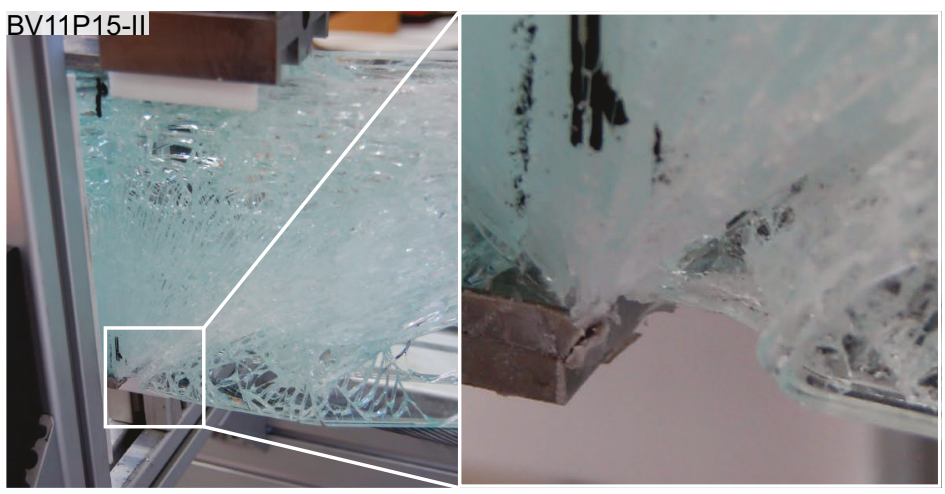

Bild 98

Klaffende Lücke zwischen zwei Rissflanken im Prüfkörper BV 11. Die Zwischenfolie überbrückt den Riss mit großer Dehnung.

Polycarbonat-Zwischenschicht eine weiterhin intakte Druckzone an der Oberkante und eine andersartige „Bruchzwiebel“, deren Bruchlinienenden nach innen ausliefen (Bild 99, mittig). In einer Verbundglasvariante mit teilvorgespanntem Glas wurden Bruchausläufer nach außen mit der für TVG typischen Verästelung an der Trägerunterkante beobachtet (Bild 99, rechts). Dabei konnte die PolycarbonatZwischenschicht eine Zuglastübertragung über die Rissflanken gewährleisten.

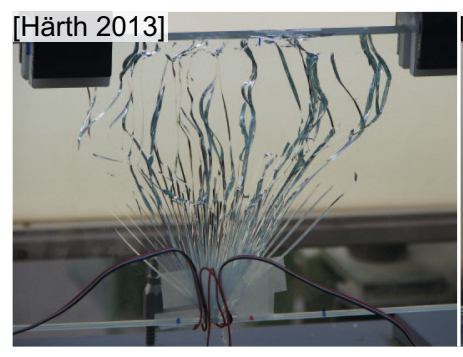

Louter [2011] zeigt die Rissentwicklung in schlaff bewehrten Glasträgern. Auch hier entstand ausgehend von einem Rissursprung ein lokaler Rissfächer im Floatglas. Eine Weiterentwicklung des Rissbildes war durch das zunehmende Auftreten weiterer Rissfächer im Bereich der maximalen Biegebelastung charakterisiert. Zudem beeinflussten die Eigenschaften des Zwischenmaterials auch die Ausbildung des Risses im Verbundglas. Dabei wurde eine Wirkung als Rissbarriere beschrieben, sodass der Anriss einer Glasschicht nicht unmittelbar an gleicher Stelle in die danebenliegende Verbundglas-Schicht übertragen wurde - es kam zu einer Verteilung einzelner Rissfächer über die Trägerlänge.

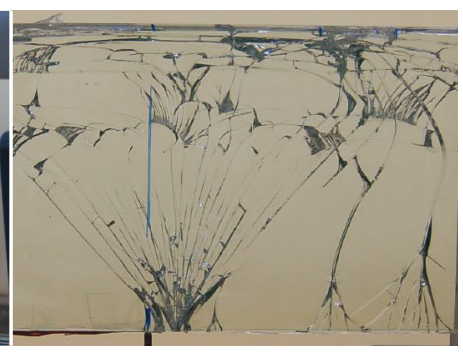

Bild 99

Typische Rissfächer in Verbundglasträgern aus Floatglas (links) und „Bruchzwiebel“ (mittig) in Hybridträgern nach Härth [2013] sowie ein typischer Bruchfächer in einem VSG-Träger aus TVG im dazugehörigen Forschungsprojekt am Institut für Baukonstruktion. 
Demgegenüber zeigten sich durchgehende Rissursprünge in allen Schichten im Verbundglas bei einer steifen, flächigen Verklebung ${ }^{349}$, sodass sich an dieser Stelle eine ungünstige Gelenkwirkung ausbilden konnte. Die verwendete Stahlbewehrung konnte, aufgrund ihrer im Vergleich zur Zwischenschicht deutlich größeren Steifigkeit, eine effektive Überbrückung der Rissflanken und damit eine signifikante Zuglastüberleitung sicherstellen.

Als typisches Beispiel der Rissentwicklung mechanisch vorgespannter Floatglasträger zeigen Louter et al. [2014] typisierte Bruchbilder nach dem Versagen der Prüfkörper. ${ }^{350}$ Darin folgten einem ersten lokalen Anriss in der Regel weitere Einzelrissfächer und somit eine Rissverteilung mit einem finalen Zerdrücken der Bruchstücke in der Druckzone und in dessen weiterer Folge ein Versagen des Querschnitts (Bild 96, mittig). Die metallische Bewehrung war zu einer Überbrückung der Rissflanken in der Lage.

Die Zusammenstellung zeigt, dass das Rissbild in Glasträgern vornehmlich durch die Wahl des Glas- und Zwischenschichtmaterials und nur in geringerem Maße durch die Bewehrung oder eine Vorspannung beeinflusst wird. Eine Bewehrung kann eine Brücke zwischen zwei Rissflanken bilden, sodass das Versagen der Träger vermieden wird.

\section{Spannbetonträger}

Trost et al. [1984] beschreiben das Rissverhalten verbundlos vorgespannter Betonträger. Dabei wurde ein schneller, jedoch nicht schlagartiger Anstieg vertikaler Einzelrisse festgestellt, sodass es zu einer raschen Einschnürung der Druckzone kam. Das damit verbundene Auftreten großer Druckverzerrungen verursachte ein Ablenken der Risswurzeln in horizontaler Richtung als Folge des begleitenden Querzuges (Bild 96, unten).

${ }^{349}$ Es wurde der als Zwischenschichtmaterial unübliche UV-härtende Acrylatklebstoff DELO® GB368® verwendet.

350 Ähnliche Berichte finden sich in [Bos et al. 2004], [Louter et al. 2006], [Louter 2013], [Louter et al. 2013b], [Louter et al. 2013a] und [Louter 2013]. 


\section{Vergleichende Beurteilung}

Allen Rissbildern ist gemein, dass ein Risswachstum in Form eines Einzelrisses oder Rissfächers ausgehend von der vorgedrückten, zugbeanspruchten Kante initiiert wird. Die Rissenden werden in der Druckzone horizontal abgelenkt. Entsprechend wird eine vergrößerte Druckzonenhöhe, beispielsweise durch eine mechanische Vorspannung, mit einem vergrößerten verbleibenden Querschnitt assoziiert. Dies wurde im Glasbau bisher nur qualitativ beobachtet, sodass die weiterführende Forschung die Beschreibung der Druckzonenhöhe vor und nach dem Erstriss beinhalten sollte.

Anders als im Betonbau kann ein um die starke Achse biegebeanspruchter Verbundglasquerschnitt einen Rissfortschritt über die gesamte Trägerbreite vermeiden (Bild 100). Dies kann günstig wirken, da so ein größerer Querschnittsanteil ungerissen verbleibt. Jedoch besteht die Gefahr, dass ein schlanker Glasquerschnitt auf diese Weise einen erheblichen Anteil seiner Biegesteifigkeit um die schwache Achse verliert und somit die Tendenz zum seitlichen Versagen infolge einer Lastexzentrizität vorherrscht.
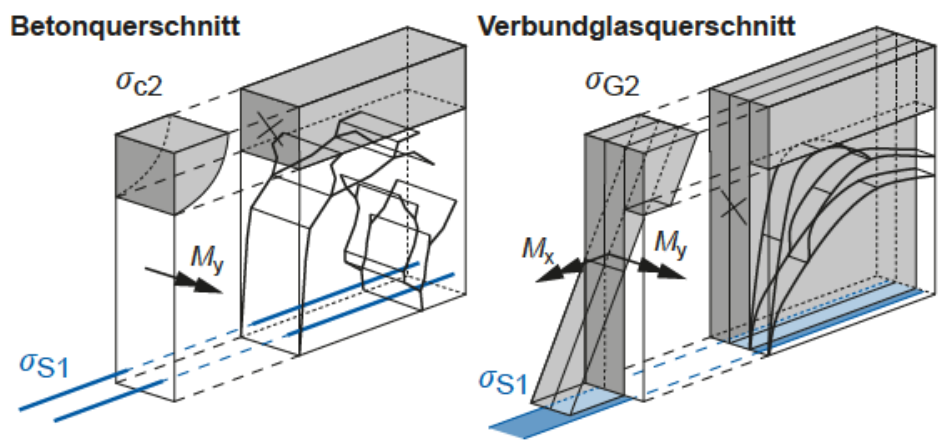

Bild 100

Risssperre.

Während ein Riss in einem Stahlbeton- oder Spannbetonbauteil (links) als über die Trägerbreite durchgehend angenommen wird, kann er in einem Verbundglasträger (rechts) auf eine oder mehrere Einzelscheiben begrenzt bleiben. Dies führt zu einem andersartigen Tragmechanismus.

Während in anderen Untersuchungen überwiegend thermisch entspanntes Floatglas verwendet wird, wurden die Spannglasträger dieser Arbeit mit teilvorgespanntem Glas konzipiert. Demnach stellt sich die Frage nach der Wahl eines passenden Glaswerkstoffes, um eine graduelle Rissentwicklung zu ermöglichen und somit ein „explosionsartiges“ Versagen zu vermeiden. 
Im Fall einer zentrischen Vorspannung unter alleiniger Wirkung der Vorspannkraft steht der gesamte Glasquerschnitt dauerhaft unter Druckbeanspruchung, sodass ein Versagen als Folge einer lokalen Zugfestigkeitsüberschreitung ${ }^{351}$ oder einem Stabilitätsversagen erfolgt (Tafel 33). Für Erstere wird angenommen, dass sie durch eine entsprechende konstruktive Maßnahme ausgeschlossen wird, während der zweite Fall des Stabilitätsversagens unabhängig von der Festigkeit des Grundmaterials ist. Damit erreicht ein mechanisch vorgespannter Floatglasträger die gleiche Tragfähigkeit wie ein unbewehrter, thermisch vorgespannter Träger, wenn die Differenz zur Kurzzeitfestigkeit $R_{\text {d.kurz }}$ durch die mechanische Vorspannung ausgeglichen wird. Dafür sind $29,2 \mathrm{~N} / \mathrm{mm}^{2}$ (TVG) beziehungsweise $62,5 \mathrm{~N} / \mathrm{mm}^{2}$ (ESG) nötig, sodass ein Vorspanngrad des Floatglasträgers von mindestens 0,65 beziehungsweise 1,39 resultiert.

Die nötigen Vorspanngrade wurden jedoch bisher in keiner Anwendung erreicht ${ }^{352}$, sodass davon auszugehen ist, dass die Nutzung unbewehrter, thermisch vorgespannter Glasträger in diesem Fall bevorzugt wird. Darüber hinaus zeigt Bos [2009], dass die im thermischen Vorspannprozess gespeicherte Energie mehrere Zehnerpotenzen größer ist als die eingebrachte Energie aus einer äußeren Belastung. Verbleibt also der mechanische Vorspanngrad deutlich kleiner, so ist damit zu rechnen, dass im Bruchfall kein prägnant feineres Bruchbild auftritt. Einzig bisher nicht näher quantifizierte Sicherheitserwägungen in Bezug auf das zu erreichende Bruchbild können im Resultat die Verwendung von thermisch entspannten Floatglasträgern rechtfertigen. Daher wird vorgeschlagen, im Rahmen weiterführender Forschungsaktivitäten ein Duktilitätskriterium für bewehrte und vorgespannte Glasträger zu definieren. ${ }^{353}$

Bei exzentrischer Vorspannung muss der Vorspanngrad eines Floatglasträgers unterhalb von $11 \%$ bleiben, um eine Überschreitung des Dauerwiderstandes der vorgezogenen Druckkante auszuschließen. Davon ausgehend, dass die eingebrachte Druckspannung aus dem Normalkraftanteil der

\footnotetext{
351 Beispielsweise an der Lasteinleitungsstelle.

352 Vergleiche Tafel 8, Seite 98.

353 Vergleiche Gleichung 50, Seite 112.
} 


\begin{tabular}{|c|c|c|c|c|c|c|c|}
\hline \multirow{2}{*}{\multicolumn{2}{|c|}{$\begin{array}{l}\text { Glaskantenspannung } \\
{\left[\mathrm{N} / \mathrm{mm}^{2}\right]}\end{array}$}} & \multicolumn{3}{|c|}{ zentrische Vorspannung } & \multicolumn{3}{|c|}{ exzentrische Vorspannung } \\
\hline & & \multicolumn{2}{|c|}{$\begin{array}{ll}\text { Floatglas } & \text { TVG } \\
<0 & <0\end{array}$} & $\begin{array}{l}\text { ESG } \\
<0\end{array}$ & $\frac{\text { Floatglas }}{5,0}$ & $\frac{\text { TVG }}{46,7}$ & $\frac{E S G}{80,0}$ \\
\hline 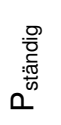 & $\begin{array}{l}E_{\text {d.OK }}=R_{\text {d.ständig }} \\
E_{\text {d.UK }}=R_{\text {d.ständig }} \\
K=E_{\text {d.min }} / f_{k}\end{array}$ & $\begin{array}{l}<0 \\
<0 \\
-\end{array}$ & $\begin{array}{l}<0 \\
<0 \\
-\end{array}$ & $\begin{array}{l}<0 \\
<0 \\
-\end{array}$ & $\begin{array}{l}5,0 \\
<0 \\
0,11\end{array}$ & $\begin{array}{l}46,7 \\
<0 \\
0,67\end{array}$ & $\begin{array}{l}80,0 \\
<0 \\
0,67\end{array}$ \\
\hline$\frac{\stackrel{N}{\frac{N}{3}}}{\stackrel{+}{+}}$ & $\begin{array}{l}E_{\text {d.OK }}=R_{\text {d.kurz }} \\
E_{\text {d.UK }}=R_{\text {d.kurz }} \\
K=E_{\text {d.min }} / f_{k}\end{array}$ & $\begin{array}{l}<0 \\
14,0 \\
0,31\end{array}$ & $\begin{array}{l}<0 \\
46,7 \\
0,67\end{array}$ & $\begin{array}{l}<0 \\
80,0 \\
0,67\end{array}$ & $\begin{array}{l}<0 \\
14,0 \\
(0,31)\end{array}$ & $\begin{array}{l}<0 \\
46,7 \\
0,67\end{array}$ & $\begin{array}{l}<0 \\
80,0 \\
0,67\end{array}$ \\
\hline
\end{tabular}

\begin{abstract}
Vorspannkraft deutlich kleiner ist, als der Anteil aus dem Biegemoment, muss zunächst eine mechanische Druckvorspannung von $-5,0 \mathrm{~N} / \mathrm{mm}^{2}$ zuzüglich der Kurzzeitfestigkeit von $14,0 \mathrm{~N} / \mathrm{mm}^{2}$ dekomprimiert werden ${ }^{354}$, bis die resultierende Beanspruchbarkeit von 19,0 N/mm² erreicht wird. Demgegenüber liegt die Beanspruchbarkeit unbewehrter, thermisch vorgespannter Glasträger mehr als doppelt so hoch. Somit wird eine exzentrische mechanische Vorspannung von Floatglasträgern gegenüber unbewehrter thermisch vorgespannter Konstruktionen keine ausreichenden Vorteile erbringen.
\end{abstract}

Es verbleibt die Möglichkeit, die Tragfähigkeit eines thermisch vorgespannten Glases in einer Trägerkonstruktion durch eine exzentrische mechanische Vorspannung zu vergrößern. Hier sind maximale Vorspanngrade von 0,67 gemäß Tafel 33 möglich. Die zusätzlich eingebrachte Energie aus der mechanischen Vorspannung wird laut Bos [2009] keine deutliche Veränderung der Rissbildung hervorrufen, sodass sich hier theoretisch eine sinnvolle konstruktive Variante ergibt.

\section{Kritik}

Als Kritik bleibt zu vermerken, dass eine Vermessung der Rissbreiten in Analogie zum Stahlbeton- und Spannbetonbau nicht erfolgt ist. Dies wurde angesichts des kritischen Belastungszustandes als zu gefährlich beurteilt. Zudem traten, insbesondere im Bereich der Bruchfächer, eine große

\footnotetext{
354 Die Bemessungswerte des Tragwiderstandes ergeben sich für Gläser ohne planmäßige Vorspannung bei ständiger und kurzer Zugbeanspruchung der Kanten nach [DIN 18008-1, Kap. 8.3].
}

Tafel 33

Maximale Bemessungswerte der Auswirkung an einer Trägeroberkante $(\mathrm{OK})$ und -unterkante (UK) (nach [DIN 18008-1] mit $\mathrm{k}_{\mathrm{c}}=1,0$ ) sowie der zugehörige (charakteristische) Vorspanngrad $k$ nach Gleichung 2, Seite 45. 
Anzahl dicht nebeneinander liegender Einzelrisse auf, sodass die Möglichkeit zur Messung der Breite eingeschränkt war. Darüber hinaus werden Rissbreiten in Glasträgern durch die thermische Vorspannung beeinflusst. Es ist allgemein bekannt, dass deren Verteilung über die Glasoberfläche, entgegen dem theoretischen Ansatz ${ }^{355}$, inhomogen ist. Sie ist entsprechend durch eine vorangestellte Quantifizierung ${ }^{356}$ einzubeziehen.

\section{Weiterer Forschungsbedarf}

Es gilt ausblickend sicherzustellen, dass die Risse wirksam überbrückt werden. Eine vergrößerte intakte Druckzone wirkt sich dafür günstig aus. Daher müssen zukünftige Konstruktionen sowohl die anfallende Energie im Rissfall sicher aufnehmen, als auch die entstehenden Risse an ihrem Ursprung effektiv überbrücken. Es wird daher vorgeschlagen, die verbundlose Vorspannung von Glasträgern um die Option des nachträglichen Verbundes zu erweitern. ${ }^{357}$ Durch den Verbund kann eine unmittelbare Überbrückung von Rissflanke zu Rissflanke erfolgen. Zusätzlich sorgt der Zeitpunkt der Herstellung der Verbindung dafür, dass der Verbundwerkstoff nicht dauerhaft belastet ist.

Außerdem sollte die Rissbreitenentwicklung nach dem Erstriss und anschließender Entlastung dokumentiert werden. Daraus resultiert die Entwicklung der Druckzonenhöhe, welche zum Lastabtrag beiträgt.

355 Die thermische Vorspannung wird nach Bild 11 (Seite 32) für die Bemessung als homogen verteilt angenommen und wird als Mindestwertes interpretiert.

356 Dies kann mit spannungsoptischen Methoden erfolgen. Eine detaillierte Beschreibung für die Anwendung im Glasbau ist beispielsweise in [Feldmann et al. 2012] zusammengestellt.

357 Vergleiche Kapitel 6.7.1, ab Seite 262. 


\subsubsection{Spannungszuwachs in der Bewehrung unter Biegebelastung}

\section{Spannglasträger}

Die Seilkraft änderte sich unter Biegung für alle Spannglasprüfkörper zunächst linear. Der zugehörige Anstieg war auf unterschiedliche Seildurchmesser zurückzuführen. Das Verhalten kann mit der analytischen Rechnung nachvollzogen werden. Daraus wurde eine Spannungsänderung von $123 \mathrm{~N} / \mathrm{mm}^{2}$ bis $165 \mathrm{~N} / \mathrm{mm}^{2}$ errechnet. ${ }^{358}$ Dies entspricht weniger als $10 \%$ der charakteristischen Festigkeit des Seilmaterials. Es wurde beobachtet, dass die Seilkraft nahe der Versagenslast der Prüfkörper mitunter stagnierte oder gar abfiel. Zudem wurde der Maximalwert der Seilkraft nach dem Erstriss im Glas verzeichnet, sodass dieser Lastzustand bemessungsrelevant wird.

\section{Glasträger mit vorgespannter Bewehrung}

Die exemplarischen Ergebnisse für Seildurchmesser von $8 \mathrm{~mm}$ und $10 \mathrm{~mm}$ in [Louter et al. 2014] und [Louter et al. 2013a] zeigten einen mit zunehmender Belastung flacher werdenden Spannungszuwachs in der Bewehrung. Dies war wahrscheinlich eine Folge der kontinuierlichen Führung der Spannglieder entlang der oberen und unteren zurückgesetzten Verbundglaskante. Dabei folgte die Seillinie stets der Trägerkrümmung, sodass eine andere Verträglichkeitsbedingung anzusetzen ist. ${ }^{359}$ Eine sprunghafte Änderung der Seilkraft infolge der Rissbildentwicklung im Glas ist der Ergebnisdarstellung nicht zu entnehmen. Vielmehr wurde die Bewehrung nach dem Erstriss mit kleiner werdender Kraftän-

\footnotetext{
358 Vergleiche Gleichung 97, Seite 305. Unter Annahme eines linearen Verhaltens bis zur Bruchlast ergeben sich rechnerisch größere Seilkräfte und Seilkraftänderungen als unter experimentellen Bedingungen, sodass das Ergebnis auf der sicheren Seite liegt.

359 Ein geradliniger Verlauf zwischen den Verankerungspunkten ist hierfür keine realistische Annahme.
} 
derung belastet. Insgesamt ergaben sich Spannungsänderungen von circa $60 \mathrm{~N} / \mathrm{mm}^{2}$ bis $150 \mathrm{~N} / \mathrm{mm}^{2} .{ }^{360}$

Jordão et al. [2014] zeigen ebenso exemplarisch einen linearen Anstieg der Seilkraft mit der Biegebelastung, welche sich nach dem Erstriss deutlich schneller steigert. In dieser Untersuchung wurde eine Spannungsänderung von circa $80 \mathrm{~N} / \mathrm{mm}^{2}$ gemessen, welche sich nach dem Auftreten des ersten Risses im Glas auf bis zu $580 \mathrm{~N} / \mathrm{mm}^{2}$ steigerte. ${ }^{361}$ Darüber hinaus weisen Firmo et al. [2015] auf eine mögliche unsymmetrische Verteilung der Kräfte in den nebeneinander liegenden Spanngliedern hin.

\section{Spannbetonträger}

Trost et al. [1984] und Weller [1988] beschreiben einen linearen Spannungszuwachs in der Bewehrung von Betonträgern im Zustand I, gefolgt von einer raschen Steigerung mit beginnender Rissbildung. Es ergab sich ein nahezu bilinearer Zusammenhang zwischen der Last und der Spannung in der Bewehrung. Jedoch wird darauf hingewiesen, dass ein vergleichsweise großer Spannungzuwachs bis zu $550 \mathrm{~N} / \mathrm{mm}^{2}$ beobachtet wurde, da die initiale Vorspannkraft gering war. ${ }^{362}$ In den heute gültigen Bemessungsregeln darf vereinfachend ein Spannungszuwachs von $100 \mathrm{~N} / \mathrm{mm}^{2}$ angenommen werden, wenn keine genauere Berechnung durchgeführt wird. ${ }^{363}$

\section{Vergleichende Beurteilung}

Die Ergebnisse für Spannglasträger sowie anderer Beispiele aus dem Glasbau und dem Spannbetonbau stimmen darin überein, dass sich die Spannung in der Bewehrung zu-

\footnotetext{
360 Ablesewerte aus Ergebnisdiagrammen in [Louter et al. 2013a]. Unter Annahme einer charakteristischen Festigkeit von 500 bis $700 \mathrm{~N} / \mathrm{mm}^{2}$ resultiert eine Auslastung von $9 \%$ bis $30 \%$.

361 Ablesewerte aus dem Ergebnisdiagramm in [Jordão et al. 2014]. Eine Materialangabe liegt nicht vor.

362 Gemessen an den seinerzeit gültigen Bemessungsregeln wurden nur $55 \%$ der Stahlfestigkeit ausgenutzt.

${ }^{363}$ Vergleiche $\Delta \sigma_{\mathrm{p}, \mathrm{ULS}}=100 \mathrm{~N} / \mathrm{mm}^{2}$ in [DIN EN 1992-1-1, Kap. 5.10.8(2)].
} 
nächst linear mit steigender Belastung ändert. Wenn nach dem Erstriss eine weitere Laststeigerung möglich ist, so tritt die maximale Kraft in der Bewehrung in diesem zweiten Lastzweig auf.

In den hier gezeigten Beispielen ergaben sich bereits Spannungsänderungen von mehr als $100 \mathrm{~N} / \mathrm{mm}^{2}$. Die vereinfachende Annahme aus [DIN EN 1992-1-1] zum pauschalen Ansatz der Spannungsänderung kann nicht verallgemeinernd übernommen werden. Diese Vorgehensweise wurde nur unter besonderen Annahmen abgeleitet, deren Gültigkeit entsprechend nachzuweisen ist. ${ }^{364} \mathrm{Da}$ diese für den Glasbau nicht existieren, die entsprechenden Berechnungsmethoden jedoch als Stand der Technik beschrieben werden können, wird empfohlen, die Seilkraftänderung explizit zu berechnen und die Bewehrung entsprechend auszulegen.

\section{Kritik}

Die Messung der Seilkraft erfolgte lediglich am Spannanker, sodass die maximale Belastung ohne zeitabhängige oder konstruktive Verluste aufgezeichnet wurde. Alternativ ist die Messung am Festanker zusätzlich sinnvol| ${ }^{365}$, um Umlenkkraftverluste in jedem Versuch individuell zu ermitteln. Die für diese Arbeit verwendete Versuchsanordnung wurde schließlich aus praktischen Gründen während der Durchführung gewählt und Verluste beispielhaft ermittelt. Hierin zeigten sich keine maßgebenden Umlenkkraftverluste. ${ }^{366}$

\section{Weiterer Forschungsbedarf}

Es ergibt sich die Notwendigkeit, den Einfluss der Konstruktion auf die Entwicklung der Bewehrungskraft für Glasträger in der weiterführenden Forschung allgemeingültig zu beschreiben. Damit sollen ungünstige und ungewollte Effekte, beispielsweise aus unzulässiger Schiefstellung, vermieden

\footnotetext{
364 Vergleiche [Trost et al. 1984].

365 Dies wurde im Projekt „Spannglasbrücke“ durchgeführt. Vergleiche [Engelmann \& Weller 2016]

366 Vergleiche Kapitel 3.3.5, insbesondere Bild 48, Seite 118.
} 
werden. Damit wird die Seilkraftänderung mechanisch vorgespannter Glasträger einer sicheren Auslegung und Ausnutzung zugänglich gemacht.

\subsection{Tragverhalten unter Dauer- und Temperaturbelastung}

\section{Spannglasträger}

In den eigenen experimentellen Untersuchungen zeigten sich Spannkraftverluste zwischen $19 \%$ und $27 \%$ innerhalb der Versuchslaufzeit von 1000 h. ${ }^{367}$ Die Seilkraftverläufe wurden mit einer Regressionsfunktion analytisch beschrieben und stehen so für eine weiterführende Analyse zur Verfügung. Daneben zeigte sich das in Analogie zum Spannbetonbau abgeleitete Berechnungsmodell als nicht passend. Als Ursache wurde mittels einer numerischen Studie die in der Idealisierung fehlende räumliche Durchbiegung infolge der Trägerimperfektion und das zeit- sowie temperaturabhängige Materialmodell der Zwischenschicht identifiziert. Ferner ist der Einfluss der aufgeklebten Umlenkpunkte auf die Gesamtsteifigkeit des Trägers zu berücksichtigen. ${ }^{368}$

Zusätzlich zeigte sich, dass eine Temperatursteigerung erwartungsgemäß zu einem Verlust an Vorspannkraft als Folge der unterschiedlichen Temperaturverzerrung der beteiligten Materialien führt. ${ }^{369}$ Dies konnte entsprechend der vorherrschenden Steifigkeitsverhältnisse analytisch beschrieben werden.

\section{Bewehrte und unbewehrte Glasträger}

Beim Bau bewehrter und unbewehrter Glasträger ergab sich bisher nicht die Notwendigkeit, die Durchbiegung unter Dauerlasten zu vermessen, da nicht davon auszugehen ist, dass

\footnotetext{
367 Vergleiche Kapitel 3.4, ab Seite 134.

368 Vergleiche Kapitel 4.3.5, ab Seite 199.

369 Vergleiche Kapitel 3.6, ab Seite 165.
} 
es zu einer maßgebenden Kriechverformung kommt. Dabei wird die horizontale Durchbiegung als ausreichend klein beurteilt, sodass eine maßgebende Belastung der Zwischenschicht vermieden wird. ${ }^{370}$ Die Dauerhaftigkeit der Konstruktionsbestandteile wurde nachgewiesen, um sicherzustellen, dass das Kurzzeittragverhalten auch bei gealterten Bauteilen für eine Bemessung angesetzt werden kann. ${ }^{371}$

Einzig für hybride Träger mit Polycarbonat-Zwischenschicht wurden Biegeversuche unter konstanter Biegelast durchgeführt und Aufzeichnungen der vertikalen Durchbiegungen in Feldmitte bereitgestellt. ${ }^{372}$ Härth [2013] beobachtet dabei keinen Trend zu einer Verformungszunahme. Weiterführende Aussagen zur räumlichen Durchbiegung wurden nicht gemacht.

Unter den veröffentlichten Ergebnissen zum Tragverhalten von Glasträgern mit vorgespannter Bewehrung findet sich keine Angabe zum Dauerlastverhalten, zur zeitlichen Veränderung der wirkenden Spannkraft oder zum Systemtragverhalten unter Temperaturlast. ${ }^{373}$ Damit fehlt für diese Konstruktionen weiterhin der Nachweis einer dauerhaften Wirkung der Vorspannung. Dies unterstreicht die Notwendigkeit der hier vorgestellten Ergebnisse für Spannglasträger.

\section{Spannbetonträger}

Im Spannbetonbau existiert ein normativ gefasstes Berechnungsverfahren zur Ermittlung der Spannkraftverluste in Abhängigkeit der Baustoffeigenschaften. ${ }^{374}$ Daraus wurde ein analytisches Berechnungsmodell zur Ermittlung der Seilkraftverluste von Spannglasträgern abgeleitet. ${ }^{375}$ Zusätzlich wird

370 Die Zeitabhängigkeit der Festigkeit des Glases wird im Rahmen der Bemessung berücksichtigt. Vergleiche [DIN 18008-1].

371 So untersucht Louter [2011] die Verbundkonstruktion zwischen Glas und schlaffer Bewehrung unter zyklischer Temperatur- und Feuchtebelastung sowie unter variierter Lasteinwirkungsdauer.

372 Vergleiche [Härth 2013]

373 Vergleiche [Bos et al. 2004], [Louter et al. 2006], [Louter 2013], [Louter et al. 2013b], [Louter et al. 2013a], [Jordão et al. 2014], [Cupac \& Louter 2015] und [Louter \& Cupac 2015].

374 Vergleiche [DIN EN 1992-1-1, Kap. 5.10].

375 Vergleiche Kapitel 2.7.6, ab Seite 79. 
der Einfluss einer Temperaturbelastung mit den Lastangaben des Eurocodes rechnerisch abgedeckt. ${ }^{376}$

\section{Vergleichende Beurteilung}

Für die weiteren Ausführungen wird zunächst die Annahme getroffen, dass der Spannkraftverlust in einem Spannglasträger mit dem vorgestellten Modell binnen einer Lebenszeit von 10 Jahren weniger als $15 \%$ beträgt. ${ }^{377}$ Grundsätzlich wird es als sinnvoll erachtet, auf ein späteres Nachspannen verzichten zu können.

Gegenüber den Verlusten im Spannbetonbau traten in den eigenen Untersuchungen unverhältnismäßig hohe Seilkraftveränderungen auf. Die Grenze von $15 \%$ wurde bereits nach der Versuchszeit von $1000 \mathrm{~h}$ erreicht und überschritten. Diese wurde vorab bewusst gebilligt, um ein messbares Ergebnis zu erreichen und erstmals überhaupt Spannkraftverluste in vorgespannten Glasträgern strukturiert aufzuzeichnen. Damit sind die Grundlagen gelegt, die Spannkraftverluste auf ein akzeptables Maß zu reduzieren, mit dem Ziel, dauerhafte Bauteile zu konstruieren, deren Bewehrung nicht nachgespannt werden muss. Dabei kann auf Basis der gezeigten Ergebnisse eine Extrapolation der Seilkraftverluste auf die erwartete Lebenszeit des Bauteils vorgenommen werden.

\section{Kritik}

Die eigene experimentelle Untersuchungsmethodik ist dahingehend zu kritisieren, dass ein Einfluss der Belastungskonstruktion auf die Verformung nicht vollkommen ausgeschlossen werden kann. Ein Vergleich mit unbelasteten Referenzen war infolgedessen nicht möglich. Zusätzlich ist das gewählte Belastungsmodell zu beanstanden, da eine konstante

\footnotetext{
376 Vergleiche [DIN EN 1991-1-5] sowie [DIN EN 1991-1-1, Kap. 2.3].

377 Geßner et al. [2017] stellen eine Abschätzung für das Verlustmodell der [DIN EN 1992-1-1] vor, wonach die bestimmenden Parameter gegen einen Grenzwert laufen. Dabei ergibt sich ein Spannkraftverlust von etwa $15 \%$ als Endwert $(t \rightarrow \infty)$. Für verbundlos vorgespannte Betonträger kommen Zilch \& Zehetmaier [2010] in einem Beispiel zu einem ähnlichen Ergebnis.
} 
Dauerlast keine realistische Annahme für eine tatsächliche Baukonstruktion darstellt. Entsprechend ist in Analogie zum Stahlbeton- und Spannbetonbau ein Modell unter veränderlichen äußeren Lasten abzuleiten, um eine Änderung der Trageigenschaften unter Dauerlast auszuschließen. Dabei sind natürliche Laständerungen und -schwankungen zu berücksichtigen.

Des Weiteren beruht das analytische Modell zur Berechnung der Spannkraftverluste auf der Annahme, dass sich die Schubspannung in der Zwischenschicht aus der Superpostion der Anteile aus reiner Normalkraftbeanspruchung und reiner Biegebelastung um die schwache Achse zusammensetzt. Unberücksichtigt bleiben die Anteile aus der Verdrehung des Querschnitts. Darüber hinaus zeigt sich das Materialmodell der Zwischenschicht, repräsentiert durch die zugehörige Kriechzahl, als äußerst sensitiv gegenüber der Temperatureinwirkung. Dies ist auf die Materialeigenschaften der verwendeten PVB-Zwischenschicht zurückzuführen, welche im Temperaturbereich der Dauerlastversuche ihren Tranformationsbereich aufweist. ${ }^{378}$

Entsprechend der unverhältnismäßig hohen Kriechzahlen ist davon abzuraten, das Zwischenschichtmaterial durch eine Vorspannung zu belasten. Entsprechend werden in Kapitel 6 konstruktive Gegenmaßnahmen vorgeschlagen.

\section{Weiterer Forschungsbedarf}

Es besteht die Notwendigkeit, ein Lastszenario zur realistischen Abschätzung der Seilkraftverluste weiterzuentwickeln, an angepassten Prüfbauteilen unter Laborbedingungen zu erproben und die Ergebnisse an realen Bauteilen zu bestätigen. Dabei sind Werte festzulegen, die den zulässigen Seilkraftverlust definieren.

378 Schuler [2003] gibt beispielsweise die Glasübergangstemperatur von PVB mit $20^{\circ} \mathrm{C}$ an. Ensslen [2005] bestätigt dies mit der Angabe eines Glasübergangstemperaturbereiches von $10^{\circ} \mathrm{C}$ bis $20^{\circ} \mathrm{C}$. Die eigenen Versuche fanden im Temperaturbereich zwischen $17^{\circ} \mathrm{C}$ und $30^{\circ} \mathrm{C}$ statt. 
Danach ist der analytische Ansatz zu erweitern und mit den experimentellen Ergebnissen unter dem geänderten Lastszenario in Einklang zu bringen, um eine realitätsnahe Vorhersage der Spannkraftverluste zu ermöglichen.

\subsection{Resttragfähigkeit}

\section{Spannglasträger}

Für die eigenen experimentellen Untersuchungen wurde ein Nachweiskonzept aufgestellt, welches unter einer angenommenen Biegebelastung unterhalb der vorab bestimmten Erstrisslast einen stufenweise steigenden Schädigungsgrad berücksichtigt. Es zeigt sich, dass bei moderatem Lastniveau eine Vergrößerung der Standzeit durch eine Bewehrung und Vorspannung zu erreichen ist. Demgegenüber wurden auch Fälle beobachtet, in denen die Vorspannung ungünstig wirkte und zu einem frühzeitigen Versagen führte. ${ }^{379}$ Somit ist die Untersuchung eines Teilschädigungsszenarios unter einer Belastung unterhalb der Erstrisslast zusätzlich notwendig, da sich hierbei sicherheitsrelevante Tragsituationen ergeben können, die durch eine duktile Bauteilreaktion bei höheren Lasten nicht abgebildet sind.

\section{Glasträger}

Aufgrund fehlender normativer Vorgaben werden projektspezifische Anforderungen an Glasträger in der Regel individuell gestellt und ein experimenteller Nachweis gefordert.

Hess [2000] führte Versuche an unbewehrten Trägern unter einer Gebrauchslast von $42 \%$ der Bruchspannung durch. 380 Auf diesem Lastniveau wurden eine Schädigung der Trä-

\footnotetext{
379 Vergleiche Kapitel 3.5, ab Seite 147.

380 Aus Vorversuchen wurde die minimale Bruchspannung entnommen und mit einem globalen Sicherheitsfaktor von 2,4 beaufschlagt. Vergleiche [Hess 2000].
} 
gerfläche durch einen harten $S$ to $\beta^{381}$ herbeigeführt und die verbleibende Traglast durch einen Biegeversuch festgestellt.

Für die Ermittlung der Resttragfähigkeit der unbewehrten Glasträger für die „Alte Mensa“ der TU Dresden wurden ebenso experimentelle Untersuchungen durchgeführt. ${ }^{382}$ Die Beanspruchung des Glases ergab sich dabei aus der Eigenlast und der halben Schneelast. Anschließend wurden die beiden äußeren Schichten des Vierfach-Glaslaminates mittels eines Hammerschlages gebrochen. Nach dem Ablauf einer Standzeit von $12 \mathrm{~h}$ zeigten sich keine auffälligen Verformungszuwächse, sodass die gestellten Anforderungen erfüllt wurden.

Im Vergleich dazu fanden für das Projekt „Therme Badenweiler" keine experimentellen Untersuchungen statt. ${ }^{383}$ Vielmehr erfolgte eine rechnerische Nachweisführung unter Annahme des Versagens der beiden äußeren Glasschichten aus Einscheiben-Sicherheitsglas.

Für Hybridträger führte Härth [2013] ebenso Resttragfähigkeitsuntersuchungen im Anschluss an einen Dauerlastversuch durch. ${ }^{384}$ Dabei wurden die Einzelscheiben aus thermisch entspanntem Floatglas unter einer Biegelast, die dem dreifachen Bemessungswert der Glaskantenspannung unter ständigen Lasten entspricht, schrittweise mit einem spitzen Metallwerkzeug angeschlagen und für $24 \mathrm{~h}$ Standzeit belassen. Die Hybridträger zeigten nach dem Anschlagen aller Glasschichten eine Lastumlagerung auf die PolycarbonatZwischenschicht, was eine zeitabhängige Verformung der verbleibenden Trägerkonstruktion zur Folge hatte.

381 Es wird der Aufprall einer pendelnden Stahlkugel variierter Masse und steigernder Fallhöhe benutzt. Vergleiche [Hess 2000].

382 Vergleiche [Zschippang et al. 2006] und [Prüfbericht 01.02.2005] sowie Bild 1, links oben (Seite 17)

${ }^{383}$ Vergleiche [Schober et al. 2004].

384 Zusätzlich wird das Tragverhalten nach dem Auftreten des Erstrisses zur Beschreibung der Resttragfähigkeit in [Härth 2013] verwendet. Diese Ergebnisse sind vielmehr mit dem Tragverhalten im Kurzzeit-Biegeversuch vergleichbar und eignen sich daher hier nicht für einen Vergleich. 
Damit können grundsätzlich drei unterschiedliche Vorgehensweisen zum Nachweis der Resttragfähigkeit ${ }^{385}$ benannt werden:

- Ein Nachweis erfolgt mittels einer experimentellen Untersuchung mit einer Biegebelastung unterhalb der Erstrisslast $^{386}$ und Berücksichtigung eines Schädigungsszenarios. Dabei werden die Glasschichten vor oder nach dem Aufbringen der Biegelast angeschlagen. Zusätzlich wird eine verbleibende Standzeit gefordert.

- Es wird eine Versagenslast unter einem vorab eingestellten Schädigungszustand durch Steigerung der Auflast experimentell ermittelt.

- Ein Teilschädigungszustand wird ohne experimentellen Nachweis rechnerisch betrachtet.

\section{Spannbetonträger}

Ein Nachweis der Resttragfähigkeit ist im Stahlbeton- und Spannbetonbau nicht nötig. Jedoch besteht die Anforderung, robuste Konstruktionen zu errichten, welche auch im Fall einer unplanmäßigen Beanspruchung mit Ankündigung versagen. ${ }^{387}$

\section{Vergleichende Beurteilung}

Die beiden letztgenannten Varianten zur Ermittlung der Resttragfähigkeit von Glasträgern enthalten keine Aussagen zur verbleibenden Standzeit der geschädigten Konstruktion. Zudem wird das Konzept einer "Opferscheibe“ angewandt, welches als nicht materialeffizient herausgestellt wurde. Entsprechend wird empfohlen, ein Konzept zum Nachweis der Resttragfähigkeit bewehrter und unbewehrter Glasträger zu

\footnotetext{
385 Darüber hinaus wird der Nachweis der Resttragfähigkeit für Konstruktionen, für die hinreichende Erfahrungen vorliegen, durch die Einhaltung konstruktiver Randbedingungen nachgewiesen. Vergleiche [DIN 180081, Kap. 9].

${ }^{386}$ Es ist üblich, eine Vertikalverglasung neben ihrer Eigenlast mit der Hälfte der anfallenden Nutzlast zu beanspruchen. Vergleiche [Glaserlass Hessen 2004] und [Merkblatt ZiE Bayern 2015].

387 Vergleiche Kapitel 2.8, ab Seite 90.
} 
entwickeln. Darin soll eine Biegelast unterhalb der Erstrisslast über eine anzugebende Standzeit abgetragen werden. Dafür ist ein relevantes Schädigungsszenario zu konzipieren, wozu die Ergebnisse dieser Arbeit beitragen können.

Es ist deutlich zwischen dem Nachbruchtragverhalten nach dem Überschreiten der Erstrisslast und der Beschreibung der Resttragfähigkeit zu unterscheiden. Für beide Zustände ist ein getrennter Nachweis zu erbringen, da unterschiedliche, ungünstige Auswirkungen beobachtet wurden.

\section{Kritik}

Die gewählte Reihenfolge der Glasschädigung erfüllt die eingangs gestellten Anforderungen nur teilweise. Im Einzelfall verformte sich der Träger nach einer Schädigung erneut in Richtung der Ausgangsposition. Es bleibt daher zu bedenken, dass ebenso ein Schadensszenario mit dem Versagen eines vollständigen Paketes zu berücksichtigen bleibt. Nichtsdestotrotz sollte weiterhin das Ziel angestrebt werden, auch nach dem Riss aller Schichten im Verbundglas ein Momentengleichgewicht unter Ausnutzung der Bewehrung zu finden, sodass die Wahl einer Schädigungsreihenfolge geringer gewichtet werden kann.

\section{Weiterer Forschungsbedarf}

Die Grundannahmen für die gezeigten Prüfszenarien sind derzeit in der Literatur nicht ausreichend dokumentiert und hinterlegt. Es wird daher vorgeschlagen, vorhandene Erfahrungen zusammenzufassen. Dies kann die Auswertung von Versuchen zur Erlangung einer Einzelzulassung ${ }^{388}$ sowie die Sammlung und Auswertung von Schadensfällen beinhalten Darauf aufbauend kann ein umfassendes und allgemeingültiges Konzept zum Nachweis der Resttragfähigkeit aufgebaut werden.

388 In Deutschland wird eine Zustimmung im Einzelfall benötigt. 
Daneben wird das Tragverhalten neuartiger Konstruktionen numerisch abgebildet. Diese Rechenverfahren können für den Fall des Glasbruchs anwendungstauglich erweitert werden. Es wird daher vorgeschlagen, das Rissverhalten von Glaskonstruktionen rechnerisch abzubilden, um deren Tragverhalten ökonomisch analysieren zu können. ${ }^{389}$ Dabei ist die Rissbildung infolge der thermischen Vorspannung einzubeziehen. Damit kann auch die Tragwirkung des gebrochenen Glasanteils berücksichtigt und eine effektive Nachweisführung ermöglicht werden.

\section{$5.5 \quad$ Zusammenfassung}

Zur Charakterisierung mechanisch vorgespannter Glasträger im Allgemeinen und Spannglasträgern im Besonderen ist das Tragverhalten unter Vorspann- und Biegelasten sowie unter einer Temperaturänderung zu beschreiben. Darüber hinaus soll die Dauerhaftigkeit durch die Ermittlung der Spannkraftverluste sichergestellt sein. Zusätzlich ergibt sich ein wesentlicher Sicherheitsaspekt durch den Nachweis der Resttragfähigkeit. Entsprechend werden die wesentlichen Parameter der Trägerkonstruktionen in Tafel 34 gegenübergestellt. Dies betrifft die Erstrisslast $F_{c r .1}$ im Kurzzeit-Biegeversuch, den relativen Seilkraftverlust $\Delta P_{\text {rel }}$ im Dauerlast-Versuch und die Standzeit im Versuch zur Bestimmung der Resttragfähigkeit.

Dabei zeigt sich, dass mit steigendem Bewehrungsgrad und vergrößerter Vorspannkraft eine Zunahme der Erstrisslasten beobachtet wurde. Zudem wird ein gebrochener Träger durch eine Bewehrung in Position gehalten. Insbesondere im Versuch zur Bestimmung der Resttragfähigkeit zeigen sich verlängerte Standzeiten. Jedoch kann es bei einer Überlastung des Systems zu einem frühzeitigen Versagen kommen. Des Weiteren vergrößert sich der Seilkraftverlust gleichzeitig auf unvertretbar hohe Werte. Die Gründe für dieses Verhalten wurden mit einer ergänzenden numerischen Studie untersucht und im Einzelnen diskutiert. Dabei wurden die

389 Louter [2011] schlägt dafür die sequentielle lineare Analyse vor. 


\begin{tabular}{|c|c|c|c|c|}
\hline Gruppe & $\mathrm{F}_{\mathrm{cr} .1}[\mathrm{kN}]$ & $\Delta P_{\text {rel }}[-]$ & Standzeit [h] & \multirow{7}{*}{$\begin{array}{l}\text { Tafel } 34 \\
\text { Gegenüberstellung ausge- } \\
\text { wählter Trageigenschaften. } \\
\text { Erstrisslast nach Bild } 51 \\
\text { (Seite 123), relativer Seil- } \\
\text { kraftverlust nach Tafel } 17 \\
\text { (Seite 142) und Standzeit } \\
\text { nach Bild } 71 \text { (Seite 156). }\end{array}$} \\
\hline Referenz & 24.5 bis 36.6 & - & 48.1 bis 53.1 & \\
\hline & & & & \\
\hline $\begin{array}{l}\text { Referenz } \\
\text { bewehrt }\end{array}$ & 23,7 bis 34,0 & $-1,5 \%$ & 48,3 bis 72,2 & \\
\hline $\begin{array}{l}P_{\mathrm{m} 0}=9 \mathrm{kN} \\
d_{p}=(5,0) 6,1 \mathrm{~mm}\end{array}$ & 30,9 bis 32,7 & $-3,8 \%$ & 48,5 bis 72,8 & \\
\hline $\begin{array}{l}P_{\mathrm{m} 0}=15 \mathrm{kN} \\
\mathrm{d}_{\mathrm{p}}=8,1 \mathrm{~mm}\end{array}$ & 34,1 bis 36,3 & $\begin{array}{l}-19,0 \% \\
\left(-21,9 \%^{a}\right)\end{array}$ & 48,7 bis 112,5 & \\
\hline $\begin{array}{l}P_{\mathrm{m} 0}=15 \mathrm{kN} \\
\mathrm{d}_{\mathrm{p}}=10,1 \mathrm{~mm}\end{array}$ & 35,8 bis 36,7 & $\begin{array}{l}-14,7 \% \\
\left(-26,8 \%{ }^{a}\right)\end{array}$ & $0,5^{b}$ bis $72,6^{c}$ & \\
\hline
\end{tabular}

Anwendungsgrenzen sowie günstige von ungünstigen Effekten getrennt. Damit kann der Prüfkörper dieser Arbeit nicht unverändert als Bauteil verwendet werden, da in keinem Fall ausschließlich günstige Effekte zusammentreffen. Auf Grundlage der hier gezeigten Daten kann jedoch eine Abschätzung des Tragverhalten erfolgen, sodass fortführende Untersuchungen zu einer günstigen Kombination aller notwendiger Eigenschaften führen können.

\section{Sind höhere Erstriss- und Traglasten möglich?}

Spannglasträger zeigten eine gesteigerte Erstrisslast. Somit konnte ein günstiger Effekt der mechanischen Vorspannung gezielt ausgenutzt werden. Eine geplante Steigerung der Traglast wurde jedoch nicht beobachtet. Daher muss die Konstruktion angepasst werden. Hierfür werden Vorschläge in Kapitel 6 (ab Seite 242) ausgearbeitet.

\section{Ist eine Überdimensionierung mit „Opferscheiben“ nötig?}

Die Bewehrung ersetzt die Funktion von „Opferscheiben“ und hält auch einen gebrochenen Träger an Ort und Stelle, woraus ein geringerer Materialverbrauch resultiert. Die Querschnitte sind damit schlanker und haben eine kleinere Biegesteifigkeit, sodass mit geringeren Vorspannkräften, und 
folglich einer minimierten, zusätzlich eingebrachten elastischen Energie, ein gleich hoher Vorspanngrad erreicht wird. Somit ist der Verzicht auf „Opferscheiben“ für vorgespannte Glasträger vielmehr als essenziell notwendig zu bewerten, um das Tragverhalten zu optimieren.

\section{Sind Spannglasträger sichere Bauteile?}

Die Tragsicherheit einer Glasträgerkonstruktion ergibt sich aus dem Tragverhalten, der Art und Weise des Versagens, der Duktiliät sowie der Resttragfähigkeit.

Die mechanische Vorspannung steigert das Gebrauchstauglichkeitsniveau um den Dekompressionsanteil, sodass die Rissfreiheit des Glases bis zu höheren Lasten garantiert ist. Dies bedingt den Nachteil, dass im Rissfall unter höheren Lasten eine größere Energie frei wird, welche zusätzlich durch die Wirkung der mechanischen und thermischen Vorspannung vergrößert wird. Durch weitere Forschungsarbeit ist nachzuweisen, dass ein „explosionsartiges“ Versagen ohne jegliche Ankündigung, beispielsweise durch eine Begrenzung der Vorspannkraft, sicher vermieden wird.

Mittels einer Bewehrung kann ein duktiles Bauteilverhalten mit einem Lastabtrag nach dem Erstriss ermöglicht werden. Mit diesem Nachweis kann der Erstriss als Gebrauchstauglichkeitsgrenze definiert werden, während die Tragfähigkeitsgrenze mit deutlich sichtbarem, gebrochenem Glasanteil bei höheren Lasten erreicht wird.

Die hier gezeigte Diskussion der Resttragfähigkeit lehnt sich an die experimentelle Nachweisführung für bestehende Bauprojekte einschließlich ihrer individuellen Anforderungen an. In den benannten Grenzen, insbesondere bezüglich der Belastung und des Schädigungsszenarios und unter Berücksichtigung der kritischen Diskussion, besteht die Möglichkeit, Spannglasträger als resttragsicher zu beschreiben.

Unter diesen Aspekten können Spannglasträger insgesamt als sichere Bauteile bezeichnet werden. 


\section{Sind Spannglasträger dauerhafte Bauteile?}

Zunächst kann eine Temperaturbelastung auf Spannglasträger rechnerisch berücksichtigt werden, sodass die Dauerhaftigkeit gewährleistet werden kann.

Die Untersuchung des Tragverhaltens unter Dauerlasten brachte die Größenordnung der gemessenen Spannkraftverluste als kritischen Aspekt hervor. Entsprechend kann nur eine weiterführende Untersuchung mit angepassten Konstruktionen zu einer abschließenden Wertung führen. Bei positiver Evaluation ist ein Nachweiskonzept zur Ermittlung der Spannkraftverluste zu finalisieren.

Nichtsdestotrotz können insbesondere verbundlos vorgespannte Spannglieder nachgespannt werden. Damit besteht die Möglichkeit, Spannglasträger dauerhaft auszuführen.

\section{Welche Konstruktionen sind zu empfehlen?}

Die Belastung der Zwischenschicht, ein exzentrischer Lasteintrag in die Verbundglaskante und daraus resultierende exzessive seitliche Durchbiegungen sowie das „explosionsartige" Versagen haben sich als ungünstige Eigenschaften herausgestellt - mit der Vorspannung ist vorsichtig umzugehen. Entsprechend sind solche Konstruktionen zu empfehlen, die eine mechanische Vorspannung gleichmäßig und mit minimierter elastischer Energie in das Tragwerk einbringen. Um fortführend effektive Spannglasträger zu konstruieren, soll das Verhalten allgemeingültig charakterisiert und ein nachträglicher Verbund realisiert werden. 


\section{$6 \quad$ Konstruktive Empfehlungen}

\subsection{Zielsetzung}

In diesem Kapitel werden die konstruktiven Erfahrungen aus Teilprojekten zusammengefasst, die im Rahmen der übergeordneten Forschungsarbeit abgeschlossen wurden, und in diese Empfehlungssammlung einfließen.

Dabei werden die Anforderungen an die Einzelkomponenten aufgezählt, die zugehörige Lösung beschrieben und in einen Kontext gestellt. Es werden Vor- und Nachteile benannt, um eine Ausgangsbasis für eine Weiterentwicklung bereitzustellen.

Das Zusammenspiel folgender Aspekte steht im Mittelpunkt:

- Eignung der Konstruktion - Materialwahl

- Fertigungsmöglichkeit - Verfügbarkeit von Fertigungsverfahren, Fertigungsmitteln

- Funktionssicherheit - Dimensionierung der Bauteile durch Berechnung oder andere Verfahren

\section{$6.2 \quad$ Teilprojekte}

\subsubsection{Forschungsprojekt „Bewehrte Glasträger“}

Das Forschungsprojekt „Bewehrte Glasträger“ am Institut für Baukonstruktion der TU Dresden stand am Anfang der Forschung an Glasträgern mit vorgespannter Bewehrung. ${ }^{390}$ Darin wurden erstmals verbundlos vorgespannte Glasträger mit variierten Bewehrungsführungen ${ }^{391}$ sowie einfacher und zweifach Umlenkung konstruiert und experimentell untersucht. Wesentliche Ergebnisse finden sich in [Weller \& Engelmann 2014a] und [Weller \& Engelmann 2014b]. Die geklebte Vorzugsvariante des Umlenkpunktes entstand im

390 Bundesministerium für Wirtschaft und Energie (BMWi) Zentrales Innovationsprogramm Mittelstand (ZIM), Kooperationsprojekt (KF) KF2050205HF1. Laufzeit vom 01. 04. 2012 bis 31. 07. 2014.

391 Zentrische und exzentrische Bewehrungsführung. 
Zuge dieser Entwicklung. Zudem wurde der Begriff „Spannglasträger“ in Analogie zum „Spannbetonträger“ eingeführt.

Letztlich wurde auf der Tagung „Glasbau 2015“ in Dresden ein „Spannglassteg“ auf Basis der Experimentalkonstruktion vorgestellt. Die $2 \mathrm{~m}$ weit spannende Brücke konnte durch die Konferenzbesucher über eine begehbare Verglasung direkt benutzt werden (Bild 101).

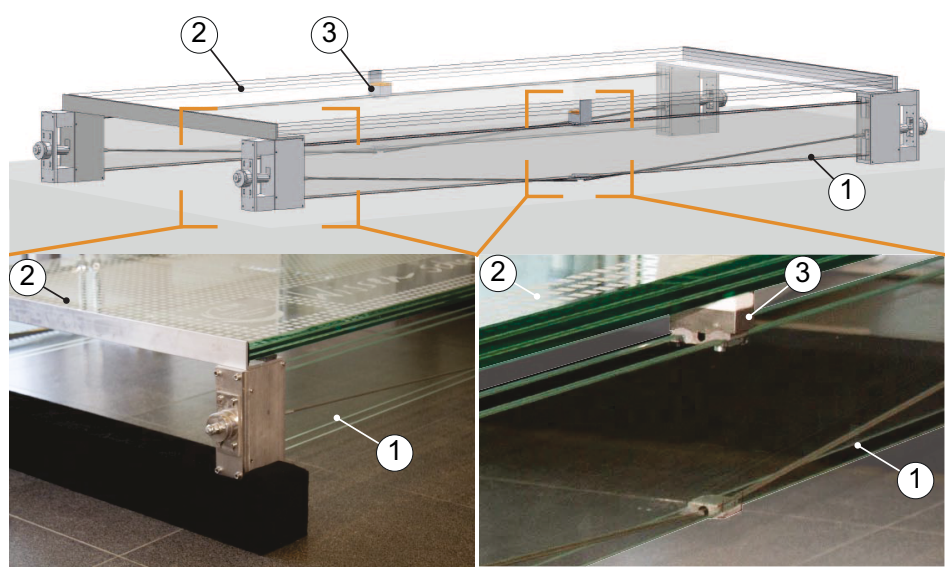

\subsubsection{Spannglasbrücke - glasstec 2014}

Der Wunsch sowohl die Spannweite zu maximieren, als auch ein nutzbares Demonstratorobjekt auf der "glasstec 2014“ in Düsseldorf vorzustellen, führte am Ende des Forschungsprojektes zur Konstruktion einer $9 \mathrm{~m}$ weit spannenden „Spannglasbrücke" (Bild 102). Vorangegangene experimentelle Untersuchungen ${ }^{392}$ zeigten die Tragfähigkeit der Hauptträger, sodass ein Nachweis der Gesamtkonstruktion einschließlich seiner Zugangstreppe, einer umlaufenden Brüstung sowie einer großflächigen begehbaren Verglasung erbracht werden konnte. ${ }^{393}$

\section{Bild 101}

Spannglassteg als Demonstrator am 19.03. und 20.03.2015 auf der „Glasbau 2015“ an der TU Dresden.

(1) Spannglasträger

(2) Begehbare Verglasung aus $8 \mathrm{~mm}$ TVG mit rutschhemmendem Siebdruck und 2x $10 \mathrm{~mm}$ TVG

(3) Zwischenauflage 


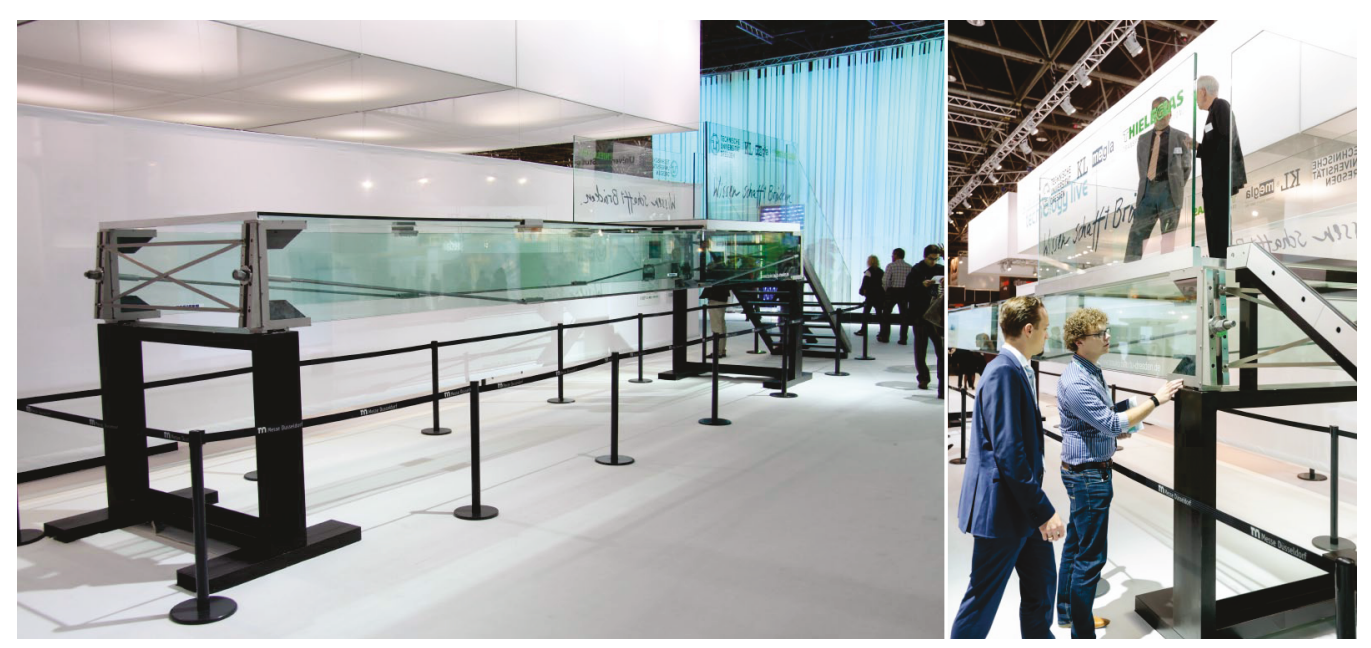

\section{Bild 102}

Spannglasbrücke als Demonstrator vom 21. 10. bis 24.10.2014 auf der „glasstec 2014“ in Düsseldorf.

Bild 103

Spannglasbrücke als Beitrag zu einem Entwurfswettbewerb für Fußgängerbrücken auf der IABSE Konferenz am 12. 05. 2015 in Nara (Japan).

\subsubsection{Fußgängerbrücke in Nara (Japan) 2015}

Darüber hinaus konnte das Konzept einer Spannglasbrücke auch erfolgreich bei einem Brückenentwurfswettbewerb am 12. 05. $2015 \mathrm{im}$ Vorfeld der IABSE Konferenz in Nara (Japan) platziert werden (Bild 103). Um die darin geforderte Spannweite von $26 \mathrm{~m}$ zu erreichen, mussten je Hauptträger drei Segmente vorgesehen werden, welche mittels eines durchlaufenden Spannseils vorgespannt wurden. Hierin begründet sich die erste anwendungsnahe Segmentbauweise mit Spannglasträgern. ${ }^{394}$

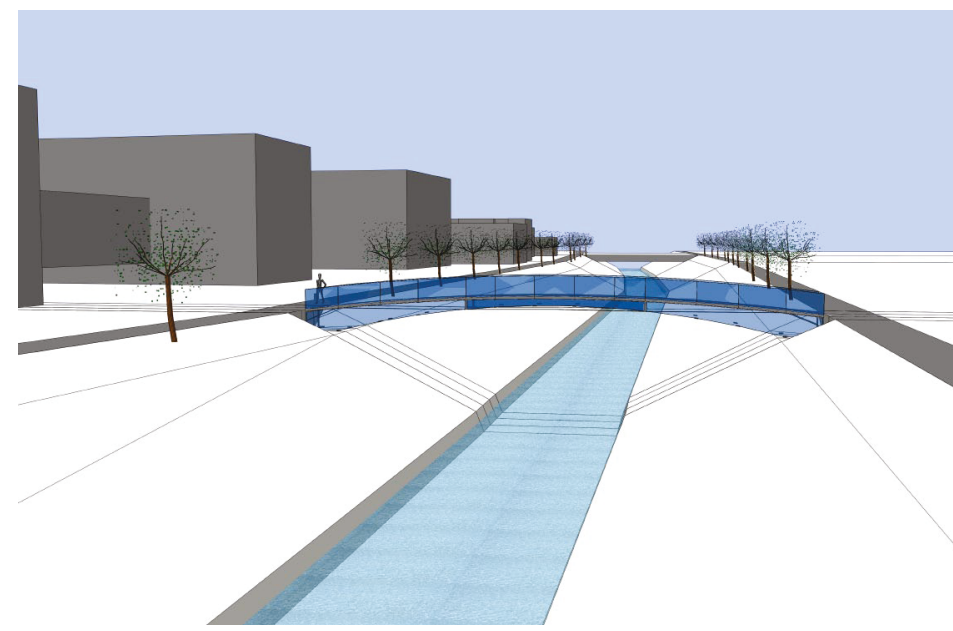

${ }^{394}$ Vergleiche [Engelmann 2015]. 


\subsection{Verankerungen}

\subsubsection{Tragfähigkeit der Verankerung}

Eine Verankerung soll eine tragfähige Verbindung zwischen der Bewehrung und dem Grundquerschnitt am Auflager ermöglichen, die Bewehrung sicher verankern ${ }^{395}$ und eine gleichmäßige Verteilung der Vorspannkraft gewährleisten.

Dazu wurde eine ausreichend steife Kopfplatte ${ }^{396}$ konstruiert und einer regelmäßigen visuellen Überprüfung auf Beschädigungen und unzulässige Verformungen unterzogen. Während der experimentellen Studien erwies sich die Konstruktion als schadensfrei und funktionstüchtig. Die Seilenden wurden mittels verpresster Gewindefittinge ${ }^{397}$ und einer Spannmutter mit der Verankerungskonstruktion verschraubt. Diese Methode ist im Seilbau üblich, verfügbar und leicht zu handhaben. Darüber hinaus ist diese Verbindung problemlos zu öffnen und wiederzuverwenden.

Grundsätzlich werden Verankerungen im Spannbetonbau in zwei wesentliche Gruppen, die Klemm- und die Keilverankerung, eingeteilt. 398

Eine Keilverankerung kam für die experimentelle Studie nicht in Betracht, da ein Nachspannen nur bedingt möglich ist und ein Schlupf beim Absetzen der Vorspannkraft auf die Ankerplatte („Nachlassweg“) einen zusätzlichen Seilkraftverlust bedeutet. Darüber hinaus kann es zum Versagen der Verankerung bei vollständiger Entlastung der Bewehrung kommen.

Eine Klemmverankerung ist im Spannbetonbau bei Nutzung einer Stahlbewehrung nicht mehr üblich. Plant man jedoch

395 Insbesondere sollen die Trageigenschaften so beschaffen sein, dass die Bewehrung auf freier Länge versagt. Vergleiche [ETAG 013].

${ }^{396}$ Es wurde hochlegierter Stahl (1.4571) einer Mindestdicke von $15 \mathrm{~mm}$ verbaut.

397 Vergleiche [ETA-11/0160].

398 Vergleiche [Leonhardt 1980] und [Rombach 2010]. Eine Verankerung im Verbund ist im Glasbau nicht möglich (Vergleiche Kapitel 2.3.3, ab Seite 35). Darüber hinaus stellen Verankerungen mit Presshülen, Stauchköpfen und Schlaufen spezifische Ausführungen im Spannbetonbau dar, die für Glasträger nach heutigem Wissensstand als nicht relevant beurteilt werden. 
das Vorspannen einer Faserbewehrung, stellt diese Option eine interessante Alternative dar. Darüber hinaus ist ein Verguss zwischen Faserbewehrung und Ankerkörper üblich, um eine kontinuierliche Lastüberleitung zu ermöglichen. ${ }^{399}$

\subsubsection{Seilkrafteinleitung}

Wie im Spannbetonbau müssen beim Bau vorgespannter Glasträger (hohe) Seilkräfte materialgerecht in die Glaskante eingeleitet werden, um Spannungsspitzen und unzulässig hohe Spaltzugkräfte im Bereich der Lasteinleitung zu vermeiden.

Um dieser Anforderung gerecht zu werden wurde eine Vorspannklotzung aus dem im Glasbau üblichen POM-C mithilfe der Ergebnisse aus [Ebert 2014] konstruiert. Dafür gewährleistete eine Führungsnut in der Kopfplatte die Lagesicherheit der Klotzung gegenüber der Glaskante (Bild 104).

Dabei kam es jedoch im Einzelfall zum Verrutschen der Vorspannklotzung (Bild 104 (3)). Entsprechend ist im Rahmen der Montage sicherzustellen, dass die Klotzung in der geplanten Endlage verbleibt. Dies ist bereits mit der hier verwendeten Konstruktion möglich, da der oben abschließende Deckel der Auflagerkonstruktion abnehmbar gestaltet wurde (Bild 104 (5)).

Zudem kommen auch andere Kunststoff-Klotzungen aus Polyetherimid (PEI), Polyetheretherketon (PEEK) oder Polyethylenterephthalat (PET) für diese Anwendung in Betracht. ${ }^{400}$ Zudem ist die Verwendung eines Injektionsmörtels ${ }^{401}$ als weitere Alternative denkbar.

\footnotetext{
${ }^{399}$ Eine Übersicht über Verankerungen von CFK-Zugelementen findet sich in [Schlaich et al. 2012].

400 Vergleiche [Ebert 2014]

401 Zur Lagerung punktförmig gehaltener Verglasungen kommt ein Vergussmaterial in Betracht. Vergleiche [DIN 18008-3, Anhang A] und [Z-21.3-1830 2011].
} 


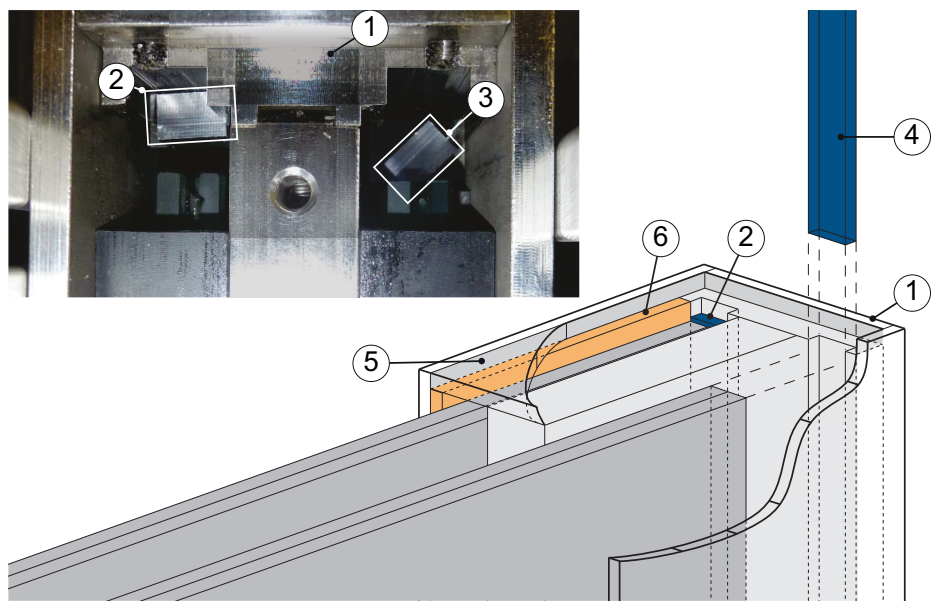

\subsubsection{Toleranzausgleich}

\section{Toleranzausgleich zwischen VSG-Paketen}

Daneben kommt der Verankerungskonstruktion die Aufgabe zu, einen Toleranzausgleich zu ermöglichen. Dabei muss insbesondere die ungleiche Länge zweier VSG-Pakete im Querschnitt egalisiert werden, um eine intolerable Schiefstellung zu vermeiden (Bild 105).
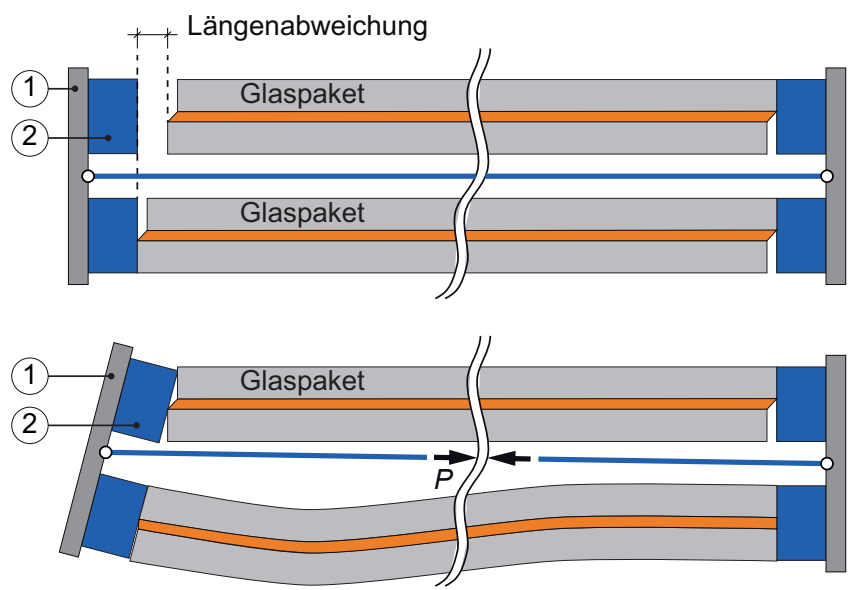

Beim Vorliegen eines unzulässigen Längenunterschiedes fand ein Ausgleich durch die Anpassung der Klotzungsdicke mittels einer spanenden Nachbearbeitung einzelner Vorspannklötze statt. Alternativ wurden Zulagen zwischen der
Bild 104

Ausschnitt der Verankerungskonstruktion mit verrutschter Vorspannklotzung.

(1) Kopfplatte

(2) Vorspannklotzung in Führung

(3) verrutschter Vorspannklotz

(4) Einbau der Vorspannklotzung nach der Positionierung der VSG-Pakete

(5) Deckel (abnehmbar)

(6) EPDM Zwischenlage

\section{Bild 105}

Schiefstellung der Auflagerkonstruktion infolge eines Längenunterschiedes beider VSG-Pakete im Doppelquerschnitt. Das Vorspannen des Seils verursacht die Schiefstellung des Trägers.
(1) Kopfplatte
(2) Kontaktklotz 


\section{Bild 106}

Schiefstellung eines Prüfkörpers aus dem Projekt "Spannglasbrücke“ (links) und zugehöriges Auflagerdetail (rechts).

(1) Vorspannklotzung

(2) ergänzte Klotzung (blau)

(3) offener Spalt zwischen Glaskante und Vorspannklotzung
Klotzung und der Glaskante vorgesehen, um den aufgetretenen Spalt zu schließen (Bild 106).

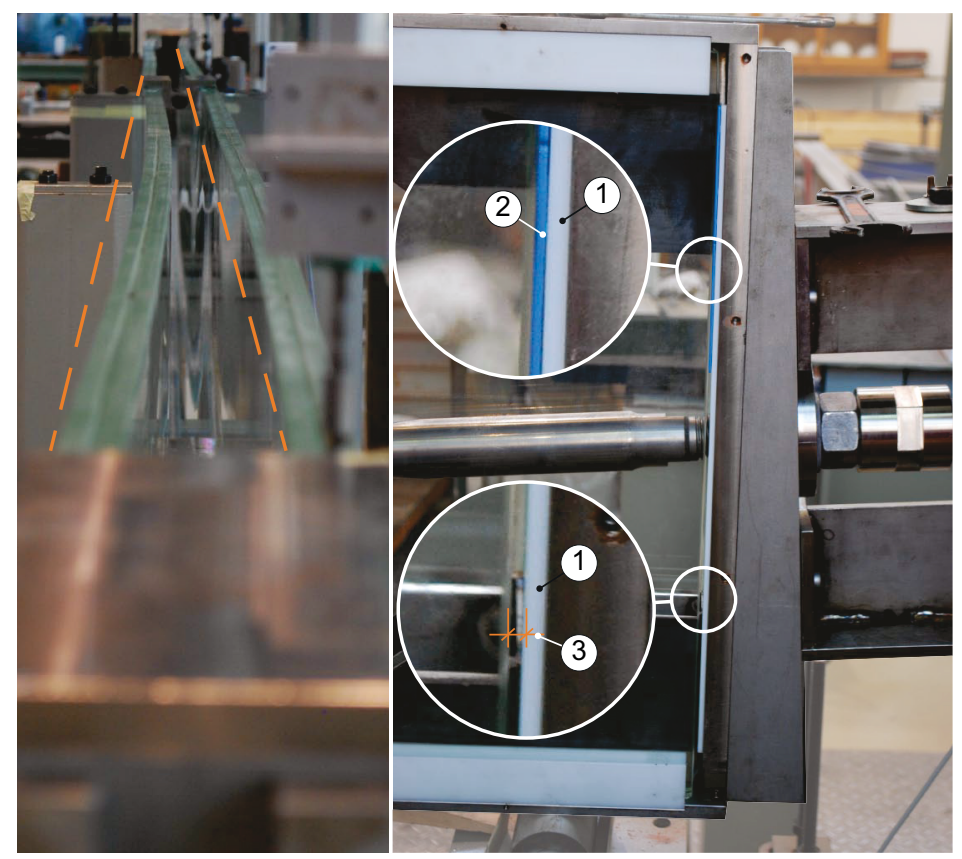

Als Alternative besteht die Möglichkeit eines automatischen Toleranzausgleichs. So schlägt [Ebert 2014] dafür eine Keilkonstruktion vor. Darüber hinaus ist ein bewegliches beziehungsweise einstellbares Scharniergelenk denkbar, welches einen aufgetretenen Spalt überbrückt und dennoch beide Glaspakete gleichmäßig am Gesamtlastabtrag beteiligt (Bild 107). Beiden Varianten ist gemein, dass dafür eine größere Anzahl mechanischer Kleinteile notwendig sind, die ihrerseits schadenursächlich werden können.

\section{Bild 107}

Toleranzausgleich mit keilfömigem Futterstück (links) und Funktionsskizze eines Scharniergelenkes in Anlehnung an [Ebert 2014] (rechts).

(1) Kopfplatte

(2) Keil mit Lagesicherung (nicht dargestellt)

(3) keilförmiges Futterstück mit Lagerschale für Kontaktklotz

(4) Scharniergelenk
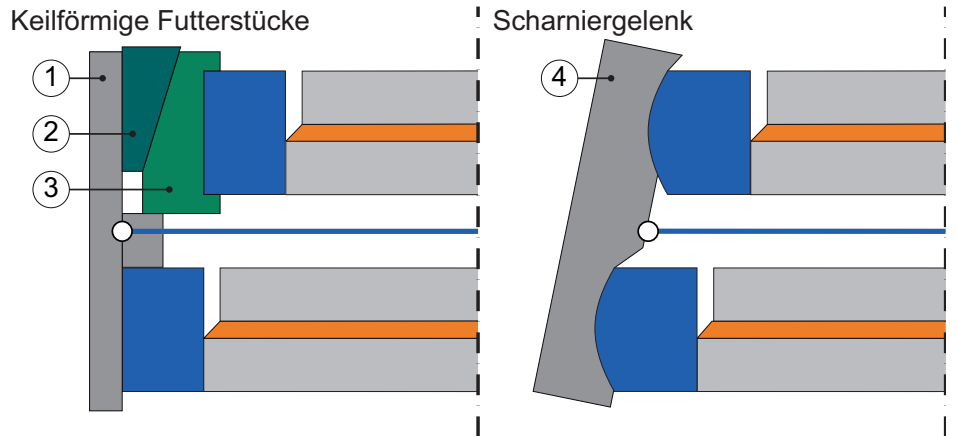

Insbesondere für die Klotzung langer Kanten können alternativ segmentierte Vorspannklotzungen vorgesehen werden, 
die beispielsweise an zwei Stellen im oberen und unteren Kantendrittel lokal vorgesehen werden. Diese Klotzung verläuft nicht über die gesamte Höhe der Kante, sodass ein Pendelarm konstruiert werden kann, der die anfallenden Lasten gleichmäßig auf die Kontaktpunkte zum Glas verteilt. ${ }^{402}$ Zur Festlegung der Klotzungsdimensionen ist ausblickend die Lasteinleitung in die Verbundglaskante genauer zu untersuchen, um Spannungskonzentrationen und eine lokale Überlastung im Glas und im Glaskontaktmaterial zu vermeiden. In Fortentwicklung der Analogiebetrachtung zum Stahlbeton- und Spannbetonbau ist dann der Nachweis einer Teilflächenbelastung zu erbringen. ${ }^{403}$

\section{Toleranzausgleich im Verbundglas}

Zusätzlich werden die Kanten eines Verbundglases produktionsbedingt einen tolerablen Kantenversatz ${ }^{404}$ aufweisen, welcher als Ursache für folgenschwere horizontale Durchbiegungen sowie der zusätzlichen Belastung der Zwischenschicht identifiziert wurde (Bild 106). In dessen Folge kommt es zum frühzeitigen Tragversagen sowie zu vergrößerten Seilkraftverlusten. Um dies zu vermeiden ${ }^{405}$, werden konstruktive Gegenmaßnahmen aufgezählt und verglichen (Bild 108 und Tafel 35).

Um diesen Kantenversatz konstruktiv zu tilgen, werden mehrere Lösungsansätze vorgeschlagen. Zum einen können Verbundglaskanten plangeschliffen werden (Bild 108 (a)). Dies ist derzeit in Deutschland nur für thermisch entspanntes Floatglas zulässig. ${ }^{406}$ Thermisch vorgespannte Glasarten müssen über einen entsprechenden Nachweis verfügen, der in der Regel eine Reduzierung der Festigkeit zur Folge hat. ${ }^{407}$ Zudem kann auch bei diesem Arbeitsschritt nicht garantiert werden, dass gegenüberliegende Kanten parallel verlaufen, sodass hierfür ein entsprechender Ausgleich zu

\footnotetext{
402 Als Vorbild kann die Lagerung der Traverse nach Bild 45 (Seite 108) dienen.

403 Vergleiche [DIN EN 1992-1-1, Kap. 5.10, 6.4 und 6.7].

404 Vergleiche [DIN EN ISO 12543-1].

405 Vergleiche auch [DIN 18008-1, Kap. 10.1].

406 Vergleiche [DIN EN 572].

407 [DIN EN 1863], [DIN EN 12150-1] sowie [Weller et al. 2016].
} 
konstruieren ist. Der Vorteil dieser Variante ist die Möglichkeit weiterhin übliche Klotzungsmaterialien zu verwenden, sodass Bauteile ohne stoffschlüssige Verbindungen erstellt werden können. Dadurch kann die Demontage sowie ein Austausch von Glaselementen problemlos erfolgen.

(a) Planschliff

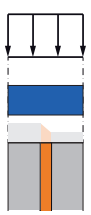

(b) Scharniergelenk
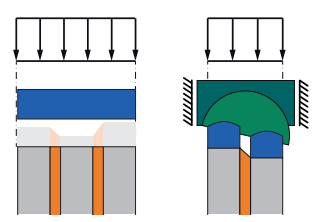

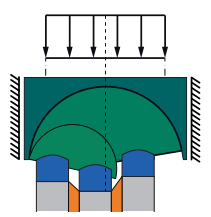

(c) individuelle Klotzung

(d) Klebung
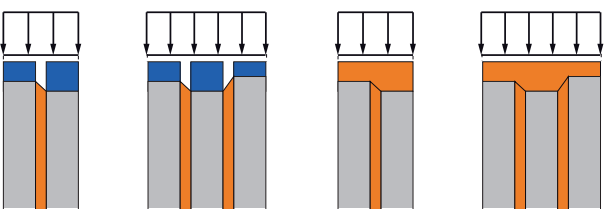

Bild 108

Konstruktive Möglichkeiten zum Ausgleich eines Versatzes der Kanten eines Verbund-Sicherheitsglases.

Des Weiteren ist eine Lastverteilung auf die Kanten der Einzelscheiben durch ein Scharniergelenk möglich (Bild 108 (b)). Solche Konstruktionen schlägt Ebert [2014] vor. Dabei handelt es sich entsprechend der verwendeten Glasdicke um kleinteilige mechanische Komponenten. Deren Funktionsweise wird mit steigender Schichtenanzahl im Verbundglas zunehmend komplex, sodass eine praktische Anwendung im Vergleich zu den übrigen, in Bild 108 vorgestellten Varianten, als weniger aussichtsreich beurteilt wird.

Außerdem kann eine Klotzung individuell angepasst werden (Bild 108 (c)). Dazu ist es denkbar, die betreffende Kantenoberfläche zu vermessen und beispielsweise individuelle Klotzungen mit additiven Fertigungsverfahren herzustellen.

Letztlich besteht die Möglichkeit, den Spalt zwischen einer Kopfplatte und der Verbundglaskante vollständig zu füllen und eine stoffschlüssige Verbindung herzustellen (Bild 108 (d)). Dazu stehen Injektionsmörtel sowie Klebstoffe zur Verfügung. Darüber hinaus kann vorgesehen werden, den Spalt während des Laminierprozesses mit dem Zwischenschichtmaterial auszufüllen. Man spricht hierbei von „einlaminierten“ Verbindungen. Als vorteilhaft kann die vollständige Tilgung eines Versatzes ohne eine Nachbearbeitung der Verbundglaskante gesehen werden. Jedoch bedingen die unterschiedlichen Dicken der Verfüllung verschiedenartige Steifigkeiten, sodass es weiterhin zum inhomogenen Lasteintrag 
kommen kann. ${ }^{408}$ Zudem ist die Frage der Materialverträglichkeit zu klären.

Derzeit liegen keine systematischen Untersuchungen zum Lasteintrag in die Verbundglaskante vor. ${ }^{409}$ Dies bildet damit ein offenes Forschungsthema.

\begin{tabular}{|c|c|c|c|c|}
\hline & Planschliff & $\begin{array}{l}\text { Scharnier- } \\
\text { gelenk }\end{array}$ & $\begin{array}{l}\text { individuelle } \\
\text { Klotzung }\end{array}$ & Klebung \\
\hline \multicolumn{5}{|c|}{ Eignung und Materialwahl } \\
\hline Glasart & $\begin{array}{l}\text { nur thermisch } \\
\text { entspanntes Glas }\end{array}$ & \multicolumn{3}{|c|}{ thermisch entspanntes und vorgespanntes Glas } \\
\hline $\begin{array}{l}\text { Glaskontakt- } \\
\text { werkstoff }\end{array}$ & \multicolumn{2}{|c|}{ gängige Klotzungsmaterialien } & $\begin{array}{l}\text { Klotzungsmaterial } \\
\text { sowie Werkstof- } \\
\text { fe zur additiven } \\
\text { Fertigung }\end{array}$ & $\begin{array}{l}\text { Injektionsmörtel, } \\
\text { Zwischenschicht- } \\
\text { materialien oder } \\
\text { Klebverbindungen }\end{array}$ \\
\hline \multicolumn{5}{|c|}{ Fertigungsmöglichkeit } \\
\hline $\begin{array}{l}\text { Montage } \\
\text { und } \\
\text { Demontage }\end{array}$ & \multicolumn{3}{|c|}{ günstig, keine stoffschlüssige Verbindung } & $\begin{array}{l}\text { ungünstig, Tren- } \\
\text { nung stoffschlüs- } \\
\text { siger Verbindung } \\
\text { nötig }\end{array}$ \\
\hline \multicolumn{5}{|c|}{ Funktionssicherheit und Dimensionierung } \\
\hline Glasart & $\begin{array}{l}\text { zusätzliche Nach- } \\
\text { weise für thermisch } \\
\text { vorgespanntes Glas }\end{array}$ & \multicolumn{3}{|c|}{ keine weiteren Anforderungen an die Glasart } \\
\hline $\begin{array}{l}\text { Glaskontakt- } \\
\text { werkstoff }\end{array}$ & \multicolumn{3}{|c|}{ Dimensionierung der Klotzung z. B. nach [Ebert 2014] } & $\begin{array}{l}\text { offenes For- } \\
\text { schungsthema }\end{array}$ \\
\hline \multirow[t]{2}{*}{ Weiteres } & $\begin{array}{l}\text { Parallelität gegen- } \\
\text { überliegender }\end{array}$ & $\begin{array}{l}\text { begrenzte Erfahrun- } \\
\text { gen vorhanden; }\end{array}$ & \multicolumn{2}{|c|}{$\begin{array}{l}\text { unterschiedliche Steifigkeit aufgrund } \\
\text { unterschiedlicher Materialdicke }\end{array}$} \\
\hline & $\begin{array}{l}\text { Kanten nicht } \\
\text { garantiert }\end{array}$ & $\begin{array}{l}\text { kleinteilige Kon- } \\
\text { struktion }\end{array}$ & $\begin{array}{l}\text { keine Erfahrungen } \\
\text { verfügbar }\end{array}$ & $\begin{array}{l}\text { nur Nutzung von } \\
\text { Injektionsmörteln } \\
\text { erprobt; Material- } \\
\text { verträglichkeit ist } \\
\text { vorab zu klären }\end{array}$ \\
\hline
\end{tabular}

Tafel 35

Vergleich konstruktiver Maßnahmen zum Ausgleich des Kantenversatzes im

\subsubsection{Neigungsausgleich} Verbund-Sicherheitsglas.

Während der Biegung des Trägers kommt es zur Verdrehung der Verankerung um die starke sowie um die schwache Biegeachse. Entsprechend der Verträglichkeitsbedingung kann

408 Luible [2004] gibt einen zugehörigen Exzentrizitätswert an.

409 Eine numerische Vorstudie findet sich in [Bukieda 2015]. 
es zu einer Relativverdrehung zwischen Glas und dem Seil kommen. Um lokale Biegebeanspruchungen im Seil beziehungsweise in dessen Gewindefitting zu vermeiden, muss die auftretende Verformung durch die Verankerungskonstruktion sicher aufgenommen werden.

Dieser Anforderung wurde mit der Konstruktion einer Kugelkalotte begegnet, die einen richtungsunabhängigen Ausgleich ermöglicht (Bild 109). Damit stellte sich die Grundfläche zum Absetzen der Spannmutter selbstständig senkrecht zur Seilachse ein. Darüber hinaus wurden Kugelscheiben und Kegelpfannen zum Ausgleich kleinerer Winkel ${ }^{410}$ verwendet. Als weitere Alternative ist ebenso ein stufenlos einstellbares Spanneisen (Bild 109 (5)) möglich.

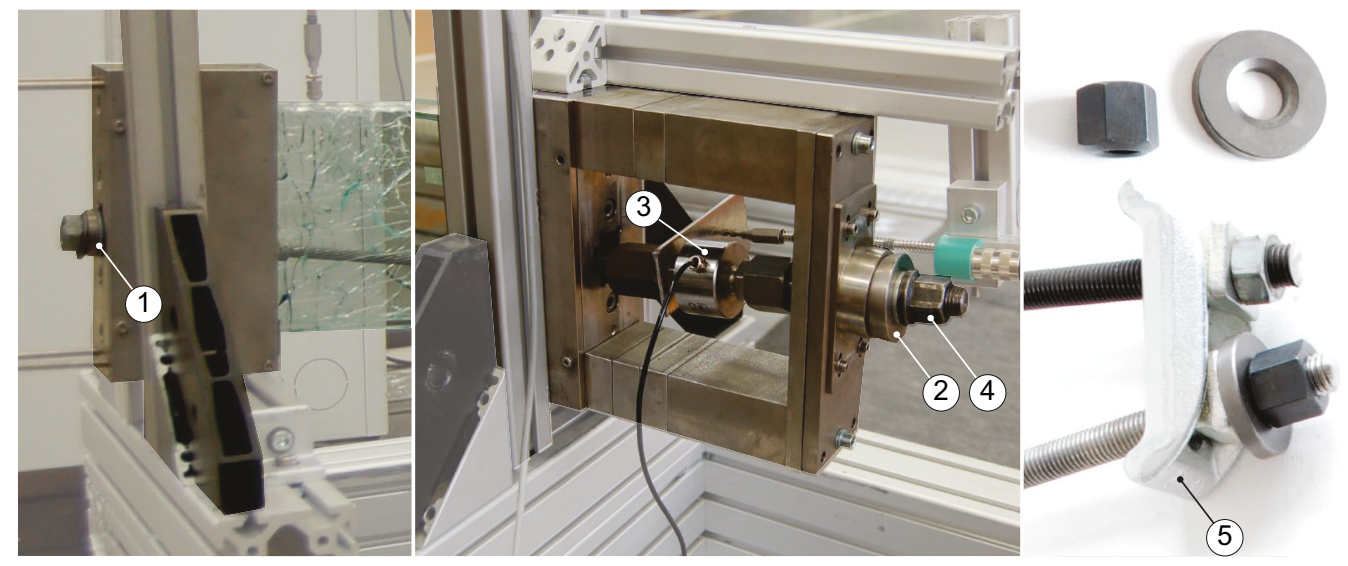

Bild 109

Möglichkeiten des Neigungsausgleichs an der Verankerung.

(1) Kugelscheibe/Kegelpfanne

(2) Kugelkalotte

(3) Kraftmessdose

(4) Spannmutter

(5) Spanneisen
Für das Projekt „Spannglasbrücke“ hingegen wurden individuell angepasste Winkelstreifen in Verbindung mit einer Kugelscheibe und Kegelpfanne verwendet (Bild 110). Die zugehörigen experimentellen Untersuchungen haben gezeigt, dass die Einstellung der Zielgeometrie problemlos funktioniert. Es bedarf hierfür jedoch eines vergleichsweise hohen Fertigungsaufwandes.

Mit der gleichen Konstruktion kann darüber hinaus eine Einstellung unterschiedlicher Seilneigungen entsprechend der gewählten Geometrievariante und Bewehrungsführung ermöglicht werden.

${ }^{410}$ Nach [DIN 6319] ist ein Winkelausgleich bis $3^{\circ}$ möglich. 


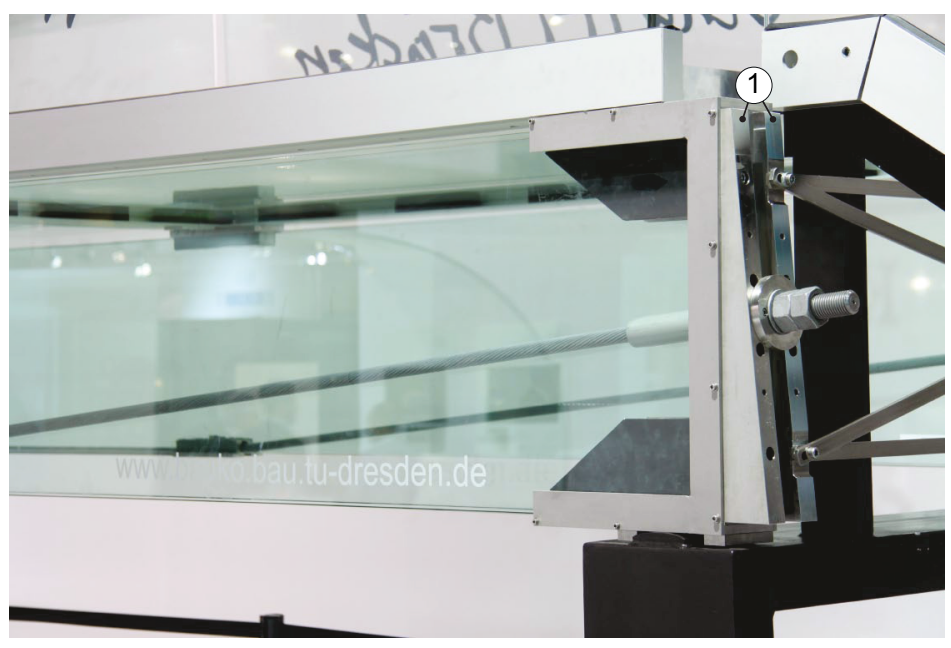

Bild 110

Winkelstreifen (1) im Projekt „Spannglasbrücke“ zum Ausgleich der Seilneigung gegenüber der Glasträgerachse.

\subsection{Vorspannverfahren}

Durch das Vorspannverfahren soll die Vorspannung (schrittweise) als Einwirkung auf das Spannglied und den Grundquerschnitt aufgebracht werden. Dabei wird der angestrebte Tragmechanismus durch Verankerung, Umlenkung und Ausbildung eines Verbundes realisiert.

Zur Erfüllung dieser Anforderung wurden einerseits ein manuelles, andererseits ein hydraulisches Spannverfahren erprobt.

Die Anwendung eines manuellen Vorspannverfahrens durch das Anziehen einer Spannmutter mit einem Ring- oder Maulschlüssel zeigt bereits Bild 109 (4). Alternativ wurde ein hydraulisches Vorspannverfahren im Rahmen des Projektes „Spannglasbrücke“ gewählt (Bild 111). Eine temporäre Hilfskonstruktion hinter der Spannmutter (1) verlängerte das Seil (4) zur Verankerung hinter einem Hohlkolbenzylinder (5, 6 und 7). Eine ähnliche Konstruktion wird auch zur Herstellung vorgespannter Glasträger in der Literatur vorgestellt. ${ }^{411}$

Für beide Varianten war die Konstruktion eines Spannstuhls

(8) notwendig, welcher eine Spannmutter beziehungsweise eine Spannpresse aufnehmen kann. Dabei konnte die

411 Vergleiche [Louter 2013], [Louter et al. 2013b], [Louter et al. 2013a] [Louter et al. 2014]. 
Messung der Seilkraft mittels einer Kraftmessdose direkt im Lastpfad eingepasst werden (Bild 109 (3)).

Bild 111

Hydraulisches Vorspannen der Bewehrung eines Spannglasträgers.

(1) Spannmutter

(2) Koppelstück

(3) Kugelscheibe/Kegelpfanne

(4) temporäre Seilverlängerung

(5) Hohlkolbenzylinder

(6) Kugelscheibe/Kegelpfanne

(7) temporäre Spannmutter

(8) Spannstuhl

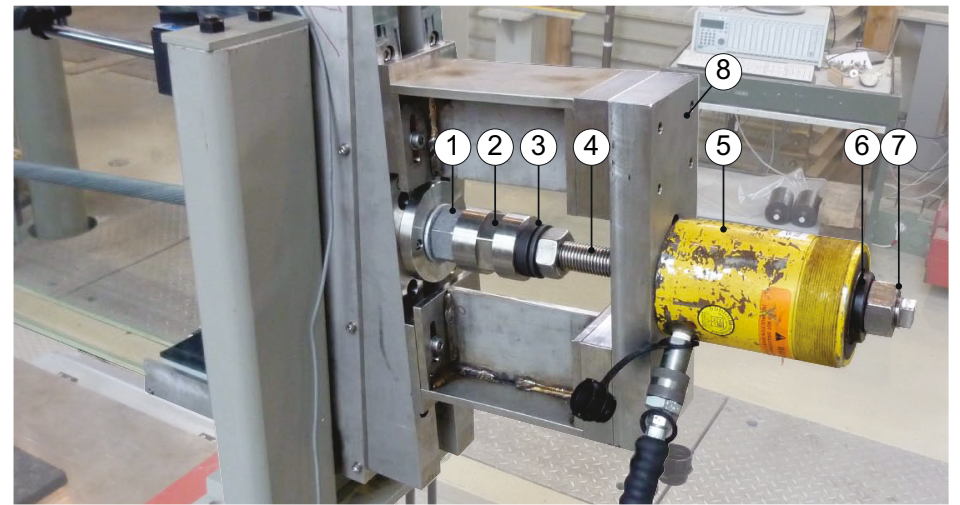

Weiterführende Spannverfahren, wie das Vorspannen durch Vorverformung, die Scheitelauspressung eines Bogentragwerks, das Vorspannen gegen Widerlager und das Vorspannen durch Spreizen, sind in Analogie zum Spannbetonbau denkbar. ${ }^{412}$ Das manuelle und hydraulische Vorspannen von Glasträgern hat sich jedoch als zweckmäßig erwiesen, sodass eine Weiterentwicklung mit heutigem Stand nicht als erstrebenswert beurteilt wird.

\subsection{Umlenkpunkte}

Ein Umlenkpunkt soll die auftretenden Umlenkkräfte materialund lastgerecht in die Verbundglaskante einleiten. Darüber hinaus führt er das Seil bei minimierter Seilreibung im geplanten Umlenkwinkel. Dabei ist insbesondere eine lokale Verzerrung des Bewehrungsmaterials zu vermeiden. Zusätzlich soll der Umlenkpunkt in Spannglasträgern dazu beitragen die lokale Torsionssteifigkeit des Doppelquerschnitts zu beeinflussen.

\footnotetext{
412 Vergleiche [Leonhardt 1980] und [Rombach 2010].
} 
Dazu wurden stählerne Umlenkpunkte als Einbauteil ${ }^{413}$ gefertigt und ein Sattelradius sowie Umlenkwinkel nach Bild 43 (Seite 105) berücksichtigt. ${ }^{414}$

\subsubsection{Geklotzte Umlenkpunkte}

Klotzungen können ausschließlich Druckkontaktlasten übertragen $^{415}$, sodass der metallische Umlenkpunkt die Glaskante vollständig umgreifen muss. Erste Konstruktionen mit „weicher" Klotzung (Bild 112 (a)) zeigten dabei auffällige große Längsverdrehungen zum Glas (Bild 113, links).

(a)

(b)

(c)

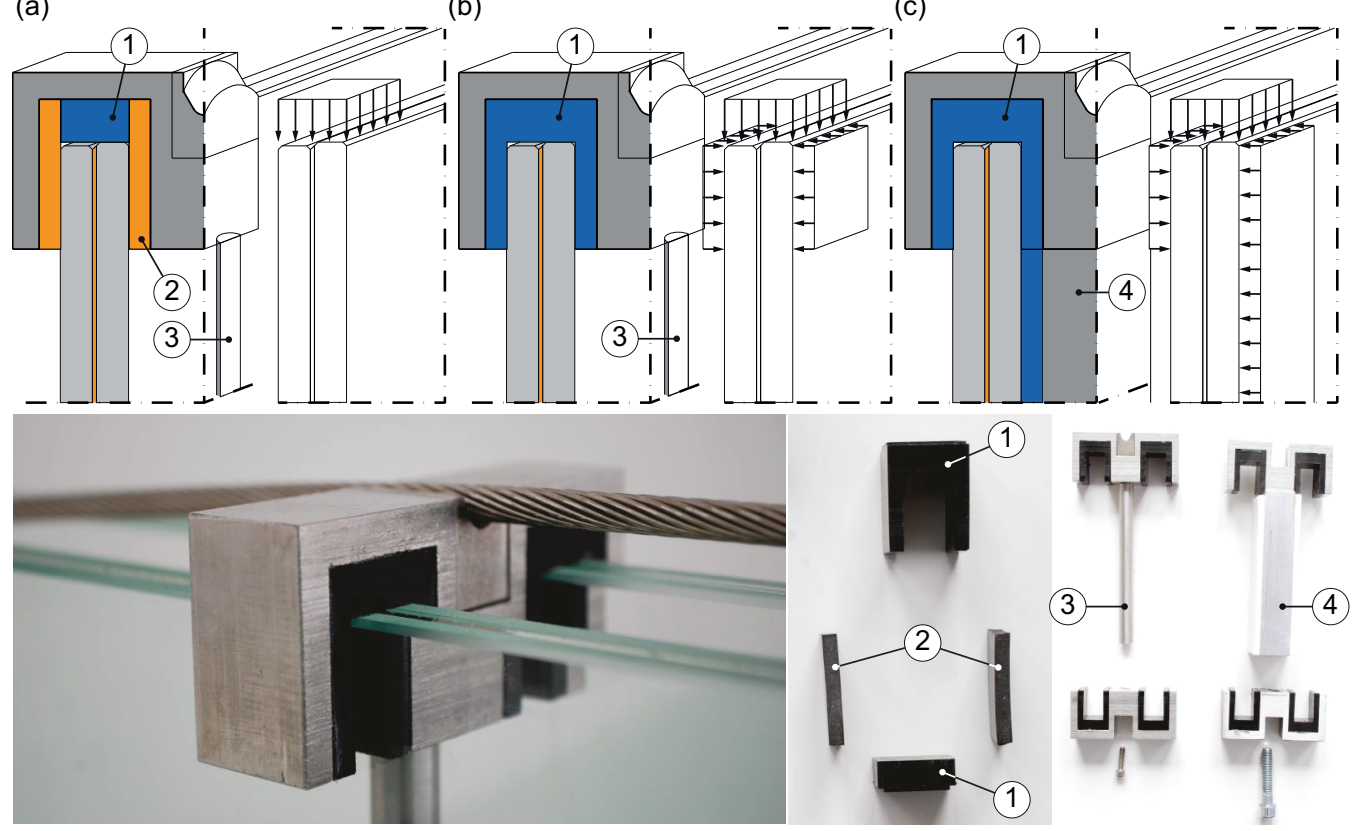

Bild 112

Geklotzte Umlenkkonstruktionen.

${ }^{413}$ Auch [Rombach 2010] verweist auf die hohen Anforderungen an Um-

(1) Klotzung „steif“ (POM-C) lenkkonstruktion, die auch im Betonbau üblicherweise als gesonderte Einbauteil gefertigt werden.

(2) Klotzung „weich“ (EPDM)

414 Die Werte unterschreiten die Angaben in [DIN EN 1993-1-11, Kap. 6.3], was aufgrund der experimentellen Verkleinerung der Prüfkörper als gerechtfertigt angenommen wurde.

(3) Verbindungsstab

(4) breiter Verbindungsstab "Schott"

415 [Ebert 2014] verweist darauf, dass der Reibkoeffizient zwischen Glas und Klotzung bemessungsrelevant werden kann. Daher sollten nur Klotzungswerkstoffe gewählt werden, die einen geringen Reibwert aufweisen. 
Es wurde geschlussfolgert, dass mit dieser Konstruktion nur eine Lagesicherung möglich war, jedoch kein gezielter Lastabtrag erfolgte. Entsprechend wurde auch die umgreifenden Klotzungsanteile „steif“ aufgeführt (Bild 112 (b) und (c)).

Darüber hinaus können Klotzungen keine Schublasten übertragen, sodass es zum Verschieben der Umlenkpunkte kommen kann, wenn Umlenkkräfte mit Lastkomponenten entlang der Glaskante wirken. Als Beispiel dafür können die zweifach umgelenkten Trägerkonfigurationen dieser Arbeit benannt werden. ${ }^{416}$
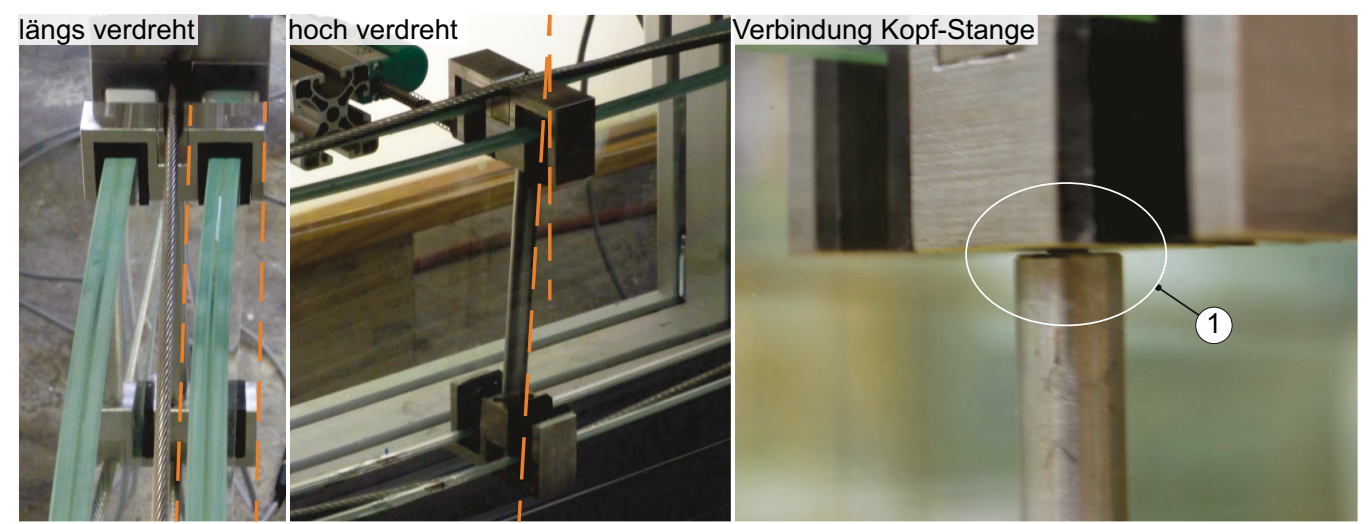

\section{Bild 113}

Problemdetails geklotzter Umlenkkonstruktionen.

Links: Glas gegenüber dem Umlenkbock verdreht. Mitte: gegenläufige horizontale Verschiebung des oberen und unteren Umlenkpunktes.

Rechts: geringe Steifigkeit der Schraubverbindung zwischen Umlenkkopf und Verbindungsstange ermöglicht unerwünschte Verschiebungen (1).
Daher wurde eine Verbindungsstange zwischen dem oberen und unteren Umlenkpunkt vorgesehen, welcher statisch notwendig, jedoch optisch unerwünscht war. Damit zeigte sich eine weiterhin mögliche horizontale Relativverschiebung des oberen zum unteren Umlenkpunkt (Bild 113, mittig) aufgrund möglicher Spalte zur Verbindungsstange (Bild 113 (1)). Daher wurde die Stange zum „Schott“ erweitert (Bild 112, rechts), wodurch jedoch keine wesentliche Verbesserung der Tragfähigkeit erzielt und die Variante verworfen wurde.

416 Vergleich Bild 42, Seite 104. 


\subsubsection{Geklebte Umlenkpunkte}

Mit auf die Glasträger aufgeklebter Umlenkpunkte war zusätzlich ein planmäßiger Abtrag von Schublasten möglich (Bild 114), weshalb auf die Verbindungsstange verzichtet werden konnte. Mit umgreifenden Enden als Nothalterung bestand die Möglichkeit einer Kaltverformung der Träger in Längsrichtung, was jedoch zu keinem nennenswerten Vorteil führte. ${ }^{417}$
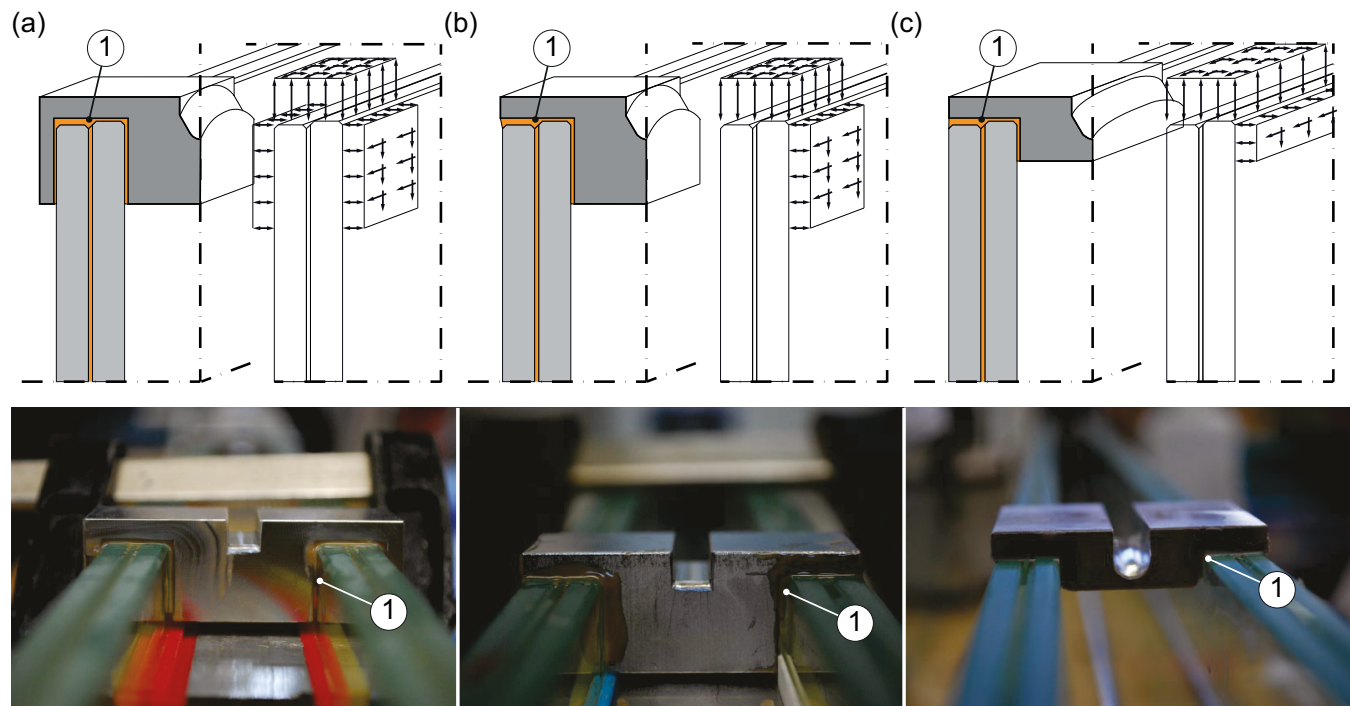

Bild 114

Geklebte Umlenkkonstruktionen mit einer Klebfuge (1)

Um im Weiteren eine vollständige ebene Glasfläche der Spannglasträger zu erreichen, wurde auf eine umgreifende von $1 \mathrm{~mm}$ Dicke. Umlenkung verzichtet, was zu ästhetischen Vorteilen aufgrund eines vollständig minimierten Stahlanteils führte. Dabei zeigte sich jedoch, dass ein Versagen der Klebung zum Abreißen der Umlenkung vom Glas führen kann (Bild 115, links). Darüber hinaus kam es zu Spannungsspitzen aufgrund des minimierten Stahlanteils und der Verwendung eines vergleichsweise steifen Epoxidharzklebstoffs. ${ }^{418}$ Dies zeigte sich im Polfilterbild während des Biegeversuchs. Im Vergleich zu einer geklotzten Konstruktion ohne wesentliche

417 Vergleiche [Weller \& Engelmann 2014b].

418 Gegenüber dem verwendeten Klebstoff SikaFast $\AA^{8} 5211$ NT mit einer Zugscherfestigkeit von circa $10 \mathrm{~N} / \mathrm{mm}^{2}$ bei Raumtemperatur, wird der hier verwendete Epoxidharzklebstoff 3M Scotch-Weld® 9323 durch einen Wert von circa $36-40 \mathrm{~N} / \mathrm{mm}^{2}$ bestimmt. Vergleiche [SikaFast 5211NT] und [Scotch Weld 9323]. 
Spannungsspitzen und horizontal verlaufenden Farblinien (Bild 115, rechts) zeigt Bild 115 (mittig) ein frühzeitiges Glasversagen an.
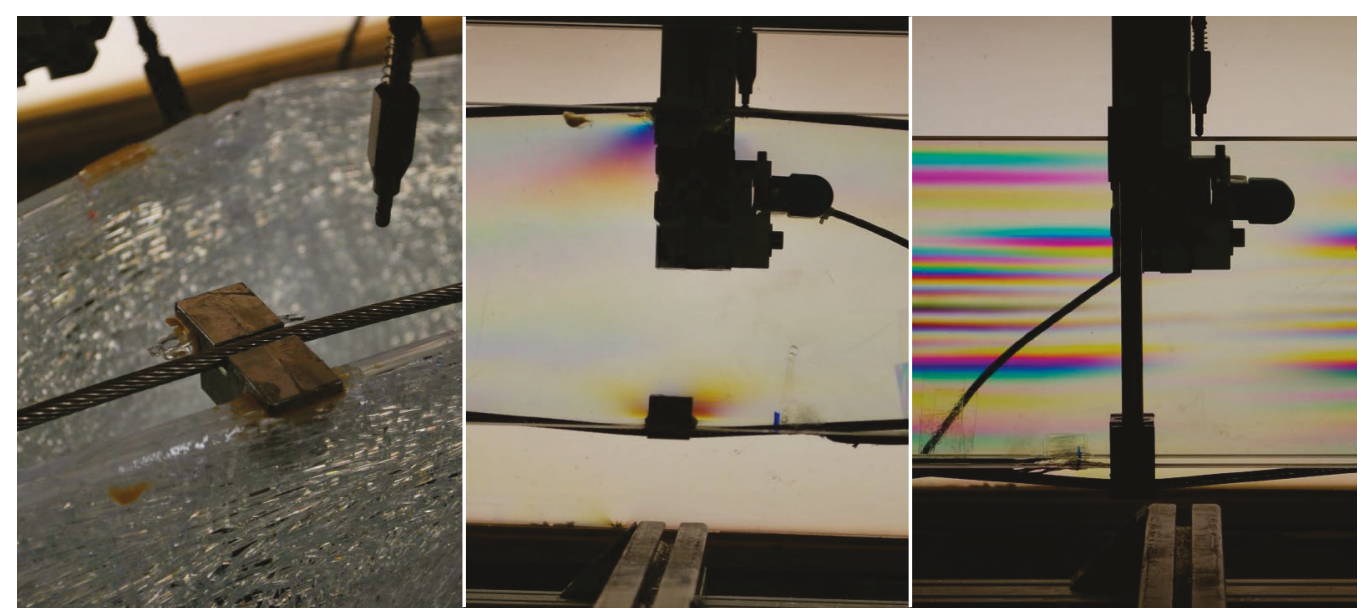

\section{Bild 115}

Problemstellen geklebter Umlenkkonstruktionen.

Links: stoffschlüssig gefügte Verbindungen können ohne Nothalterung abreißen

Mittig: Es sind Spannungskonzentrationen bei ungünstiger Klebstoffwahl möglich. Im Vergleich dazu rechts ein Polfilterbild einer geklotzten Konstruktion nach Bild 112 (b)

ohne erkennbare

Spannungskonzentrationen.
Die ästhetischen Vorteile sollten jedoch erhalten bleiben, sodass als Kompromisslösung die Vorzugsvariante der Prüfkörper dieser Arbeit entstand. Darin wurde die Länge der Umlenkpunkte in Längsrichtung verdoppelt und ein weicherer Acrylatklebstoff gewählt, wodurch der gewünschte Trageffekt erreicht wurde.

Die Detailentwicklung des Umlenkpunktes war zugunsten der Erforschung des Tragverhaltens der Gesamtkonstruktion zurückgesetzt. Entsprechend kann eine Weiterentwicklung des konstruktiven Details angestrebt werden, um vergrößerte Tragvorteile zu erzielen.

\subsection{Montage}

Durch die Montage soll gewährleistet werden, dass die gewünschte Funktionalität fehlerfrei erreicht wird. Gleichzeitig soll ein wirtschaftliches Verfahren sicherstellen, dass eine konkurrenzfähige Konstruktion erstellt wird.

Dafür wurde eine schrittweise Aufbauanweisung erstellt und erprobt: 
1. Bereitstellung aller Einzelteile

2. Aufstecken der Auflagerklotzungen auf die Glaspakete

3. Einstellen der Glaspakete in die Auflagerkonstruktion

4. Einstecken der Vorspannklotzung

5. Einhängen der Bewehrung am Festanker und durchführen bis zum Spannanker

6. zentrisches Vorspannen der Bewehrung zum Schließen aller Lücken zwischen Glaspaketen und Vorspannklotzungen

7. Herstellen der Klebverbindung entsprechend der unten vorgestellten Klebanweisung

8. Entspannen der Bewehrung und Umlenken entsprechend der geplanten Seilführung

9. Erneutes Einhängen des Seils am Spannanker und Aufbau der Vorspanntechnik (Kalotten, Spannpressen, Messtechnik)

Ergänzend wurd eine Verfahrensanleitung zum Aufkleben der Umlenkpunkte erarbeitet (Bild 116)

1. Bereitstellen aller Einzelteile und Materialien

2. Reinigen der Klebflächen auf dem Glas und den Umlenkpunkten sowie der Kleblehre ${ }^{419}$

3. Aufbringen der Abstandhalter auf dem Umlenkpunkt (Bild 117

(1))

4. Abkleben benachbarter Bereiche, die keinen Kontakt zum Klebstoff haben sollen

5. Positionieren einer Kleblehre (a)

6. Aufbringen des Klebstoffs auf die zu klebenden Flächen der Umlenkpunkte

7. Positionieren des Umlenkpunktes auf der Kleblehre (b)

8. Positionierung der zweiten Kleblehre (c) und finales Andrücken des Umlenkpunktes bis zum Anschlag auf der Kleblehre. Eine konstante und gewünschte Klebfugendicke wird durch die geometrische Gestaltung erreicht. Es bietet sich an, die Klebstelle nach diesem Schritt beispielsweise mit einer Klemme oder Schraubzwinge zu fixieren.

${ }^{419} \mathrm{Ab}$ diesem Arbeitsschritt sind zum Arbeitsschutz Handschuhe zu tragen. Eine erneute Verschmutzung der aufzuklebenden Flächen ist zu vermeiden. Die Sicherheitshinweise des Klebstoffherstellers sind zu beachten. 
9. Abstreichen überschüssigen Klebstoffs (d). Während dieses Schrittes sollte kontrolliert werden, ob über die gesamte Fugenlänge ausreichend Klebstoff austritt. Gegebenenfalls ist zusätzlicher Klebstoff einzufüllen. Bei Klebstoffen mit merklicher Schrumpfneigung sollte eine Wulst überstehen bleiben.

10. Aushärten des Klebstoffs nach Herstellerangaben
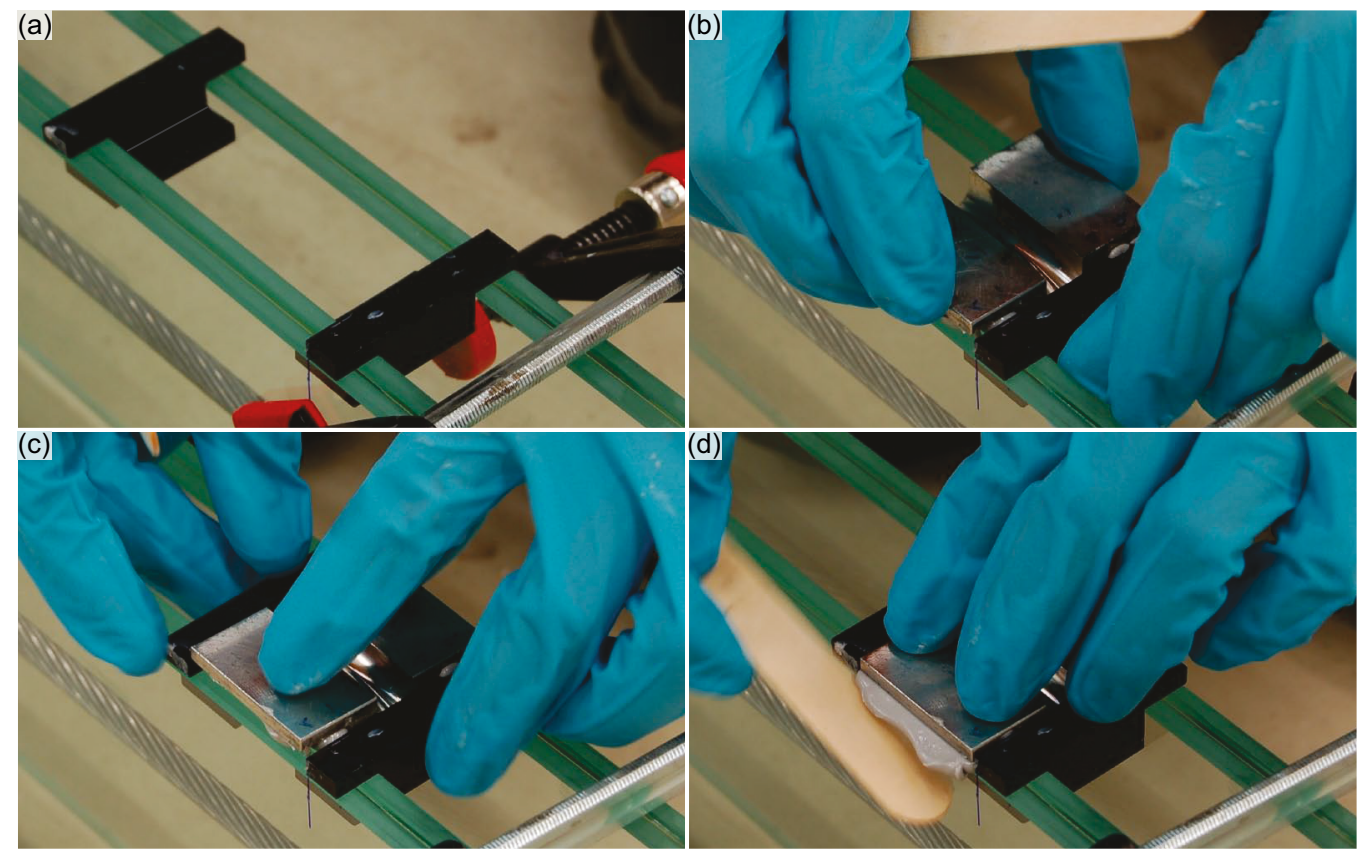

Bild 116

Verfahrensschritte zum Aufkleben der Umlenkpunkte.

Insbesondere die Wahl der Abstandhalter zeigte sich als

(a) Positionieren einer Kleblehre

(b) Positionieren des Umlenkpunktes

(c) Positionierung der zweiten Kleblehre

(d) Abstreichen überschüssigen Klebstoffs wesentlich, da sie eine konstante Klebfugendicke und eine korrekte Positionierung der Umlenkpunkte sicherstellen. Zudem wurden sie zur Kontrolle verwendet: waren die Abstandhalter (Bild 117, oben links) durch die transparenten Seiten des Trägers nach dem Verkleben zu sehen (Bild 117, oben rechts), so konnte davon ausgegangen werden, dass die Fuge die gewünschte Geometrie erreicht hat. Zudem war die Wahl des Materials der Abstandhalter entscheidend. Insbesondere im Projekt „Spannglasbrücke“ mussten sie die bedeutende Eigenlast der voluminösen stählernen Umlenkpunkte tragen, um das vollständige Herausdrücken des Klebstoffs zu vermeiden (Bild 117, unten). 


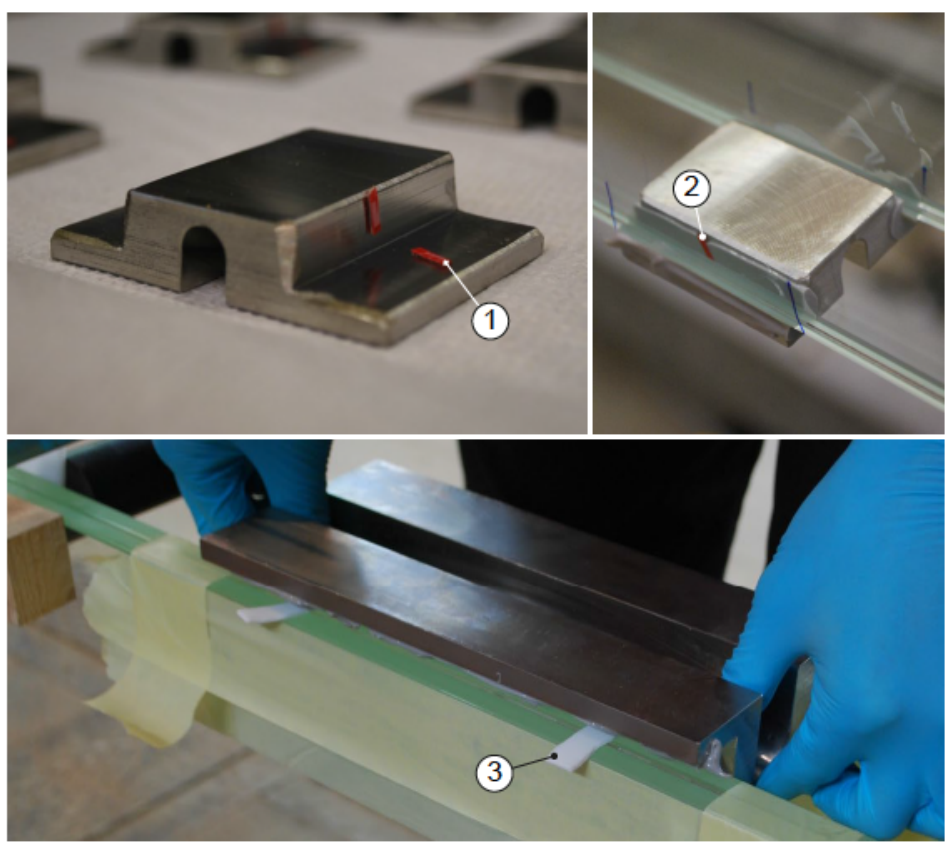

\subsection{Weiterführende Konstruktionen}

Neben der Anwendung als Dachbinder oder konventioneller Fassadenpfosten ist das Konzept eines verbundlos vorgespannten Trägers für den Einsatz in Seilnetzfassen denkbar. Darin werden Glasflächen durch Seilkonstruktionen versteift, welche für diesen Zweck vorgespannt und an einer Unterkonstruktion verankert werden müssen. Es ist denkbar, die dabei Vorspannkräfte direkt in der Glasebene zu belassen, sodass die Steifigkeit der Fassade erhalten bleibt, die zugehörige Unterkonstruktion jedoch entlastet wird (Bild 118). Diese Idee einer "Spannglasfassade" sollte anhand von Fallbeispielen statisch und konstruktiv überprüft werden.

\section{Seilnetzfassade}

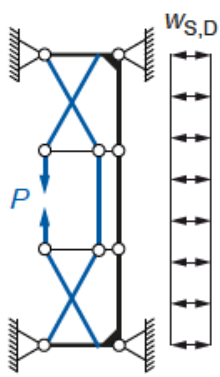

\section{Spannglasfassade}
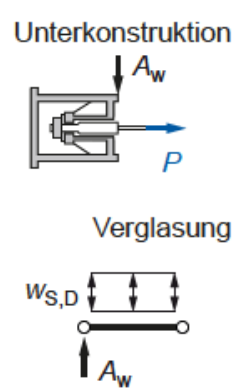

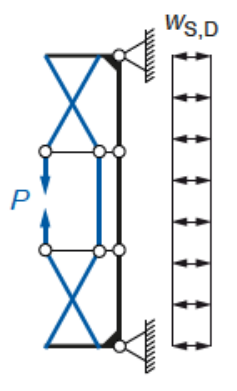

Unterkonstruktion

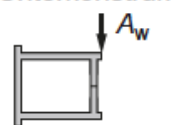

Verglasung

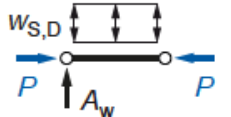

\section{Bild 117}

Umlenkpunkte mit aufgebrachten Abstandhaltern vor dem Kleben.

Bei der Auswahl der Abstandhalter (1), (2) ist die Eigenlast der Umlenkpunkte zu beachten. Die voluminösen Umlenkpunkte aus dem Projekt „Spannglasbrücke" $(8 \mathrm{~kg})$ wurde mit steiferen Abstandhaltersteifen aus PTFE (3) aufgeklebt, um das vollständige Herausdrücken der Klebstoffs aus der Fuge und einen daraus folgenden, möglichen Metall-Glaskontakt aufgrund der Eigenlast des Umlenkpunktes zu vermeiden.

\section{Bild 118}

Prinzipskizze Spannglasfassade.

Während die Vorspannkraft in einer Seilnetzfassade über die Unterkonstruktion abgetragen wird, kann eine Spannglasfassade die Druckfestigkeit der Verglasungsebene ausnutzen. Somit wird die Unterkonstruktion entlastet. 
Entsprechend dem eingangs gewählten Analogieansatz wird mittels einer Betrachtung des heutigen Anwendungsspek-

trums verbundlos vorgespannter Betonträger ein Ausblick auf mögliche weiterführende Konstruktionen gegeben. Auf Basis der aufgefundenen Ergebnisse werden zwei konstruktive, als aussichtsreich beurteilte, Ansätze näher erläutert.

- Nachträgliche Verstärkung und Instandsetzung bestehender Bauwerke ${ }^{420}$, auch durch die Ausnutzung der Möglichkeiten neuartiger Spanngliedmaterialien ${ }^{421}$

- Brückenbau: beispielsweise Schrägseil-, Extra-Dosed- und Spannbandbrücken ${ }^{422}$

- Spannglasträger mit nachträglichem Verbund (Kapitel 6.7.1)

- Segmentbauweise (Kapitel 6.7.2)

\subsubsection{Spannglasträger mit nachträglichem Verbund}

Als wesentliches Problem der vorgestellten Spannglasträger zeigten sich eine vergleichsweise geringe Fähigkeit, nach dem Erstriss weitere Lasten abzutragen. Dies wurde mit einem ungünstigen Versagensmechanismus in Verbindung gebracht. Dabei konnte die verwendete Bewehrungskonstruktion keine ausreichende Überbrückung zwischen einzelnen Rissflanken sicherstellen. Infolge des feinen Bruchbildes ergab sich eine große Anzahl von Rissen zwischen zwei Verankerungs- oder Umlenkpunkten in der Trägerkonstruktion. Zwischen zwei Rissflanken ist nur eine Übertragung von Druckbeanspruchungen möglich, welche durch die Zugbeanspruchung im Spannglied überdrückt wird. Eine Reihe von einzelnen Bruchstücken zeigt sich dabei jedoch nicht als stabil und neigt zum Verlust der Tragfähigkeit durch seitliches Ausweichen.

Damit, und im Vergleich zu anderen Konstruktionen, wird vorgeschlagen, einen nachträglichen Verbund zwischen der

\footnotetext{
420 Beispiele sind unter anderem in [Novák et al. 2015], [Pelke \& Zichner 2015] und [Alex \& Berlin 2011] zu finden.

421 So werden Lamellen und Spannglieder aus carbonfaserverstärkter Kunststoffen (CFK) verwendet. Vergleiche unter anderem [Suter \& Jungo 2001], [Köhler \& Schimetta 2002] und [Vorwagner et al. 2013].

422 Einen Einblick bieten beispielsweise Gehlen et al. [2002].
} 
Bewehrung und dem Grundquerschnitt herzustellen. Damit ist eine unmittelbare Überbrückung der Risse möglich. Dabei wird der Verbundwerkstoff im Sinne der im Konstruktiven Glasbau üblichen Fügetechnologien nicht einer Dauerbelastung aus Vorspannung und Eigenlast ausgesetzt.

Diese Idee führt jedoch zur Aufgabe des Konzeptes „Doppelquerschnitt", da hierin keine ausreichenden geometrischen Möglichkeiten zur Ausbildung des Verbundes vorhanden sind. Zudem ist ein Nachspannen oder ein Austausch der Bewehrung nicht mehr möglich.

Nichtsdestotrotz kann diese Option eine interessante Alternative für zukünftige Glasträgerkonstruktionen bieten, da den erkannten Nachteilen zweckmäßig begegnet wird.

\subsubsection{Segmentbauweise}

Die möglichen Spannweiten einzelner Glasträger sind produktionsbedingt begrenzt. Auch der zu verzeichnende Entwicklungstrend der letzten Jahren zu Glasprodukten mit Abmessungen von mehr als $9 \mathrm{~m}$ kann diesem Problem nicht langfristig entgegen wirken, da mit steigender Spannweite der logistische Aufwand zur Herstellung, Transport und Montage überproportional steigt. Entsprechend kann eine effektive Fügetechnologie zum Stoß einzelner Spannglassegmente zu weitspannenden Konstruktionen führen.

Es wird daher vorgeschlagen, das Spannglied über eine Mehrzahl von Einzelträgern durchzuführen und die KopfstöBe über die einzubringende Vorspannkraft zu überdrücken. Dieser Ansatz führt zu einer Segmentbauweise im Konstruktiven Glasbau, welche es ermöglicht nahezu beliebige Spannweiten zu erreichen (Bild 119). Zusätzlich besteht die Möglichkeit einer umfassenden Vorfertigung, sodass nahezu vollständige Bauteile an den Bauort verbracht und unverzüglich installiert werden können. Dies wirkt sich positiv auf das Qualitätsniveau und die Dauer des Bauprozesses aus. 
Bild 119

Prinzipskizze Segmentbauweise mit Spannglasträgern.

Mittels der Vorspannung wird die Koppelfuge vollständig überdrückt.
Bei dieser Variante verbleibt die konstruktive Aufgabe, eine materialgerechte Stoßkonstruktion zu erstellen und effektive Tragsysteme aus Spannglasträgern zu entwickeln.

Zusammenfassend können so neuartige Tragwerke entwickelt und dem Drang nach steigender Transparenz durch ein effektives Tragsystem begegnet werden.

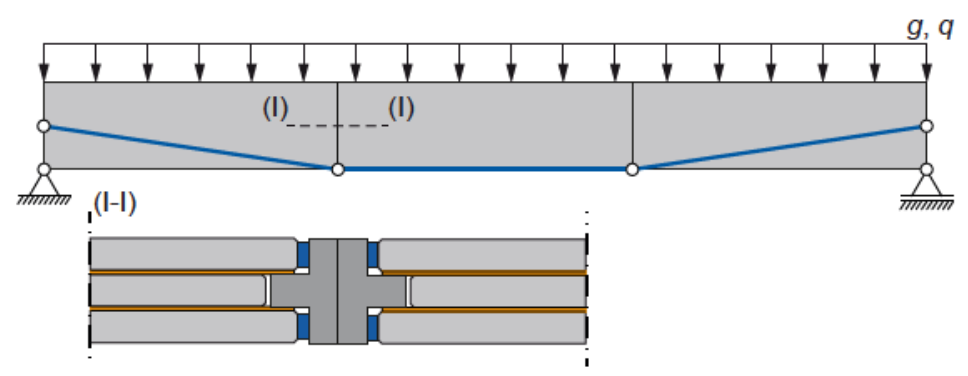

\subsection{Zusammenfassung}

In diesem Kapitel wurden ausgehend von der Beschreibung der begleitenden Forschungsprojekte konstruktive Empfehlungen zur Ausbildung einzelner Bestandteile sowie Herstellungsverfahren von Spannglasträgern beschrieben. Damit werden die Erfahrungen bei der Entwicklung von Spannglasträgern zusammengefasst und können als Grundlage für die Entwicklung fortgeschrittener Tragsysteme genutzt werden.

Am zielführendsten erscheint derzeit sowohl einen vorgespannten Glasträger mit Bewehrung im nachträglichen Verbund, als auch eine Segmentbauweise zu entwickeln, um die ungünstigen Eigenschaften der Baukonstruktion zu tilgen und zukünftig effizientere sowie transparentere Tragwerke zu gestalten. 


\subsection{Zusammenfassung}

Der Gedanke, maximale Transparenz in ein Bauwerk zu bringen, war Inspirationsquelle für Architekten und Ingenieure des letzten Jahrhunderts. Dabei wurde insbesondere die Gebäudehülle zunehmend mit neuen Aufgaben bedacht. Es sollten großflächige und vom Haupttragwerk losgelöste, fast selbstständig „schwebende“ Konstruktionen entstehen, die dem natürlichen Licht keinerlei Barriere entgegen setzen. Für den Ingenieur heißt die Anforderung, das Tragwerk so zu gestalten, dass es ebenso maximal transparent wird und als solches kaum in Erscheinung tritt. Daraus entwickelte sich der moderne Konstruktive Glasbau mit dem Anspruch, auch die Materialeigenschaften des Glases für einen gezielten Lastabtrag heranzuziehen, um dadurch eine effektivere Gesamttragwirkung zu erreichen.

Konventionelle Fassadenpfosten oder Dachbalken aus Metall oder Holz werden daher vielfach durch Glasträger ersetzt. Dabei wird der Glasquerschnitt nunmehr nicht ausschließlich als statische Platte beansprucht, sondern um seine starke Achse gebogen, sodass der Grenzzustand der Tragfähigkeit durch eine Überschreitung der Glaskantenfestigkeit oder einen Stabilitätsverlust charakterisiert ist. Zudem erfolgt ein Versagen spröde und ohne Vorankündigung. Um dieser ungünstigen Eigenschaft zu begegnen, werden konventionelle Glasträger überdimensioniert, also mit zusätzlichen, gering ausgenutzten „Opferscheiben“ versehen, sodass ein Bruch praktisch ausgeschlossen wird. Damit manifestierte sich eine weniger effiziente Bauweise.

Im Gegensatz dazu wird ein weiterer spröder Baustoff im Bauwesen fast universell eingesetzt - Beton. Dessen Sprödigkeit wird mit der Bewehrung des Grundquerschnitts begegnet. Dieser Ansatz wurde mit der Entwicklung bewehrter Glasträger bereits in vielfältigen Forschungsprojekten aufgegriffen. Dabei zeigte sich nach dem weiterhin spröden Glasbruch die Möglichkeit, einen Lastabtrag über die entstandenen Rissflanken sicherzustellen, sodass ein duktiles Bauteilverhalten erreicht wurde. Dabei verbleibt die Heraus- 
forderung, eine dauerhaft leistungsfähige Verbindung zwischen dem Glas und der Bewehrung herzustellen, da ein vergleichbares „einbetonieren“ im Glasbau nicht möglich ist.

Auch eine mechanische Vorspannung von Glasträgern wurde analog zum Spannbetonbau bereits vorgeschlagen und in einzelnen Referenzobjekten gebaut. Damit ist zu erwarten, dass die Erstrisslast vergrößert, der Durchbiegung aktiv entgegen gewirkt und ein redundanter Lastabtragspfad bereitgestellt wird. In der Literatur sind jedoch nur Ergebnisse einzelner Sondierungsuntersuchungen verfügbar, die das Tragverhalten mechanisch vorgespannter Glasträger beschreiben. Dabei zeigte sich zum ersten eine große Anzahl von Konstruktionsvarianten und zum zweiten die Herausforderung, die zusätzlich eingebrachte elastische Energie im Bruchfall sicher abzutragen.

Damit sind weder eine umfassende Systematik, noch ausreichende Konstruktionserfahrungen verfügbar, die eine allgemeingültige Charakterisierung mechanisch vorgespannter Glasträger ermöglichen. Zusätzlich wurde bisher ausschließlich auf die notwendige Beschreibung und Beeinflussung des Tragverhaltens unter kurzzeitig wirkenden Lasten abgezielt. Eine Beschreibung möglicher Spannkraftverluste bei einer dauerhaften Belastung oder der im Glasbau notwendige Nachweis der Resttragfähigkeit wurde, auch mangels allgemeingültiger Vorgaben, nicht explizit geführt. Doch erst durch diese zusätzlichen Überlegungen kann ein hinreichender Nachweis der Funktionsweise ermöglicht werden.

Die theoretisch angestrebten, günstigen Trageigenschaften mechanisch vorgespannter Glasträger sollen praktisch in einer Baukonstruktion umsetzbar werden. Entgegen dem Vorgehen eines planenden Ingenieurs wurde in dieser Arbeit ausgehend vom idealisierten Tragwerk eine konstruktive Detailentwicklung gezeigt und erprobt. Im Vergleich beider Ergebnisse ergab sich die Machbarkeit des Konzeptes sowie eine Reihe konstruktiver Empfehlungen.

Es wurden die Fragen beantwortet, ob Glasträger mit verbundlos vorgespannter Bewehrung - Spannglasträger - sichere und dauerhafte Bauteile sind, deren Weiterentwicklung 
zu effizienteren und weiterhin transparenten Glaskonstruktionen führen kann.

Daher wurde zunächst der Stand des Wissens im Konstruktiven Glasbau anhand einer Analogiebetrachtung zum Stahlbeton- und Spannbetonbau zusammengestellt. Sie beinhaltete einen Vorschlag zur umfassenden Systematisierung und Bezeichnungsweise derartiger Konstruktionen. Es erfolgte eine Eingruppierung ausgeführter Bauten und verfügbarer in der Forschung untersuchter Konstruktionen, sodass die vorgeschlagene Nomenklatur ihre Anwendbarkeit zeigte. Das Tragverhalten konnte in Analogie zum Spannbetonbau mittels der linearen Balkentheorie beschrieben werden. Zusätzlich wurde die These vertreten, dass sich die Spannkraftverluste im Konstruktiven Glasbau ebenso wie im Betonbau rechnerisch erfassen lassen.

Den benannten Herausforderungen beim Bau von Spannglasträgern wurde anschließend durch ein mehrgliedriges Konzept begegnet. Die auftretenden Biegezugspannungen an der Glaskante werden durch die mechanische Vorspannung überdrückt, sodass eine Vergrößerung der Erstrisslast erfolgte. Zudem wurde der Stabilitätsproblematik durch die Entwicklung eines „Doppelquerschnitts“ begegnet, der eine maximale Torsionssteifigkeit bei minimalem Glasanteil ermöglichte. Dadurch werden Spannglasträger, insbesondere für größere Spannweiten, wirtschaftlich herstellbar. Darüber hinaus sind deutlich geringere Vorspannkräfte nötig, um eine signifikant hohe Druckvorspannung an der Biegezugkante zu erreichen. Dabei wird eine minimale elastische Energie ins Bauteil eingetragen, welche im Bruchfall zu einem verringerten Zerstörungsgrad des Glases führt und damit als sicher zu beurteilen ist.

Diese theoretischen Überlegungen wurden mittels einer vierstufigen, experimentellen Studie untermauert, durch eine numerische Berechnung ergänzt und bildeten damit erstmals eine umfassende und systematische Datengrundlage für die Beschreibung des Tragverhaltens von Spannglasträgern unter kurzzeitig und dauerhaft wirkenden Lasten als Kern dieser Arbeit. 
Zunächst wurden 15 Prüfkörper variierter Bewehrungsgrade zur Beschreibung des Tragverhaltens unter Vorspannbelastung, unter steigender Biegebelastung bis zum Erstriss sowie weiterführend bis zum Tragversagen belastet. Dabei zeigte sich eine Steigerung der Erstrisslast, welche jedoch durch die vergleichsweise große Streuung der Glasfestigkeit überlagert war. Das dabei beobachtete Rissverhalten wurde in drei Gruppen eingeteilt. Neben dem sofortigen, spröden Versagen ergab sich eine degressive oder progressive Weiterbelastung. Letzteres Verhalten wurde als günstig herausgestellt und geschlussfolgert, dass das Tragverhalten durch eine vorgespannte Bewehrung sowohl günstig als auch ungünstig beeinflusst werden kann. Nach dem vollständigen Bruch wurden alle bewehrten Träger vom Seil gehalten. Die dabei aufgetretene Versagensform bedingte jedoch einen vollständigen Verlust der Vorspannung. Damit ergibt sich die Grundlage, einen Spannglasträger so auszulegen, dass er gegenüber einer konventionellen Glaskonstruktionen die erwarteten Vorteile zeigt.

Im zweiten Schritt wurden 24 Prüfkörper für jeweils 1000 Stunden mit einer Gebrauchslast beaufschlagt und die resultierenden Spannkraftverluste in Abhängigkeit der Bewehrungskonstruktion und Vorspannkraft ermittelt. Hierbei zeigten sich überproportional große Spannkraftverluste, welche insbesondere durch die Belastung der Zwischenschicht, als auch durch die horizontale Durchbiegung verursacht wurden. Entsprechend wurde empfohlen, die Belastung der Zwischenschicht konstruktiv auszuschließen und die seitliche Durchbiegung zu begrenzen.

Des Weiteren wurde die Resttragfähigkeit von Spannglasträgern in einem eigens entwickelten Versuch mit steigendem Teilzerstörungsgrad beschrieben. Es zeigte sich, dass eine Steigerung der Standzeit möglich war, die Vorspannung jedoch auch ungünstig wirken kann. Insbesondere bei einem unsymmetrischen Zerstörungszustand bewirkte die Vorspannung eine zusätzliche Verformung, welche zum frühzeitigen Tragversagen führte. Dieser sicherheitsrelevante Tragaspekt kann durch einen reinen Duktilitätsnachweis im Traglastversuch nicht beschrieben werden und begründet damit die Notwendigkeit einer gesonderten Untersuchung. 
Grundsätzlich zeigten bewehrte Träger unter Anwendung der neu entwickelten Versuchsmethode gegenüber unbewehrten Konstruktionen vergrößerte Standzeiten, sodass ein Spannglasträger im Grundsatz als resttragfähig zu beschreiben ist.

Letztlich bestätigte eine ergänzende Untersuchung, dass es zu einem Verlust an Vorspannkraft infolge unterschiedlicher Wärmedehnzahlen der beteiligten Werkstoffe bei einer Temperaturänderung kam. Dieser Verlust konnte mit einer analytischen Berechnung ermittelt werden und ist somit für die Auslegung von Spannglasträgern verwendbar. Insgesamt verblieb eine ausreichend hohe Seilkraft im System, sodass ein Spannglasträger im baupraktisch üblichen Temperaturbereich seine Trageigenschaften bewahrte.

Schließlich wurden alle untersuchten Eigenschaften, die Tragfähigkeit, Dauerhaftigkeit und Resttragfähigkeit in einer umfassenden Diskussion gemeinsam betrachtet, um einen hinreichenden Machbarkeitsnachweis für Spannglasträger zu erbringen. Dabei erbrachten vergrößerte Vorspannkräfte eine steigende Erstrisslast. Dem entgegengesetzt wurden steigende Seilkraftverluste und übermäßige horizontale Durchbiegungswerte infolge einer Überlastung beobachtet. Damit ergibt sich die Notwendigkeit, die Vorspannkraft auf einen Glasträger sowie die daraus resultierenden Spannungen und Verformungen zu begrenzen. Insbesondere der konstruktive Ausschluss einer Belastung des Zwischenschichtmaterials kann zu einer gesteigerten Erstrisslast bei gleichzeitig akzeptablen Spannkraftverlusten führen.

Zudem wurde gezeigt, dass die Trag- und Sicherheitseigenschaften sowie die Leistungsfähigkeit von Spannglasträgern maßgeblich von der Wahl des Grundmaterials abhängen. Während exzentrisch bewehrte Träger aus thermisch entspanntem Floatglas nur geringfügig mechanisch vorgespannt werden dürfen, um eine Überlastung des Glases zu vermeiden, werden oftmals thermisch vorgespannte Glasarten eingesetzt. Diese wiederum zeigen im Fall des Glasbruchs aufgrund der umzuwandelnden gespeicherten Energie aus der thermischen Vorspannung und der äußeren mechanischen Last eine feineres Bruchbild, welches sich ungünstig auf die Resttragfähigkeit auswirkt. Daher muss 
sichergestellt werden, dass der vollständig gebrochene Verbundglasanteil der Trägerkonstruktion durch die verwendete Bewehrung gehalten und als Einzelelement die Systemtragfähigkeit einer großfächigen Konstruktion nicht gefährdet. Als Weiterentwicklung wurde daher vorgeschlagen, einen Glasträger mit Vorspannung im nachträglichen Verbund zu entwickeln. Es wird erwartet, dass diese Konstruktionsart die aufgezeigten ungünstigen Versagenseigenschaften kompensieren kann.

Als wesentliche Beiträge zur Forschung auf dem Wissensgebiet des Konstruktiven Glasbaus können somit erstmalig die Entwicklung einer Bezeichnungsweise und einer Berechnungsmethode des Tragverhaltens in Analogie zum Spannbetonbau, die Entwicklung einheitlicher experimenteller Prüfmethoden und die Bereitstellung eines einheitlichen Datensatzes für eine konkrete Ausführungsvariante mechanisch vorgespannter Glasträger benannt werden.

Neben der notwendigen Beschreibung des Kurzzeittragverhaltens wurden in dieser Arbeit zudem die Aspekte der Spannkraftverluste unter Dauer- und Temperaturlasten sowie Fragen der Resttragfähigkeit thematisiert. Damit ist erstmals eine umfassende Charakterisierung von Spannglasträgern verfügbar. Es bleibt zu beachten, dass eine mechanische Vorspannung neben den nachgewiesenen günstigen Eigenschaften auch ungünstig wirken kann und so das Tragkonzept infrage stellt. Auf Grundlage der vorgestellten Untersuchungen ist es nun möglich günstige Eigenschaften gezielt zu betonen und trageffiziente Konstruktionen zu entwerfen.

\subsection{Ausblick}

Mit dieser Arbeit wurde ein erster umfassender und systematischer Einblick in das Tragverhalten von Glasträgern mit verbundlos vorgespannter Bewehrung vorgestellt. Darin zeigte sich eine vergrößerte Erstrisslast, ein in der Regel robustes Verhalten in nicht kalkulierbaren Situationen, die zum Glasbruch führen und ein erwarteter Vorspannkraftverlust unter Dauer- und Temperaturlasten. 
Als wesentliches Versagenskriterium wurde der Stabilitätsverlust herausgestellt. Diesem wurde mit dem Konzept des Doppelquerschnitts einschließlich geklebter Umlenkpunkte begegnet, welche sich als wesentlich für die Beschreibung des Gesamttragverhaltens zeigten. Derzeit ist jedoch keine anwendungstaugliche Methode verfügbar, eine Bemessung der Klebkonstruktion durchzuführen. Dies ist jedoch für eine sichere Anwendung nötig und sollte daher ein Forschungsthema der näheren Zukunft werden.

Insbesondere die ergänzende numerische Studie hat gezeigt, dass die Tragfähigkeit der Umlenkkonstruktion einen wesentlichen Einfluss auf das Gesamttragverhalten des Systems hat. Entsprechend können zusätzliche Potenziale mit der Weiterentwicklung dieses baukonstruktiven Details erschlossen werden. Das Kleben als Fügetechnologie wurde hierin zwar angewendet, stand aber nicht im Fokus der Betrachtung, sodass eine fortführende Forschung auf diesem Spezialgebiet des Konstruktiven Glasbaus auch die Anwendungsmöglichkeiten für Spannglasträger ausweiten und deren sichere Verwendung ermöglichen kann.

Zudem zeigte sich die Detaillierung des Lasteintrages in die Verbundglaskante als wesentlich. Dieser Systempunkt wir heute auf Basis von Erfahrungswerten konstruiert - eine Bemessung von Glas oder Klotzungsmaterial findet nicht statt. Derzeit ein stetiger Trend zu großformatigen Isolierverglasungen mit Verbundglasanteilen sichtbar. Deren Eigenlasten müssen über effiziente Klotzungen in die Unterkonstruktion eingeleitet werden. Daneben wird Verbundglas als primär lastabtragendes Bauteil, und der Anforderung an den materialgerechten Lastübertrag zwischen benachbarten Verbundglaskanten, eingesetzt. Entsprechend ist ein großes Anwendungsfeld absehbar, welches die Erforschung des Lasteintrages in die Verbundglaskante und deren Bemessung notwendig macht.

Ein weiteres Ziel bei der Entwicklung neuartiger Glasträgerkonstruktionen ist das Erreichen eines duktilen Bauteilversagens. Hierfür sind jedoch keinerlei Grenzwerte oder allgemeingültige Nachweismethoden verfügbar. Mit den Definitionen und Ergebnissen dieser Arbeit können, in Verbindung 
mit darüber hinaus verfügbaren Informationen, Duktilitätsanforderungen ausblickend quantifiziert werden.

Schließlich sollte die Konstruktionsweise auf weiterführende Anwendungen übertragen werden. Mit der Entwicklung einer Segmentbauweise, der Herstellung von Spannglasträgern im nachträglichen Verbund und der Verwendung für die Konstruktion von Seilnetzfassaden sind einige Ansätze abgeleitet worden. Darüber hinaus sollen die vorgestellten Ideen und Ansätze dazu anregen, weiterführende Anwendungen zu erschließen. 


\subsection{Fachbücher und Fachaufsätze}

Abel \& Krautwald 2001

Abeln \& Preckwinkel 2011

Ahrens 2016

Alex \& Berlin 2011

Baraldi et al. 2016

Bedon \& Louter 2016

Belis et al. 2004

Belis et al. 2006

Belis et al. 2009a

Belis et al. 2009b

Belis et al. 2011
Abel, M.; Krautwald, W: Ruhrtalbrücke Rumbeck - Extern vorgespannte Taktschiebebrücke mit Hilfspylon. In: Beton- und Stahlbetonbau 96 (2001), Nr. 7, S. 497-502. DOl10.1002/best.200100600.

Abeln, B.; Preckwinkel, E.: Entwicklung hybrider Stahl-Glas-Träger. In: Stahlbau 80 (2011), Nr. 4, S. 218-225. DOl10.1002/stab.201101410.

Ahrens, C. G.: Katalog. 2016. - http://drahtseiltechnik.cgahrens.de/ Kataloge-Downloads/Christian_G_Ahrens_Katalog.pdf, letzter Zugriff am 18.02.2016.

Alex, J.; Berlin, M.: Methodik und Beispiel einer Brückenertüchtigung durch externe Vorspannung. In: Beton- und Stahlbetonbau 106 (2011), Nr. 2, S. 129-136. DOI10.1002/best.201000075.

Baraldi, D.; Cecchi, A.; Foraboschi, P.: Broken tempered laminated glass: Non-linear discrete element modeling. In: Composite Structures 140 (2016), S. 278-295. DOI10.1016/j.compstruct.2015.12.050.

Bedon, C.; Louter, C.: Finite-Element Numerical Simulation of the Bending Performance of Post-Tensioned Structural Glass Beams with Adhesively Bonded CFRP Tendons. In: American Journal of Engineering and Applied Sciences 9 (2016). DOI10.3844/ajeassp.2016.680.691.

Belis, J.; Van Impe, R.; De Meester, B.; u. a.: Stability approach of the dimensioning of glass beams. In: International Symposium on the Application of Architectural Glass-Engineering and architectural design of glass 2004 (ISAAG), München, Deutschland, 2004.

Belis, J.; Louter, C.; Verfaillie, K.; u. a.: The effect of post-tensioning on the buckling behaviour of a glass T-beam. In: International Symposium on the Application of Architectural Glass-Engineering and architectural design of glass 2006 (ISAAG), München, Deutschland, 2006, S. 129-136.

Belis, J; Callewaert, D.; Delincé, D.; u. a.: Experimental failure investigation of a hybrid glass/steel beam. In: Engineering Failure Analysis 16 (2009), Nr. 4, S. 1163-1173. DOl10.1016/j.engfailanal.2008.07.011.

Belis, J.; Depauw, J.; Callewaert, D.; u. a.: Failure mechanisms and residual capacity of annealed glass/SGP laminated beams at room temperature. In: Engineering Failure Analysis 16 (2009), Nr. 6, S. 1866-1875. DOI10.1016/j.engfailanal.2008.09.023.

Belis, J.; Mocibob, D.; Luible, A.; u. a.: On the size and shape of initial out-of-plane curvatures in structural glass components. In: Con- 
struction and Building Materials 25 (2011), Nr. 5, S. 2700-2712.

DOI10.1016/j.conbuildmat.2010.12.021.

Betonbau 11/2015

BIV 2004

Bünemann 1997

Bohmann 1999

Bos 2009

Bos et al. 2004

Bucak et al. 2009a

Bucak et al. 2009b

Bukieda 2015

Bukieda 2016

Cagnacci et al. 2009

Correia et al. 2011

Cruz \& Pequeno 2008
Beton- und Stahlbetonbau (2015): Sonderdruck Elementdecken mit Gitterträgerns. Deckblatt. (2015), Nr. 11.

BIV: Bundesinnungsverband des Glashandwerks: Technische Richtlinien des Glaserhandwerks. Mehrteilig. Düsseldorf: Verlagsanstalt Handwerk GmbH, 2004.

Bünemann, B.: Umbau der Lindener Volksbank in Hannover. In: Glas. Architektur und Technik 2. Jahrgang (1997), Nr. 1, S. 19-24.

Bohmann, D.: Ein numerisches Verfahren zur Berechnung von Verbundglasscheiben. Dissertation, Rheinisch-Westfälische Technische Hochschule Aachen, 1999

Bos, F. P.: Safety Concepts in Structural Glass Engineering. Dissertation, Technische Universiteit Delft, 2009.

Bos, F. P.; Veer, F. A.; Hobbelman, G. J.; u. a.: Stainless steel reinforced and post-tensioned glass beams. In: Proceedings of the 12th International Conference of Experimental Mechanics (ICEM12), Bari, Italien, 2004.

Bucak, Ö.; Bues, M.; Illguth, M.; u. a.: Bonded steel glass hybrid beams. In: Tagungsband Glass Performance Days (GPD), Tampere, Finnland, 2009, S. 318-320.

Bucak, Ö.; Geiß, P. L.; Kötter, M. P.; u. a.: Geklebte Stahl-GlasVerbundtragwerke. In: Stahlbau Spezial - Konstruktiver Glasbau 78 (2009), Nr. 1, S. 36-40. DOl10.1002/stab.200910028.

Bukieda, P.: FEM Studie zur Lasteinleitung in eine Verbundglaskante. Projektarbeit, Institut für Baukonstruktion, Technische Universität Dresden (unveröffentlicht). 2015

Bukieda, P.: Spannglasträger unter Temperaturlast. Diplomarbeit, Technische Universität Dresden (unveröffentlicht). 2016.

Cagnacci, E.; Orlando, M.; Spinelli, P.: Experimental campaign and numerical simulation of the behavior of reinforced glass beams. In: Tagungsband Glass Performance Days (GPD), Tampere, Finnland, 2009, S. 484-487.

Correia, J. R.; Valarinho, L.; Branco, F. A.: Post-cracking strength and ductility of glass-GFRP composite beams. In: Composite Structures 93 (2011), Nr. 9 , S. 2299-2309. DOI10.1016/j.compstruct.2011.03.018.

Cruz, P.; Pequeno, J.: Timber-glass composite beams: mechanical behaviour $\&$ architectural solutions. In: Tagungsband Challenging Glass - Conference 
on Architectural and Structural Applications of Glass (CGC), Delft, Niederlande, 2008, S. 439-448.

Cruz \& Valente 2013

Cupac \& Louter 2015

DAfStb 2012

Fremdwörterbuch 2006

BRL A 2015/2

MLTB 2015

DuPont SGP 2008

Ebert 2014

Engelmann 2015

Engelmann \& Weller 2016

Engelmann et al. 2016

Ensslen 2005

ETA-11/0160
Cruz, P. J. S.; Valente, I.: Experimental analysis on steel reinforced glass beams at different. In: Tagungsband COST Action TU0905, Mid-term Conference on Structural Glass, Poreč, Kroatien. CRC Press, Taylor \& Francis Group, 2013, S. 261. DOI10.1201/b14563-37.

Cupac, J.; Louter, C.: Post-Tensioned Structural Glass Beams - Comparative Experimental Study. In: Tagungsband Advanced Building Skins, Graz, Österreich (2015), Nr. EPFL-CONF-207873, S. 166-172.

DAfStb: Deutscher Ausschuss für Stahlbeton - DAfStb (Hrsg.): Erläuterungen zu DIN EN 1992-1-1 und DIN EN 1992-1-1/NA (Eurocode 2). 1. Auflage. Berlin: Beuth, 2012.

Der Duden - Band 5: Das Fremdwörterbuch. 9. Auflage. Dudenverlag, 2006.

Deutsches Institut für Bautechnik (DIBt): Bauregelliste A (BRL). (2015/2).

Deutsches Institut für Bautechnik (DIBt): Muster-Liste der Technischen Baubestimmungen (MLTB). (Juni 2015).

DuPont SGP: DuPont: SentryGlas Plus Elastic Properties (SGP 5000). 2008.

Ebert, J.: Einleitung hoher Lasten in Glaskanten - Ein Beitrag zum Einsatz von Kunststoffen als Klotzungsmaterial. Dissertation, Technische Universität Dresden, 2014.

Engelmann, M.: Spannglass-Bridge. Footbridge-design competition contribution. In: Young Engineers Workshop at IABSE Conference Nara (2015). DOI10.13140/RG.2.1.4379.2480.

Engelmann, M.; Weller, B.: Post-Tensioned Glass Beams for a $9 \mathrm{~m}$ Spannglass Bridge. In: Structural Engineering International 26 (2016), Nr. 2, S. 103-113. DOl10.2749/101686616X14555428759000.

Engelmann, M.; Bukieda, P.; Weller, B.: Experimental Investigation on Posttensioned Spannglass Beams during Temperature Loads. In: Tagungsband Challenging Glass 5 - Conference on Architectural and Structural Applications of Glass (CGC5), Gent, Belgien, 2016.

Ensslen, F.: Zum Tragverhalten von Verbund-Sicherheitsglas unter Berücksichtigung der Alterung der Polyvinylbutyral-Folie. Dissertation, RuhrUniversität Bochum, 2005.

ETA-11/0160: Europäische Technische Zulassung ETA-11/0160: PFEIFER Seil-Zugglieder. DIBt, 2011. 
Fahlbusch 2007

Feirabend 2010

Feldmann et al. 2012

Feldmann et al. 2014

Fingerloos et al. 2012

Firmo et al. 2015

Freytag 2001

Freytag et al. 2012

Froli \& Lani 2010

Gehlen et al. 2002

Geßner et al. 2017

Göppert \& Paech 2008
Fahlbusch, M.: Zur Ermittlung der Resttragfähigkeit von Verbundsicherheitsglas am Beispiel eines Glasbogens mit Zugstab. Dissertation, Technische Universität Darmstadt, 2007.

Feirabend, S.: Steigerung der Resttragfähigkeit von Verbundsicherheitsglas mittels Bewehrung in der Zwischenschicht. Dissertation, Universität Stuttgart, 2010.

Feldmann, M.; Kasper, R.; Langosch, K.: Glas für tragende Bauteile. Werner Verlag, 2012.

Feldmann, M.; Kasper, R.; Abeln, B.; u. a.: Guidance for European structural design of glass components. Publications Office of the European Union, 2014.

Fingerloos, F.; Hegger, J.; Zilch, K.: Eurocode 2 für Deutschland: DIN EN 1992-1-1 Bemessung und Konstruktion von Stahlbeton-und Spannbetontragwerken-Teil 1-1: Allgemeine Bemessungsregeln und Regeln für den Hochbau mit Nationalem Anhang. Kommentierte Fassung. Beuth Verlag, 2012

Firmo, F.; Jordão, S.; Neves, L. C.; u. a.: Structural Behaviour of Simple and Pre-stressed Hybrid Steel-Glass Beams. In: International Conference on Recent Advances in Rehabilitation and Sustainability of Structures, Azoren, Portugal, 2015

Freytag, B.: Glas-Beton Verbundbauweise. (2001), Nr. 4, S. 268 DOI10.1002/best.200100340.

Freytag, B.; Santner, G.; Sparowitz, L.; u. a.: Structures made of Glass and Ultra High Performance Concrete - Current Research. In: Tagungsband Challenging Glass 3 - Conference on Architectural and Structural Applications of Glass (CGC3), Delft, Niederlande, 2012, S. 653-668. DOI10.3233/978-1-61499-061-1-653.

Froli, M.; Lani, L.: Glass Tensegrity Trusses. In: Structural Engineering International 20 (2010), Nr. 4, S. 436-441. DOl10.2749/101686610793557564.

Gehlen, B.; Nützel, O.; Brand, W.; u. a.: Hochfeste Zugglieder im Brückenbau - Anforderungen und Ausführungsbeispiele. In: Beton- und Stahlbetonbau 97 (2002), Nr. 10, S. 512-521. DOl10.1002/best.200202430.

Geßner, S.; Niedermeier, R.; Ahrens, M.A.; u. a.: Spannbetonbau - Entwicklung, Bemessung und Konstruktion. In: Bergmeister, K. (Hrsg.); Fingerloos, F. (Hrsg.); Wörner, J.-D. (Hrsg.): Beton-Kalender 2017, Ernst \& Sohn, 2017 DOl10.1002/9783433606803.ch1.

Göppert, K.; Paech, C.: Gläsernes Mahnmal zur Erinnerung an die Terrorattentate vom 11. März 2004 in Madrid. In: Stahlbau Spezial - Konstruktiver Glasbau 77 (2008), Nr. 1, S. 42-46. DOI10.1002/stab.200810031. 
Haas \& Haldimann 2004

Haldimann et al. 2008

Hess 2000

Glaserlass Hessen 2004

Hightec Glas 2014

Hobbelmann et al. 2001

Holberndt 2006

Härth 2013

Interpane 2011

Invernizzi et al. 201

Jordão et al. 2014

Josef Gartner \& Co 1991

Kaltenbach 2003

Kasper 2005
Haas, C.; Haldimann, M.: Entwurf und Bemessung von Tragelementen aus Glas - Wissensbericht. Rapport ICOM 493. 2004.

Haldimann, M.; Luible, A.; Overend, M.: Structural use of glass. Bd. 10. International Association for Bridge and Structural Engineering (IABSE), 2008.

Hess, R.: Glasträger: Forschungsbericht. Bd. 20. vdf Hochschulverlag AG, 2000.

Hessisches Ministerium für Wirtschaft, Verkehr und Landesentwicklung: Anwendung nicht geregelter Bauarten nach $\S 20$ der Hessischen Bauordnung (HBO) im Bereich der Glaskonstruktionen. hier: Anforderungen an Bauarten im Zustimmungsverfahren und Freistellung vom Erfordernis der Zustimmung im Einzelfall nach $\S 20$ Abs. 1 HBO. (2004).

Hightec Glas 2014: Träume von tragender Transparenz. 2014. - http://www. hitecglas.de/downloads/hitecglas.pdf, letzter Zugriff am 22.04.2016.

Hobbelmann, G.J.; Zijl, G.P.A.G. van; Veer, F.A.; u. a.: New Structural Material by Architectural Demand. In: Zingoni, A. (Hrsg.): Structural Engineering, Mechanics and Computation (Vol. 1). Elsevier, Amsterdam, 2001, S. 455 462.

Holberndt, T.: Entwicklung eines Bemessungskonzeptes für den Nachweis von stabilitätsgefährdeten Glasträgern unter Biegebeanspruchung. Dissertation, Technische Universität Berlin, 2006.

Härth, K.: Beitrag zum Tragverhalten hybrider Träger aus Glas und Kunststoff. Dissertation, Technische Universität Dresden, 2013.

Interpane: Interpane Handbuch: Gestalten mit Glas. Lauenförde: Eigenverlag, 2011.

Invernizzi, S.; Trovato, D.; Hendriks, M.A.N.; u. a.: Sequentially linear modelling of local snap-back in extremely brittle structures. In: Engineering Structures 33 (2011), S. 1617 - 1625. DOI10.1016/j.engstruct.2011.01.031.

Jordão, S.; Pinho, M.; Martin, J. P.; U. a.: Behaviour of laminated glass beams reinforced with pre-stressed cables. In: Steel Construction 7 (2014), Nr. 3, S. 204-207. DOI10.1002/stco.201410027.

Josef Gartner \& Co: Gesicherter Glasträger. Erfinder: Antrag auf Nichtnennung. Deutsches Patentamt. DE4140537A1, 1991. - 09.12.1991.

Kaltenbach, F.: Transluzente Materialien: Glas-Kunststoff-Metall. Walter de Gruyter, 2003.

Kasper, R.: Tragverhalten von Glasträgern. Dissertation, RheinischWestfälische Technische Hochschule Aachen, 2005. 
Köhler \& Schimetta 2002

Kothe 2013

Kott 2006

Langosch 2012

Langosch et al. 2013

Laufs 2000

Leonhardt 1980

Louter 2008

Louter 2009

Louter 2011

Louter 2013

Louter \& Cupac 2015

Louter \& Veer 2007a

Louter \& Veer 2007b
Köhler, C.; Schimetta, G.: Schwieriger Umbau einer Stadtbrücke - Brückentrennung, Verstärkung mit externer Vorspannung, CFK Lamellen. In: Beton- und Stahlbetonbau 97 (2002), Nr. 2, S. 115-120. DOI10.1002/best.200200390.

Kothe, M.: Alterungsverhalten von polymeren Zwischenschichtmaterialien im Bauwesen. Dissertation, Technische Universität Dresden, 2013.

Kott, A.: Zum Trag- und Resttragverhalten von Verbundsicherheitsglas. Dissertation, Eidgenössische Technische Hochschule Zürich, 2006.

Langosch, K.: Das Tragverhalten von Glasstützen mit Mono- und Verbundquerschnitten. Dissertation, Rheinisch-Westfälische Technische Hochschule Aachen, 2012.

Langosch, K.; Feldmann, M.; Hoffmeister, B.: Wandartige Monoglasstützen unter axialen Drucklasten und Biegung. In: Stahlbau 82 (2013), Nr. 1, S. 143-155. DOI10.1002/stab.201390060.

Laufs, W.: Ein Bemessungskonzept zur Festigkeit thermisch vorgespannter Gläser. Dissertation, Rheinisch-Westfälische Technische Hochschule Aachen, 2000

Leonhardt, F.: Vorlesungen über Massivbau - Teil 5: Spannbeton. Springer, 1980.

Louter, C.: Bewehrte Glasträger. In: Stahlbau Spezial - Konstruktiver Glasbau 77 (2008), Nr. 1, S. 23-27. DOI10.1002/stab.200810028.

Louter, P. C.: High-strength fibre rods as embedded reinforcement in SentryGlas-laminated glass beams. In: Tagungsband Glass Performance Days (GPD), Tampere, Finnland, 2009, S. 285-289.

Louter, P. C.: Fragile yet ductile: structural aspects of reinforced glass beams. Dissertation, Technische Universiteit Delft, 2011.

Louter, C.: Reinforced and post-tensioned glass beams. In: Tagungsband Glass Performance Days (GPD), Tampere, Finnland, 2013, S. 353-355.

Louter, C.; Cupac, J.: Comparative Study of Post-tensioned Glass Beams with Bonded Tendons. In: Tagungsband Glass Performance Days (GPD), Tampere, Finnland, 2015, S. $383-386$

Louter, C.; Veer, F.: Experimental research on scale 1:4 models of an $18 \mathrm{~m}$ reinforced glass beam, part I. In: Tagungsband Glass Performance Days (GPD), Tampere, Finnland, 2007, S. 93-97.

Louter, C.; Veer, F.: Large span reinforced glass beams, prototype research. In: Tagungsband IASS Symposium 2007. Shell and Spacial Structures: 
Structural Architecture - Towards the future looking to the past, Venedig, Italien, 2007, S. 1-10.

Louter et al. 2005

Louter et al. 2006

Louter et al. 2007

Louter et al. 2010

Louter et al. 2012

Louter et al. 2013a

Louter et al. 2013b

Louter et al. 2014

Ludwig \& Seele 1995

Luible 2004

Martens et al. 2015a
Louter, P. C.; Belis, J.; Bos, F. P.; u. a.: Reinforced glass cantilever beams. In: Tagungsband Glass Processing Days (GPD), Tampere, Finnland, 2005, S. 429-433.

Louter, C.; Heusden, J. van; Veer, F.; u. a.: Post-Tensioned Glass Beams. 2006. In: Fracture of Nano and Engineering Materials and Structures. Springer Science + Business Media, 2006. DOI10.1007/1-4020-4972-2_296, S. 597-598.

Louter, P. C.; Veer, F. A.; Hobbelman, G. J.: Reinforcing glass, effects of reinforcement geometry and bonding technology. In: Tagungsband Glass Processing Days (GPD), Tampere, Finnland, 2007, S. 87-92.

Louter, C.; Leung, C.; Kolstein, H.; u. a.: Structural glass beams with embedded glass fibre reinforcement. In: Tagungsband Challenging Glass 2 Conference on Architectural and Structural Applications of Glass (CGC2), Delft, Niederlande, 2010, S. 439-448.

Louter, C.; Belis, J.; Veer, F.; u. a.: Structural response of SG-laminated reinforced glass beams; experimental investigations on the effects of glass type, reinforcement percentage and beam size. In: Engineering Structures (2012), Nr. 36, S. 292-301. DOI10.1016/j.engstruct.2011.12.016.

Louter, C.; Nielsen, J. H.; Belis, J.: Exploratory experimental investigations on post-tensioned structural glass beams. In: Tagungsband International Conference Structures and Architecture (ICSA), Guimarães, Portugal, 2013, S. $358-365$.

Louter, C.; Pérez, A.; Jordan, T.; u. a.: Post-tensioned structural glass beams - Experimental investigations. In: Tagungsband COST Action TU0905, Midterm Conference on Structural Glass, Poreč, Kroatien. CRC Press, Taylor \& Francis Group, 2013, S. 277-284. DOI10.1201/b14563-39.

Louter, C.; Cupac, J.; Lebet, J.-P.: Exploratory experimental investigations on post-tensioned structural glass beams. In: Journal of Facade Design and Engineering 2 (2014). DOI10.3233/FDE-130012.

Ludwig, J. J.; Seele, G.: Gebäude-Tragkonstruktion mit mindestens einem Tragkörper aus Glas, welcher durch ein Zugelement verstärkt ist. Deutsches Patentamt. DE19530572A1, 1995. - 19.08.1995.

Luible, A.: Stabilität von Tragelementen aus Glas. Dissertation, Ecole Polytechnique Fédérale de Lausanne, 2004.

Martens, K.; Belis, J.; Caspeele, R.; u. a.: Numerical investigation of reinforced laminated glass beams. In: IABSE Symposium Report Bd. 104 International Association for Bridge and Structural Engineering, International 
Association for Bridge and Structural Engineering (IABSE), 2015, S. 1-8. DOI10.2749/222137815815774278.

Martens et al. $2015 b$

MM TN-504-1 2010

Miyasato et al. 2013

Musterbauordnung 2002

Neugebauer 2005

Nielsen 2009

Nijsse 2015

Novák et al. 2015

Merkblatt ZiE Bayern 2015

Ølgaard et al. 2009

Pelke \& Zichner 2015

Petersen 1982
Martens, K.; Caspeele, R.; Belis, J.: Development of Reinforced and Posttensioned Glass Beams: Review of Experimental Research. In: Journal of Structural Engineering (ASCE) 142 (2015), Nr. 5. DOI10.1061/(ASCE)ST.1943$541 X .0001453$

MICRO-MEASUREMENTS Tech Note TN-504-1: Thermisches Ausgangssignal aus DMS und K-Faktor-Änderung mit der Temperatur. 2010. - https://www.me-systeme.de/docs/de/basics/TechNotes/TN-504-1_ sebstkompensation.pdf, letzter Zugriff am 18.02.2016.

Miyasato, N.; Okada, A.; Hiroishi, S.; u. a.: An Application Evaluation of Hybrid Glass Panel System as Structural Member. In: Tagungsband Glass Performance Days (GPD), Tampere, Finnland, 2013, S. 110-116.

Musterbauordnung: Musterbauordnung (MBO) Fassung November 2002. Informationssystem Bauministerkonferenz, 2002.

Neugebauer, J.: A special fixation with which the broken laminated safety glass is prevent from falling down. In: Tagungsband Glass Performance Days (GPD), Tampere, Finnland, 2005, S. 8-12.

Nielsen, J. H.: Tempered Glass - bolted connections and related problems. Dissertation, Danmarks Tekniske Universitet, 2009.

Nijsse, R.: The bearable lightness of all glass structures. In: IABSE Symposium Report Bd. 104 International Association for Bridge and Structural Engineering, 2015, S. 1-8. DOl10.2749/222137815815774304.

Novák, B.; Pelke, E.; Boros, V.; u. a.: Endverankerungen bei Ertüchtigung von Brücken mit externer Vorspannung. In: Beton- und Stahlbetonbau 110 (2015), Nr. 2, S. 138-154. DOI10.1002/best.201400103.

Oberste Baubehörde im Bayerischen Staatsministerium des Innern, für Bau und Verkehr: Merkblatt ZiE-Nr. 3A. Hinweise zur Erlangung einer Zustimmung im Einzelfall (ZiE) gemäß Art. 18 Abs. 1 und Art. 19 Abs. 1 Bayerische Bauordnung (BayBO). Bereich Konstruktiver Glasbau. (2015).

Ølgaard, A. B.; Nielsen, J. H.; Olesen, J. F.: Design of Mechanically Reinforced Glass Beams: Modelling and Experiments. In: Structural Engineering International (2009). DOl10.2749/101686609788220169.

Pelke, E.; Zichner, T.: Ertüchtigung der Nibelungenbrücke Worms. In: Beton- und Stahlbetonbau 110 (2015), Nr. 2, S. 113-130. DOI10.1002/best.201400082.

Petersen, C.: Statik und Stabilität der Baukonstruktionen. Vieweg, 1982 (2., durchgesehene Auflage). 
Plumb 1989

Prüfbericht 01.02.2005

Prüfbericht 11.07.2013

Scotch Weld 9323

SikaFast 5211NT

Puller 2012

R Core Team 2014

Rombach 2010

Sackmann 2008

Schlaich et al. 2012

SBP 2014

Schober et al. 2004

Schollglas 2006

Schuler 2003
Plumb, R. C.: Antique windowpanes and the flow of supercooled liquids. In: Journal of Chemical Education (1989). DOl10.1021/ed066p994.

Prüfbericht 01.02.05: TU Dresden, Institut für Baukonstruktion - Prüfbericht: Trag- und Resttragfähigkeitsprüfung der Glasträger Umbau Mensa/Rektorat der Technischen Universität Dresden. (unveröffentlicht). 2005.

Prüfbericht 11.07.13: TU Dresden, Institut für Baukonstruktion - Prüfbericht: Zugversuche in Anlehnung an DIN EN ISO 15630-3. (unveröffentlicht). 2013.

Produkt-Information: Scotch-Weld®9323 B/A. 06/2002.

Produktdatenblatt Version 1: SikaFast@-5211 NT. 05/2013.

Puller, K.: Untersuchung des Tragverhaltens von in die Zwischenschicht von Verbundglas integrierten Lasteinleitungselementen. Dissertation, Universität Stuttgart, 2012. DOI10.18419/opus-462.

R Core Team: R: A Language and Environment for Statistical Computing. R Foundation for Statistical Computing, Wien, Österreich. http://www. R-project.org. 2014.

Rombach, G.: Spannbetonbau. 2. aktualisierte Auflage. Wiley-Blackwell, 2010.

Sackmann, V.: Untersuchungen zur Dauerhaftigkeit des Schubverbunds in Verbundsicherheitsglas mit unterschiedlichen Folien aus Polyvinylbutyral. Dissertation, Technische Universität München, 2008.

Schlaich, M.; Zwingmann, B.; Liu, Y.; u. a.: Zugelemente aus CFK und ihre Verankerungen. In: Bautechnik 89 (2012), Nr. 12, S. 841 - 850. DOI10.1002/bate.201200057.

Schlaich Bergermann Partner: Projekte - Glasdach und Glasfassade Badenweiler Therme. 2014. - http://www.sbp.de/projekt/ glasdach-und-glasfassade-badenweiler-therme/, letzter Zugriff am 08.02.2017.

Schober, H.; Gerber, H.; Schneider, J.: Ein Glashaus für die Therme in Badenweiler. In: Stahlbau 73 (2004), Nr. 11, S. 886-892.

DOI10.1002/stab.200490213.

Schollglas: Verglasungsrichtlinien. Version 1.1. Barsinghausen: Eigenverlag, 2006.

Schuler, C.: Einfluss des Materialverhaltens von Polyvinylbutyral auf das Tragverhalten von Verbundsicherheitsglas in Abhängigkeit von Temperatur und Belastung. Dissertation, Technische Universität München, 2003. 
Sedlacek et al. 2003

Sobek et al. 2000

Sobek et al. 2006

Sofistik AG 2014

Speranzini \& Agnetti 2013

Stockes 1999

Suter \& Jungo 2001

Trost et al. 1984

Ungermann et al. 2010

Veer et al. 2003

Vorwagner et al. 2013

Waagner Biro 2014
Sedlacek, G.; Wellershoff, F.; Düser, H.: Bemessungsschubmodul für PVBFolien in Verbundsicherheitsglas unter Klimalasten in Deutschland. 2003.

Sobek, W.; Kutterer, M.; Messmer, R.: Untersuchungen zum Schubverbund bei Verbundsicherheitsglas - Ermittlung des zeit-und temperaturabhängigen Schubmoduls von PVB. In: Bauingenieur 75 (2000), Nr. 1, S. 41-46.

Sobek, W.; Kutterer, M.; Feirabend, S.; u. a.: Teil 2 Grundlagen. Konstruieren mit Glas - Festigkeit und Tragverhalten. In: Schittich, C. (Hrsg.); Staib, G. (Hrsg.); Balkow, D. (Hrsg.); u. a.: Glasbau Atlas Bd. 2. überarbeitete und erweiterte Auflage, Institut für internationale Architektur-Dokumentation $\mathrm{GmbH}$ \& Co. KG, 2006.

Sofistik AG: ASE Manual, Version 2014.4 - Allgemeine Statik Finiter Element Strukturen. SOFiSTiK AG, Oberschleissheim. 2014.

Speranzini, E.; Agnetti, S.: Post-cracking behaviour of reinforced glass beams. In: Tagungsband COST Action TU0905, Mid-term Conference on Structural Glass, Poreč, Kroatien. CRC Press, Taylor \& Francis Group, 2013, S. 285-292. DOI10.1201/b14563-40.

Stockes, Y. M.: Flowing windowpanes: fact or fiction? In: Proceedings of the Royal Society of London. A Mathematical, Physical and Engineering Sciences (1999), Nr. 455, S. 2751-2756. DOl10.1098/rspa.1999.0425.

Suter, R.; Jungo, D.: Vorgespannte CFK-Lamellen zur Verstärkung von Bauwerken. In: Beton- und Stahlbetonbau 96 (2001), Nr. 5, S. 350-358. DOI10.1002/best.200100370.

Trost, H.; Cordes, H.; Weller, B.: Untersuchungen zur Vorspannung ohne Verbund. In: Deutscher Ausschuss für Stahlbeton (DAfStb). Heft 355. (1984), S. $7-70$.

Ungermann, D.; Preckwinkel, E.: Structural behaviour of hybrid steel-glass beams. In: Tagungsband Challenging Glass 2 - Conference on Architectural and Structural Applications of Glass (CGC2), Delft, Niederlande, 2010, S. 485-496.

Veer, F. A.; Rijgersberg, H.; Ruytenbeek, D.; u. a.: Composite glass beams, the third chapter. In: Tagungsband Glass Performance Days (GPD), Tampere, Finnland, 2003, S. 307-310.

Vorwagner, A.; Burtscher, S. L.; Grass, G.: Effizientes Vorspannen von CFKLamellen. In: Beton- und Stahlbetonbau 108 (2013), Nr. 3, S. 188-197. DOI10.1002/best.201200055.

Waagner Biro 2014: Tower Place. 2014. - http://www.waagner-biro.com/de/ sparten/stahl-glas-technik/referenzen/referenz/tower-place, letzter Zugriff am 14.04.2014. 
Wagner 2002

Weimar 2011

Weller 1988

Weller \& Engelmann 2013

Weller \& Engelmann 2014a

Weller \& Engelmann 2014b

Weller \& Engelmann 2015

Weller et al. 2009a

Weller et al. $2009 b$

Weller et al. 2010a

Weller et al. 2010b

Weller et al. $2010 \mathrm{c}$

Weller et al. 2016
Wagner, E.: Glasschäden: Oberflächenbeschädigungen, Glasbrüche in Theorie und Praxis, 2002.

Weimar, T.: Untersuchungen zu Glas-Polycarbonat-Verbundtafeln. Dissertation, Technische Universität Dresden, 2011.

Weller, B.: Experimentelle Untersuchungen zum Biegetragverhalten von Durchlaufträgern mit Vorspannung ohne Verbund. In: Deutscher Ausschuss für Stahlbeton (DAfStb). Heft 391. (1988), S. 73 - 125.

Weller, B.; Engelmann, M.: Novel Reinforced Spannglass Beams. In: Tagungsband Glass Performance Days (GPD), Tampere, Finnland, 2013, S. 282-284.

Weller, B.; Engelmann, M.: Deformation of Spannglass Beams Subject to Post-Tensioning. In: Tagungsband Challenging Glass 4 - Conference on Architectural and Structural Applications of Glass (CGC4) \& COST Action TU0905 Final Conference, Lausanne, Schweiz, 2014, S. 285-294. DOI10.1201/b16499-42.

Weller, B.; Engelmann, M.: Spannglasträger - Glasträger mit vorgespannter Bewehrung. In: Stahlbau 83 (2014), Nr. 1, S. 193-203. DOI10.1002/stab.201490059.

Weller, B.; Engelmann, M.: 9 m Spannglasbrücke auf der glasstec 2014. In: Stahlbau 84 (2015), Nr. 1, S. 455 - 464. DOl10.1002/stab.201590102.

Weller, B.; Härth, K.; Werner, F.; u. a.: Hybridbauteile im Konstruktiven Glasbau. In: Stahlbau Spezial - Konstruktiver Glasbau 78 (2009), Nr. S1, S. 2935. DOI10.1002/stab.200910027.

Weller, B.; Weimar, T.; Krampe, P.; u. a.: Glass Steel Beams - Development of Hybrid Structures. In: Tagungsband Glass Performance Days (GPD), Tampere, Finnland, 2009, S. 435-439.

Weller, B.; Meier, A.; Weimar, T.: Glass-Steel Beams as Structural Members of Façades. In: Tagungsband Challenging Glass 2 - Conference on Architec tural and Structural Applications of Glass (CGC2), Delft, Niederlande, 2010, S. $517-524$

Weller, B.; Meier, A.; Weimar, T.; u. a.: Hybride Glasträger als Fassadenelemente. In: Stahlbau Spezial - Konstruktiver Glasbau 79 (2010), Nr. 1, S. 41-45. DOI10.1002/stab.201001306.

Weller, B.; Weimar, T.; Meier, A.: Hybrid facade system made of glass and steel. In: Weller, B. (Hrsg.); Schneider, J. (Hrsg.): Tagungsband engineered transparency conference 2010, Düsseldorf, Deutschland, 2010, S. 149-158.

Weller, B.; Lohr, K.; Engelmann, M.: Influence of Regrinding Depth on Edge Strength of Tempered Glass. In: Tagungsband Challenging Glass 5 - Con- 
ference on Architectural and Structural Applications of Glass (CGC5), Gent, Belgien, 2016.

Wellershoff \& Sedlacek 2003

Wellershoff et al. 2011

Wölfel 1987

Wörner et al. 2001

Z-21.3-1830 2011

Zanotta 1998

Zilch \& Weiher 2008

Zilch \& Zehetmaier 2010

Zimmermann 1988

Zschippang et al. 2006
Wellershoff, F.; Sedlacek, G.: Structural Use of Glass in Hybrid Elements: Steel-Glass-Beams, Glass-GFRP-Plates. In: Tagungsband Glass Performance Days (GPD), Tampere, Finnland, 2003, S. 268-270.

Wellershoff, F.; Sendelbach, M.; Schmitt, F.: Einsatz von tragenden Glaselementen in Glasbrücken und Glaspavillions. In: Stahlbau Spezial - Konstruktiver Glasbau (2011), Nr. 1, S. 13 - 23. DOI10.1002/stab.201120003.

Wölfel, E.: Nachgiebiger Verbund - Eine Näherungslösung und deren Anwendungsmöglichkeiten. In: Stahlbau 56 (1987), Nr. 6, S. 173-180.

Wörner, J. D.; Schneider, J.; Fink, A.: Glasbau: Grundlagen, Berechnung, Konstruktion. Springer, 2001.

Z-21.3-1830: Allgemeine bauaufsichtliche Zulassung Z-21.3-1830: Hilti Injektionsanker System HIT-HY 70 zur Verankerung in Mauerwerk. DIBt, 2011.

Zanotta, E. D.: Do cathedral glasses flow? In: American Journal of Physics (1998), Nr. 66, S. 392-395. DOI10.1119/1.19026.

Zilch, K.; Weiher, H.: Ingenieurbaukunst: 120 Jahre Spannbetonbau - von Doehring und Jackson bis heute. In: Beton- und Stahlbetonbau 103 (2008), Nr. 6, S. 422-430. DOI10.1002/best.200802012.

Zilch, K.; Zehetmaier, G.: Bemessung im Konstruktiven Betonbau. Nach DIN 1045-1 (Fassung 2008) und EN 1992-1-1 (Eurocode 2). 2., neu bearbeitete und erweiterte Auflage. Springer, 2010.

Zimmermann, J.: Biegetragverhalten und Bemessung von Träger mit Vorspannung ohne Verbund. In: Deutscher Ausschuss für Stahlbeton (DAfStb). Heft 391. (1988), S. $5-71$.

Zschippang, S.; Wies, W.; Weller, B.; u. a.: Glasdach Mensa und Rektorat der Technischen Universität Dresden. In: Stahlbau 75 (2006), S. 428 - 432. DOI10.1002/stab.200610044.

\subsection{Normen und Richtlinien}

DIN 1249-10

DIN 17022
DIN 1249-10, August 1990: Flachglas im Bauwesen - Teil 10: Chemische und physikalische Eigenschaften. Deutsche Norm. Berlin: Beuth, 1990.

DIN 17022: Wärmebehandlung von Eisenwerkstoffen, Verfahren der Wärmebehandlung. Teile 1 bis 5. Deutsche Norm. Berlin: Beuth, 1989 - 2000. 
DIN 18008-1

DIN 18008-1 (NA 1419)

DIN 18008-2

DIN 18008-3

DIN 18008-7

DIN 18008

DIN 4227

DIN 51067-1

DIN 6319

DIN EN 10052

DIN EN 10080

DIN EN 10138

DIN EN 10264-4

DIN EN 12150-1
DIN 18008-1, Dezember 2010: Glas im Bauwesen - Bemessungs- und Konstruktionsregeln - Teil 1: Begriffe und allgemeine Grundlagen. Deutsche Norm. Berlin: Beuth, 2010.

DIN 18008-1, NA 005-09-25 AA N 1419: Glas im Bauwesen - Bemessungsund Konstruktionsregeln - Teil 1: Begriffe und allgemeine Grundlagen. Norm Manusskript, 2016. - unveröffentlicht.

DIN 18008-2, Dezember 2010: Glas im Bauwesen - Bemessungs- und Konstruktionsregeln - Teil 2: Linienförmig gelagerte Verglasungen. Deutsche Norm. Berlin: Beuth, 2010.

DIN 18008-3, Juli 2013: Glas im Bauwesen - Bemessungs- und Konstruktionsregeln - Teil 3: Punktförmig gelagerte Verglasungen. Deutsche Norm. Berlin: Beuth, 2013.

DIN 18008-7: Glas im Bauwesen - Bemessungs- und Konstruktionsregeln Teil 7: Sonderkonstruktionen. (in Vorbereitung).

DIN 18008, Dezember 2010: Glas im Bauwesen - Bemessungs- und Konstruktionsregeln. Mehrteilig. Deutsche Norm. Berlin: Beuth, 2010.

DIN 4227: Spannbeton. Teile 1 bis 6. Deutsche Norm. Berlin: Beuth, $1979-$ 1991. - zurückgezogen.

DIN 51067-1, Mai 1977: Prüfung keramischer Roh- und Werkstoffe; Bestimmung der Druckfestigkeit bei Raumtemperatur (KDF) an feuerfesten Werkstoffen mit einer Gesamtporosität bis $45 \%$. Deutsche Norm. Berlin: Beuth, 1977. - zurückgezogen.

DIN 6319, Oktober 2001: Kugelscheiben. Kegelpfannen. Deutsche Norm. Berlin: Beuth, 2001.

DIN EN 10052, Januar 1994: Begriffe der Wärmebehandlung von Eisenwerkstoffen. Deutsche Norm. Berlin: Beuth, 1994.

DIN EN 10080, August 2005: Stahl für die Bewehrung von Beton - Schweiß geeigneter Betonstahl - Allgemeines. Deutsche Norm. Berlin: Beuth, 2005.

DIN EN 10138: Spannstähle. Teile 1 bis 4. Deutsche Norm. Berlin: Beuth, 2000.

DIN EN 10264-4, März 2012: Stahldraht und Drahterzeugnisse - Stahldraht für Seile - Teil 4: Draht aus nichtrostendem Stahl. Deutsche Norm. Berlin: Beuth, 2012.

DIN EN 12150-1, November 2000: Glas im Bauwesen - Thermisch vorgespanntes Kalknatron-Einscheibensicherheitsglas - Teil 1: Definition und Beschreibung. Deutsche Norm. Berlin: Beuth, 2000. 
DIN EN 12385-1

DIN EN 12385-10

DIN EN 12385-2

DIN EN 12390

DIN EN 1288-3

DIN EN 13670

DIN EN 1863

DIN EN 1990

DIN EN 1991-1-1

DIN EN 1991-1-5

DIN EN 1991

DIN EN 1992

DIN EN 1992-1-1

DIN EN 1992-1-1 NA
DIN EN 12385-1, Januar 2009: Drahtseile aus Stahldraht - Sicherheit Teil 1: Allgemeine Anforderungen. Deutsche Norm. Berlin: Beuth, 2009.

DIN EN 12385-10, Juli 2008: Drahtseile aus Stahldraht - Sicherheit - Teil 10: Spiralseile für den allgemeinen Baubereich. Deutsche Norm. Berlin: Beuth, 2008.

DIN EN 12385-2, Juli 2008: Drahtseile aus Stahldraht - Sicherheit - Teil 2: Begriffe, Bezeichnung und Klassifizierung. Deutsche Norm. Berlin: Beuth, 2008.

DIN EN 12390: Prüfung von Festbeton. Teile 1 bis 11. Deutsche Norm. Berlin: Beuth, $2000-2015$

DIN EN 1288-3, September 2000: Glas im Bauwesen - Bestimmung der Biegefestigkeit von Glas - Teil 3: Prüfung von Proben bei zweiseitiger Auflagerung (Vierschneiden-Verfahren). Deutsche Norm. Berlin: Beuth, 2000.

DIN EN 13670, März 2011: Ausführung von Tragwerken aus Beton. Deutsche Norm. Berlin: Beuth, 2011.

DIN EN 1863: Glas im Bauwesen - Teilvorgespanntes Kalknatronglas. Teile 1 und 2. Deutsche Norm. Berlin: Beuth, 2012.

DIN EN 1990, Dezember 2010: Eurocode: Grundlagen der Tragwerksplanung. Deutsche Norm. Berlin: Beuth, 2010.

DIN EN 1991-1-1, Dezember 2010: Eurocode 1: Einwirkungen auf Tragwerke - Teil 1-1: Allgemeine Einwirkungen auf Tragwerke - Wichten, Eigengewicht und Nutzlasten im Hochbau. Deutsche Norm. Berlin: Beuth, 2010.

DIN EN 1991-1-5, Dezember 2010: Eurocode 1: Einwirkungen auf Tragwerke - Teil 1-5: Allgemeine Einwirkungen auf Tragwerke - Temperatureinwirkungen. Deutsche Norm. Berlin: Beuth, 2010.

DIN EN 1991, Dezember 2010: Eurocode 1: Einwirkungen auf Tragwerke. Mehrteilig. Deutsche Norm. Berlin: Beuth, 2010.

DIN EN 1992: Eurocode 2: Bemessung und Konstruktion von Stahlbetonund Spannbetontragwerken. Mehrteilig. Deutsche Norm. Berlin: Beuth, 2010-2015.

DIN EN 1992-1-1, Januar 2011: Eurocode 2: Bemessung und Konstruktion von Stahlbeton- und Spannbetontragwerken - Teil 1-1: Allgemeine Bemessungsregeln für den Hochbau. Deutsche Norm. Berlin: Beuth, 2011.

DIN EN 1992-1-1/NA, April 2013: Nationaler Anhang - National festgelegte Parameter - Eurocode 2: Bemessung und Konstruktion von Stahlbeton- und Spannbetontragwerken - Teil 1-1: Allgemeine Bemessungsregeln für den Hochbau. Deutsche Norm. Berlin: Beuth, 2013. 
DIN EN 1993-1-11

DIN EN 206-1

DIN EN 572-1

DIN EN 572-3

DIN EN 572

DIN EN ISO 12543-1

DIN EN ISO 12543-5

DIN EN ISO 15630-3

E DIN EN 1863-1/A1

ETAG 013
DIN EN 1993-1-11, Dezember 2010: Eurocode 3: Bemessung und Konstruktion von Stahlbauten - Teil 1-11: Bemessung und Konstruktion von Tragwerken mit Zuggliedern aus Stahl. Deutsche Norm. Berlin: Beuth, 2010.

DIN EN 206-1, Juli 2001: Beton - Teil 1: Festlegung, Eigenschaften, Herstellung und Konformität. Deutsche Norm. Berlin: Beuth, 2001.

DIN EN 572-1, November 2012: Glas im Bauwesen - Basiserzeugnisse aus Kalk-Natronsilicatglas - Teil 1: Definitionen und allgemeine physikalische und mechanische Eigenschaften. Deutsche Norm. Berlin: Beuth, 2012.

DIN EN 572-3, November 2012: Glas im Bauwesen - Baiserzeugnisse aus Kalk-Natronsilicatglas - Teil 3: Poliertes Drahtglas. Deutsche Norm. Berlin: Beuth, 2012.

DIN EN 572, November 2012: Glas im Bauwesen - Basiserzeugnisse aus Kalk-Natronsilicatglas. Mehrteilig. Deutsche Norm. Berlin: Beuth, 2012.

DIN EN ISO 12543-1, Dezember 2012: Glas im Bauwesen - Verbundglas und Verbund-Sicherheitsglas - Teil 1: Definition und Beschreibung von Bestandteilen. Deutsche Norm. Berlin: Beuth, 2012.

DIN EN ISO 12543-5, Dezember 2011: Glas im Bauwesen - Verbundglas und Verbund-Sicherheitsglas - Teil 5: Maße und Kantenbearbeitung. Deutsche Norm. Berlin: Beuth, 2011.

DIN EN ISO 15630-3, Februar 2011: Stähle für das Vorspannen von Beton Prüfverfahren - Teil 3: Spannstähle. Deutsche Norm. Berlin: Beuth, 2011.

E DIN EN 1863-1/A1: Glas im Bauwesen - Teilvorgespanntes Kalknatronglas - Teil 1: Definition und Beschreibung. Deutsche Norm. Berlin: Beuth, 2015.

ETAG 013: Guideline for European technical approval of post-tensioning kits for prestressing of structures. EOTA. Brüssel., 2002. 


\section{Bezeichnungen}

\section{Lateinische Buchstaben}

\begin{tabular}{|c|c|}
\hline$a, b, c$ & Regressionsparameter \\
\hline A & Fläche \\
\hline B & Breite (des gesamten Doppelquerschnitts) \\
\hline d & Dicke, Schichtdicke (Dicke der Zwischenschicht, Glasdicke) \\
\hline E & Elastizitätsmodul \\
\hline $\mathrm{F}$ & Festigkeit im Allgemeinen oder Einzelkraft \\
\hline$f_{p}$ & Zugfestigkeit Spannbewehrung (Spannstahl) \\
\hline $\begin{array}{l}f_{p 0,1} \\
F\end{array}$ & $\begin{array}{l}\text { Wert der } 0,1 \% \text { Dehngrenze der Spannbewehrung (Spannstahl) } \\
\text { Kraft, Auflast }\end{array}$ \\
\hline g & Eigenlast \\
\hline$h_{z}$ & Zugzonenhöhe \\
\hline $\mathrm{H}$ & Höhe (des Doppelquerschnitts) \\
\hline I & Trägheitsmoment zweiter Ordnung, Flächenträgheitsmoment \\
\hline $\mathrm{L}$ & Länge, Abstand oder Spannweite \\
\hline M & Biegemoment \\
\hline $\mathrm{N}$ & Normalkraft \\
\hline$P$ & Vorspannkraft \\
\hline$P_{\max }$ & Vorspannkraft am Ende des Spannvorgangs ( $\mathrm{t}=0$; Mittelwert) \\
\hline q & Verkehrslast \\
\hline Q & Querkraft \\
\hline$R^{2}$ & Bestimmtheitsmaß \\
\hline S & empirische Standardabweichung \\
\hline t & Zeitvariable \\
\hline $\mathrm{T}$ & Temperatur \\
\hline $\mathrm{T}_{\mathrm{g}}$ & Glasübergangstemperatur \\
\hline $\mathrm{v}$ & empirischer Variationskoeffizient \\
\hline$v_{y}$ & horizontale Durchbiegung in y-Richtung \\
\hline V & Querkraft nach [DIN EN 1992-1-1] \\
\hline W & Trägheitsmoment erster Ordnung \\
\hline$\overline{\mathrm{x}}$ & empirische Standardabweichung \\
\hline$w_{z}$ & vertikale Durchbiegung in z-Richtung \\
\hline $\begin{array}{l}w_{Z . T} \\
Z_{B k}\end{array}$ & $\begin{array}{l}\text { Traversenweg in z-Richtung } \\
\text { charakteristische Zugtragfähigkeit }\end{array}$ \\
\hline
\end{tabular}

Griechische Buchstaben

$\begin{array}{ll}\alpha_{T} & \text { Wärmedehnzahl } \\ \delta & \text { Verschiebung } \\ \gamma & \text { Gleitwinkel } \\ \varepsilon & \text { Verzerrung } \\ \varepsilon_{\text {komp }} & \text { kompensierte Verzerrung } \\ \varepsilon_{\text {mech }} & \text { mechanische Verzerrung } \\ \varepsilon_{\text {therm }} & \text { thermische Verzerrung } \\ \eta & \text { Viskosität } \\ \eta_{\mathrm{p}} & \text { Verlustfaktor } \\ \kappa & \text { Vorspanngrad } \\ \mu & \text { Reibungsbeiwert } \\ \rho & \text { Relaxationsbeiwert } \\ \sigma & \text { Spannung } \\ \varphi & \text { (End-)Kriechzahl, Kriechbeiwert }\end{array}$


Indizes

$\begin{array}{ll}\text { A } & \text { Außergewöhnliche Einwirkung oder Auswirkung } \\ \text { B } & \text { Beton } \\ \text { b } & \text { bond, Verbund } \\ \text { c } & \text { compression (beispielsweise Beton-Druckfestigkeit in [DIN EN 1992-1-1]) } \\ \text { c } & \text { creepage, Kriechen } \\ \text { cr } & \text { crack, gerissen } \\ \text { d } & \text { Bemessungswert } \\ \text { el } & \text { elastisch } \\ \text { F } & \text { bezüglich der Auflast } \\ \text { g } & \text { Eigenlast } \\ \text { G } & \text { Glas, Glasquerschnitt } \\ \text { ges } & \text { gesamt; im Sinne einer Summe aus Einzelanteilen } \\ \text { k } & \text { charakteristischer Wert } \\ \text { K } & \text { Klotzung beziehungsweise Kontaktmaterial } \\ \text { m } & \text { Mittelwert } \\ \text { M } & \text { bezüglicher der Trägermitte } \\ \text { max; min } & \text { Maximalwert; Minimalwert } \\ \text { OK; UK } & \text { bezüglich der Oberkante/Unterkante des Trägers } \\ \text { p } & \text { Spannbewehrung (Spannstahl) } \\ \text { P } & \text { bezüglich der Vorspannkraft } \\ \text { r } & \text { Kurzzeitrelaxation (zeitunabhängig) } \\ \text { rel } & \text { Langzeitrelaxation; visko-elastisch (zeitabhängig) } \\ \text { sl } & \text { Schlupf } \\ \text { S } & \text { Bewehrungsquerschnitt (schlaff oder vorgespannt) } \\ \text { T } & \text { Torsion } \\ \text { v } & \text { viskos } \\ \text { VG } & \text { bezüglich des Verbundglases } \\ \text { X, y, z } & \text { bezüglich Längsachse, starker und schwacher Querschnittsachse } \\ \text { Z } & \text { Zwischenschicht } \\ \text { O; 1000h } & \text { bezüglich dem Zeitpunkt t = 0 h beziehungsweise t = 1000 h } \\ & \end{array}$

Abkürzungen

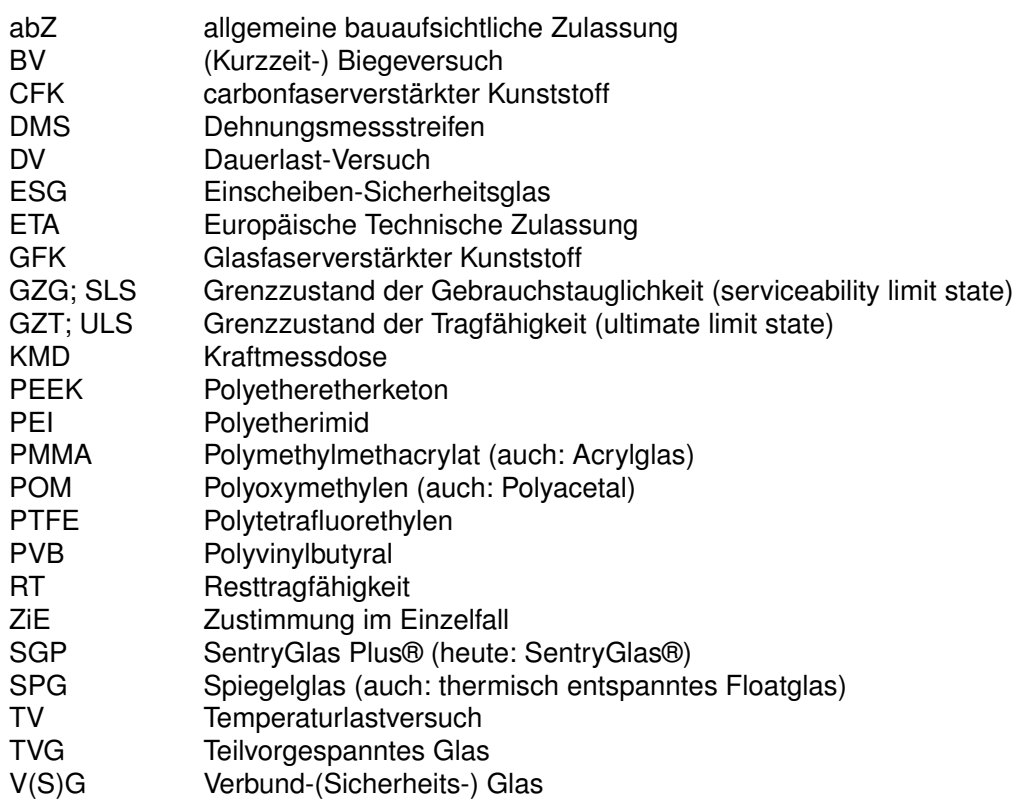




\section{Abbildungsverzeichnis und -nachweis}

Foto oben links: Glasdach der „Alten Mensa“, Institut für Baukonstruktion, TU Dresden Foto oben rechts: Glasbrücke im Eaton Centre, Calgary, Kanada. [Wellershoff et al. 2011], gespiegelte Darstellung

Foto unten links: Vertikale Glasträger als Fassadenschwerter im Kunstmuseum Stuttgart. HASCHER JEHLE Architektur, Berlin, Svenja Bockhop

Foto unten rechts: Horizontale Glasträger als Fassadenschwerter im Richard Bradshaw Amphitheatre at the Four Seasons Centre for the Performing Arts, Toronto, Kanada. Diamond and Schmitt Architects Inc., Steven Evans Photography

(n)

Flächige Bewehrung eines Verbundglases.

Foto: [Feirabend 2010, Seite 74]

Foto links: [Bos et al. 2004, Fig. 9]

6 Spannglasbrücke auf der glasstec 2014

Foto: Messe Düsseldorf/Tillmann

Einordnung der Glasbaunormung in das Konzept der Eurocodes

Foto oben: [Louter 2013, Seite 353]

Foto unten: [Firmo et al. 2015, Figure 20]

Foto oben: Santarsiero, M., ohne Jahr

15 Beispiel: sofortiger Verbund

Foto links: Schmal, M. https://commons.wikimedia.org/wiki/File:

SpannbetonFertigdecke_Herstellung.jpg, letzter Zugriff 17. 06. 2015

Foto rechts: [Cupac \& Louter 2015]

Foto oben: [Abel \& Krautwald 2001]

Foto mittig: [Bos et al. 2004, Seite 5]

Foto oben links: BBV Systems GmbH http://www.bbv-systems.com/referenzen/ spannverfahren/brueckenbau/, letzter Zugriff 14.03. 2017

Beispiel: flächige Bewehrung

Foto rechts: [Feirabend 2010, Seite 147]

Foto links: [Betonbau 11/2015]

Foto rechts: [Neugebauer 2005, Seite 9]

Foto oben: http://www waagner-biro.com/de/sparten/stahl-glas-technik/

referenzen/referenz/tower-place, letzter Zugriff 14. 04. 2014 
Foto oben: Michael Rasche

Übersichtsschema nach Lage und Verlauf der Bewehrung 43

Last-Verzerrungs-Diagramm für Beton und Glas $\quad 50$

Kriechverformung der Glaskonstruktion $\quad 53$

Kriechzahlen einer PVB Zwischenschicht $\quad 56$

Kriechzahlen für Klotzungswerkstoffe $\quad 59$

$\begin{array}{ll}\text { Verankerungen und Kopplungen } & 61\end{array}$

Doppelquerschnitt $\quad 62$

Imperfekter Querschnitt $\quad 66$

Systembild zentrischer Seilvorspannung $\quad 69$

Systembild Seilbelastung in Seilrichtung $\quad 71$

Last-Verformungskurve belasteter Seile $\quad 71$

Systembild Kraftgrößenmethode $\quad 72$

Beispiel: Stabwerkmodelle $\quad 75$

Resttragfähigkeit nach [Kott 2006] 94

Übersicht notwendiger experimenteller Untersuchungen 101

Querschnitt, Abmessungen und Bezeichnungen innerhalb der Prüfkörper $\quad 104$

Umlenksattel $\quad 105$

Explosionszeichnung der Auflagerkonstruktion 105

Vierpunkt-Biegeversuchsstand für kurzzeitige Biegebelastung 108

Spannweg beispielhaft $\quad 114$

Verhältnis von Seilkraft und Durchbiegung beispielhaft 115

Umlenkkraftverlust $\quad 118$

$\begin{array}{lr}\text { Vorspannsteifigkeit } & 120\end{array}$

Veränderung der horizontalen Durchbiegung 122

Erstrisslast 123

Typisiertes Bruchversagen $\quad 125$

Rissentwicklung $1 \quad 126$

Rissentwicklung $2 \quad 126$

Rissentwicklung $3 \quad 127$

Nachbruch unbewehrter und bewehrter Träger 130

$\begin{array}{ll}\text { Nachbruchknick im Kurzzeit-Biegeversuch } & 131\end{array}$

Glasabplatzung im Kurzzeit-Biegeversuch 131

Vierpunkt-Biegeversuchsstand für dauerhafte Biegebelastung $\quad 135$

$\begin{array}{lr}\text { Verlauf der Seilkraft über die gesamte Versuchszeit } & 138\end{array}$

Regressionsfehler im Dauerversuch (Gleichung 51) 139

Regressionsfehler im Dauerversuch (Gleichung 53) 141

$\begin{array}{ll}\text { Durchbiegungsänderungen im Dauerversuch } & 144\end{array}$

Absolute Verschiebung im Dauerversuch $\quad 145$

Biegeversuchsstand zur Ermittlung der Resttragfähigkeit $\quad 148$

Verfahren im Versuch zur Ermittlung der Resttragfähigkeit $\quad 150$

Charakteristisches Rissbild im Versuch zur Ermittlung der Resttragfähigkeit 152

Verlauf des Resttragfähigkeitsversuchs am Beispiel des Prüfkörpers RT19 P15-II 153

Änderung der Seilkraft vor und nach dem Anschlagen einer Glasschicht $\quad 154$

Seilkraft, Glasverzerrung und Durchbiegung für Prüfkörper RT 21

Reststandzeit im Resttragfähigkeitsversuch $\quad 156$

Abweichendes Rissbild im Resttragfähigkeitsversuch $\quad 158$

Rissbilder RT 20 P15-II 159 
Mehrwellige Versagensfom mit flächigem Rissbild und abgerissenem Umlenkpunkt 162 Abscherversagen im Resttragfähigkeitsversuch im Prüfkörper RT 15 163

Referenzglaskörper

Vorgehen bei der Auswertung der Biegeversuche unter Temperaturlast Temperaturversuch TV01-P6F5

Einfluss der Drehsteifigkeit der Umlenkungen auf den Eigenwert

Foto mittig: [Bos et al. 2004, Fig. 11].

Foto rechts: Precast/Prestressed Concrete Institute (PCI) und Civil and Mineral Engineering at the University of Toronto: Beam Test...watch beam failure in slowmotion! 2014. https://www.youtube.com/watch?v=6ycbDCnoO8M, veröffentlicht am 04. 07. 2014, letzter Zugriff am 08. 02. 2017

Foto mittig: [Louter et al. 2014, Seite 13]

Foto unten: [Weller 1988, Seite 93]

Foto mittig: [Härth 2013, Seite 143]

Foto rechts: Institut für Baukonstruktion

Fotos unten: Institut für Baukonstruktion, TU Dresden

Foto rechts: Messe Düsseldorf/Tillmann 
Verfahrensschritte zum Aufkleben der Umlenkpunkte

260

Umlenkpunkte mit aufgebrachten Abstandhaltern vor dem Kleben

261 


\section{Tabellenverzeichnis}

Allgemeine Regelungen der [DIN EN 1990]

$2 \quad$ Alternative Verbundglasaufbauten 30

Untersuchte Einwirkungen und Übersicht über zugehörige Untersuchungsmethoden

Charakteristische Eigenschaften von Glas und Beton 48

Idealisierung der Tragwerke nach [DIN EN 1992-1-1]

Zustände im Grenzzustand der Gebrauchstauglichkeit 93

Ausschluss eines unangekündigten Versagens vorgespannter Konstruktionen 95

Übersicht vorgespannter Glasträger mit linearer Bewehrung $\quad 98$

$9 \quad$ Eigenschaften der verwendeten Bewehrung 106

10 Prüfkörper Kurzzeit-Biegeversuche 107

11 Regressionsparameter zur Beschreibung der Verzerrungsänderung im Vorspannversuch 117

12 Regressionsparameter zur Beschreibung der Verzerrungsänderung im Kurzzeit-Biegeversuch 121

Biegelast und Traversenweg beim ersten, zweiten und dritten Rissereignis

Prüfkörper Dauerversuche 134

Regressionsparameter des Seilkraftverlaufes über die Zeit im Dauerlast-Biegeversuche $\quad 139$

Alternative Regressionsparameter im Dauerlast-Biegeversuche $\quad 141$

Ergebnisse Dauerlast-Biegeversuche $\quad 142$

Vergleich des absoluten Seilkraftverlustes $\Delta \mathrm{P}$ nach Anzahl der Umlenkungen $\quad 142$

$\begin{array}{ll}\text { Prüfkörper Resttragfähigkeits-Versuche } & 147\end{array}$

Prüfkörper Temperatur-Biegeversuche 165

$\begin{array}{ll}\text { Versuchsmatrix Temperatur-Biegeversuche } & 165\end{array}$

Wärmedehnzahl des Referenzglaskörpers 172

Berechnungsergebnisse des Stabwerkmodells und Vergleich mit der analytischen Lösung $\quad 188$

Horizontale Durchbiegung im numerischen Vorspannversuch 192

Vertikale Durchbiegung im numerischen Vorspannversuch 193

Numerisch ermittelte Biegesteifigkeit $a_{F}$ in Abhängigkeit von der Imperfektionsannahme $\quad 195$

Seilkraftänderung ap im FEM Modell in Abhängigkeit von der Imperfektionsannahme $\quad 196$

Änderung der seitlichen Durchbiegung im numerischen Biegeversuch 197

$\begin{array}{ll}\text { Vergleich der Seilkräfte } & 200\end{array}$

Seilkraftänderungen nach $1000 \mathrm{~h}$ im numerischen Modell 203

$\begin{array}{ll}\text { Vergleich der Duktilitätskennwerte } & 217\end{array}$

Maximale Seilkräfte unter Kurzzeit-Biegeversuch und Resttragfähigkeitsversuch $\quad 217$

Maximale Bemessungswerte der Auswirkung an einer Trägeroberkante und -unterkante $\quad 225$

Gegenüberstellung ausgewählter Trageigenschaften 239

Vergleich konstruktiver Maßnahmen zum Ausgleich des VSG-Kantenversatzes 251

$\begin{array}{ll}\text { Seilvorspannung zuzüglich sofortiger Verluste } & 308\end{array}$

Erwartete Biegekraft- und Biegespannungsänderung 311

Seilkraftverluste $\Delta \mathrm{P}$ nach $1000 \mathrm{~h} \quad 314$

Effektive Glasdicken und Biegesteifigkeit um die schwache Achse $\quad 316$

Effektive Seilsteifigkeit in Abhängigkeit zur Belastungszeit 317

Seilkräfte unter Biege- und Vorspannbelastung im Resttragversuch 317

Reine Glasdurchbiegung im Versuch zur Ermittlung der Resttragfähigkeit 318

Seilkraft nach einer Temperaturänderung von $10 \mathrm{~K} \quad 318$ 


\section{A Analytische Schnittgrößenberechnung}

\section{Zielsetzung}

Die Schnittkräfte und Verformungsgrößen der innerlich statisch unbestimmten Spannglasträger dieser Arbeit werden analytisch mittels der Kraftgrößenmethode berechnet. Dabei wird die lineare Balkentheorie I. Ordnung angewendet und folglich die horizontale Durchbiegung als Folge einer geometrischen Imperfektion sowie ein mögliches Biegedrillknicken vernachlässigt. Die zugehörige Lösung dient der vereinfachten Abschätzung. ${ }^{423}$ Dabei werden die erwarteten Ergebnisse zur Gegenüberstellung mit den experimentell ermittelten Werten berechnet. Die resultierende Abweichung erlaubt eine Wertung der Anwendungsgrenzen.

Das Tragsystem wird in ein statisch bestimmtes Hauptsystem unter der Biegebelastung $F$, der Vorspannbelastung $P_{0}$ und der Temperaturbelastung $\Delta T$ sowie ein System unter Belastung durch die statisch unbestimmte Größe $X_{1}$ aufgeteilt (Bild 120). Beide Anteile werden über die Verträglichkeitsbedingung ${ }^{424}$ zusammengeführt.

\section{Statisch bestimmtes Hauptsystem „0“}

\section{Biegebelastung (F)}

Aus der Biegebelastung $\mathrm{F}$ resultiert ein Momentenverlauf in zwei Abschnitten:

$$
\begin{aligned}
& M_{F .0 . I}\left(x_{I}\right)=\frac{F}{2} \cdot \frac{L_{S}}{3} \cdot \frac{3 \cdot x_{I}}{L_{S}}=\frac{F}{2} \cdot x_{I} \\
& M_{F .0 . I I}\left(x_{I I}\right)=\frac{F}{2} \cdot \frac{L_{S}}{3}=\frac{F \cdot L_{S}}{6} \\
& 423 \text { Insbesondere fehlende Angabe zur Steifigkeit des hier verwendeten } \\
& \text { Doppelquerschnitts um dessen schwache Achse erschwert zunächst } \\
& \text { weiterführende Angaben. } \\
& 424 \text { Vergleiche Gleichung } 75 \text {, Seite } 298 .
\end{aligned}
$$


Bild 120

Freikörperbild.

Für die Berechnung wird die Spannweite $\left(L_{S}\right)$, die Länge des Glases $\left(L_{G}\right)$ und die Länge des Spannseils $\left(L_{p}\right)$ unterschieden.

Zudem wird die Wirkung des Seils durch die Ersatzkräfte an den Verankerungen $\left(P_{0}\right)$ und an den Umlenkungen an den Drittelspunkten $\left(U=2 \cdot P_{0} \cdot \sin (\alpha / 2)\right)$ angenommen. statisch unbestimmtes System

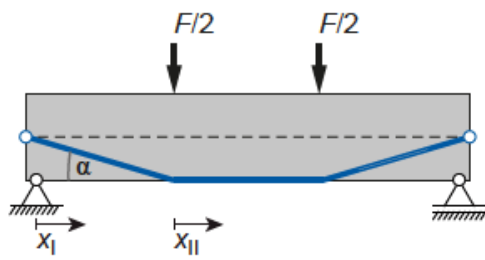

$0 \leq x_{1} \leq L / 3$

$0 \leq x_{\| 1} \leq L / 6$

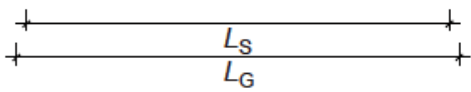

"1" - System

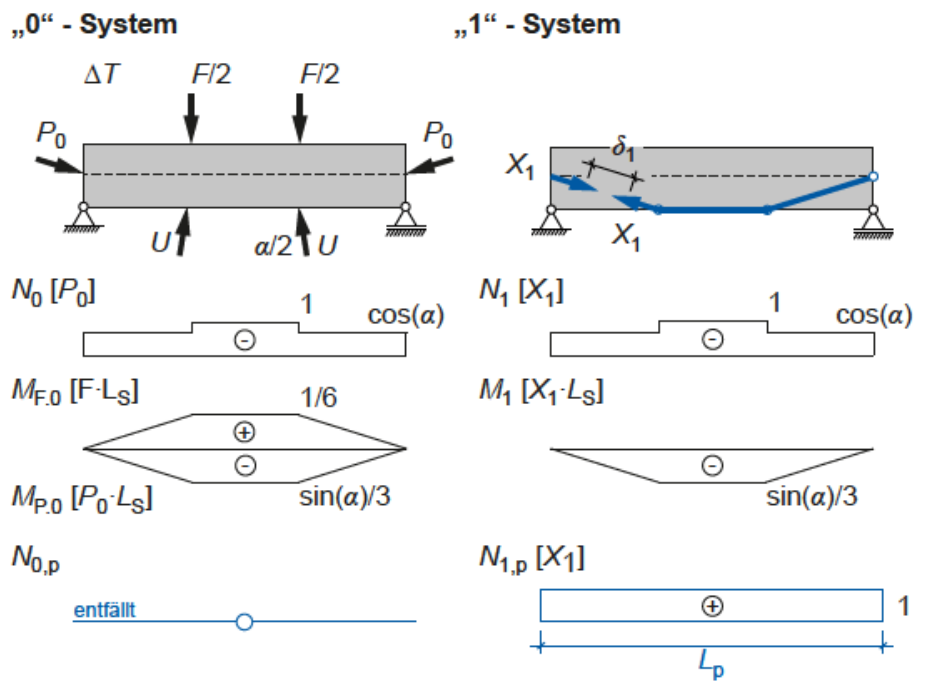

Aus der Biegebelastung resultiert kein Normalkraftanteil. Zudem wird die Schubverformung aus der Querkraftwirkung vernachlässigt.

\section{Biegebelastung $\left(P_{0}\right)$}

Aus der Vorspannung $\mathrm{P}_{0}$ resultiert ein Momenten- und Normalkraftverlauf in zwei Abschnitten. Die Schubverformung aus der Querkraftwirkung wird vernachlässigt.

$$
\begin{aligned}
M_{P .0 . I}\left(x_{l}, \alpha\right) & =-P_{0} \cdot \sin (\alpha) \cdot \frac{L_{S}}{3} \cdot \frac{3 \cdot x_{I}}{L_{S}} \\
& =-P_{0} \cdot \sin (\alpha) \cdot x_{I}
\end{aligned}
$$




$$
M_{P .0 . I I}\left(x_{\| l}, \alpha\right)=-P_{0} \cdot \sin (\alpha) \cdot \frac{L_{S}}{3}
$$

$$
N_{P .0 . I}\left(x_{l}\right)=-P_{0} \cdot \cos (\alpha)
$$

Gleichung 68

$$
N_{P .0 . I I}\left(x_{I I}\right)=-P_{0}
$$

\section{Temperaturbelastung $(\Delta T)$}

Im statisch bestimmten (ungezwängten) Hauptsystem ergeben sich keine Schnittkräfte infolge einer über die Querschnittshöhe konstanten Temperaturbelastung.

\section{Statisch bestimmtes Hauptsystem „1“}

Aus der statisch Überzähligen $\mathrm{X}_{1}$ resultiert ein Momentenund Normalkraftverlauf in zwei Abschnitten.

$$
\begin{array}{ll}
M_{X .1 . I}\left(x_{I}\right)=-X_{1} \cdot \sin (\alpha) \cdot \frac{L_{S}}{3} \cdot \frac{3 \cdot x_{I}}{L_{S}}=-X_{1} \cdot \sin (\alpha) \cdot x_{I} & \text { Gleichung } 70 \\
& \\
M_{X .1 . I I}\left(x_{I I}\right)=-X_{1} \cdot \sin (\alpha) \cdot \frac{L_{S}}{3} & \text { Gleichung } 71
\end{array}
$$


Gleichung 72

Gleichung 73

Gleichung 74

Gleichung 75
$N_{X .1 . I}\left(X_{l}\right)=-X_{1} \cdot \cos (\alpha)$

$N_{X .1 . / I}\left(X_{I I}\right)=-X_{1}$

Dabei repräsentiert $X_{1}$ die Seilkraft, welche notwendig ist, um die Verträglichkeitsbedingung zu erfüllen:

$N_{X .1 . p}\left(X_{p}\right)=X_{1}$

Mit Gleichung 74 wird die statisch Unbestimmte $X_{1}$ als Änderung der Normalkraft im Seil interpretierbar. Die Schubverformung aus der Querkraftwirkung wird vernachlässigt.

\section{Arbeitsgleichung}

Mit Bild 120 wird die Verträglichkeitsbedingung in Gleichung 75 formuliert.

$$
\delta_{1}=\delta_{10}+X_{1} \cdot \delta_{11}=0
$$


Deren Anteile werden mit der Arbeitsgleichung als Summe der Biege- und Normalkraftanteile aus F, $\mathrm{P}_{0}, \mathrm{X}_{1}$ sowie der Temperaturänderung bestimmt:

$$
\begin{aligned}
\delta_{i j} & =\int_{\left(L_{S}\right)} \frac{M_{i} \cdot M_{j}}{E_{G} \cdot I_{y \cdot G}} \mathrm{~d} x_{G(I+I l)}+\int_{\left(L_{G}\right)} \frac{N_{i} \cdot N_{j}}{E_{G} \cdot A_{G}} \mathrm{~d} x_{G(I+I l)} \\
& +\int_{\left(L_{G}\right)} \Delta T \cdot \alpha_{T, G} \cdot N_{G, j} \mathrm{~d} x_{G(I+I)} \\
& +\int_{\left(L_{p}\right)} \frac{N_{i} \cdot N_{j}}{E_{p} \cdot A_{p}} \mathrm{~d} x_{p}+\int_{\left(L_{p}\right)} \Delta T \cdot \alpha_{T, p} \cdot N_{p, j} \mathrm{~d} x_{p}
\end{aligned}
$$

Gleichung 76

Die Lösungen der Integrale über die Laufvariablen bei unveränderlichen Querschnitts- und Materialkennwerten sind als Tafelwerte verfügbar, sodass im Weiteren die einzelnen Summanden aus Gleichung 76 mit den zugehörigen Faktoren aufgeschrieben werden. Dabei wird die Symmetrie des Tragsystems ausgenutzt.

\section{Einflusszahl $\delta_{11}$}

Im Ergebnis werden die Anteil aus dem Biegemoment im Glas (Gleichung 77 und 78), der Normalkraft im Glas (Gleichung 79 und 80) und der Seilkraft (Gleichung 81) ermittelt. Darüber hinaus besteht kein Anteil aus der Temperaturlast im „1“-System.

$$
\begin{aligned}
\delta_{11 . M . I} & =\frac{1}{3} \cdot \frac{L_{S}}{3 \cdot E_{G} \cdot I_{y . G}} \cdot M_{X .1 . I}\left(X_{I}=\frac{L_{S}}{3}\right)^{2} \\
& =\frac{L_{S}^{3} \cdot X_{1}^{2} \cdot \sin ^{2}(\alpha)}{81 \cdot E_{G} \cdot I_{y . G}}
\end{aligned}
$$


Gleichung 78

Gleichung 79

Gleichung 80

Gleichung 81

Gleichung 82

$$
\begin{aligned}
\delta_{11 . N . I} & =\frac{L_{G}}{3 \cdot E_{G} \cdot A_{G}} \cdot N_{X .1 . I}\left(X_{I}=\frac{L_{G}}{3}\right)^{2} \\
& =\frac{L_{G} \cdot X_{1}^{2} \cdot \cos ^{2}(\alpha)}{3 \cdot E_{G} \cdot A_{G}}
\end{aligned}
$$

$$
\begin{aligned}
\delta_{11 . N . I l} & =\frac{L_{G}}{6 \cdot E_{G} \cdot A_{G}} \cdot N_{X .1 . I I}\left(x_{\| l}=0\right)^{2} \\
& =\frac{L_{G} \cdot X_{1}^{2}}{6 \cdot E_{G} \cdot A_{G}}
\end{aligned}
$$

Unter Berücksichtigung der Symmetrie ergibt sich der Anteil $\delta_{11} \mathrm{zu}$ :

$$
\begin{aligned}
\delta_{11} & =2 \cdot\left(\delta_{11 . M . I}+\delta_{11 . M . I I}+\delta_{11 . N . I}+\delta_{11 . N . I I}+\delta_{11 . N . p}\right) \\
& =\frac{L_{G} \cdot X_{1}^{2}}{3 \cdot E_{G} \cdot A_{G}}+\frac{L_{p} \cdot X_{1}^{2}}{E_{p} \cdot A_{p}} \\
& +\frac{5 \cdot L_{S}^{3} \cdot \sin ^{2}(\alpha) \cdot X_{1}^{2}}{81 \cdot E_{G} \cdot I_{y . G}}+\frac{2 \cdot L_{G}^{3} \cdot \cos ^{2}(\alpha) \cdot X_{1}^{2}}{3 \cdot E_{G} \cdot A_{G}}
\end{aligned}
$$


Bei geringer Neigung des Seils sind die Länge des Glases $\left(L_{G}\right)$, die Länge des Seils $\left(L_{p}\right)$ sowie die Spannweite $\left(L_{S}\right)$ nahezu identisch. Sie werden durch $L_{\text {ges }}$ ersetzt. Zudem gilt bei kleinen Winkeln die Vereinfachung $\sin ^{2}(\alpha) \sim \alpha$ und $\cos ^{2}(\alpha) \sim 1$. Jedoch zeigte eine Parameterstudie mit den Material- und Geometriekennwerten dieser Arbeit, dass der Ersatz von $\sin ^{2}(\alpha)$ zu Abweichungen von bis zu $4 \%$ führt, was als unzulässig hoch beurteilt wird. Dem gegenüber hat der Summand mit $\cos ^{2}(\alpha)$ keinen bestimmenden Einfluss.

Daher verbleibt für $\delta_{11}$ die folgende Vereinfachung:

$$
\delta_{11}=\left(\frac{L_{g e s}}{E_{G} \cdot A_{G}}+\frac{L_{g e s}}{E_{p} \cdot A_{p}}+\frac{5 \cdot L_{g e s}^{3} \cdot \sin ^{2}(\alpha)}{81 \cdot E_{G} \cdot I_{y}}\right) \cdot X_{1}^{2}
$$

\section{Einflusszahl $\delta_{10}$}

Ebenso werden die Beiträge der Einflusszahl $\delta_{10}$ als Anteil aus dem Biegemoment im Glas (Gleichung 84 und 85), der Normalkraft im Glas (Gleichung 86 und 87 ) sowie dem Anteil aus der Temperaturlast in Glas und Seil (Gleichung 88 bis 90 ) berechnet.

$$
\begin{aligned}
\delta_{10 . M . I} & =\frac{1}{3} \cdot \frac{L_{S}}{3 \cdot E_{G} \cdot I_{y . G}} \cdot M_{X .1 . I}\left(x_{I}=\frac{L_{S}}{3}\right) \cdot\left(M_{F .0 . I}\left(x_{I}=\frac{L_{S}}{3}\right)+M_{P .0 . I}\left(x_{I}=\frac{L_{S}}{3}\right)\right) \\
& =\frac{L_{S}^{2} \cdot X_{1} \cdot \sin (\alpha)}{27 \cdot E_{G} \cdot I_{y . G}} \cdot\left(\frac{F \cdot L_{S}}{6}-\frac{L_{S} \cdot P_{0} \cdot \sin (\alpha)}{3}\right)
\end{aligned}
$$

Gleichung 84 


$$
\begin{aligned}
\delta_{10 . M . I I} & =\frac{L_{S}}{6 \cdot E_{G} \cdot I_{y . G}} \cdot M_{X .1 . I I}\left(x_{\| I}=0\right) \cdot\left(M_{F .0 . I I}\left(x_{\| I}=0\right)+M_{P .0 . I I}\left(x_{\| I}=0\right)\right) \\
& =\frac{L_{S}^{2} \cdot X_{1} \cdot \sin (\alpha)}{18 \cdot E_{G} \cdot I_{y \cdot G}} \cdot\left(\frac{F \cdot L_{S}}{6}-\frac{L_{S} \cdot P_{0} \cdot \sin (\alpha)}{3}\right)
\end{aligned}
$$

Gleichung 85

$$
\begin{aligned}
\delta_{10 . N . I} & =\frac{L_{G}}{3 \cdot E_{G} \cdot A_{G}} \cdot N_{X .1 . I}\left(x_{I}=\frac{L_{G}}{3}\right) \cdot\left(N_{F .0}\left(X_{I}=\frac{L_{G}}{3}\right)+N_{P .0 . I}\left(x_{I}=\frac{L_{G}}{3}\right)\right) \\
& =\frac{L_{G} \cdot \cos (\alpha)^{2} \cdot P_{0} \cdot X_{1}}{3 \cdot E_{G} \cdot A_{G}}
\end{aligned}
$$

Gleichung 86

$$
\begin{aligned}
\delta_{10 . N . I I} & =\frac{L_{G}}{6 \cdot E_{G} \cdot A_{G}} \cdot N_{X .1 . I I}\left(x_{I I}=0\right) \cdot\left(N_{F .0}\left(x_{I I}=0\right)+N_{P .0 . I I}\left(x_{I I}=0\right)\right) \\
& =\frac{L_{G} \cdot P_{0} \cdot X_{1}}{6 \cdot E_{G} \cdot A_{G}}
\end{aligned}
$$

Gleichung 87

Gleichung 88

$$
\begin{aligned}
\delta_{10 . N T . I} & =N_{X .1 . I}\left(X_{I}=\frac{L_{G}}{3}\right) \cdot \Delta T \cdot \alpha_{T . G} \cdot \frac{L_{G}}{3} \\
& =-\frac{L_{G}}{3} \cdot X_{1} \cdot \Delta T \cdot \alpha_{T . G} \cdot \cos (\alpha)
\end{aligned}
$$




$$
\begin{aligned}
\delta_{10 . N T . I I} & =N_{X .1 . I I}\left(X_{I}=0\right) \cdot \Delta T \cdot \alpha_{T . G} \cdot \frac{L_{G}}{6} \\
& =-\frac{L_{G}}{6} \cdot X_{1} \cdot \Delta T \cdot \alpha_{T . G} \\
\delta_{10 . N T . p} & =N_{X .1 . p}\left(X_{p}\right) \cdot \Delta T \cdot \alpha_{T . p} \cdot \frac{L_{p}}{2} \\
& =\frac{L_{p}}{2} \cdot X_{1} \cdot \Delta T \cdot \alpha_{T . p}
\end{aligned}
$$

Unter Berücksichtigung der Symmetrie ergibt sich der Anteil $\delta_{10} \mathrm{zu}$ :

$$
\begin{aligned}
\delta_{10} & =2 \cdot\left(\delta_{10 . M . I}+\delta_{10 . M . I I}+\delta_{10 . N . I}+\delta_{10 . N . I I}+\delta_{10 . N T . I}+\delta_{10 . N T . I I}+\delta_{10 . N T . p}\right) \\
& =\left(-\frac{5 \cdot L_{S}^{3} \cdot \sin (\alpha)}{162 \cdot E_{G} \cdot I_{y . G}}\right) \cdot F \cdot X_{1} \\
& +\left(\frac{L_{G}}{3 \cdot E_{G} \cdot A_{G}}+\frac{5 \cdot L_{S}^{3} \cdot \sin ^{2}(\alpha)}{81 \cdot E_{G} \cdot I_{y . G}}+\frac{2 \cdot L_{G} \cdot \cos ^{2}(\alpha)}{3 \cdot E_{G} \cdot A_{G}}\right) \cdot P_{0} \cdot X_{1} \\
& +\left(L_{p} \cdot \alpha_{T . p}-\frac{L_{G} \cdot \alpha_{T . G}}{3}-\frac{2 \cdot L_{G} \cdot \alpha_{T . G} \cdot \cos (\alpha)}{3}\right) \cdot \Delta T \cdot X_{1}
\end{aligned}
$$

Gleichung 91

Auch hier werden aufgrund der geringen Seilneigung die Länge des Glases $\left(L_{G}\right)$, die Länge des Seils $\left(L_{p}\right)$ sowie die Spannweite $\left(L_{S}\right)$ durch $L_{\text {ges }}$ ersetzt. Ein Ersatz von $\cos ^{2}(\alpha) \sim$ 1 wird erneut durchgeführt. Ebenso führt ein Ersatz von $\cos (\alpha) \sim 1$ nur zu unwesentlichen Änderungen im Ergebnis. Letztlich wird ein Ersatz von $\sin ^{2}(\alpha)$ nicht durchgeführt, 
da auch hier unzulässig hohe Abweichungen auftreten, sodass folgende Vereinfachung verwendet wird:

Gleichung 92

$$
\begin{aligned}
\delta_{10} & =\left(\left[\frac{1}{E_{G} \cdot A_{G}}+\frac{5 \cdot L_{g e s}^{2} \cdot \sin ^{2}(\alpha)}{81 \cdot E_{G} \cdot I_{y}}\right] \cdot P_{0}\right. \\
& \left.-\frac{5 \cdot L_{g e s}^{2} \cdot \sin (\alpha)}{162 \cdot E_{G} \cdot I_{y . G}} \cdot F+\left(\alpha_{T . p}-\alpha_{T . G}\right) \cdot \Delta T\right) \cdot L_{g e s} \cdot X_{1}
\end{aligned}
$$

\section{Ermittlung der Überzähligen $\mathrm{X}_{1}$}

Unter Berücksichtigung der oben ermittelten Ergebnisse und Vereinfachungen wird die Verträglichkeitsbedingung ${ }^{425}$ ausgewertet und die Überzählige $X_{1}$ in Abhängigkeit der Belastungswerte $\mathrm{P}_{0}, \mathrm{~F}$ und $\Delta \mathrm{T}$ angegeben:

Gleichung 93

$$
X_{1}=-\frac{\delta_{10}}{\delta_{11}}=x_{1 . p} \cdot P_{0}+x_{1 . F} \cdot F+x_{1 . T} \cdot \Delta T
$$

$$
x_{1 . p}=-\frac{E_{p} \cdot A_{p} \cdot\left[27 \cdot I_{y} \cdot\left(2 \cdot \cos ^{2}(\alpha)+1\right)+5 \cdot A_{G} \cdot L_{g e s}^{2} \cdot \sin ^{2}(\alpha)\right]}{E_{p} \cdot A_{p} \cdot\left[27 \cdot I_{y} \cdot\left(2 \cdot \cos ^{2}(\alpha)+1\right)+5 \cdot A_{G} \cdot L_{g e s}^{2} \cdot \sin ^{2}(\alpha)\right]+81 \cdot E_{G} \cdot A_{G} \cdot I_{y}}
$$

Gleichung 94

Gleichung 95

$$
x_{1 . F}=\frac{5 \cdot A_{G} \cdot E_{p} \cdot A_{p} \cdot L_{g e s}^{2} \cdot \sin (\alpha)}{162 \cdot I_{y \cdot G} \cdot\left(E_{G} \cdot A_{G}+E_{p} \cdot A_{p}\right)}
$$

${ }^{425}$ Vergleiche Gleichung 75, Seite 298. 


$$
x_{1 . T}=\frac{E_{G} \cdot A_{G} \cdot E_{p} \cdot A_{p} \cdot\left(\alpha_{T . p}-\alpha_{T . G}\right)}{E_{G} \cdot A_{G}+E_{p} \cdot A_{p}}
$$

Für die Prüfkörper dieser Arbeit werden die Materialkennwerte und Geometrieangaben nach Kapitel 3.2 mit $\mathrm{L}_{\text {ges }}=$ $2000 \mathrm{~mm}$ verwendet:

$$
\begin{aligned}
& X_{1}\left(d_{p}=5,0 \mathrm{~mm}\right)=-0,020 \frac{\mathrm{kN}}{\mathrm{kN}} \cdot P_{0}+0,057 \frac{\mathrm{kN}}{\mathrm{kN}} \cdot F-13,5 \frac{\mathrm{N}}{\mathrm{K}} \cdot \Delta T \\
& X_{1}\left(d_{p}=6,1 \mathrm{~mm}\right)=-0,029 \frac{\mathrm{kN}}{\mathrm{kN}} \cdot P_{0}+0,083 \frac{\mathrm{kN}}{\mathrm{kN}} \cdot F-19,8 \frac{\mathrm{N}}{\mathrm{K}} \cdot \Delta T \\
& X_{1}\left(d_{p}=8,1 \mathrm{~mm}\right)=-0,061 \frac{\mathrm{kN}}{\mathrm{kN}} \cdot P_{0}+0,178 \frac{\mathrm{kN}}{\mathrm{kN}} \cdot F-18,3 \frac{\mathrm{N}}{\mathrm{K}} \cdot \Delta T \\
& X_{1}\left(d_{p}=10,1 \mathrm{~mm}\right)=-0,091 \frac{\mathrm{kN}}{\mathrm{kN}} \cdot P_{0}+0,270 \frac{\mathrm{kN}}{\mathrm{kN}} \cdot F-27,7 \frac{\mathrm{N}}{\mathrm{K}} \cdot \Delta T
\end{aligned}
$$

Gleichung 97

\section{Schnittgrößen im statisch unbestimmten System}

\section{Kraftgrößen in Feldmitte}

Die Schnittgrößen im statisch unbestimmten System ergeben sich aus der Superposition der beiden in Bild 120 dargestellten Teilsysteme unter Zuhilfenahme der nunmehr bekannten Lösung für die Überzählige $X_{1}$ aus Gleichung 93. 
Dabei ergibt sich das maximale Biegemoment in Feldmitte nach Gleichung 98, die Normalkraft nach Gleichung 100.

$$
\begin{aligned}
M_{g e s}\left(x_{\|}=\frac{L}{6}\right) & =M_{F .0 . I I}\left(x_{\| l}=\frac{L}{6}\right)+M_{P .0 . I I}\left(x_{\| I}=\frac{L}{6}\right)+M_{X .1 . I I}\left(x_{\| l}=\frac{L}{6}\right) \\
& =\left(1+x_{1 . p}\right) \cdot \frac{L_{g e s}}{3} \cdot-\sin (\alpha) \cdot P_{0} \\
& +\left(\frac{1}{2}-\sin (\alpha) \cdot x_{1 . F}\right) \cdot \frac{L_{g e s}}{3} \cdot F-\sin (\alpha) \cdot \frac{L_{g e s}}{3} \cdot x_{1 . T} \cdot \Delta T
\end{aligned}
$$

Gleichung 98

$$
\text { Gleichung 99 } \begin{aligned}
M_{\text {ges }}\left(d_{p}=5,0 \mathrm{~mm}\right) & =-0,073 \mathrm{~m} \cdot P_{0}+0,329 \mathrm{~m} \cdot F+1,01 \frac{\mathrm{N} \cdot \mathrm{m}}{\mathrm{K}} \cdot \Delta T \\
M_{\text {ges }}\left(d_{p}=6,1 \mathrm{~mm}\right) & =-0,072 \mathrm{~m} \cdot P_{0}+0,327 \mathrm{~m} \cdot F+1,48 \frac{\mathrm{N} \cdot \mathrm{m}}{\mathrm{K}} \cdot \Delta T \\
M_{\text {ges }}\left(d_{p}=8,1 \mathrm{~mm}\right) & =-0,070 \mathrm{~m} \cdot P_{0}+0,320 \mathrm{~m} \cdot F+1,36 \frac{\mathrm{N} \cdot \mathrm{m}}{\mathrm{K}} \cdot \Delta T \\
M_{\text {ges }}\left(d_{p}=10,1 \mathrm{~mm}\right) & =-0,068 \mathrm{~m} \cdot P_{0}+0,313 \mathrm{~m} \cdot F+2,07 \frac{\mathrm{N} \cdot \mathrm{m}}{\mathrm{K}} \cdot \Delta T
\end{aligned}
$$

Gleichung 100

$$
\begin{aligned}
N_{\text {ges }}\left(x_{\| l}=\frac{L}{6}\right) & =N_{P .0 . I I}\left(x_{\| I}=\frac{L}{6}\right)+N_{X .1 . I I}\left(x_{\| l}=\frac{L}{6}\right) \\
& =-\left(\left(1+x_{1 . p}\right) \cdot P_{0}+x_{1 . F} \cdot F+x_{1 . T} \cdot \Delta T\right)
\end{aligned}
$$

$$
\text { Gleichung } 101 \quad \begin{aligned}
N_{g e s}\left(d_{p}=5,0 \mathrm{~mm}\right) & =-0,980 \frac{\mathrm{kN}}{\mathrm{kN}} \cdot P_{0}-0,057 \frac{\mathrm{kN}}{\mathrm{kN}} \cdot F+13,5 \frac{\mathrm{N}}{\mathrm{K}} \cdot \Delta T \\
& N_{g e s}\left(d_{p}=6,1 \mathrm{~mm}\right)=-0,971 \frac{\mathrm{kN}}{\mathrm{kN}} \cdot P_{0}-0,083 \frac{\mathrm{kN}}{\mathrm{kN}} \cdot F+19,8 \frac{\mathrm{N}}{\mathrm{K}} \cdot \Delta T \\
& N_{g e s}\left(d_{p}=8,1 \mathrm{~mm}\right)=-0,939 \frac{\mathrm{kN}}{\mathrm{kN}} \cdot P_{0}-0,178 \frac{\mathrm{kN}}{\mathrm{kN}} \cdot F+18,3 \frac{\mathrm{N}}{\mathrm{K}} \cdot \Delta T \\
& N_{g e s}\left(d_{p}=10,1 \mathrm{~mm}\right)=-0,909 \frac{\mathrm{kN}}{\mathrm{kN}} \cdot P_{0}-0,270 \frac{\mathrm{kN}}{\mathrm{kN}} \cdot F+27,7 \frac{\mathrm{N}}{\mathrm{K}} \cdot \Delta T
\end{aligned}
$$


Dabei wird die Überzählige $X_{1}$ als Änderung der Seilkraft infolge der innerlich statischen Unbestimmtheit interpretiert, sodass sich die Seilkraft nach dem Vorspannen ergibt:

$$
\begin{aligned}
N_{p . g e s} & =P_{0}+X_{1} \\
& =\left(1+x_{1 . p}\right) \cdot P_{0}+x_{1 . F} \cdot F+x_{1 . T} \cdot \Delta T
\end{aligned}
$$

$$
\begin{aligned}
& N_{\text {p.ges }}\left(d_{p}=5,0 \mathrm{~mm}\right)=0,980 \frac{\mathrm{kN}}{\mathrm{kN}} \cdot P_{0}+0,057 \frac{\mathrm{kN}}{\mathrm{kN}} \cdot F-13,5 \frac{\mathrm{N}}{\mathrm{K}} \cdot \Delta T \\
& N_{\text {p.ges }}\left(d_{p}=6,1 \mathrm{~mm}\right)=0,971 \frac{\mathrm{kN}}{\mathrm{kN}} \cdot P_{0}+0,083 \frac{\mathrm{kN}}{\mathrm{kN}} \cdot F-19,8 \frac{\mathrm{N}}{\mathrm{K}} \cdot \Delta T \\
& N_{\text {p.ges }}\left(d_{p}=8,1 \mathrm{~mm}\right)=0,939 \frac{\mathrm{kN}}{\mathrm{kN}} \cdot P_{0}+0,178 \frac{\mathrm{kN}}{\mathrm{kN}} \cdot F-18,3 \frac{\mathrm{N}}{\mathrm{K}} \cdot \Delta T \\
& N_{p . \text { ges }}\left(d_{p}=10,1 \mathrm{~mm}\right)=0,909 \frac{\mathrm{kN}}{\mathrm{kN}} \cdot P_{0}+0,270 \frac{\mathrm{kN}}{\mathrm{kN}} \cdot F-27,7 \frac{\mathrm{N}}{\mathrm{K}} \cdot \Delta T
\end{aligned}
$$

Darin reduziert der stets negative Summand $x_{1 . p}$ die Seilkraft in Abhängigkeit der gewählten Vorspannkraft $\left(N_{\text {p.ges }}(F=0\right.$ und $\Delta T=0)$ ) sodass er als sofortiger elastischer Seilkraftverlust interpretierbar ist. Während der experimentellen Studie wird die Seilkraft unmittelbar gemessen und der theoretische Wechsel zwischen zwei unterschiedlichen Tragsystemen ${ }^{426}$ verläuft stattdessen kontinuierlich. Dies bedeutet, dass für das rechnerische Ergebnis die Seilkraft $\mathrm{N}_{\mathrm{p}}(\mathrm{t}=0)$ zuzüglich des zu erwartenden sofortigen Verlustes nach Tafel 36 mit dem experimentellen Ergebnis verglichen wird. ${ }^{427}$

426 Vergleiche Bild 35, Seite 69.

427 Die hier ermittelten Werte der Vorspannkraft sind gleichzeitig neue Eingangswerte für ihre Berechnung, sodass eine mehrschrittige Iteration nötig wird. Die hier abgedruckten Ergebnisse ohne Iteration unterscheiden sich jedoch weniger als $1 \%$ vom verfeinerten Ergebnis. 
Tafel 36

Seilvorspannung zuzüglich sofortiger Verluste für den Vergleich zwischen experimentellem und rechnerischem Ergebnis.

\begin{tabular}{|c|c|c|c|c|}
\hline $\mathbf{P}_{\mathrm{m} 0}$ & $3 \mathrm{kN}$ & $6 \mathrm{kN}$ & 9 kN & $15 \mathrm{kN}$ \\
\hline $5,0 \mathrm{~mm}$ & $3,06 \mathrm{kN}$ & $6,12 \mathrm{kN}$ & $9,18 \mathrm{kN}$ & $15,30 \mathrm{kN}$ \\
\hline $6,1 \mathrm{~mm}$ & $3,09 \mathrm{kN}$ & $6,17 \mathrm{kN}$ & $9,26 \mathrm{kN}$ & $15,44 \mathrm{kN}$ \\
\hline $8,1 \mathrm{~mm}$ & $3,18 \mathrm{kN}$ & $6,37 \mathrm{kN}$ & $9,55 \mathrm{kN}$ & $15,92 \mathrm{kN}$ \\
\hline $10,1 \mathrm{~mm}$ & $3,27 \mathrm{kN}$ & $6,55 \mathrm{kN}$ & $9,82 \mathrm{kN}$ & $16,37 \mathrm{kN}$ \\
\hline
\end{tabular}

\section{Biegespannung in Feldmitte}

Die Biegespannung in Feldmitte ergibt sich aus den Schnittgrößen im Allgemeinen und für die Glasunterkante (UK) in Abhängigkeit vom Bewehrungsdurchmesser:

$$
\begin{aligned}
& \sigma_{\text {ges }}=\frac{N_{\text {ges }}\left(x_{l l}=\frac{L}{6}\right)}{A_{G}} \pm \frac{M_{\text {ges }}\left(x_{l l}=\frac{L}{6}\right)}{W_{y}} \\
& \sigma_{\text {ges.UK }}\left(d_{p}=5,0 \mathrm{~mm}\right)=-0,108 \frac{1}{\mathrm{~cm}^{2}} \cdot P_{0}+0,364 \frac{1}{\mathrm{~cm}^{2}} \cdot F+1,50 \frac{\mathrm{N}}{\mathrm{K} \cdot \mathrm{cm}^{2}} \cdot \Delta T \\
& \sigma_{\text {ges.UK }}\left(d_{p}=6,1 \mathrm{~mm}\right)=-0,107 \frac{1}{\mathrm{~cm}^{2}} \cdot P_{0}+0,361 \frac{1}{\mathrm{~cm}^{2}} \cdot F+2,19 \frac{\mathrm{N}}{\mathrm{K} \cdot \mathrm{cm}^{2}} \cdot \Delta T \\
& \sigma_{\text {ges.UK }}\left(d_{p}=8,1 \mathrm{~mm}\right)=-0,104 \frac{1}{\mathrm{~cm}^{2}} \cdot P_{0}+0,351 \frac{1}{\mathrm{~cm}^{2}} \cdot F+2,02 \frac{\mathrm{N}}{\mathrm{K} \cdot \mathrm{cm}^{2}} \cdot \Delta T \\
& \sigma_{\text {ges.UK }}\left(d_{p}=10,1 \mathrm{~mm}\right)=-0,100 \frac{1}{\mathrm{~cm}^{2}} \cdot P_{0}+0,340 \frac{1}{\mathrm{~cm}^{2}} \cdot F+3,07 \frac{\mathrm{N}}{\mathrm{K} \cdot \mathrm{cm}^{2}} \cdot \Delta T
\end{aligned}
$$

Gleichung 104

Gleichung 105

\section{Durchbiegung in Feldmitte}

Die Durchbiegung in Feldmitte ist nach linearer Biegetheorie I. Ordnung nicht von der Normalkraft abhängig, sodass 
die Lösung aus gängigen Tafelwerten mit bekannten Belastungsgliedern ermittelt wird:

$$
w_{z . g e s}=\frac{3 \cdot L_{\text {ges }}^{3}}{76,8 \cdot E_{G} \cdot l_{y}} \cdot\left(\frac{F}{2}-\sin (\alpha) \cdot\left(P_{0}+X_{1}\right)\right) \cdot\left(1-\frac{0,8}{3^{2}}-\frac{0,2}{3^{4}}\right)
$$

Gleichung 106

$$
\begin{aligned}
& w_{z . \text { ges }}\left(d_{p}=5,0 \mathrm{~mm}\right)=-0,066 \frac{\mathrm{mm}}{\mathrm{kN}} \cdot P_{0}+0,297 \frac{\mathrm{mm}}{\mathrm{kN}} \cdot F+0,091 \frac{\mathrm{mm}}{100 \cdot \mathrm{K}} \cdot \Delta T \\
& w_{z . \text { ges }}\left(d_{p}=6,1 \mathrm{~mm}\right)=-0,065 \frac{\mathrm{mm}}{\mathrm{kN}} \cdot P_{0}+0,295 \frac{\mathrm{mm}}{\mathrm{kN}} \cdot F+0,133 \frac{\mathrm{mm}}{100 \cdot \mathrm{K}} \cdot \Delta T \\
& w_{z . \text { ges }}\left(d_{p}=8,1 \mathrm{~mm}\right)=-0,063 \frac{\mathrm{mm}}{\mathrm{kN}} \cdot P_{0}+0,289 \frac{\mathrm{mm}}{\mathrm{kN}} \cdot F+0,123 \frac{\mathrm{mm}}{100 \cdot \mathrm{K}} \cdot \Delta T \\
& w_{z . \text { ges }}\left(d_{p}=10,1 \mathrm{~mm}\right)=-0,061 \frac{\mathrm{mm}}{\mathrm{kN}} \cdot P_{0}+0,282 \frac{\mathrm{mm}}{\mathrm{kN}} \cdot F+0,186 \frac{\mathrm{mm}}{100 \cdot \mathrm{K}} \cdot \Delta T
\end{aligned}
$$

Die berechneten Faktoren in Gleichung 107 machen deutlich, dass die Durchbiegung im Wesentlichen durch die Biegelast und in geringerem Maße durch die Vorspannung beeinflusst wird. Beide Anteile wirken entgegengesetzt. Dabei muss die Vorspannkraft ungefähr den dreifachen Wert der Biegelast erreichen, um diese vollständig auszugleichen. Die Temperaturänderung hat im relevanten Temperaturbereich demgegenüber keinen maßgebenden Einfluss auf die vertikale Durchbiegung. 


\section{Erwartungswerte im Kurzzeit-Biegeversuch (BV)}

\section{Belastungsgeschwindigkeit}

Die Belastungsgeschwindigkeit wird ohne die Wirkung des statisch unbestimmten Anteils des Seil berechnet, um für alle Prüfkörper - Referenzen und vorgespannte Träger ${ }^{428}$ - eine einheitliche Basis zu bieten:

Gleichung 108

$$
\begin{aligned}
& \frac{d}{d t} F=\frac{d}{d t} \sigma \cdot \frac{2 \cdot B \cdot H^{2}}{3 \cdot\left(L_{S}-L_{b}\right)}=\frac{2 \cdot 2 \cdot 4 \cdot 6 \cdot 150^{2}}{3 \cdot(2000-666)} \cdot \frac{\mathrm{N}}{\mathrm{s}}=540 \frac{\mathrm{N}}{\mathrm{s}} \\
& d F / d t \quad \text { Lastrate } \\
& d \sigma / d t \quad \text { Spannungsrate } \\
& L_{S} \quad \text { Spannweite (Bild 45, Seite 108) } \\
& L_{b} \quad \text { Traversenbreite (Bild 45, Seite 108) }
\end{aligned}
$$

Daraus ergibt sich die Traversengeschwindigkeit bezüglich der Trägermitte:

$$
\begin{aligned}
\frac{d}{d t} w_{z . T} & =\frac{3 \cdot L_{G}^{3}}{2 \cdot 76,8 \cdot E_{G} \cdot I_{y, G}} \cdot \frac{d}{d t} F \cdot\left(1-\frac{0,8}{3^{2}}-\frac{0,2}{3^{4}}\right) \\
& =\frac{3 \cdot 2000^{3} \cdot 12}{2 \cdot 76,8 \cdot 70000 \cdot 4 \cdot 6 \cdot 150^{3}} \cdot 540 \cdot \frac{368}{405} \frac{\mathrm{mm}}{\mathrm{s}} \\
& =0,16 \frac{\mathrm{mm}}{\mathrm{s}} \\
\frac{d}{d t} w_{z . T} & =0,19 \frac{\mathrm{mm}}{\mathrm{s}}(+15 \%) \\
d w_{z . T} / d t & \text { Traversen-Belastungsgeschwindigkeit }
\end{aligned}
$$

Im Prüfaufbau wirkt diese Traversengeschwindigkeit an den Lasteinleitungspunkten. In der Feldmitte ist die Durchbiegung demgegnüber nach linearer Balkentheorie $8 \%\left(=\frac{69}{64}\right)$ größer. Zudem wird ein noch unbekannter Anteil der Gesamtlast zu einer Stauchung der Auflagerklotzung führen

${ }^{428}$ Mit Querschnittswerten nach Bild 42, Seite 104. 
und die verbleibende Durchbiegungsgeschwindigkeit und damit die Spannungsrate im Glas reduzieren. Daher wird die Traversengeschwindigkeit zusätzlich um einen gewählten Wert von $15 \%$ vergrößert. Die tatsächliche Spannungsrate wird aufgezeichnet und ausgewertet.

Dabei ergeben sich für bewehrte Träger aufgrund der Wirkung des Seils, unabhängig vom Wert der Vorspannkraft und bei gleichbleibender Traversengeschwindigkeit nach Gleichung 109, folgende Biegekraft- (Gleichung 106) und Biegespannungsänderung (Gleichung 104) sowie Biegesteifigkeit.

\begin{tabular}{lllll}
\hline $\begin{array}{l}\mathbf{d}_{\mathbf{p}} \\
{[\mathbf{m m}]}\end{array}$ & $\begin{array}{l}\mathbf{d w} \mathbf{z} / \mathbf{d t} \\
{[\mathbf{m m} / \mathbf{s}]}\end{array}$ & $\begin{array}{l}\mathbf{d F} / \mathbf{d t} \\
{[\mathbf{N} / \mathbf{s}]}\end{array}$ & $\begin{array}{l}\mathbf{d} \boldsymbol{\sigma} / \mathbf{d t} \\
{\left[\mathbf{N} /\left(\mathbf{m m}^{2} \cdot \mathbf{s}\right)\right]}\end{array}$ & $\begin{array}{l}\mathbf{d F} / \mathbf{d w} \mathbf{w}_{\mathbf{z}} \\
{[\mathbf{k N} / \mathbf{m m}]}\end{array}$ \\
\hline \multirow{2}{*}{ unbewehrt } & 0,16 & 540 & 2,00 & 3,33 \\
\multirow{2}{*}{5,0} & 0,19 & 621 & 2,30 & 3,33 \\
6,1 & 0,19 & 630 & 2,29 & 3,37 \\
8,1 & 0,19 & 634 & 2,29 & 3,39 \\
10,1 & 0,19 & 648 & 2,27 & 3,47 \\
\hline
\end{tabular}

Tafel 37

Erwartete Biegekraft- und Biegespannungsänderung infolge einer gleichbleibenden weggesteuerten Belastung.

$\Delta T=0$.

Die Abweichungen der Spannungsrate liegen im Zielbereich von 2,0 $\pm 0,4 \mathrm{~N} /\left(\mathrm{mm}^{2} \cdot \mathrm{s}\right)$ nach [DIN EN 1288-3], sodass die Annahme zur Belastungsgeschwindigkeit als nutzbar beurteilt wird.

\section{Spannweg im Vorspannversuch}

Der Spannweg $w_{P}$ ergibt sich aus der Seillängung sowie der Stauchung der Vorspannklotzung und des Verbundglases. Im Vergleich zur Längung des Seils ist die Stauchung des Verbundglases und der Vorspannklotzungen dieser Konstruktion klein, wie sich am Beispiel eines Spannglasträgers 
dieser Arbeit mit 8,1 mm Seildurchmesser unter Einheitslast zeigt:

Gleichung 110

$$
\begin{aligned}
& \frac{P \cdot L_{p}}{E_{p} \cdot A_{p}}>\frac{2 \cdot P \cdot L_{K}}{4 \cdot E_{K} \cdot A_{K}} \text { und } \frac{P \cdot d_{Z}}{2 \cdot G_{Z} \cdot A_{Z}} \\
& \frac{1 \cdot 2000 \mathrm{~mm}}{160000 \frac{\mathrm{N}}{\mathrm{mm}^{2}} \cdot 39 \mathrm{~mm}^{2}}=\frac{1}{3} \cdot \frac{\mathrm{mm}}{\mathrm{kN}} \\
& \frac{2 \cdot 1 \cdot 8 \mathrm{~mm}}{4 \cdot 4356 \frac{\mathrm{N}}{\mathrm{mm}^{2}} \cdot 900 \mathrm{~mm}^{2}}=\frac{1}{980} \cdot \frac{\mathrm{mm}}{\mathrm{kN}} \\
& \frac{1 \cdot 0,76 \mathrm{~mm}}{2 \cdot 0,86 \frac{\mathrm{N}}{\mathrm{mm}^{2}} \cdot 0,30 \mathrm{~m}^{2}}=\frac{1}{679} \cdot \frac{\mathrm{mm}}{\mathrm{kN}}
\end{aligned}
$$

Unter Vernachlässigung der Anteile aus Klotzung und Verbundglas kann die Relation zwischen Seilkraft und Spannweg $w_{P}$ wie folgt angegeben werden:

Gleichung 111

$$
\begin{array}{ll}
N_{p}\left(w_{P}\right) & =\frac{w_{P}}{L_{p}} \cdot E_{p} \cdot A_{p} \\
N_{p}\left(d_{p}=5,0 \mathrm{~mm}\right) & =0,98 \frac{\mathrm{kN}}{\mathrm{mm}} \\
N_{p}\left(d_{p}=6,1 \mathrm{~mm}\right) & =1,43 \frac{\mathrm{kN}}{\mathrm{mm}} \\
N_{p}\left(d_{p}=8,1 \mathrm{~mm}\right) & =3,12 \frac{\mathrm{kN}}{\mathrm{mm}} \\
N_{p}\left(d_{p}=10,1 \mathrm{~mm}\right) & =4,80 \frac{\mathrm{kN}}{\mathrm{mm}}
\end{array}
$$

\section{Biegeversuch}

Im weggesteuerten Biegeversuch resultiert eine Biegelast aus dem Traversenweg. Dieser wird als Biegesteifigkeit der Konstruktion $\mathrm{dF} / \mathrm{dw}_{\mathrm{z}}$ nach Tafel 37 angegeben. 
Die Seile werden infolge der Biegebelastung gestreckt, was zu einer Steigerung der Seilkraft mit Vergrößerung des Biegeweges führt. Diese Belastungsgeschwindigkeit des Seils ergibt sich aus der Seilkraftänderung nach Gleichung 102 unter der Steifigkeit $\mathrm{dF} / \mathrm{dw}_{\mathrm{z}}$ nach Tafel 37 bei konstanter Temperatur $(\Delta T=0) z u$ :

$$
\begin{aligned}
\frac{d N_{p}}{d w_{z . T}} & =N_{p . g e s}\left(P_{0}, F=\frac{d F}{d w_{z . T}}\right)-N_{p . g e s}\left(P_{0}, F=0\right) \\
& =\left(1+x_{1 . p}\right) \cdot P_{0}+x_{1 . F} \cdot 0 \\
& -\left(1+x_{1 . p}\right) \cdot P_{0}+x_{1 . F} \cdot \frac{d F}{d w_{z . T}} \\
& =x_{1 . F} \frac{d F}{d w_{z . T}}
\end{aligned}
$$

Sie ist unabhängig von der Vorspannkraft, jedoch durch die gewählten Seileigenschaften bestimmt:

$$
\begin{aligned}
& \frac{d N_{p}}{d w_{z . T}}\left(d_{p}=5,0 \mathrm{~mm}\right)=0,057 \frac{\mathrm{kN}}{\mathrm{kN}} \cdot 3,37 \frac{\mathrm{kN}}{\mathrm{mm}}=0,19 \frac{\mathrm{kN}}{\mathrm{mm}} \\
& \frac{d N_{p}}{d w_{z . T}}\left(d_{p}=6,1 \mathrm{~mm}\right)=0,083 \frac{\mathrm{kN}}{\mathrm{kN}} \cdot 3,39 \frac{\mathrm{kN}}{\mathrm{mm}}=0,28 \frac{\mathrm{kN}}{\mathrm{mm}} \\
& \frac{d N_{p}}{d w_{z . T}}\left(d_{p}=8,1 \mathrm{~mm}\right)=0,178 \frac{\mathrm{kN}}{\mathrm{kN}} \cdot 3,47 \frac{\mathrm{kN}}{\mathrm{mm}}=0,62 \frac{\mathrm{kN}}{\mathrm{mm}} \\
& \frac{d N_{p}}{d w_{z . T}}\left(d_{p}=10,1 \mathrm{~mm}\right)=0,270 \frac{\mathrm{kN}}{\mathrm{kN}} \cdot 3,54 \frac{\mathrm{kN}}{\mathrm{mm}}=0,96 \frac{\mathrm{kN}}{\mathrm{mm}}
\end{aligned}
$$

Damit werden die weggesteuerten experimentellen Ergebnisse mit dem analytischen Modell verknüpft. 


\section{Erwartungswerte im Dauerversuch (DV)}

Die Seilkraftverluste des Dauerversuchs sind nach Gleichung 37 (Seite 89) in Tafel 38 in Abhängigkeit der Größenordnung der Biegebelastung zusammengefasst.

\section{Tafel 38}

Seilkraftverluste $\Delta \mathrm{P}$ nach $1000 \mathrm{~h}$ Versuchsdauer nach Gleichung 37 (Seite 89).

Mit $\varphi_{\mathrm{G}}=0$ und $\Delta \mathrm{T}=0$.
Bild 121

Die Durchbiegung des Laminates (links) entspricht der Durchbiegung eines monolithischen Trägers effektiver Dicke (rechts).

Gleichung 114

\begin{tabular}{llll}
\hline $\mathbf{d}_{\mathbf{p}}$ & $\mathbf{P}_{\mathbf{m} \mathbf{0}}$ & $\mathbf{F}=\mathbf{0} \mathbf{k N}$ & $\mathbf{F}=\mathbf{1 0} \mathbf{~ N N}$ \\
\hline $5,0 \mathrm{~mm}$ & $9,0 \mathrm{kN}$ & $0,11 \mathrm{kN}$ & $0,12 \mathrm{kN}$ \\
$8,1 \mathrm{~mm}$ & $15,0 \mathrm{kN}$ & $0,59 \mathrm{kN}$ & $0,66 \mathrm{kN}$ \\
$10,1 \mathrm{~mm}$ & $15,0 \mathrm{kN}$ & $0,90 \mathrm{kN}$ & $1,06 \mathrm{kN}$ \\
\hline
\end{tabular}

Darüber hinaus wird die seitliche Durchbiegung eines Rechteckquerschnitts mit schubweicher Zwischenschicht für die numerischen Untersuchungen in Kapitel 4 berechnet. Werden zwei Umlenkpunkte verwendet, so wird die Biegung um die schwache Laminatachse als Vierpunkt-Biegung beschreibbar. Es wird daher jene Dicke eines monolithischen Biegebalkens gesucht, welcher sich genauso weit durchbiegt, wie der zugehörige Laminatquerschnitt ${ }^{429}$ (Bild 121). Dabei werden beide Durchbiegungen analytisch nach Gleichung 114 und Gleichung 115 berechnet. Das Umstellen nach der effektiven Dicke liefert den Zusammenhang mit dem zeit- und temperaturabhängigen Schubmodul der Zwischenschicht nach Gleichung 115.

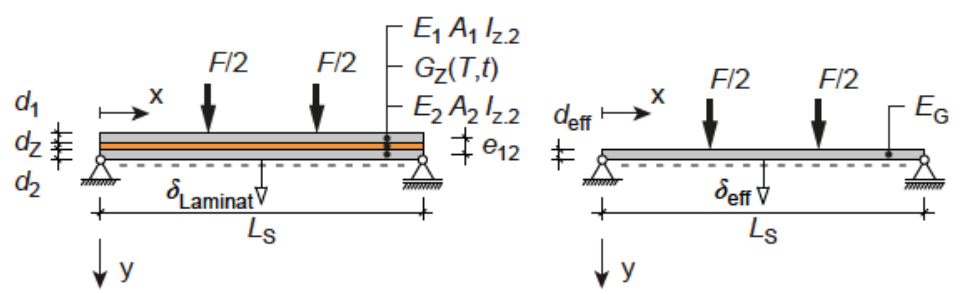

$$
\delta=\frac{23 \cdot F \cdot L_{S}^{3}}{108 \cdot E_{G} \cdot H \cdot d_{e f f}^{3}}
$$

$\delta$

\footnotetext{
${ }^{429}$ Vergleiche [Schuler 2003] und [Ensslen 2005] für die Anwendung auf Verbundglas.
} 
$\delta\left(G_{Z}(T, t)\right)=\frac{\left(1+9,6 \cdot \frac{B_{V}}{S_{Q}\left(G_{Z}(T, t)\right) \cdot L_{S}}\right) \cdot M_{Z}\left(G_{Z}(T, t)\right) \cdot L_{S}^{2}}{24 \cdot B_{V}} \cdot\left(3-4 \cdot \beta^{2}\right)$

$\begin{array}{ll}B_{V} & \text { Biegesteifigkeit des Laminats } \\ S_{Q} & \text { Schubsteifigkeit des Laminats } \\ M_{Z} & \text { Biegemomentenanteil der Zwischen- } \\ \beta & \text { Schicht } \\ \beta & \text { Lastangriffspunkt, hier } \beta=1 / 3\end{array}$

Gleichung 115

$B_{V}=\frac{E_{1} \cdot A_{1} \cdot E_{2} \cdot A_{2} \cdot e_{12}^{2}}{E_{1} \cdot A_{1}+E_{1} \cdot A_{1}}=\frac{E_{G}^{2} \cdot A_{G}^{2} \cdot e_{12}^{2}}{2 \cdot E_{G} \cdot A_{G}}$

Gleichung 116

$B_{V}$

Biegesteifigkeit des Laminats

$B_{D}=E_{1} \cdot I_{z .1}+E_{2} \cdot I_{z .2}=2 \cdot E_{G} \cdot I_{z . G}$

Gleichung 117

$B_{D} \quad$ Biegesteifigkeit der gläsernen Deckschichten

$S_{Q}\left(G_{Z}(T, t)\right)=\frac{G_{Z}(T, t) \cdot e_{12}^{2} \cdot H}{d_{z}}$

Gleichung 118

$S_{Q} \quad$ Schubsteifigkeit des Laminats

$k\left(G_{Z}(T, t)\right)=\frac{24}{3-4 \cdot \beta^{2}} \cdot \frac{B_{V}}{S_{Q}\left(G_{Z}(T, t)\right) \cdot L_{S}^{2}}$

Gleichung 119

k

Beiwert 


$$
\begin{gathered}
M_{Z}\left(G_{Z}(T, t)\right)=\left(1-\frac{B_{D}}{B_{D}+\frac{B_{V}}{1+k\left(G_{Z}(T, t)\right)}}\right) \cdot \frac{F \cdot L_{S}}{6} \\
M_{Z} \begin{array}{l}
\text { Biegemomentenanteil der Zwischen- } \\
\text { schicht }
\end{array}
\end{gathered}
$$

Damit wir die effektive Glasdicke in Abhängigkeit der Belastungszeit angegeben (Tafel 39).

Tafel 39

Effektive Glasdicken und Biegesteifigkeit um die schwache Achse in Abhängigkeit zur Belastungszeit mit $\mathrm{G}_{\mathrm{Z}}\left(\mathrm{t}, \mathrm{T}=23^{\circ} \mathrm{C}\right)$ nach Gleichung 5.

\begin{tabular}{llll}
\hline $\mathbf{t}$ & $\mathbf{G}_{\mathbf{z}}$ & $\mathbf{d}_{\text {eff }}$ & $\mathbf{I}_{\mathbf{z . e f f}}$ \\
{$[-]$} & {$\left[\mathbf{N} / \mathbf{m m}^{\mathbf{2}}\right]$} & $\begin{array}{l}\mathbf{m m}] \\
{\left[\mathbf{1 0} \mathbf{~ m m}^{\mathbf{4}}\right]}\end{array}$ \\
\hline 0 (mit Verbund) & $\infty$ & 12,00 & $21,60(100 \%)$ \\
$1 \mathrm{~s}$ & 0,616 & 10,71 & $15,34(71 \%)$ \\
$1 \mathrm{~min}$ & 0,473 & 10,46 & $14,30(66 \%)$ \\
$1 \mathrm{~h}$ & 0,330 & 10,09 & $12,84(59 \%)$ \\
$50 \mathrm{~h}$ & 0,194 & 9,52 & $10,77(50 \%)$ \\
$100 \mathrm{~h}$ & 0,170 & 9,37 & $10,30(48 \%)$ \\
$250 \mathrm{~h}$ & 0,138 & 9,16 & $9,60(44 \%)$ \\
$500 \mathrm{~h}$ & 0,114 & 8,97 & $9,02(42 \%)$ \\
$750 \mathrm{~h}$ & 0,100 & 8,84 & $8,65(40 \%)$ \\
$1000 \mathrm{~h}$ & 0,090 & 8,75 & $8,37(39 \%)$ \\
ohne Verbund & 0 & 7,56 & $5,40(25 \%)$ \\
\hline
\end{tabular}

Darüber hinaus wird die zeitabhängige Wirkung auf die Zwischenenschicht und die Klotzung im numerischen Modell durch eine reduzierte Seilsteifigkeit nach Gleichung 121 ausgedrückt. Tafel 40 gibt die Einzelwerte, getrennt nach gemeinsamer $\left(\eta_{p}(K Z)\right)$ sowie alleiniger zeitabhängiger Wirkung von Klotzung $\left(\eta_{p}(K)\right.$ für $\left.E_{p \text {.eff }}\left(\Delta P_{Z, c}=0\right)\right)$ und Zwischenschicht $\left(\eta_{p}(Z)\right.$ für $\left.E_{p . e f f}\left(\Delta P_{K, c}=0\right)\right)$, an.

$$
\begin{aligned}
& E_{p . e f f}=\frac{P_{c}-\Delta P_{K, c}-\Delta P_{Z, c}}{P_{c}} \cdot E_{p}=\eta_{p} \cdot E_{p} \\
& E_{p . \text { eff }} \quad \text { effektive Seilsteifigkeit } \\
& \triangle P_{K, c} \quad \text { Seilkraftverlust aus Kriechen Klotzung } \\
& \Delta P_{Z, c} \quad \text { Seilkraftverlust aus Kriechen Zwi- } \\
& \eta_{p} \quad \begin{array}{l}
\text { schenschicht (Gleichung } 26 \\
\text { rechnerischer Verlustfaktor }
\end{array}
\end{aligned}
$$




\begin{tabular}{|c|c|c|c|c|c|c|c|c|c|}
\hline \multirow[t]{2}{*}{$\begin{array}{l}\mathbf{t} \\
{[-]}\end{array}$} & \multicolumn{3}{|c|}{$\begin{array}{l}P_{\mathbf{m} 0}=9,0 \mathbf{k N} \\
d_{p}=5,0 \mathrm{~mm}\end{array}$} & \multicolumn{3}{|c|}{$\begin{array}{l}\mathbf{P}_{\mathrm{m} 0}=15, \mathbf{0 k N} \\
\mathrm{d}_{p}=8,1 \mathrm{~mm}\end{array}$} & \multicolumn{3}{|c|}{$d_{p}=10,1 \mathrm{~mm}$} \\
\hline & $\eta_{p}(K Z)$ & $\eta_{p}(K)$ & $\eta_{p}(Z)$ & $\eta_{p}(K Z)$ & $\eta_{p}(K)$ & $\eta_{p}(Z)$ & $\eta_{p}(K Z)$ & $\eta_{p}(K)$ & $\eta_{p}(Z)$ \\
\hline 0 & $100,0 \%$ & $100,0 \%$ & $100,0 \%$ & $100,0 \%$ & $100,0 \%$ & $100,0 \%$ & $100,0 \%$ & $100,0 \%$ & $100,0 \%$ \\
\hline $1 \mathrm{~s}$ & $100,0 \%$ & $100,0 \%$ & $100,0 \%$ & $99,9 \%$ & $100,0 \%$ & $100,0 \%$ & $99,8 \%$ & $99,9 \%$ & $99,8 \%$ \\
\hline $1 \mathrm{~min}$ & $99,9 \%$ & $100,0 \%$ & $99,9 \%$ & $99,7 \%$ & $100,0 \%$ & $100,0 \%$ & $99,6 \%$ & $99,7 \%$ & $99,6 \%$ \\
\hline $1 \mathrm{~h}$ & $99,8 \%$ & $100,0 \%$ & $99,8 \%$ & $99,3 \%$ & $99,9 \%$ & $99,8 \%$ & $99,0 \%$ & $99,4 \%$ & $99,2 \%$ \\
\hline $50 \mathrm{~h}$ & $99,5 \%$ & $99,9 \%$ & $99,6 \%$ & $98,3 \%$ & $99,6 \%$ & $99,3 \%$ & $97,4 \%$ & $98,8 \%$ & $98,1 \%$ \\
\hline $100 \mathrm{~h}$ & $99,4 \%$ & $99,8 \%$ & $99,5 \%$ & $98,0 \%$ & $99,5 \%$ & $99,2 \%$ & $97,0 \%$ & $98,5 \%$ & $97,7 \%$ \\
\hline $250 \mathrm{~h}$ & $99,2 \%$ & $99,8 \%$ & $99,4 \%$ & $97,5 \%$ & $99,4 \%$ & $99,1 \%$ & $96,1 \%$ & $98,1 \%$ & $97,1 \%$ \\
\hline $500 \mathrm{~h}$ & $99,0 \%$ & $99,8 \%$ & $99,2 \%$ & $96,9 \%$ & $99,3 \%$ & $99,0 \%$ & $95,3 \%$ & $97,6 \%$ & $96,3 \%$ \\
\hline $750 \mathrm{~h}$ & $98,9 \%$ & $99,8 \%$ & $99,1 \%$ & $96,5 \%$ & $99,3 \%$ & $98,9 \%$ & $94,6 \%$ & $97,2 \%$ & $95,7 \%$ \\
\hline $1000 \mathrm{~h}$ & $98,8 \%$ & $99,8 \%$ & $99,0 \%$ & $96,1 \%$ & $99,3 \%$ & $98,9 \%$ & $94,0 \%$ & $96,8 \%$ & $95,1 \%$ \\
\hline $10000 \mathrm{~h}$ & $88,8 \%$ & $99,6 \%$ & $89,2 \%$ & $64,2 \%$ & $98,7 \%$ & $98,0 \%$ & $44,9 \%$ & $65,5 \%$ & $46,9 \%$ \\
\hline
\end{tabular}

Tafel $\mathbf{4 0}$

Effektive Seilsteifigkeit $\eta_{p}$ in Abhängigkeit von der Belastungszeit mit $\mathrm{G}_{\mathrm{Z}}\left(\mathrm{t}, \mathrm{T}=23^{\circ} \mathrm{C}\right)$ nach Gleichung 5 (Seite 53) für die numerische Berechnung der Seilkraftverluste in

Während der experimentellen Bestimmung der ResttragfäKapitel 4.3.5, ab Seite 199. higkeit ändert sich die Trägersteifigkeit durch das Brechen einzelner Scheiben im Verbundglas. Dafür wird eine Steifigkeitsreduktion von $25 \%$ je gebrochener Scheibe angesetzt. Daraus resultiert eine gesteigerte Seilkraft nach Gleichung 103 in Tabelle 41.

\begin{tabular}{lcccrr}
\hline $\begin{array}{l}\mathbf{d}_{\mathbf{p}} \\
\text { [mm] }\end{array}$ & $\begin{array}{l}\mathbf{P}_{\mathbf{m} 0} \\
{[\mathbf{k N}]}\end{array}$ & $\begin{array}{l}\mathbf{l}_{\mathbf{y}} \text { und } \mathbf{A}_{\mathbf{G}} \\
100 \%\end{array}$ & \multicolumn{1}{c}{$75 \%$} & \multicolumn{1}{c}{$50 \%$} & \multicolumn{1}{c}{$25 \%$} \\
\hline 8,1 & 0 & $1,88 \mathrm{kN}$ & $2,49 \mathrm{kN}$ & $3,68 \mathrm{kN}$ & $7,03 \mathrm{kN}$ \\
5,0 & 9 & $9,60 \mathrm{kN}$ & $9,73 \mathrm{kN}$ & $10,01 \mathrm{kN}$ & $10,83 \mathrm{kN}$ \\
8,1 & 15 & $16,83 \mathrm{kN}$ & $17,14 \mathrm{kN}$ & $17,76 \mathrm{kN}$ & $19,65 \mathrm{kN}$ \\
10,1 & 15 & $17,74 \mathrm{kN}$ & $18,21 \mathrm{kN}$ & $19,16 \mathrm{kN}$ & $21,99 \mathrm{kN}$ \\
\hline
\end{tabular}

Tafel 41

Seilkräfte unter Biege- und Vorspannbelastung (Tafel 36) im Resttragversuch (RT) bei veränderlicher Biegesteifigkeit ly und Fläche des Glasquerschnitts $A_{G}$.

$\mathrm{F}=10,6 \mathrm{kN}$ und $\Delta \mathrm{T}=0$.

Im Umkehrschluss werden die gemessenen Seilkraftänderungen verwendet, um eine tatsächliche Biegesteifigkeit zu errechnen. Im Unterschied zu den hier angegebenen Ergebnissen ist die Tragwirkung der gebrochenen Schichten in der Druckzone im Versuchsergebnis bereits enthalten. Die Differenz zwischen dem analytischen und experimentellen Ergebnis wird als unberücksichtigte Tragreserve gewertet. 
Tafel 42

Reine Glasdurchbiegung unter Biege- und Vorspannbelastung (Tafel 36) im Versuch zur Ermittlung der Resttragfähigkeit (RT) bei veränderlicher Biegesteifigkeit ly und Fläche des Glasquerschnitts $A_{G}$.

$\mathrm{F}=10,6 \mathrm{kN}$ und $\Delta \mathrm{T}=0$.

\begin{tabular}{|c|c|c|c|c|c|}
\hline $\begin{array}{l}d_{p} \\
{[\mathrm{~mm}]}\end{array}$ & $\begin{array}{l}P_{\mathrm{mo}} \\
{[\mathrm{kN}]}\end{array}$ & $\begin{array}{l}\mathbf{I}_{\mathbf{y}} \text { und } \mathbf{A}_{\mathbf{G}} \\
100 \%\end{array}$ & $75 \%$ & $50 \%$ & $25 \%$ \\
\hline \multicolumn{2}{|c|}{ unbewehrt } & $3,18 \mathrm{~mm}$ & $4,25 \mathrm{~mm}$ & $6,37 \mathrm{~mm}$ & $12,74 \mathrm{~mm}$ \\
\hline 8,1 & 0 & $3,06 \mathrm{~mm}$ & $4,02 \mathrm{~mm}$ & $5,88 \mathrm{~mm}$ & $10,85 \mathrm{~mm}$ \\
\hline 5,0 & 9 & $2,54 \mathrm{~mm}$ & $3,38 \mathrm{~mm}$ & $5,02 \mathrm{~mm}$ & $9,83 \mathrm{~mm}$ \\
\hline 8,1 & 15 & $2,05 \mathrm{~mm}$ & $2,71 \mathrm{~mm}$ & $3,98 \mathrm{~mm}$ & $7,46 \mathrm{~mm}$ \\
\hline 10,1 & 15 & $1,99 \mathrm{~mm}$ & $2,62 \mathrm{~mm}$ & $3,80 \mathrm{~mm}$ & $6,83 \mathrm{~mm}$ \\
\hline
\end{tabular}

\section{Erwartungswerte im Temperaturversuch (TV)}

Letztlich wird der erwartete Seilkraftverlust bei einer Temperaturänderung ermittelt. Hierbei kommt in allen Versuchen ein Seil des Durchmessers 8,1 mm nach Tafel 9 zum Einsatz.

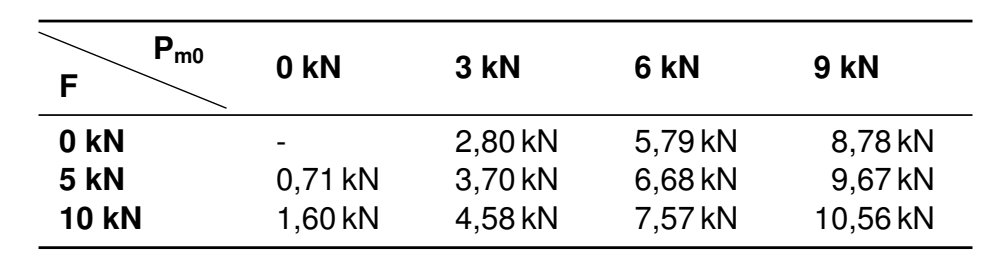

Tafel 43

Seilkraft nach einer Temperaturänderung von $10 \mathrm{~K}$.

Rechnerische Vorspannbelastung nach Tafel 36 (Seite 308). 


\section{B Kurzzeit-Biegeversuche}

In dieser Anlage werden die Ergebnisse der Kurzzeit-Biegeversuche gemäß Kapitel 3.2 dokumentiert.

Die folgende Tabelle gibt einen Überblick. Darauf folgend wird zunächst das Vorspannen jeder Trägergruppe dargestellt.

Anschließend wird das Tragverhalten jedes einzelnen Prüfkörpers bis zum Bruch wieder-

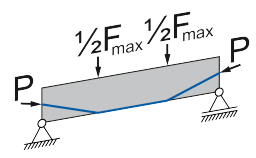
gegeben und das Versagen charakterisiert.

Als wesentliches Ergebnis ergibt sich die Bruchlast in Abhängigkeit der Belastung aus Vorspannung des Seils und Biege belastung im Vierpunkt-Biegeversuch.

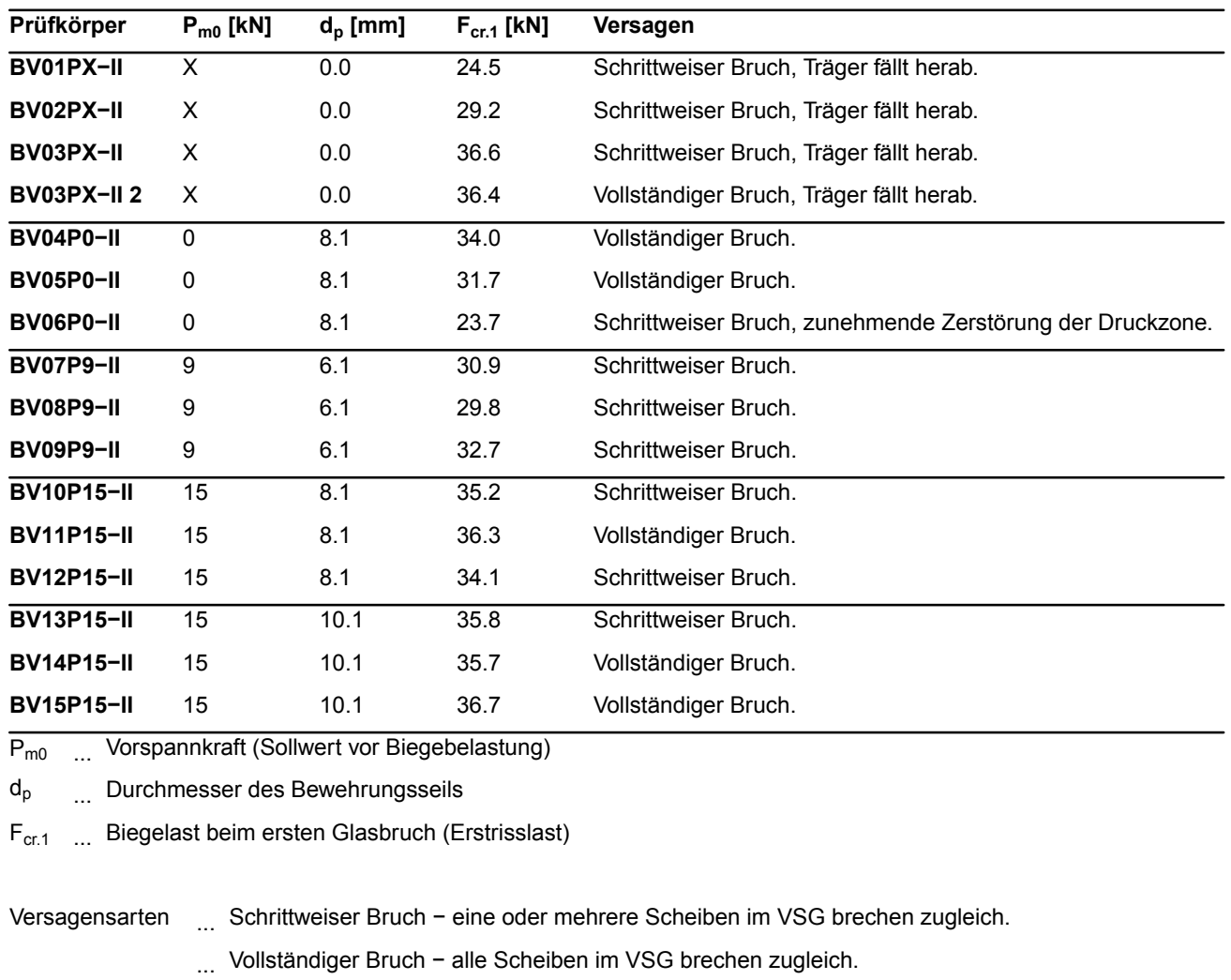




\section{Bruchversuch Referenzträger unbewehrt}

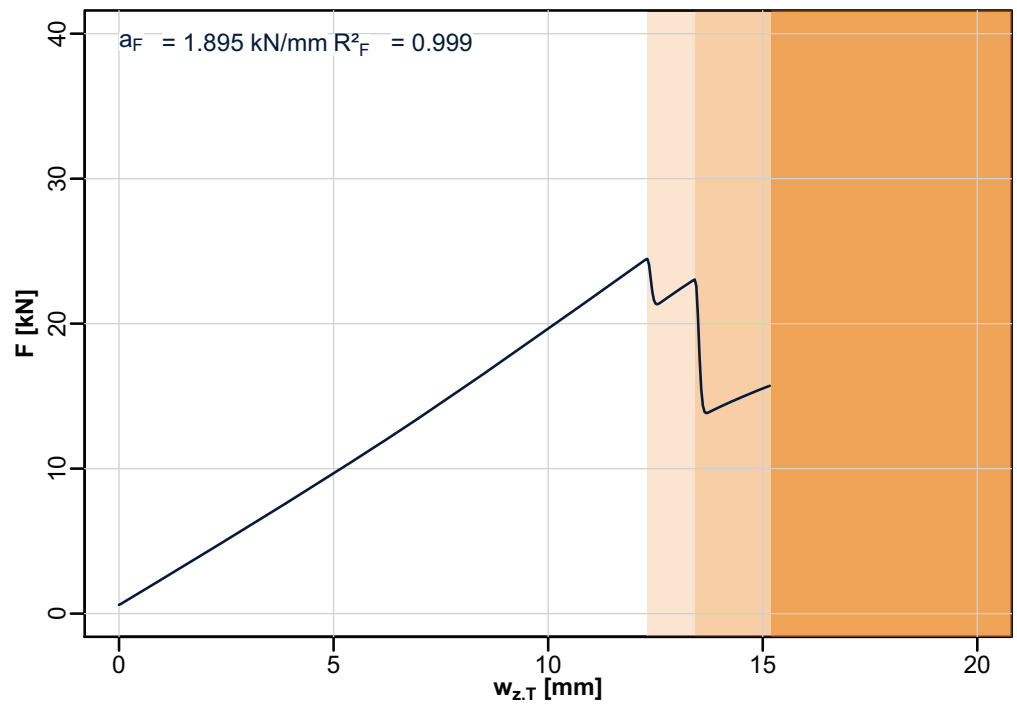

Kraftänderung

BV01PX-II

- Prüfkraft $F$

$\square \quad$ ungebrochen

$\square \quad$ Teilbruch

$\square \quad$ Versagen Schrittweiser Bruch Träger fällt herab.
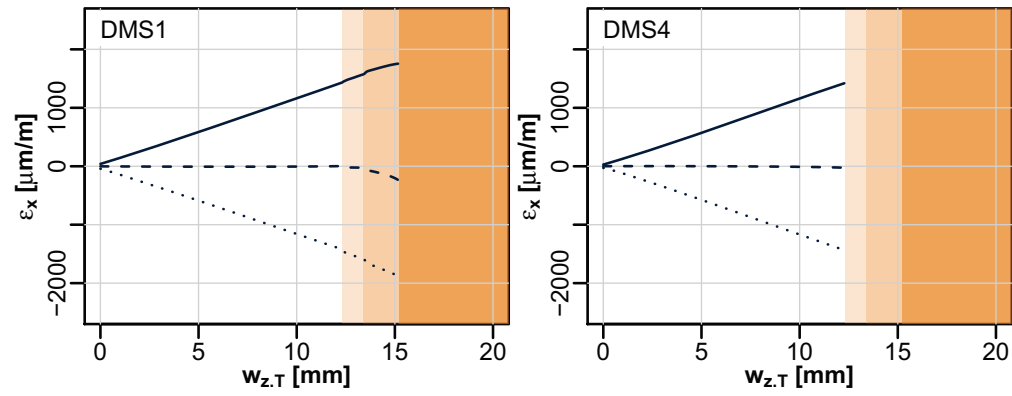

Glasverzerrung

... Oberkante

- Mitte

- Unterkante
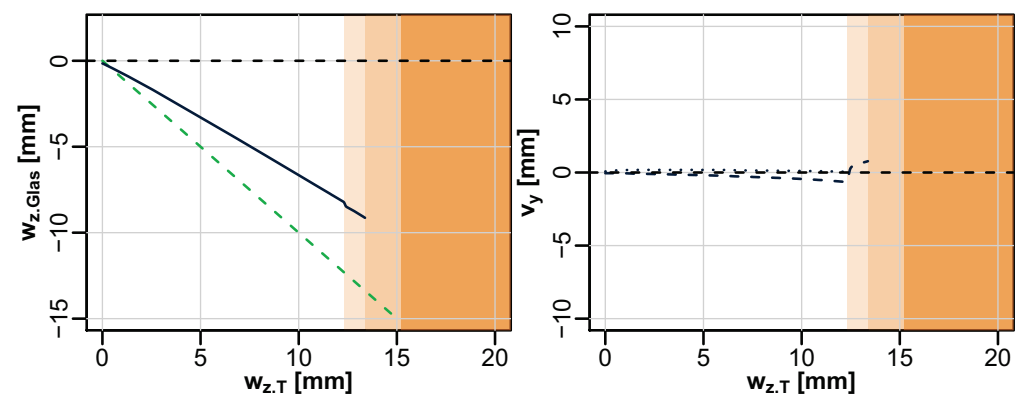

Durchbiegung

- $\mathrm{w}_{\text {zGlas }}$ Unterkante

- Lastrate

- - $\mathrm{V}_{\mathrm{y}}$ Oberkante

$\mathrm{v}_{\mathrm{y}}$ Unterkante

\begin{tabular}{|c|c|c|c|c|c|}
\hline $\mathrm{F}_{\mathrm{cr.i}}[\mathrm{kN}]$ & $\mathrm{N}_{\mathrm{p}}\left(\mathrm{F}_{\text {cr.i. }}\right)[\mathrm{kN}]$ & Funktion & $\mathbf{a}_{\varepsilon}[\mu \mathrm{m} /(\mathbf{m} \times \mathbf{m m})]$ & $\mathbf{b}_{\varepsilon}[\mu \mathrm{m} / \mathrm{m}]$ & $\mathbf{R}_{\varepsilon \cdot \min }^{2}[-]$ \\
\hline 24.5 & - & $\varepsilon_{1 \mid 4 . \text { oben }}\left(P_{0}, w_{z . T}\right)$ & -112.3 & -30.3 & 1.000 \\
\hline 23.0 & - & $\varepsilon_{1 \mid 4 . \text { unten }}\left(P_{0}, w_{z . T}\right)$ & 112.9 & 26.0 & 1.000 \\
\hline 15.8 & - & Funktion & $a_{i}[\mathrm{~mm} / \mathrm{mm}]$ & $\overline{b_{i}[\mathrm{~mm}]}$ & $\mathbf{R}_{\text {i.min }}$ [-] \\
\hline \multirow[t]{3}{*}{-} & - & $\mathrm{w}_{\mathrm{z} . \mathrm{Glas}}\left(\mathrm{P}_{0}, \mathrm{w}_{\mathrm{z} . \mathrm{T}}\right)$ & -0.66 & -0.036 & 1.000 \\
\hline & & $\mathrm{v}_{\mathrm{y} \text {.oben }}\left(\mathrm{P}_{0}, \mathrm{w}_{\mathrm{z} . \mathrm{T}}\right)$ & -0.039 & -0.008 & 0.982 \\
\hline & & $v_{y . \text { unten }}\left(P_{0}, w_{z . T}\right)$ & -0.004 & 0.18 & 0.235 \\
\hline
\end{tabular}




\section{Bruchversuch Referenzträger unbewehrt}

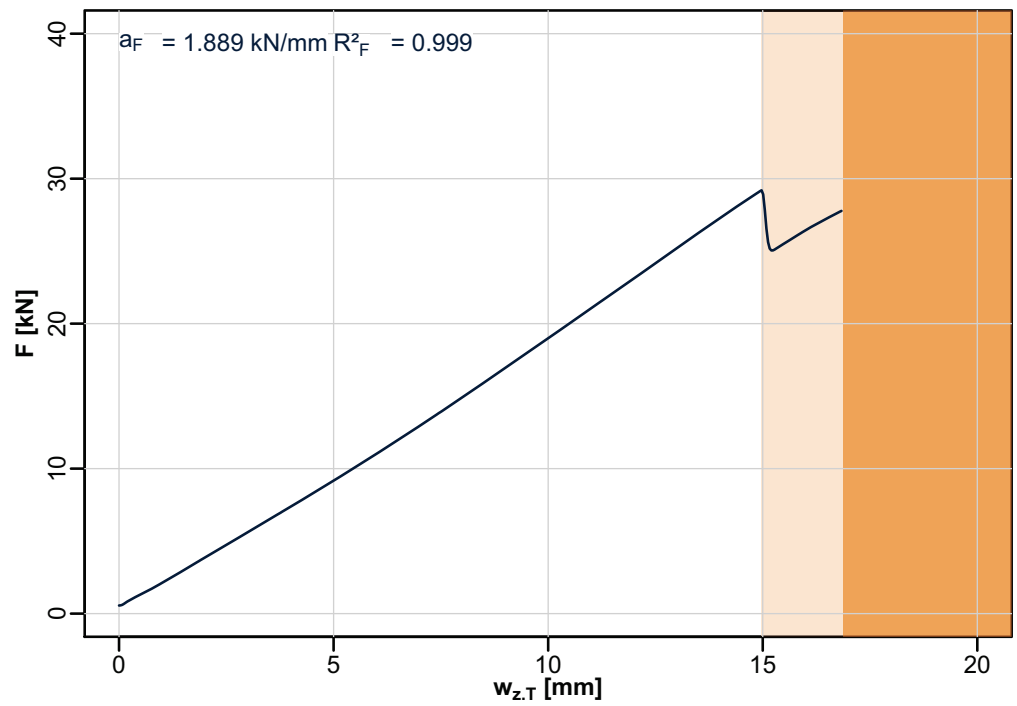

Kraftänderung BV02PX-II

- Prüfkraft $F$

$\square \quad$ ungebrochen

$\square \quad$ Teilbruch

$\square \quad$ Versagen Schrittweiser Bruch, Träger fällt herab.
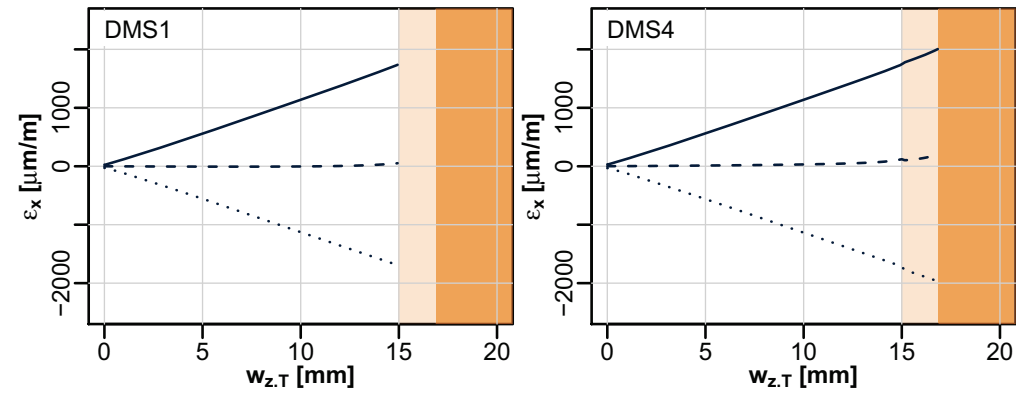

Glasverzerrung

... Oberkante

- Mitte

- Unterkante

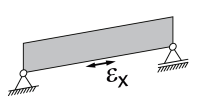

Durchbiegung

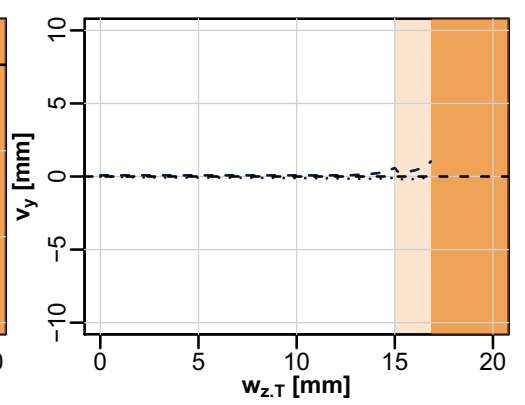

$\mathrm{w}_{\mathrm{zGlas}}$ Unterkante

_ Lastrate

- vy $_{\text {y }}$ Oberkante

$\mathrm{v}_{\mathrm{y}}$ Unterkante

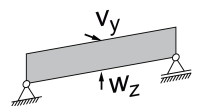

\begin{tabular}{|c|c|c|c|c|c|}
\hline $\mathrm{F}_{\mathrm{cr.i}}[\mathrm{kN}]$ & $\mathbf{N}_{\mathrm{p}}\left(\mathrm{F}_{\mathrm{cr.i}}\right)[\mathrm{kN}]$ & Funktion & $a_{\varepsilon}[\mu m /(m \times m m)]$ & $\mathbf{b}_{\varepsilon}[\mu \mathrm{m} / \mathrm{m}]$ & $\mathbf{R}_{\varepsilon . \min }^{2}[-]$ \\
\hline 29.2 & - & $\varepsilon_{1 \mid 4 . \text { oben }}\left(P_{0}, W_{z . T}\right)$ & -112.2 & -2.2 & 1.000 \\
\hline 27.8 & - & $\varepsilon_{1 \mid 4 . \text { unten }}\left(P_{0}, w_{z . T}\right)$ & 113.5 & -1.5 & 1.000 \\
\hline- & - & Funktion & $a_{i}[\mathrm{~mm} / \mathrm{mm}]$ & $b_{i}[\mathrm{~mm}]$ & $\mathbf{R}^{2}{ }_{\text {i.min }}[-]$ \\
\hline \multirow[t]{3}{*}{-} & - & $\mathrm{w}_{\mathrm{z} . \mathrm{Glas}}\left(\mathrm{P}_{0}, \mathrm{w}_{\mathrm{z} . \mathrm{T}}\right)$ & -0.66 & 0.016 & 1.000 \\
\hline & & $\mathrm{v}_{\mathrm{y} . \text { oben }}\left(\mathrm{P}_{0}, \mathrm{w}_{\mathrm{z} . \mathrm{T}}\right)$ & 0.00 & 0.082 & 0.620 \\
\hline & & $v_{y . \text { unten }}\left(P_{0}, w_{z . T}\right)$ & -0.008 & -0.034 & 0.910 \\
\hline
\end{tabular}




\section{Bruchversuch Referenzträger unbewehrt}
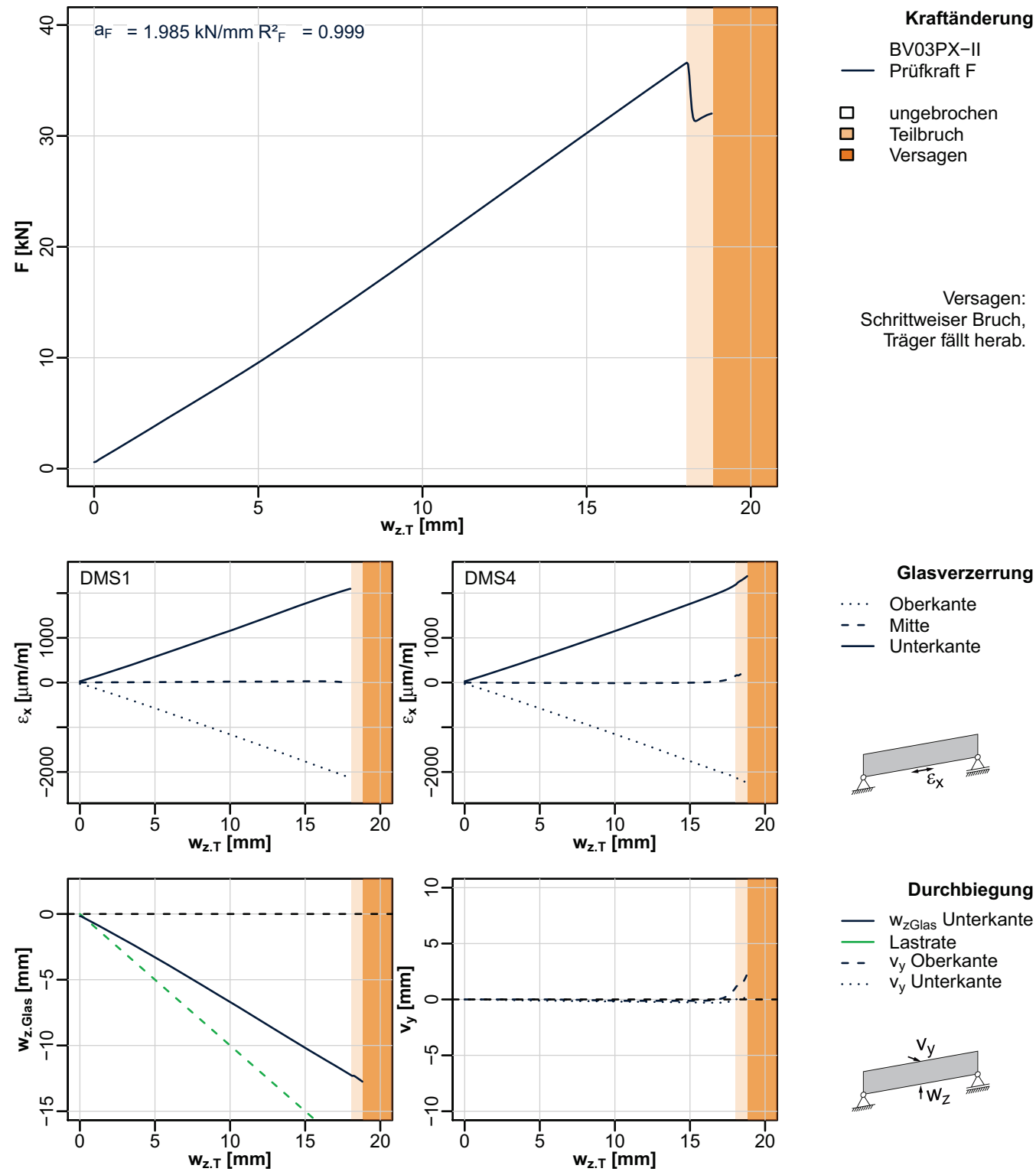

Durchbiegung

- $\mathrm{w}_{\text {zGlas }}$ Unterkante

- Lastrate

- - $\mathrm{v}_{\mathrm{y}}$ Oberkante

$v_{y}$ Unterkante

\begin{tabular}{|c|c|c|c|c|c|}
\hline $\mathrm{F}_{\mathrm{cr.i}}[\mathrm{kN}]$ & $\mathrm{N}_{\mathrm{p}}\left(\mathrm{F}_{\mathrm{cr.i}}\right)[\mathrm{kN}]$ & Funktion & $a_{\varepsilon}[\mu \mathrm{m} /(\mathbf{m} \times \mathbf{m m})]$ & $\mathbf{b}_{\varepsilon}[\mu \mathrm{m} / \mathrm{m}]$ & $\mathbf{R}_{\varepsilon \cdot \min }^{2}[-]$ \\
\hline 36.6 & - & $\varepsilon_{1 \mid 4 . \text { oben }}\left(P_{0}, W_{z . T}\right)$ & -116.4 & 0.4 & 1.000 \\
\hline 32.0 & - & $\varepsilon_{1 \mid 4 . \text { unten }}\left(P_{0}, w_{z . T}\right)$ & 116.4 & 1.0 & 1.000 \\
\hline - & - & Funktion & $a_{i}[\mathrm{~mm} / \mathrm{mm}]$ & $\mathbf{b}_{\mathrm{i}}[\mathrm{mm}]$ & $\mathbf{R}_{\text {i.min }}[-]$ \\
\hline \multirow[t]{3}{*}{-} & - & $\mathrm{w}_{\mathrm{z} . \text { Glas }}\left(\mathrm{P}_{0}, \mathrm{w}_{\mathrm{z} . \mathrm{T}}\right)$ & -0.67 & 0.021 & 1.000 \\
\hline & & $v_{y . o b e n}\left(P_{0}, w_{z . T}\right)$ & -0.012 & 0.034 & 0.871 \\
\hline & & $\mathrm{v}_{\mathrm{y} . \text { unten }}\left(\mathrm{P}_{0}, \mathrm{w}_{\mathrm{z} . \mathrm{T}}\right)$ & -0.018 & -0.002 & 0.991 \\
\hline
\end{tabular}




\section{Bruchversuch Referenzträger unbewehrt}
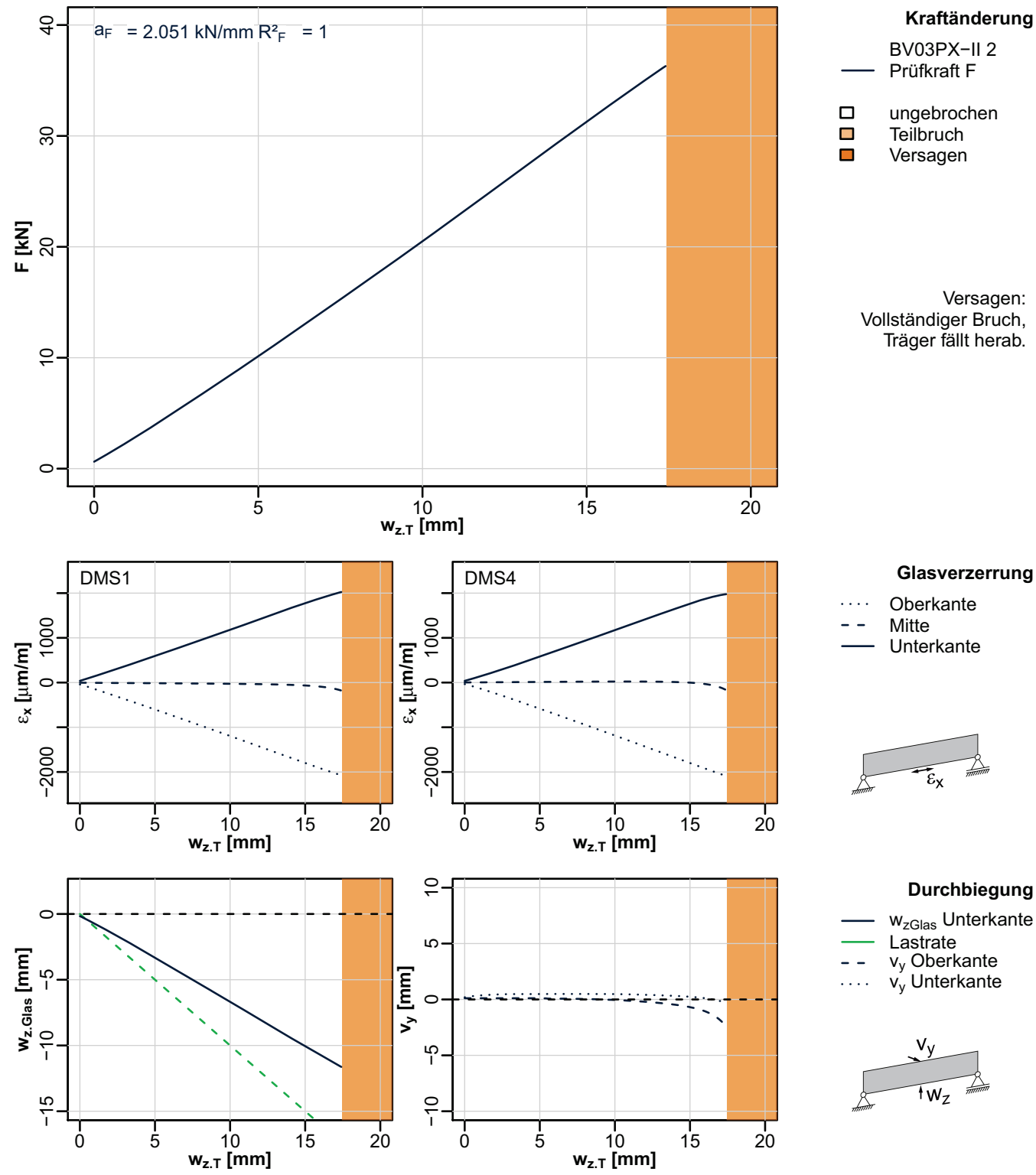

Durchbiegung

- w $_{\text {zGlas }}$ Unterkante

- Lastrate

- - $\mathrm{v}_{\mathrm{y}}$ Oberkante

$v_{y}$ Unterkante

\begin{tabular}{|c|c|c|c|c|c|}
\hline $\mathrm{F}_{\mathrm{cr.i}}[\mathrm{kN}]$ & $\mathbf{N}_{\mathrm{p}}\left(\mathrm{F}_{\mathrm{cr.i}}\right)[\mathrm{kN}]$ & Funktion & $a_{\varepsilon}[\mu m /(m \times m m)]$ & $\mathbf{b}_{\varepsilon}[\mu \mathrm{m} / \mathrm{m}]$ & $\mathbf{R}_{\varepsilon \cdot \min }^{2}[-]$ \\
\hline 36.4 & - & $\varepsilon_{1 \mid 4 . \text { oben }}\left(P_{0}, w_{z . T}\right)$ & -117.5 & -21.5 & 1.000 \\
\hline- & - & $\varepsilon_{1 \mid 4 . \text { unten }}\left(P_{0}, \mathrm{w}_{\mathrm{z.T}}\right)$ & 116.5 & 17.9 & 1.000 \\
\hline - & - & Funktion & $a_{i}[\mathrm{~mm} / \mathrm{mm}]$ & $\mathbf{b}_{\mathrm{i}}[\mathrm{mm}]$ & $\mathbf{R}^{2}{ }_{\text {i.min }}[-]$ \\
\hline \multirow[t]{3}{*}{-} & - & $\mathrm{w}_{\mathrm{z} . \text { Glas }}\left(\mathrm{P}_{0}, \mathrm{~W}_{\mathrm{z} . \mathrm{T}}\right)$ & -0.66 & -0.032 & 1.000 \\
\hline & & $v_{y . \text { oben }}\left(P_{0}, w_{z . T}\right)$ & -0.033 & 0.22 & 0.769 \\
\hline & & $v_{y . \text { unten }}\left(P_{0}, w_{z . T}\right)$ & 0.001 & 0.43 & 0.008 \\
\hline
\end{tabular}




\section{Bruchversuch Referenzträger schlaff bewehrt $d_{S}=10,1 \mathrm{~mm}$}
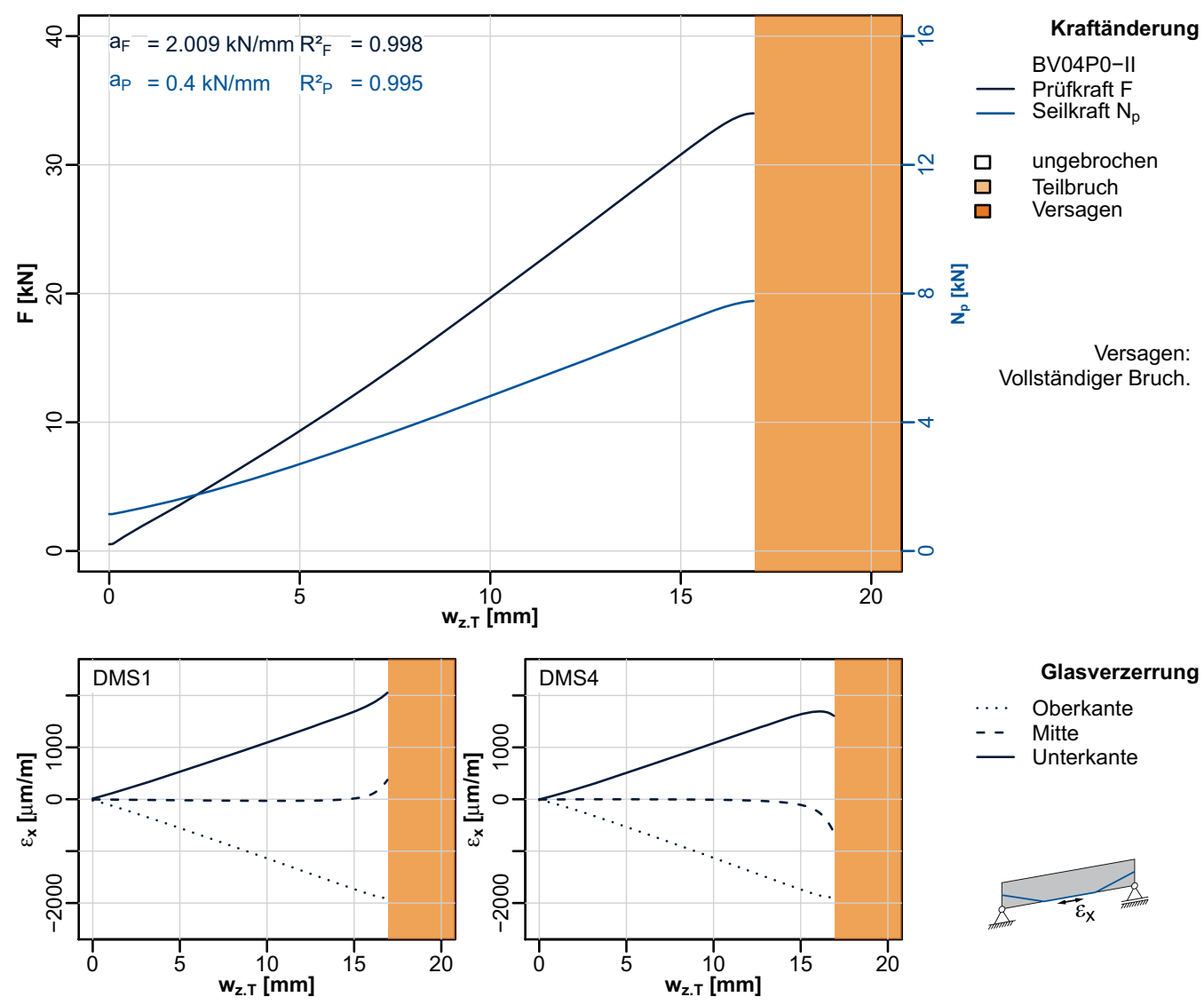

Glasverzerrung

... Oberkante

- Mitte

- Unterkante
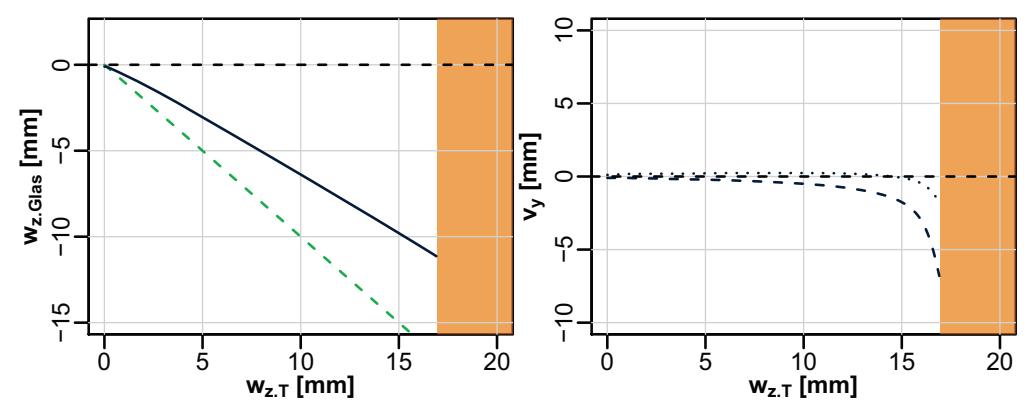

Durchbiegung

- $\mathrm{w}_{\text {zGlas }}$ Unterkante

- Lastrate

- - $v_{y}$ Oberkante

$v_{y}$ Unterkante

\begin{tabular}{|c|c|c|c|c|c|}
\hline $\mathrm{F}_{\mathrm{cr.i}}[\mathrm{kN}]$ & $\mathrm{N}_{\mathrm{p}}\left(\mathrm{F}_{\text {cr.i. }}\right)[\mathrm{kN}]$ & Funktion & $\mathbf{a}_{\varepsilon}[\mu \mathrm{m} /(\mathbf{m} \times \mathbf{m m})]$ & $\mathbf{b}_{\varepsilon}[\mu \mathrm{m} / \mathrm{m}]$ & $\mathbf{R}_{\varepsilon \cdot \min }^{2}[-]$ \\
\hline 34.0 & 7.8 & $\varepsilon_{1 \mid 4 . \text { oben }}\left(P_{0}, w_{z . T}\right)$ & -115.1 & 11.1 & 0.999 \\
\hline - & - & $\varepsilon_{1 \mid 4 . \text { unten }}\left(P_{0}, w_{z . T}\right)$ & 111.4 & -19.0 & 0.999 \\
\hline- & - & Funktion & $a_{i}[\mathrm{~mm} / \mathrm{mm}]$ & $\overline{b_{i}[\mathrm{~mm}]}$ & $\mathbf{R}_{\text {i.min }}$ [-] \\
\hline \multirow[t]{3}{*}{-} & - & $\mathrm{w}_{\mathrm{z} . \mathrm{Glas}}\left(\mathrm{P}_{0}, \mathrm{w}_{\mathrm{z} . \mathrm{T}}\right)$ & -0.66 & 0.16 & 0.999 \\
\hline & & $\mathrm{v}_{\mathrm{y} \text {.oben }}\left(\mathrm{P}_{0}, \mathrm{w}_{\mathrm{z} . \mathrm{T}}\right)$ & -0.061 & 0.043 & 0.882 \\
\hline & & $v_{y . \text { unten }}\left(P_{0}, w_{z . T}\right)$ & 0.003 & 0.19 & 0.132 \\
\hline
\end{tabular}




\section{Bruchversuch Referenzträger schlaff bewehrt $d_{S}=10,1 \mathrm{~mm}$}
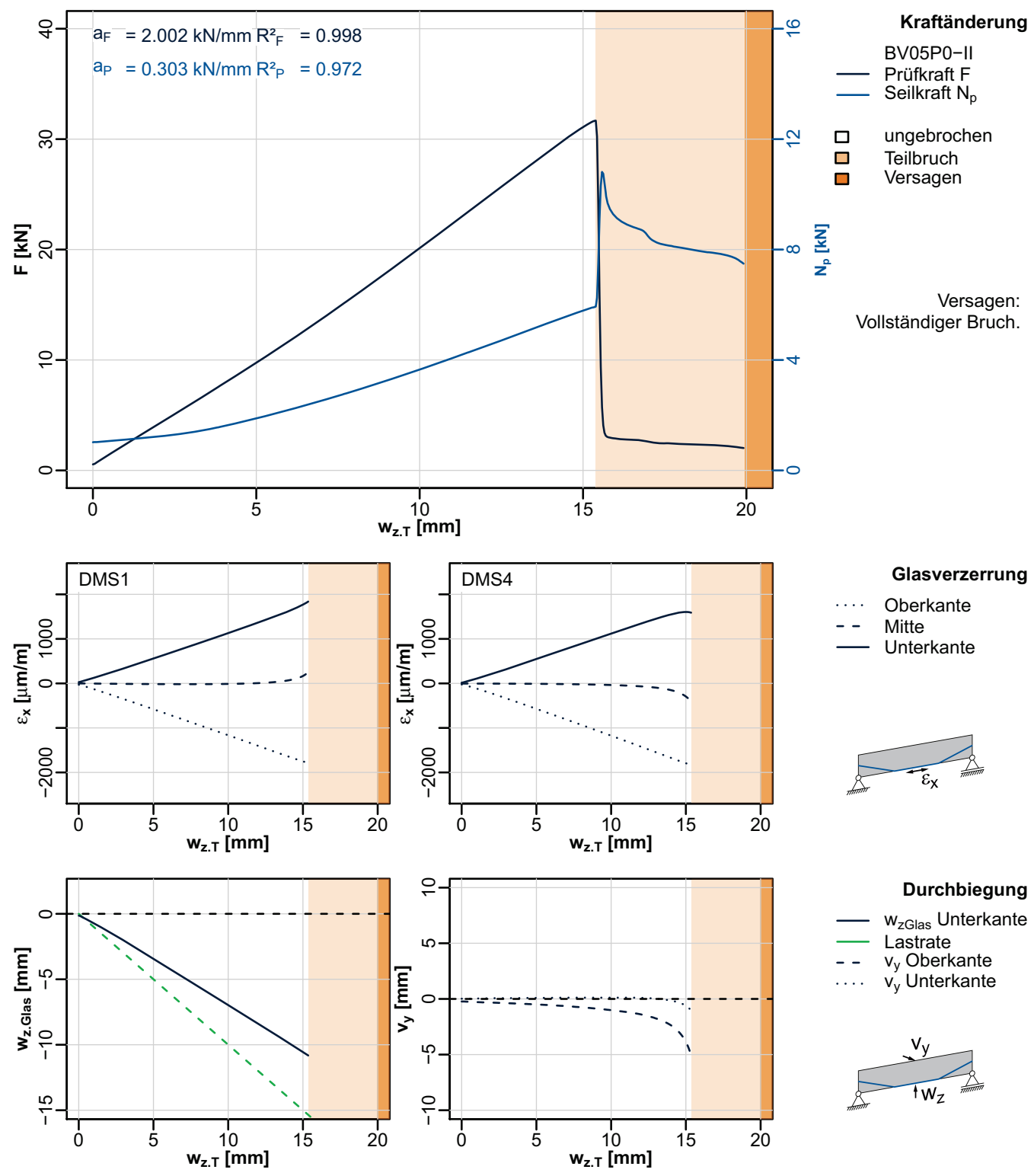

Durchbiegung

- w $_{\text {zGlas }}$ Unterkante

- Lastrate

- - $v_{y}$ Oberkante

$v_{y}$ Unterkante

\begin{tabular}{ll}
\hline $\mathbf{F}_{\text {cr.i }}[\mathbf{k N}]$ & $\mathbf{N}_{\mathrm{p}}\left(\mathbf{F}_{\text {cr.i }}\right)[\mathbf{k N}]$ \\
\hline 31.7 & 5.9 \\
2.0 & 1.1 \\
- & - \\
- & -
\end{tabular}

\begin{tabular}{|c|c|c|c|}
\hline Funktion & $a_{\varepsilon}[\mu m /(m \times m m)$ & $\mathbf{b}_{\varepsilon}[\mu \mathrm{m} / \mathrm{m}]$ & $\mathbf{R}_{\varepsilon, \min }^{2}[-]$ \\
\hline$\varepsilon_{1 \mid 4 . \text { oben }}\left(P_{0}, w_{z . T}\right)$ & -115.6 & -7.7 & 1.000 \\
\hline$\varepsilon_{1 \mid 4 . \text { unten }}\left(P_{0}, W_{z . T}\right)$ & 112.6 & 2.9 & 1.000 \\
\hline Funktion & $a_{\mathrm{i}}[\mathrm{mm} / \mathrm{mm}]$ & $\mathrm{b}_{\mathrm{i}}[\mathrm{mm}]$ & $\mathbf{R}_{\mathrm{i} \text {.min }}[-]$ \\
\hline $\mathrm{w}_{\mathrm{z} \text {.Glas }}\left(\mathrm{P}_{0}, \mathrm{w}_{\mathrm{z} . \mathrm{T}}\right)$ & -0.70 & 0.029 & 1.000 \\
\hline$v_{y . o b e n}\left(P_{0}, w_{z . T}\right)$ & -0.09 & -0.11 & 0.940 \\
\hline$v_{y \text {.unten }}\left(P_{0}, w_{z . T}\right)$ & 0.007 & 0.046 & 0.768 \\
\hline
\end{tabular}




\section{Bruchversuch Referenzträger schlaff bewehrt $d_{s}=10,1 \mathrm{~mm}$}
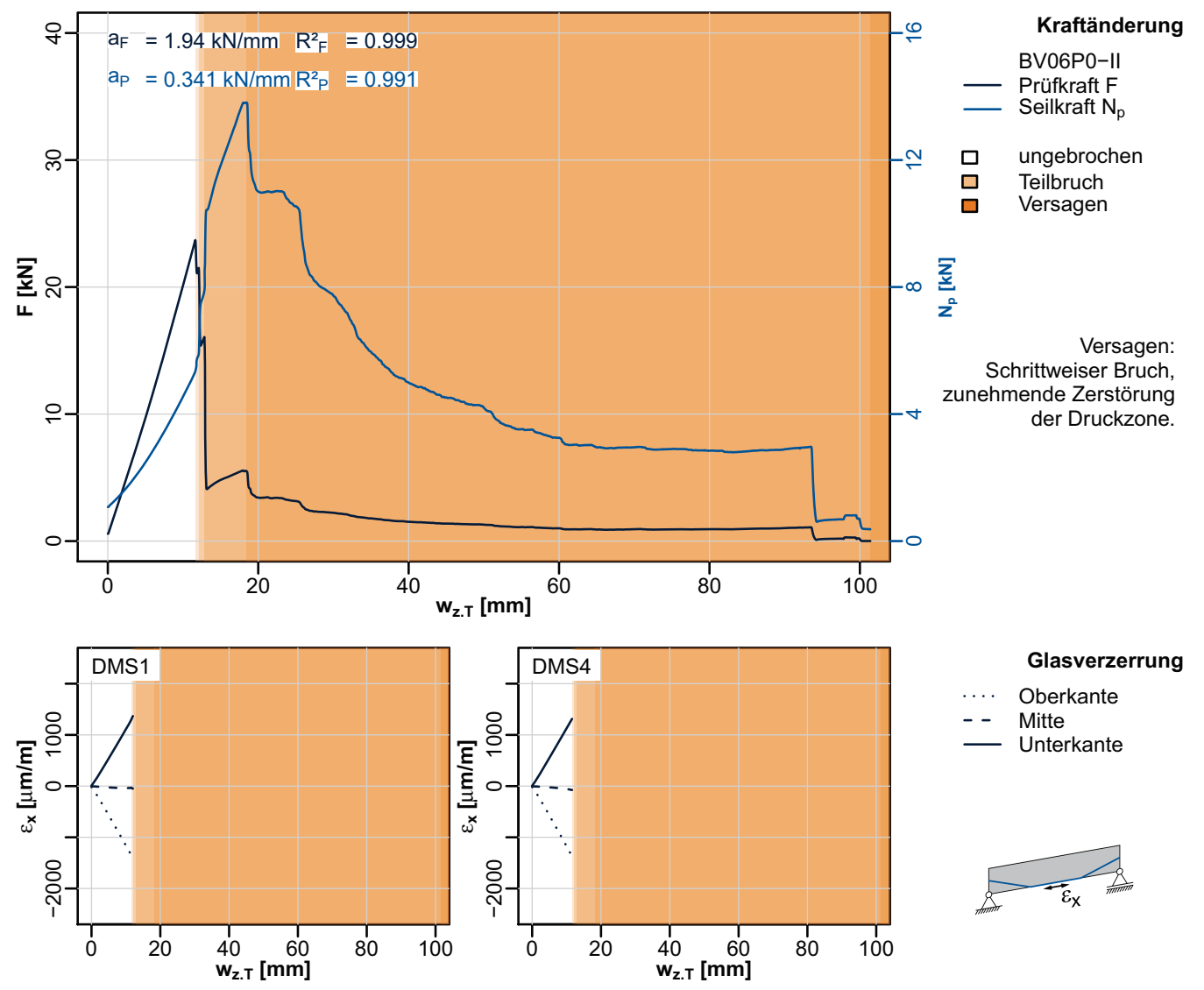

Glasverzerrung

... Oberkante

- - Mitte

- Unterkante
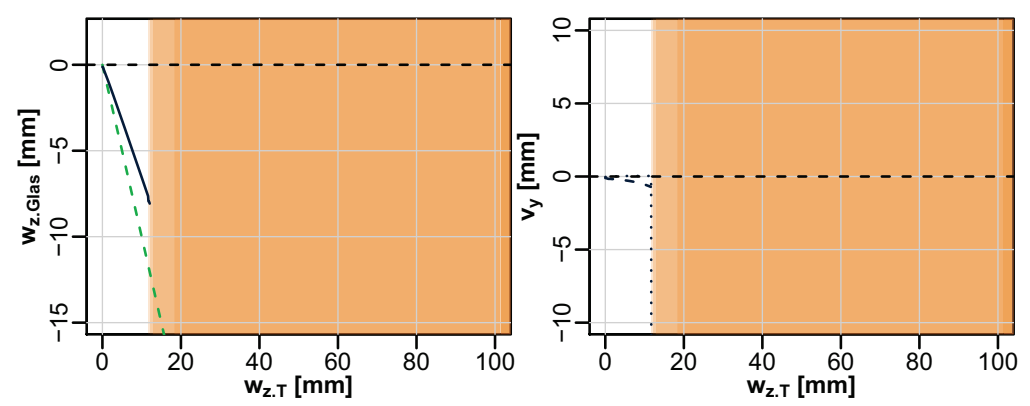

Durchbiegung

- $\mathrm{w}_{\text {zGlas }}$ Unterkante

- Lastrate

- $v_{y}$ Oberkante

.... $v_{y}$ Unterkante

\begin{tabular}{ll}
\hline $\mathbf{F}_{\text {cr. }}[\mathbf{k N}]$ & $\mathbf{N}_{\mathbf{p}}\left(\mathbf{F}_{\text {cr.i }}\right)[\mathbf{k N}]$ \\
\hline 23.7 & 5.3 \\
21.5 & 5.9 \\
16.1 & 7.9 \\
5.5 & 13.8
\end{tabular}

\begin{tabular}{|c|c|c|c|}
\hline Funktion & $\mathbf{a}_{\varepsilon}[\mu \mathbf{m} /(\mathbf{m} \times \mathbf{m m})]$ & $\mathbf{b}_{\varepsilon}[\mu \mathrm{m} / \mathrm{m}]$ & $\mathbf{R}_{\varepsilon, \min }^{2}[-]$ \\
\hline$\varepsilon_{1 \mid 4 . \text { oben }}\left(P_{0}, w_{z . T}\right)$ & -115.3 & 3.2 & 1.000 \\
\hline$\varepsilon_{1 \mid 4 . \text { unten }}\left(P_{0}, w_{z . T}\right)$ & 110.8 & -10.2 & 1.000 \\
\hline Funktion & $a_{i}[\mathrm{~mm} / \mathrm{mm}]$ & $\mathbf{b}_{\mathrm{i}}[\mathrm{mm}]$ & $\mathbf{R}_{\mathrm{i} \text {.min }}[-]$ \\
\hline $\mathrm{w}_{\mathrm{z} . \text { Glas }}\left(\mathrm{P}_{0}, \mathrm{w}_{\mathrm{z} . \mathrm{T}}\right)$ & -0.65 & 0.004 & 1.000 \\
\hline$v_{y . o b e n}\left(P_{0}, w_{z . T}\right)$ & -0.036 & -0.098 & 0.966 \\
\hline$v_{y \text {.unten }}\left(P_{0}, w_{z . T}\right)$ & 0.007 & -0.012 & 0.813 \\
\hline
\end{tabular}




\section{Vorspannen Träger mit $P_{m 0}=9 \mathrm{kN}$ und $d_{p}=6,1 \mathrm{~mm}$}

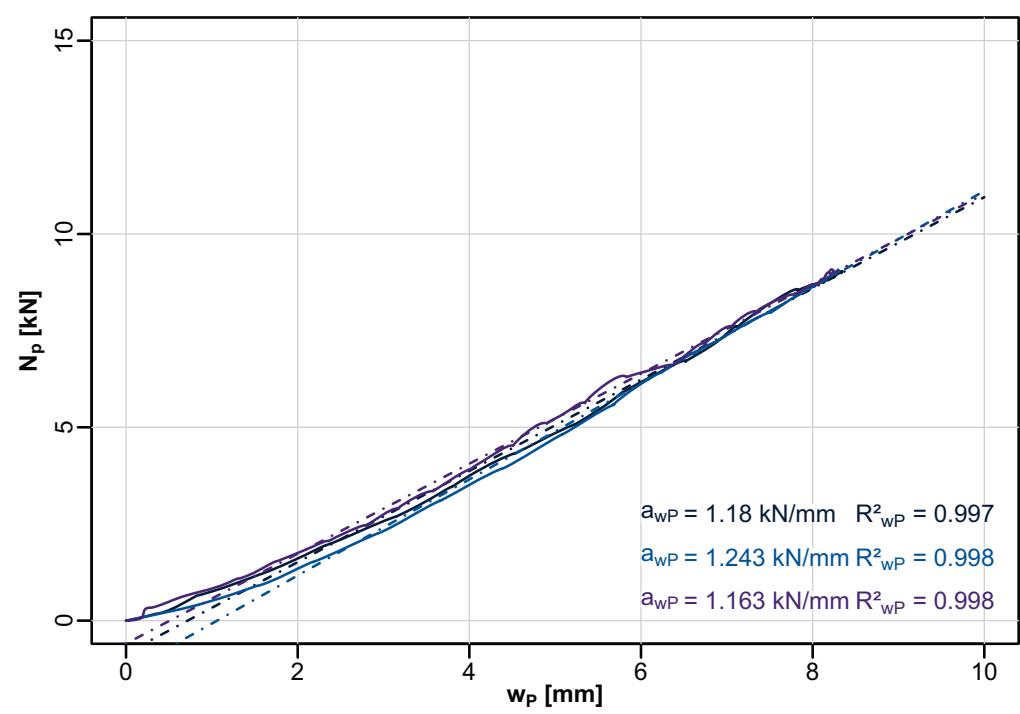

Seilkraftänderung

— BV07P9-II

- BV08P9-II

- BV09P9-II

... $a_{w P} \times w_{P}+b_{w P}$
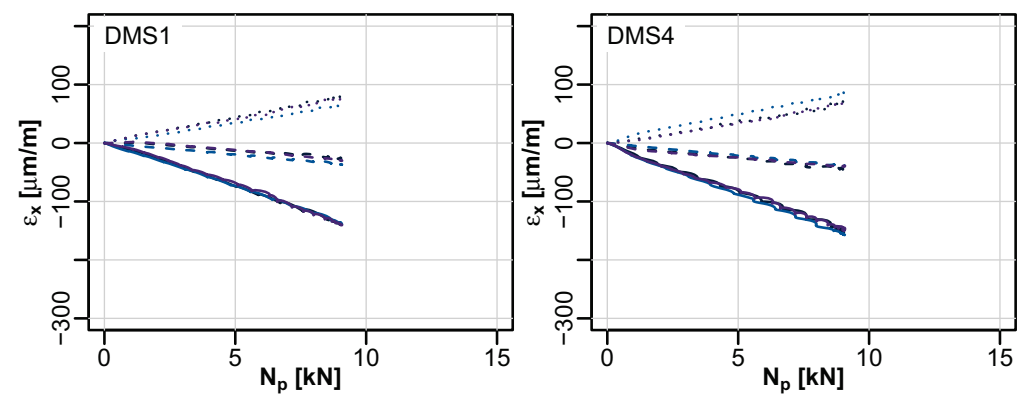

Glasverzerrung

... Oberkante

- - Mitte

- Unterkante
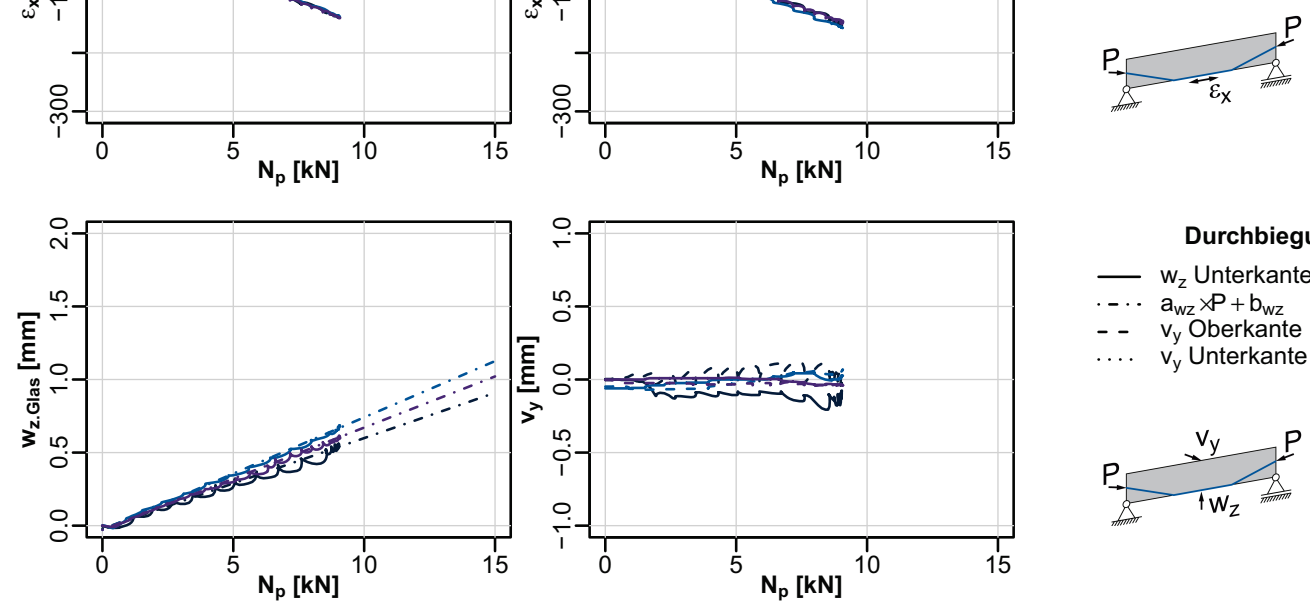

Durchbiegung

- $\mathrm{w}_{\mathrm{z}}$ Unterkante

... $a_{w z} \times P+b_{w z}$

- - $v_{y}$ Oberkante

.... $v_{y}$ Unterkante

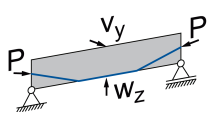

\begin{tabular}{llllll}
\hline Prüfkörper & $\mathbf{a}_{\varepsilon 1 \mid 4 . \text { oben }}$ & $\mathbf{a}_{\varepsilon 1 \mid 4 . u n t e n}$ & $\mathbf{a}_{\mathbf{w z}}$ & \multicolumn{2}{l}{$\mathbf{v}_{\mathbf{y} \text {.oben.max }} \mathbf{v}_{\mathbf{y} \text {.unten.max }}$} \\
& {$[\mu \mathbf{m} /(\mathbf{m} \times \mathbf{k N})]$} & {$[\mu \mathbf{m} /(\mathbf{m} \times \mathbf{k N})]$} & {$[\mathbf{m m} / \mathbf{k N}]$} & {$[\mathbf{m m}]$} & {$[\mathbf{m m}]$} \\
\hline BV07P9-II & $8.8\left(\mathrm{R}^{2}=0.994\right)$ & $-15.7\left(\mathrm{R}^{2}=0.998\right)$ & $0.062\left(\mathrm{R}^{2}=0.988\right)$ & -0.0758 & -0.0302 \\
BV08P9-II & $7.2\left(\mathrm{R}^{2}=0.998\right)$ & $-15.4\left(\mathrm{R}^{2}=0.999\right)$ & $0.077\left(\mathrm{R}^{2}=0.997\right)$ & 0.0273 & 0.0665 \\
BV09P9-II & $8.5\left(\mathrm{R}^{2}=0.996\right)$ & $-16.2\left(\mathrm{R}^{2}=0.997\right)$ & $0.070\left(\mathrm{R}^{2}=0.997\right)$ & -0.0394 & -0.0514 \\
\hline
\end{tabular}




\section{Bruchversuch Träger mit $P_{m 0}=9 \mathrm{kN}$ und $d_{p}=6,1 \mathrm{~mm}$}
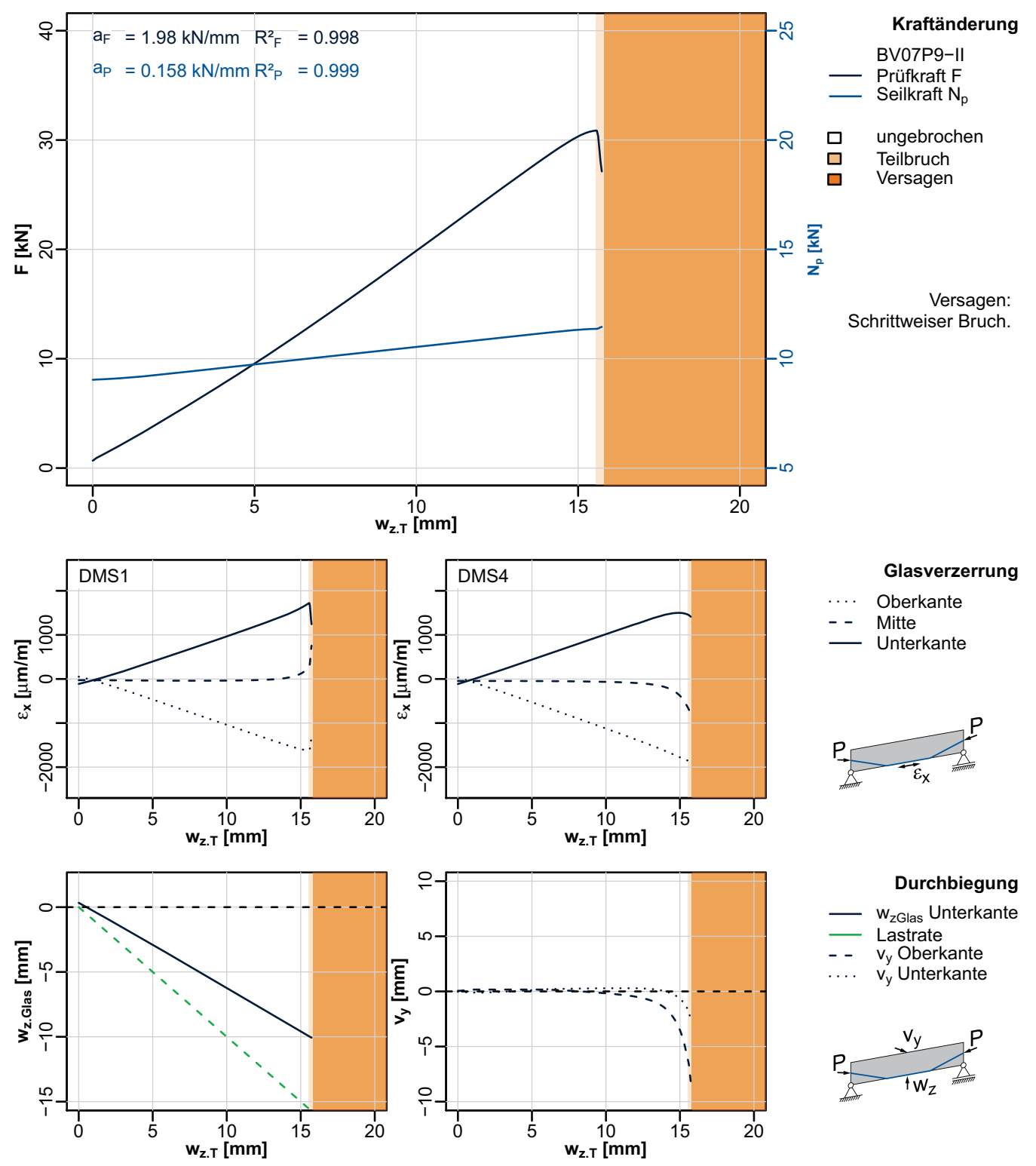

Durchbiegung

— $\mathrm{w}_{\mathrm{zGlas}}$ Unterkante

- Lastrate

- - $v_{y}$ Oberkante

.... $v_{\mathrm{y}}$ Unterkante

\begin{tabular}{|c|c|c|c|c|c|}
\hline $\mathrm{F}_{\text {cr.i }}[\mathrm{kN}]$ & $\mathbf{N}_{\mathrm{p}}\left(\mathrm{F}_{\mathrm{cr.i}}\right)[\mathrm{kN}]$ & Funktion & $a_{\varepsilon}[\mu \mathrm{m} /(\mathbf{m} \times \mathbf{m m})]$ & $\mathbf{b}_{\varepsilon}[\mu \mathrm{m} / \mathrm{m}]$ & $\mathbf{R}_{\varepsilon \cdot \min }^{2}[-]$ \\
\hline 30.9 & 11.4 & $\varepsilon_{1 \mid 4 . \text { oben }}\left(P_{0}, w_{z . T}\right)$ & -112.4 & 87.5 & 1.000 \\
\hline 22.2 & 10.7 & $\varepsilon_{1 \mid 4 . \text { unten }}\left(P_{0}, W_{z . T}\right)$ & 111.6 & -152.3 & 0.999 \\
\hline - & - & Funktion & $a_{i}[\mathrm{~mm} / \mathrm{mm}]$ & $\mathbf{b}_{\mathrm{i}}[\mathrm{mm}]$ & $\mathbf{R}^{2}{ }_{\text {i.min }}[-]$ \\
\hline \multirow[t]{3}{*}{-} & - & $\mathrm{w}_{\mathrm{z} . \text { Glas }}\left(\mathrm{P}_{0}, \mathrm{~W}_{\mathrm{z} . \mathrm{T}}\right)$ & -0.66 & 0.39 & 1.000 \\
\hline & & $v_{y . o b e n}\left(P_{0}, w_{z . T}\right)$ & -0.048 & 0.31 & 0.702 \\
\hline & & $v_{y . \text { unten }}\left(P_{0}, w_{z . T}\right)$ & 0.039 & -0.11 & 0.895 \\
\hline
\end{tabular}




\section{Bruchversuch Träger mit $P_{m 0}=9 \mathrm{kN}$ und $d_{p}=6,1 \mathrm{~mm}$}
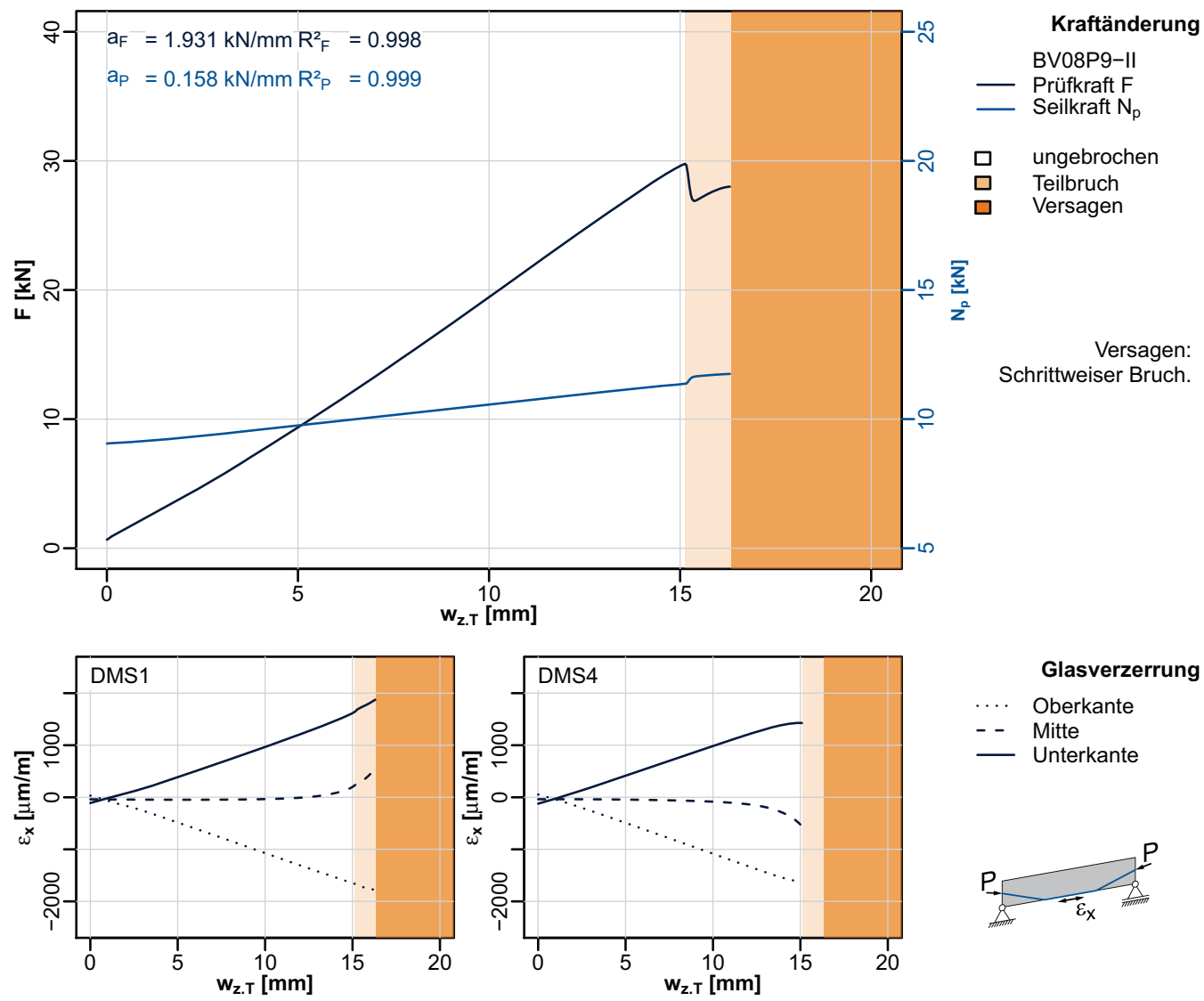

Glasverzerrung

... Oberkante

- - Mitte

- Unterkante
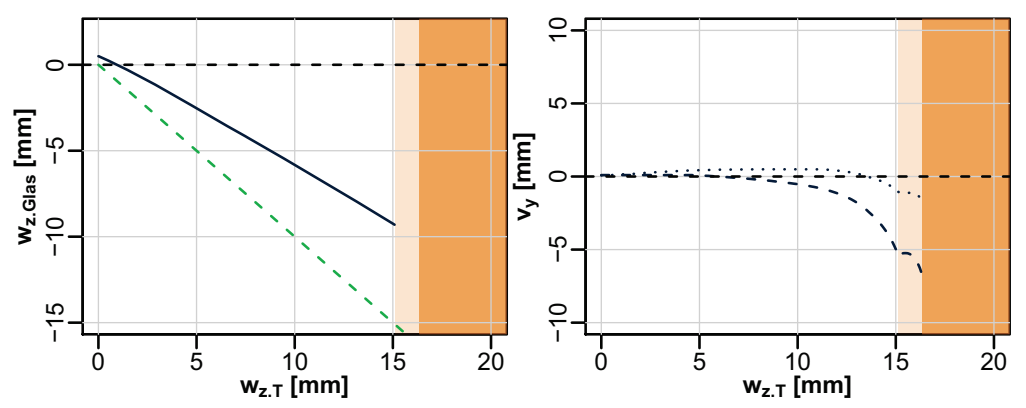

Durchbiegung

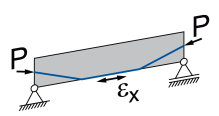

\begin{tabular}{ll}
\hline $\mathbf{F}_{\text {cr.i }}[\mathbf{k N}]$ & $\mathbf{N}_{\mathbf{p}}\left(\mathbf{F}_{\text {cr.i }}\right)[\mathbf{k N}]$ \\
\hline 29.8 & 11.4 \\
28.0 & 11.8 \\
- & - \\
- & -
\end{tabular}

\begin{tabular}{|c|c|c|c|}
\hline Funktion & $a_{\varepsilon}[\mu m /(m \times m m)]$ & $\mathbf{b}_{\varepsilon}[\mu \mathrm{m} / \mathrm{m}]$ & $\mathbf{R}_{\varepsilon \cdot \min }^{2}[-]$ \\
\hline$\varepsilon_{1 \mid 4 . \text { oben }}\left(P_{0}, w_{z . T}\right)$ & -113.4 & 68.1 & 0.999 \\
\hline$\varepsilon_{1 \mid 4 . \text { unten }}\left(P_{0}, W_{z . T}\right)$ & 111.5 & -153.5 & 0.998 \\
\hline Funktion & $a_{i}[\mathrm{~mm} / \mathrm{mm}]$ & $\mathbf{b}_{\mathrm{i}}[\mathrm{mm}]$ & $\mathbf{R}_{\text {i.min }}^{2}[-]$ \\
\hline $\mathrm{w}_{\text {z.Glas }}\left(\mathrm{P}_{0}, \mathrm{w}_{\mathrm{z} . \mathrm{T}}\right)$ & -0.65 & 0.67 & 1.000 \\
\hline$v_{y . \text { oben }}\left(P_{0}, w_{z . T}\right)$ & -0.087 & 0.35 & 0.796 \\
\hline$v_{y \text {.unten }}\left(P_{0}, w_{z . T}\right)$ & 0.032 & 0.19 & 0.734 \\
\hline
\end{tabular}




\section{Bruchversuch Träger mit $P_{m 0}=9 \mathrm{kN}$ und $d_{p}=6,1 \mathrm{~mm}$}
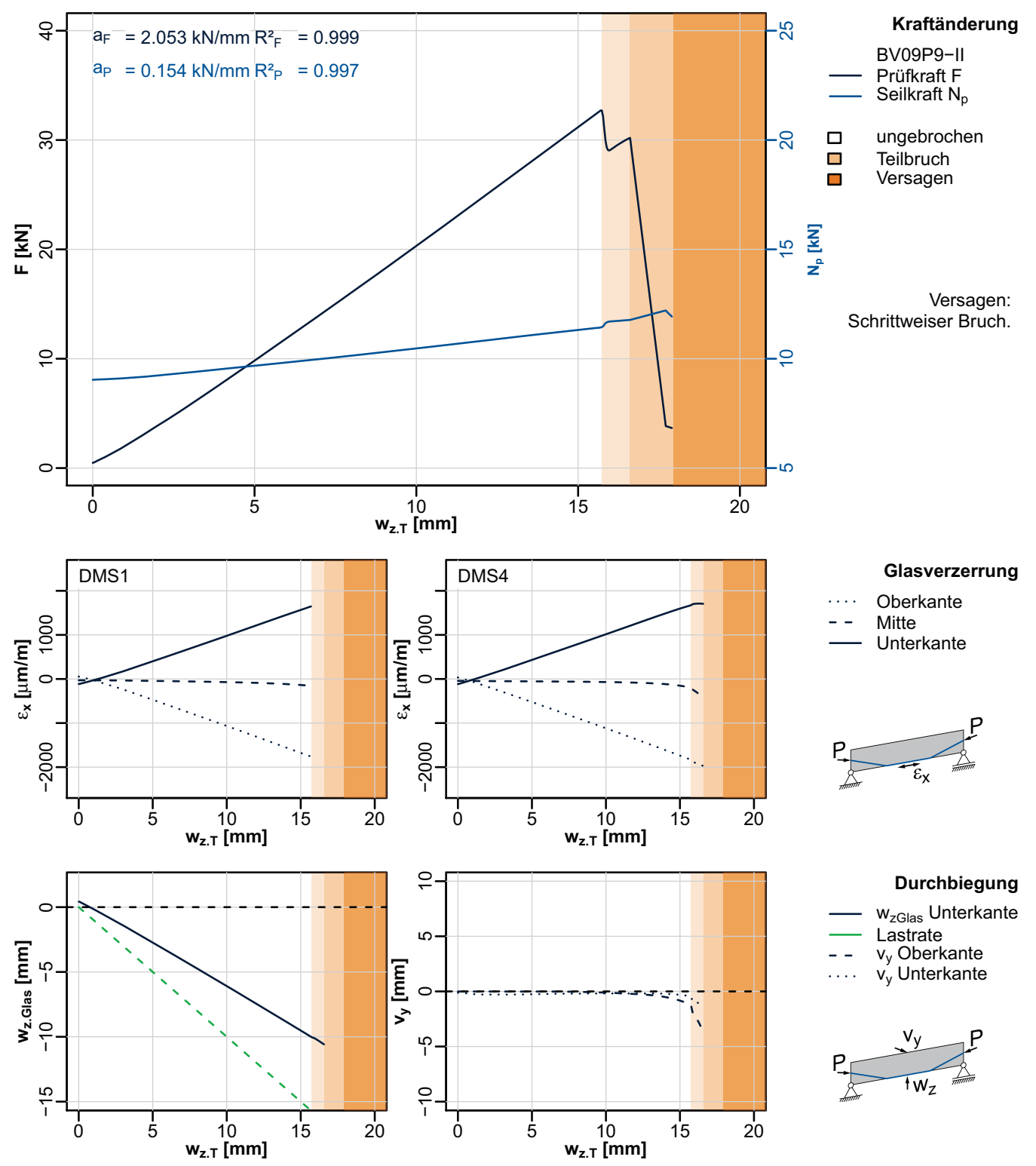

Durchbiegung

— $\mathrm{w}_{\mathrm{zGlas}}$ Unterkante

- Lastrate

- - $v_{y}$ Oberkante

… $v_{\mathrm{y}}$ Unterkante

\begin{tabular}{ll}
\hline $\mathbf{F}_{\text {cr.i }}[\mathbf{k N}]$ & $\mathbf{N}_{\mathbf{p}}\left(\mathbf{F}_{\text {cr.i }}\right)[\mathbf{k N}]$ \\
\hline 32.7 & 11.4 \\
30.2 & 11.8 \\
3.5 & 11.6 \\
- & -
\end{tabular}

\begin{tabular}{|c|c|c|c|}
\hline Funktion & $\mathbf{a}_{\varepsilon}[\mu \mathrm{m} /(\mathbf{m} \times \mathbf{m m})]$ & $\mathbf{b}_{\varepsilon}[\mu \mathrm{m} / \mathrm{m}]$ & $\mathbf{R}_{\varepsilon \cdot \min }^{2}[-]$ \\
\hline$\varepsilon_{1 \mid 4 . \text { oben }}\left(P_{0}, W_{z . T}\right)$ & -116.3 & 99.3 & 0.999 \\
\hline$\varepsilon_{1 \mid 4 . \text { unten }}\left(P_{0}, w_{z . T}\right)$ & 113.4 & -156.7 & 0.999 \\
\hline Funktion & $a_{i}[\mathrm{~mm} / \mathrm{mm}]$ & $\mathbf{b}_{\mathrm{i}}[\mathrm{mm}]$ & $\mathbf{R}_{\text {i.min }}$ [-] \\
\hline $\mathrm{W}_{\text {z.Glas }}\left(\mathrm{P}_{0}, \mathrm{~W}_{\mathrm{z} . \mathrm{T}}\right)$ & -0.66 & 0.56 & 1.000 \\
\hline $\mathrm{v}_{\mathrm{y} . \text { oben }}\left(\mathrm{P}_{0}, \mathrm{w}_{\mathrm{z} . \mathrm{T}}\right)$ & -0.017 & 0.052 & 0.683 \\
\hline$v_{y . \text { unten }}\left(P_{0}, w_{z . T}\right)$ & 0.006 & -0.26 & 0.304 \\
\hline
\end{tabular}




\section{Vorspannen Träger mit $P_{m 0}=15 \mathrm{kN}$ und $d_{p}=8,1 \mathrm{~mm}$}
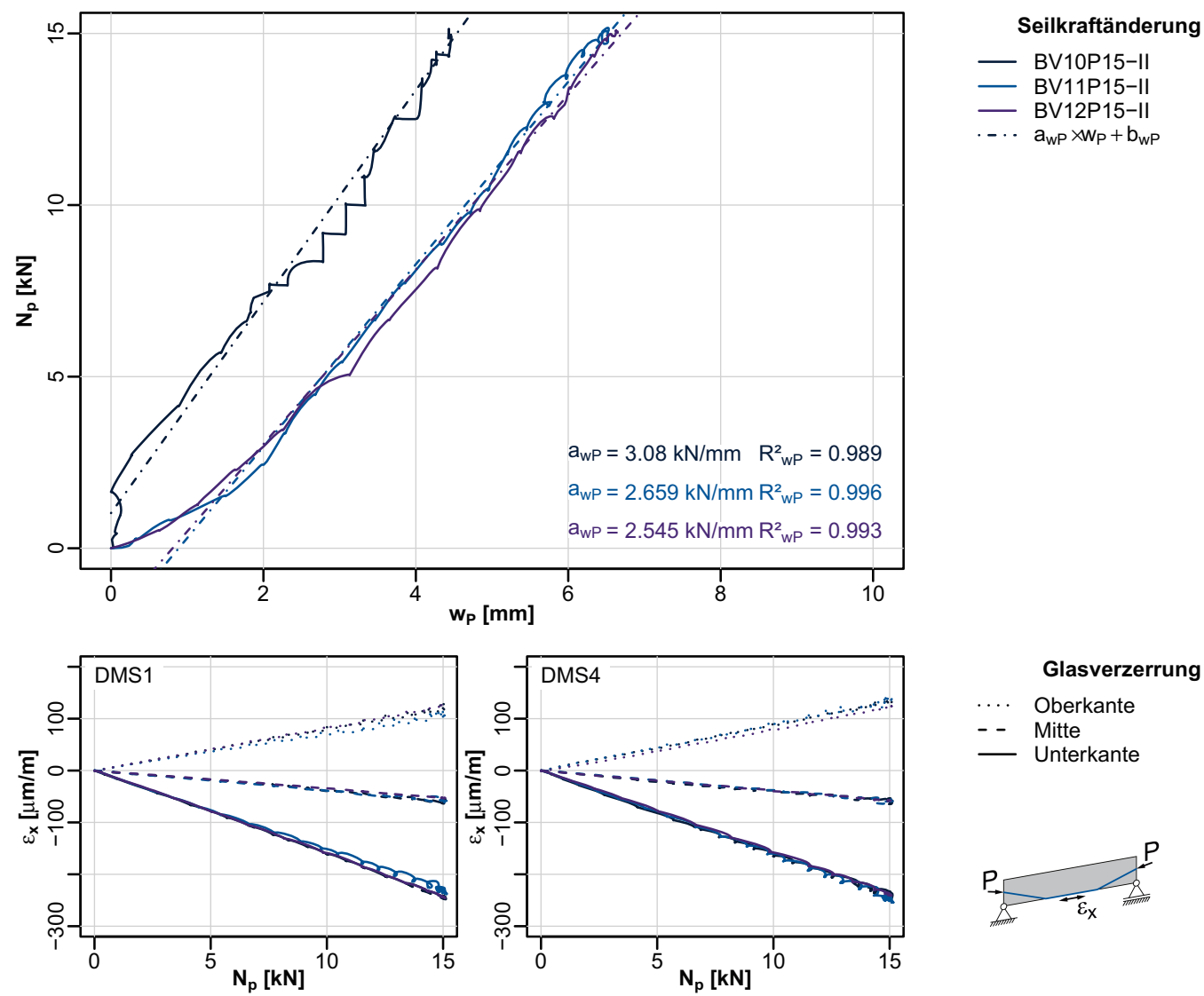
Glasverzerrung
... Oberkante
- Mitte
- Unterkante
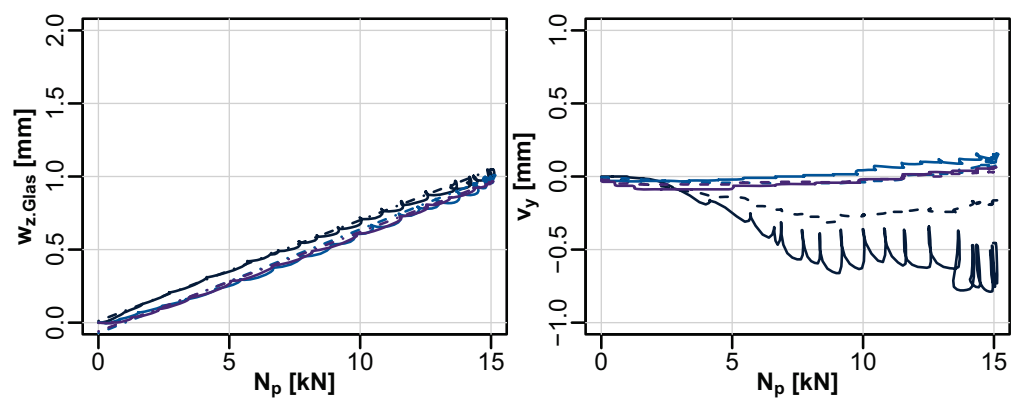

Durchbiegung

- $\mathrm{w}_{\mathrm{z}}$ Unterkante

$\cdots a_{w z} \times P+b_{w z}$

- - $v_{y}$ Oberkante

.... $v_{y}$ Unterkante

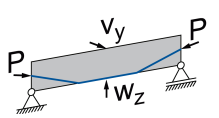

\begin{tabular}{llllll}
\hline Prüfkörper & $\mathbf{a}_{\varepsilon 1 \mid 4 . o b e n}$ & $\mathbf{a}_{\varepsilon 1 \mid 4 . u n t e n}$ & $\mathbf{a}_{\mathbf{w z}}$ & \multicolumn{2}{l}{$\mathbf{v}_{\mathbf{y} \text {.oben.max }} \mathbf{v}_{\mathbf{y} \text {.unten.max }}$} \\
& {$[\mu \mathrm{m} /(\mathbf{m} \times \mathbf{k N})]$} & {$[\mu \mathbf{m} /(\mathbf{m} \times \mathbf{k N})]$} & {$[\mathbf{m m} / \mathbf{k N}]$} & {$[\mathbf{m m}]$} & {$[\mathbf{m m}]$} \\
\hline BV10P15-II & $8.4\left(R^{2}=0.995\right)$ & $-16.2\left(R^{2}=0.998\right)$ & $0.069\left(R^{2}=0.997\right)$ & -0.455 & -0.151 \\
BV11P15-II & $7.7\left(R^{2}=0.992\right)$ & $-15.7\left(R^{2}=0.998\right)$ & $0.071\left(R^{2}=0.995\right)$ & 0.152 & 0.0847 \\
BV12P15-II & $8.4\left(R^{2}=0.999\right)$ & $-16.4\left(R^{2}=1.000\right)$ & $0.068\left(R^{2}=0.999\right)$ & 0.0637 & 0.0242 \\
\hline
\end{tabular}




\section{Bruchversuch Träger mit $P_{m 0}=15 \mathrm{kN}$ und $d_{p}=8,1 \mathrm{~mm}$}
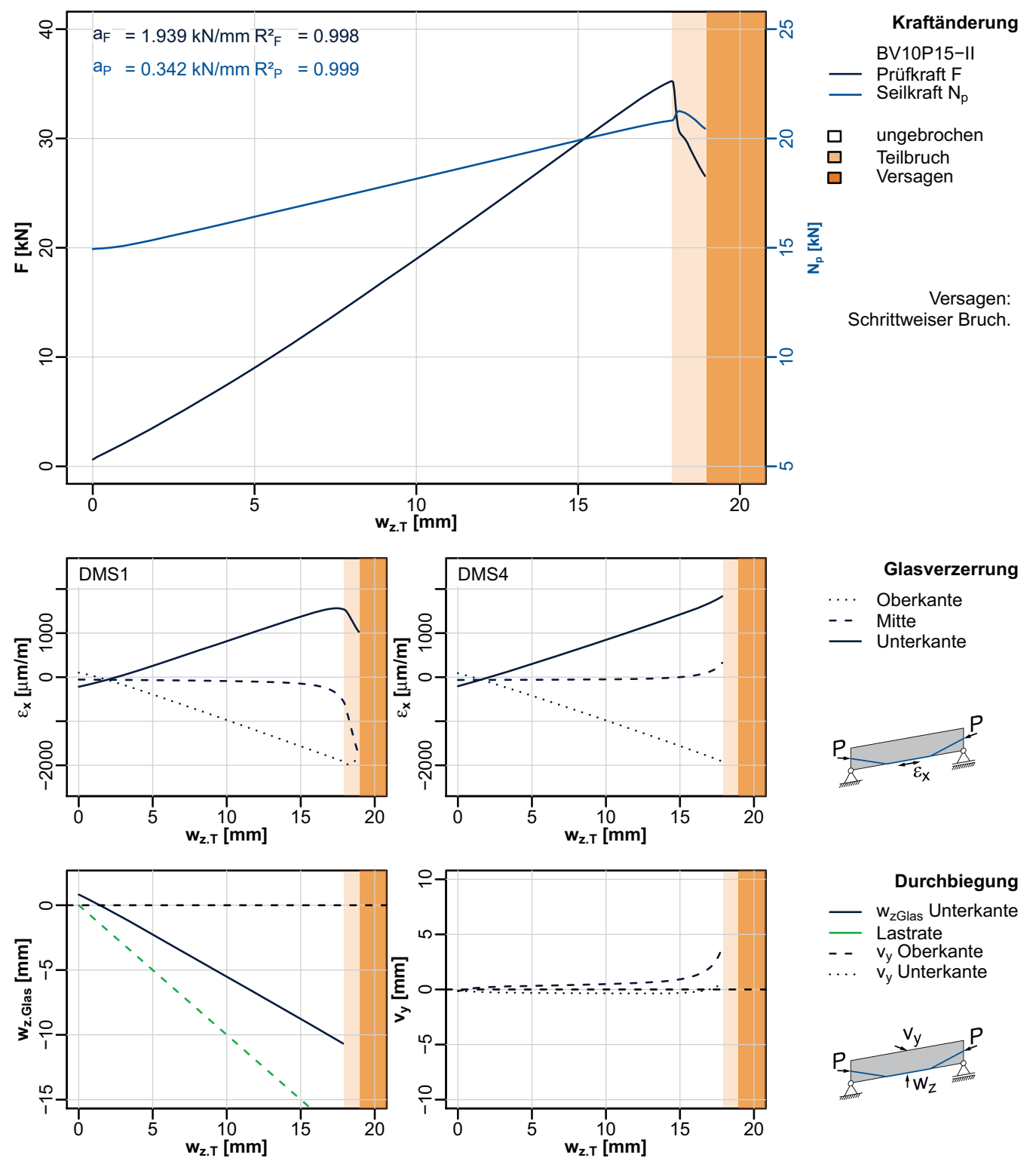

Durchbiegung

- w $_{\text {zGlas }}$ Unterkante

- Lastrate

- - $v_{y}$ Oberkante

.... $v_{\mathrm{y}}$ Unterkante

\begin{tabular}{ll}
\hline $\mathbf{F}_{\text {cr.i }}[\mathbf{k N}]$ & $\mathbf{N}_{\mathbf{p}}\left(\mathbf{F}_{\text {cr.i }}\right)[\mathbf{k N}]$ \\
\hline 35.2 & 20.8 \\
26.2 & 20.3 \\
- & - \\
- & -
\end{tabular}

\begin{tabular}{|c|c|c|c|}
\hline Funktion & $a_{\varepsilon}[\mu m /(m \times m m)]$ & $\mathbf{b}_{\varepsilon}[\mu \mathrm{m} / \mathrm{m}]$ & $\mathbf{R}_{\varepsilon \cdot \min }^{2}[-]$ \\
\hline$\varepsilon_{1 \mid 4 . \text { oben }}\left(P_{0}, W_{z . T}\right)$ & -113.5 & 159.7 & 0.999 \\
\hline$\varepsilon_{1 \mid 4 . \text { unten }}\left(P_{0}, W_{z . T}\right)$ & 108.9 & -272.2 & 0.999 \\
\hline Funktion & $a_{i}[\mathrm{~mm} / \mathrm{mm}]$ & $\mathbf{b}_{\mathrm{i}}[\mathrm{mm}]$ & $\mathbf{R}_{\text {i.min }}[-]$ \\
\hline $\mathrm{W}_{\mathrm{z} . \mathrm{Glas}}\left(\mathrm{P}_{0}, \mathrm{~W}_{\mathrm{z} . \mathrm{T}}\right)$ & -0.64 & 0.94 & 1.000 \\
\hline$v_{y . o b e n}\left(P_{0}, w_{z . T}\right)$ & 0.044 & 0.074 & 0.943 \\
\hline$v_{y . \text { unten }}\left(P_{0}, w_{z . T}\right)$ & -0.012 & -0.22 & 0.772 \\
\hline
\end{tabular}




\section{Bruchversuch Träger mit $P_{m 0}=15 \mathrm{kN}$ und $d_{p}=8,1 \mathrm{~mm}$}
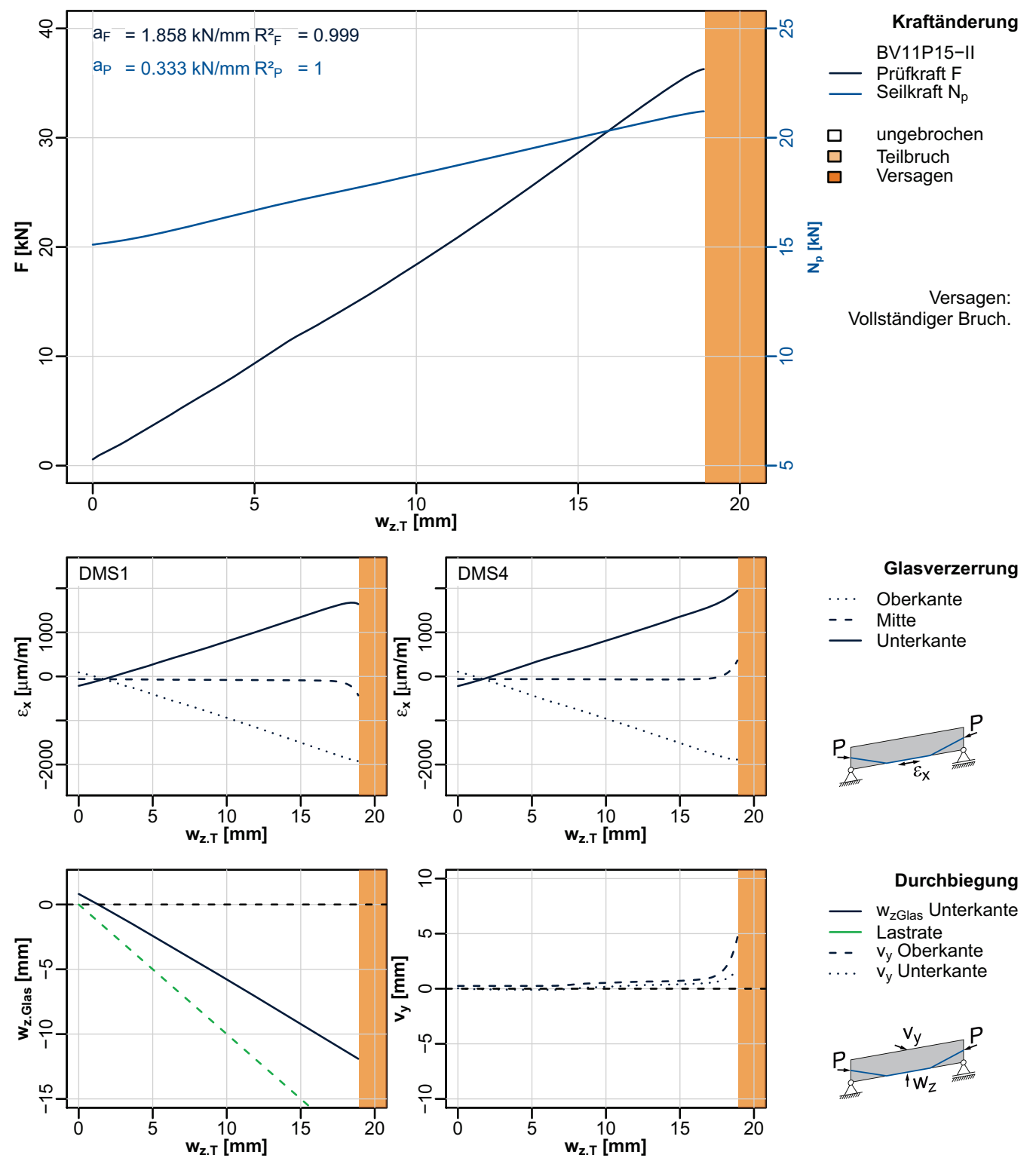

Durchbiegung

_ w $_{\text {zGlas Unterkante }}$

- Lastrate

- - $v_{y}$ Oberkante

.... $v_{\mathrm{y}}$ Unterkante

\begin{tabular}{ll}
\hline $\mathbf{F}_{\text {cr.i }}[\mathbf{k N}]$ & $\mathbf{N}_{\mathbf{p}}\left(\mathrm{F}_{\text {cr.i }}\right)[\mathbf{k N}]$ \\
\hline 36.3 & 21.2
\end{tabular}

\begin{tabular}{|c|c|c|c|}
\hline Funktion & $a_{\varepsilon}[\mu \mathrm{m} /(\mathbf{m} \times \mathbf{m m})]$ & $\mathbf{b}_{\varepsilon}[\mu \mathrm{m} / \mathrm{m}]$ & $\mathbf{R}_{\varepsilon . \min }^{2}[-]$ \\
\hline$\varepsilon_{1 \mid 4 . \text { oben }}\left(P_{0}, w_{z . T}\right)$ & -107.1 & 124.8 & 1.000 \\
\hline$\varepsilon_{1 \mid 4 . \text { unten }}\left(P_{0}, W_{z . T}\right)$ & 104.8 & -245.5 & 0.999 \\
\hline Funktion & $a_{i}[\mathrm{~mm} / \mathrm{mm}]$ & $b_{i}[\mathrm{~mm}]$ & $\left.\mathbf{R}_{\text {i.min }}{ }^{-}{ }^{-}\right]$ \\
\hline $\mathrm{W}_{\text {z.Glas }}\left(\mathrm{P}_{0}, \mathrm{~W}_{\mathrm{z} . \mathrm{T}}\right)$ & -0.67 & 0.91 & 1.000 \\
\hline$v_{y . o b e n}\left(P_{0}, w_{z . T}\right)$ & 0.037 & 0.14 & 0.895 \\
\hline$v_{y . \text { unten }}\left(P_{0}, w_{z . T}\right)$ & 0.036 & -0.18 & 0.783 \\
\hline
\end{tabular}




\section{Bruchversuch Träger mit $P_{m 0}=15 \mathrm{kN}$ und $d_{p}=8,1 \mathrm{~mm}$}
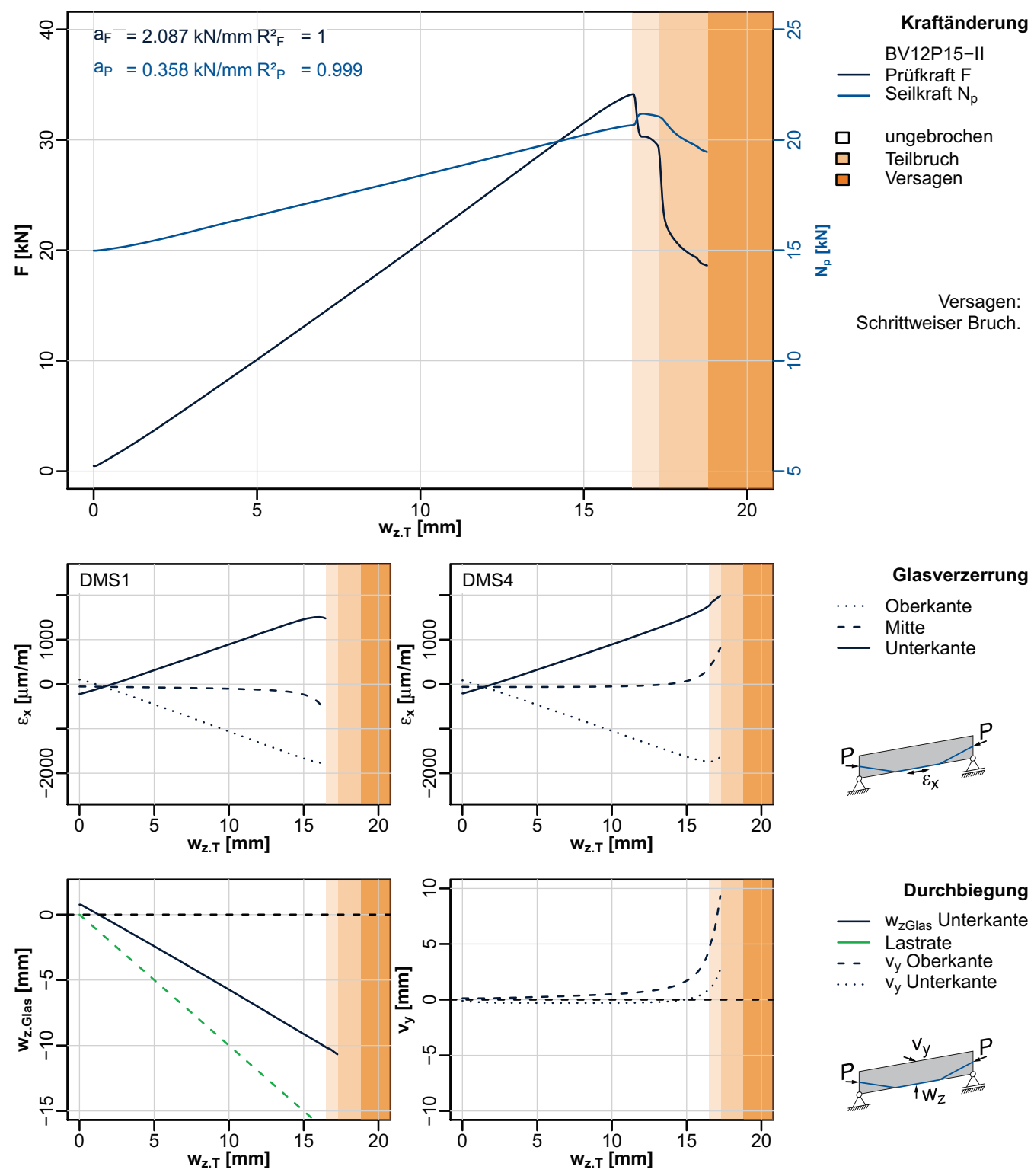

Durchbiegung

— $\mathrm{w}_{\mathrm{zGlas}}$ Unterkante

- Lastrate

- - $v_{y}$ Oberkante

.... $v_{\mathrm{y}}$ Unterkante

\begin{tabular}{ll}
\hline $\mathbf{F}_{\text {cr.i }}[\mathbf{k N}]$ & $\mathbf{N}_{\mathbf{p}}\left(\mathbf{F}_{\text {cr.i }}\right)[\mathbf{k N}]$ \\
\hline 34.1 & 20.7 \\
29.3 & 21.1 \\
16.2 & 17.1 \\
- & -
\end{tabular}

\begin{tabular}{|c|c|c|c|}
\hline Funktion & $\mathbf{a}_{\varepsilon}[\mu \mathrm{m} /(\mathbf{m} \times \mathbf{m m})]$ & $\mathbf{b}_{\varepsilon}[\mu \mathrm{m} / \mathrm{m}]$ & $\mathbf{R}_{\varepsilon, \min }^{2}[-]$ \\
\hline$\varepsilon_{1 \mid 4 . \text { oben }}\left(P_{0}, W_{z . T}\right)$ & -119.9 & 137.4 & 1.000 \\
\hline$\varepsilon_{1 \mid 4 . \text { unten }}\left(P_{0}, W_{z . T}\right)$ & 114.4 & -251.9 & 1.000 \\
\hline Funktion & $a_{i}[\mathrm{~mm} / \mathrm{mm}]$ & $\mathbf{b}_{\mathrm{i}}[\mathrm{mm}]$ & $\mathbf{R}_{\text {i.min }}$ [-] \\
\hline $\mathrm{w}_{\mathrm{z} . \mathrm{Glas}}\left(\mathrm{P}_{0}, \mathrm{w}_{\mathrm{z} . \mathrm{T}}\right)$ & -0.66 & 0.86 & 1.000 \\
\hline $\mathrm{v}_{\mathrm{y} . \text { oben }}\left(\mathrm{P}_{0}, \mathrm{w}_{\mathrm{z} . \mathrm{T}}\right)$ & 0.053 & 0.01 & 0.920 \\
\hline$v_{y . \text { unten }}\left(P_{0}, w_{z . T}\right)$ & -0.003 & -0.25 & 0.104 \\
\hline
\end{tabular}


Vorspannen Träger mit $P_{m 0}=15 \mathrm{kN}$ und $d_{p}=10,1 \mathrm{~mm}$
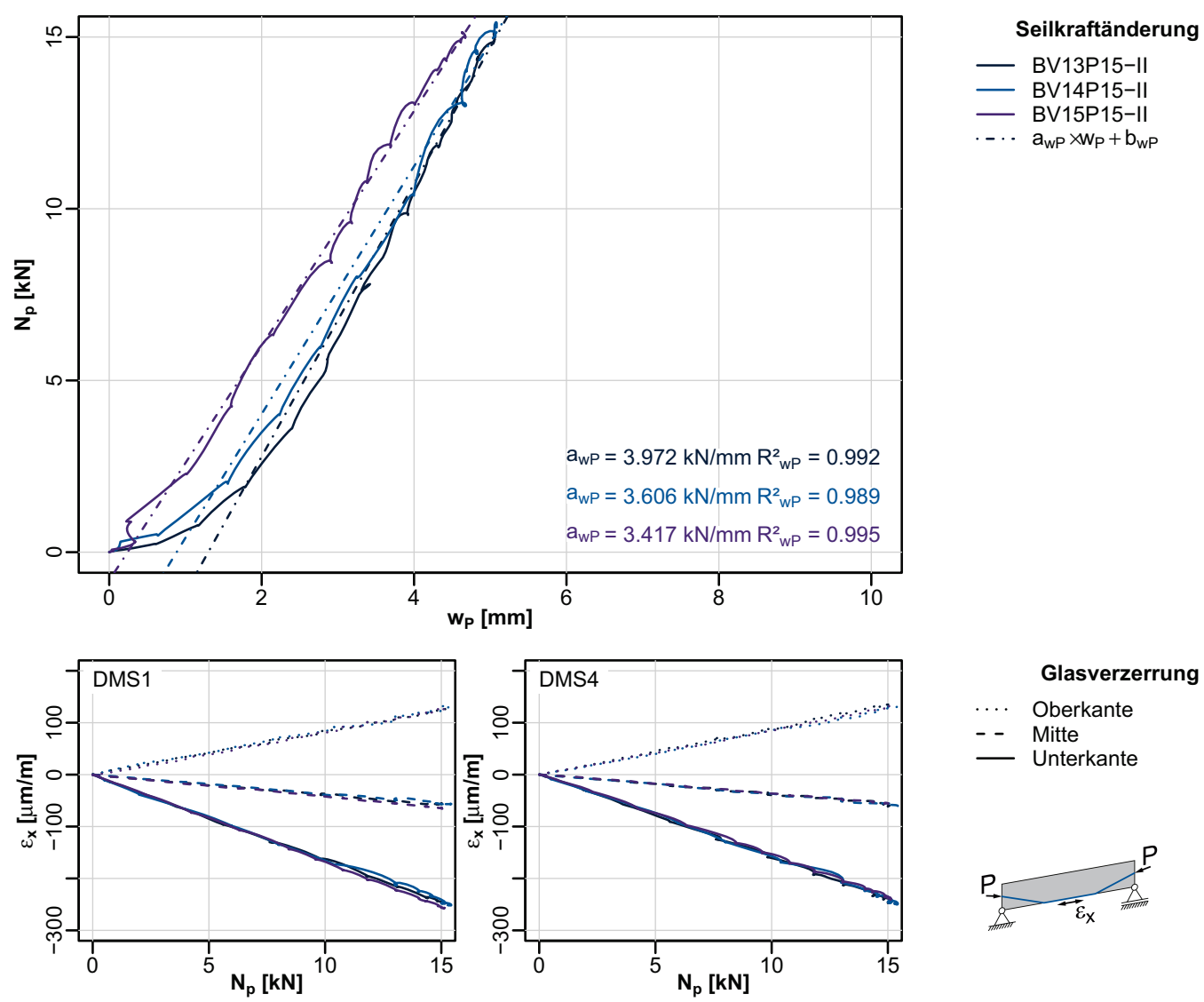
Glasverzerrung
… Oberkante
- Mitte
- Unterkante
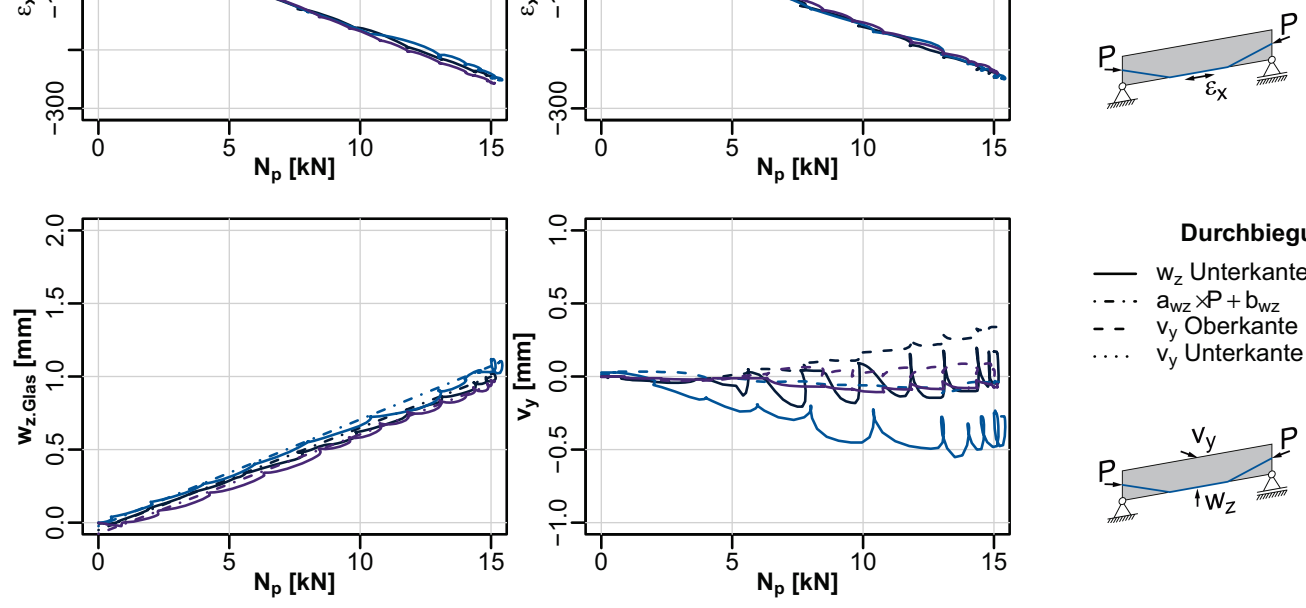

Durchbiegung

- $\mathrm{w}_{\mathrm{z}}$ Unterkante

-.. $a_{w z} \times P+b_{w z}$

- - $v_{y}$ Oberkante

.... $v_{y}$ Unterkante

\begin{tabular}{llllll}
\hline Prüfkörper & $\mathbf{a}_{\varepsilon 1 \mid 4 . o b e n}$ & $\mathbf{a}_{\varepsilon 1 \mid 4 . u n t e n}$ & $\mathbf{a}_{\mathbf{w z}}$ & \multicolumn{2}{l}{$\mathbf{v}_{\mathbf{y} \text {.oben.max }} \mathbf{v}_{\mathbf{y} \text {.unten.max }}$} \\
& {$[\mu \mathrm{m} /(\mathbf{m} \times \mathbf{k N})]$} & {$[\mu \mathrm{m} /(\mathbf{m} \times \mathbf{k N})]$} & {$[\mathbf{m m} / \mathbf{k N}]$} & {$[\mathbf{m m}]$} & {$[\mathbf{m m}]$} \\
\hline BV13P15-II & $8.4\left(\mathrm{R}^{2}=0.998\right)$ & $-16.3\left(\mathrm{R}^{2}=0.999\right)$ & $0.070\left(\mathrm{R}^{2}=0.996\right)$ & 0.170 & 0.336 \\
BV14P15-II & $8.6\left(\mathrm{R}^{2}=0.997\right)$ & $-16.2\left(\mathrm{R}^{2}=0.999\right)$ & $0.073\left(\mathrm{R}^{2}=0.996\right)$ & -0.273 & -0.0363 \\
BV15P15-II & $8.6\left(\mathrm{R}^{2}=0.999\right)$ & $-17.0\left(\mathrm{R}^{2}=0.999\right)$ & $0.068\left(\mathrm{R}^{2}=0.995\right)$ & -0.0515 & 0.00302 \\
\hline
\end{tabular}




\section{Bruchversuch Träger mit $P_{m 0}=15 \mathrm{kN}$ und $d_{p}=10,1 \mathrm{~mm}$}
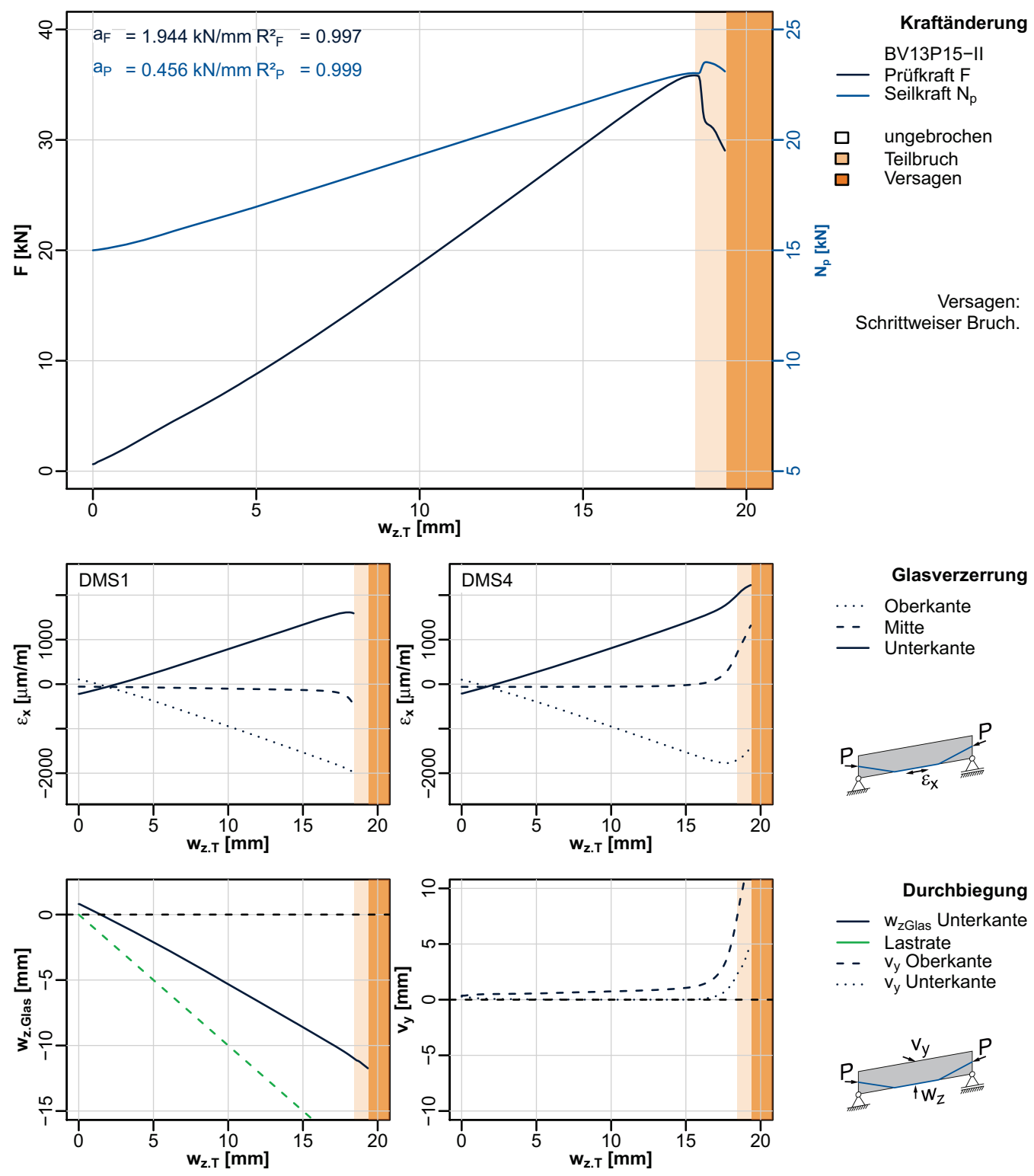

Durchbiegung

- $\mathrm{w}_{\text {zGlas }}$ Unterkante

- Lastrate

- - $v_{y}$ Oberkante

.... $v_{y}$ Unterkante

\begin{tabular}{ll}
\hline $\mathbf{F}_{\text {cr.i }}[\mathbf{k N}]$ & $\mathbf{N}_{\mathbf{p}}\left(\mathbf{F}_{\text {cr.i }}\right)[\mathbf{k N}]$ \\
\hline 35.8 & 23.0 \\
28.7 & 23.0 \\
- & - \\
- & -
\end{tabular}

\begin{tabular}{|c|c|c|c|}
\hline Funktion & $a_{\varepsilon}[\mu m /(m \times m m)]$ & $\mathbf{b}_{\varepsilon}[\mu \mathrm{m} / \mathrm{m}]$ & $\mathbf{R}_{\varepsilon \cdot \min }^{2}[-]$ \\
\hline$\varepsilon_{1 \mid 4 . \text { oben }}\left(P_{0}, w_{z . T}\right)$ & -111.2 & 161.4 & 0.999 \\
\hline$\varepsilon_{1 \mid 4 . \text { unten }}\left(P_{0}, W_{z . T}\right)$ & 105.7 & -269.4 & 0.999 \\
\hline Funktion & $a_{i}[\mathrm{~mm} / \mathrm{mm}]$ & $\mathbf{b}_{\mathrm{i}}[\mathrm{mm}]$ & $\mathbf{R}^{2}{ }_{\text {i.min }}[-]$ \\
\hline $\mathrm{W}_{\mathrm{z} . \mathrm{Glas}}\left(\mathrm{P}_{0}, \mathrm{~W}_{\mathrm{z} . \mathrm{T}}\right)$ & -0.63 & 0.99 & 0.999 \\
\hline$v_{y . o b e n}\left(P_{0}, w_{z . T}\right)$ & 0.037 & 0.40 & 0.975 \\
\hline$v_{y . \text { unten }}\left(P_{0}, w_{z . T}\right)$ & -0.01 & 0.10 & 0.564 \\
\hline
\end{tabular}




\section{Bruchversuch Träger mit $P_{m 0}=15 \mathrm{kN}$ und $d_{p}=10,1 \mathrm{~mm}$}
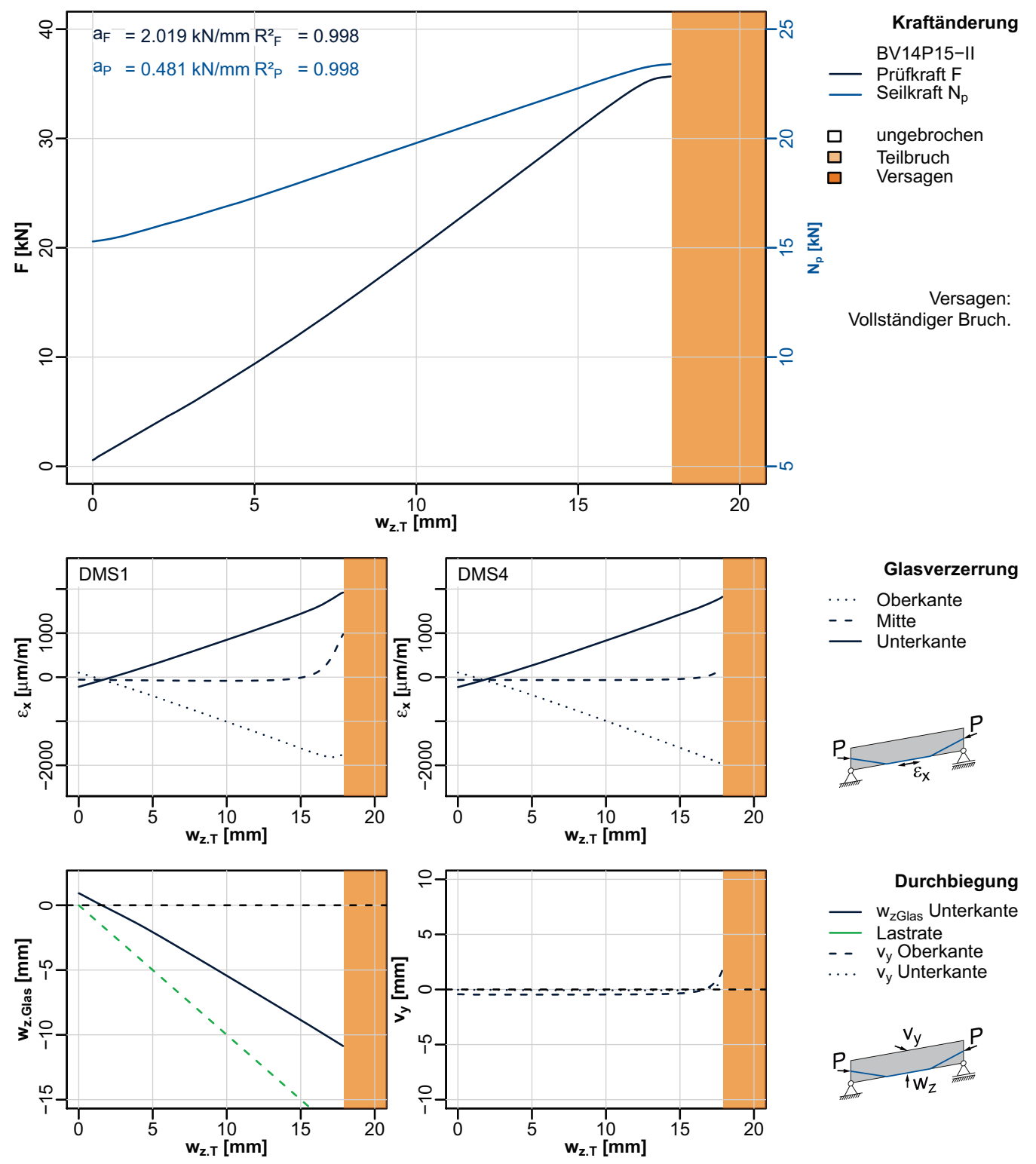

Durchbiegung

_ w $_{\text {zGlas Unterkante }}$

- Lastrate

- - $v_{y}$ Oberkante

.... $v_{\mathrm{y}}$ Unterkante

\begin{tabular}{|c|c|c|c|c|c|}
\hline $\mathrm{F}_{\text {cr.i }}[\mathrm{kN}]$ & $\mathbf{N}_{\mathrm{p}}\left(\mathrm{F}_{\mathrm{cr.i}}\right)[\mathrm{kN}]$ & Funktion & $a_{\varepsilon}[\mu \mathrm{m} /(\mathbf{m} \times \mathbf{m m})]$ & $\mathbf{b}_{\varepsilon}[\mu \mathrm{m} / \mathrm{m}]$ & $\mathbf{R}_{\varepsilon \cdot \min }^{2}[-]$ \\
\hline 35.7 & 23.4 & $\varepsilon_{1 \mid 4 . \text { oben }}\left(P_{0}, W_{z . T}\right)$ & -114.9 & 135.3 & 0.999 \\
\hline - & - & $\varepsilon_{1 \mid 4 . \text { unten }}\left(P_{0}, w_{z . T}\right)$ & 110.4 & -252.3 & 0.999 \\
\hline- & - & Funktion & $a_{i}[\mathrm{~mm} / \mathrm{mm}]$ & $\mathbf{b}_{\mathrm{i}}[\mathrm{mm}]$ & $\mathbf{R}^{2}{ }_{\text {i.min }}[-]$ \\
\hline \multirow[t]{3}{*}{-} & - & $\mathrm{w}_{\mathrm{z} . \text { Glas }}\left(\mathrm{P}_{0}, \mathrm{w}_{\mathrm{z} . \mathrm{T}}\right)$ & -0.66 & 1.11 & 0.999 \\
\hline & & $\mathrm{v}_{\mathrm{y} . \text { oben }}\left(\mathrm{P}_{0}, \mathrm{w}_{\mathrm{z} . \mathrm{T}}\right)$ & 0.002 & -0.47 & 0.421 \\
\hline & & $v_{y . \text { unten }}\left(P_{0}, w_{z . T}\right)$ & -0.003 & -0.038 & 0.769 \\
\hline
\end{tabular}




\section{Bruchversuch Träger mit $P_{m 0}=15 \mathrm{kN}$ und $d_{p}=10,1 \mathrm{~mm}$}
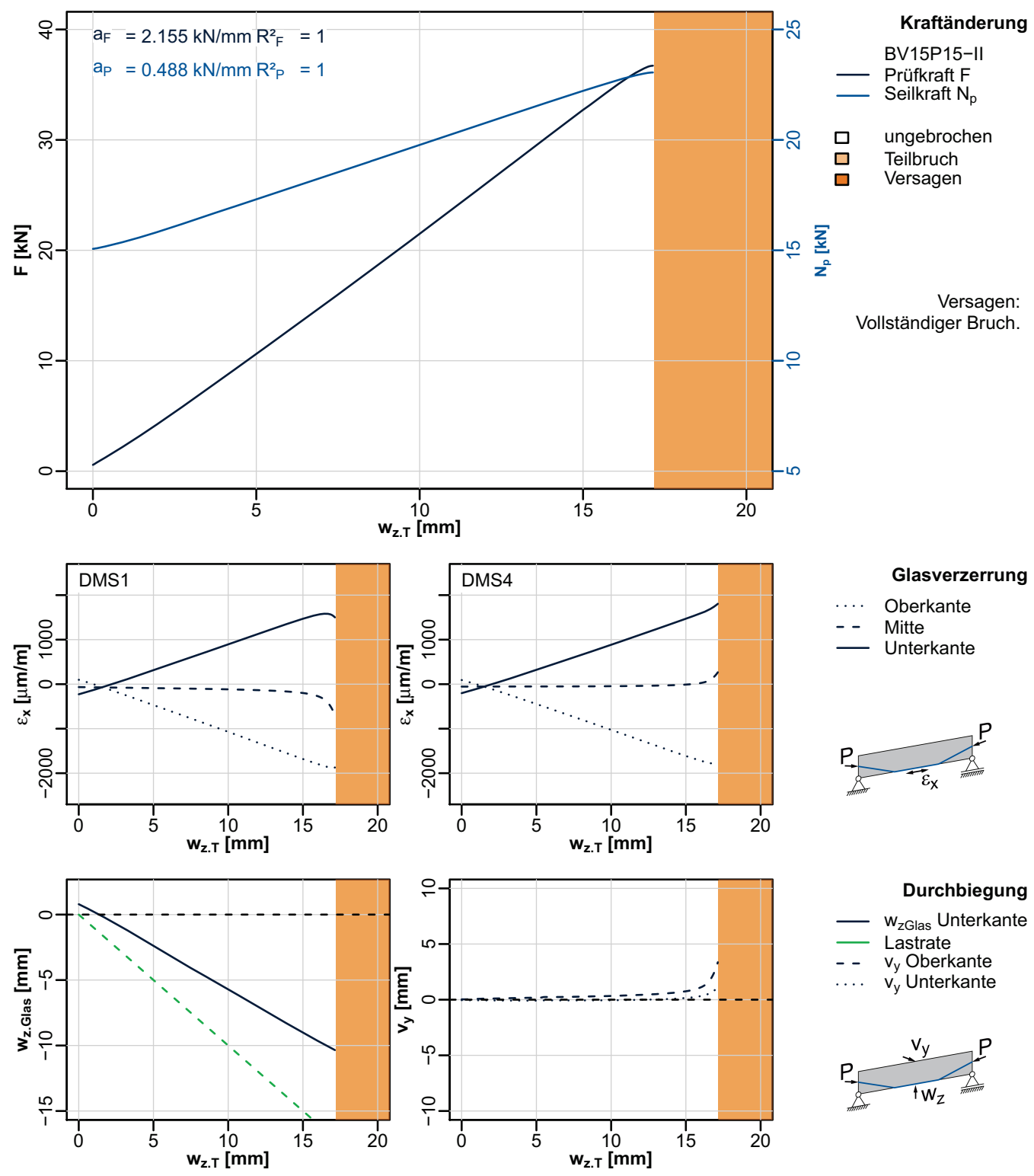

Durchbiegung

- w $_{\text {zGlas }}$ Unterkante

- Lastrate

- - $v_{y}$ Oberkante

.... $v_{\mathrm{y}}$ Unterkante

\begin{tabular}{|c|c|c|c|c|c|}
\hline $\mathrm{F}_{\mathrm{cr.i}}[\mathrm{kN}]$ & $\mathbf{N}_{\mathrm{p}}\left(\mathrm{F}_{\mathrm{cr. \textrm {i }}}\right)[\mathrm{kN}]$ & Funktion & $a_{\varepsilon}[\mu \mathrm{m} /(m \times m m)]$ & $\mathbf{b}_{\varepsilon}[\mu \mathrm{m} / \mathrm{m}]$ & $\mathbf{R}_{\varepsilon \cdot \min }^{2}[-]$ \\
\hline 36.7 & 23.1 & $\varepsilon_{1 \mid 4 . \text { oben }}\left(P_{0}, w_{z . T}\right)$ & -119.6 & 123.4 & 1.000 \\
\hline - & - & $\varepsilon_{1 \mid 4 . \text { unten }}\left(P_{0}, W_{z . T}\right)$ & 114.8 & -254.2 & 1.000 \\
\hline - & - & Funktion & $a_{i}[\mathrm{~mm} / \mathrm{mm}]$ & $\mathbf{b}_{\mathrm{i}}[\mathrm{mm}]$ & $\mathbf{R}^{2}{ }_{\text {i.min }}[-]$ \\
\hline \multirow[t]{3}{*}{ - } & - & $\mathrm{w}_{\mathrm{z} . \mathrm{Glas}}\left(\mathrm{P}_{0}, \mathrm{w}_{\mathrm{z} . \mathrm{T}}\right)$ & -0.66 & 0.90 & 1.000 \\
\hline & & $\mathrm{v}_{\mathrm{y} . \text { oben }}\left(\mathrm{P}_{0}, \mathrm{w}_{\mathrm{z} . \mathrm{T}}\right)$ & 0.034 & 0.023 & 0.978 \\
\hline & & $v_{y . \text { unten }}\left(P_{0}, w_{z . T}\right)$ & 0.006 & -0.089 & 0.488 \\
\hline
\end{tabular}




\section{Dauerversuche $1000 \mathrm{~h}$}

In dieser Anlage werden die Ergebnisse der Dauerversuche gemäß Kapitel 3.4 dokumentiert.

Die folgende Tabelle gibt einen Überblick. Darauf folgend werden die zeitabhängige Seilkraft und Verformung für jeden Versuchsdurchgang mit je vier Prüfkörpern dargestellt.

Als wesentliches Ergebnis ergibt sich der Spannkraftverlust nach $1.000 \mathrm{~h}$ in Abhängigkeit der Belastung aus Vorspannung des Seils und Biegebelastung im Vierpunkt-Biegeversuch.

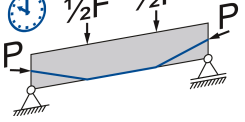

Die zugehörigen Verformungen sind als Kontrollwerte aufgenommen.

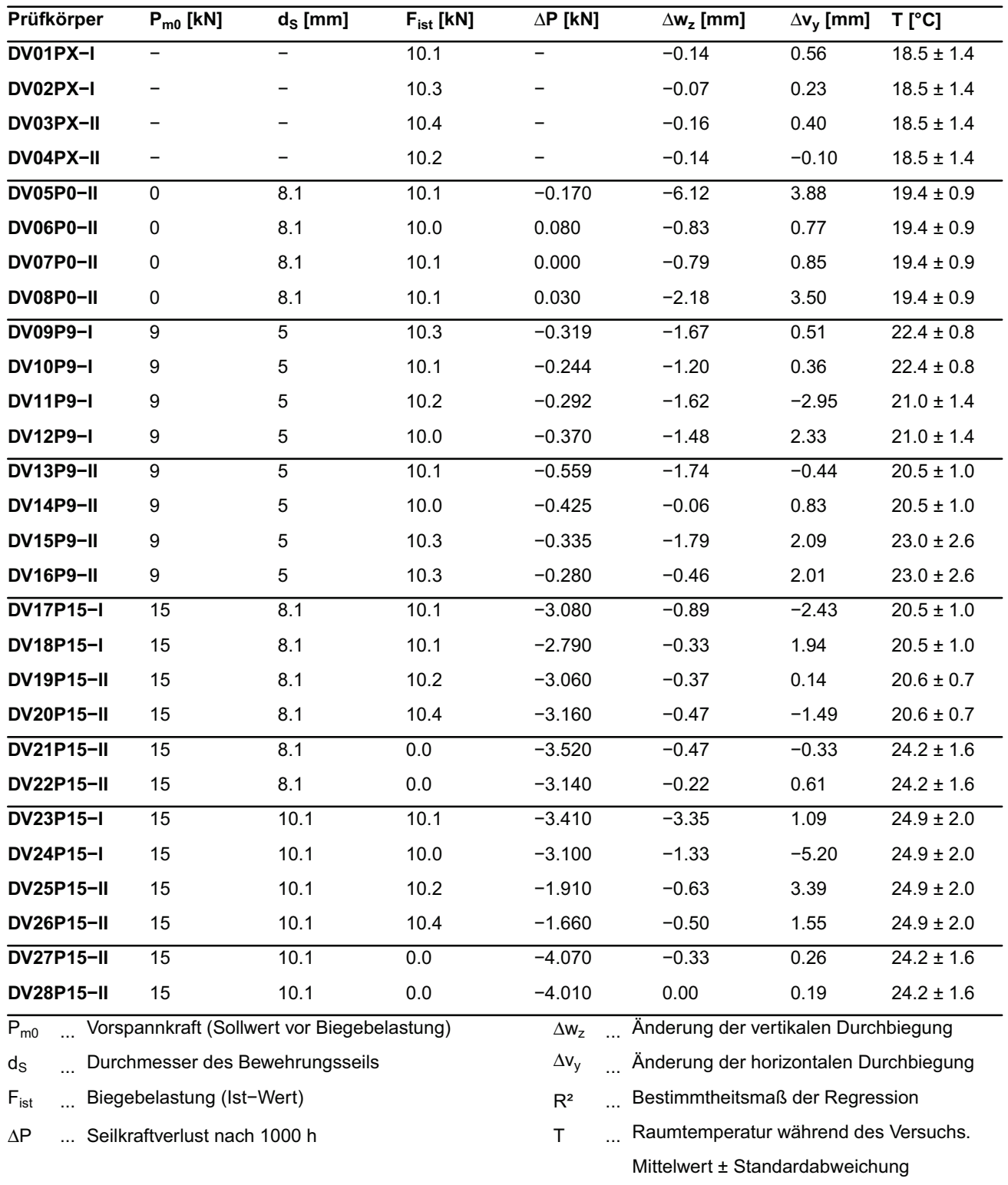


In der folgenden Tabelle ist der Zusammenhang zwischen Seilkraft und Zeit als logarithmische Regression mit den Parametern a und b sowie als Potenzfunktion mit den Parametern a, b und $c$ abgedruckt.

Das zugehörige Bestimmtheitsmaß $\mathrm{R}^{2}$ beschreibt die Güte der Regression.

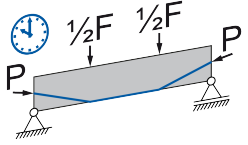

\begin{tabular}{|c|c|c|c|c|c|c|c|}
\hline Prüfkörper & $a_{\log }[k N]$ & $b_{\log }[k N]$ & $R^{2}[-]$ & $a_{\text {pot }}[k N]$ & $\mathbf{b}_{\text {pot }}[-]$ & $\mathrm{c}_{\mathrm{pot}}[\mathrm{kN}]$ & $R^{2}[-]$ \\
\hline DV01PX-I & - & - & - & - & - & - & - \\
\hline DV02PX-I & - & - & - & - & - & - & - \\
\hline DV03PX-II & - & - & - & - & - & - & - \\
\hline DV04PX-II & - & - & - & - & - & - & - \\
\hline DV05P0-II & -0.045 & 1.552 & 0.610 & - & - & - & - \\
\hline DV06P0-II & 0.008 & 1.556 & 0.640 & - & - & - & - \\
\hline DV07P0-II & -0.004 & 1.147 & 0.260 & - & - & - & - \\
\hline DV08P0-II & 0.007 & 1.185 & 0.410 & - & - & - & - \\
\hline DV09P9-I & -0.033 & 9.248 & 0.980 & -0.289 & 0.0896 & 9.543 & 0.993 \\
\hline DV10P9-I & -0.027 & 9.371 & 0.970 & -0.119 & 0.148 & 9.499 & 0.992 \\
\hline DV11P9-I & -0.036 & 9.517 & 0.770 & -0.0286 & 0.323 & 9.516 & 0.935 \\
\hline DV12P9-I & -0.035 & 9.410 & 0.890 & -0.335 & 0.0805 & 9.746 & 0.904 \\
\hline DV13P9-II & -0.051 & 8.861 & 0.980 & -0.452 & 0.0904 & 9.332 & 0.996 \\
\hline DV14P9-II & -0.046 & 8.826 & 0.930 & -0.157 & 0.167 & 8.981 & 0.998 \\
\hline DV15P9-II & -0.028 & 9.391 & 0.740 & -0.0106 & 0.475 & 9.396 & 0.957 \\
\hline DV16P9-II & -0.035 & 9.299 & 0.650 & - & - & - & - \\
\hline DV17P15-I & -0.371 & 15.651 & 0.960 & -1.762 & 0.129 & 17.279 & 0.997 \\
\hline DV18P15-I & -0.324 & 15.233 & 0.930 & -1.220 & 0.156 & 16.417 & 0.996 \\
\hline DV19P15-II & -0.402 & 15.776 & 0.970 & -1.573 & 0.147 & 17.198 & 0.998 \\
\hline DV20P15-II & -0.412 & 15.452 & 0.980 & -2.039 & 0.127 & 17.358 & 0.997 \\
\hline DV21P15-II & -0.321 & 13.942 & 0.980 & -2.395 & 0.107 & 16.450 & 0.999 \\
\hline DV22P15-II & -0.295 & 14.292 & 0.970 & -1.809 & 0.118 & 16.188 & 0.999 \\
\hline DV23P15-I & -0.343 & 16.411 & 0.970 & -14.829 & 0.0231 & 31.293 & 0.984 \\
\hline DV24P15-I & -0.312 & 15.008 & 0.930 & -0.971 & 0.182 & 16.055 & 0.994 \\
\hline DV25P15-II & -0.239 & 17.712 & 0.850 & -0.180 & 0.340 & 17.711 & 0.996 \\
\hline DV26P15-II & -0.201 & 17.297 & 0.830 & -0.141 & 0.357 & 17.330 & 0.990 \\
\hline DV27P15-II & -0.383 & 13.738 & 0.980 & -3.085 & 0.0958 & 16.918 & 0.999 \\
\hline DV28P15-II & -0.364 & 13.659 & 0.980 & -3.205 & 0.089 & 16.961 & 0.998 \\
\hline
\end{tabular}

$\mathrm{a}_{\log } ; \mathrm{b}_{\log } \quad \ldots$ Regressionsparameter $\mathrm{P}(\mathrm{t})=\mathrm{a} \rtimes \mathrm{n}(\mathrm{t} / \mathrm{h})+\mathrm{b} \quad \mathrm{a}_{\mathrm{pot}} ; \mathrm{b}_{\text {pot }} ; \mathrm{c}_{\mathrm{pot}} \quad \ldots$ Regressionsparameter $\mathrm{P}(\mathrm{t})=\mathrm{a} \times(\mathrm{t} / \mathrm{h})^{\mathrm{b}}+\mathrm{c}$

$\mathrm{R}^{2} \quad$...zugehöriges Bestimmtheitsmaß $\quad \mathrm{R}^{2} \quad$... zugehöriges Bestimmtheitsmaß 


\section{Dauerversuch Referenzträger unbewehrt}

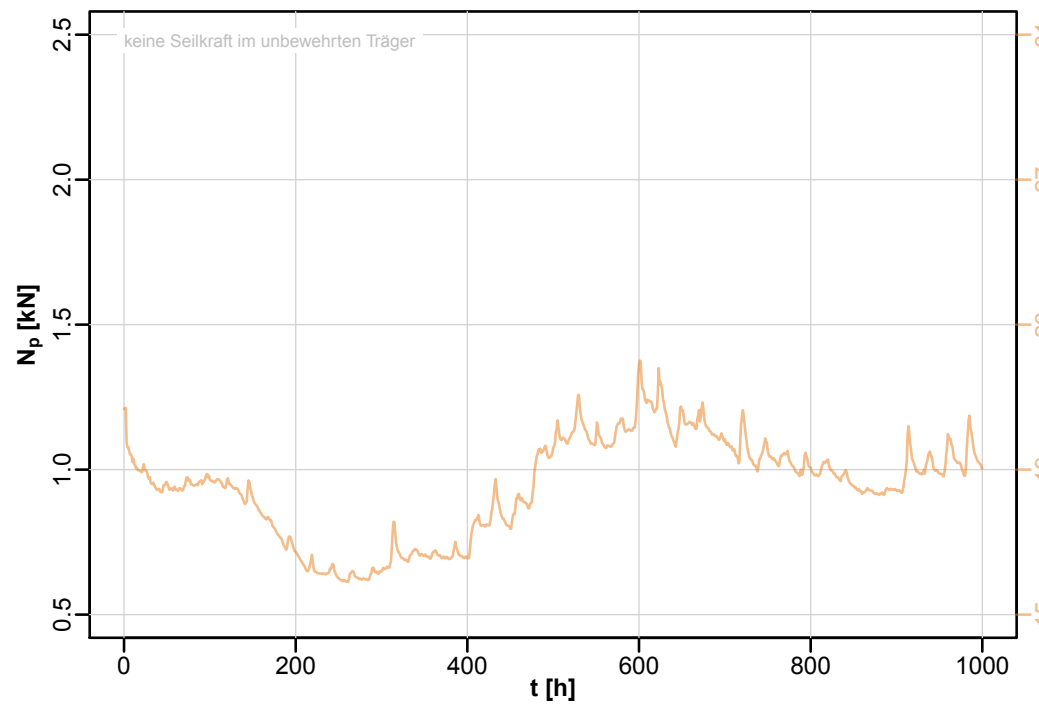

Seilkraftänderung

- DV01PX-I

- DV02PX-1

- DV03PX-II

- DV04PX-II

$\cdots a \times n(t / h)+b$

Raumtemperatur

$18.5^{\circ} \mathrm{C} \pm 1.4{ }^{\circ} \mathrm{C}$

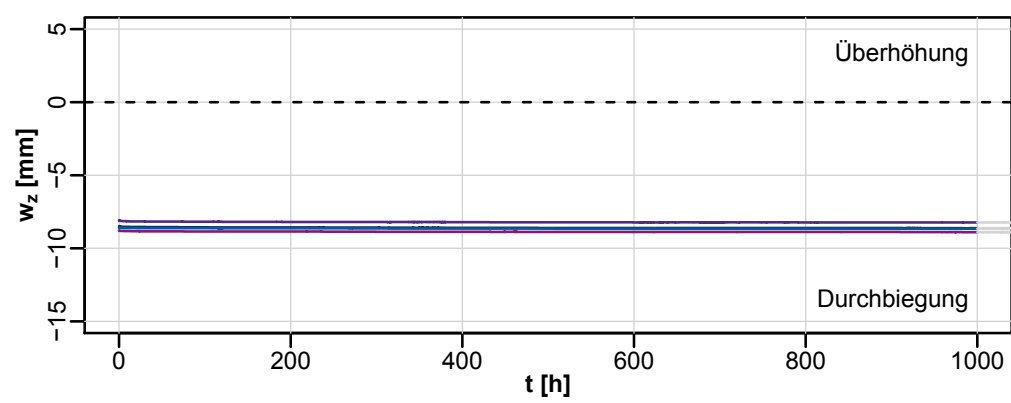

Verformung vertika

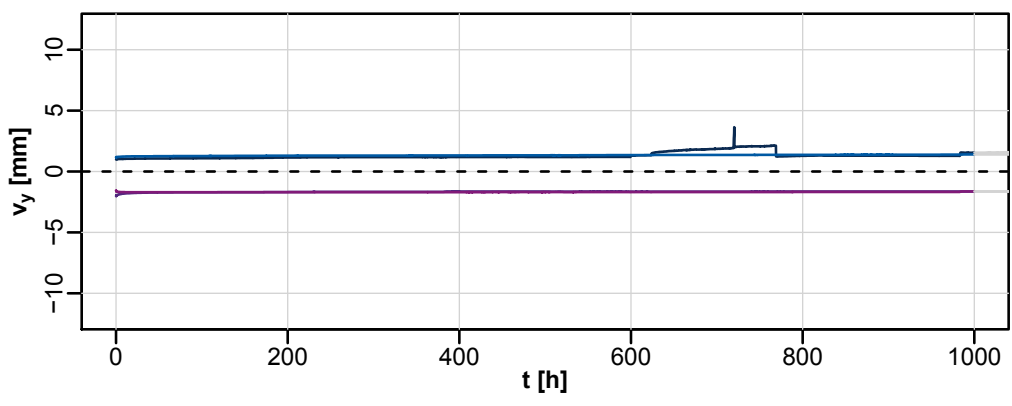

Verformung horizonta
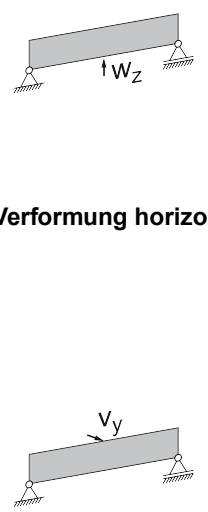

\begin{tabular}{lllllll}
\hline Prüfkörper & $\mathbf{N}_{\mathbf{p} . \max }[\mathrm{kN}]$ & $\mathbf{N}_{\mathbf{p . 1 0 0 0 ~ h}}[\mathbf{k N}]$ & $\mathbf{w}_{\mathbf{z . 0}}[\mathrm{mm}]$ & $\mathbf{w}_{\mathbf{z . 1 0 0 0} \mathbf{h}}[\mathrm{mm}]$ & $\mathbf{v}_{\mathbf{y} . \mathbf{0}}[\mathrm{mm}]$ & $\mathbf{v}_{\mathbf{y} .1000 \mathrm{~h}}[\mathrm{~mm}]$ \\
\hline DV01PX-I & - & - & -8.48 & -8.62 & 0.99 & 1.55 \\
DV02PX-I & - & - & -8.59 & -8.66 & 1.17 & 1.40 \\
DV03PX-II & - & - & -8.07 & -8.23 & -2.04 & -1.64 \\
DV04PX-II & - & - & -8.76 & -8.90 & -1.54 & -1.64 \\
\hline
\end{tabular}




\section{Dauerversuch Referenzträger schlaff bewehrt $d_{s}=8,1 \mathrm{~mm}$}
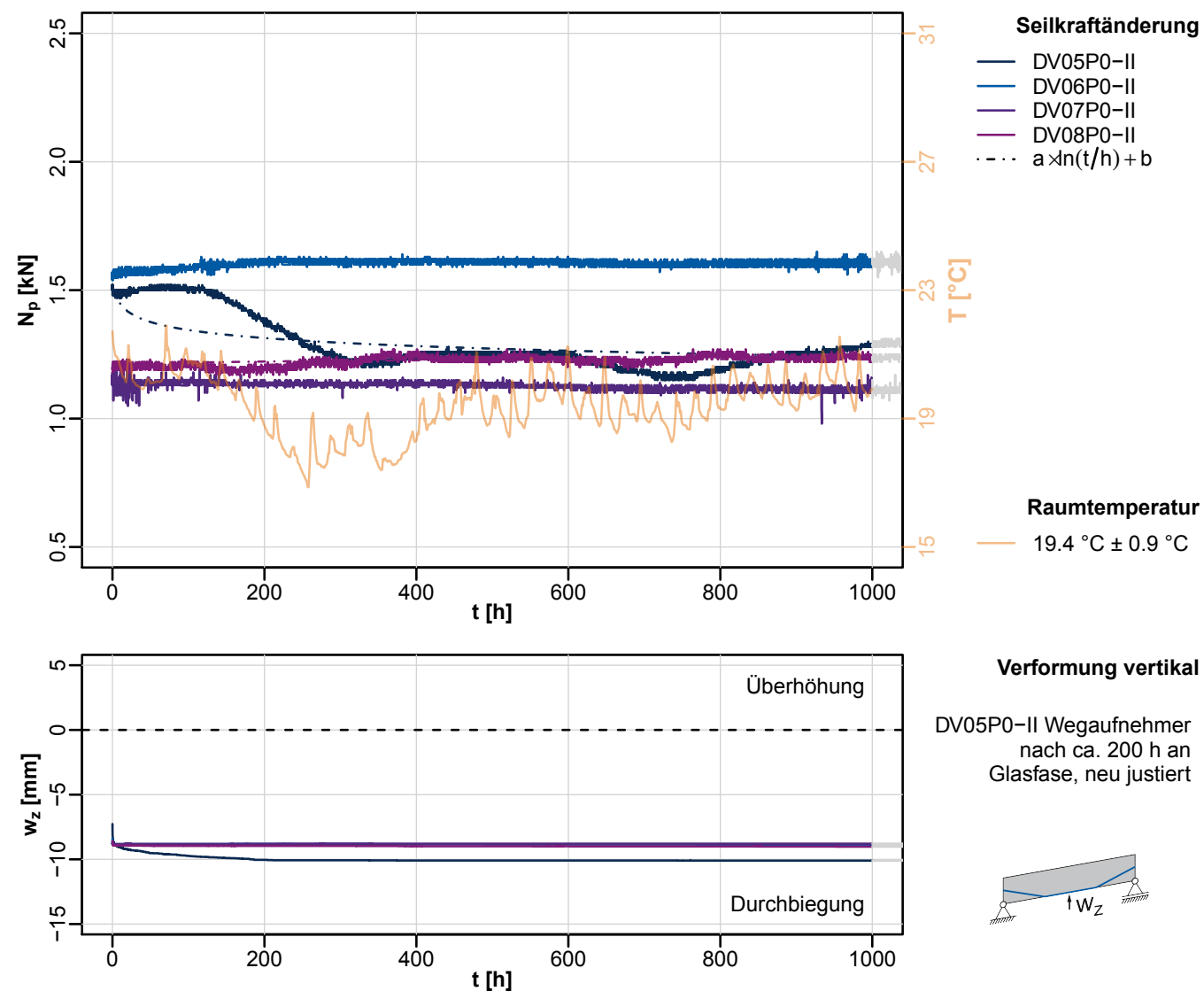

Verformung vertika

DV05P0-II Wegaufnehmer nach ca. $200 \mathrm{~h}$ an Glasfase, neu justiert
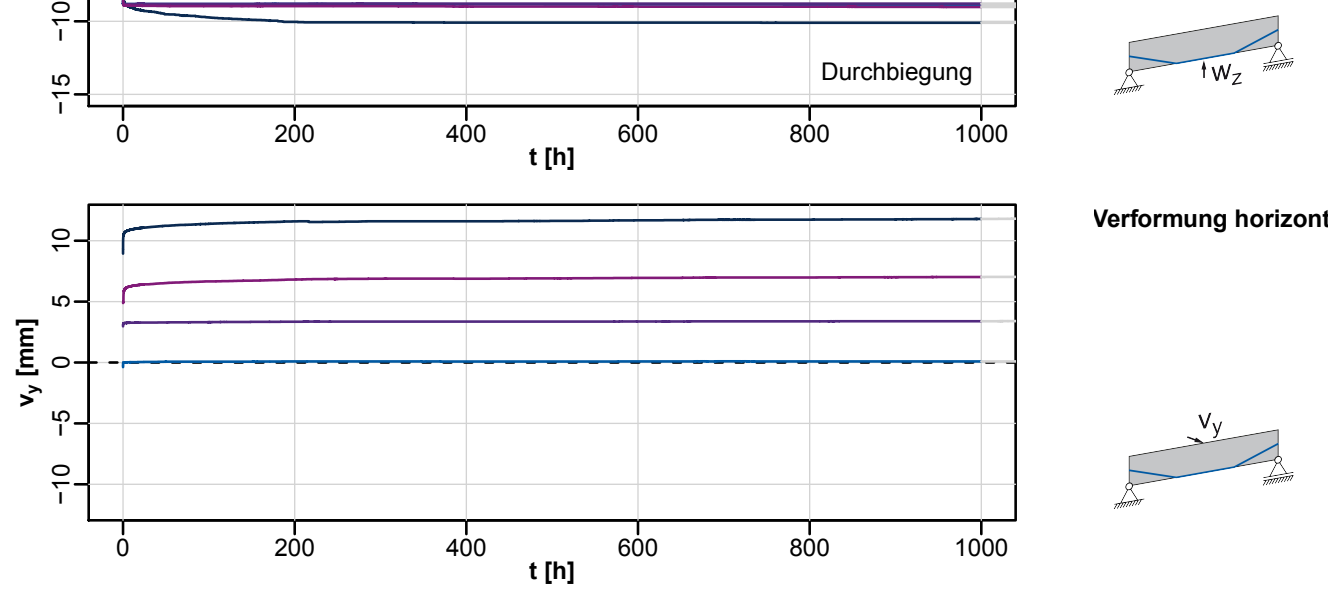

Verformung horizontal

\begin{tabular}{lllllll}
\hline Prüfkörper & $\mathbf{N}_{\mathbf{p} . \max }[\mathbf{k N}]$ & $\mathbf{N}_{\mathbf{p . 1 0 0 0 ~ h}}[\mathbf{k N}]$ & $\mathbf{w}_{\mathbf{z . 0}}[\mathbf{m m}]$ & $\mathbf{w}_{\mathbf{z . 1 0 0 0} \mathbf{h}}[\mathbf{m m}]$ & $\mathbf{v}_{\mathbf{y} . \mathbf{0}}[\mathrm{mm}]$ & $\mathbf{v}_{\mathbf{y} .1000 \mathrm{~h}}[\mathbf{m m}]$ \\
\hline DV05P0-II & 1.450 & 1.280 & -3.98 & -10.10 & 7.91 & 11.79 \\
DV06P0-II & 1.540 & 1.620 & -8.03 & -8.86 & -0.68 & 0.09 \\
DV07P0-II & 1.150 & 1.150 & -8.00 & -8.79 & 2.54 & 3.39 \\
DV08P0-II & 1.210 & 1.240 & -6.84 & -9.02 & 3.52 & 7.02 \\
\hline
\end{tabular}




\section{Dauerversuch Träger mit $P_{m 0}=9 \mathrm{kN}$ und $d_{p}=5,0 \mathrm{~mm}$}
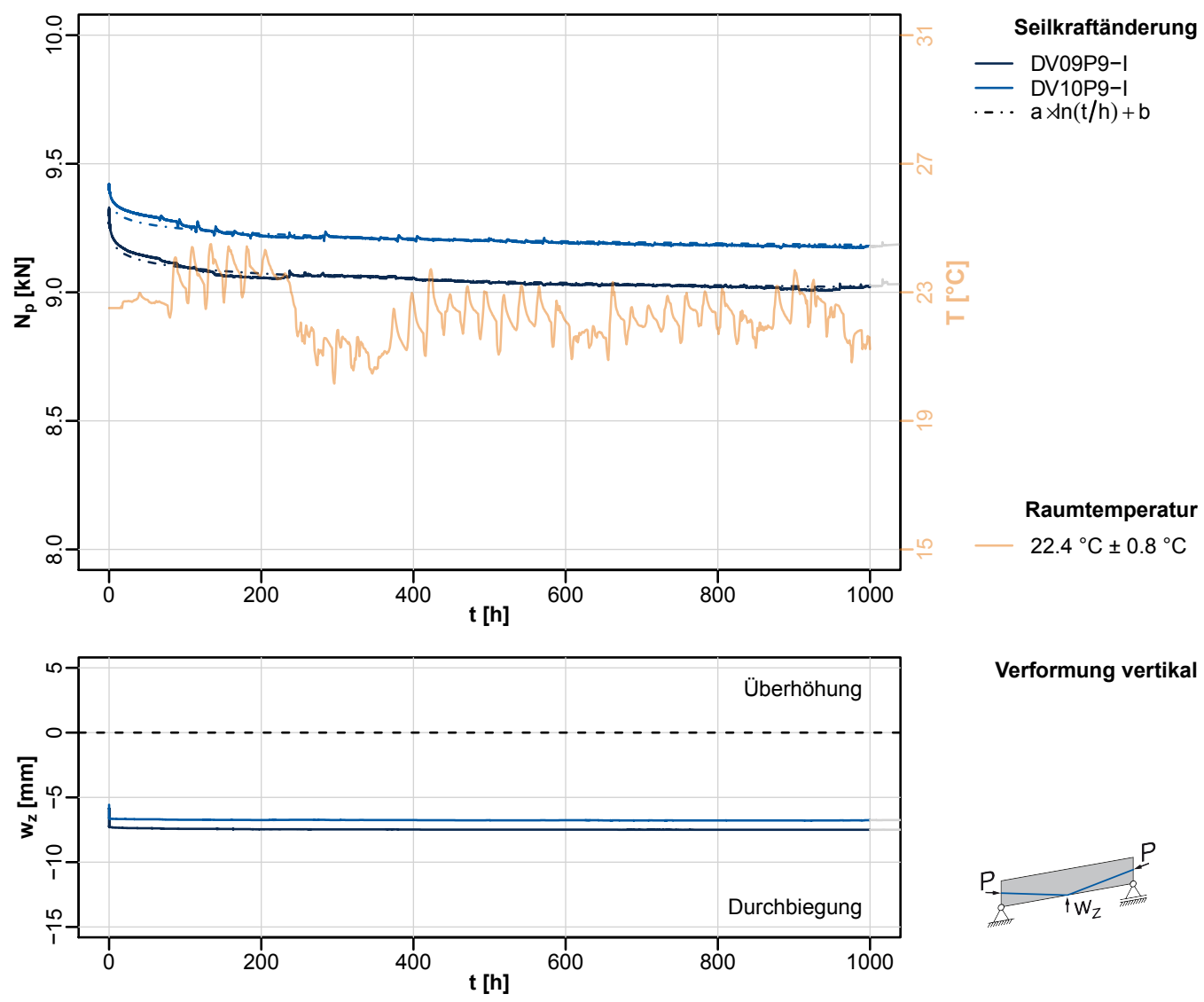

Verformung vertikal
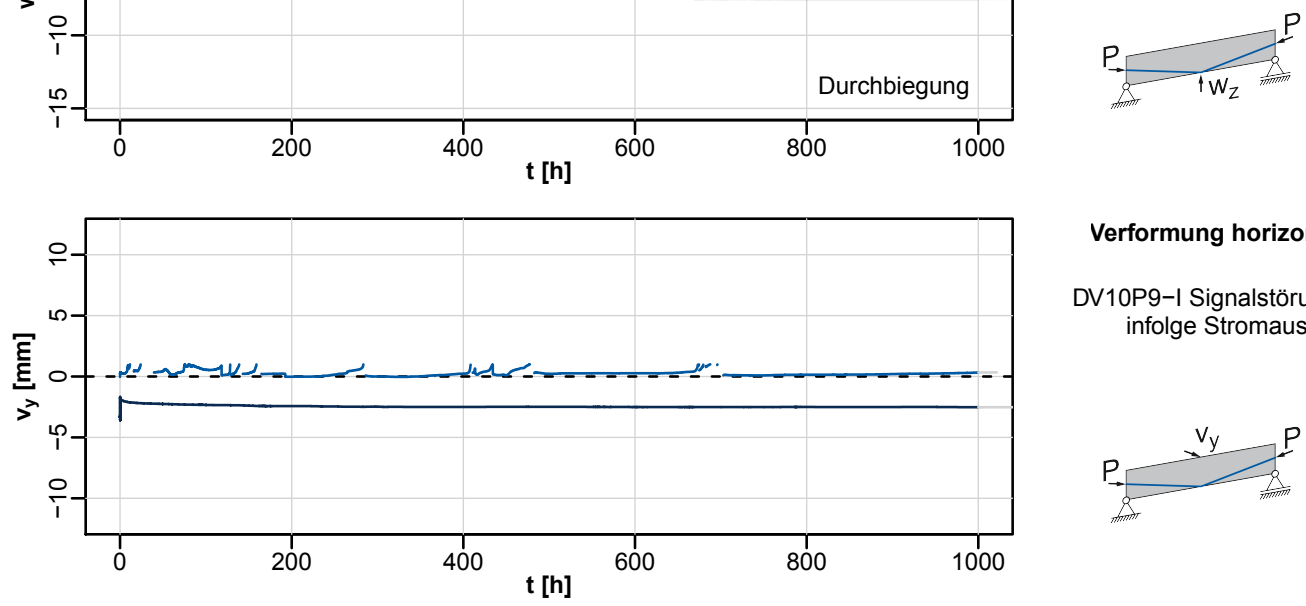

Verformung horizontal

DV10P9-I Signalstörung infolge Stromausfall

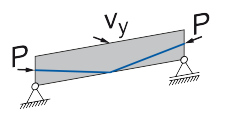

\begin{tabular}{lllllll}
\hline Prüfkörper & $\mathbf{N}_{\text {p.max }}[\mathrm{kN}]$ & $\mathbf{N}_{\mathbf{p} .1000 \mathrm{~h}}[\mathrm{kN}]$ & $\mathbf{w}_{\mathbf{z . 0}}[\mathrm{mm}]$ & $\mathbf{w}_{\mathbf{z . 1 0 0 0} \mathrm{h}}[\mathrm{mm}]$ & $\mathbf{v}_{\mathbf{y} . \mathbf{0}}[\mathrm{mm}]$ & $\mathbf{v}_{\mathbf{y} .1000 \mathrm{~h}}[\mathrm{~mm}]$ \\
\hline DV09P9-I & 9.344 & 9.025 & -5.83 & -7.50 & -3.03 & -2.52 \\
DV10P9-I & 9.422 & 9.178 & -5.55 & -6.75 & -0.03 & 0.33 \\
\hline
\end{tabular}




\section{Dauerversuch Träger mit $P_{m 0}=9 \mathrm{kN}$ und $d_{p}=5,0 \mathrm{~mm}$}
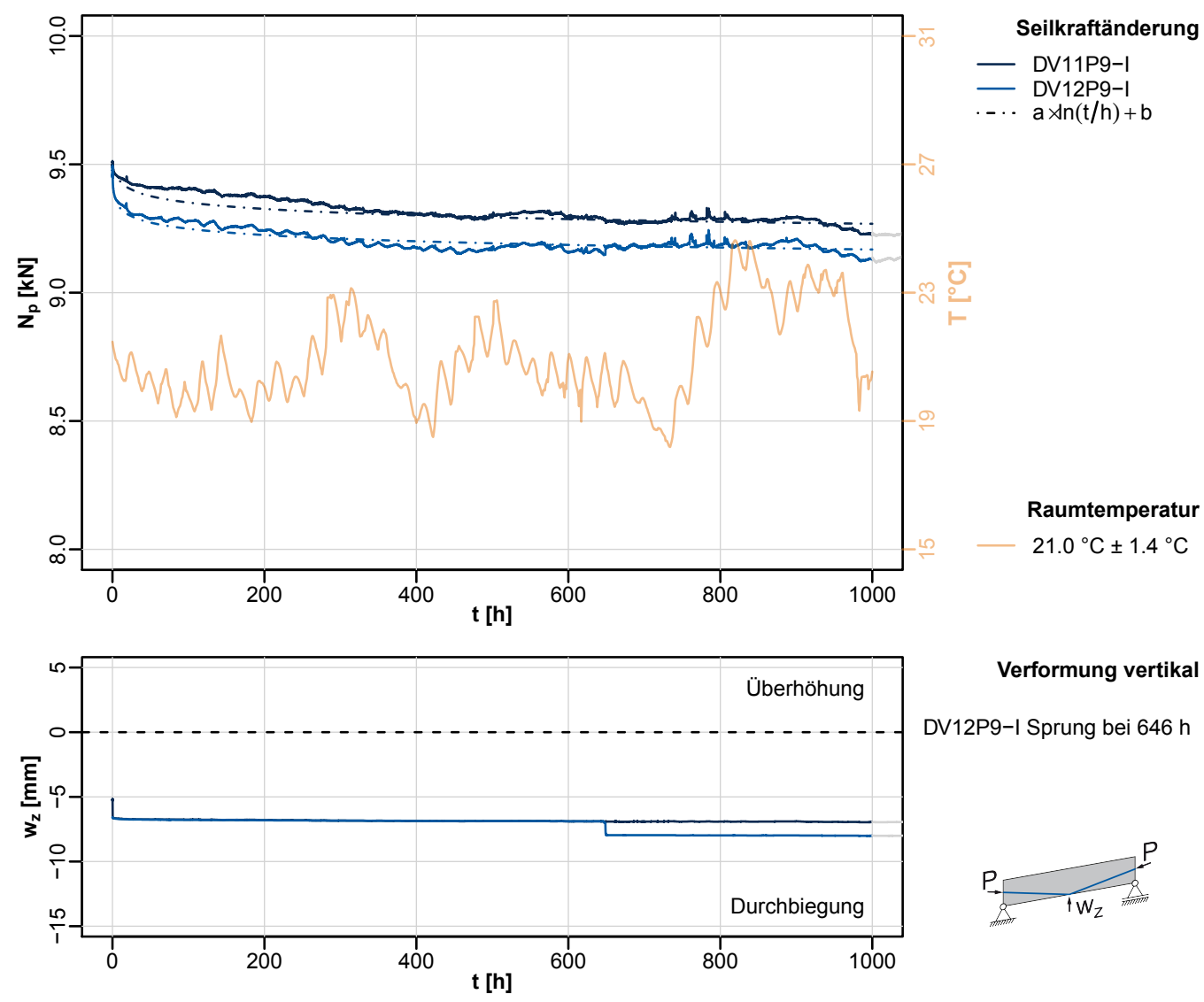

Verformung vertikal DV12P9-I Sprung bei $646 \mathrm{~h}$

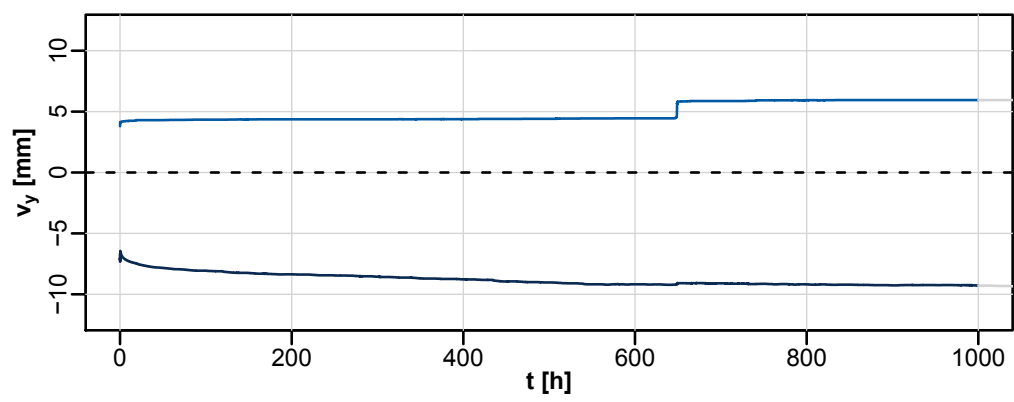

Verformung horizontal DV12P9-I Sprung bei $646 \mathrm{~h}$

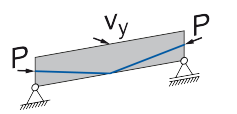

\begin{tabular}{|c|c|c|c|c|c|c|}
\hline Prüfkörper & $\mathbf{N}_{\text {p.max }}[\mathrm{kN}]$ & $N_{p .1000 h}[k N]$ & $\mathrm{w}_{\mathrm{z} .0}[\mathrm{~mm}]$ & $\mathrm{w}_{\mathrm{z} .1000 \mathrm{~h}}[\mathrm{~mm}]$ & $\mathrm{v}_{\mathrm{y} .0}[\mathrm{~mm}]$ & $v_{y .1000 h}[\mathrm{~mm}]$ \\
\hline DV11P9-I & 9.516 & 9.224 & -5.33 & -6.95 & -6.33 & -9.28 \\
\hline DV12P9-I & 9.507 & 9.137 & -6.52 & -8.00 & 3.62 & 5.95 \\
\hline
\end{tabular}




\section{Dauerversuch Träger mit $P_{m 0}=9 \mathrm{kN}$ und $d_{p}=5,0 \mathrm{~mm}$}

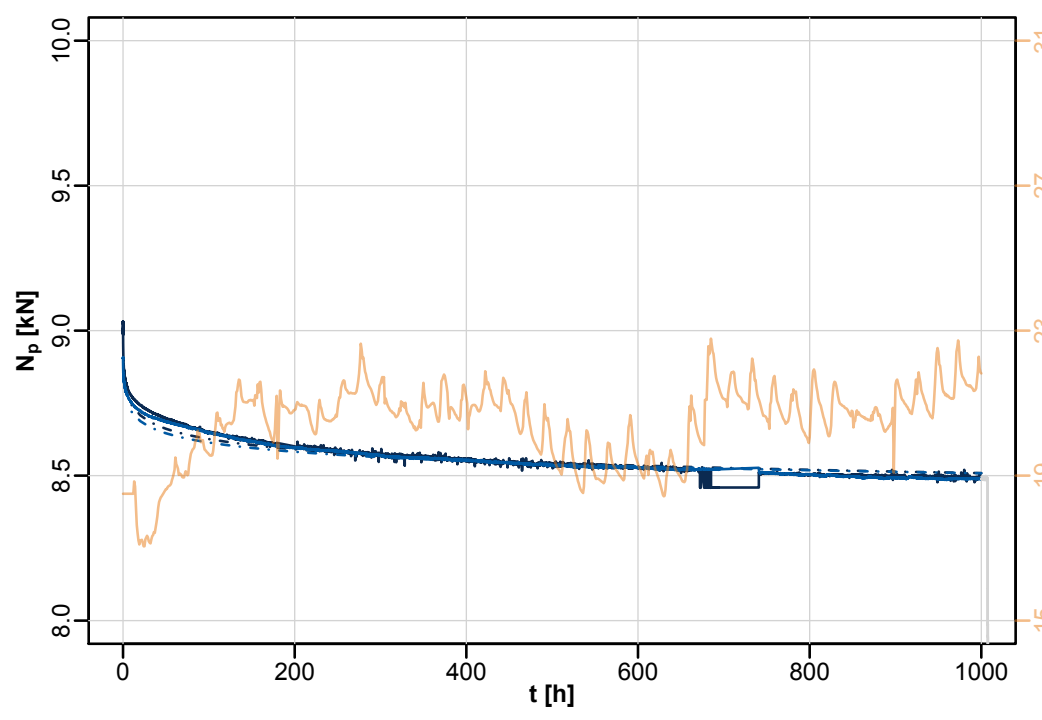

Seilkraftänderung

- DV13P9-II

DV14P9-II

- $a \times y n(t / h)+b$

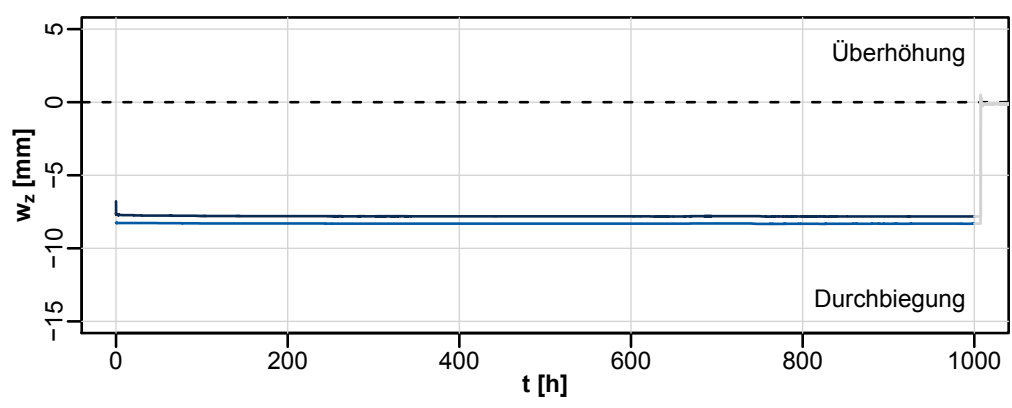

Verformung vertika

Raumtemperatur

$20.5^{\circ} \mathrm{C} \pm 1.0^{\circ} \mathrm{C}$
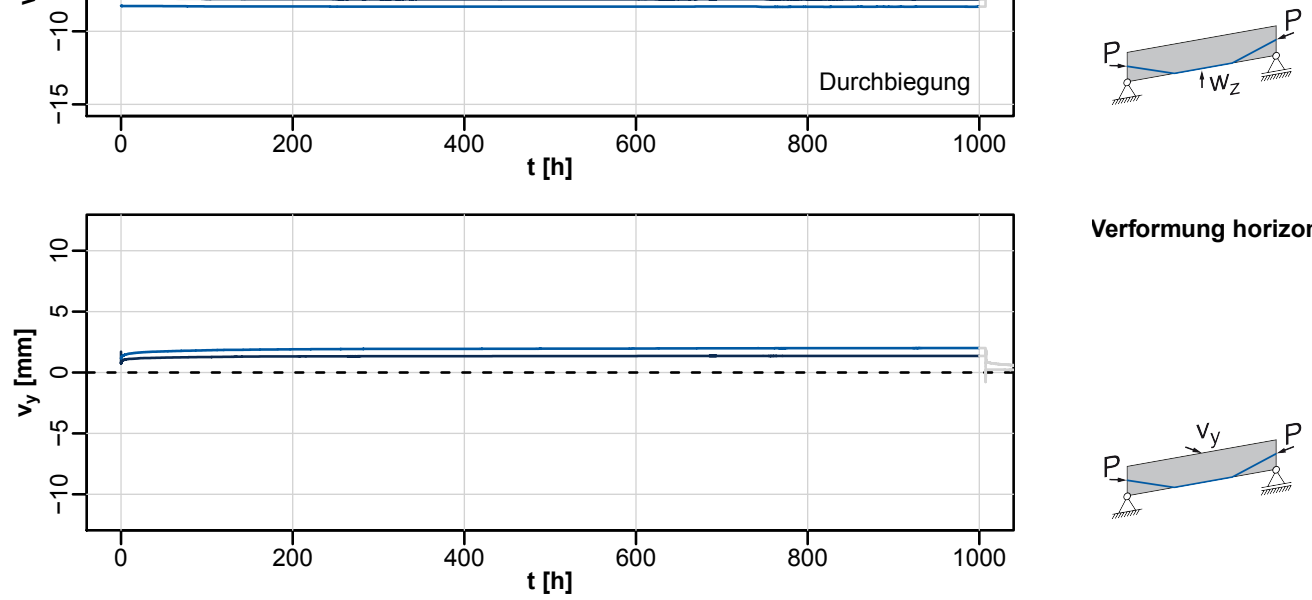

Verformung horizonta

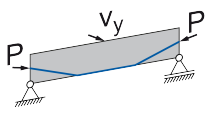

\begin{tabular}{lllllll}
\hline Prüfkörper & $\mathbf{N}_{\mathbf{p} . \max }[\mathbf{k N}]$ & $\mathbf{N}_{\mathbf{p} .1000 \mathrm{~h}}[\mathrm{kN}]$ & $\mathbf{w}_{\mathbf{z . 0}}[\mathrm{mm}]$ & $\mathbf{w}_{\mathbf{z . 1 0 0 0 h} \mathbf{h}}[\mathrm{mm}]$ & $\mathbf{v}_{\mathbf{y} . \mathbf{0}}[\mathrm{mm}]$ & $\mathbf{v}_{\mathbf{y} .1000 \mathrm{~h}}[\mathrm{~mm}]$ \\
\hline DV13P9-II & 9.053 & 8.494 & -6.08 & -7.82 & 1.80 & 1.36 \\
DV14P9-II & 8.913 & 8.488 & -8.25 & -8.31 & 1.18 & 2.01
\end{tabular}




\section{Dauerversuch Träger mit $P_{m 0}=9 \mathrm{kN}$ und $d_{p}=5,0 \mathrm{~mm}$}

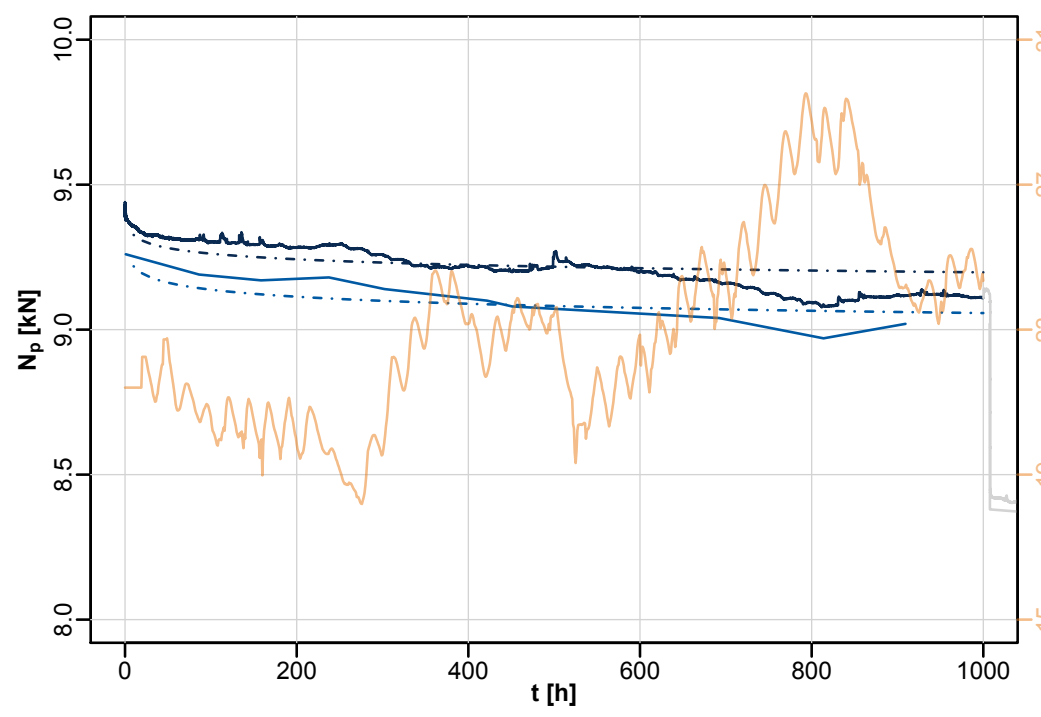

Seilkraftänderung

- DV15P9-II

DV16P9-II

- $\cdot a \rtimes n(t / h)+b$

DV16P9-II manuelle

Datenaufzeichnung

aufgrund technischen

Defekts

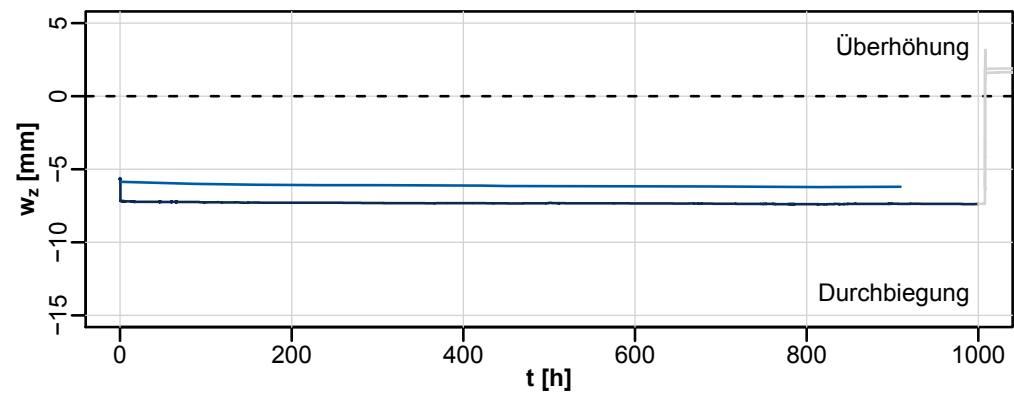

Verformung vertikal

Raumtemperatur

$23.0^{\circ} \mathrm{C} \pm 2.6^{\circ} \mathrm{C}$
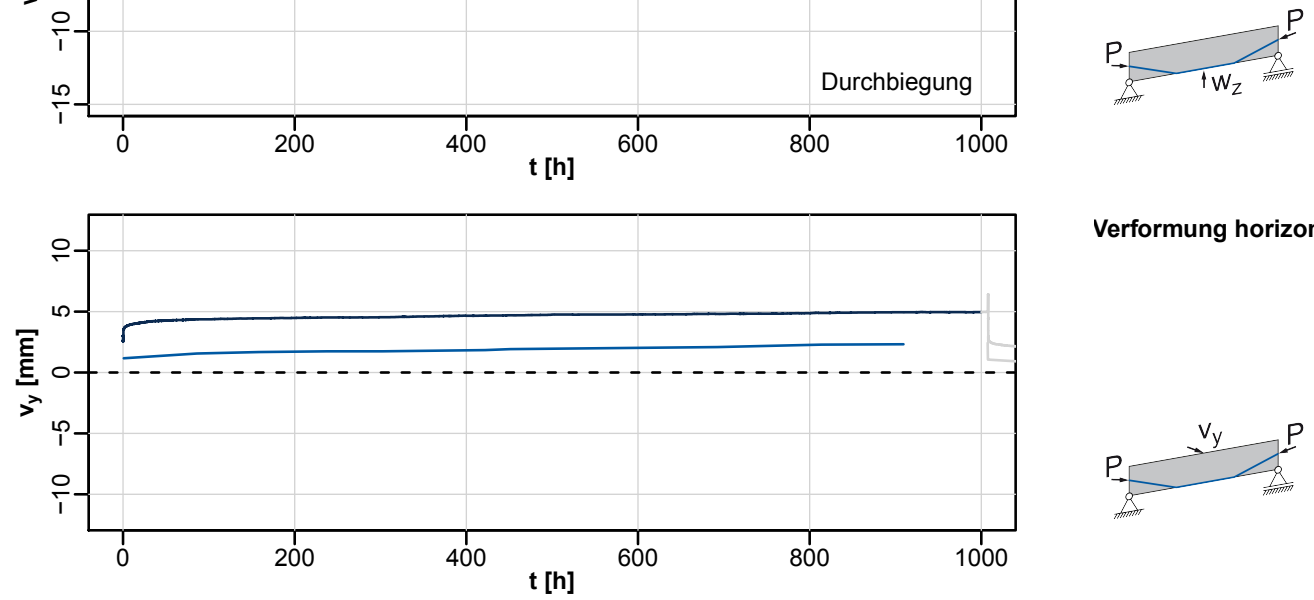

Verformung horizontal

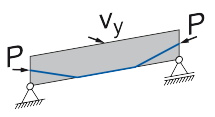

\begin{tabular}{|c|c|c|c|c|c|c|}
\hline Prüfkörper & $\mathbf{N}_{\text {p.max }}[\mathrm{kN}]$ & $N_{p .1000 h}[k N]$ & $\mathrm{w}_{\mathrm{z} .0}[\mathrm{~mm}]$ & $\mathrm{w}_{\mathrm{z} .1000 \mathrm{~h}}[\mathrm{~mm}]$ & $\mathrm{v}_{\mathrm{y} .0}[\mathrm{~mm}]$ & $\mathrm{v}_{\mathrm{y} .1000 \mathrm{~h}}[\mathrm{~mm}]$ \\
\hline DV15P9-II & 9.445 & 9.110 & -5.59 & -7.38 & 2.87 & 4.96 \\
\hline DV16P9-II & 9.300 & 9.020 & -5.79 & -6.25 & 0.42 & 2.43 \\
\hline
\end{tabular}




\section{Dauerversuch Träger mit $P_{m 0}=15 \mathrm{kN}$ und $d_{p}=8,1 \mathrm{~mm}$}
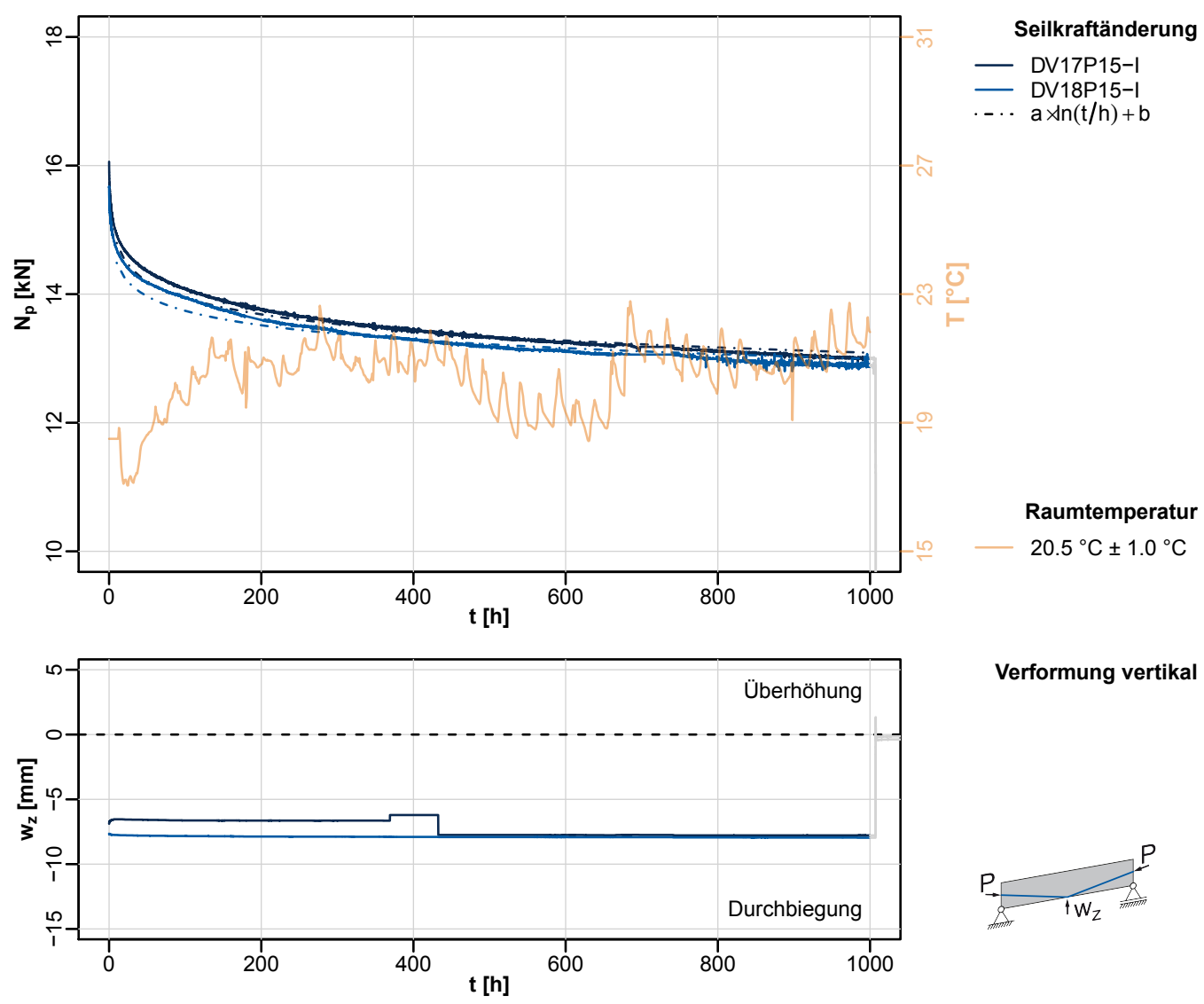

Verformung vertikal
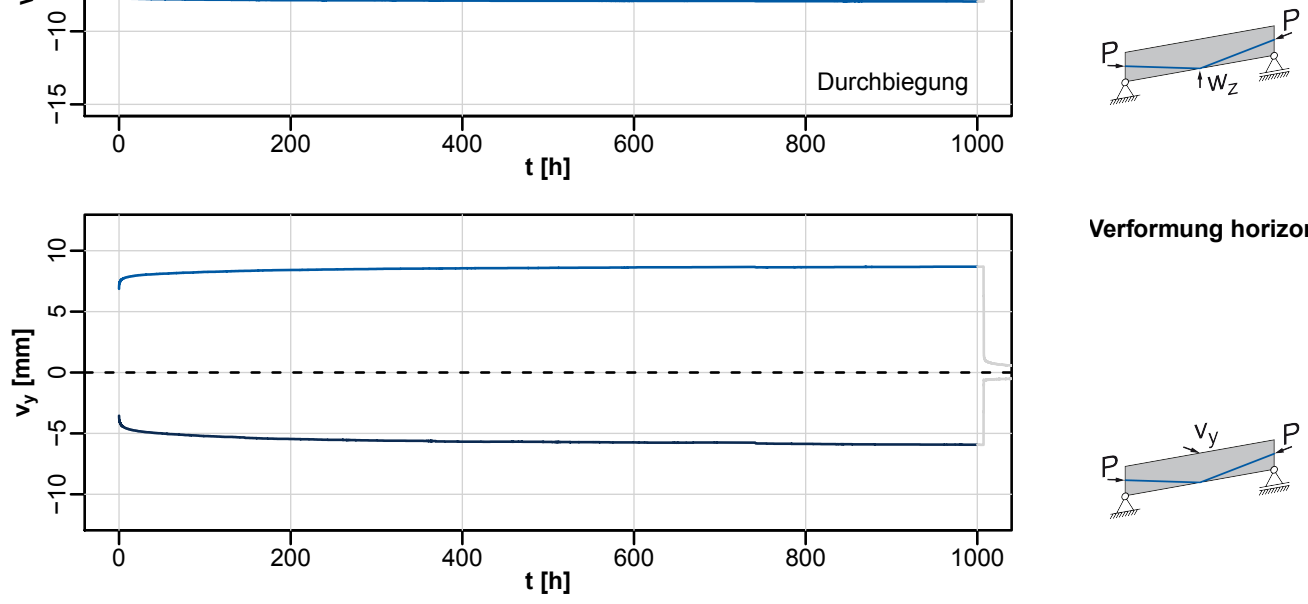

Verformung horizontal

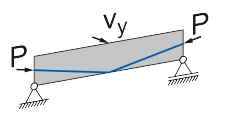

\begin{tabular}{lllllll}
\hline Prüfkörper & $\mathbf{N}_{\text {p.max }}[\mathbf{k N}]$ & $\mathbf{N}_{\mathbf{p} .1000 \mathrm{~h}}[\mathrm{kN}]$ & $\mathbf{w}_{\mathbf{z . 0}}[\mathrm{mm}]$ & $\mathbf{w}_{\mathbf{z . 1 0 0 0} \mathrm{h}}[\mathrm{mm}]$ & $\mathbf{v}_{\mathbf{y} . \mathbf{0}}[\mathrm{mm}]$ & $\mathbf{v}_{\mathbf{y} .1000 \mathrm{~h}}[\mathrm{~mm}]$ \\
\hline DV17P15-I & 16.080 & 13.000 & -6.89 & -7.78 & -3.50 & -5.93 \\
DV18P15-I & 15.690 & 12.900 & -7.63 & -7.96 & 6.75 & 8.69 \\
\hline
\end{tabular}




\section{Dauerversuch Träger mit $P_{m 0}=15 \mathrm{kN}$ und $d_{p}=8,1 \mathrm{~mm}$}
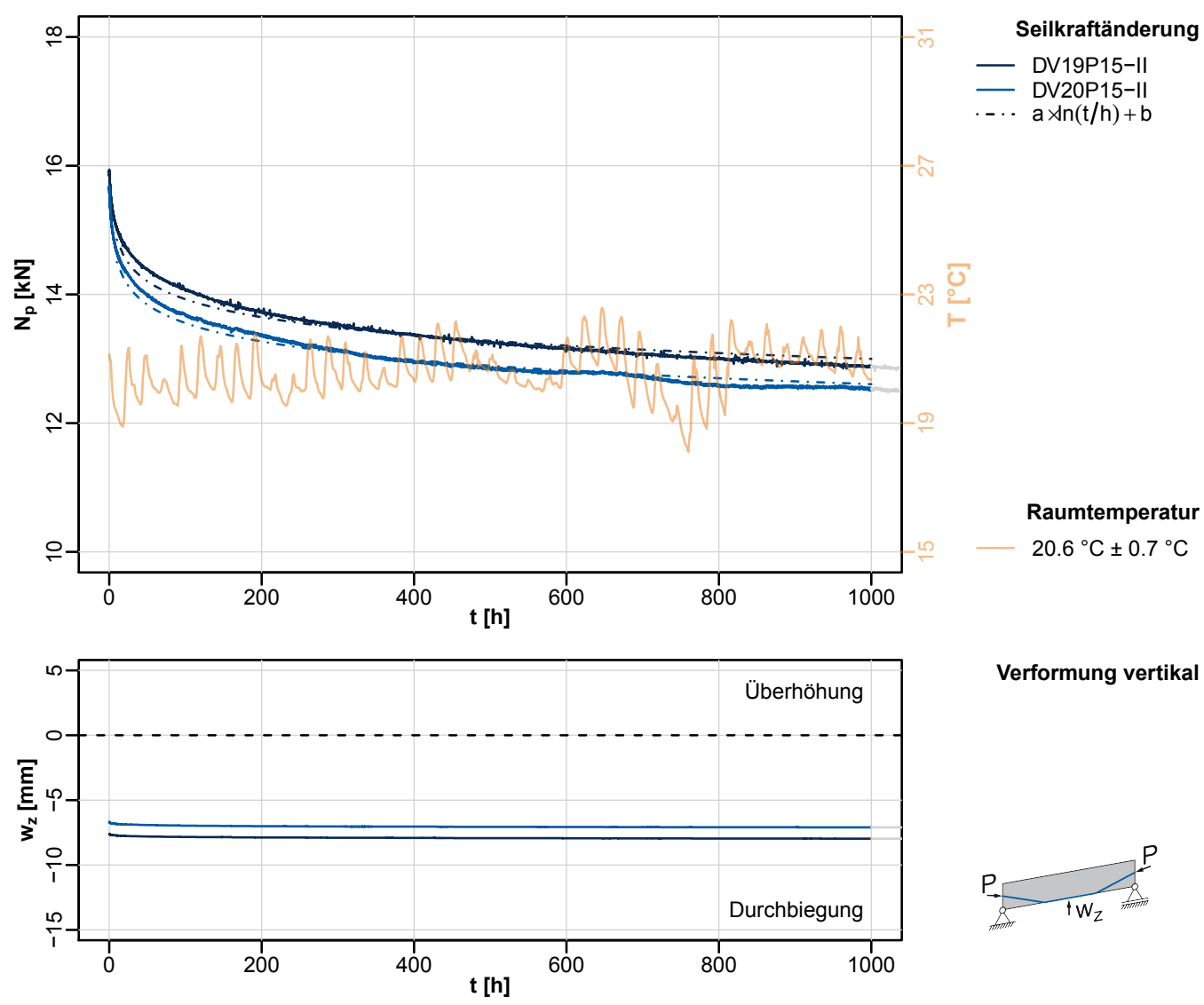

Verformung vertikal
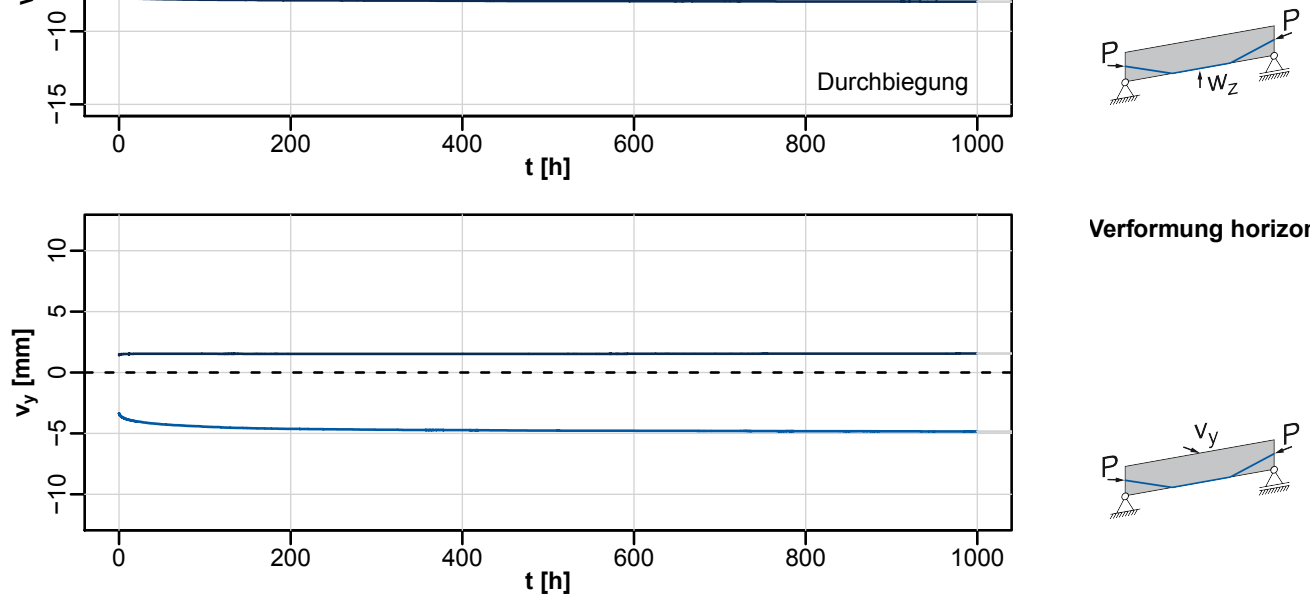

Verformung horizontal

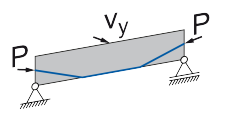

\begin{tabular}{lllllll}
\hline Prüfkörper & $\mathbf{N}_{\mathbf{p} . \max }[\mathrm{kN}]$ & $\mathbf{N}_{\mathbf{p} . \mathbf{1 0 0 0} \mathrm{h}}[\mathrm{kN}]$ & $\mathbf{w}_{\mathbf{z . 0}}[\mathrm{mm}]$ & $\mathbf{w}_{\mathbf{z . 1 0 0 0} \mathrm{h}}[\mathrm{mm}]$ & $\mathbf{v}_{\mathbf{y} . \mathbf{0}}[\mathrm{mm}]$ & $\mathbf{v}_{\mathbf{y} .1000 \mathrm{~h}}[\mathrm{~mm}]$ \\
\hline DV19P15-II & 15.930 & 12.870 & -7.60 & -7.97 & 1.42 & 1.56 \\
DV20P15-II & 15.690 & 12.530 & -6.63 & -7.10 & -3.35 & -4.84 \\
\hline
\end{tabular}


Dauerversuch Träger mit $P_{m 0}=15 \mathrm{kN}$ und $d_{p}=8,1 \mathrm{~mm}$ - ohne Auflast

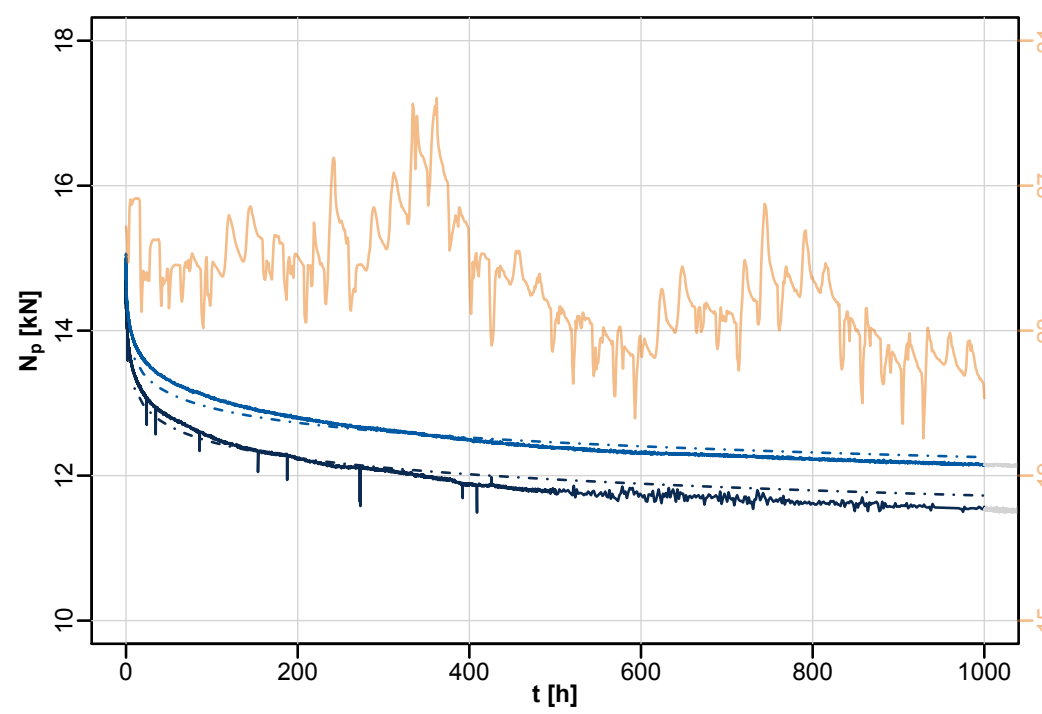

Seilkraftänderung

- DV21P15-II

- DV22P15-II

-.. $a \times n(t / h)+b$

DV21P15-II entrauscht

Raumtemperatur

$24.2^{\circ} \mathrm{C} \pm 1.6^{\circ} \mathrm{C}$

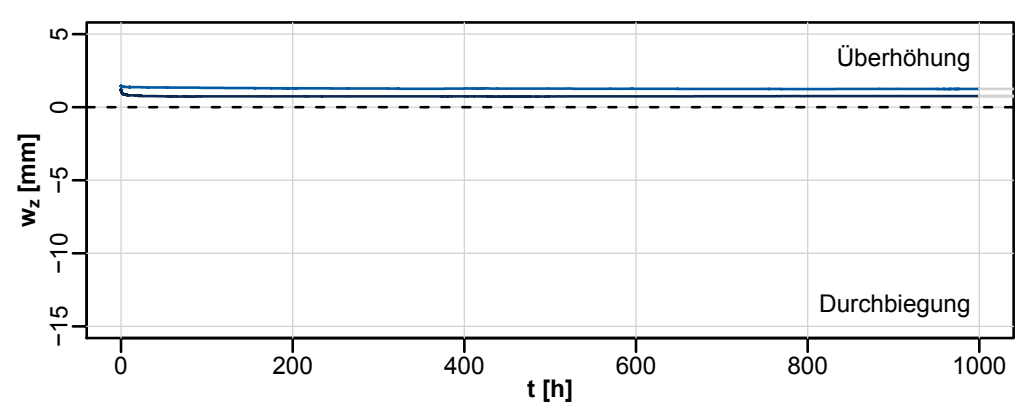

Verformung vertikal
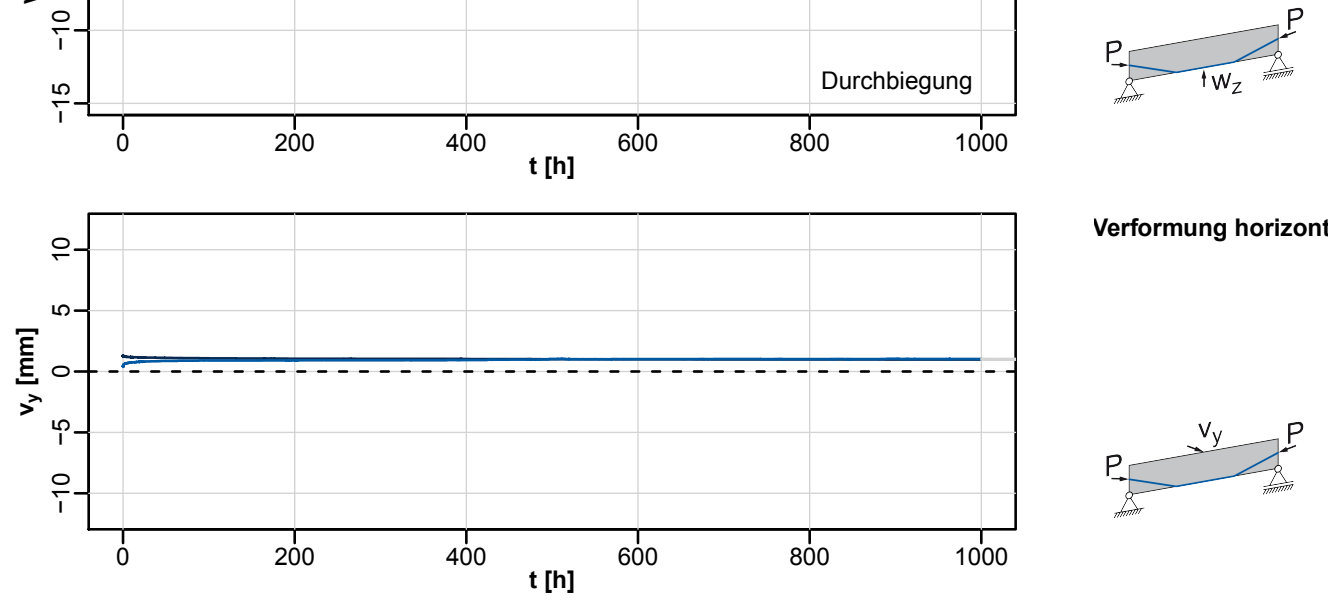

Verformung horizontal

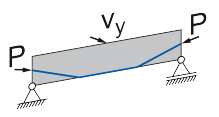

\begin{tabular}{|c|c|c|c|c|c|c|}
\hline Prüfkörper & $\mathbf{N}_{\text {p.max }}[k N]$ & $\mathbf{N}_{\mathrm{p} .1000 \mathrm{~h}}[\mathrm{kN}]$ & $\mathrm{w}_{\mathrm{z} .0}[\mathrm{~mm}]$ & $\mathrm{w}_{\mathrm{z} .1000 \mathrm{~h}}[\mathrm{~mm}]$ & $\mathrm{v}_{\mathrm{y} .0}[\mathrm{~mm}]$ & $\mathrm{v}_{\mathrm{y} .1000 \mathrm{~h}}[\mathrm{~mm}]$ \\
\hline DV21P15-II & 15.090 & 11.570 & 1.23 & 0.76 & 1.31 & 0.98 \\
\hline DV22P15-II & 15.290 & 12.150 & 1.47 & 1.25 & 0.42 & 1.03 \\
\hline
\end{tabular}




\section{Dauerversuch Träger mit $P_{m 0}=15 \mathrm{kN}$ und $d_{p}=10,1 \mathrm{~mm}$}
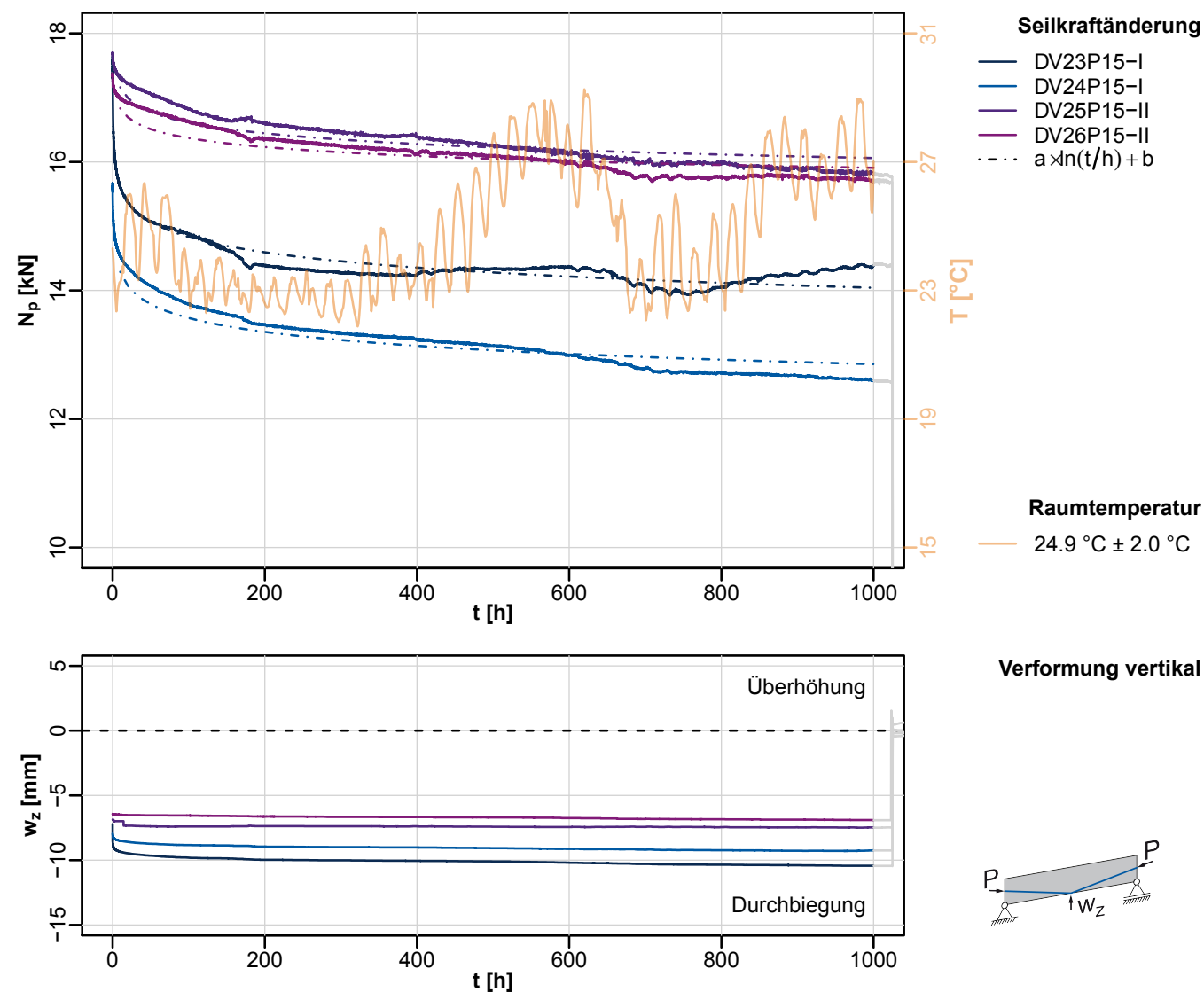

Verformung vertikal
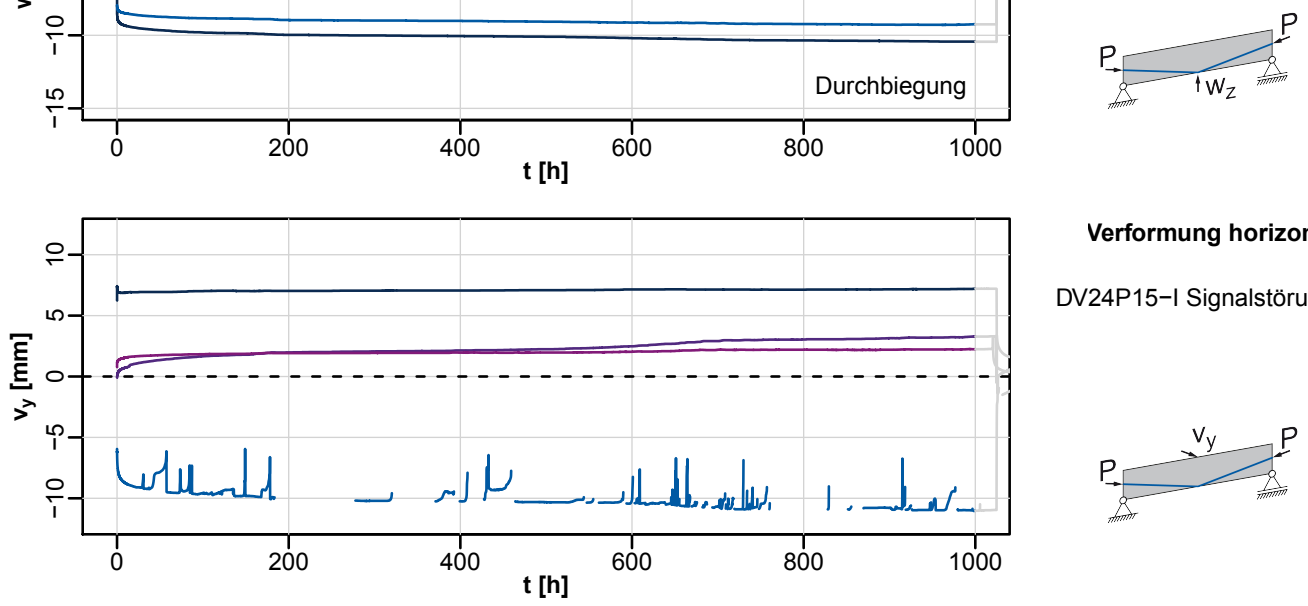

Verformung horizontal DV24P15-I Signalstörung

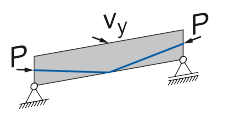

\begin{tabular}{lllllll}
\hline Prüfkörper & $\mathbf{N}_{\mathbf{p} . \max }[\mathbf{k N}]$ & $\mathbf{N}_{\mathbf{p . 1 0 0 0 ~ h}}[\mathbf{k N}]$ & $\mathbf{w}_{\mathbf{z . 0}}[\mathrm{mm}]$ & $\mathbf{w}_{\mathbf{z . 1 0 0 0 ~ h}}[\mathrm{mm}]$ & $\mathbf{v}_{\mathbf{y} . \mathbf{0}}[\mathrm{mm}]$ & $\mathbf{v}_{\mathbf{y} .1000 \mathrm{~h}}[\mathrm{~mm}]$ \\
\hline DV23P15-I & 17.800 & 14.390 & -7.09 & -10.44 & 6.12 & 7.21 \\
DV24P15-I & 15.700 & 12.600 & -7.92 & -9.25 & -5.83 & -11.03 \\
DV25P15-II & 17.720 & 15.810 & -6.85 & -7.48 & -0.11 & 3.28 \\
DV26P15-II & 17.380 & 15.720 & -6.41 & -6.91 & 0.69 & 2.24 \\
\hline
\end{tabular}




\section{Dauerversuch Träger mit $P_{m 0}=15 \mathrm{kN}$ und $d_{p}=10,1 \mathrm{~mm}$ - ohne Auflast}

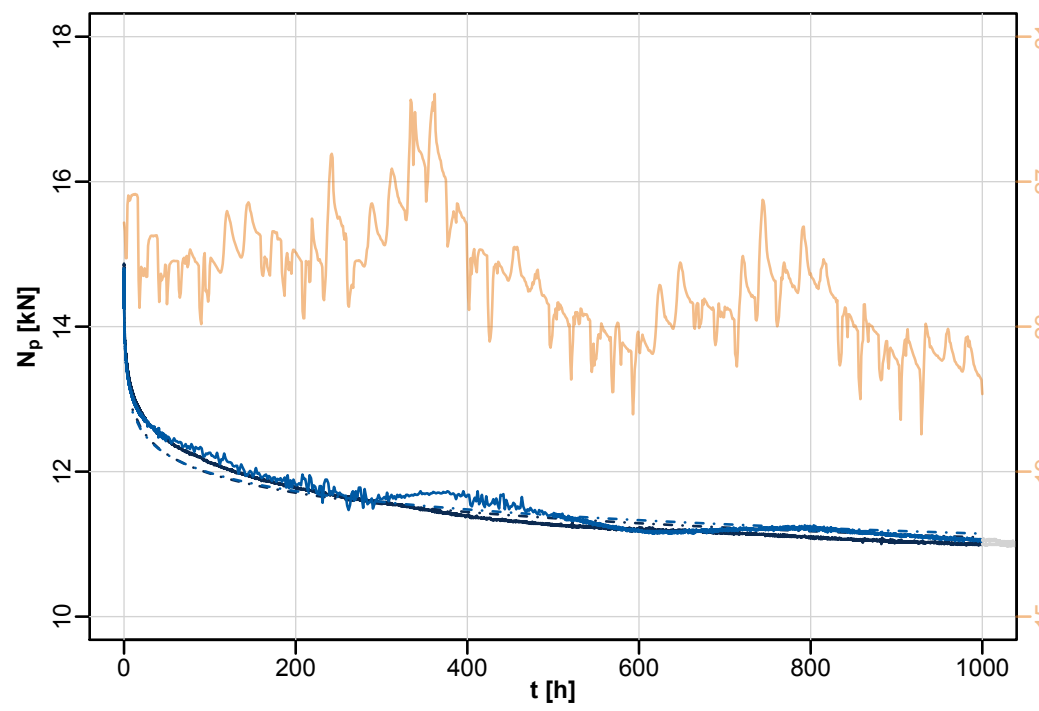

Seilkraftänderung

- DV27P15-II

- DV28P15-II

$-\cdots a \rtimes n(t / h)+b$

DV28P15-II entrauscht

Raumtemperatur

$24.2^{\circ} \mathrm{C} \pm 1.6^{\circ} \mathrm{C}$

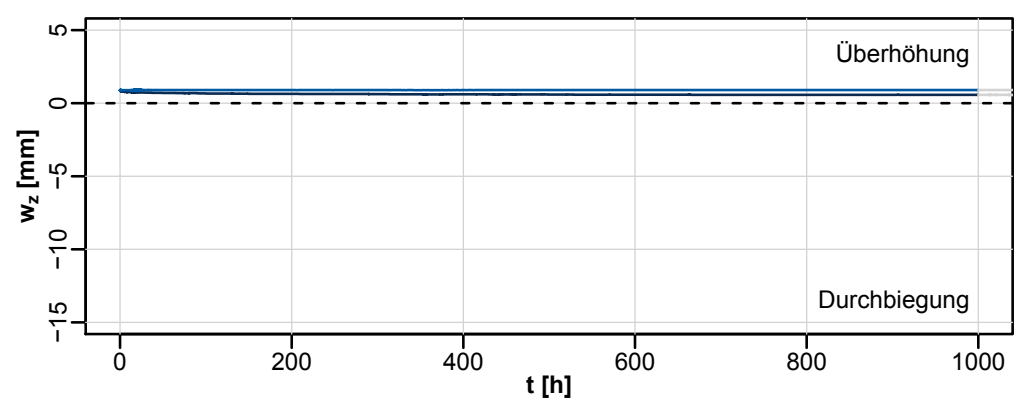

Verformung vertikal

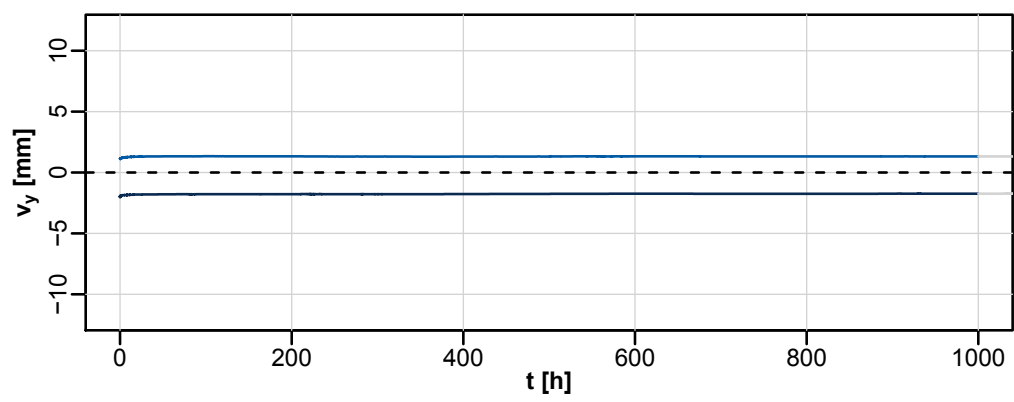

Verformung horizontal
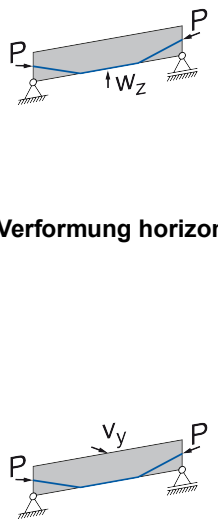

\begin{tabular}{lllllll}
\hline Prüfkörper & $\mathbf{N}_{\text {p.max }}[\mathrm{kN}]$ & $\mathbf{N}_{\mathbf{p} .1000 \mathrm{~h}}[\mathrm{kN}]$ & $\mathbf{w}_{\mathbf{z . 0}}[\mathrm{mm}]$ & $\mathbf{w}_{\mathbf{z . 1 0 0 0} \mathrm{h}}[\mathrm{mm}]$ & $\mathbf{v}_{\mathbf{y} . \mathbf{0}}[\mathrm{mm}]$ & $\mathbf{v}_{\mathbf{y} .1000 \mathrm{~h}}[\mathrm{~mm}]$ \\
\hline DV27P15-II & 15.070 & 11.000 & 0.90 & 0.57 & -2.00 & -1.74 \\
DV28P15-II & 15.060 & 11.050 & 0.90 & 0.90 & 1.13 & 1.32 \\
\hline
\end{tabular}





\section{Versuche zur Resttragfähigkeit}

In dieser Anlage werden die Ergebnisse der Resttragversuche gemäß Kapitel 3.4 dokumentiert.

Die folgende Tabelle gibt einen Überblick. Darauf folgend werden die Seikraft, Glaskantenverzerrung und Verformung für jeden Prüfkörper dargestellt.

Zu Begin und jeweils nach $24 \mathrm{~h}$ Standzeit wird eine Einzelscheibe des Verbund-Sicherheitsglases planmäßig zerstört. Dargestellt werden maximal vier Abschnitte der Standzeit zu $24 \mathrm{~h}$

Als wesentliches Ergebnis ergibt sich die Standzeit in Abhängigkeit von der Belastung aus Vorspannung des Seils und Biegebelastung im Vierpunkt-Biegeversuch.

Darüber hinaus werden die Bruchbilder aus der Fotodokumentation dargestellt.

\begin{tabular}{|c|c|c|c|c|}
\hline Prüfkörper & $\mathrm{P}_{\mathrm{m} 0}[\mathrm{kN}]$ & $\mathrm{d}_{\mathrm{s}}[\mathrm{mm}]$ & $\mathrm{F}_{\text {ist }}[\mathrm{kN}]$ & Versagenszeit [h] \\
\hline RT01PX-II & - & 0.0 & 10.6 & 53.1 \\
\hline RT02PX-II & - & 0.0 & 10.6 & 48.2 \\
\hline RT03PX-II & - & 0.0 & 10.6 & 48.1 \\
\hline RT04PX-II & - & 0.0 & 10.6 & 48.2 \\
\hline RT05P0-II & 0 & 8.1 & 10.6 & 48.3 \\
\hline RT06P0-II & 0 & 8.1 & 10.6 & 48.2 \\
\hline RT07P0-II & 0 & 8.1 & 10.6 & 72.2 \\
\hline RT08P0-II & 0 & 8.1 & 10.6 & 72.2 \\
\hline RT09P9-I & 9 & 5.0 & 10.6 & 53.8 \\
\hline RT10P9-I & 9 & 5.0 & 10.6 & 51.9 \\
\hline RT11P9-I & 9 & 5.0 & 10.4 & 71.3 \\
\hline RT12P9-I & 9 & 5.0 & 10.4 & 72.8 \\
\hline RT13P9-II & 9 & 5.0 & 10.4 & 72.7 \\
\hline RT14P9-II & 9 & 5.0 & 10.4 & 53.3 \\
\hline RT15P9-II & 9 & 5.0 & 10.4 & 48.5 \\
\hline RT16P9-II & 9 & 5.0 & 10.8 & 72.0 \\
\hline$\overline{\text { RT17P15-I }}$ & 15 & 8.1 & 10.6 & 48.7 \\
\hline RT18P15-I & 15 & 8.1 & 10.6 & 48.9 \\
\hline RT19P15-II & 15 & 8.1 & 10.6 & 88.6 \\
\hline RT20P15-II & 15 & 8.1 & 10.6 & 112.5 \\
\hline RT21P15-I & 15 & 10.1 & 10.6 & 0.5 \\
\hline RT22P15-I & 15 & 10.1 & 10.6 & 0.6 \\
\hline RT23P15-II & 15 & 10.1 & 10.6 & 72.6 \\
\hline RT24P15-II & 15 & 10.1 & 10.6 & 72.6 \\
\hline
\end{tabular}

$\mathrm{P}_{\mathrm{m} 0} \quad \ldots$ Vorspannkraft (Sollwert vor Biegebelastung)

$\mathrm{P}_{\max } \quad \ldots$ Maximale Seilkraft

$d_{s} \quad \ldots$ Durchmesser des Bewehrungsseils

$\mathrm{F}_{\text {ist }} \quad \ldots$ Biegebelastung (Ist-Wert) 


\section{Standzeit RT01PX-II Referenzträger unbewehrt}

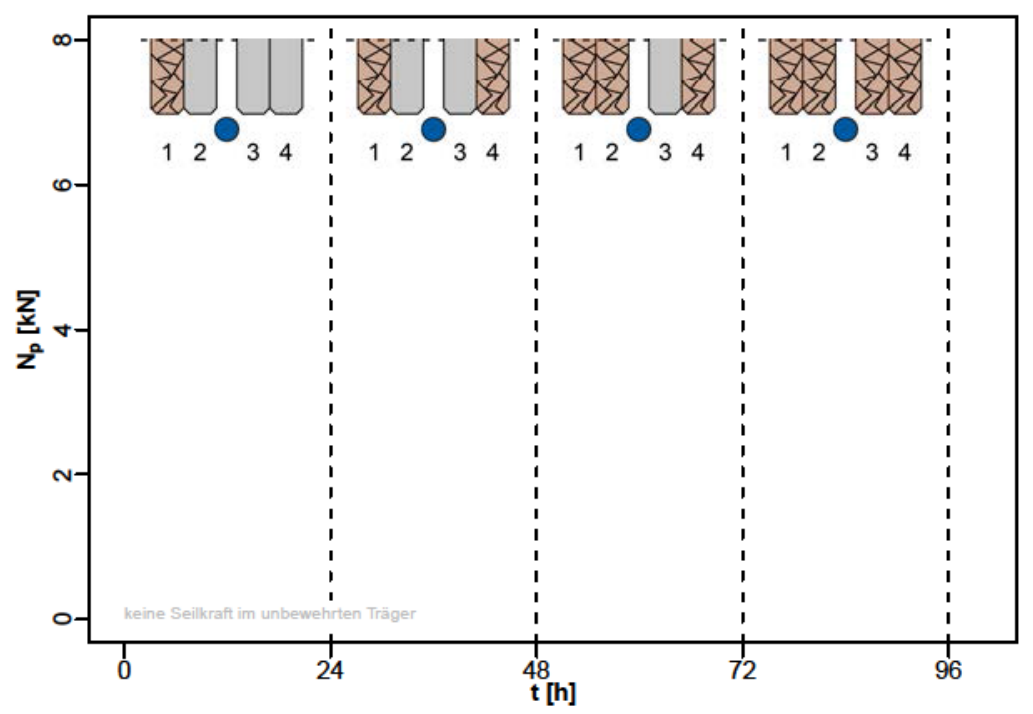

Seilkraftänderung
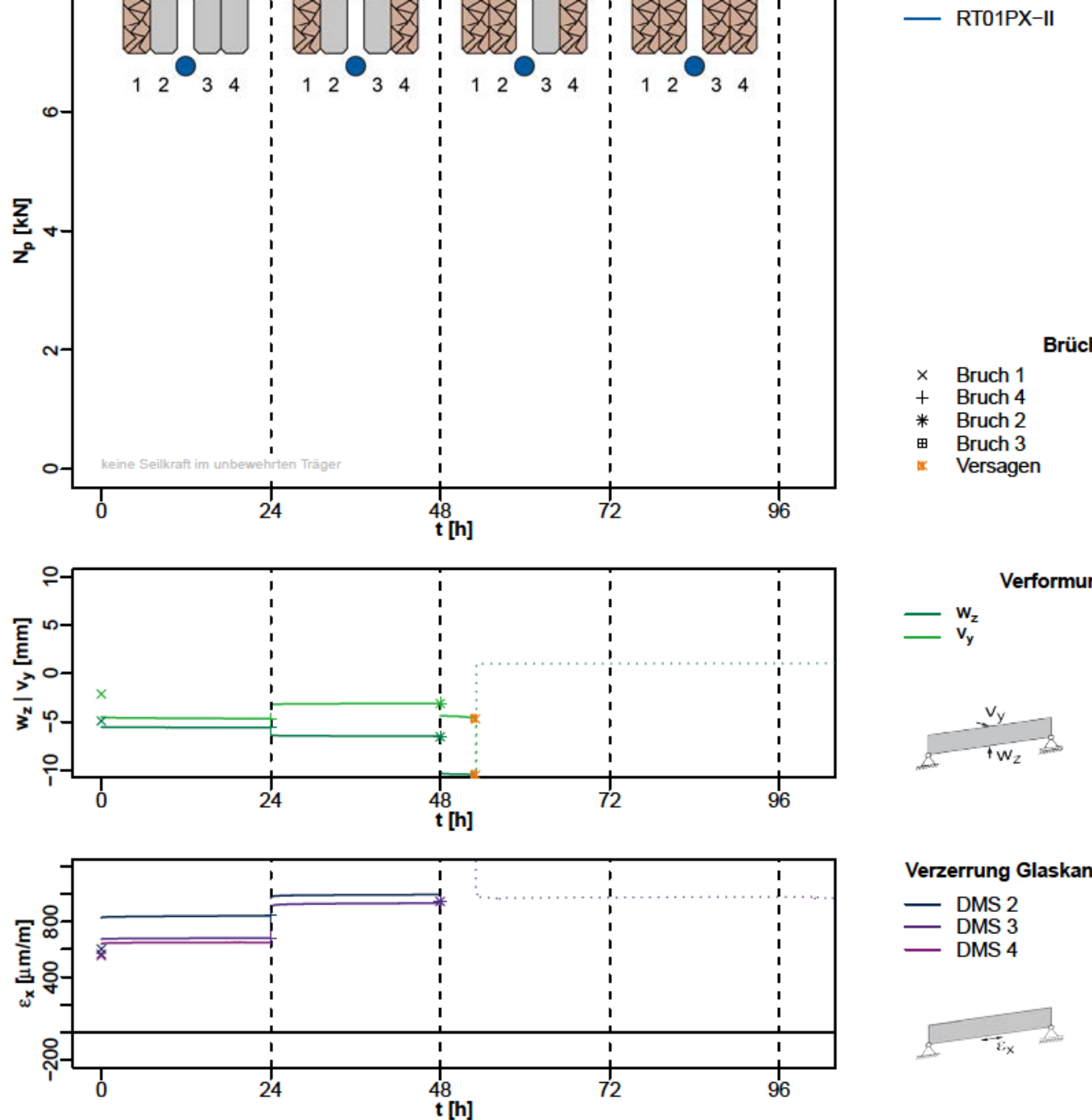

Verzerrung Glaskante

- DMS 2

- DMS 3

- DMS 4

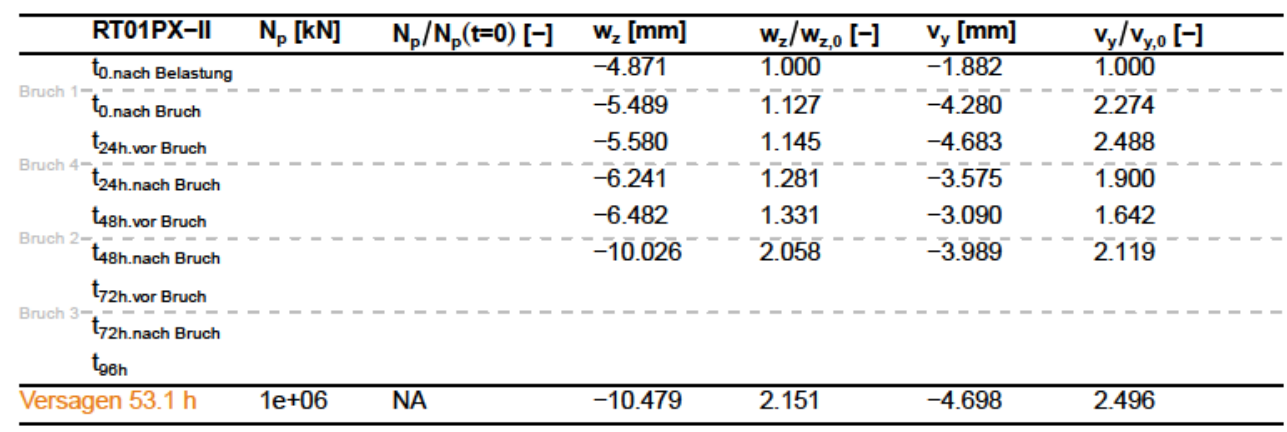


Scheibe 1

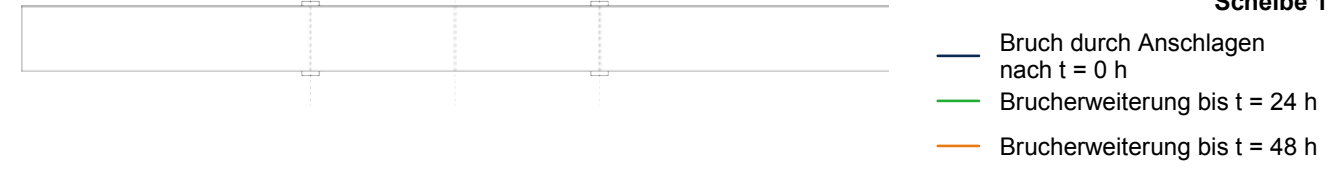

Foto nicht druckbar

Foto nicht druckbar

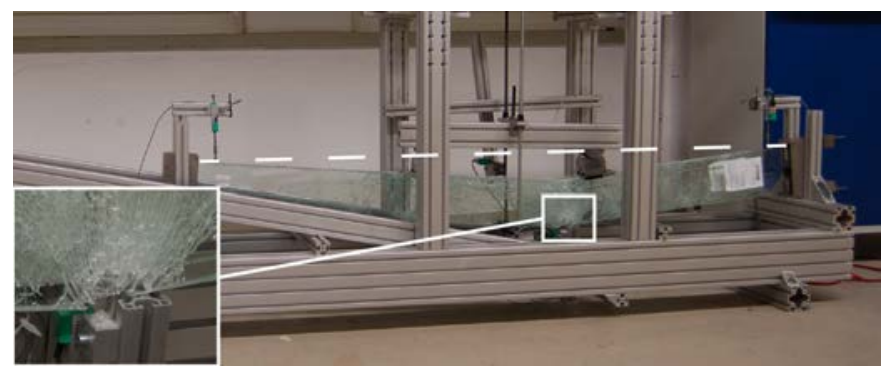

geknickter Trägerverlauf nach Versagen

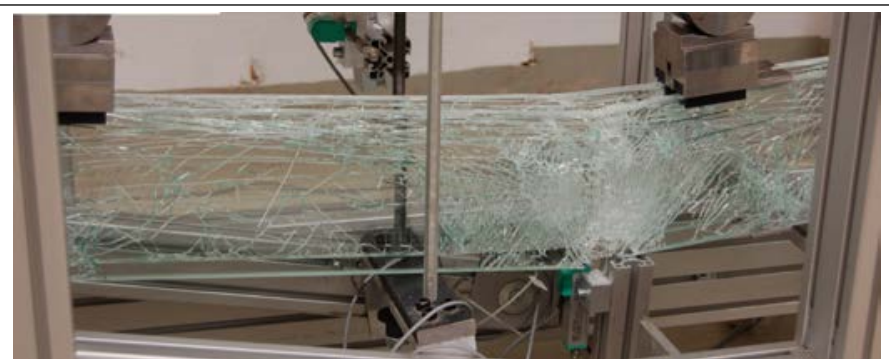

Bruch aller Scheiben

Aufklaffender Rissursprung 


\section{Standzeit RT02PX-II Referenzträger unbewehrt}

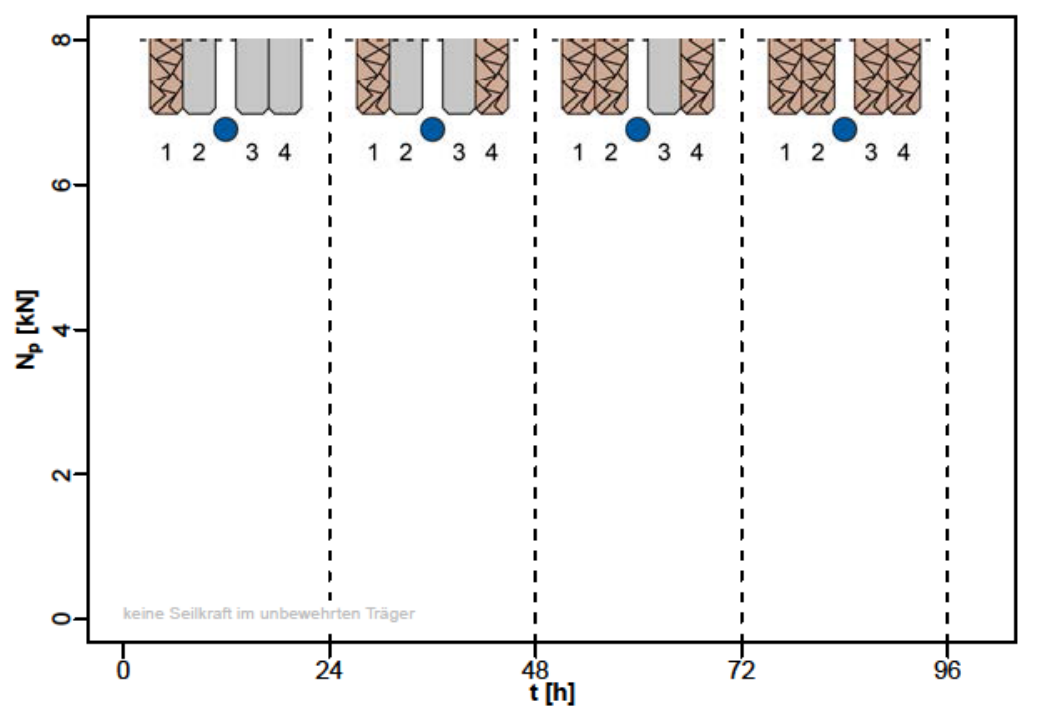

Seilkraftänderung

- RT02PX-II
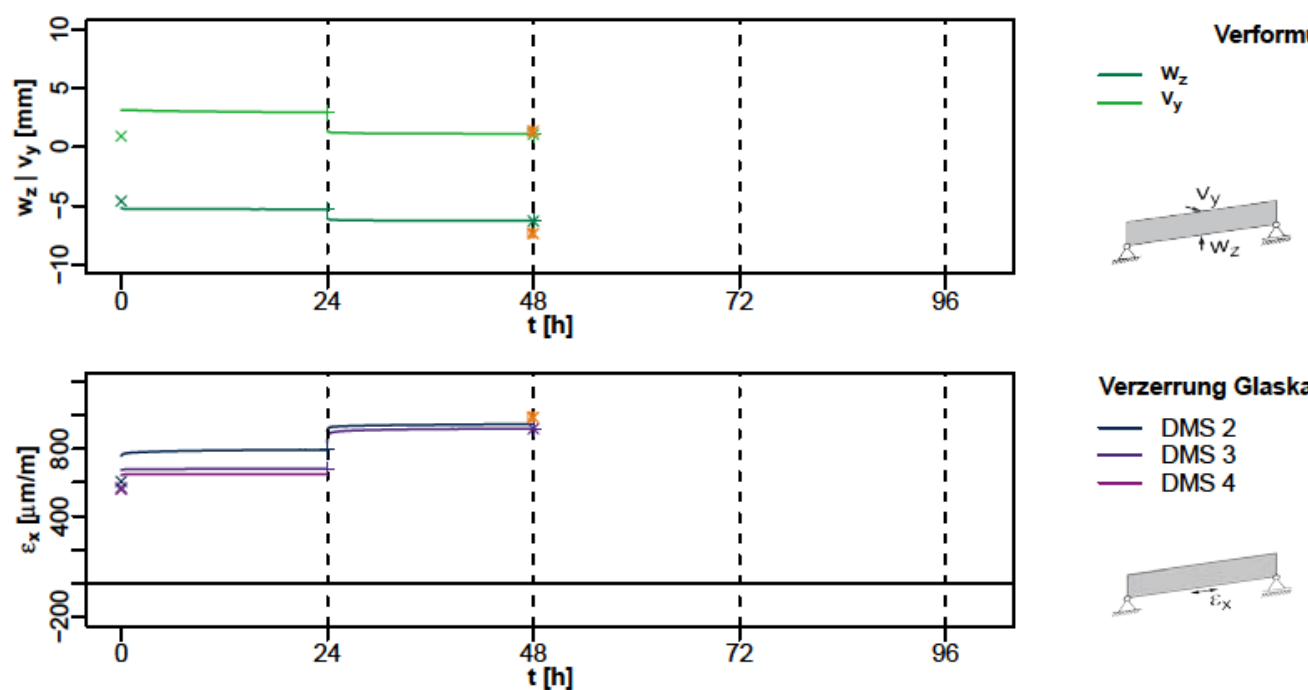

Verzerrung Glaskante

- DMS 2

- DMS 3

- DMS 4

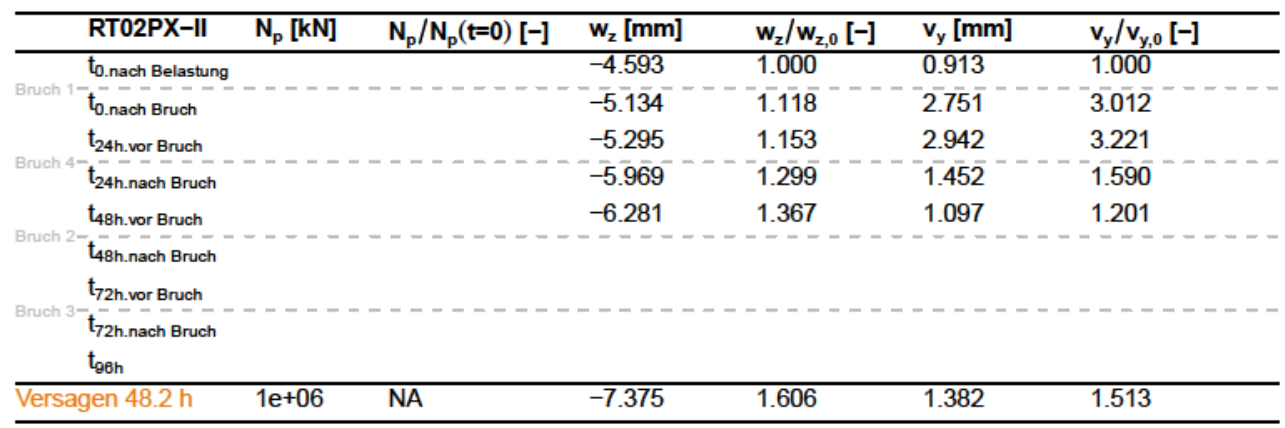




\section{Bruchbild RT02PX-II Referenzträger unbewehrt}

$h_{z}=67,8 \%$

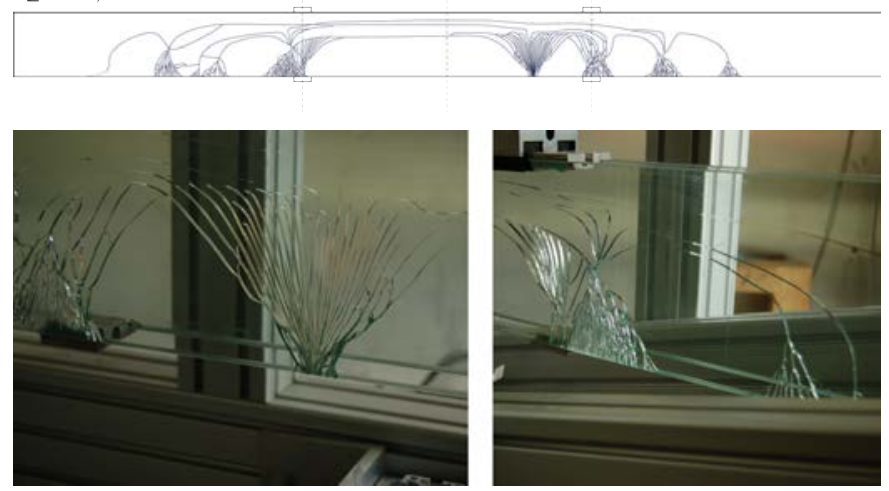

$h_{z}=64,2 \%$

Scheibe 4

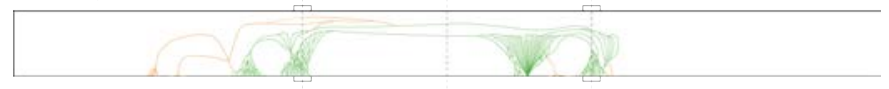
nach $\mathrm{t}=0 \mathrm{~h}$

- Brucherweiterung bis $\mathrm{t}=24 \mathrm{~h}$

Brucherweiterung bis $\mathrm{t}=48 \mathrm{~h}$
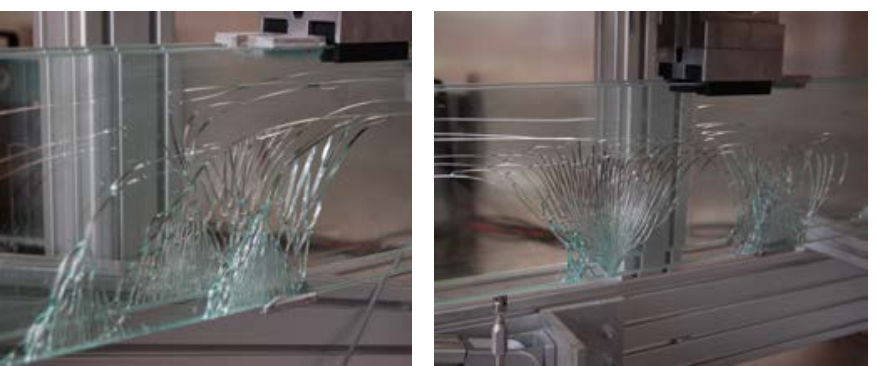

nach $\mathrm{t}=24 \mathrm{~h}$

- Brucherweiterung bis $\mathrm{t}=48 \mathrm{~h}$
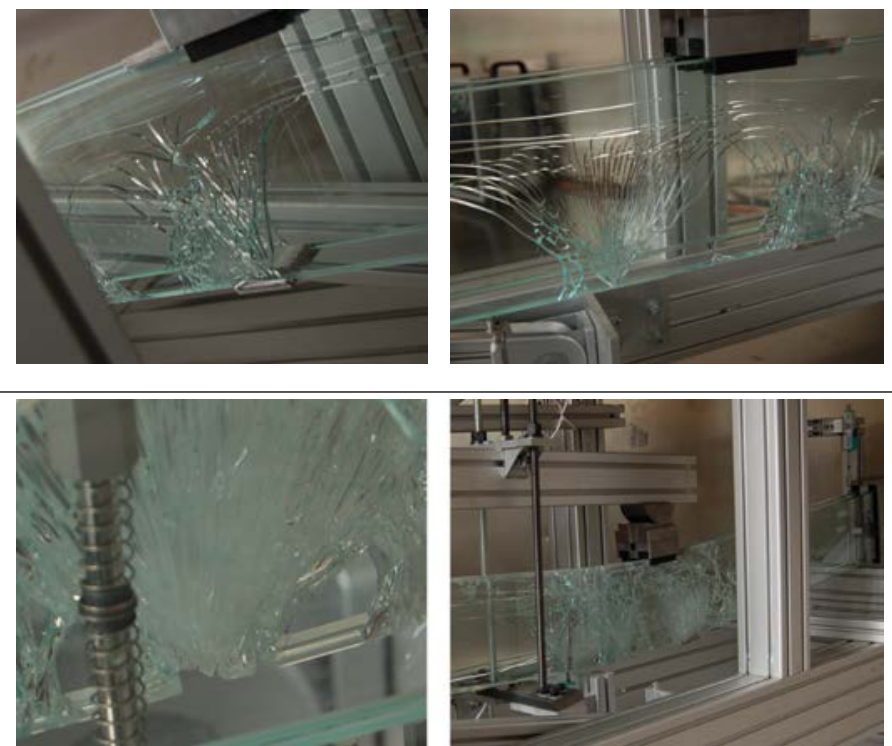

Bruch aller Scheiben

Aufklaffender Rissursprung im linken Bild

Heruntergefallener, gebrochener Träger im rechten Bild 


\section{Standzeit RT03PX-II Referenzträger unbewehrt}

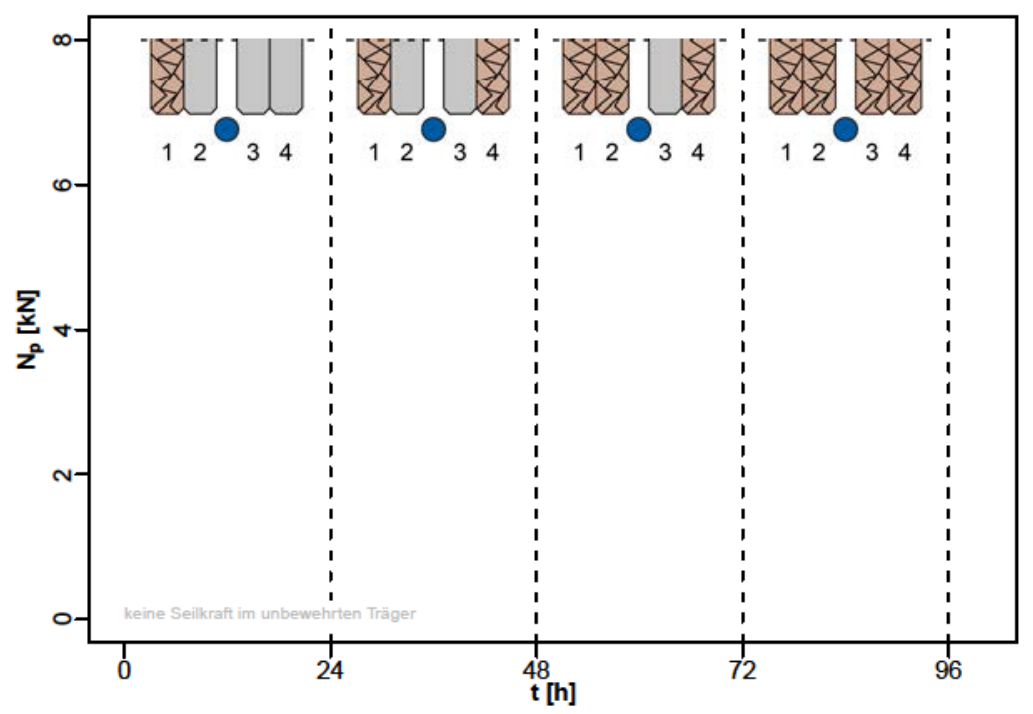

Seilkraftänderung

- RT03PX-II
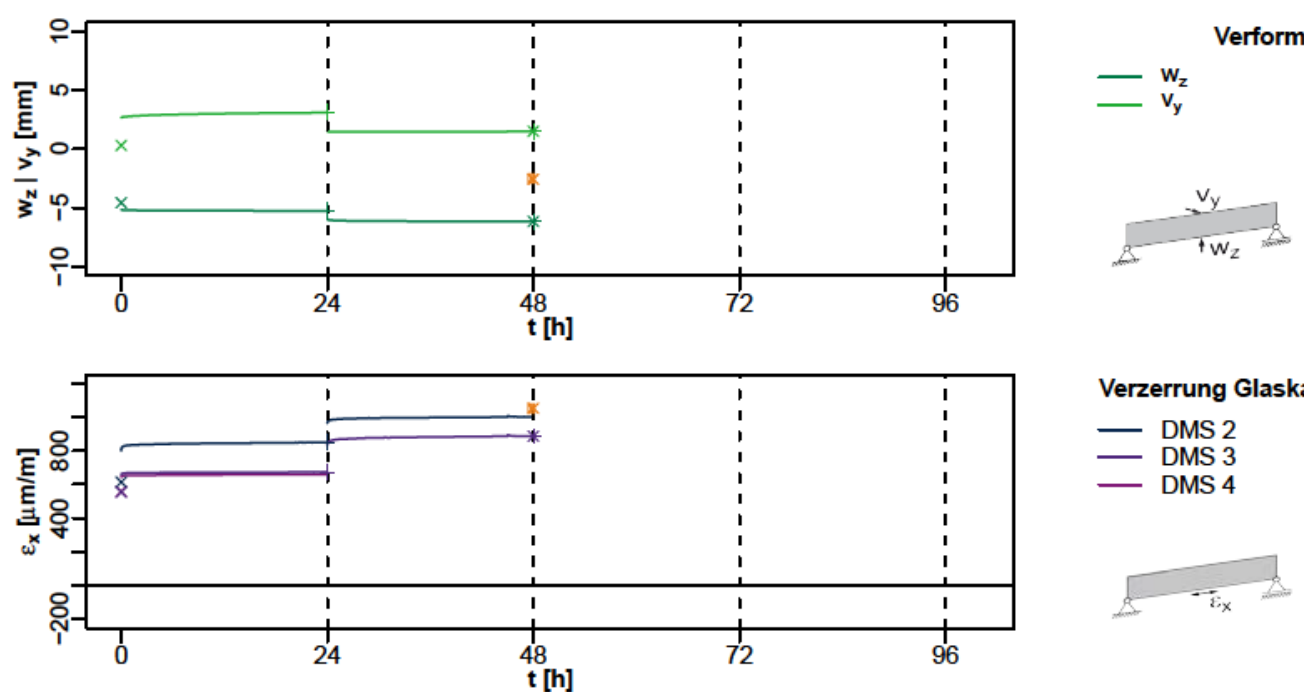

Verzerrung Glaskante

- DMS 2

- DMS 3

- DMS 4

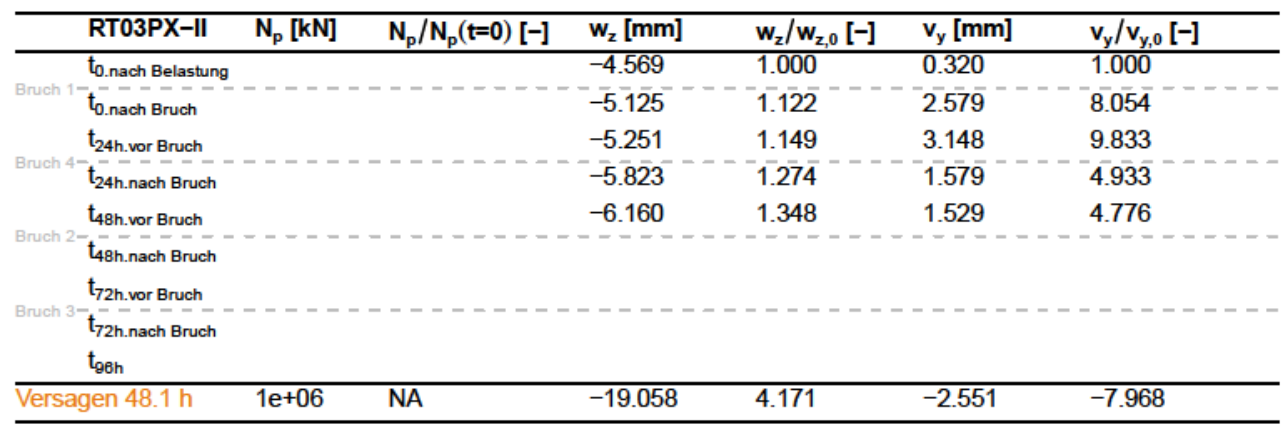


Bruchbild RT03PX-II

$h_{z}=67,7 \%$

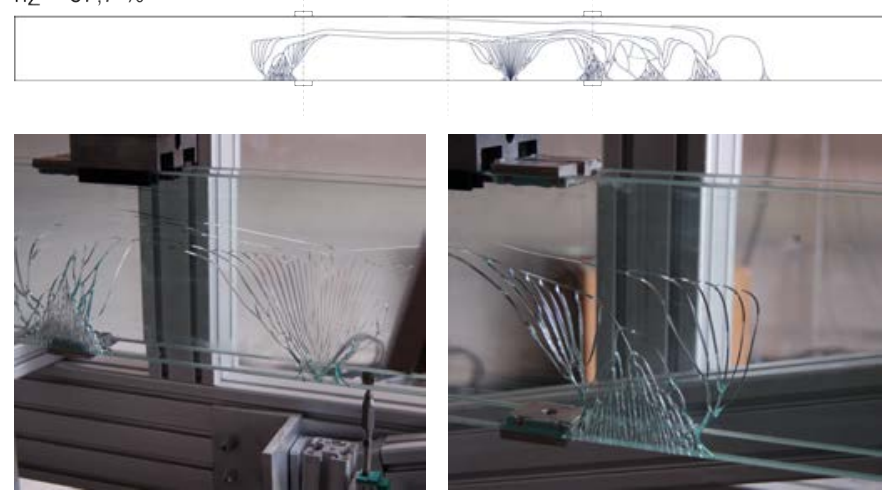

$h_{\mathrm{Z}}=62,1 \%$
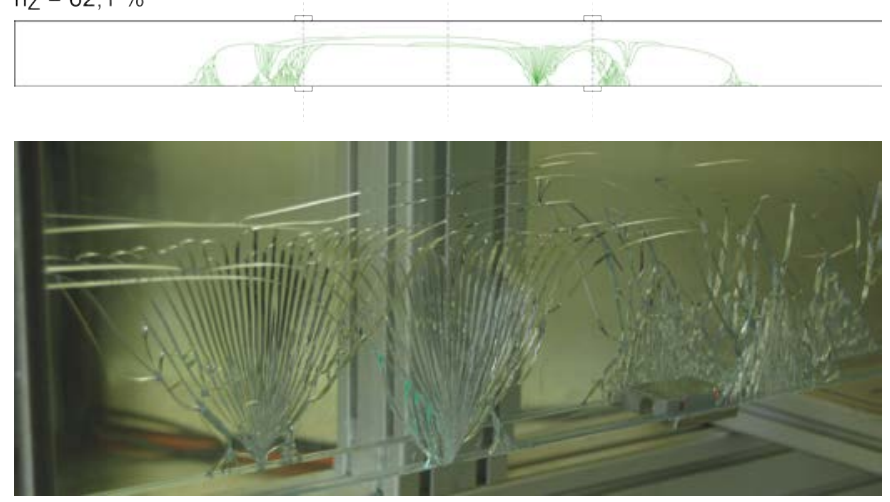

Scheibe 1

Bruch durch Anschlagen nach $\mathrm{t}=0 \mathrm{~h}$

- Brucherweiterung bis $\mathrm{t}=24 \mathrm{~h}$

- Brucherweiterung bis $\mathrm{t}=48 \mathrm{~h}$

Scheibe 4

Bruch durch Anschlagen nach $\mathrm{t}=24 \mathrm{~h}$

_ Brucherweiterung bis $\mathrm{t}=48 \mathrm{~h}$
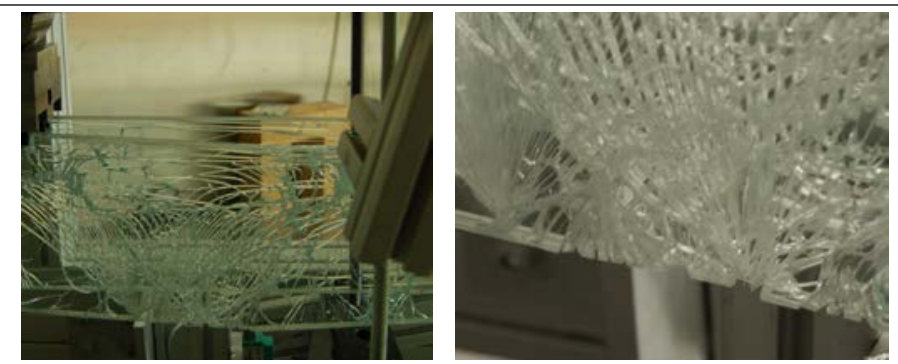

Bruch aller Scheiben

Heruntergefallener, gebrochener Träger im linken Bild Aufklaffender Rissursprung im rechten Bild 


\section{Standzeit RT04PX-II Referenzträger unbewehrt}

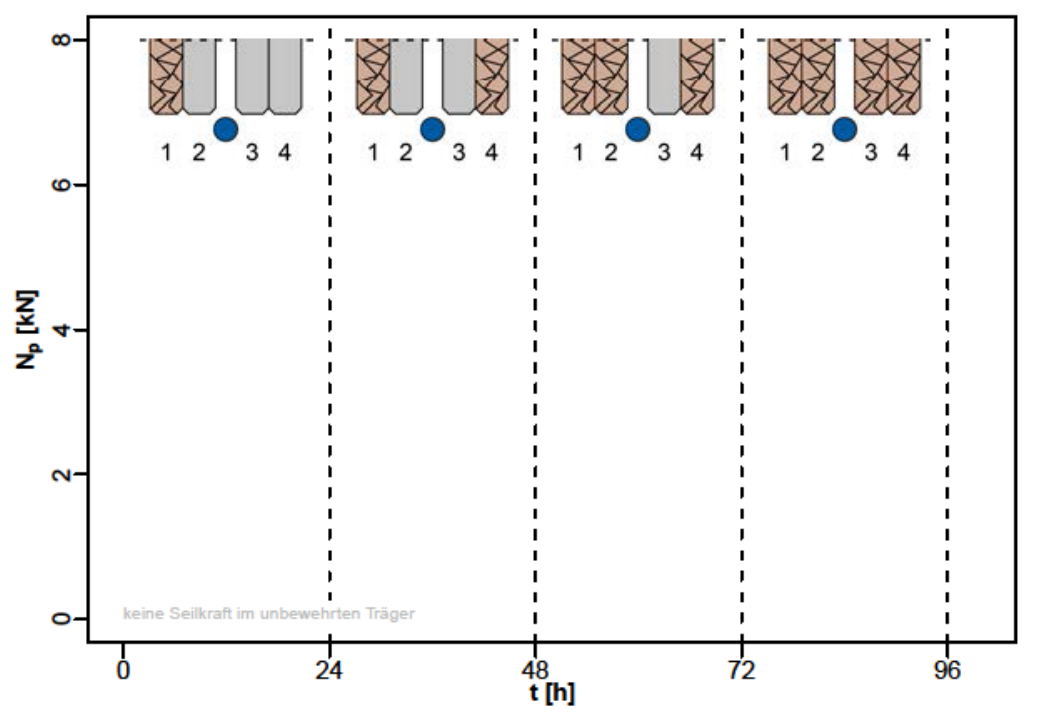

Seilkraftänderung

- RT04PX-II
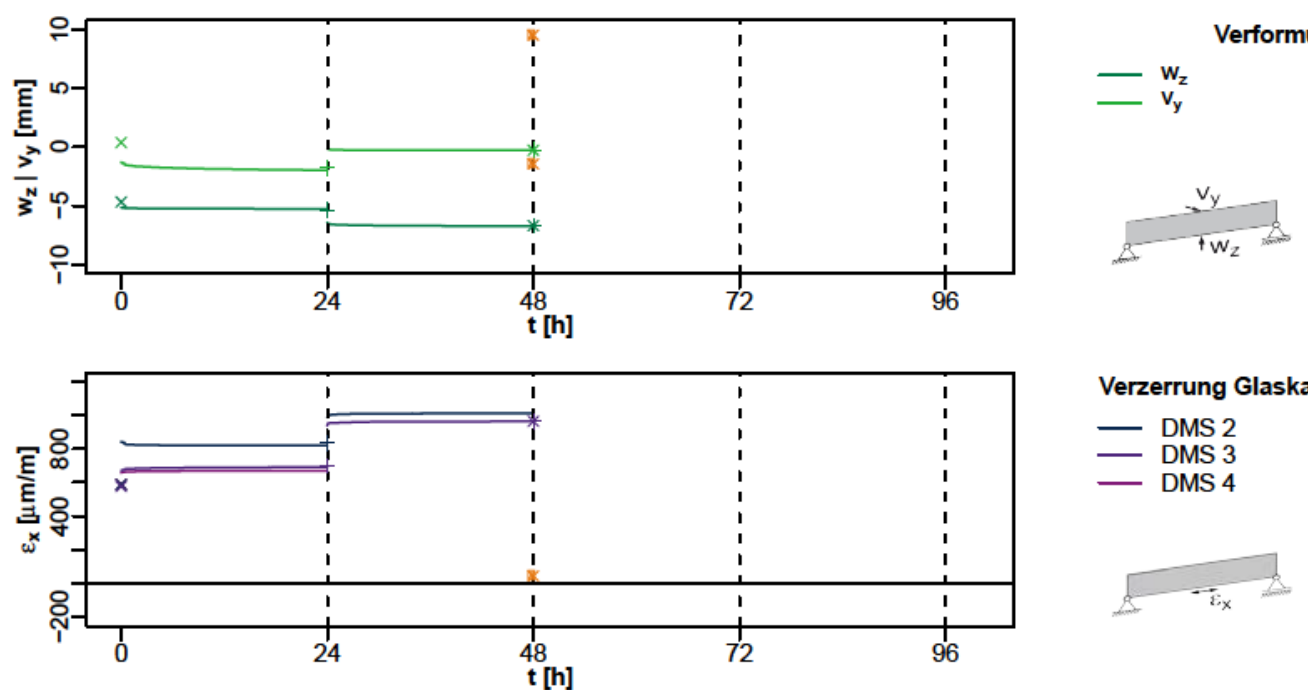

Verzerrung Glaskante

- DMS 2

- DMS 3

- DMS 4

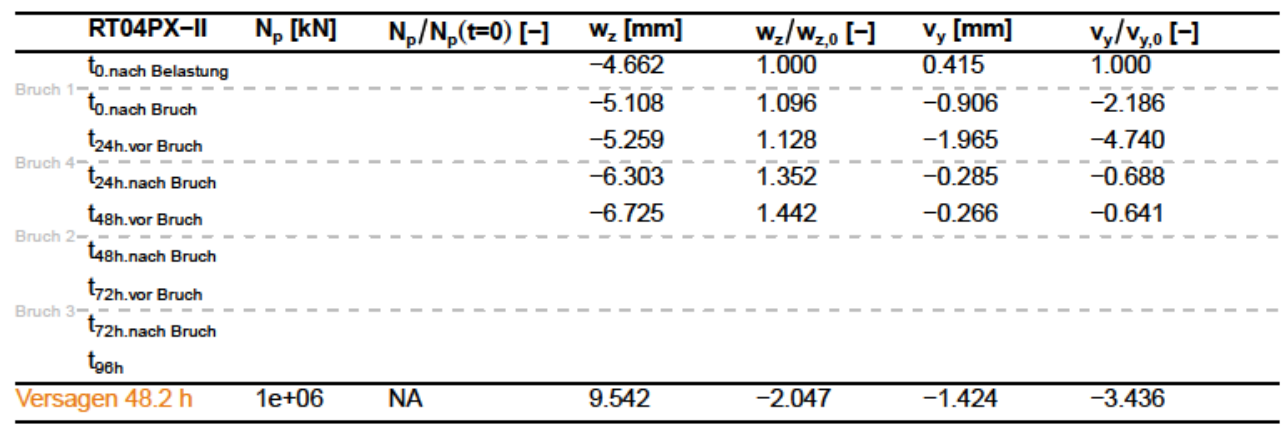




\section{Bruchbild RT04PX-II Referenzträger unbewehrt}

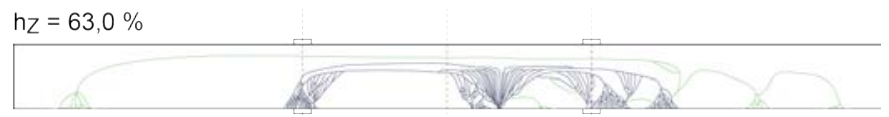

Scheibe 1

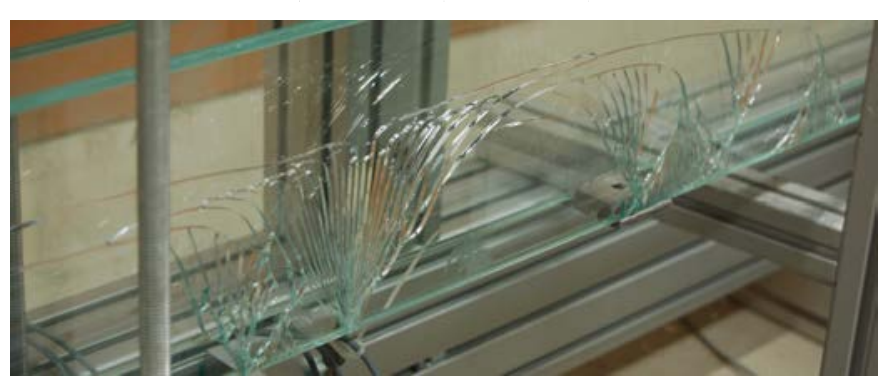

Bruch durch Anschlagen nach $\mathrm{t}=0 \mathrm{~h}$

- Brucherweiterung bis $\mathrm{t}=24 \mathrm{~h}$

- Brucherweiterung bis $\mathrm{t}=48 \mathrm{~h}$

$h_{z}=62,6 \%$

Scheibe 4

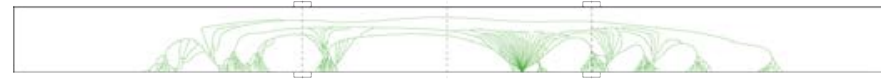

Bruch durch Anschlagen nach $\mathrm{t}=24 \mathrm{~h}$

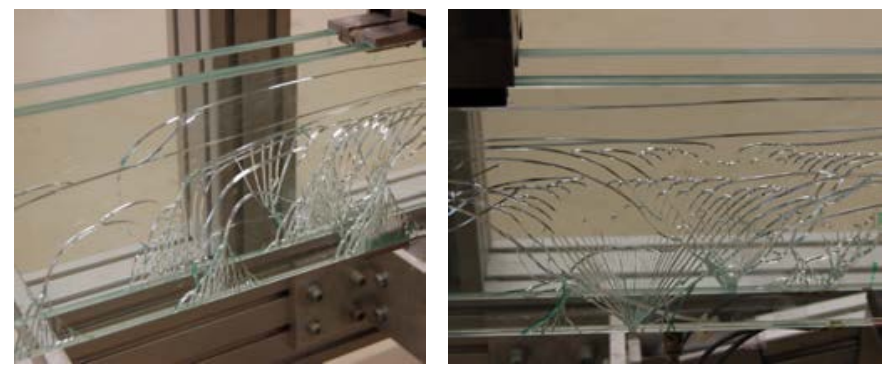

- Brucherweiterung bis $\mathrm{t}=48 \mathrm{~h}$ vielfacher Bruchfächer links

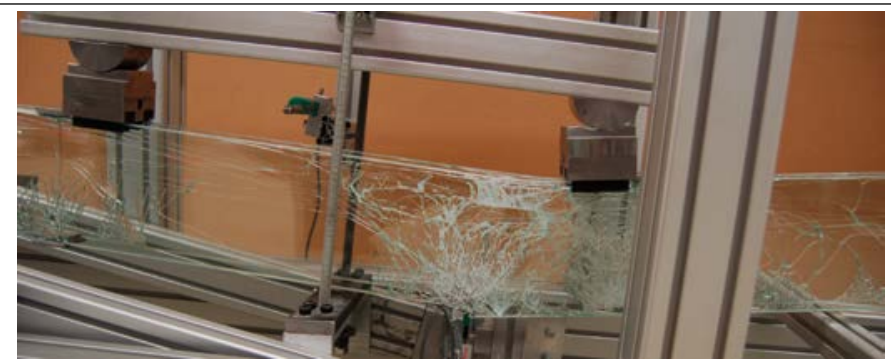

Bruch aller Scheiben

Heruntergefallener, gebrochener Träger 


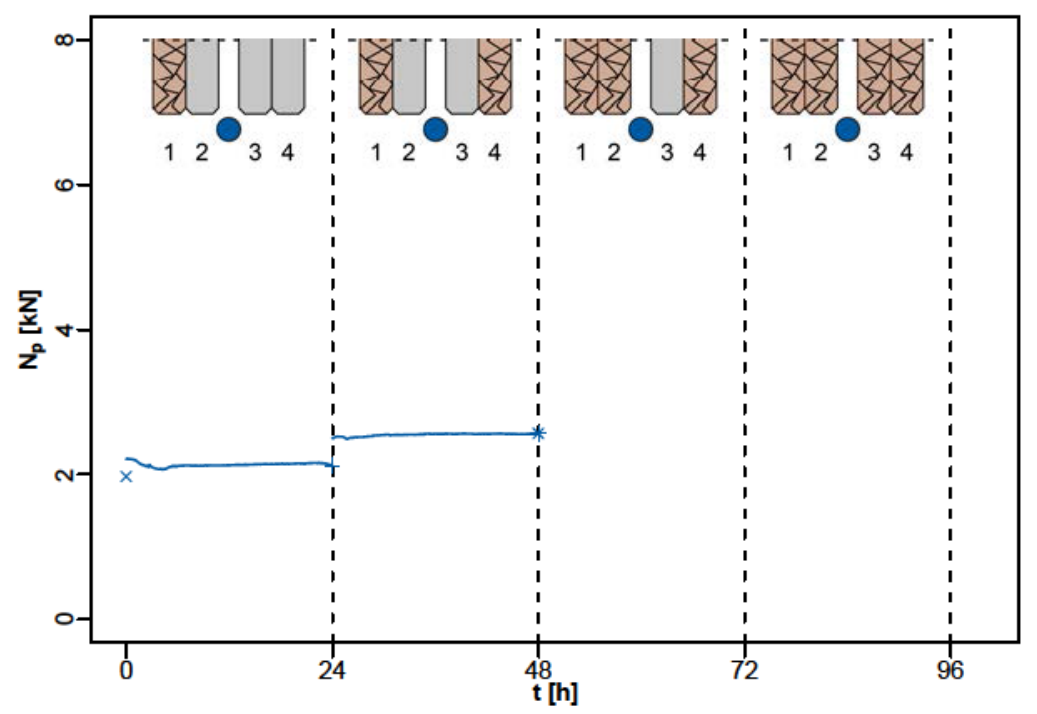

Seilkraftänderung

- RT05P0-II
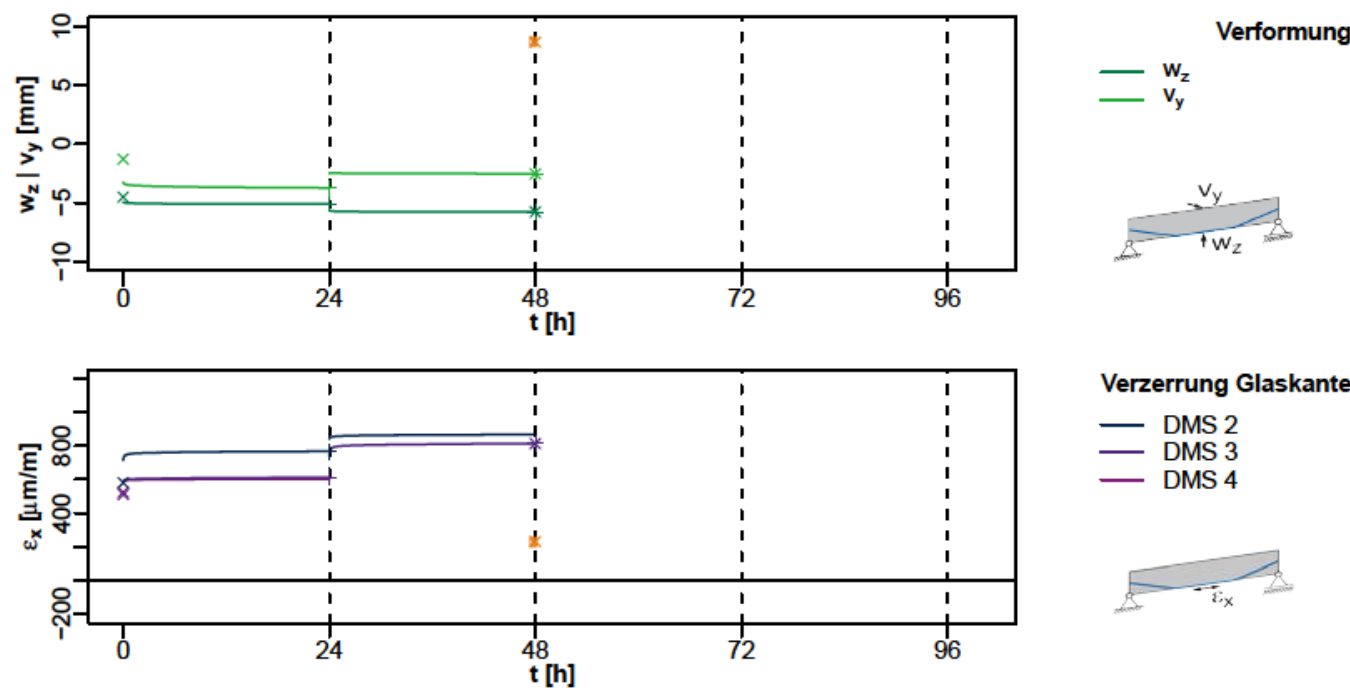

Verzerrung Glaskante

- DMS 2

- DMS 3

- DMS 4

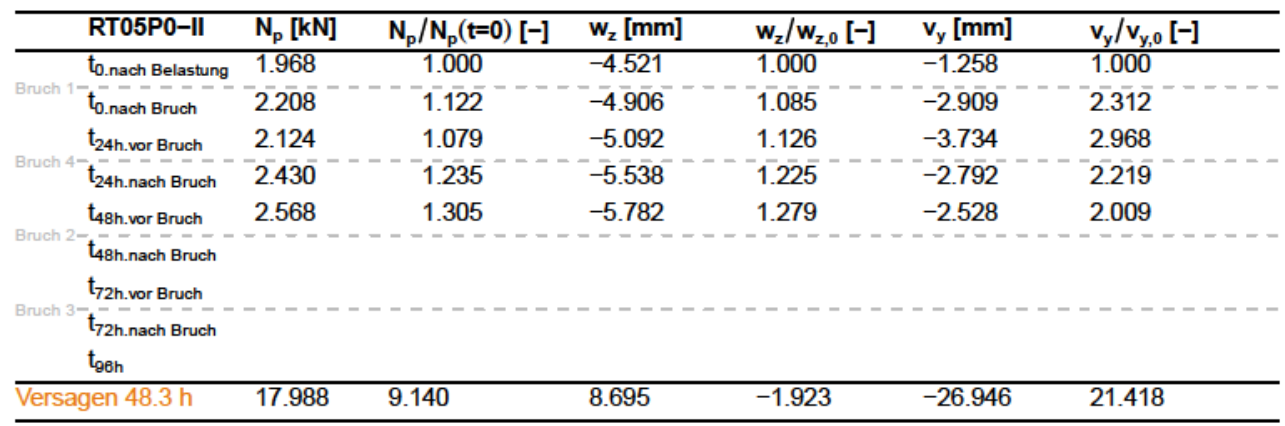


Bruchbild RT05P0-II

$h_{z}=66,4 \%$

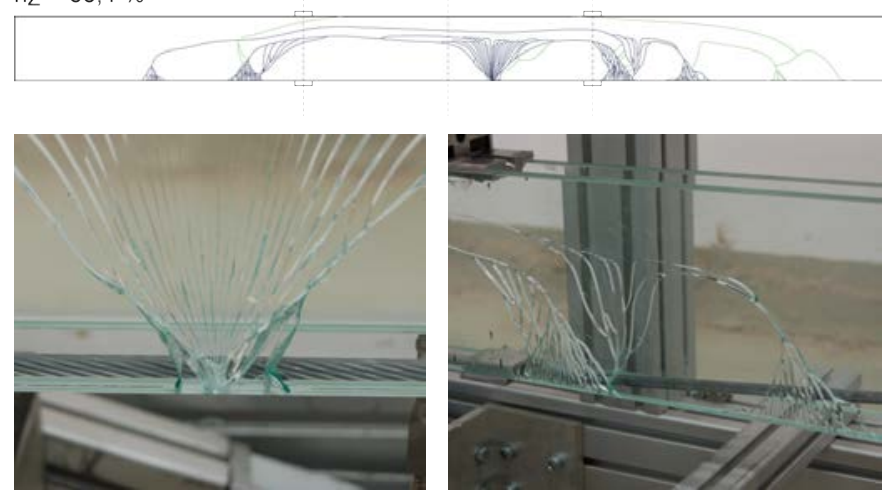

$h_{z}=61,9 \%$
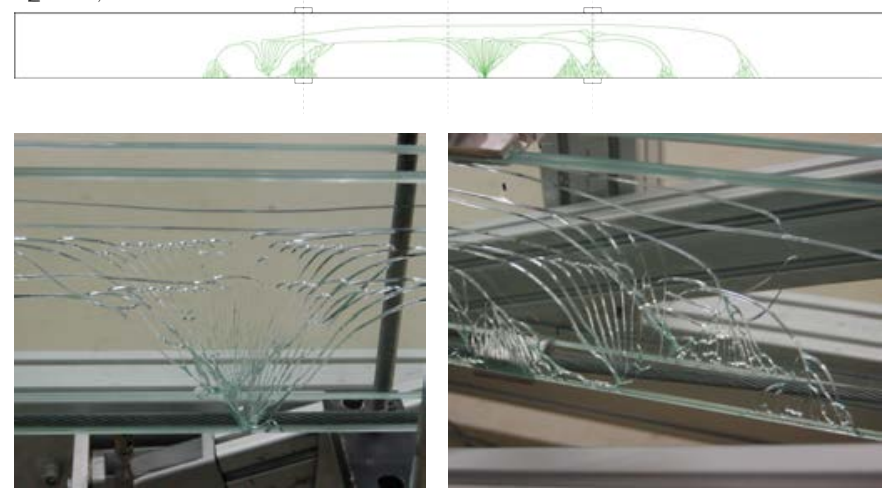

Scheibe 1

Bruch durch Anschlagen nach $\mathrm{t}=0 \mathrm{~h}$

- Brucherweiterung bis $\mathrm{t}=24 \mathrm{~h}$

Brucherweiterung bis $\mathrm{t}=48 \mathrm{~h}$

Scheibe 4

Bruch durch Anschlagen nach $\mathrm{t}=24 \mathrm{~h}$

_ Brucherweiterung bis $\mathrm{t}=48 \mathrm{~h}$
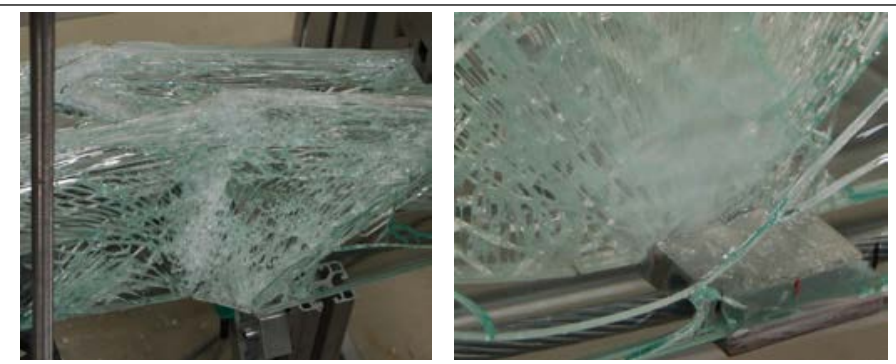

Bruch aller Scheiben

seitliches Versagen entlang der Rissursprünge (links)

zusätzlicher Bruchursprung an Umlenkung (rechts) 


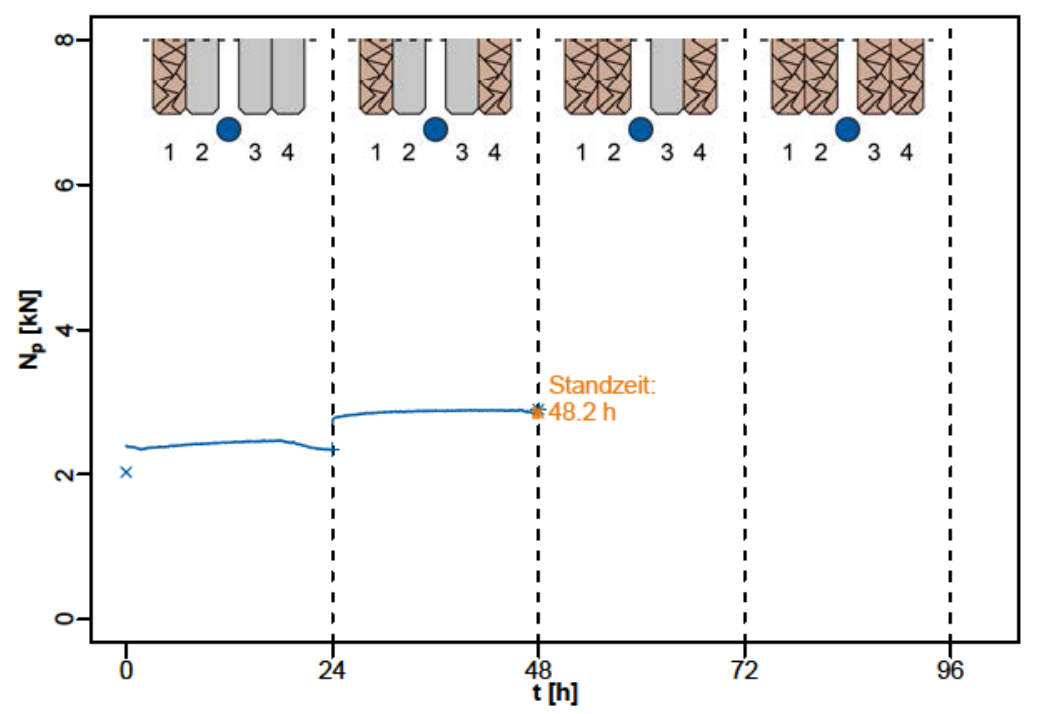

Seilkraftänderung

- RT06P0-II
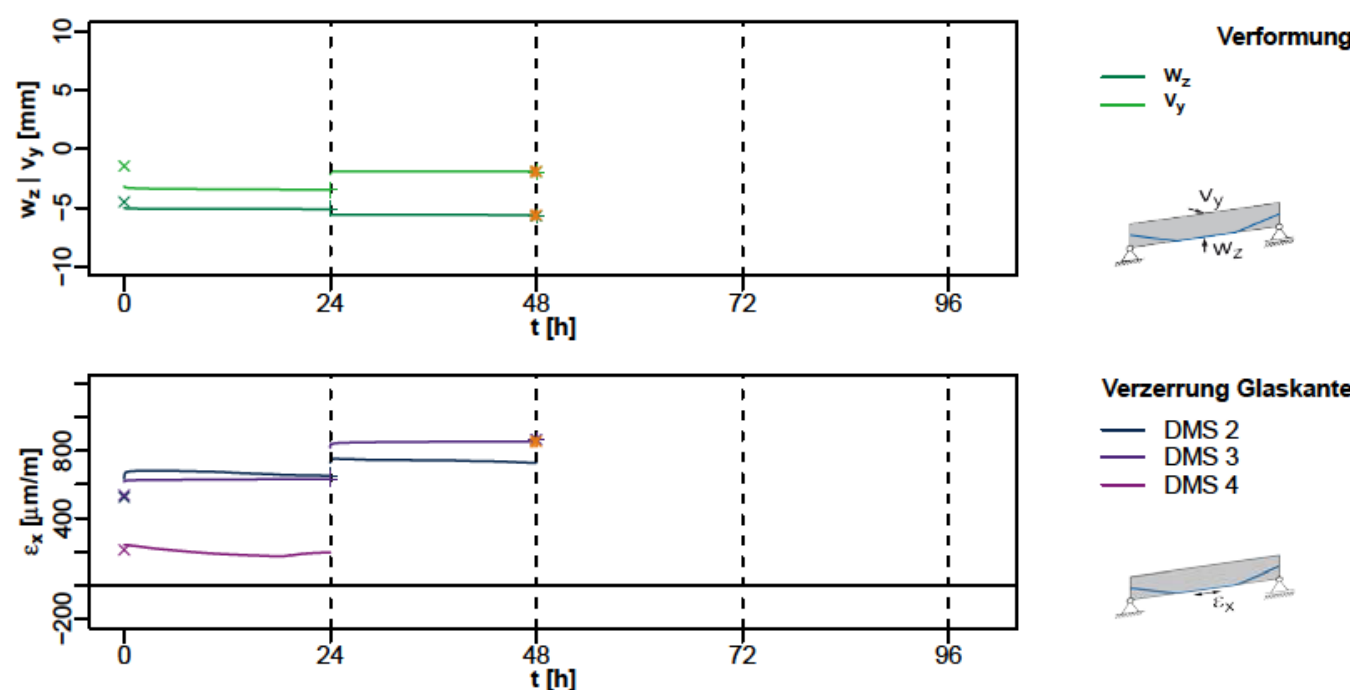

Verzerrung Glaskante

- DMS 2

- DMS 3

- DMS 4

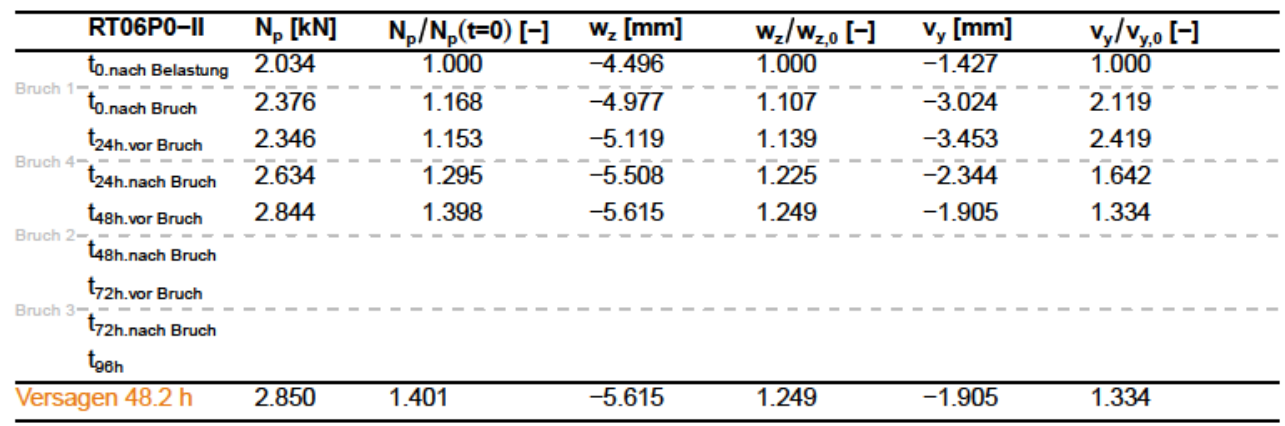




\section{Bruchbild RT06P0-II Referenzträger schlaff bewehrt $d_{S}=8,1 \mathrm{~mm}$}

$h z=66,5 \%$

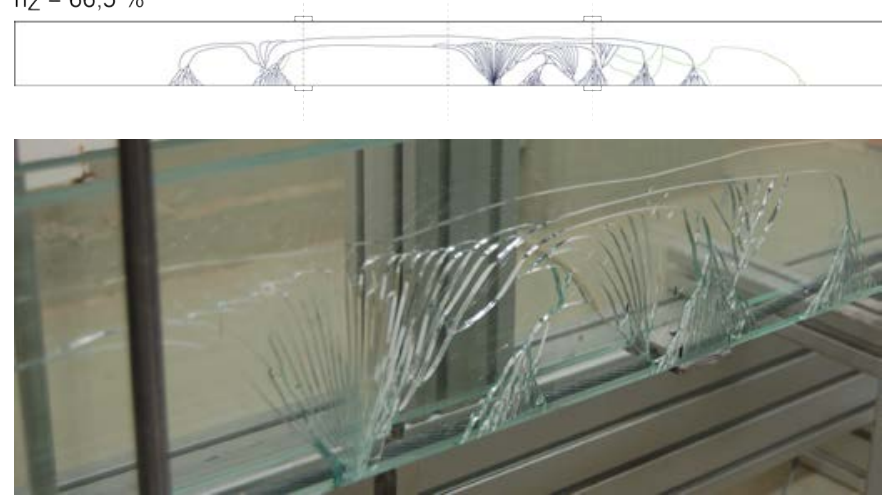

Scheibe 1

$h_{Z}=62,4 \%$

Scheibe 4

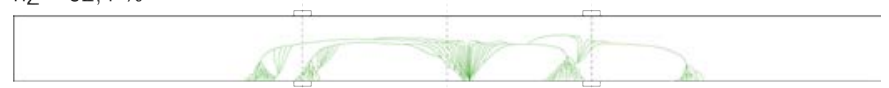

Bruch durch Anschlagen nach $\mathrm{t}=0 \mathrm{~h}$

- Brucherweiterung bis $\mathrm{t}=24 \mathrm{~h}$

Brucherweiterung bis $\mathrm{t}=48 \mathrm{~h}$
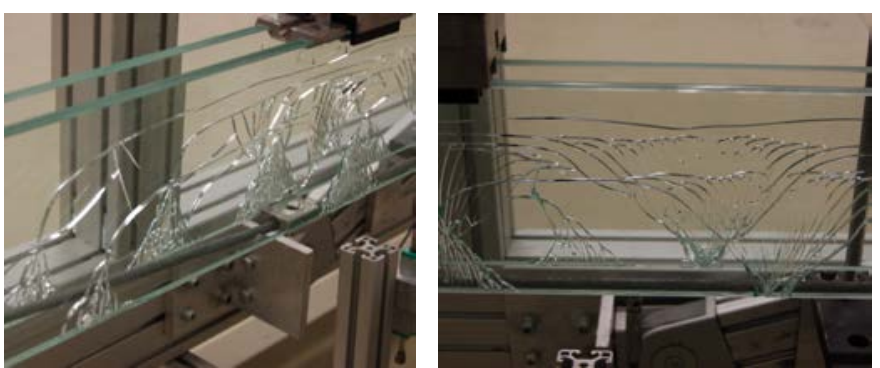

nach $\mathrm{t}=24 \mathrm{~h}$

- Brucherweiterung bis $t=48 h$
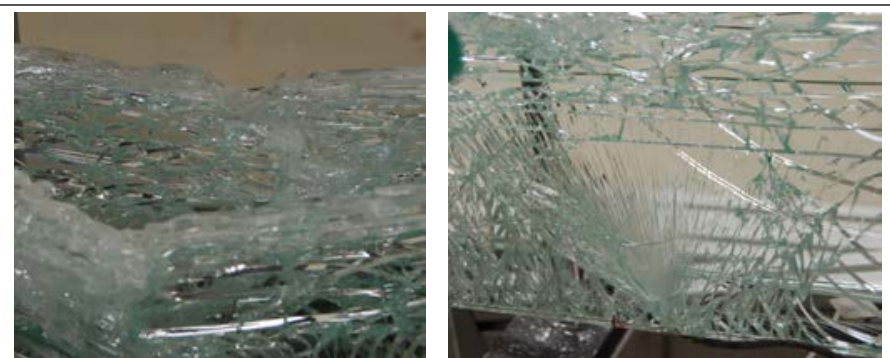

Bruch aller Scheiben

progressives, seitliches Versagen entlang der Rissursprünge Abgeplatzte Oberkante nahe der Zwischenfolie (links) zusätzlicher Bruchursprung in übrigerScheibe (rechts) 


\section{Standzeit RT07P0-II Referenzträger schlaff bewehrt $d_{S}=\mathbf{8 , 1} \mathbf{m m}$}

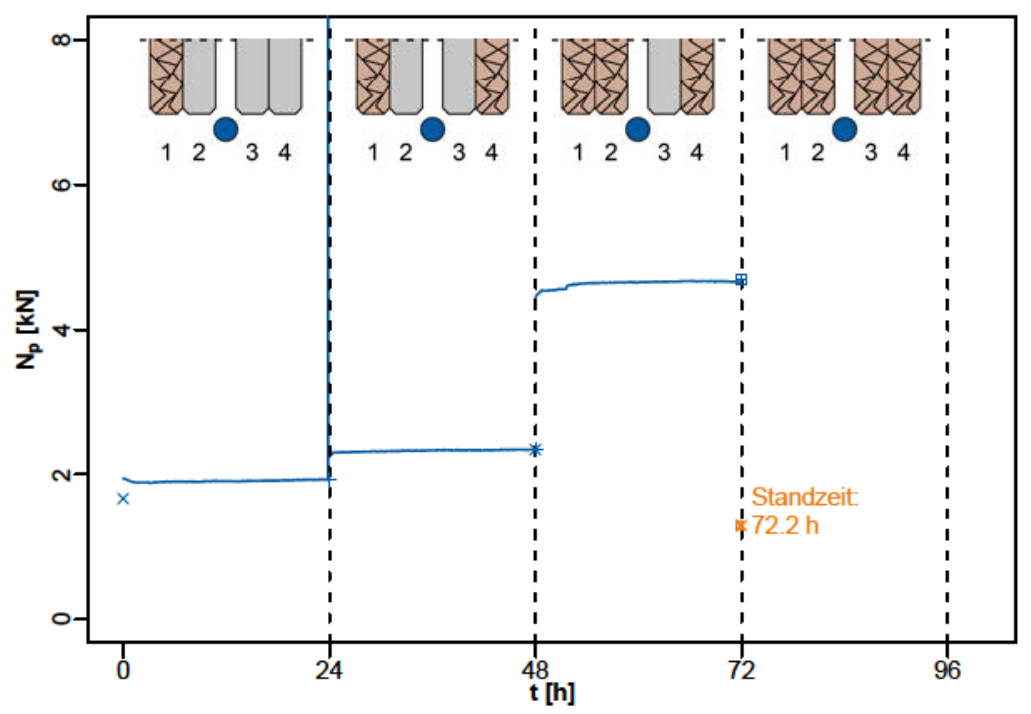

Seilkraftänderung

- RTO7P0-II
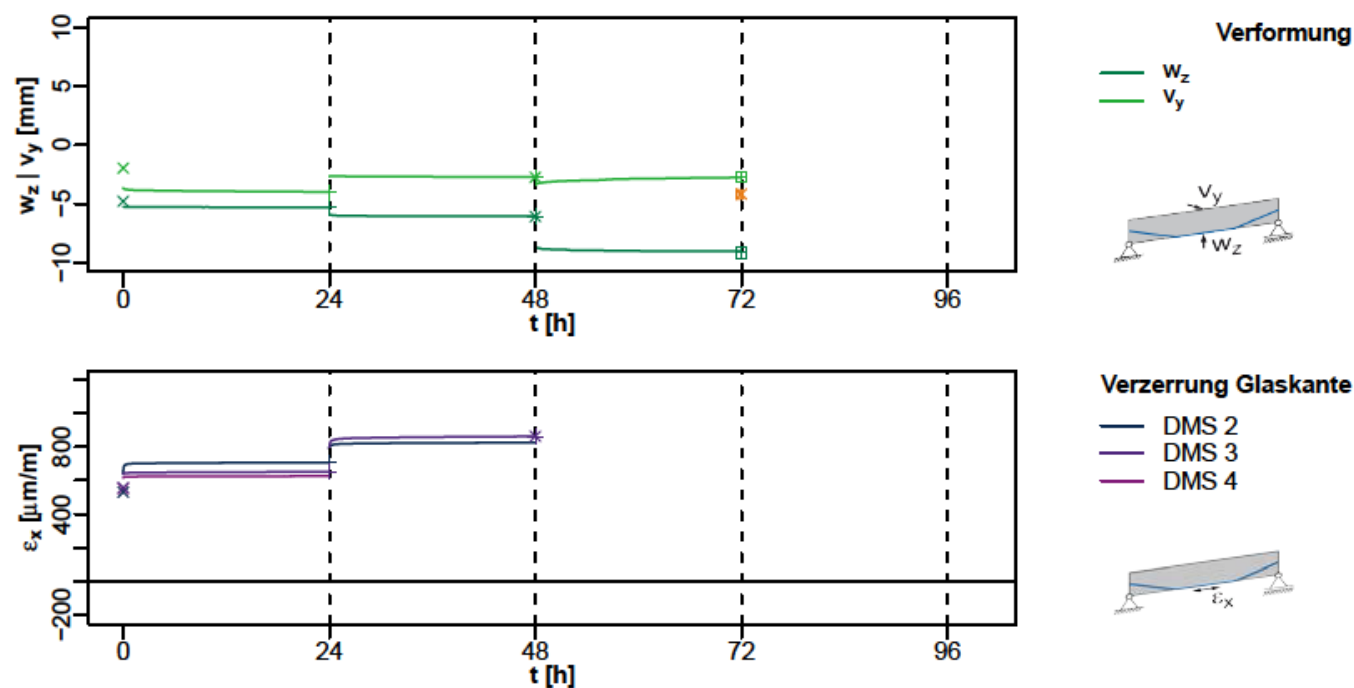

Verzerrung Glaskante

- DMS 2

- DMS 3

- DMS 4

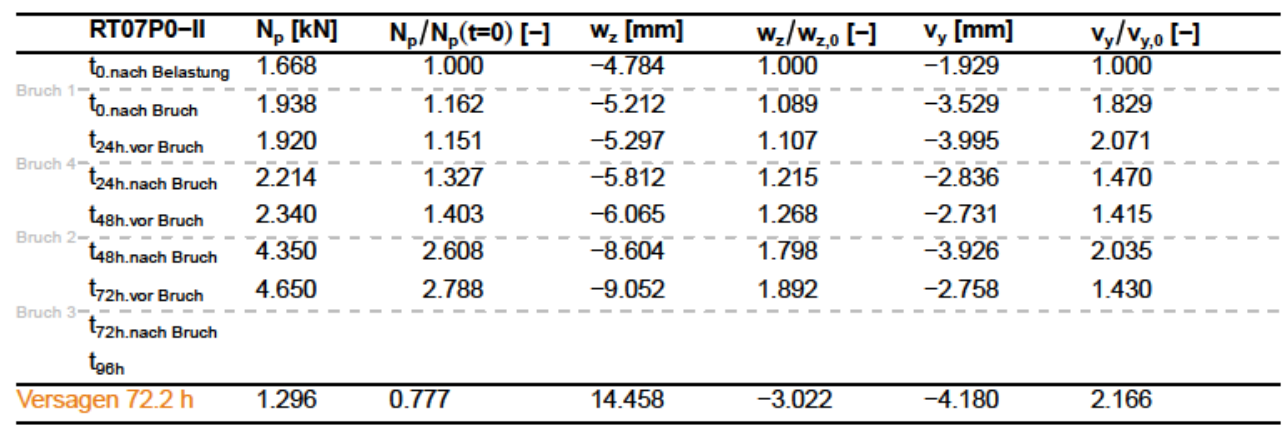


Bruchbild RT07P0-II

Referenzträger schlaff bewehrt $d_{s}=8,1 \mathrm{~mm}$

$h z=67,2 \%$

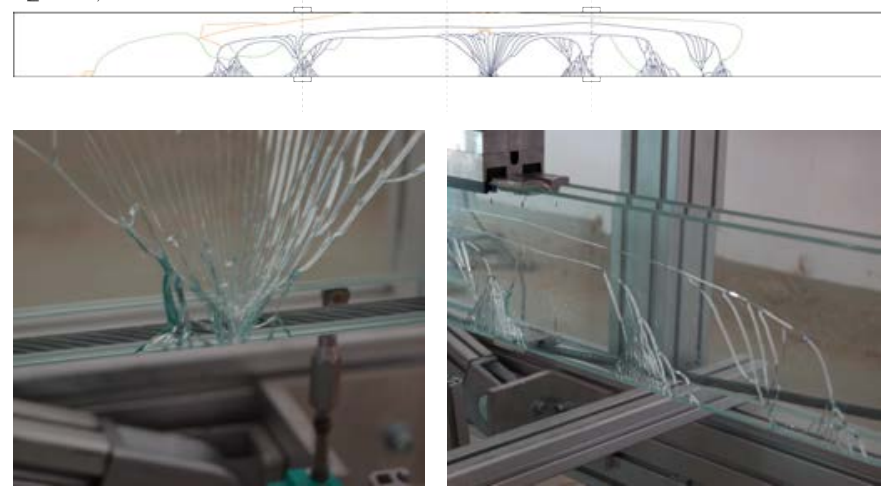

$h_{Z}=62,4 \%$
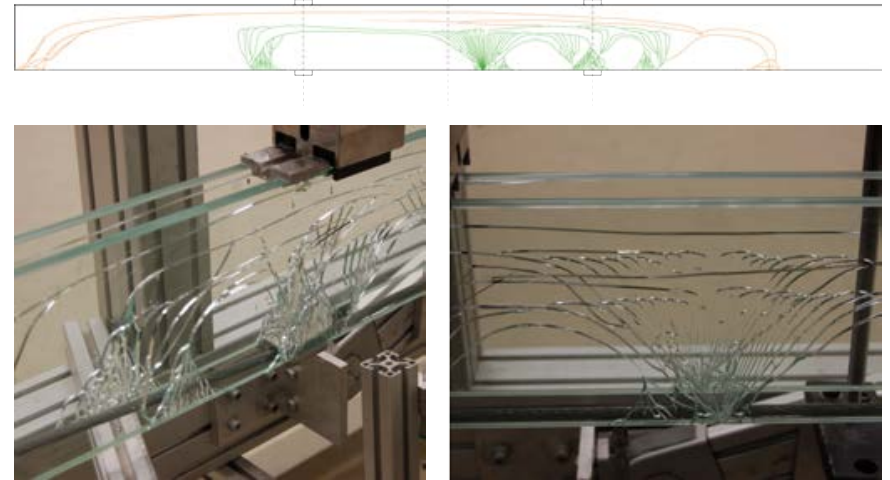

$h_{z}=83,8 \%$
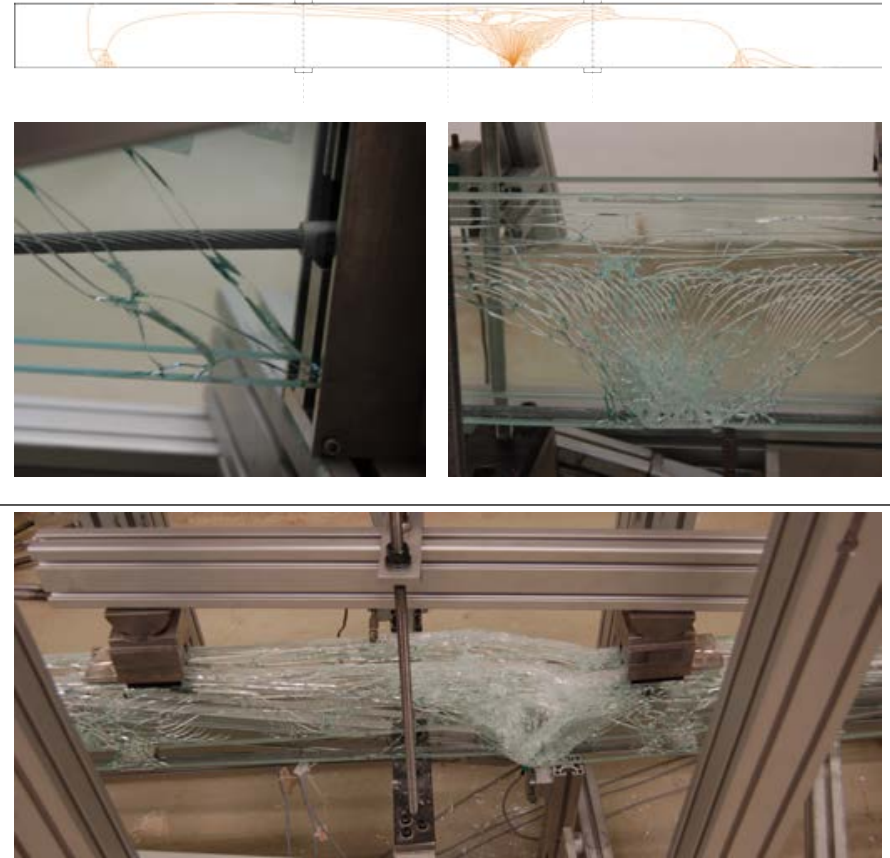

Scheibe 1

Bruch durch Anschlagen nach $\mathrm{t}=0 \mathrm{~h}$

- Brucherweiterung bis $t=24 \mathrm{~h}$

Brucherweiterung bis $\mathrm{t}=48 \mathrm{~h}$

Scheibe 4

Bruch durch Anschlagen nach $\mathrm{t}=24 \mathrm{~h}$

- Brucherweiterung bis $\mathrm{t}=48 \mathrm{~h}$

Scheibe 2

Bruch durch Anschlagen nach $\mathrm{t}=48 \mathrm{~h}$

Risserweiterung ins Auflager (links) breiter Bruchfächer und abgeplatzte Oberkante rechts

Bruch aller Scheiben

progressivesseitliches Versagen entlang der Rissursprünge 


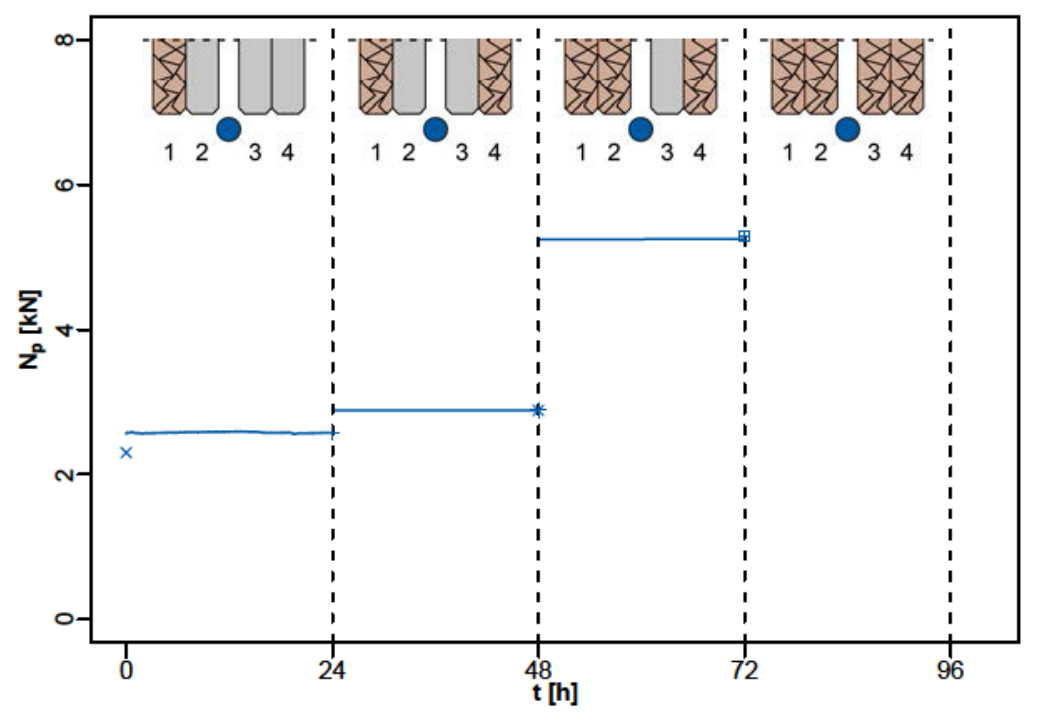

Seilkraftänderung

- RT08P0-II
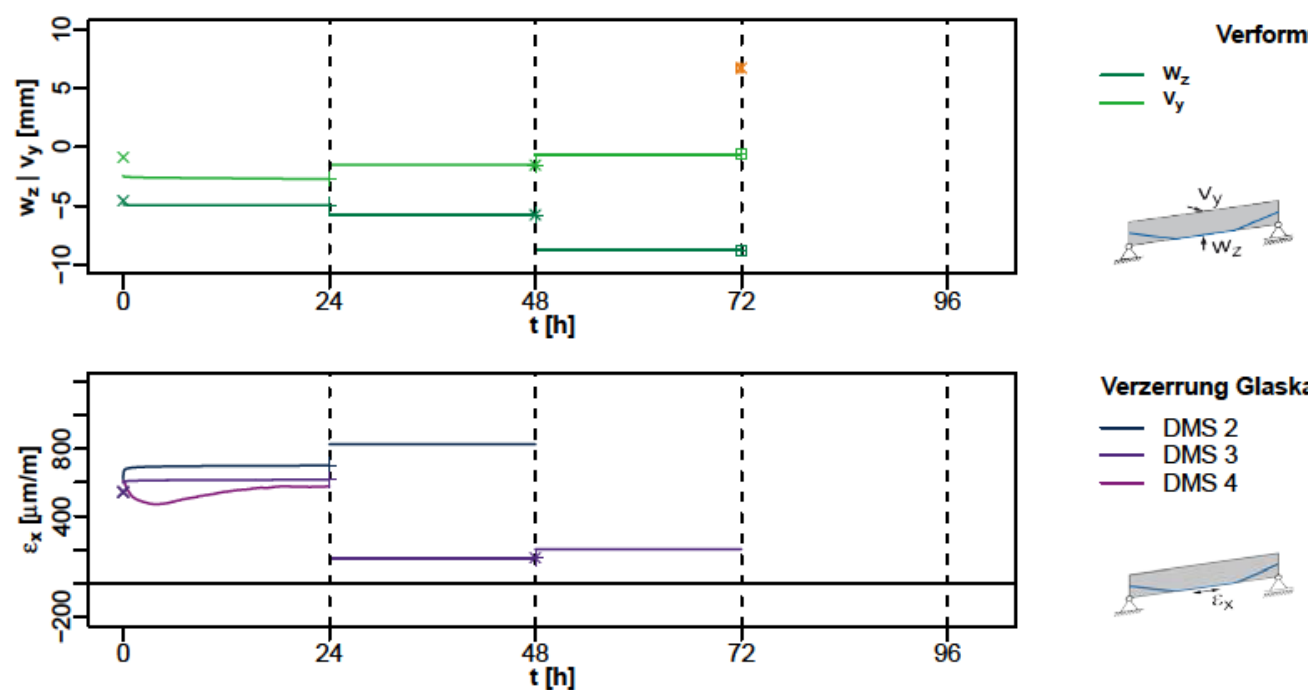

Verzerrung Glaskante

- DMS 2

- DMS 3

- DMS 4

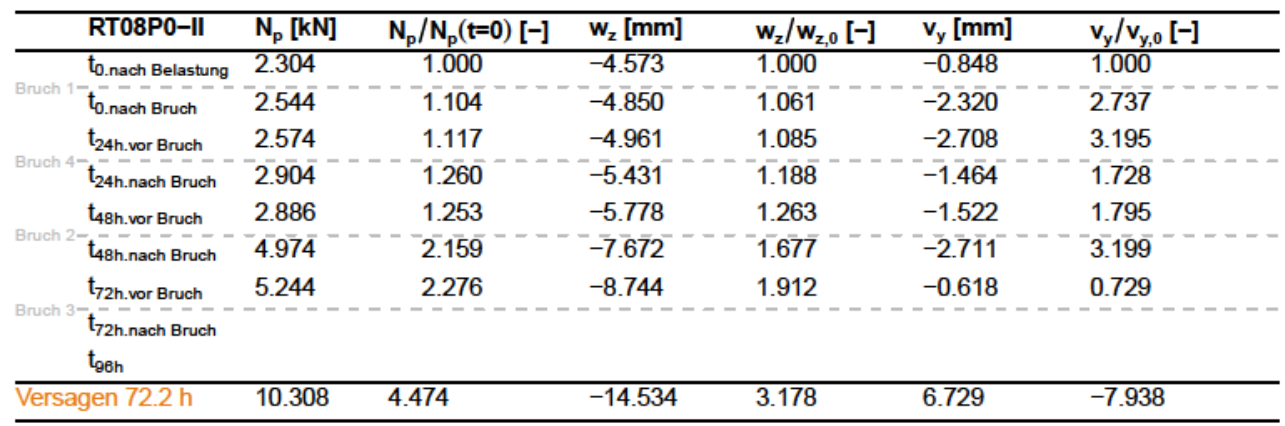


Bruchbild RT08P0-II

$h \mathrm{z}=68,8 \%$

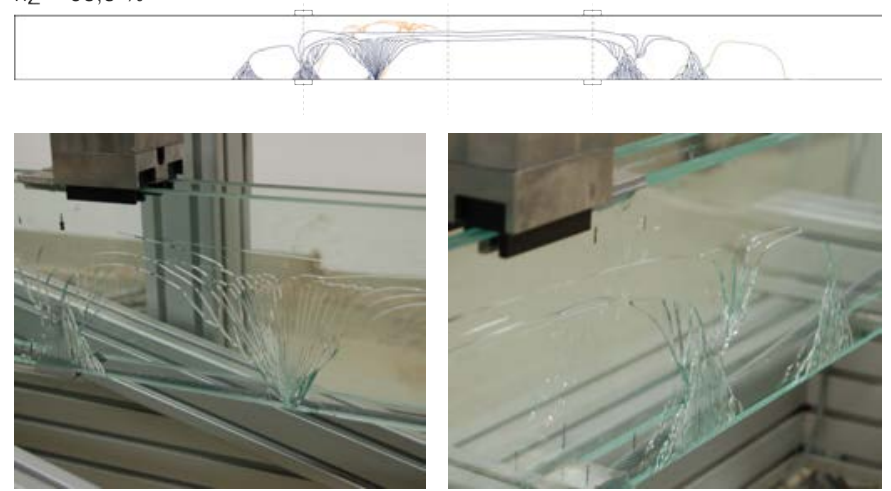

$h_{\mathrm{Z}}=65,0 \%$
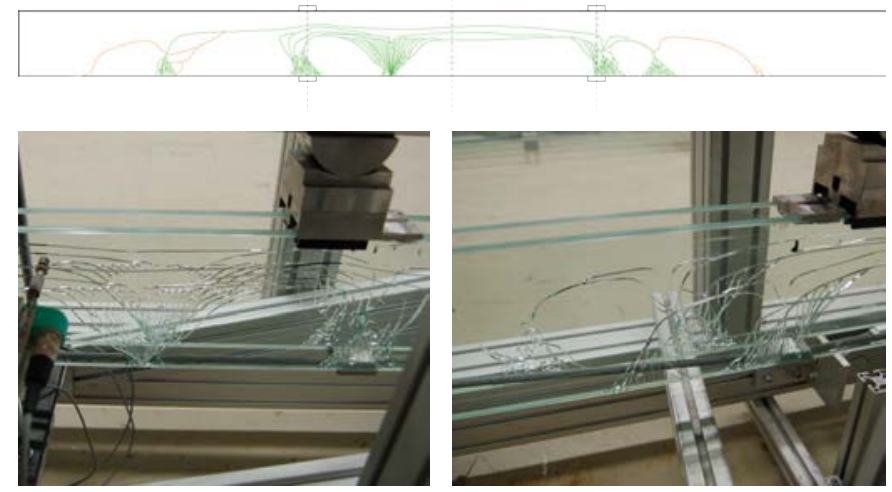

$h_{z}=73,0 \%$
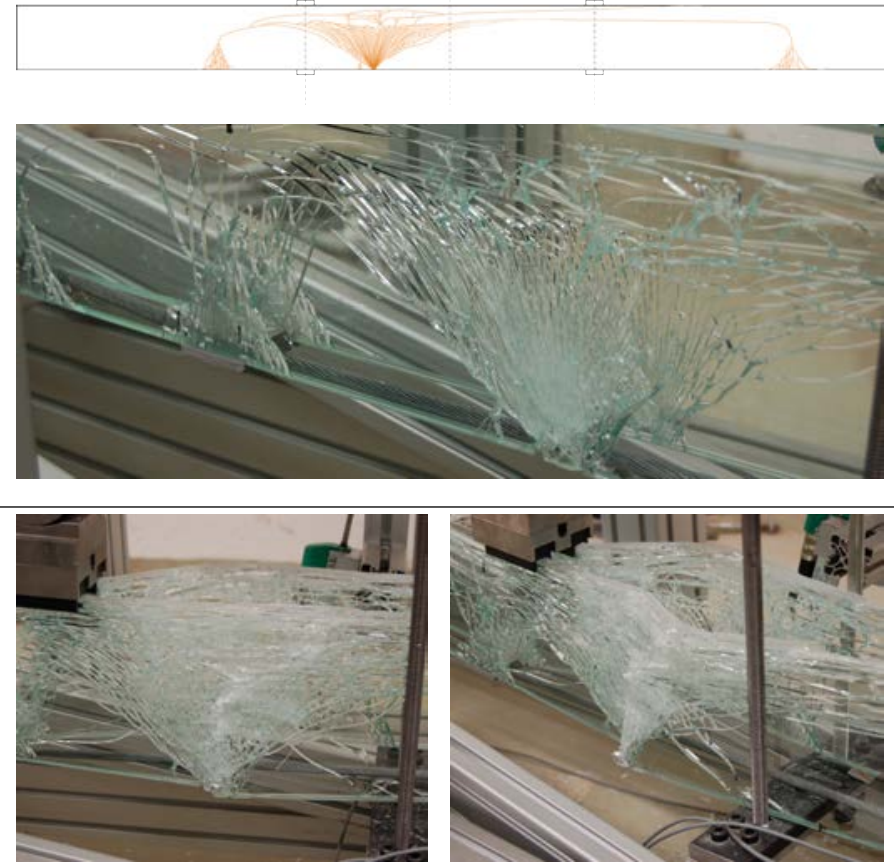

Scheibe 1

Bruch durch Anschlagen nach $\mathrm{t}=0 \mathrm{~h}$

- Brucherweiterung bis $\mathrm{t}=24 \mathrm{~h}$

_ Brucherweiterung bis $\mathrm{t}=48 \mathrm{~h}$
Scheibe 4

Bruch durch Anschlagen nach $\mathrm{t}=24 \mathrm{~h}$

_ Brucherweiterung bis $t=48 h$

Bruch aller Scheiben

zunächst hält die Bewehrung den gebrochenen Träger, anschließend progressives seitliches Versagen 


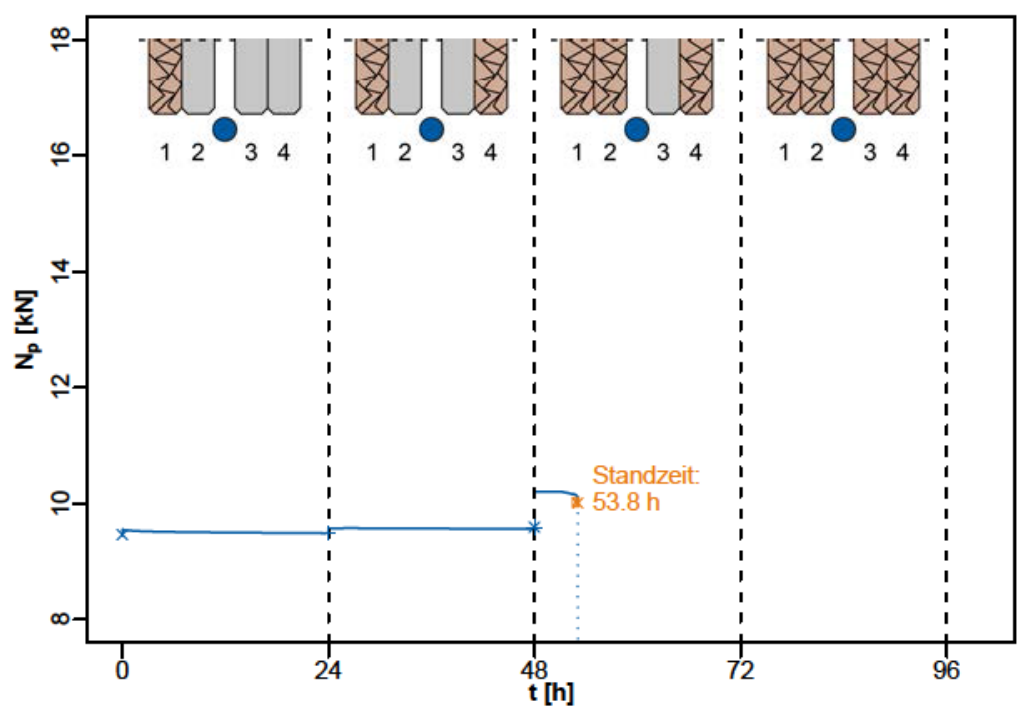

Seilkraftänderung

— RT09P9-I
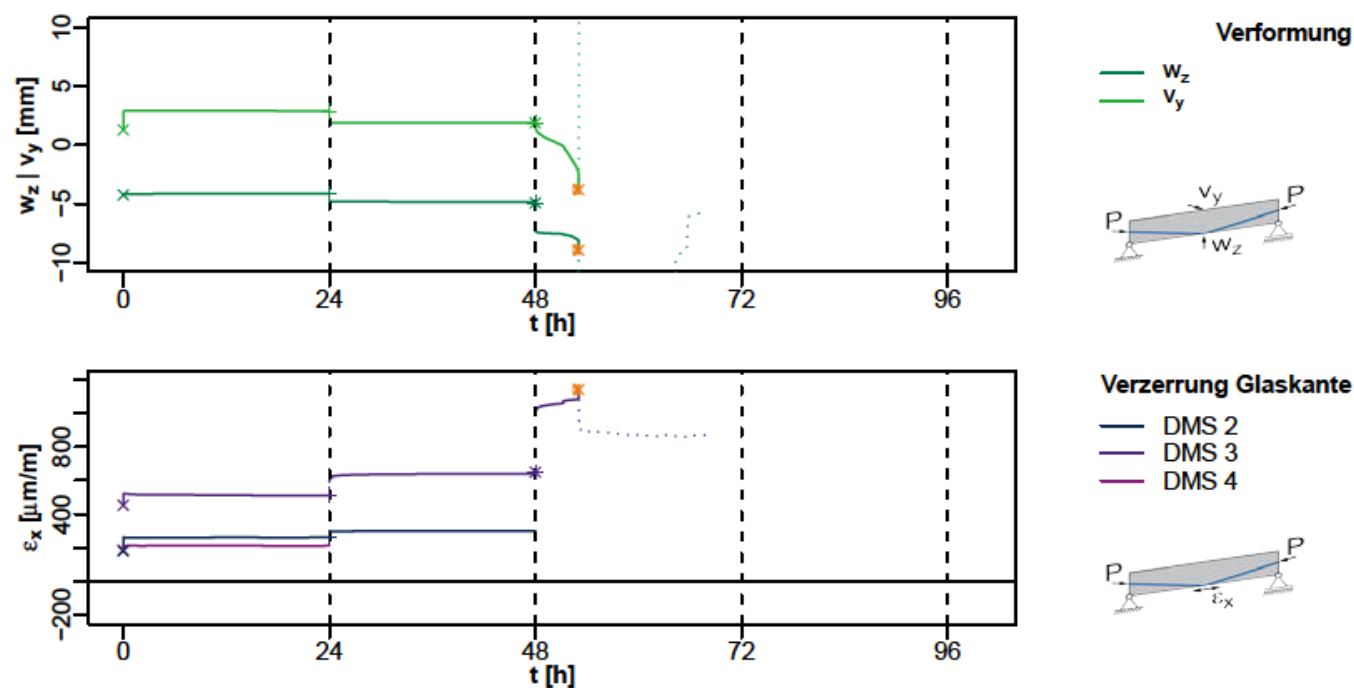

Verzerrung Glaskante

- DMS 2

- DMS 3

- DMS 4

\begin{tabular}{|c|c|c|c|c|c|c|c|}
\hline & RT09P9-I & $\mathrm{N}_{\mathrm{p}}[\mathrm{kN}]$ & $N_{p} / N_{p}(t=0)[-]$ & $w_{z}[\mathrm{~mm}]$ & $w_{z} / w_{z, 0}[-]$ & $v_{y}[\mathrm{~mm}]$ & $v_{y} / v_{y, 0}[-]$ \\
\hline & $\mathrm{t}_{\text {0.nach Belastung }}$ & 9.464 & 1.000 & -4.226 & 1.000 & 1.309 & 1.000 \\
\hline Bruch 1 & $\mathrm{t}_{0 . \text { nach Bruch }}$ & $9 . \overline{46} \overline{3}$ & 1.000 & -4.236 & $1.00 \overline{2}$ & 1.310 & $1.00 \overline{1}$ \\
\hline \multirow[b]{2}{*}{ Bruch 4} & $t_{24 h . v o r}$ Bruch & 9.490 & 1.003 & -4.141 & 0.980 & 2.920 & 2.230 \\
\hline & $\mathrm{t}_{24 \mathrm{~h} . \text { nach Bruch }}$ & 9.548 & $1.00 \overline{9}$ & -4.754 & $1.12 \overline{5}$ & $1.96 \overline{2}$ & $1.49 \overline{9}$ \\
\hline \multirow[b]{2}{*}{ Bruch 2} & 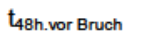 & 9.561 & 1.010 & -4.836 & 1.144 & 1.914 & 1.462 \\
\hline & $\mathrm{t}_{48 \mathrm{~h} . \text { nach Bruch }}$ & 10.187 & $1.0 \overline{7} \overline{6}$ & -7.129 & $1.68 \overline{7}$ & $1.85 \overline{6}$ & $1 . \overline{418}$ \\
\hline Bruch 3 & 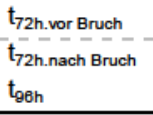 & & & & & & \\
\hline \multicolumn{2}{|c|}{ Versagen $53.8 \mathrm{~h}$} & 10.013 & 1.058 & -8.962 & 2.121 & -3.817 & -2.916 \\
\hline
\end{tabular}




\section{Bruchbild RT09P9-I Träger mit $P_{m 0}=9 \mathrm{kN}$ und $d_{p}=5,0 \mathrm{~mm}$}

$h_{z}=62,5 \%$

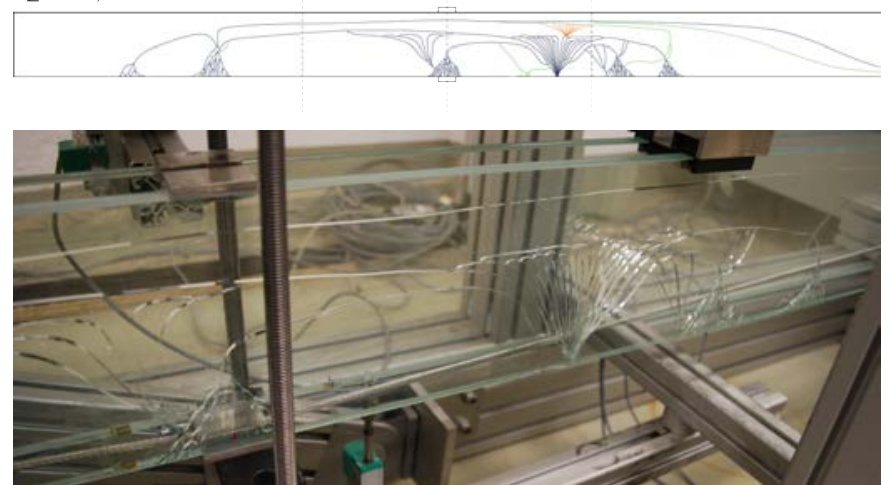

$h_{z}=60,7 \%$
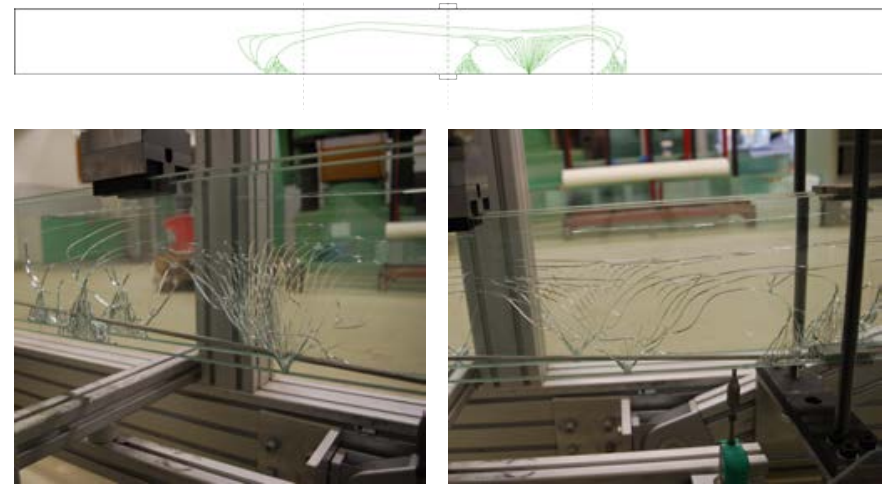

$h_{z}=69,7 \%$
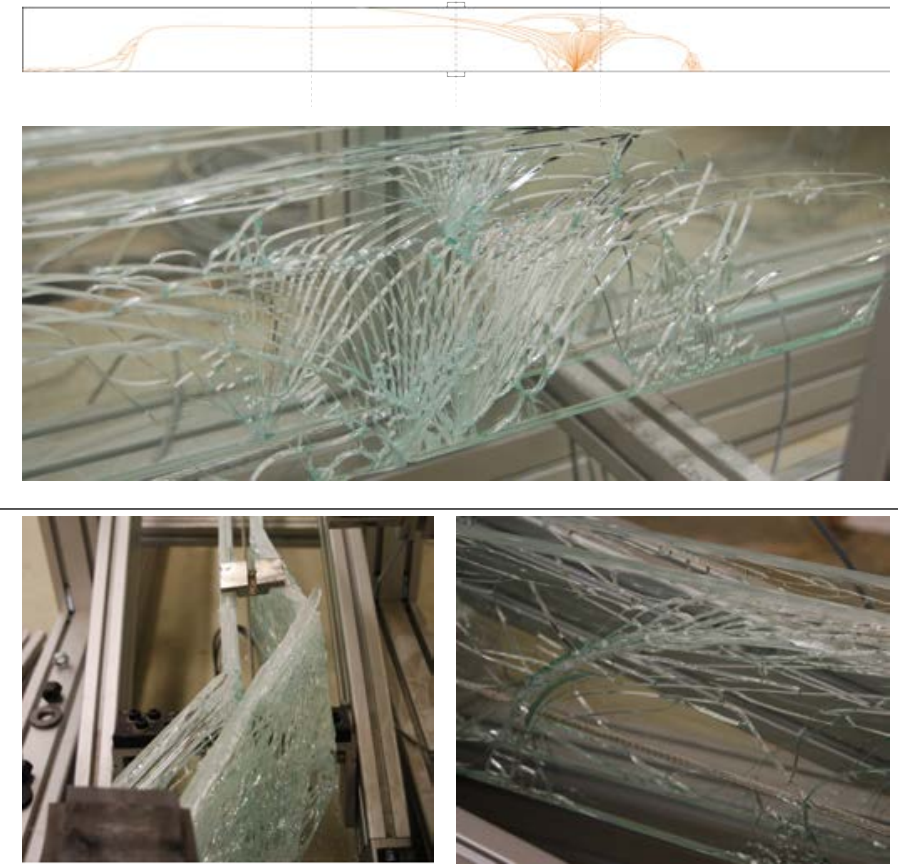

Scheibe 1

Bruch durch Anschlagen nach $\mathrm{t}=0 \mathrm{~h}$

- Brucherweiterung bis $\mathrm{t}=24 \mathrm{~h}$

- Brucherweiterung bis $\mathrm{t}=48 \mathrm{~h}$

Scheibe 4

Bruch durch Anschlagen nach $\mathrm{t}=24 \mathrm{~h}$

- Brucherweiterung bis $\mathrm{t}=48 \mathrm{~h}$

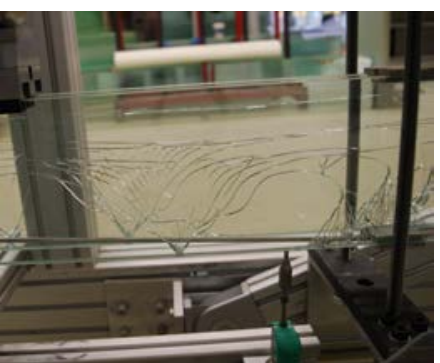

Scheibe 2 Bruch durch Anschlagen nach $\mathrm{t}=48 \mathrm{~h}$

mehrfache Bruchfächer übereinander

Bruch aller Scheiben

Einknicken der VSG-Pakete (links) markanter Riss (rechts) 


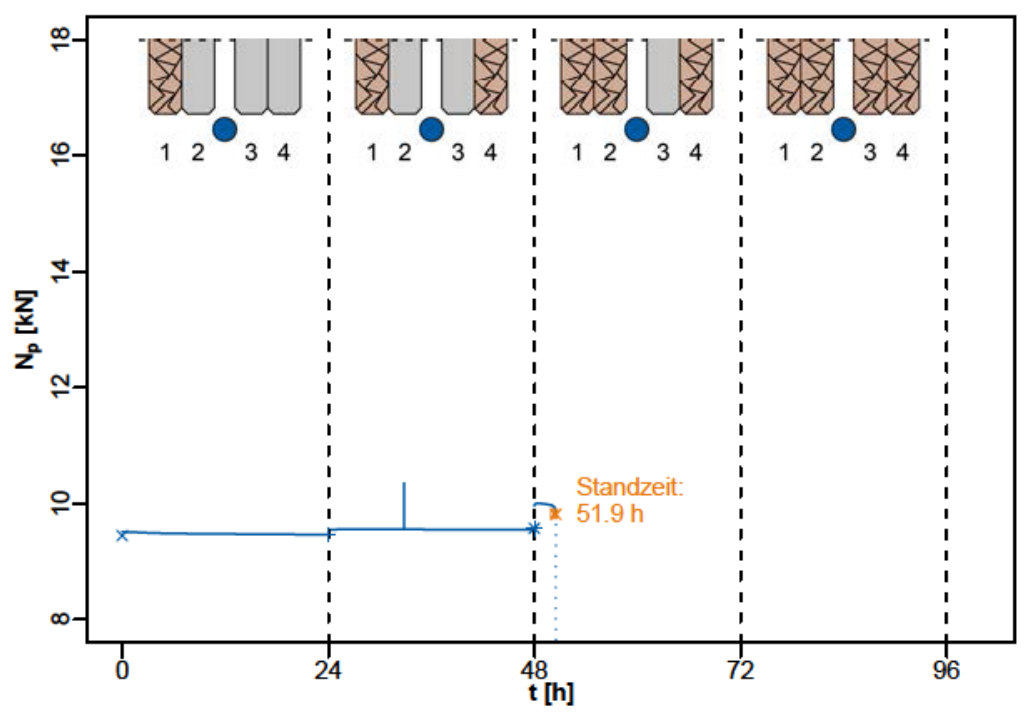

Seilkraftänderung — RT10P9-I

einmaliger Ausreißer der Seilkraft nach $33,5 \mathrm{~h}$
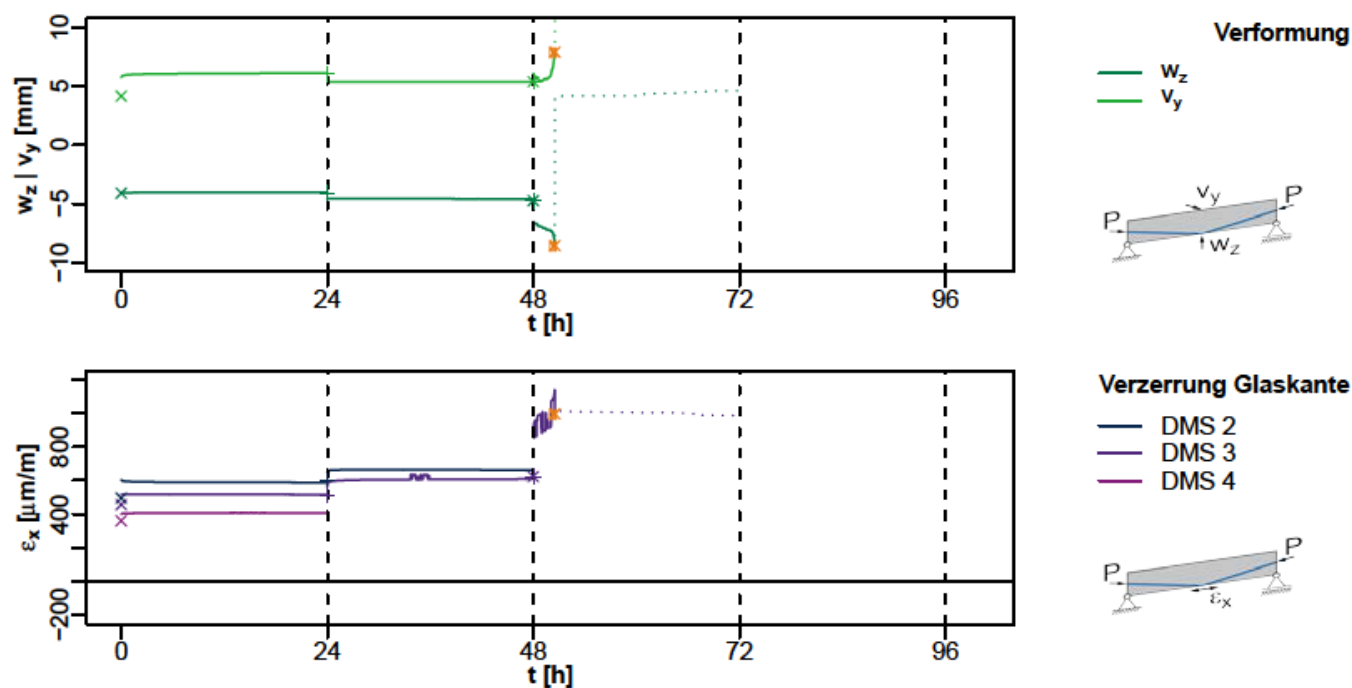

Verzerrung Glaskante - DMS 2

- DMS 3

- DMS 4

\begin{tabular}{|c|c|c|c|c|c|c|c|}
\hline & RT10P9-I & $\mathrm{N}_{\mathrm{p}}[\mathrm{kN}]$ & $N_{p} / N_{p}(t=0)[-]$ & $w_{z}[\mathrm{~mm}]$ & $w_{z} / w_{z, 0}[-]$ & $v_{y}[\mathrm{~mm}]$ & $v_{y} / v_{y, 0}[-]$ \\
\hline \multirow[b]{2}{*}{ Bruch 1} & $\mathrm{t}_{\text {0.nach Belastung }}$ & 9.405 & 1.000 & -4.056 & 1.000 & 3.453 & 1.000 \\
\hline & $\mathrm{t}_{0 . \text { nach Bruch }}$ & $9 . \overline{521}$ & $1.01 \overline{2}$ & -4.193 & $1.03 \overline{4}$ & $5.64 \overline{2}$ & $1.6 \overline{4}$ \\
\hline \multirow[b]{2}{*}{ Bruch. } & 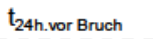 & 9.457 & 1.006 & -3.984 & 0.982 & 6.153 & 1.782 \\
\hline & $\mathrm{t}_{24 \mathrm{~h} . \text { nach Bruch }}$ & $9.4 \overline{9}$ & 1.010 & -4.463 & 1.100 & $5.4 \overline{7} \overline{1}$ & $1.5 \overline{8}$ \\
\hline \multirow[b]{2}{*}{ Bruch 2} & 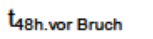 & 9.548 & 1.015 & -4.583 & 1.130 & 5.374 & 1.556 \\
\hline & $\mathrm{t}_{48 \mathrm{~h} . \text { nach Bruch }}$ & 9.948 & $1.05 \overline{8}$ & -6.505 & $1.60 \overline{4}$ & $6.19 \overline{5}$ & $1.7 \overline{9}$ \\
\hline Bruch 3 & $\begin{array}{l}\mathrm{t}_{72 \mathrm{~h} . v o r} \text { Bruch } \\
\mathrm{t}_{72 \mathrm{~h} . \text { nach Bruch }} \\
\mathrm{t}_{98 \mathrm{~h}}\end{array}$ & & & & & & - \\
\hline \multicolumn{2}{|c|}{ Versagen $51.9 \mathrm{~h}$} & 9.815 & 1.044 & -8.559 & 2.110 & 7.921 & 2.294 \\
\hline
\end{tabular}


Bruchbild RT10P9-I

$h_{z}=63,5 \%$

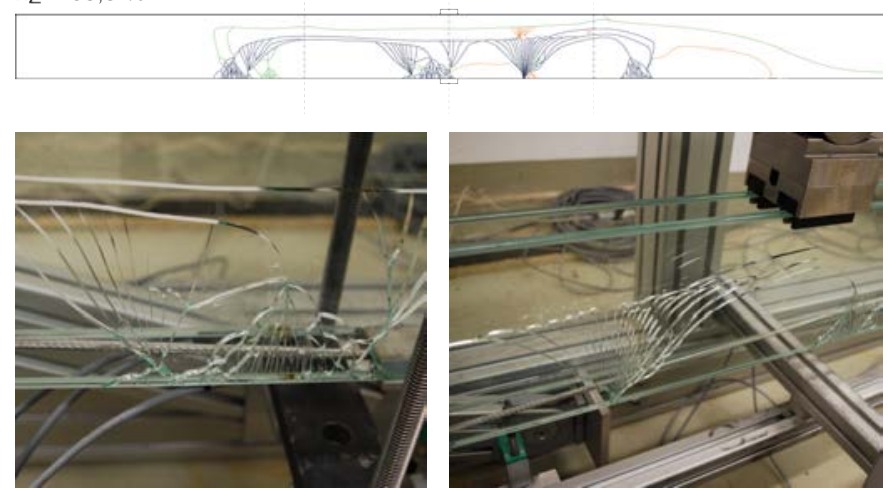

$h_{\mathrm{Z}}=55,5 \%$
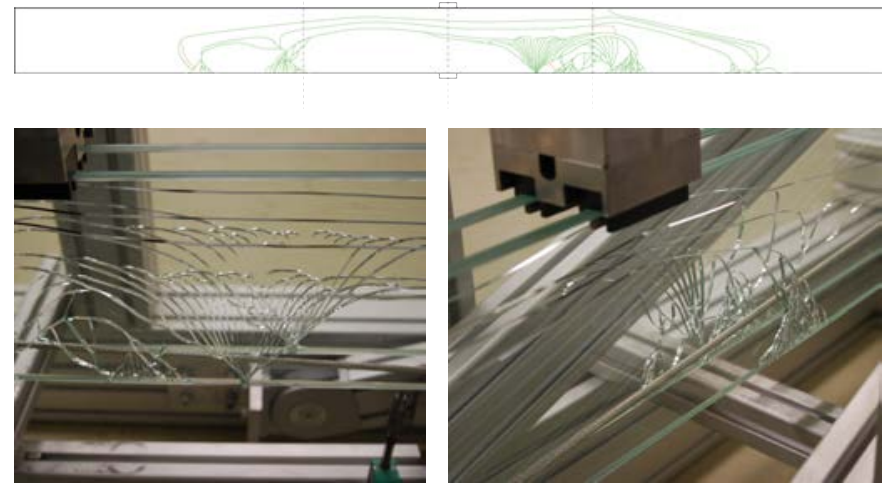

$h \mathrm{z}=72,3 \%$
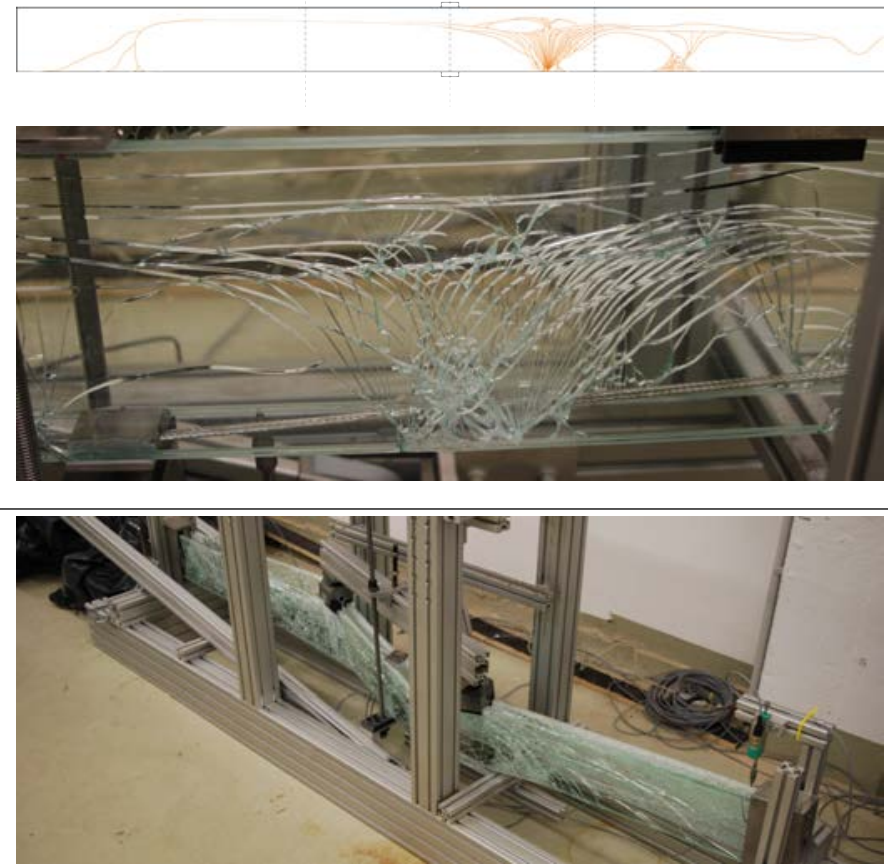

Scheibe 1

Bruch durch Anschlagen nach $\mathrm{t}=0 \mathrm{~h}$

- Brucherweiterung bis $\mathrm{t}=24 \mathrm{~h}$

_ Brucherweiterung bis $\mathrm{t}=48 \mathrm{~h}$

Scheibe 4

Bruch durch Anschlagen nach $\mathrm{t}=24 \mathrm{~h}$

- Brucherweiterung bis $\mathrm{t}=48 \mathrm{~h}$

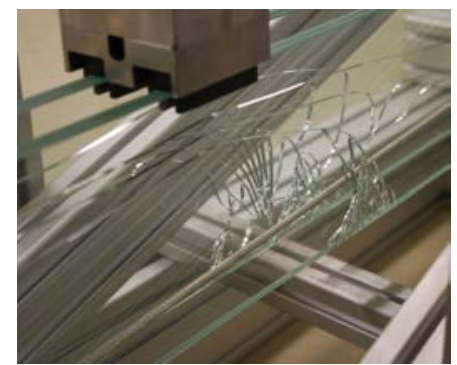

Scheibe 2

Bruch durch Anschlagen nach $\mathrm{t}=48 \mathrm{~h}$

Bruch aller Scheiben

Einknicken der VSG-Pakete Abreißen beider Umlenkpunkte 


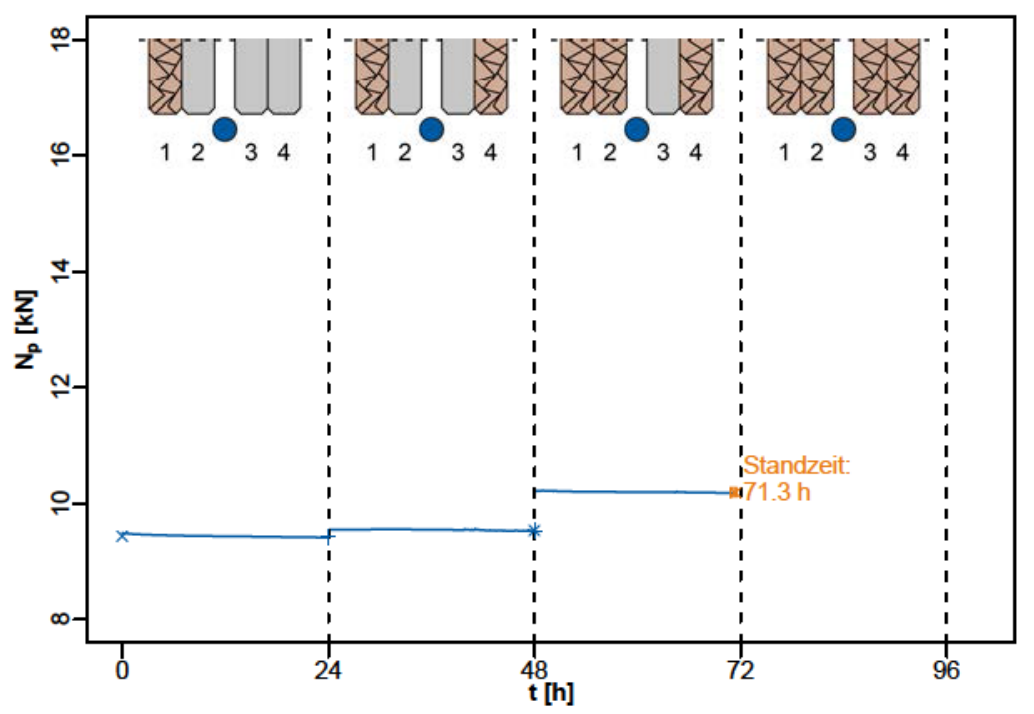

Seilkraftänderung RT11P9-I

DMS3 defekt

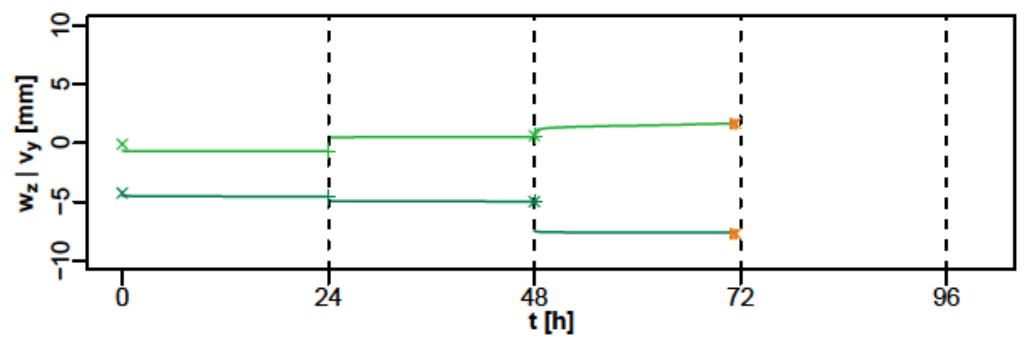

$-\mathrm{w}_{\mathrm{z}}$

Verformung

+ Bruch

* Bruch 2

$\rightarrow$ Bruch 3

k. Versagen

Brüche

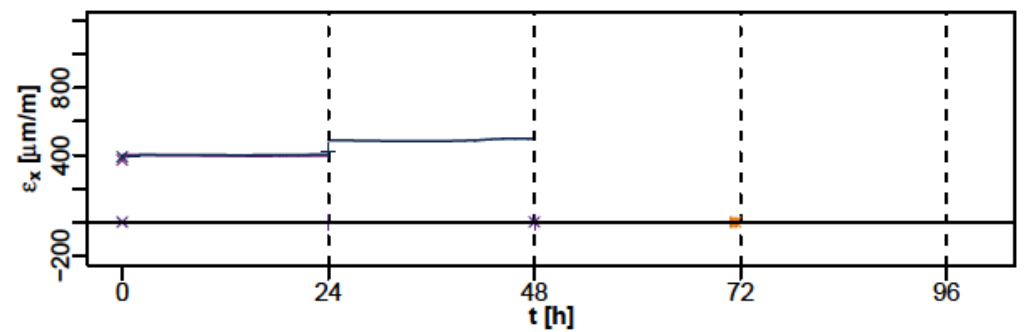

Verzerrung Glaskante

- DMS 2

- DMS 3

- DMS 4

\begin{tabular}{|c|c|c|c|c|c|c|c|}
\hline & RT11P9-I & $\mathrm{N}_{\mathrm{p}}[\mathrm{kN}]$ & $N_{p} / N_{p}(t=0)[-]$ & $\mathrm{w}_{\mathrm{z}}[\mathrm{mm}]$ & $w_{z} / w_{z, 0}[-]$ & $\mathrm{v}_{\mathrm{y}}[\mathrm{mm}]$ & $v_{y} / v_{y, 0}[-]$ \\
\hline & $\mathrm{t}_{0 . \text { nach Belastung }}$ & 9.439 & 1.000 & -4.245 & 1.000 & -0.0832 & 1.000 \\
\hline Bruch & $\mathrm{t}_{0 . \text { nach Bruch }}$ & $\overline{9} \overline{48 \overline{1}}$ & $1.00 \overline{4}$ & -4.371 & 1.030 & -0.450 & $5.40 \overline{7}$ \\
\hline & $t_{24 h . v o r}$ Bruch & 9.417 & 0.998 & -4.578 & 1.078 & -0.752 & 9.037 \\
\hline Bruch & $\mathrm{t}_{24 \mathrm{~h} . \text { nach Bruch }}$ & $9.52 \overline{9}$ & 1.010 & -4.843 & $1.14 \overline{1}$ & $0.50 \overline{2}$ & -6.037 \\
\hline & 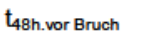 & 9.531 & 1.010 & -4.977 & 1.172 & 0.576 & -6.926 \\
\hline Bruch & 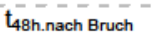 & 10.221 & $1.0 \overline{8}$ & -7.325 & $1.7 \overline{2} \overline{5}$ & 0.465 & -5.593 \\
\hline uch & 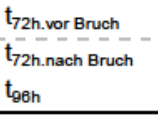 & & & & & & \\
\hline$\overline{\text { Versad }}$ & gen $71.3 \mathrm{~h}$ & 10.187 & 1.079 & -7.690 & 1.811 & 1.689 & -20.296 \\
\hline
\end{tabular}


Bruchbild RT11P9-I

Träger mit $P_{m 0}=9 \mathrm{kN}$ und $d_{p}=5,0 \mathrm{~mm}$

$h_{z}=68,8 \%$

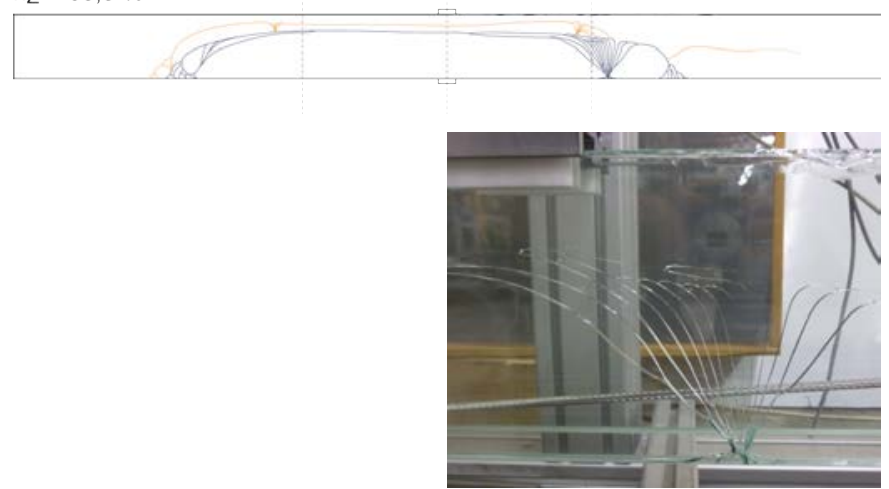

Scheibe 1

Bruch durch Anschlagen nach $\mathrm{t}=0 \mathrm{~h}$

- Brucherweiterung bis $\mathrm{t}=24 \mathrm{~h}$

Brucherweiterung bis $t=48 h$

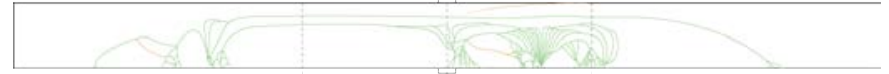

Bruch durch Anschlagen nach $\mathrm{t}=24 \mathrm{~h}$

_ Brucherweiterung bis $\mathrm{t}=48 \mathrm{~h}$

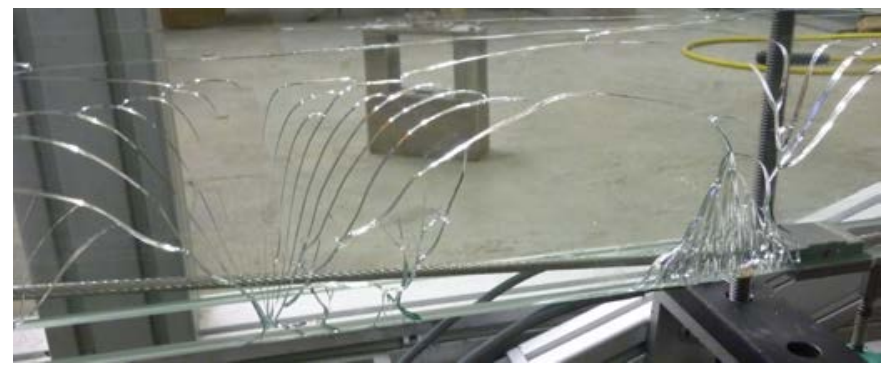

$h_{z}=72,5 \%$

Scheibe 2

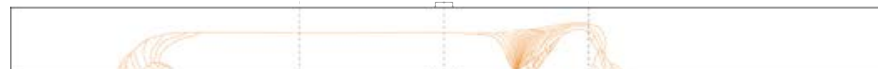

Bruch durch Anschlagen nach $\mathrm{t}=48 \mathrm{~h}$
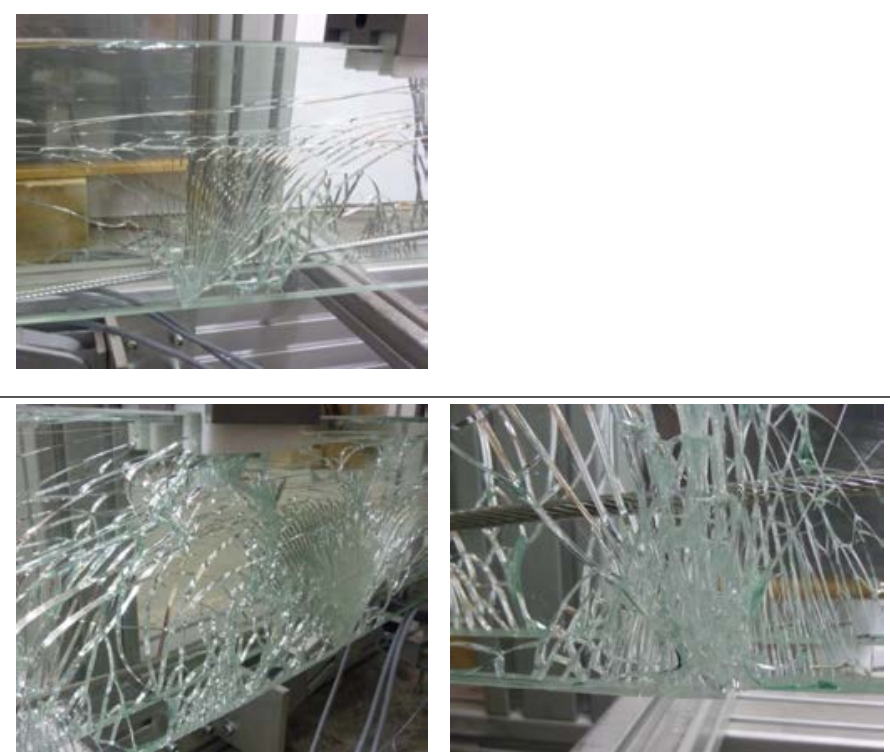

Bruch aller Scheiben

Versatz der Unterkante im rechten Bild 


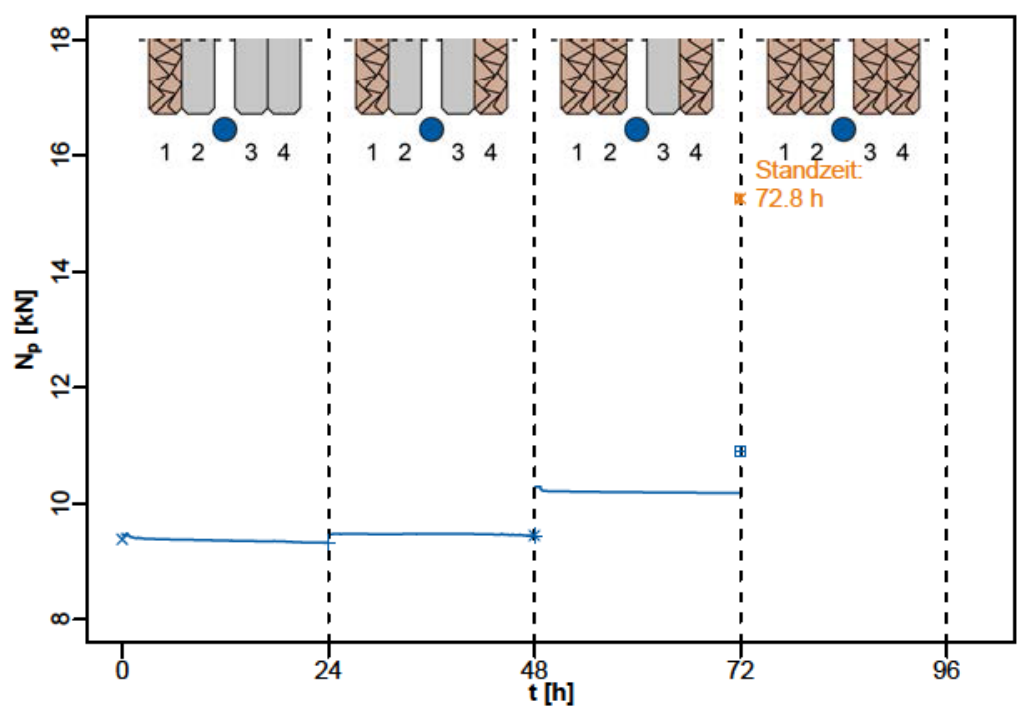

Seilkraftänderung RT12P9-I
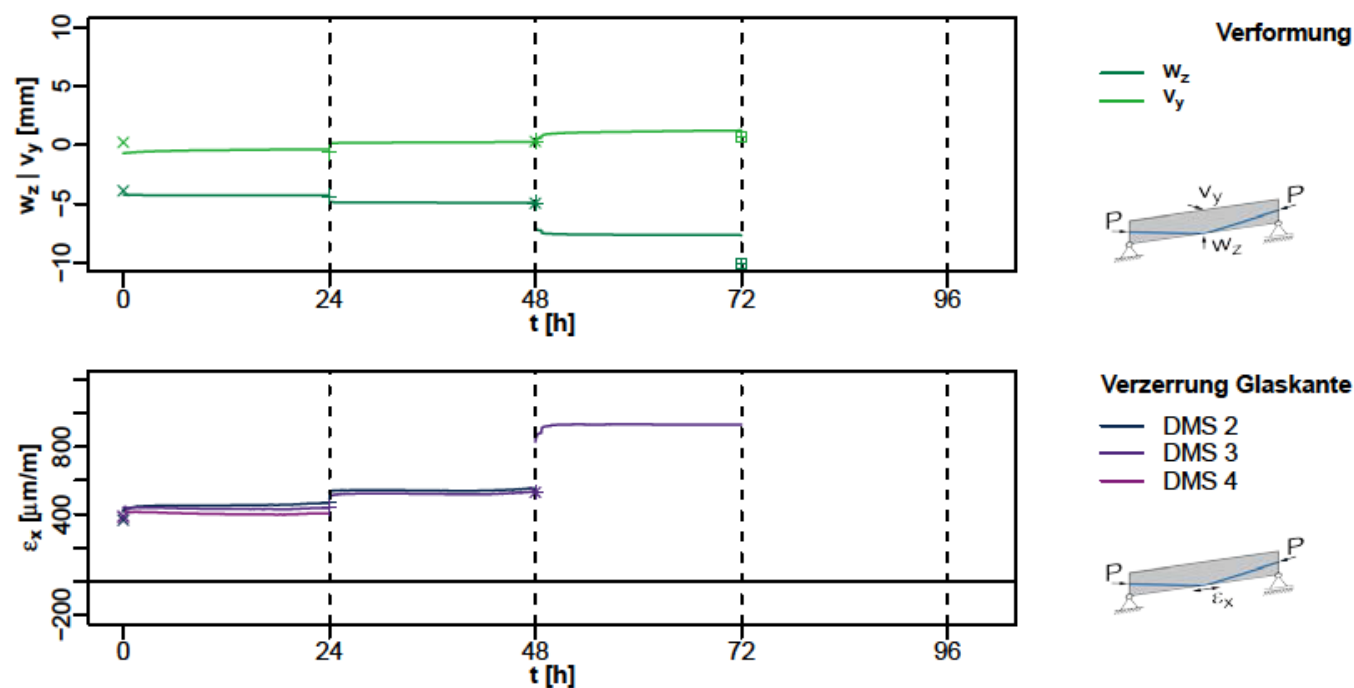

Verzerrung Glaskante - DMS 2

- DMS 3

- DMS 4

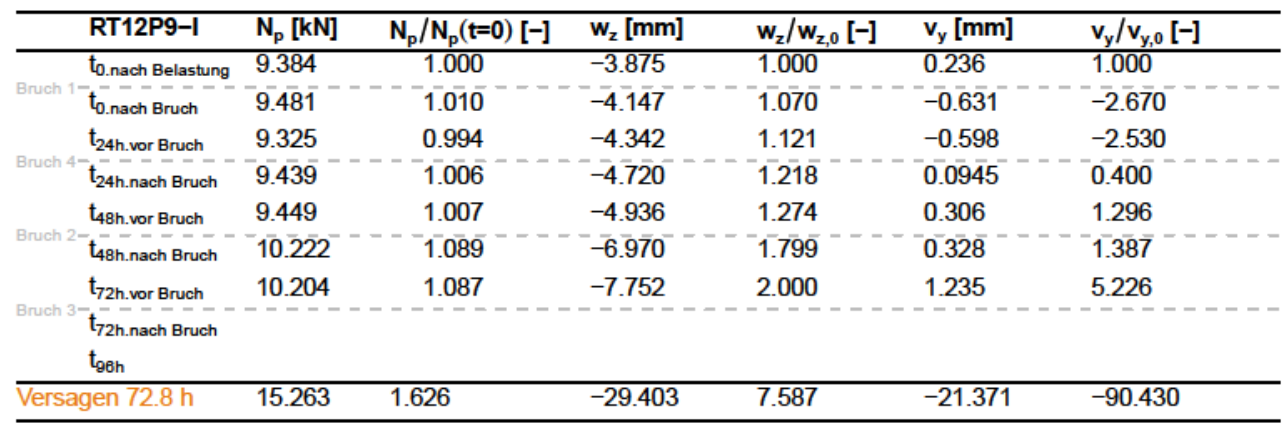


Bruchbild RT12P9-I

$h_{z}=58,6 \%$

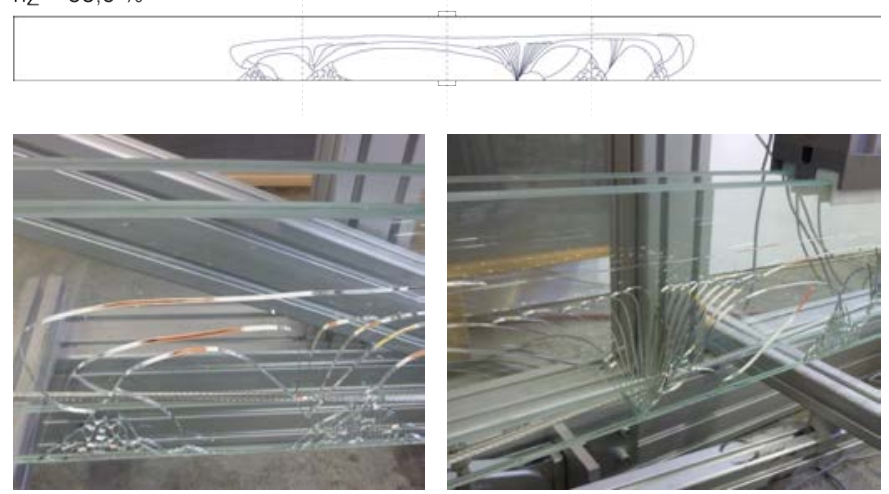

$h \mathrm{z}=64,8 \%$
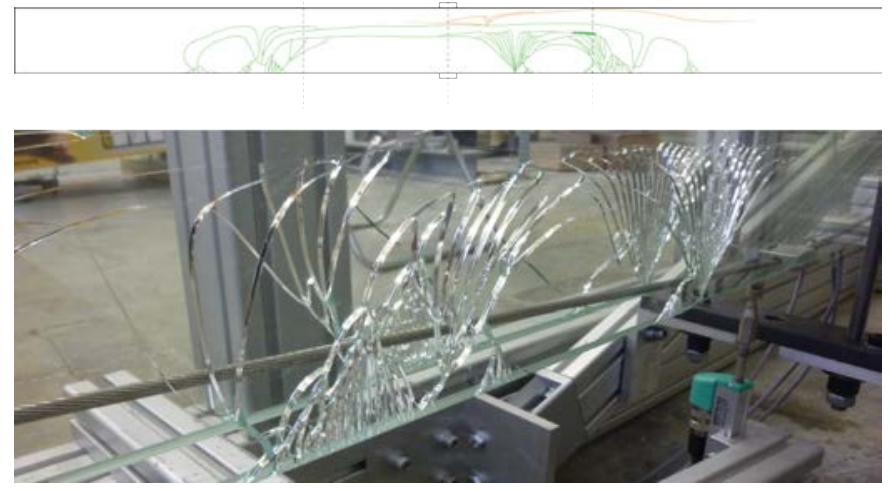

$h_{z}=74,4 \%$
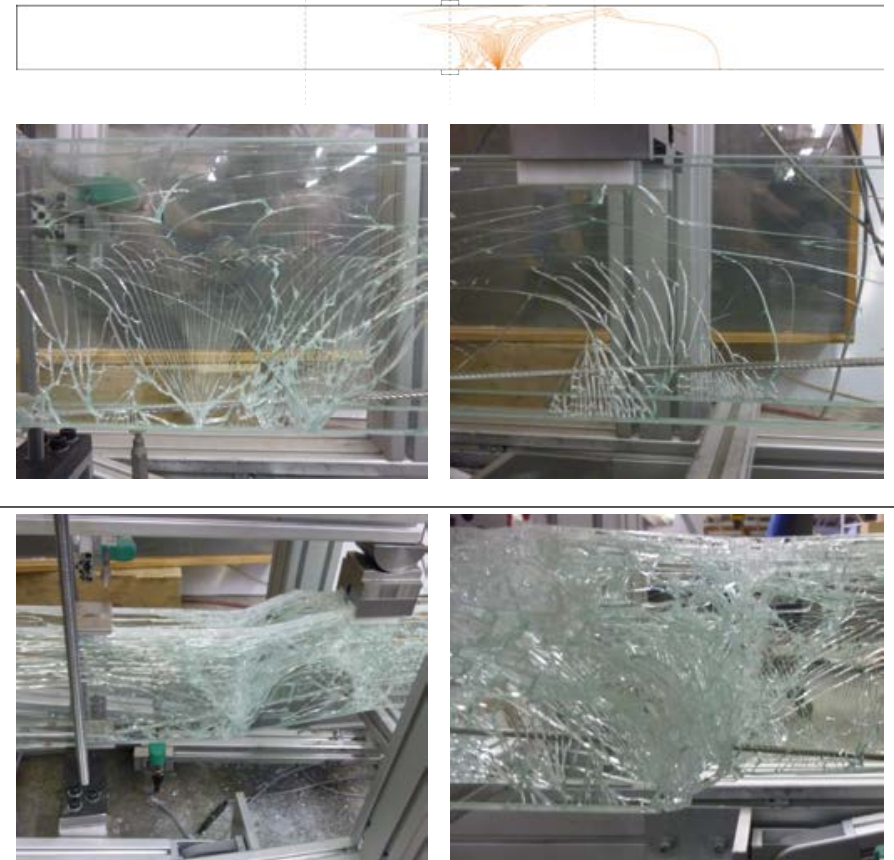

Scheibe 1

Bruch durch Anschlagen nach $\mathrm{t}=0 \mathrm{~h}$

- Brucherweiterung bis $\mathrm{t}=24 \mathrm{~h}$

Brucherweiterung bis $\mathrm{t}=48 \mathrm{~h}$

Scheibe 4

Bruch durch Anschlagen nach $\mathrm{t}=24 \mathrm{~h}$

_ Brucherweiterung bis $\mathrm{t}=48 \mathrm{~h}$

Scheibe 2

Bruch durch Anschlagen nach $\mathrm{t}=48 \mathrm{~h}$
Bruch aller Scheiben

Einknicken der VSG-Pakete 


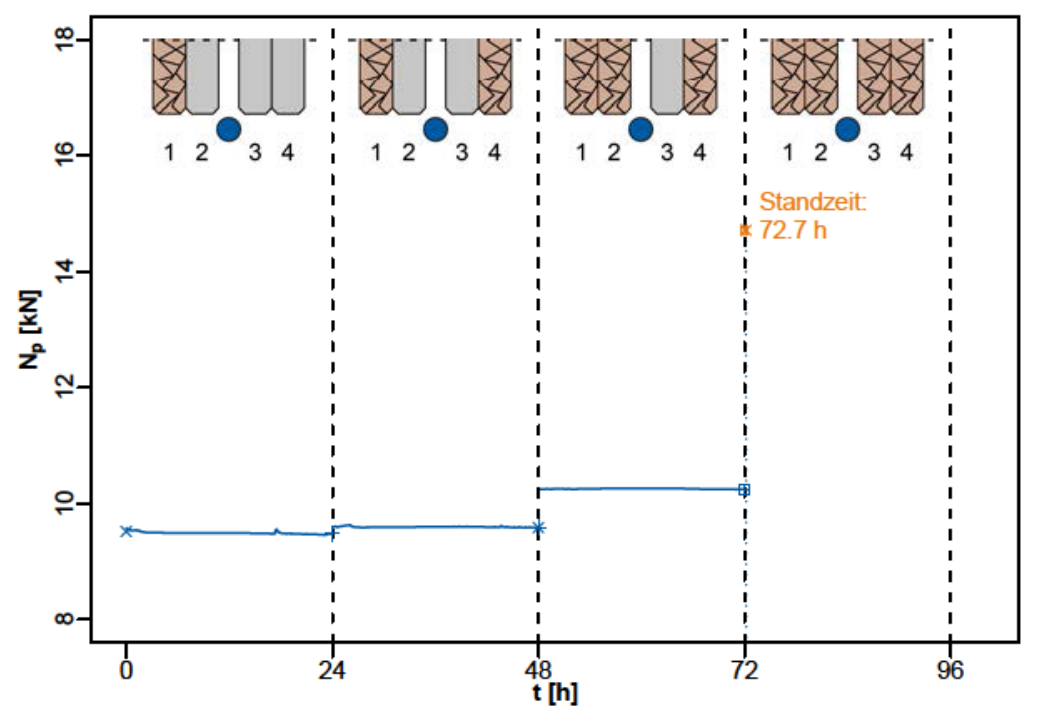

Seilkraftänderung

— RT13P9-II
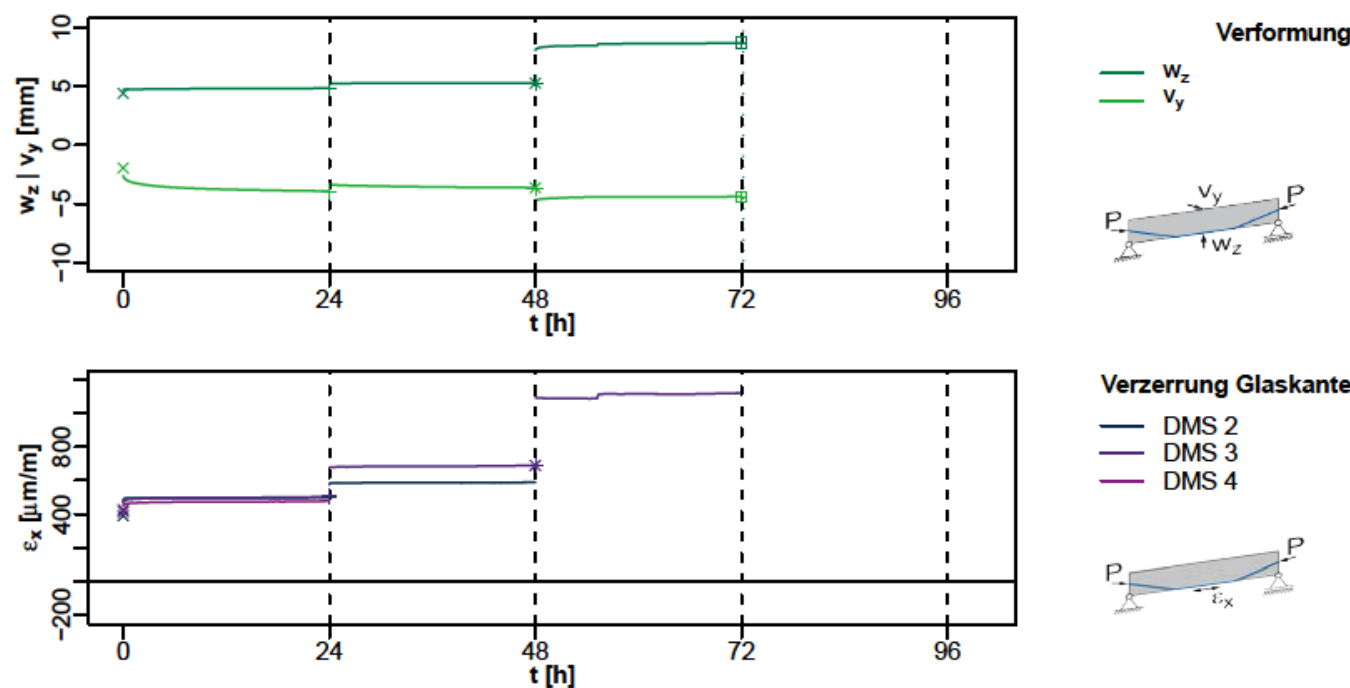

Verzerrung Glaskante

- DMS 2

- DMS 3

- DMS 4

\begin{tabular}{|c|c|c|c|c|c|c|c|}
\hline & RT13P9-II & $\mathrm{N}_{\mathrm{p}}[\mathrm{kN}]$ & $N_{p} / N_{p}(t=0)[-]$ & $\mathrm{w}_{\mathrm{z}}[\mathrm{mm}]$ & $w_{z} / w_{z, 0}[-]$ & $\mathrm{v}_{\mathrm{y}}[\mathrm{mm}]$ & $v_{y} / v_{y, 0}[-]$ \\
\hline \multirow[b]{2}{*}{ Bruch 1. } & $\mathrm{t}_{\text {0.nach Belastung }}$ & 9.518 & 1.000 & 4.403 & 1.000 & -1.950 & 1.000 \\
\hline & $\mathrm{t}_{\text {0.nach Bruch }}$ & $9 . \overline{7} \overline{8}$ & $1 . \overline{006}$ & $4 . \overline{614}$ & 1.048 & -2.563 & $1.3 \overline{14}$ \\
\hline \multirow{2}{*}{ Bruch 4 . } & 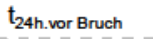 & 9.480 & 0.996 & 4.845 & 1.100 & -3.922 & 2.011 \\
\hline & 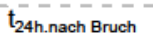 & $9.5 \overline{7} \overline{7}$ & $1.00 \overline{6}$ & $5.1 \overline{7} \overline{3}$ & $1.1 \overline{7} \overline{5}$ & -3.245 & 1.664 \\
\hline \multirow{2}{*}{ Bruch 2.} & $\mathrm{t}_{48 \mathrm{~h} . \text { vor Bruch }}$ & 9.587 & 1.007 & 5.292 & 1.202 & -3.640 & 1.867 \\
\hline & t48h.nach Bruch & 10.201 & $1.0 \overline{7} \overline{2}$ & $7.79 \overline{7}$ & $1.7 \overline{1}$ & -4.660 & 2.390 \\
\hline \multirow{2}{*}{ Bruch 3} & 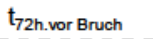 & 10.245 & 1.076 & 8.712 & 1.979 & -4.387 & 2.249 \\
\hline & 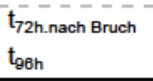 & 15.065 & $1.58 \overline{3}$ & 26.511 & $6.02 \overline{1}$ & -23.368 & $\overline{11.983}$ \\
\hline \multicolumn{2}{|c|}{ Versagen $72.7 \mathrm{~h}$} & 14.715 & 1.546 & 26.613 & 6.044 & -23.368 & 11.983 \\
\hline
\end{tabular}


Bruchbild RT13P9-II

$h_{z}=69,8 \%$

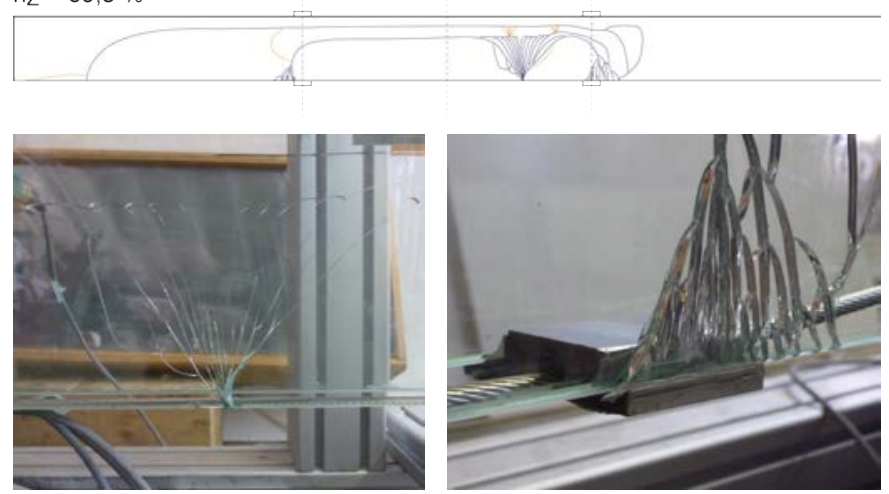

$h_{z}=60,5 \%$
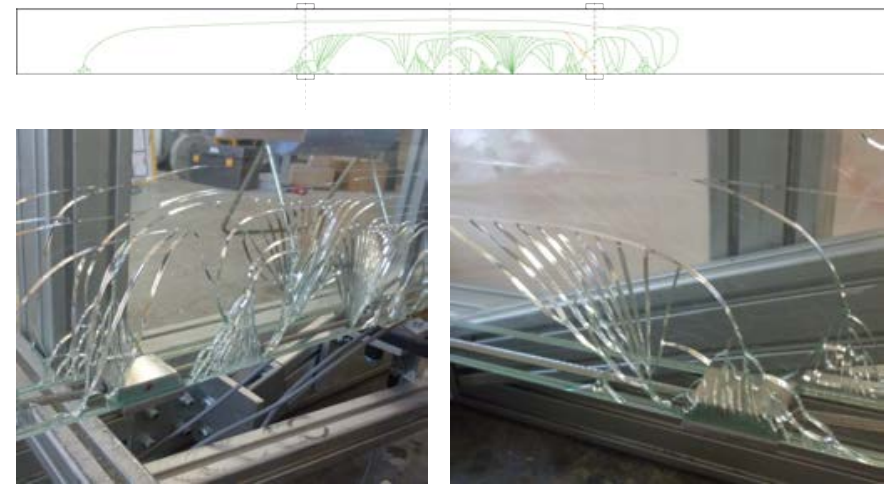

$h z=75,0 \%$
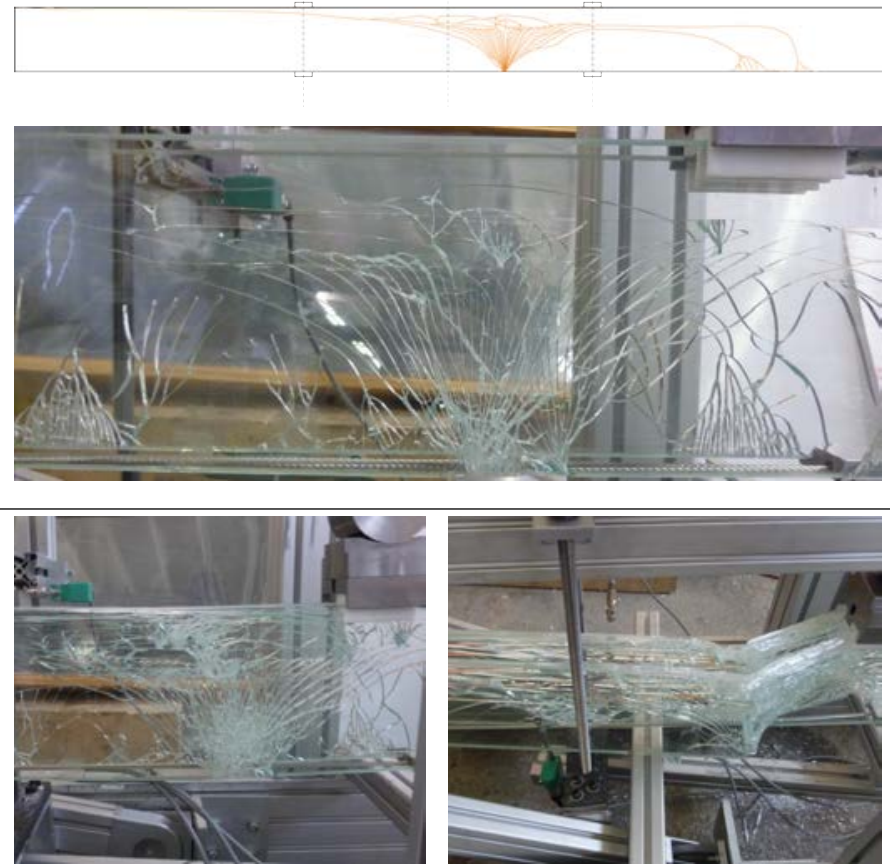

Scheibe 1

Bruch durch Anschlagen nach $\mathrm{t}=0 \mathrm{~h}$

- Brucherweiterung bis $\mathrm{t}=24 \mathrm{~h}$

Brucherweiterung bis $\mathrm{t}=48 \mathrm{~h}$

Bruchfächer am Umlenkpunkt im rechten Bild

Scheibe 4

Bruch durch Anschlagen nach $\mathrm{t}=24 \mathrm{~h}$

Brucherweiterung bis $t=48 \mathrm{~h}$

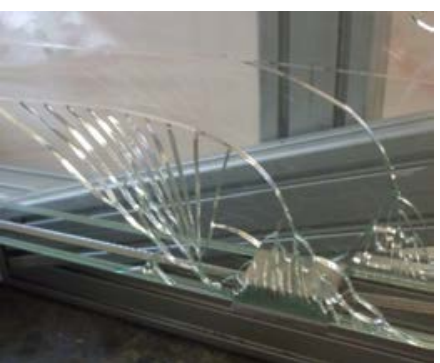

Scheibe 2

Bruch durch Anschlagen nach $\mathrm{t}=48 \mathrm{~h}$
Bruch aller Scheiben

Einknicken der VSG-Pakete im rechten Bild 


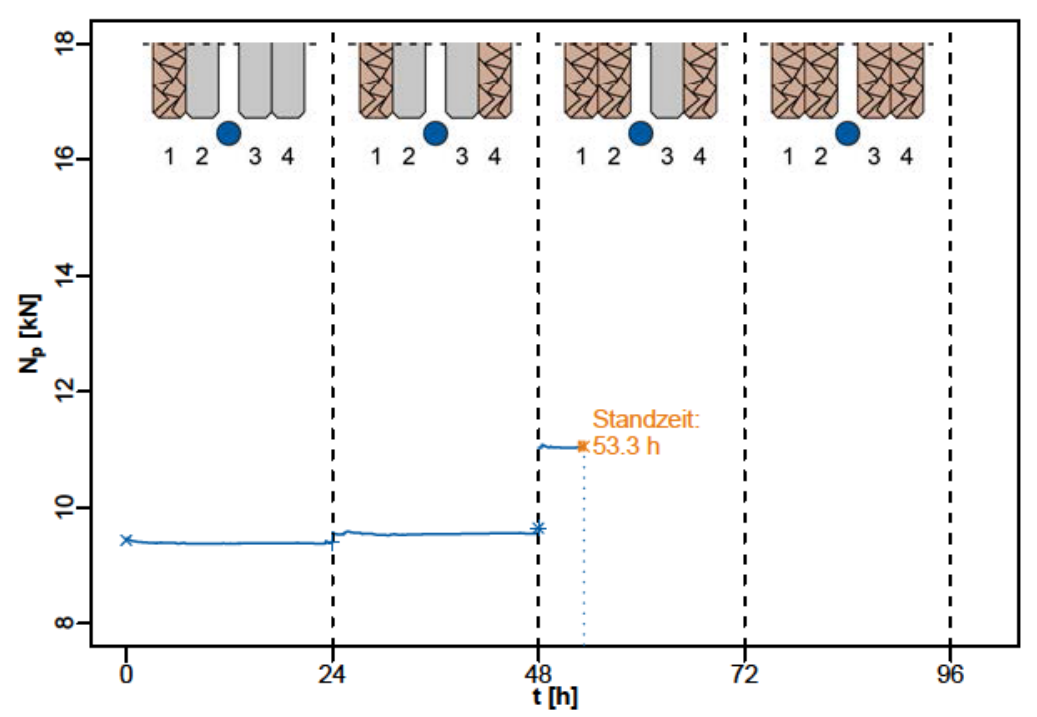

Seilkraftänderung RT14P9-II
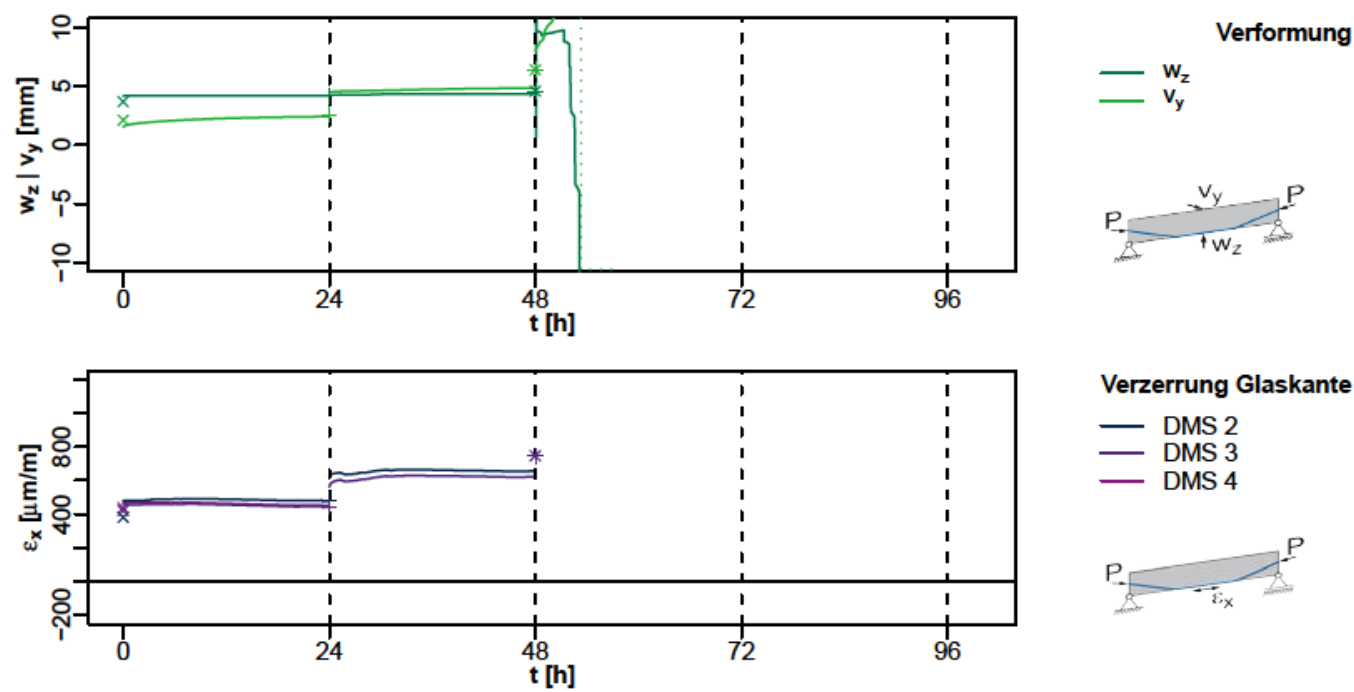

Verzerrung Glaskante - DMS 2

- DMS 3

- DMS 4

\begin{tabular}{|c|c|c|c|c|c|c|c|}
\hline & RT14P9-II & $\mathrm{N}_{\mathrm{p}}[\mathrm{kN}]$ & $N_{p} / N_{p}(t=0)[-]$ & $w_{z}[\mathrm{~mm}]$ & $w_{z} / w_{z, 0}[-]$ & $v_{y}[\mathrm{~mm}]$ & $v_{y} / v_{y, 0}[-]$ \\
\hline & $\mathrm{t}_{\text {0.nach Belastung }}$ & 9.434 & 1.000 & 3.660 & 1.000 & 2.205 & 1.000 \\
\hline Bruch & $\mathrm{t}_{\text {0.nach Bruch }}$ & $9 . \overline{4} \overline{8}$ & $1.00 \overline{5}$ & 4.148 & 1.134 & 1.550 & 0.703 \\
\hline & $t_{24 h . v o r}$ Bruch & 9.391 & 0.995 & 4.225 & 1.154 & 2.495 & 1.132 \\
\hline Bruof & $\mathrm{t}_{24 \mathrm{~h} . \text { nach Bruch }}$ & $9.45 \overline{2}$ & $1.00 \overline{2}$ & 4.200 & $1.14 \overline{8}$ & $3.20 \overline{6}$ & $1.4 \overline{4}$ \\
\hline & 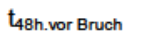 & 9.633 & 1.021 & 4.490 & 1.227 & 6.307 & 2.861 \\
\hline Bruch & $t_{48 h \text {.nach Bruch }}$ & 10.276 & $1.08 \overline{9}$ & 7.208 & $1.97 \overline{0}$ & $5.64 \overline{1}$ & $2.55 \overline{9}$ \\
\hline Bruch & $\begin{array}{l}\mathrm{t}_{72 \mathrm{~h} . v o r} \text { Bruch } \\
\mathrm{t}_{72 \mathrm{~h} . \text { nach Bruch }} \\
\mathrm{t}_{98 \mathrm{~h}}\end{array}$ & & & & & & \\
\hline Versa & gen $53.3 \mathrm{~h}$ & 11.054 & 1.172 & -11.815 & -3.228 & 21.514 & 9.758 \\
\hline
\end{tabular}


Bruchbild RT14P9-II

$h_{z}=55,2 \%$

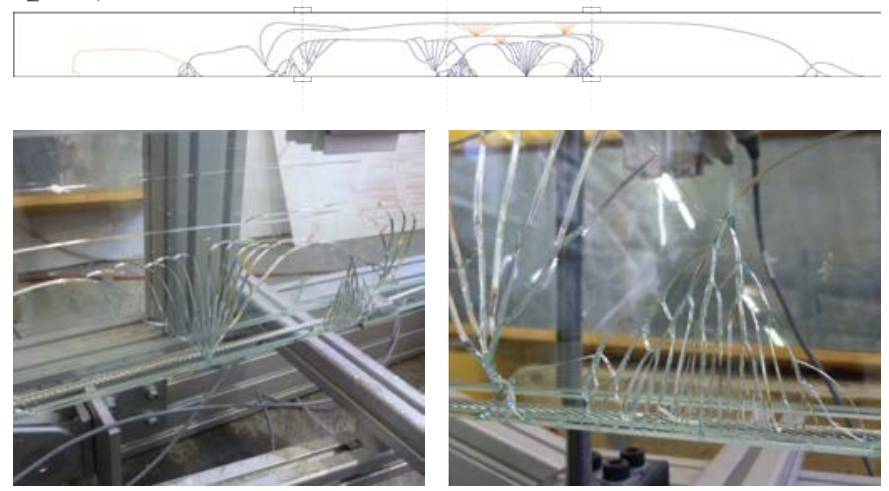

$h_{\mathrm{Z}}=60,4 \%$
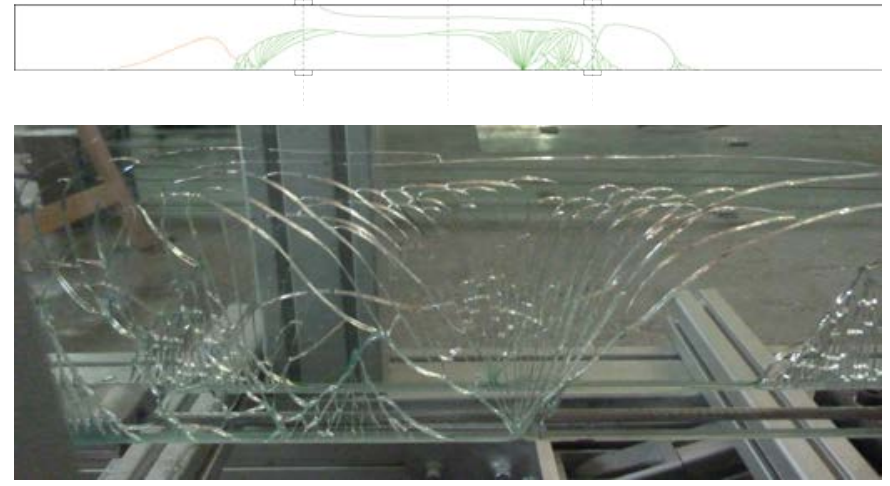

$h_{z}=66,2 \%$
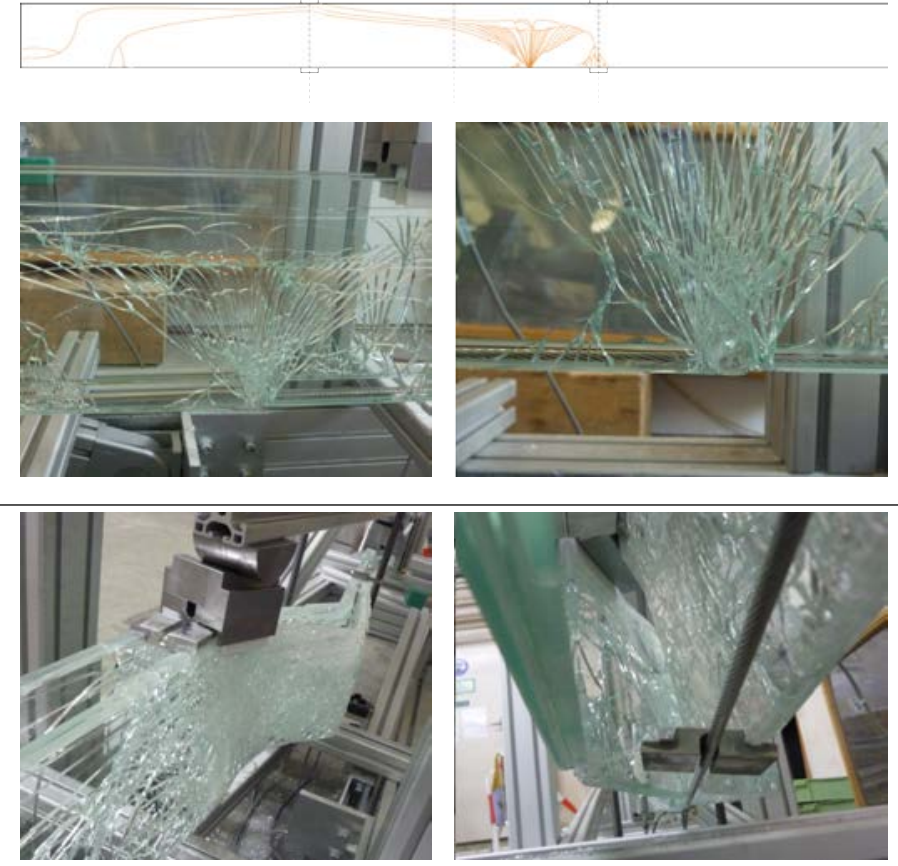

Scheibe 1

Bruch durch Anschlagen nach $\mathrm{t}=0 \mathrm{~h}$

- Brucherweiterung bis $\mathrm{t}=24 \mathrm{~h}$

- Brucherweiterung bis $\mathrm{t}=48 \mathrm{~h}$

Scheibe 4

Bruch durch Anschlagen nach $\mathrm{t}=24 \mathrm{~h}$

- Brucherweiterung bis $\mathrm{t}=48 \mathrm{~h}$ einzelner Bruch in Glasoberkante

Scheibe 2 Bruch durch Anschlagen nach $\mathrm{t}=48 \mathrm{~h}$

Versatz der Unterkanten im rechten Bild 


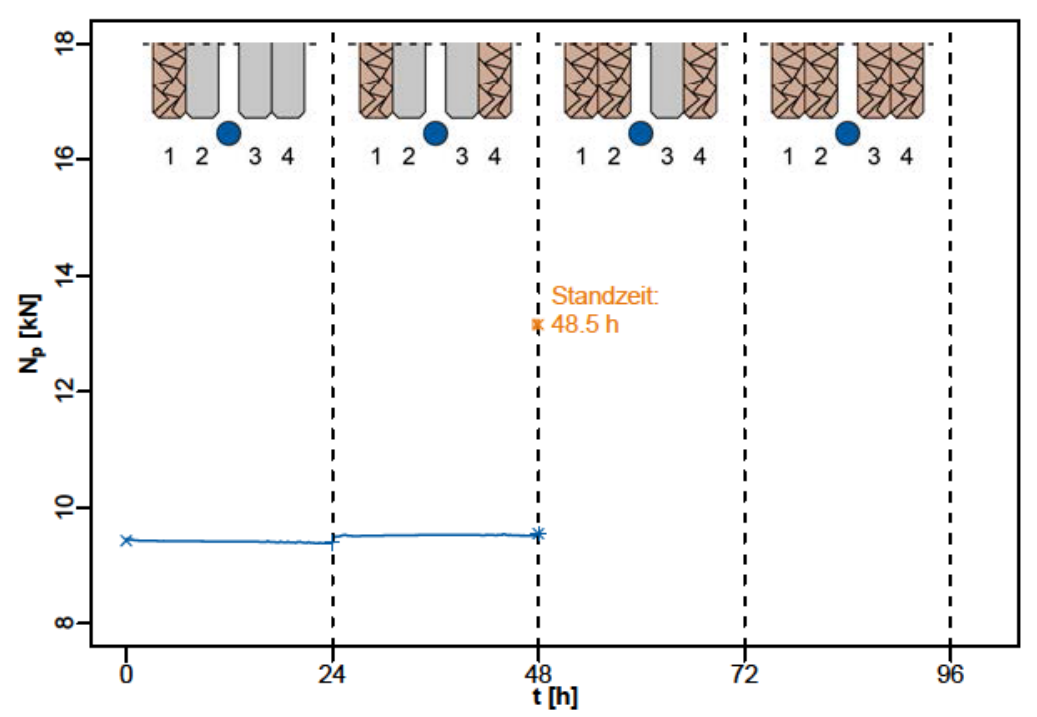

Seilkraftänderung RT15P9-II
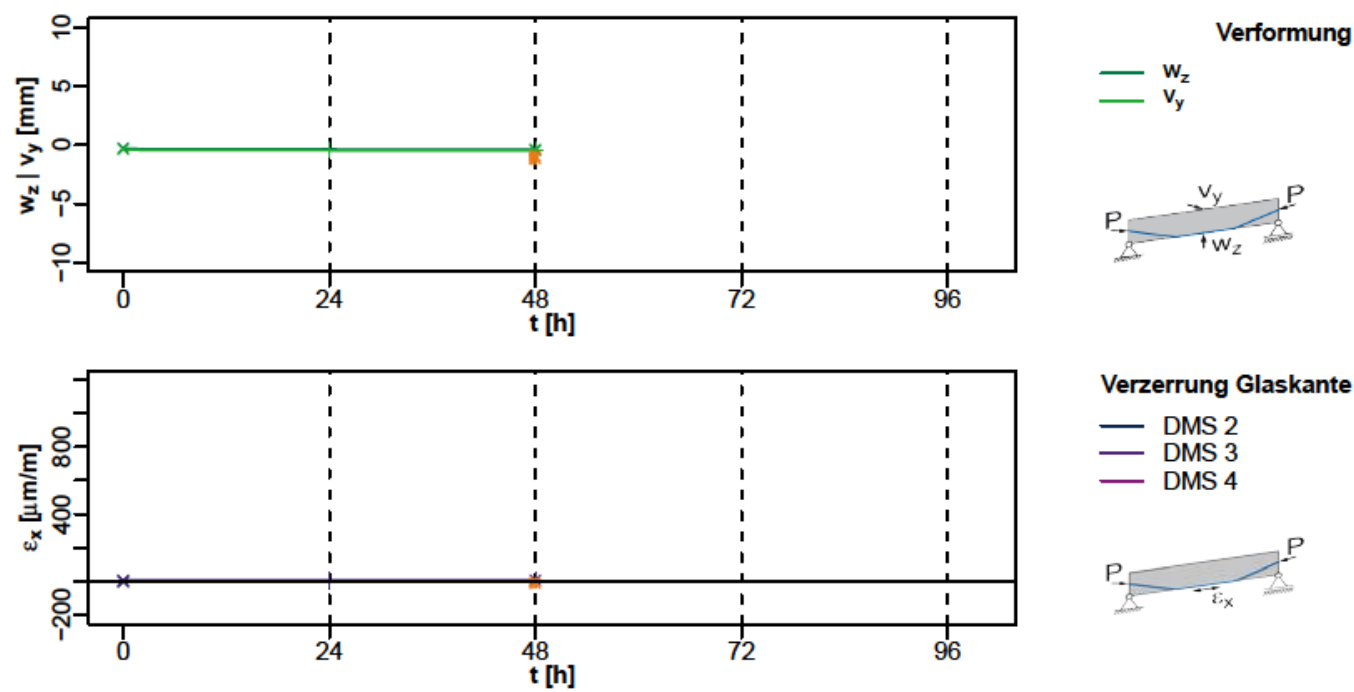

Verzerrung Glaskante - DMS 2

- DMS 3

- DMS 4

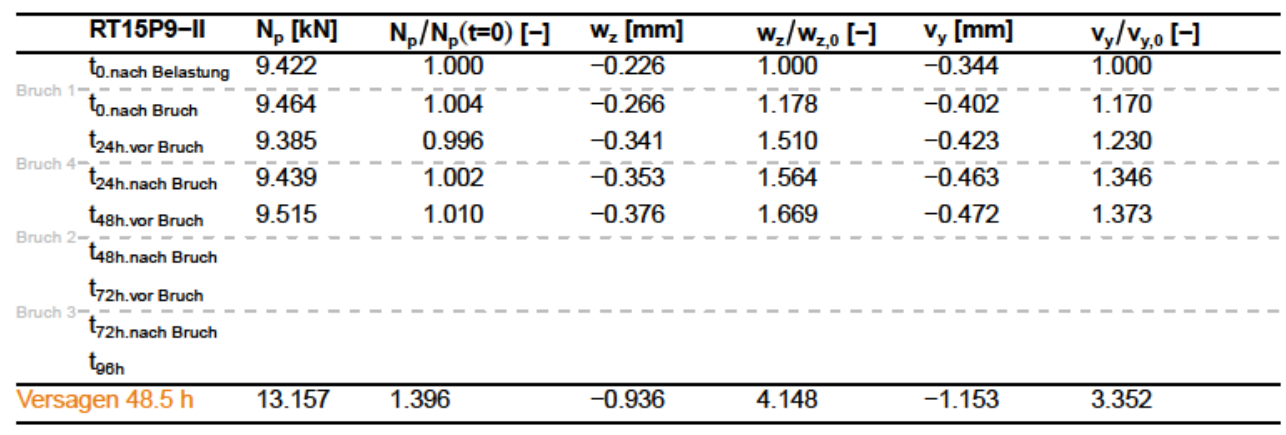


Bruchbild RT15P9-II

$h_{z}=63,1 \%$

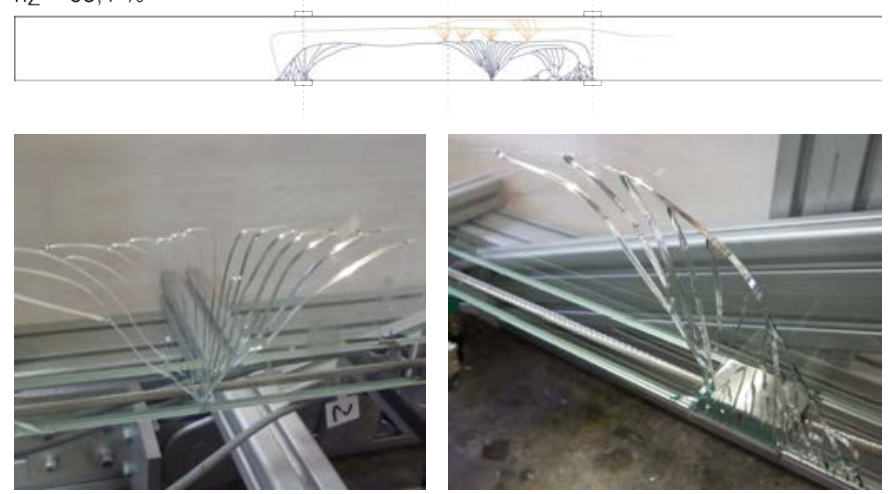

$h_{z}=53,6 \%$
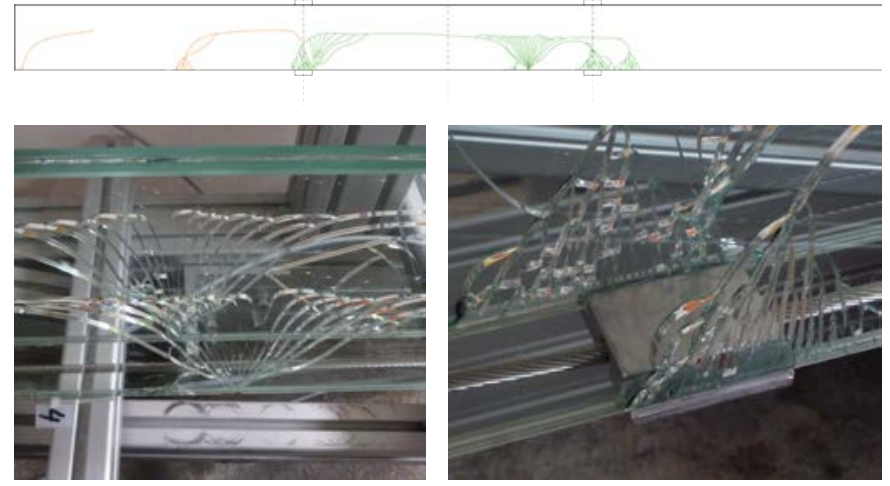

$h z=67,3 \%$
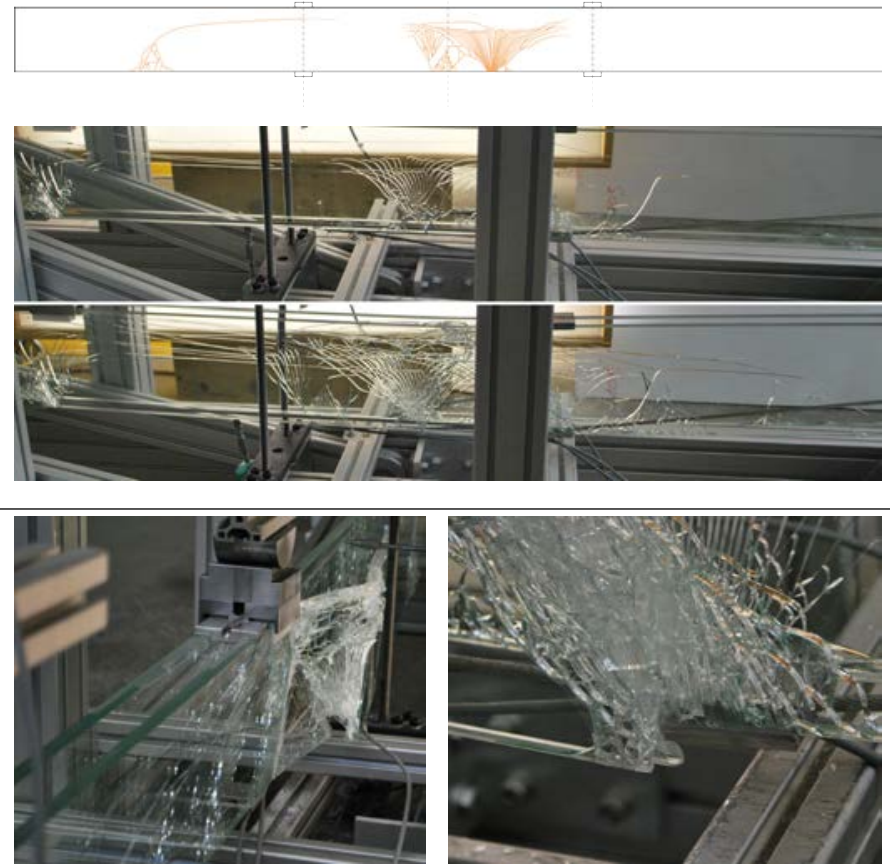

Träger mit $P_{m 0}=9 \mathrm{kN}$ und $d_{p}=5,0 \mathrm{~mm}$

Scheibe 1

Bruch durch Anschlagen nach $\mathrm{t}=0 \mathrm{~h}$

- Brucherweiterung bis $\mathrm{t}=24 \mathrm{~h}$

Brucherweiterung bis $\mathrm{t}=48 \mathrm{~h}$

Scheibe 4

Bruch durch Anschlagen nach $\mathrm{t}=24 \mathrm{~h}$

_ Brucherweiterung bis $\mathrm{t}=48 \mathrm{~h}$

Scheibe 2

Bruch durch Anschlagen nach $\mathrm{t}=48 \mathrm{~h}$

zunächst erwartetes Bruchbild, (oberes Bild)

im Anschluss Absacken infolge Bruch am Umlenktpunk (unteres Bild)

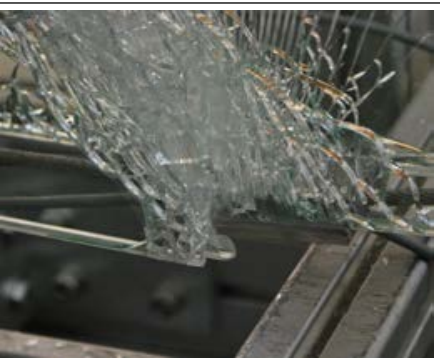

Bruch aller Scheiben

Einknicken eines VSG-Paketes im linken Bild Abscheren am Umlenkpunkt (rechtes Bild) 


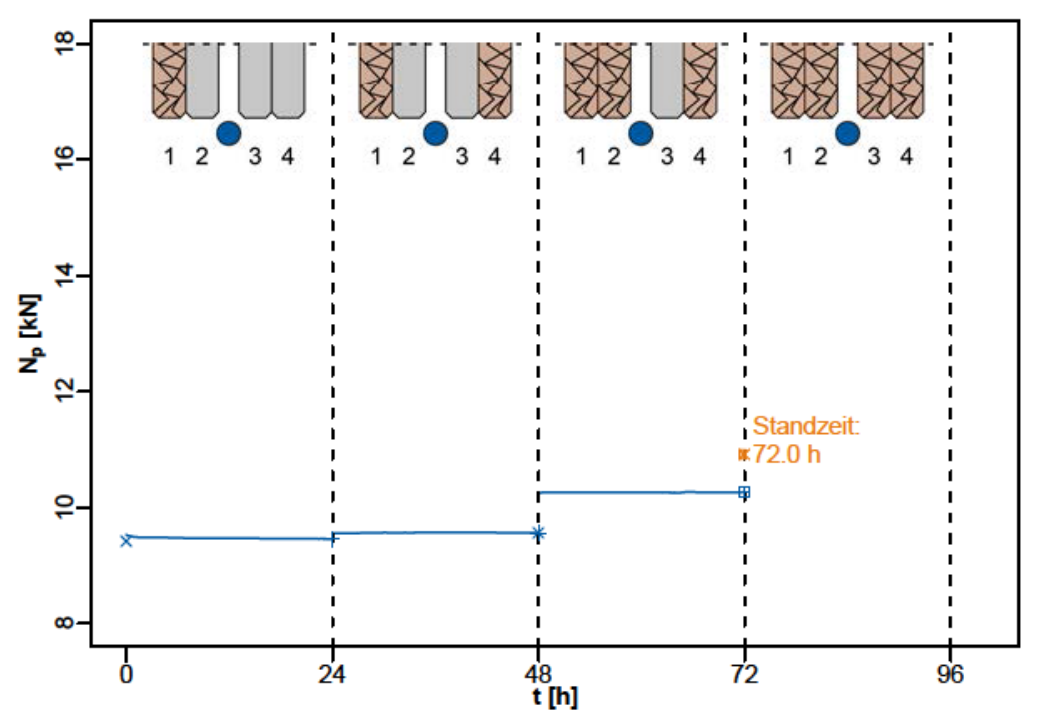

Seilkraftänderung

— RT16P9-II
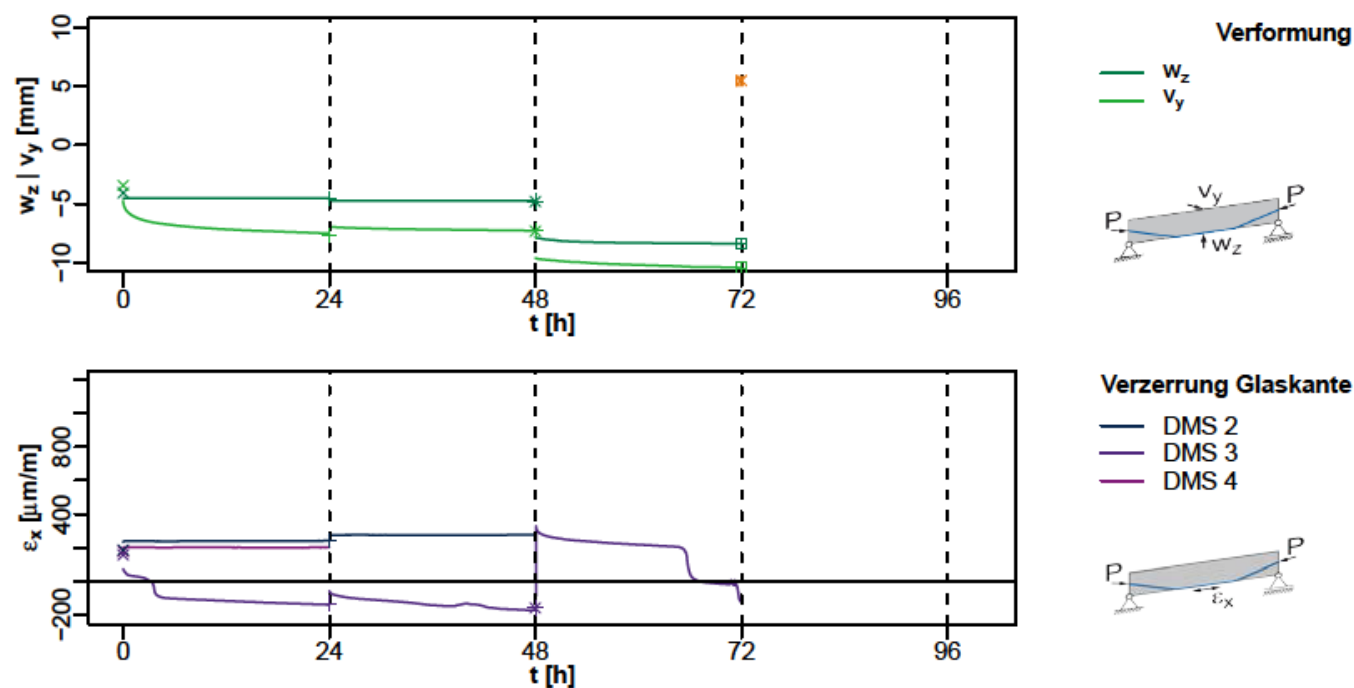

Verzerrung Glaskante

- DMS 2

- DMS 3

- DMS 4

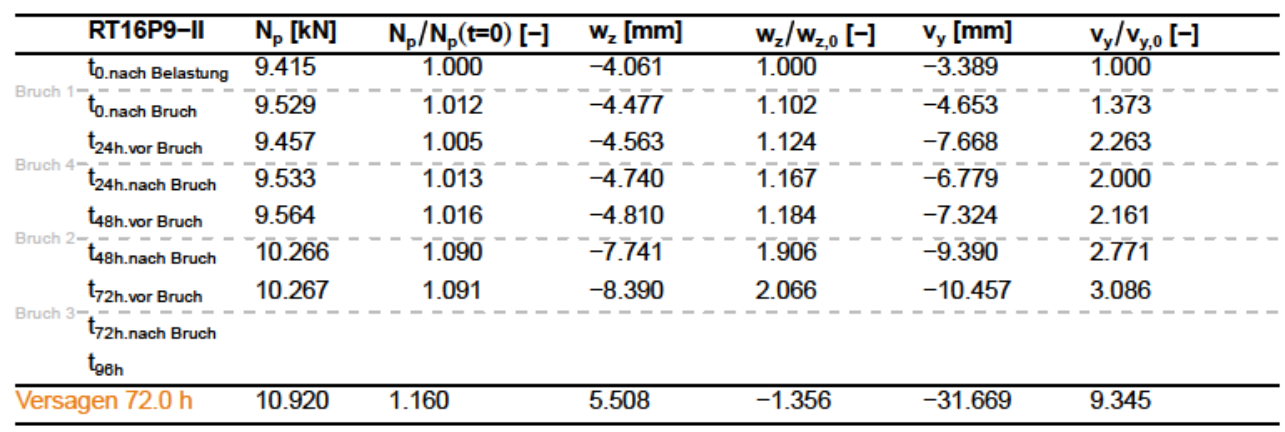


Bruchbild RT16P9-II

$h z=64,2 \%$

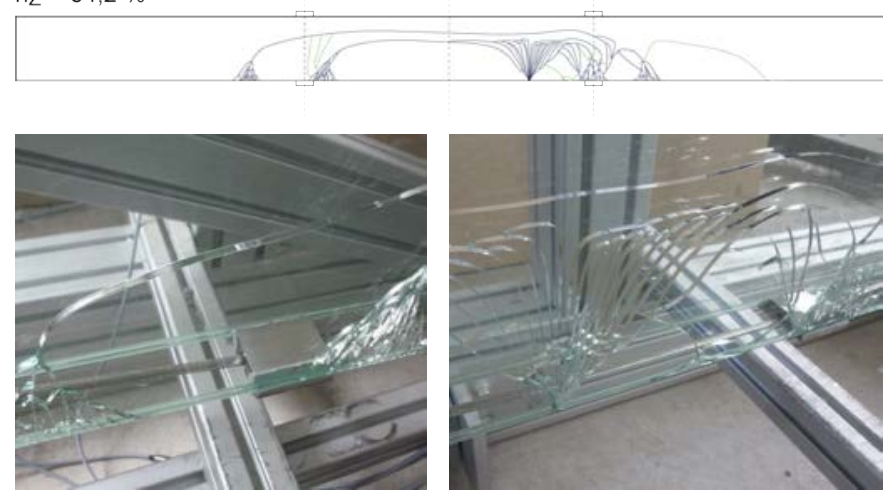

$h_{z}=58,4 \%$
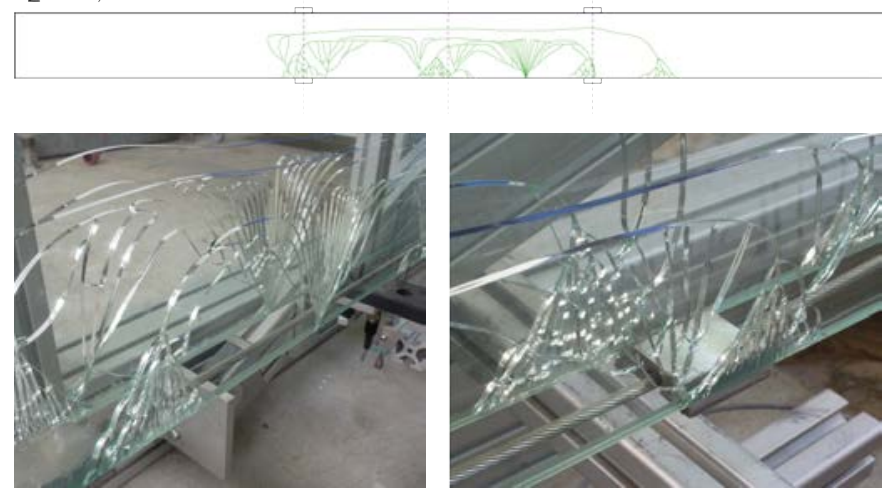

$h_{z}=73,3 \%$
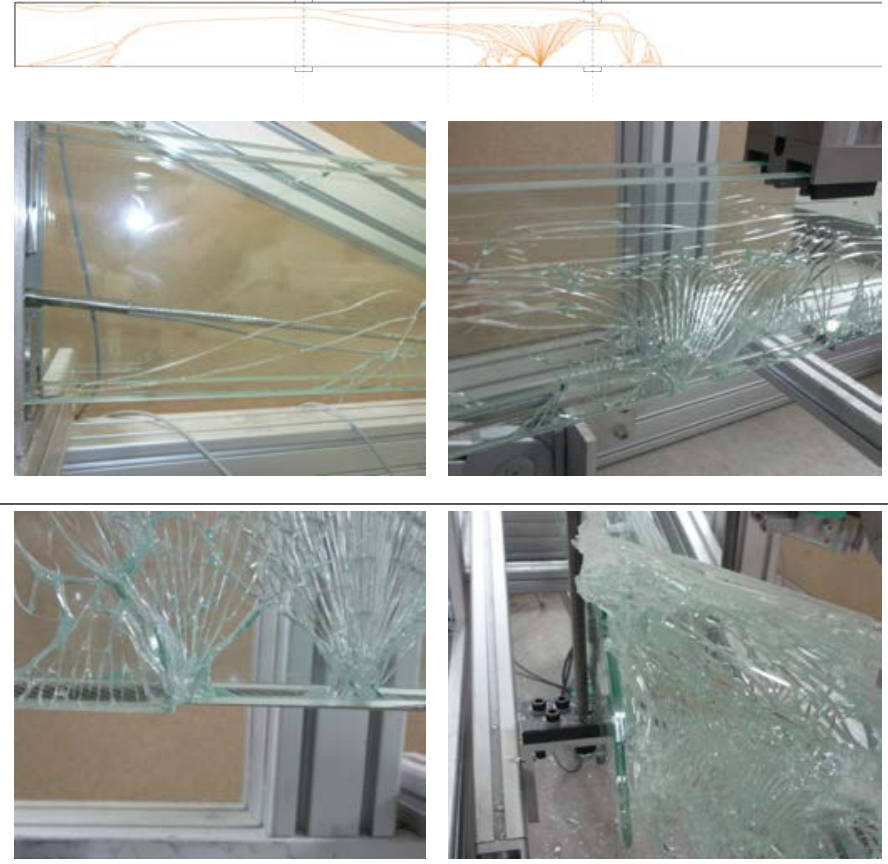

Scheibe 1

Bruch durch Anschlagen nach $\mathrm{t}=0 \mathrm{~h}$

- Brucherweiterung bis $\mathrm{t}=24 \mathrm{~h}$

- Brucherweiterung bis $\mathrm{t}=48 \mathrm{~h}$

Scheibe 4

Bruch durch Anschlagen nach $\mathrm{t}=24 \mathrm{~h}$

Brucherweiterung bis $\mathrm{t}=48 \mathrm{~h}$

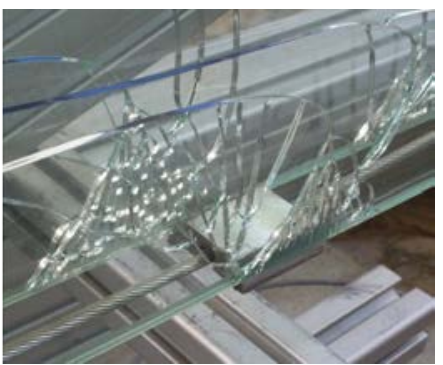

Scheibe 2

Bruch durch Anschlagen nach $\mathrm{t}=48 \mathrm{~h}$

Bruch ins Auflager im linken Bild

Bruch aller Scheiben Abscheren (linkes Bild)
Einknicken der VSG-Pakete (rechtes Bild) 
Standzeit RT17P15-I Träger mit $P_{m 0}=15 \mathrm{kN}$ und $d_{p}=8,1 \mathrm{~mm}$

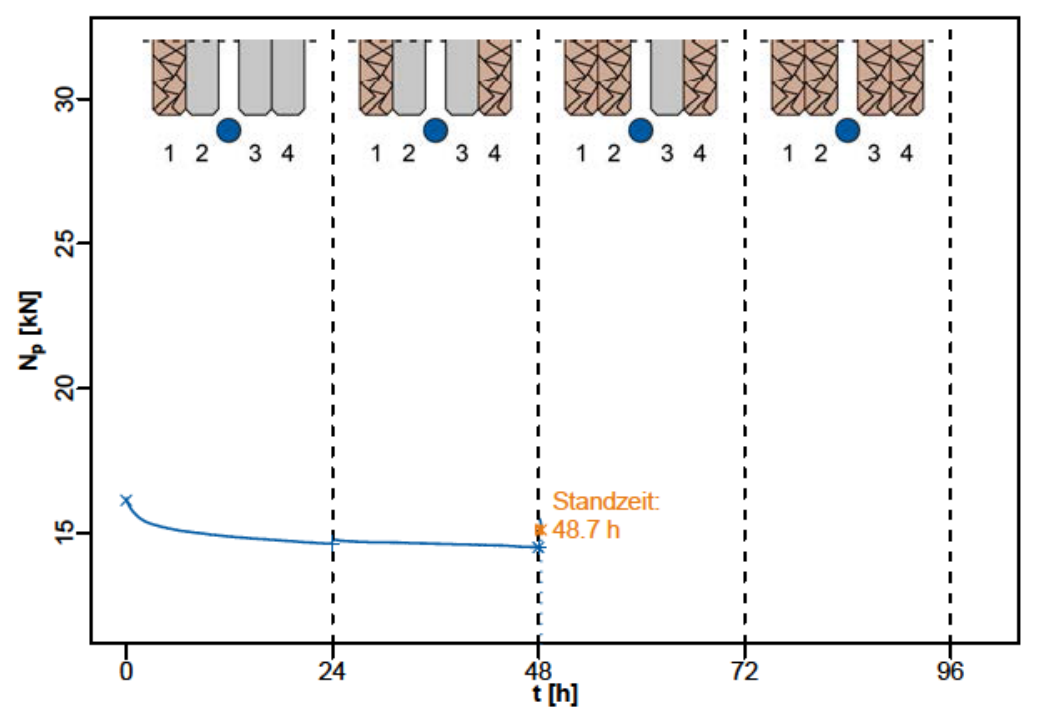

Seilkraftänderung

- RT17P15-I
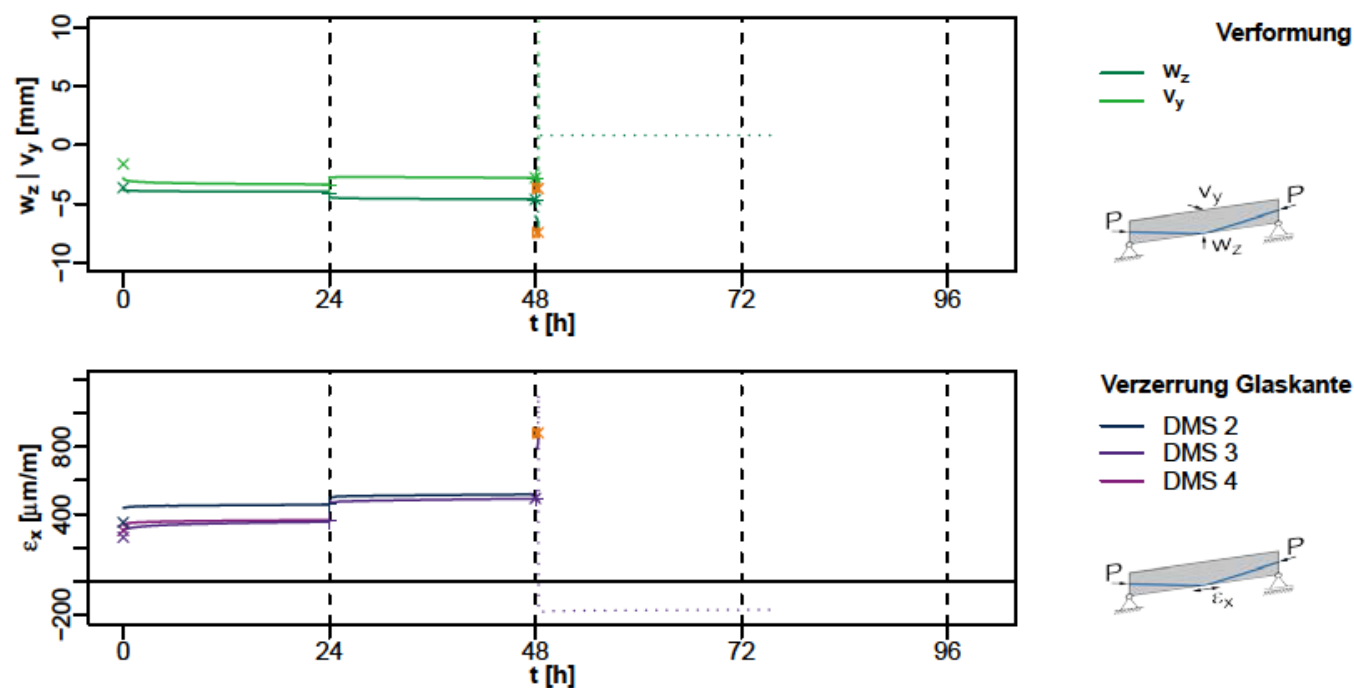

Verzerrung Glaskante

- DMS 2

- DMS 3

- DMS 4

\begin{tabular}{|c|c|c|c|c|c|c|c|}
\hline & RT17P15-I & $\mathrm{N}_{\mathrm{p}}[\mathrm{kN}]$ & $N_{p} / N_{p}(t=0)[-]$ & $\mathrm{w}_{\mathrm{z}}[\mathrm{mm}]$ & $w_{z} / w_{z, 0}[-]$ & $\mathrm{v}_{\mathrm{y}}[\mathrm{mm}]$ & $v_{y} / v_{y, 0}[-]$ \\
\hline & $\mathrm{t}_{\text {0.nach Belastung }}$ & 16.128 & 1.000 & -3.600 & 1.000 & -1.467 & 1.000 \\
\hline Bruch 1 & $\mathrm{t}_{0 . \text { nach Bruch }}$ & 16.272 & $1.00 \overline{9}$ & -3.797 & $1.05 \overline{5}$ & $-2.5 \overline{3} 1$ & $1 . \overline{724}$ \\
\hline \multirow[b]{2}{*}{ Bruch 4} & $t_{24 h . v o r}$ Bruch & 14.652 & 0.908 & -3.982 & 1.106 & -3.383 & 2.306 \\
\hline & $\mathrm{t}_{24 \mathrm{~h} . \text { nach Bruch }}$ & 14.772 & 0.916 & -4.355 & 1.210 & -2.793 & $1.90 \overline{3}$ \\
\hline \multirow{2}{*}{ Bruch 2} & 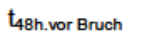 & 14.520 & 0.900 & -4.619 & 1.283 & -2.790 & 1.901 \\
\hline & $\mathrm{t}_{48 \mathrm{~h} . \text { nach Bruch }}$ & 15.534 & $0.96 \overline{3}$ & -5.866 & 1.630 & -2.756 & $1.8 \overline{7} \overline{8}$ \\
\hline Bruch 3 & 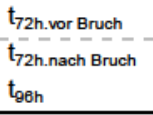 & & & & & & \\
\hline \multicolumn{2}{|c|}{ Versagen 48.7 h } & 15.126 & 0.938 & -7.435 & 2.065 & -3.696 & 2.519 \\
\hline
\end{tabular}




\section{Bruchbild RT17P15-I Träger mit $P_{m 0}=15 \mathrm{kN}$ und $d_{p}=8,1 \mathrm{~mm}$}
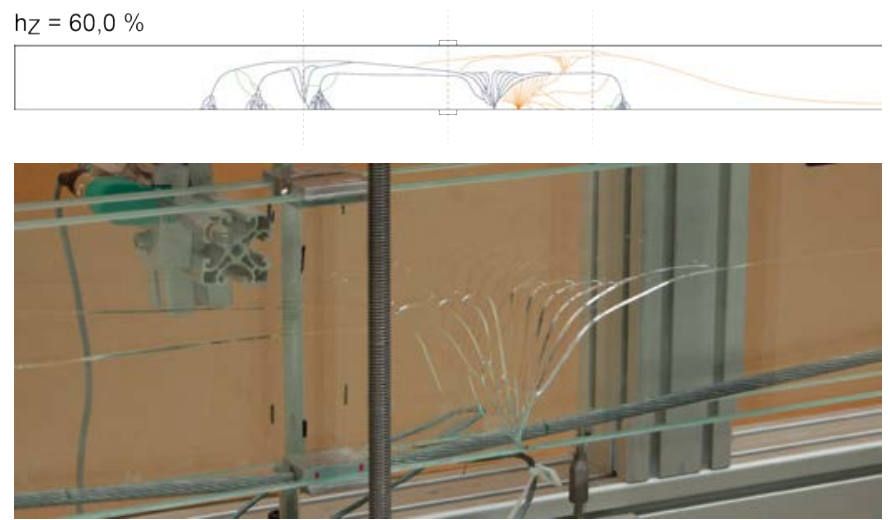

Scheibe 1

$h_{z}=59,5 \%$

Scheibe 4
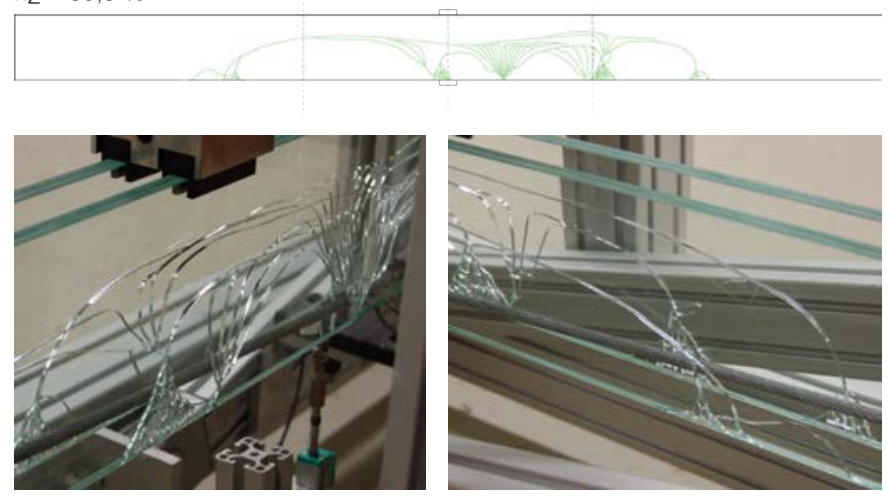

$\mathrm{hz}=71,7 \%$

Scheibe 2

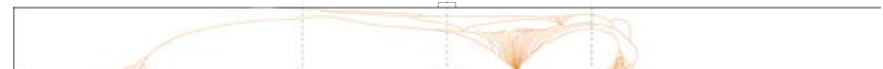

Bruch durch Anschlagen nach $\mathrm{t}=0 \mathrm{~h}$

- Brucherweiterung bis $\mathrm{t}=24 \mathrm{~h}$

- Brucherweiterung bis $\mathrm{t}=48 \mathrm{~h}$
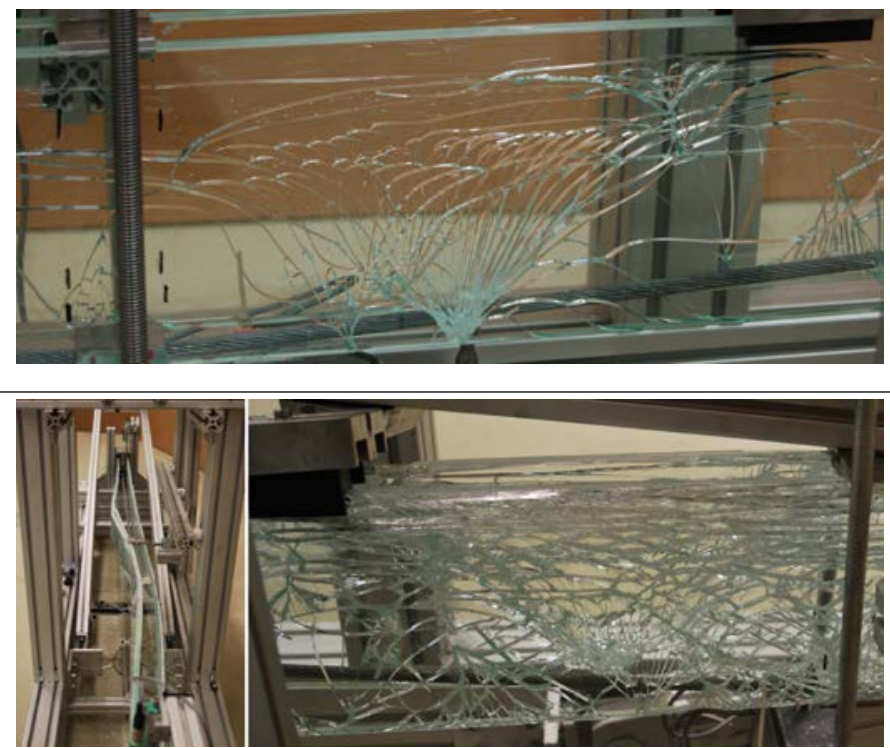

Bruch aller Scheiben

as, Umlenkung teilweise abgerissen 
Standzeit RT18P15-I Träger mit $P_{m 0}=15 \mathrm{kN}$ und $d_{p}=8,1 \mathrm{~mm}$

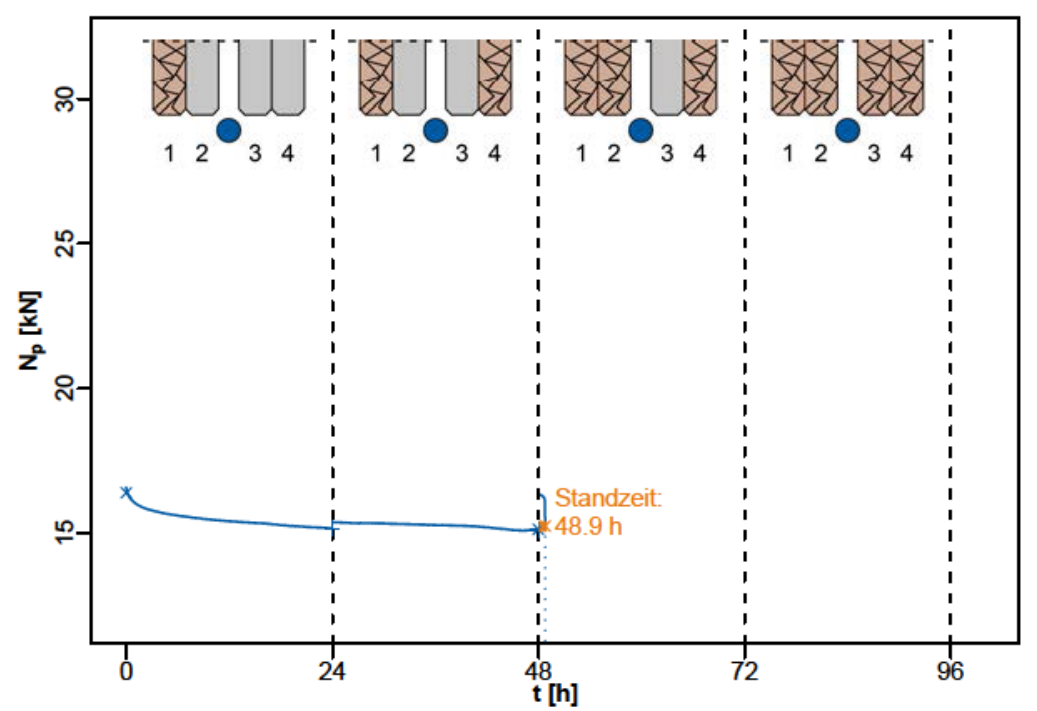

Seilkraftänderung

- RT18P15-I
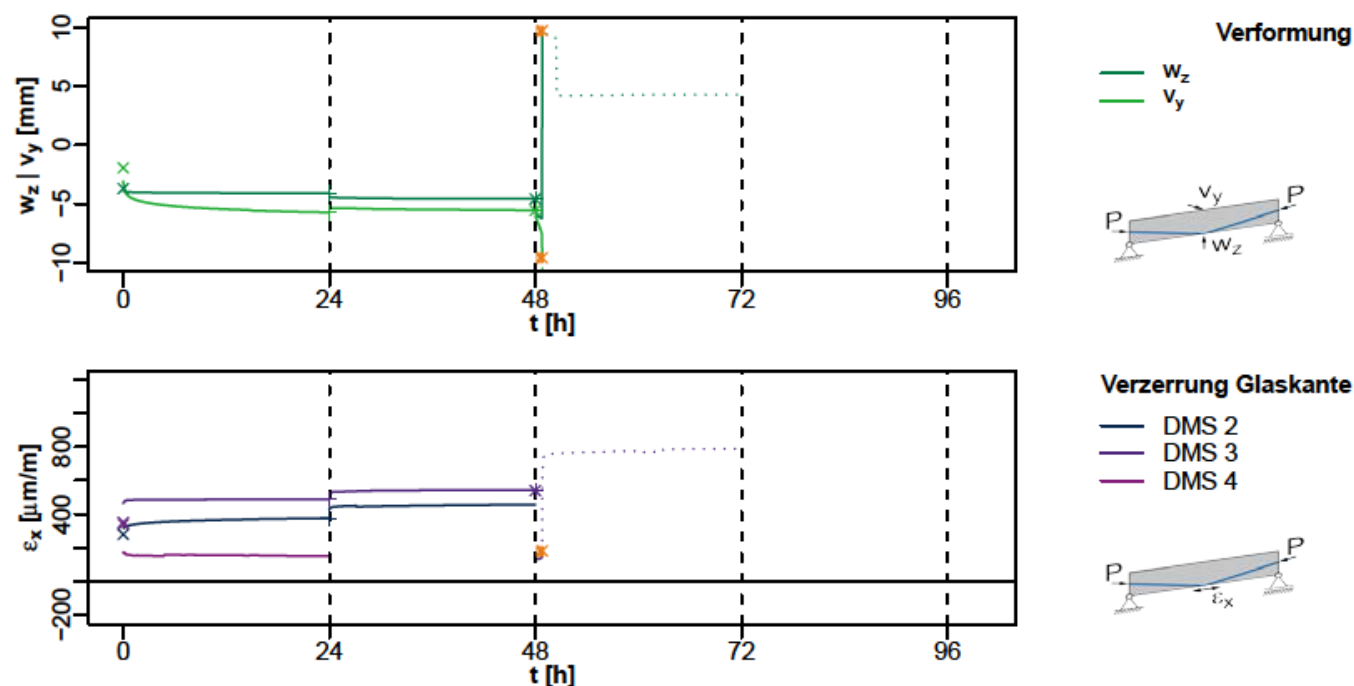

Verzerrung Glaskante

- DMS 2

- DMS 3

- DMS 4

\begin{tabular}{|c|c|c|c|c|c|c|c|}
\hline & RT18P15-I & $\mathrm{N}_{\mathrm{p}}[\mathrm{kN}]$ & $N_{p} / N_{p}(t=0)[-]$ & $\mathrm{w}_{\mathrm{z}}[\mathrm{mm}]$ & $w_{z} / w_{z, 0}[-]$ & $\mathrm{v}_{\mathrm{y}}[\mathrm{mm}]$ & $v_{y} / v_{y, 0}[-]$ \\
\hline & $\mathrm{t}_{\text {0.nach Belastung }}$ & 16.404 & 1.000 & -3.708 & 1.000 & -1.904 & 1.000 \\
\hline Bruch 1 & $\mathrm{t}_{0 . \text { nach Bruch }}$ & 16.620 & $1.01 \overline{1}$ & -3.877 & $1.04 \overline{6}$ & -2.950 & 1.550 \\
\hline \multirow[b]{2}{*}{ Bruch 4} & $t_{24 h . v o r}$ Bruch & 15.180 & 0.925 & -4.086 & 1.102 & -5.746 & 3.018 \\
\hline & $\mathrm{t}_{24 \mathrm{~h} . \text { nach Bruch }}$ & 15.348 & $0.93 \overline{6}$ & -4.289 & $1.15 \overline{7}$ & -5.502 & 2.890 \\
\hline \multirow{2}{*}{ Bruch 2} & 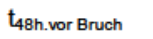 & 15.144 & 0.923 & -4.574 & 1.234 & -5.552 & 2.916 \\
\hline & $\mathrm{t}_{48 \mathrm{~h} . \text { nach Bruch }}$ & 16.308 & $0 . \overline{9} \overline{4}$ & -5.792 & $1.56 \overline{2}$ & -6.223 & $3.26 \overline{9}$ \\
\hline Bruch 3 & 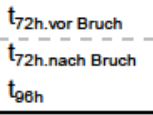 & & & & & & \\
\hline \multicolumn{2}{|c|}{ Versagen $48.9 \mathrm{~h}$} & 15.258 & 0.930 & 9.749 & -2.629 & -9.599 & 5.043 \\
\hline
\end{tabular}




\section{Bruchbild RT18P15-I Träger mit $P_{m 0}=15 \mathrm{kN}$ und $d_{p}=8,1 \mathrm{~mm}$}

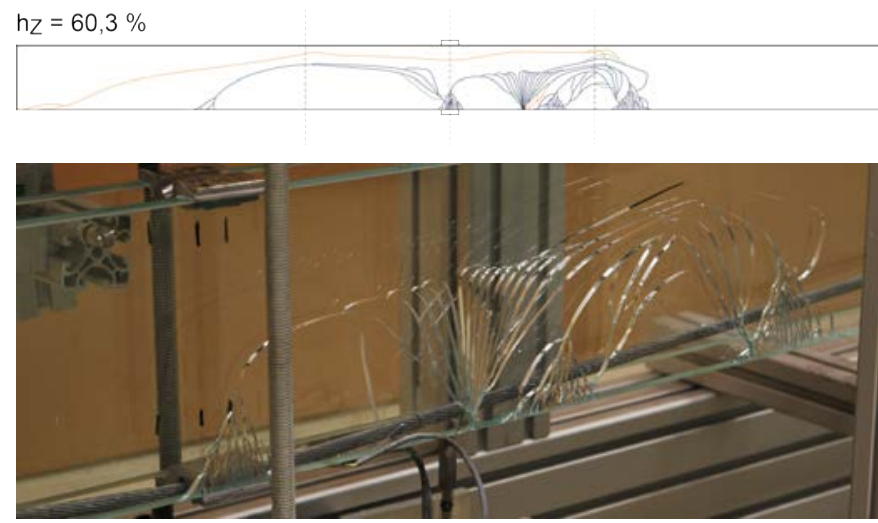

$h_{\mathrm{Z}}=55,8 \%$

Scheibe 4
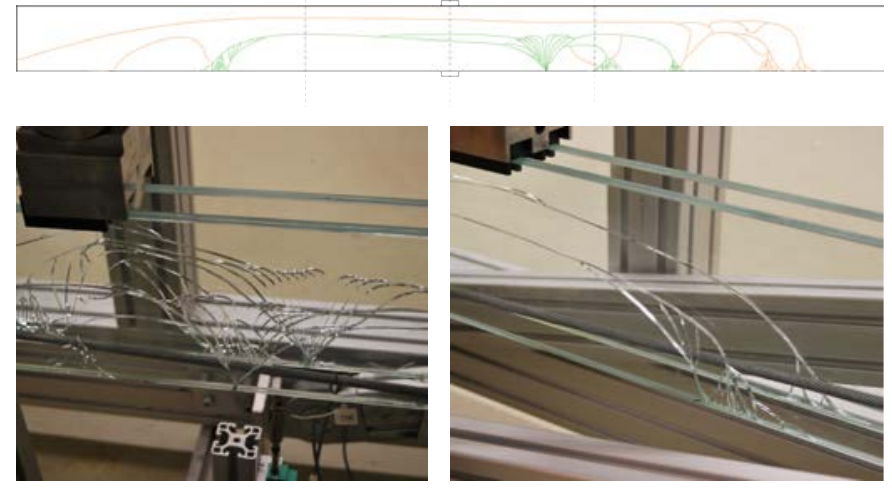

$h z=68,0 \%$

Scheibe 2

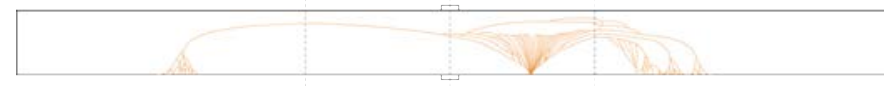

Bruch durch Anschlagen nach $\mathrm{t}=0 \mathrm{~h}$

- Brucherweiterung bis $\mathrm{t}=24 \mathrm{~h}$

- Brucherweiterung bis $\mathrm{t}=48 \mathrm{~h}$
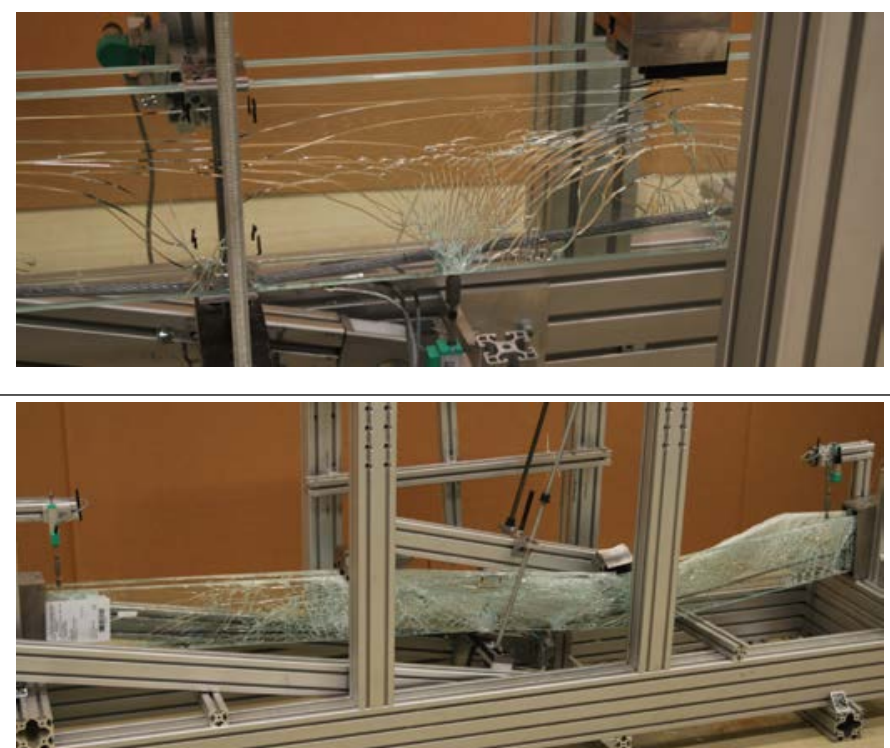

Bruch aller Scheiben 


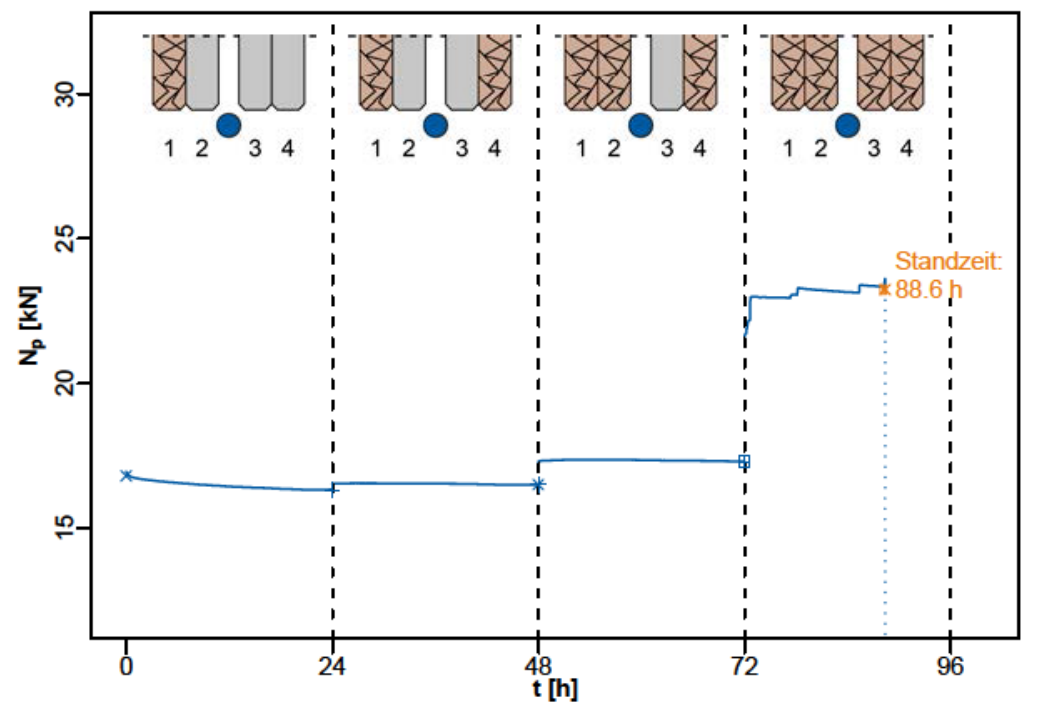

Seilkraftänderung

- RT19P15-II
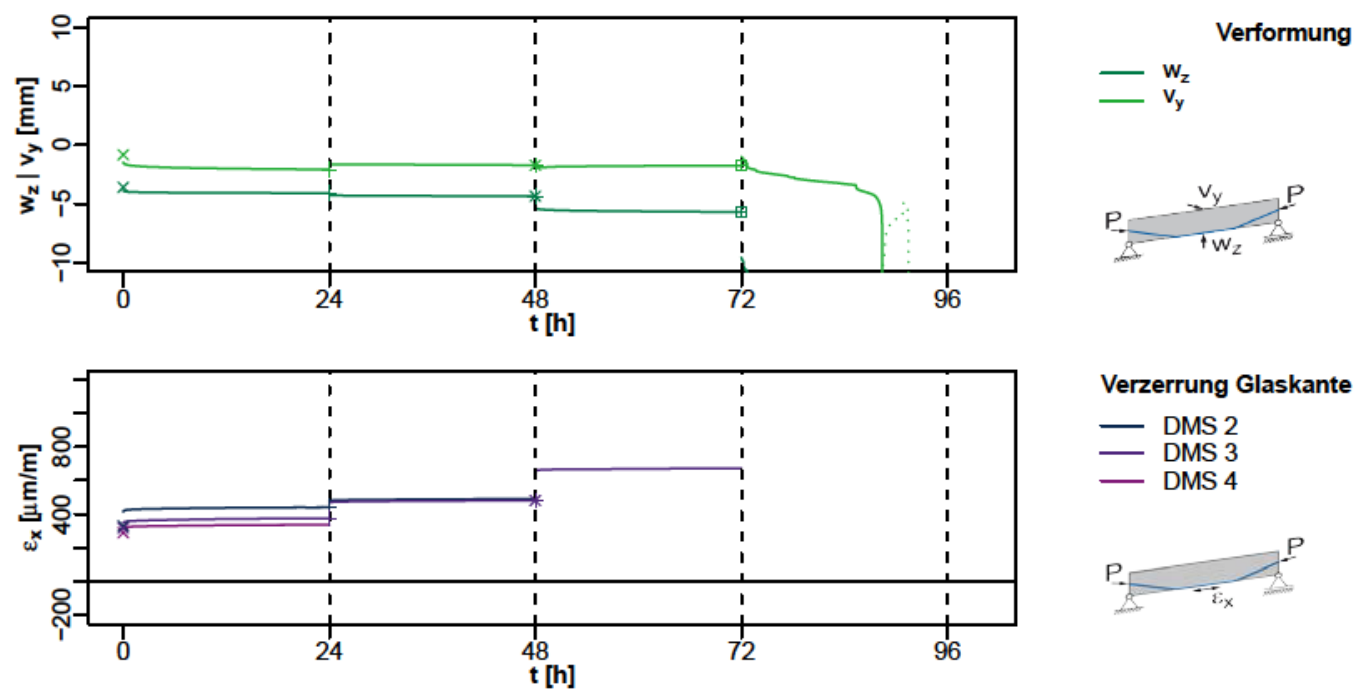

Verzerrung Glaskante

- DMS 2

- DMS 3

- DMS 4

\begin{tabular}{|c|c|c|c|c|c|c|c|}
\hline & RT19P15-II & $\mathrm{N}_{\mathrm{p}}[\mathrm{kN}]$ & $N_{p} / N_{p}(t=0)[-]$ & $w_{z}[\mathrm{~mm}]$ & $w_{z} / w_{z, 0}[-]$ & $\mathrm{v}_{\mathrm{y}}[\mathrm{mm}]$ & $v_{y} / v_{y, 0}[-]$ \\
\hline \multirow[b]{2}{*}{ Bruch 1. } & $t_{0 . \text { nach Belastung }}$ & 16.818 & 1.000 & -3.591 & 1.000 & -0.788 & 1.000 \\
\hline & $\mathrm{t}_{0 . \text { nach Bruch }}$ & $1 \overline{6} . \overline{6} 2$ & $1 . \overline{00} \overline{9}$ & -3.815 & $1.06 \overline{2}$ & -1.391 & $1 \overline{76} \overline{5}$ \\
\hline \multirow{2}{*}{ Bruch 4} & 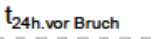 & 16.338 & 0.971 & -4.091 & 1.139 & -2.091 & 2.653 \\
\hline & 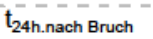 & 16.464 & $0.9 \overline{7} \overline{9}$ & -4.195 & $1.16 \overline{8}$ & $-1.6 \overline{7} 5$ & $2.12 \overline{5}$ \\
\hline \multirow{2}{*}{ Bruch 2 . } & $\mathrm{t}_{48 \mathrm{~h} . \text { vor Bruch }}$ & 16.518 & 0.982 & -4.353 & 1.212 & -1.710 & 2.169 \\
\hline & 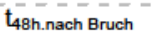 & 17.160 & 1.020 & -5.137 & 1.430 & -1.890 & 2.398 \\
\hline \multirow{2}{*}{ Bruch 3} & 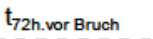 & 17.310 & 1.029 & -5.694 & 1.586 & -1.741 & 2.209 \\
\hline & 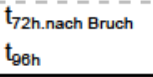 & 21.126 & 1.256 & -8.835 & 2.460 & -0.667 & $0.84 \overline{6}$ \\
\hline \multicolumn{2}{|c|}{ Versagen $88.6 \mathrm{~h}$} & 23.244 & 1.382 & -16.668 & 4.642 & -16.437 & 20.857 \\
\hline
\end{tabular}


Bruchbild RT19P15-II

$h_{z}=56,8 \%$

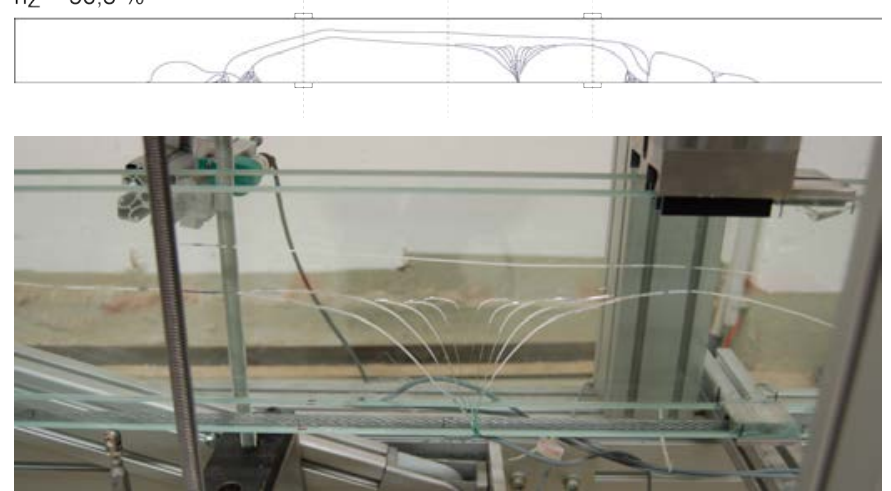

$h_{Z}=54,9 \%$
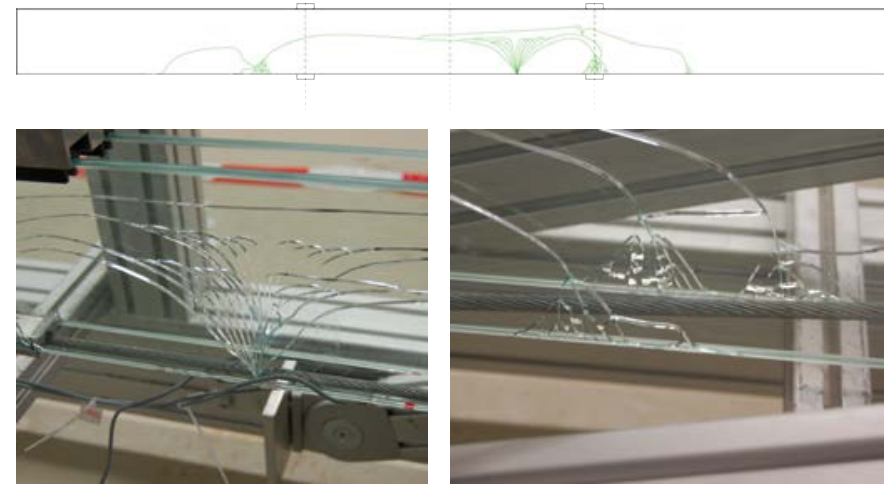

$h_{z}=67,3 \%$
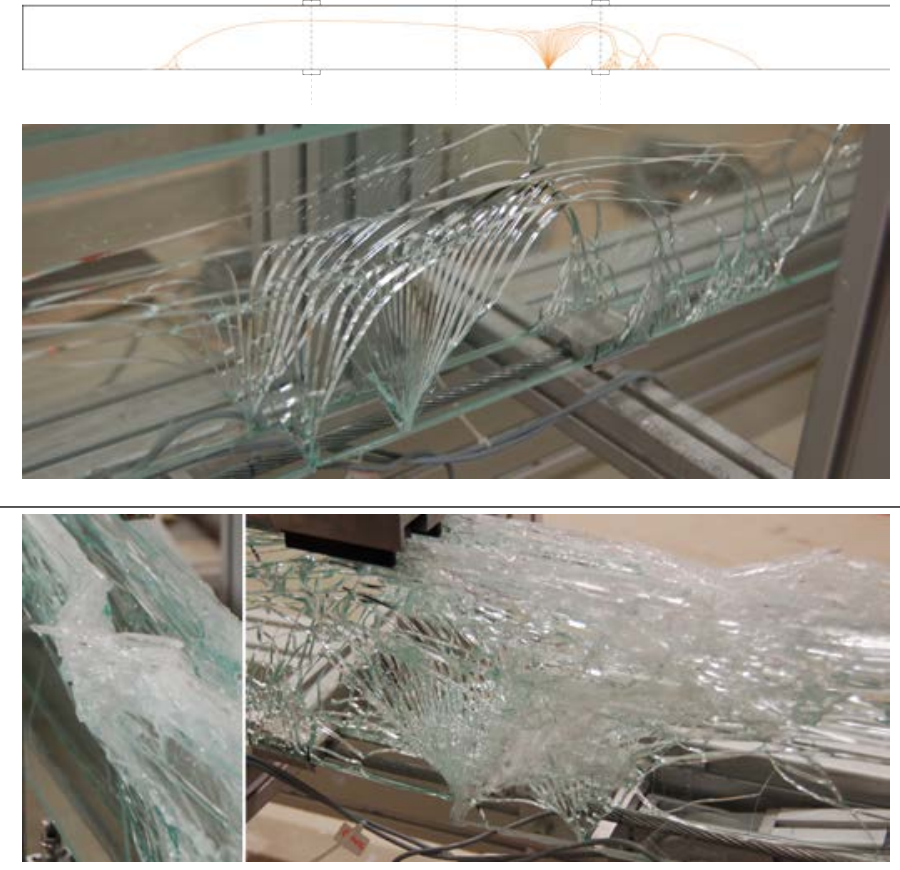

Scheibe 1

Bruch durch Anschlagen nach $\mathrm{t}=0 \mathrm{~h}$

- Brucherweiterung bis $\mathrm{t}=24 \mathrm{~h}$

Brucherweiterung bis $\mathrm{t}=48 \mathrm{~h}$
Bruch durch Anschlagen nach $\mathrm{t}=24 \mathrm{~h}$

_ Brucherweiterung bis $\mathrm{t}=48 \mathrm{~h}$
Scheibe 2

Bruch durch Anschlagen nach $\mathrm{t}=48 \mathrm{~h}$

Bruch aller Scheiben

Einknicken nahe der Oberkante Abscheren der Glasschichten

im rechten Bild 


\section{Standzeit RT20P15-II Träger mit $P_{m 0}=15 \mathrm{kN}$ und $d_{p}=8,1 \mathrm{~mm}$}

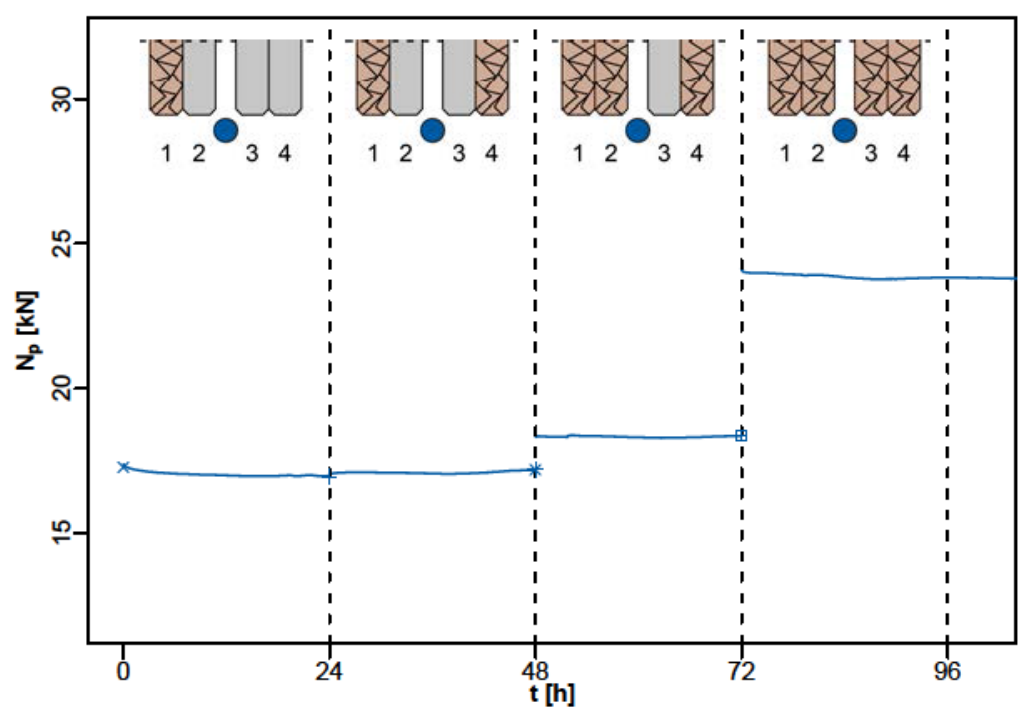

Seilkraftänderung

- RT20P15-II
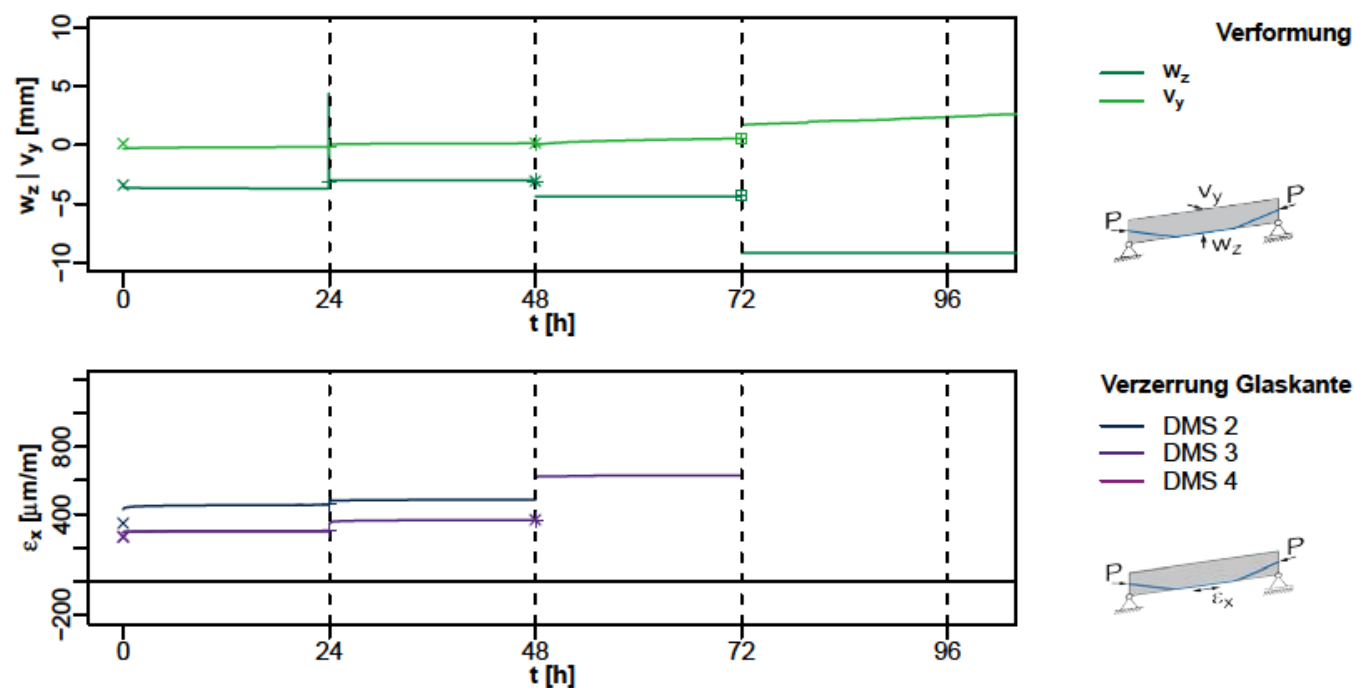

Verzerrung Glaskante

- DMS 2

- DMS 3

- DMS 4

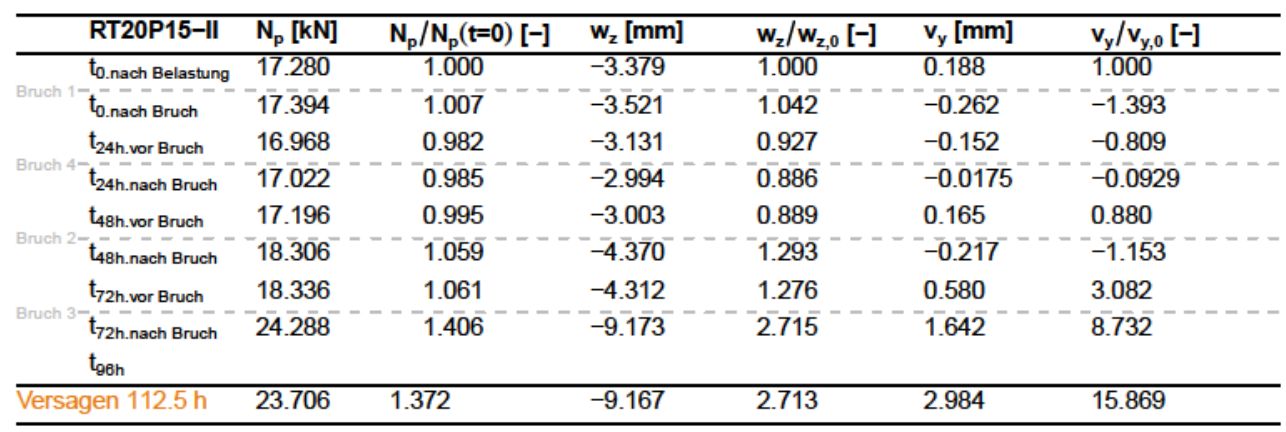




\section{Bruchbild RT20P15-II Träger mit $P_{m 0}=15 \mathrm{kN}$ und $d_{p}=8,1 \mathrm{~mm}$}

$\mathrm{hz}=57.9 \%$

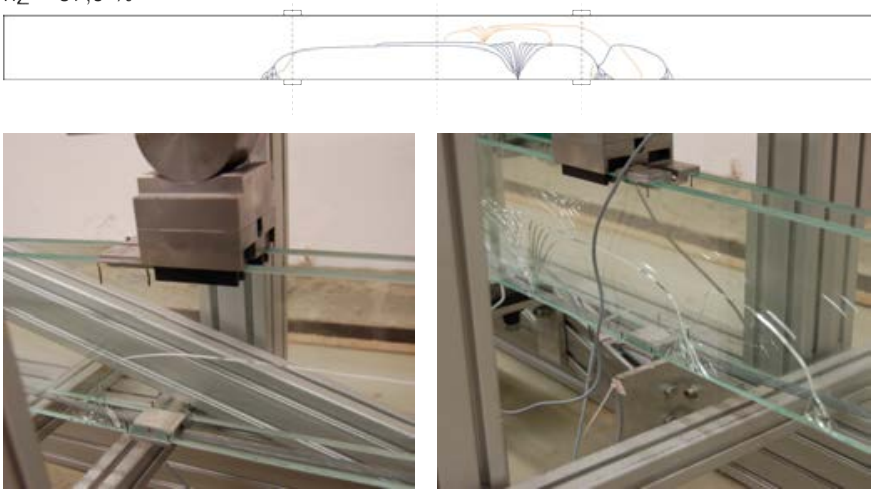

Scheibe 1

$h_{Z}=58,8 \%$

Scheibe 4

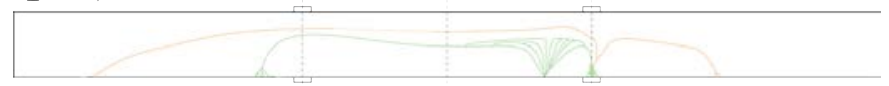

Bruch durch Anschlagen nach $\mathrm{t}=24 \mathrm{~h}$

- Brucherweiterung bis $\mathrm{t}=48 \mathrm{~h}$

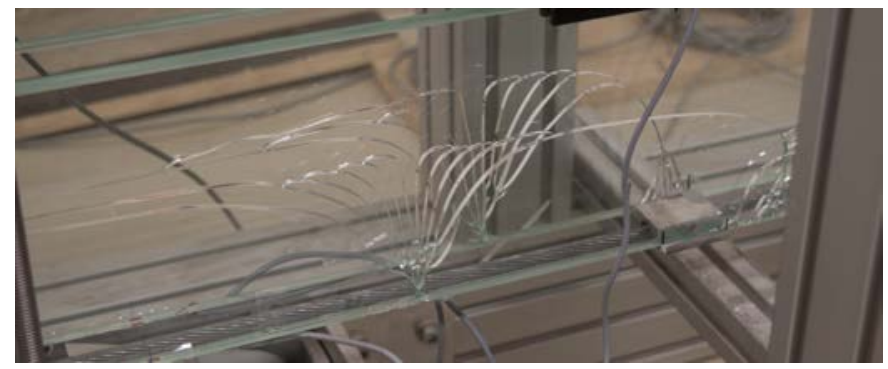

$h_{z}=73,6 \%$

Scheibe 2

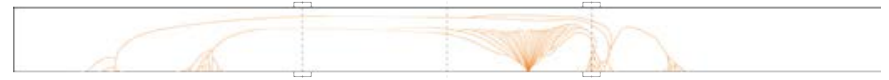

Bruch durch Anschlagen nach $\mathrm{t}=0 \mathrm{~h}$

- Brucherweiterung bis $t=24 \mathrm{~h}$

Brucherweiterung bis $t=48 h$
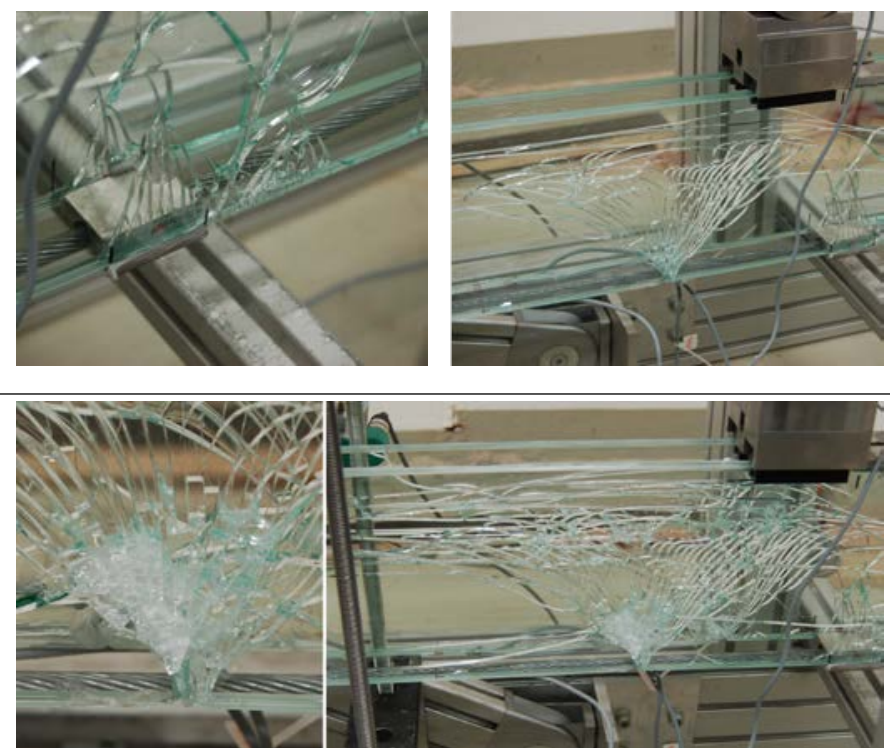

Bruch aller Scheiben

zunehmendes Zerdrücken einzelner Bruchstücke 


\section{Standzeit RT21P15-I Träger mit $P_{m 0}=15 \mathrm{kN}$ und $d_{p}=10,1 \mathrm{~mm}$}

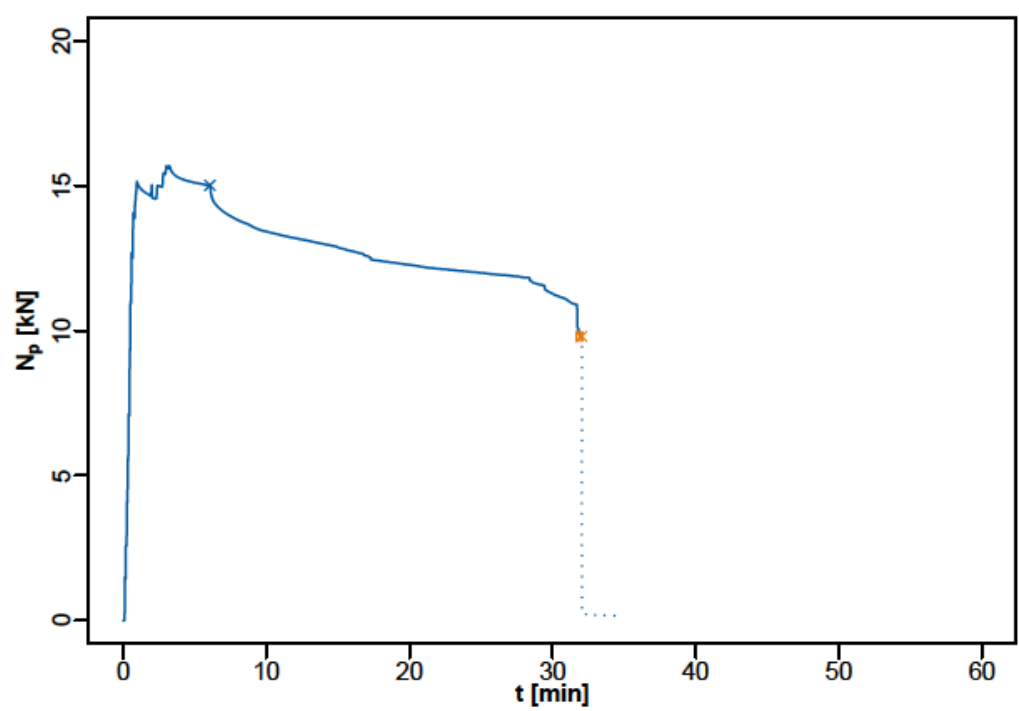

Seilkraftänderung

— RT21P15-I
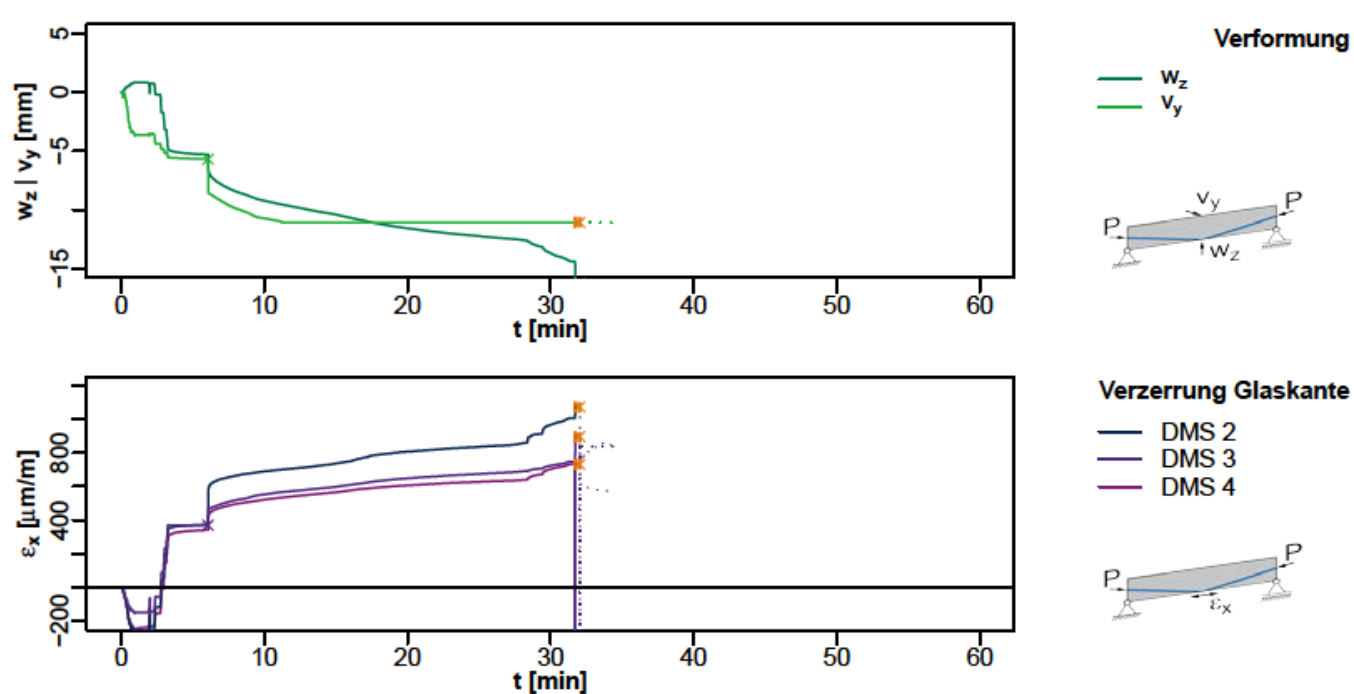

Verzerrung Glaskante

- DMS 2

- DMS 3

- DMS 4

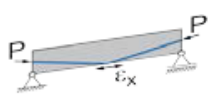

Brüche

$\times \quad$ Bruch 1

Bruch 4

* Bruch 2

*. Versagen 
Bruchbild RT21P15-I Träger mit $P_{m 0}=15 \mathrm{kN}$ und $d_{p}=10,1 \mathrm{~mm}$

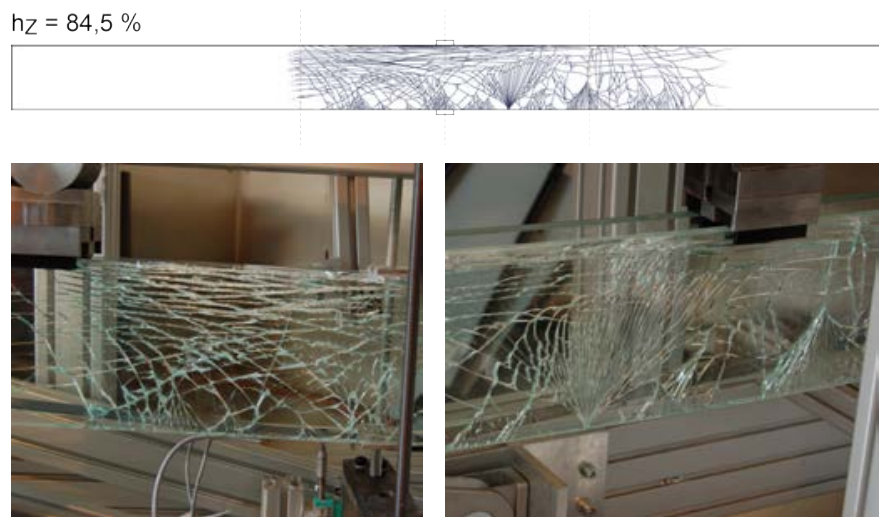

Scheibe 1

Bruch durch Anschlagen nach $\mathrm{t}=0 \mathrm{~h}$

- Brucherweiterung bis $\mathrm{t}=24 \mathrm{~h}$

- Brucherweiterung bis $\mathrm{t}=48 \mathrm{~h}$

deutlich sichtbare, sich progressiv vergrößernde horizontale Durchbiegung

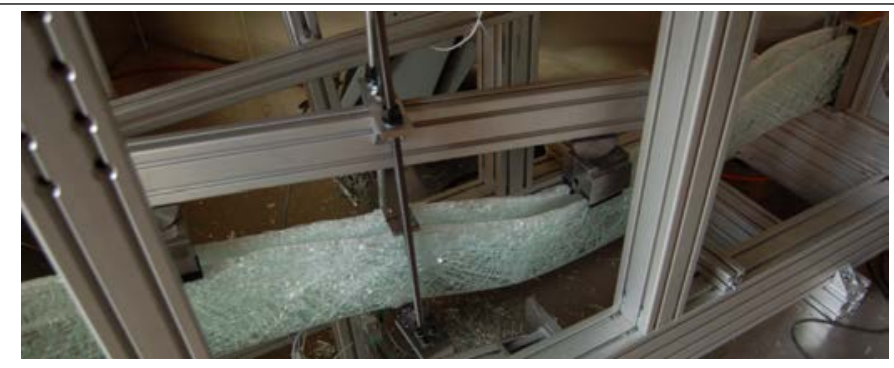

Bruch aller Scheiben

feines Bruchbild nach Versagen 


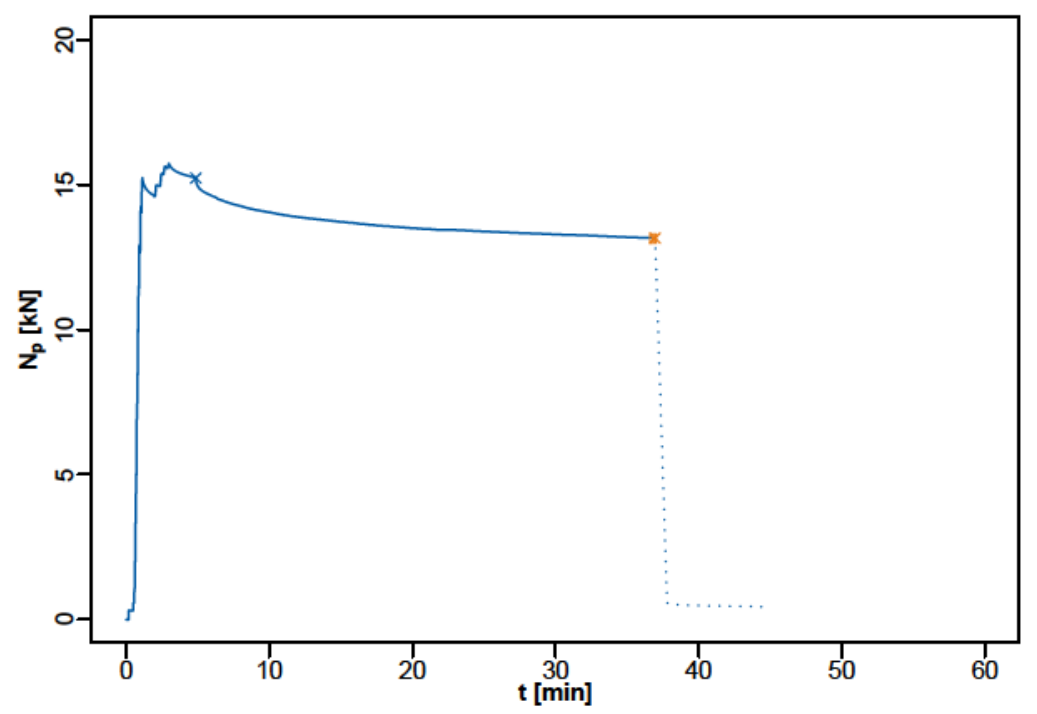

Seilkraftänderung

— RT22P15-I
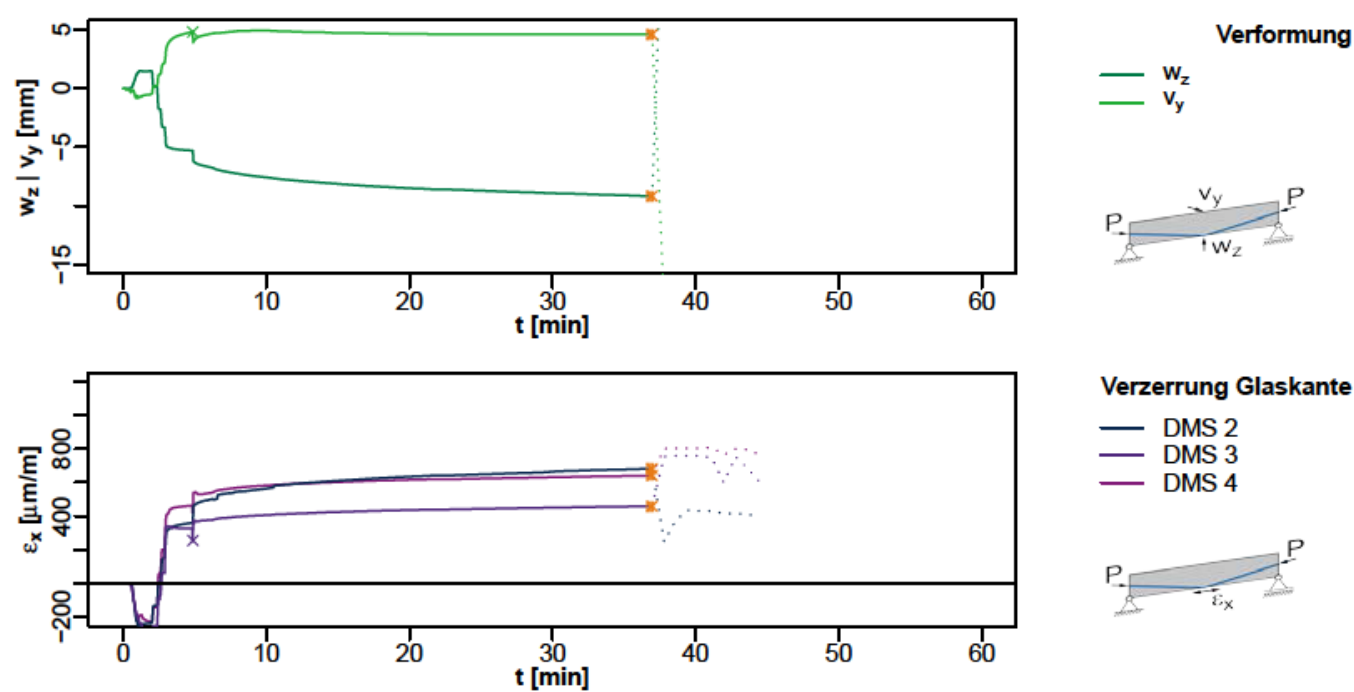

Verzerrung Glaskante

- DMS 2

- DMS 3

- DMS 4

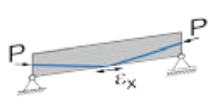

Brüche

$\times$ Bruch 1

$+\quad$ Bruch 4

$*$
$* \quad$ Bruch 2
$*$ Bruch 3

i. Versagen 


\section{Bruchbild RT22P15-I Träger mit $P_{m 0}=15 \mathrm{kN}$ und $d_{p}=10,1 \mathrm{~mm}$}

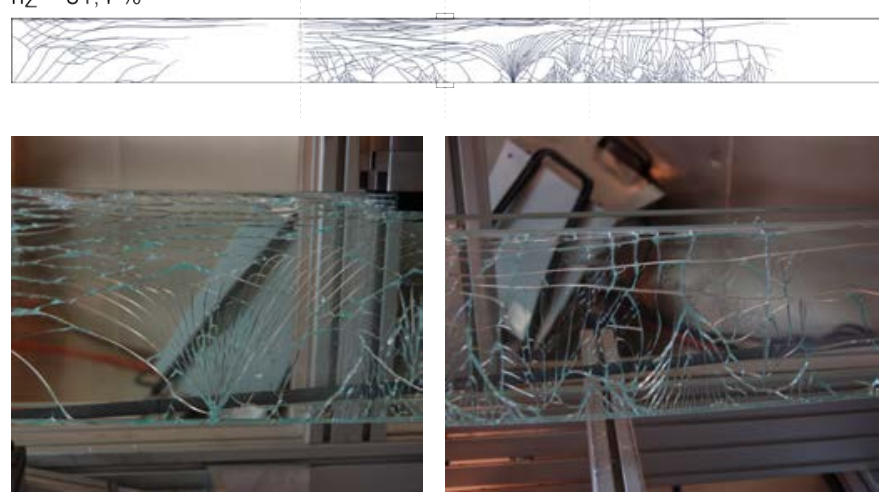

Scheibe 1

Bruch durch Anschlagen

nach $\mathrm{t}=\mathrm{Oh}$

- Brucherweiterung bis $t=24 \mathrm{~h}$

- Brucherweiterung bis $t=48 \mathrm{~h}$

deutlich sichtbare, sich progressiv vergrößernde horizontale Durchbiegung

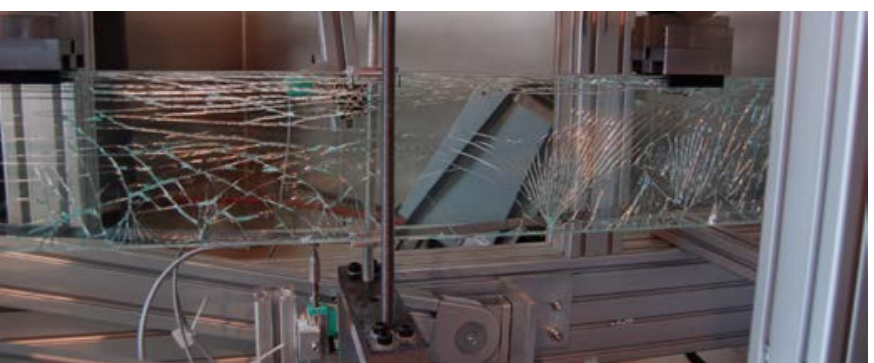

großflächiges Bruchbild
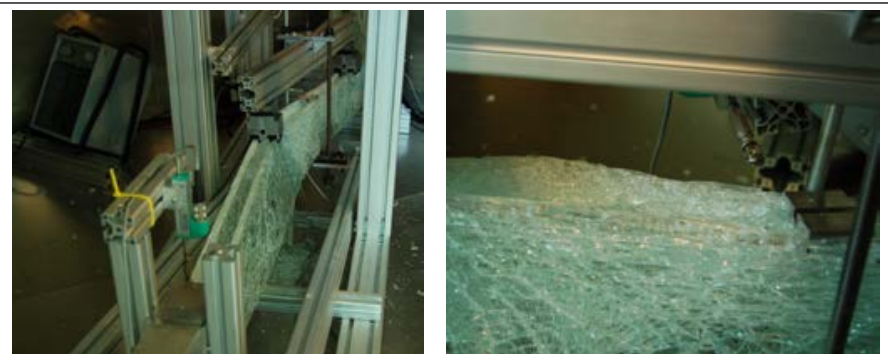

Bruch aller Scheiben

feines Bruchbild nach Versagen Abgeplatztes Glas in der

Trägeroberkante nahe der Zwischenfolie 


\section{Standzeit RT23P15-II Träger mit $P_{m 0}=15 \mathrm{kN}$ und $d_{p}=10,1 \mathrm{~mm}$}

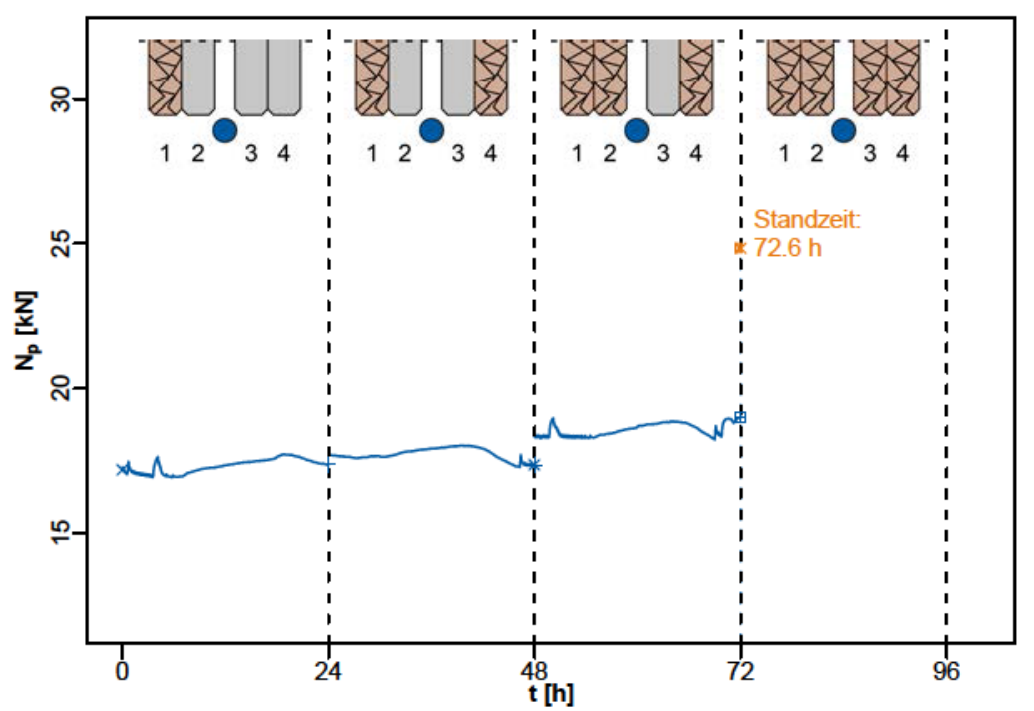

Seilkraftänderung

- RT23P15-II
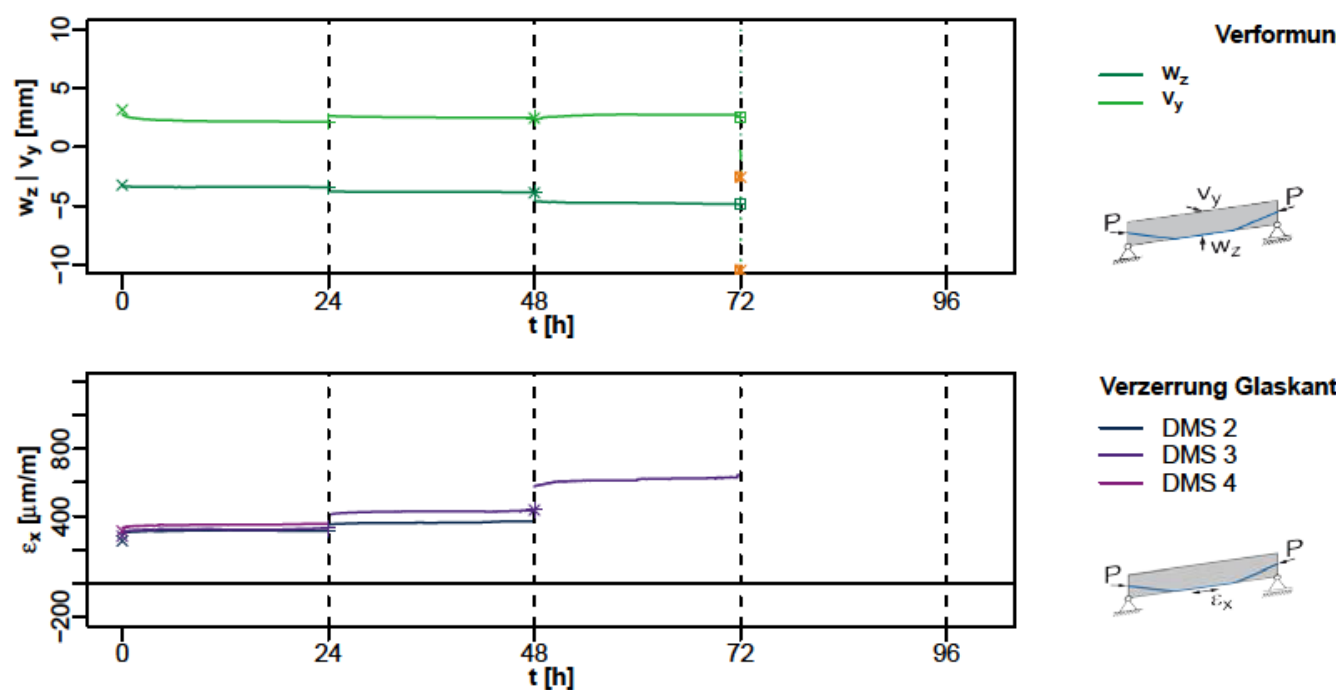

Verzerrung Glaskante

- DMS 2

- DMS 3

- DMS 4

\begin{tabular}{|c|c|c|c|c|c|c|c|}
\hline & RT23P15-II & $\mathrm{N}_{\mathrm{p}}[\mathrm{kN}]$ & $N_{p} / N_{p}(t=0)[-]$ & $\mathrm{w}_{\mathrm{z}}[\mathrm{mm}]$ & $w_{z} / w_{z, 0}[-]$ & $\mathrm{v}_{\mathrm{y}}[\mathrm{mm}]$ & $v_{y} / v_{y, 0}[-]$ \\
\hline \multirow[b]{2}{*}{ Bruch 1. } & $\mathrm{t}_{0 . \text { nach Belastung }}$ & 17.196 & 1.000 & -3.226 & 1.000 & 3.191 & 1.000 \\
\hline & $\mathrm{t}_{0 . \text { nach Bruch }}$ & $17 . \overline{304}$ & $1.00 \overline{6}$ & -3.317 & 1.028 & $2.88 \overline{2}$ & 0.903 \\
\hline \multirow{2}{*}{ Bruch 4} & $t_{24 h . v o r \text { Bruch }}$ & 17.364 & 1.010 & -3.414 & 1.058 & 2.139 & 0.670 \\
\hline & 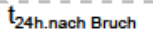 & 17.652 & 1.027 & -3.680 & 1.141 & 2.726 & 0.854 \\
\hline \multirow[b]{2}{*}{ Bruch 2 . } & $\mathrm{t}_{48 \mathrm{~h} . \text { vor Bruch }}$ & 17.370 & 1.010 & -3.872 & 1.201 & 2.477 & 0.776 \\
\hline & $\mathrm{t}_{48 \mathrm{~h} . \text { nach Bruch }}$ & 18.330 & $1.06 \overline{6}$ & -4.557 & $1.4 \overline{1}$ & $2.19 \overline{4}$ & 0.688 \\
\hline \multirow{2}{*}{ Bruch 3 . } & 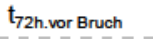 & 18.996 & 1.105 & -4.876 & 1.512 & 2.604 & 0.816 \\
\hline & 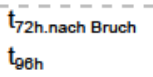 & 25.104 & $1 . \overline{460}$ & -10.042 & $3.11 \overline{3}$ & $1.48 \overline{6}$ & $0.46 \overline{6}$ \\
\hline \multicolumn{2}{|c|}{ Versagen $72.6 \mathrm{~h}$} & 24.840 & 1.445 & -10.494 & 3.253 & -2.569 & -0.805 \\
\hline
\end{tabular}




\section{Bruchbild RT23P15-II Träger mit $P_{m 0}=15 \mathrm{kN}$ und $d_{p}=10,1 \mathrm{~mm}$}

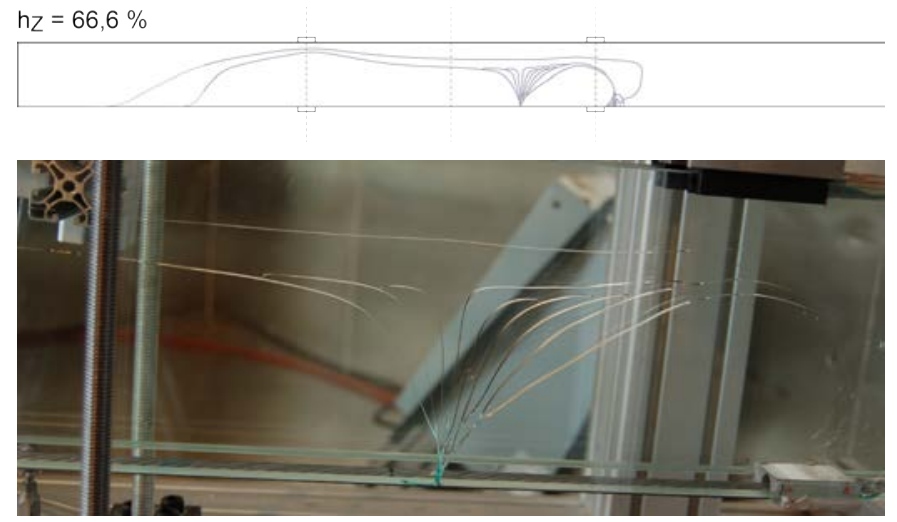

$h_{z}=70,2 \%$

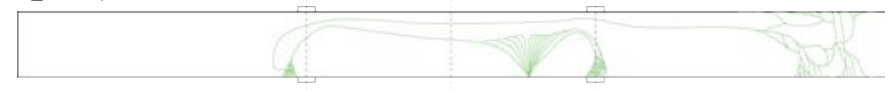

Bruch durch Anschlagen nach $\mathrm{t}=24 \mathrm{~h}$
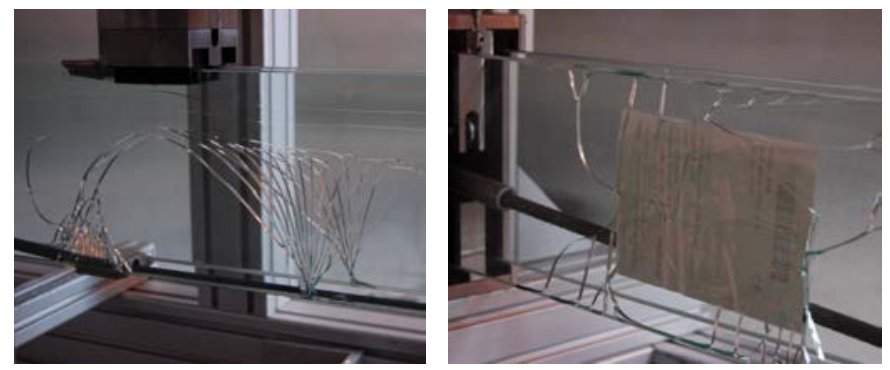

$h_{z}=67,4 \%$

Scheibe 2

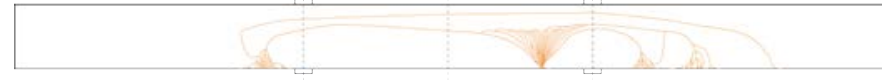

- Brucherweiterung bis $\mathrm{t}=48 \mathrm{~h}$

flächiges Bruchbild in Auflagernähe
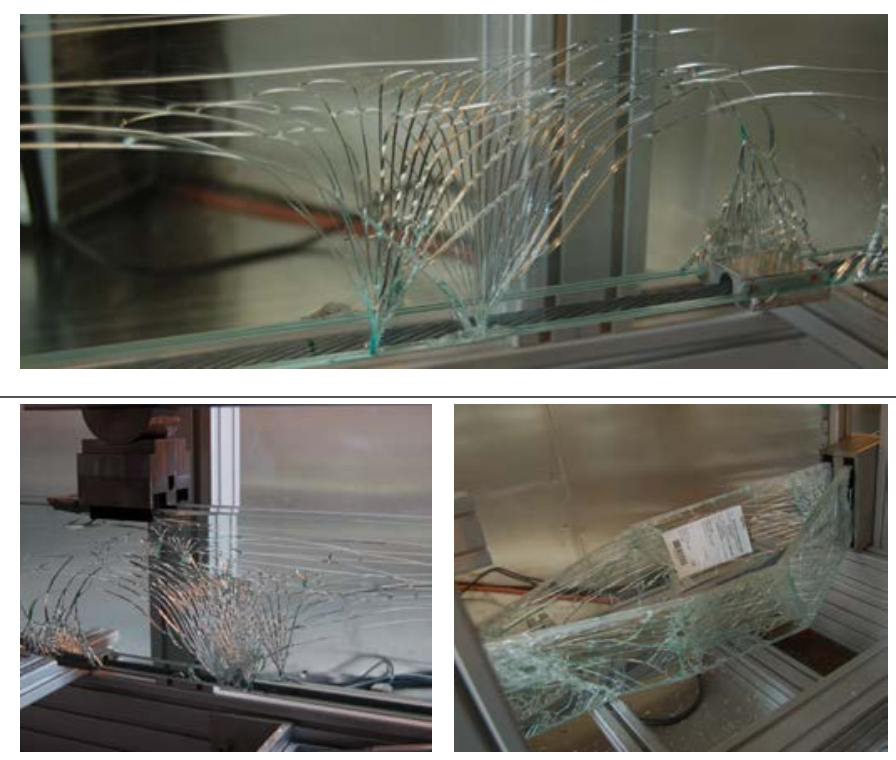

Bruch aller Scheiben

Bruchbild nach Bruch der vierten Schicht (linkes Bild)

Versagen durch seitliches Abknicken in Auflagernähe (rechts Bild) 


\section{Standzeit RT24P15-II Träger mit $P_{m 0}=15 \mathrm{kN}$ und $d_{p}=10,1 \mathrm{~mm}$}

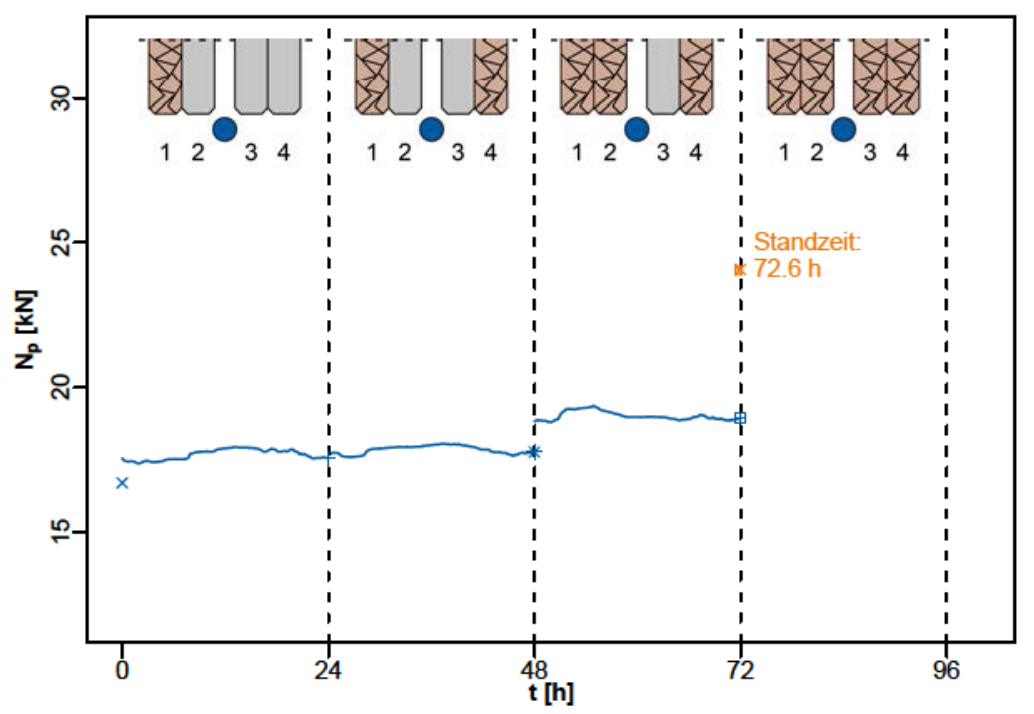

Seilkraftänderung

- RT24P15-II
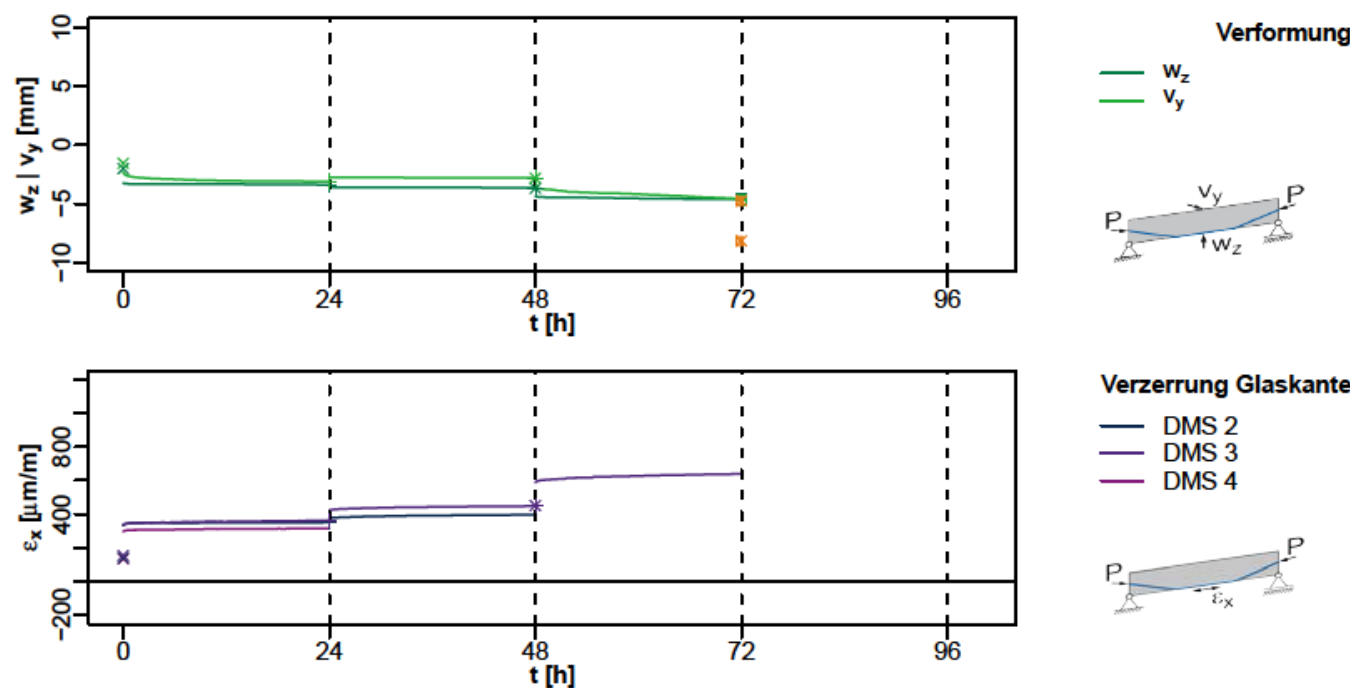

Verzerrung Glaskante

- DMS 2

- DMS 3

- DMS 4

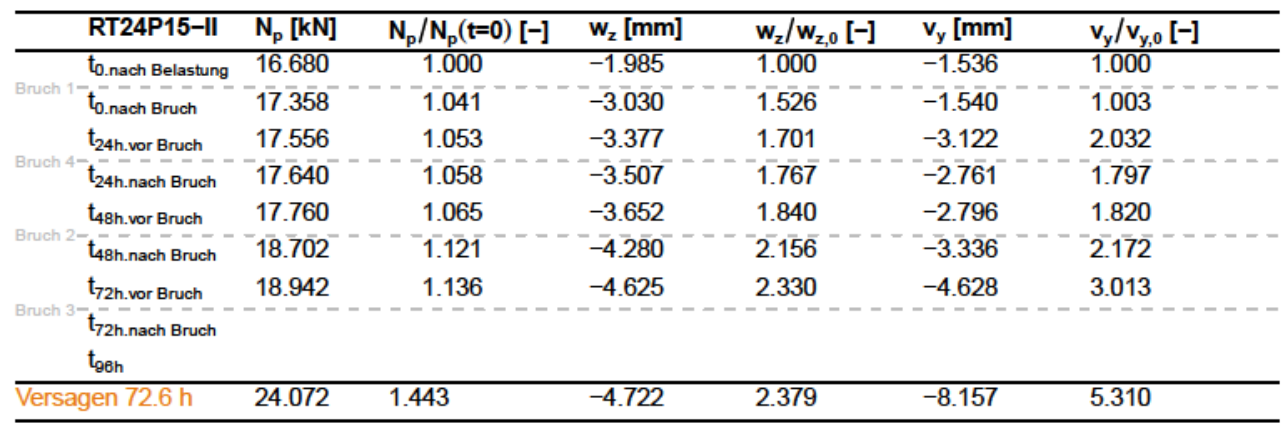


Bruchbild RT24P15-II

$h_{z}=59,2 \%$

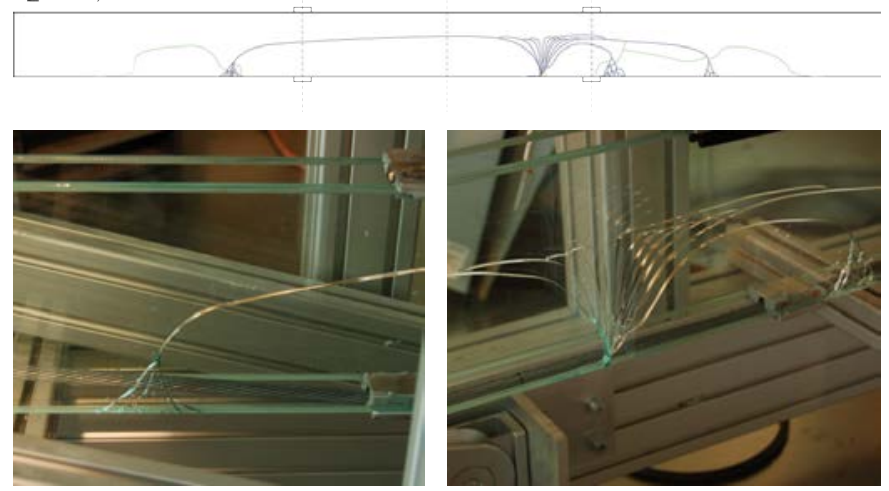

$h_{Z}=57,8 \%$
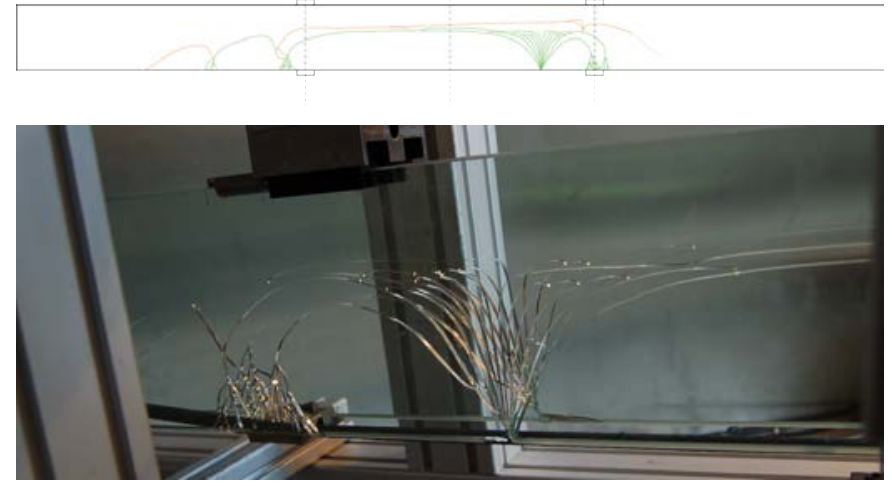

$h_{z}=66,3 \%$
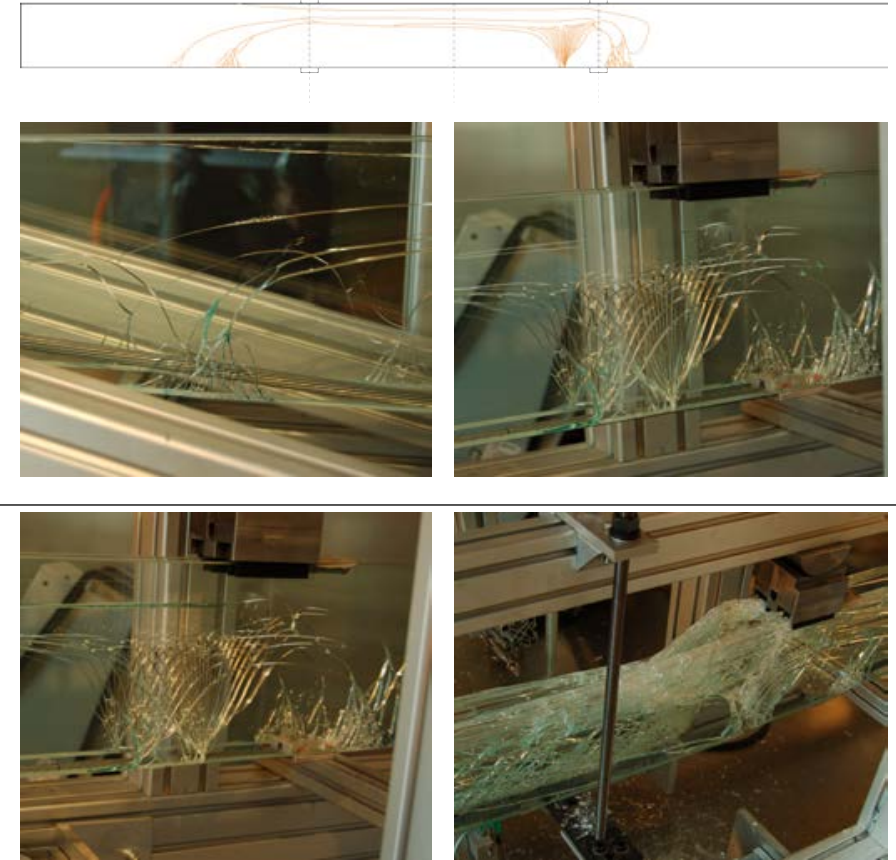

Scheibe 1

Bruch durch Anschlagen nach $\mathrm{t}=0 \mathrm{~h}$

- Brucherweiterung bis $\mathrm{t}=24 \mathrm{~h}$

Brucherweiterung bis $\mathrm{t}=48 \mathrm{~h}$

Scheibe 4

Bruch durch Anschlagen nach $\mathrm{t}=24 \mathrm{~h}$

_ Brucherweiterung bis $\mathrm{t}=48 \mathrm{~h}$

Scheibe 2 Bruch durch Anschlagen nach $\mathrm{t}=48 \mathrm{~h}$
Bruch aller Scheiben

Bruchbild nach Bruch der vierten Schicht (linkes Bild)

Versagen durch Auffalten nahe der Lasteinleitung (rechts Bild) 


\section{E Biegeversuche unter Temperaturlast}

In dieser Anlage werden die Ergebnisse der Biegeversuche unter Temperaturlast gemäß Kapitel 3.6 dokumentiert.

Die folgende Tabelle gibt einen Überblick. Darauf folgend werden die Änderung der Seilkraft, Durchbiegung und kompensierte Glasverzerrung für jeden Versuch dargestellt. Anschließend folgt eine Tabelle mit wesentlichen Einzelwerten aus den graphischen Darstellungen

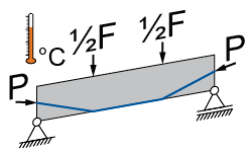

Als wesentliches Ergebnis ergibt sich die Seilkraftänderung sowie die Glasverzerrung infolge der Temperaturänderung als Mittelwert aus drei Dehnungsmessstreifen an je einer Glaskante.

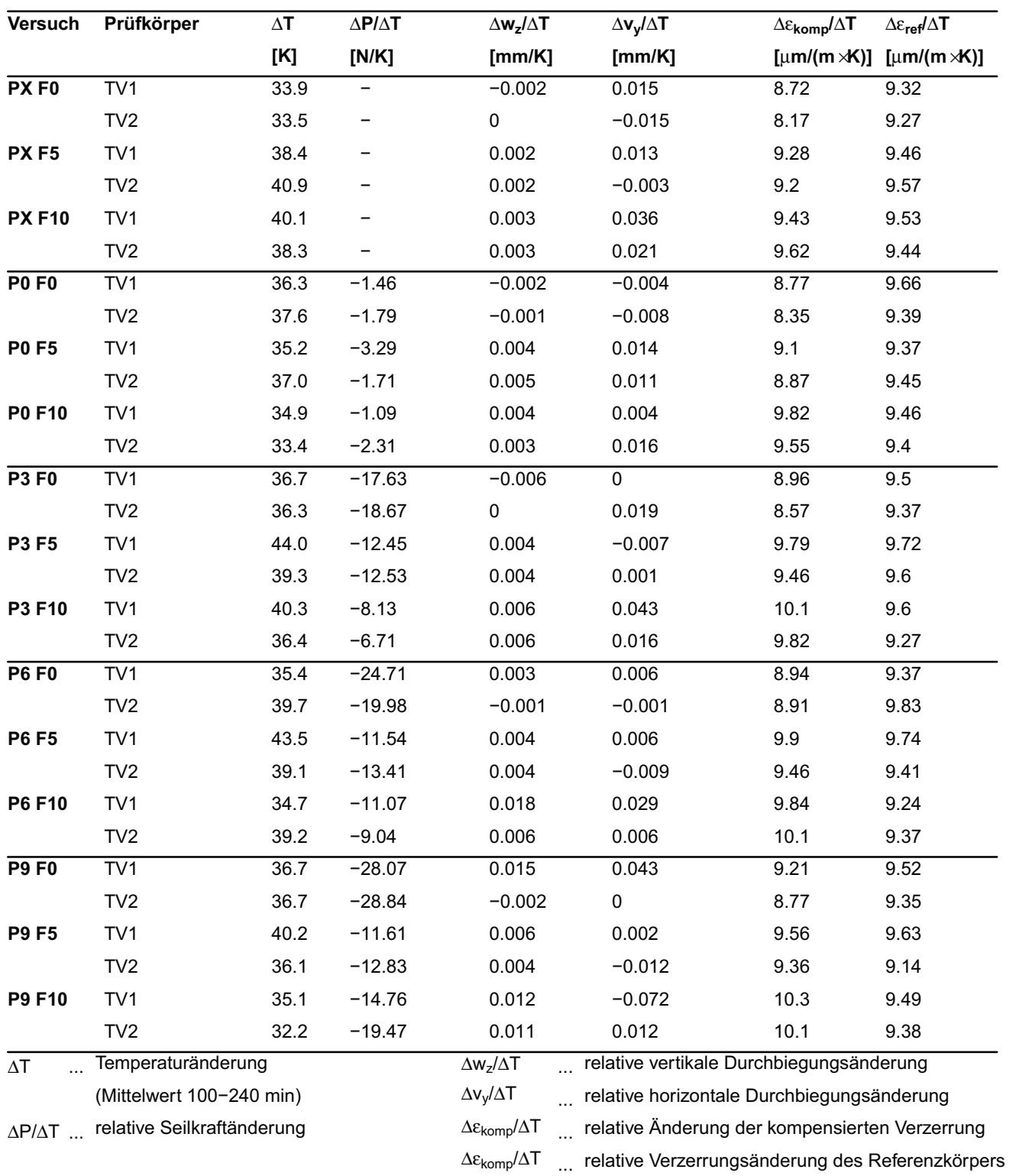


In der folgenden Tabelle sind die Werte zu Beginn der Heizphase $(t=0)$ und die Mittelwerte aus dem Zeitbereich zwischen 100 und 240 Minuten einschließlich der zugehörigen Standardabweichungen abgedruckt.

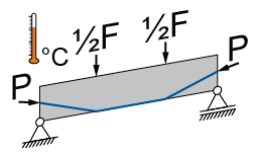

\begin{tabular}{|c|c|c|c|c|c|c|c|c|c|}
\hline \multirow[t]{2}{*}{ Versuch } & \multirow[t]{2}{*}{ Prüfkörper } & \multicolumn{2}{|c|}{$\mathrm{N}_{\mathrm{p}}[\mathrm{kN}]$} & \multicolumn{2}{|l|}{$\mathrm{w}_{\mathrm{z}}[\mathrm{mm}]$} & \multicolumn{2}{|c|}{$\mathrm{v}_{\mathrm{y}}[\mathrm{mm}]$} & \multicolumn{2}{|c|}{$\varepsilon_{\text {komp }}[\mu \mathrm{m} / \mathrm{m}]$} \\
\hline & & $t=0$ & $t>100$ & $t=0$ & $t>100$ & $t=0$ & $t>100$ & $t=0$ & $t>100$ \\
\hline \multirow[t]{2}{*}{$\overline{\text { PX F0 }}$} & TV1 & - & - & 0.00051 & $-0.057 \pm 0.02$ & 0.00 & $0.52 \pm 0.05$ & -1 & $295 \pm 28$ \\
\hline & TV2 & - & - & 0.021 & $0.028 \pm 0.02$ & -0.20 & $-0.71 \pm 0.04$ & -1 & $273 \pm 22$ \\
\hline \multirow[t]{2}{*}{ PX F5 } & TV1 & - & - & 2.57 & $2.63 \pm 0.02$ & 1.64 & $2.14 \pm 0.02$ & 272 & $628 \pm 30$ \\
\hline & TV2 & - & - & 2.87 & $2.95 \pm 0.01$ & 0.32 & $0.21 \pm 0.06$ & 275 & $651 \pm 33$ \\
\hline \multirow[t]{2}{*}{ PX F10 } & TV1 & - & - & 5.21 & $5.33 \pm 0.04$ & 0.76 & $2.19 \pm 0.2$ & 587 & $966 \pm 34$ \\
\hline & TV2 & - & - & 5.28 & $5.41 \pm 0.02$ & 0.21 & $1.01 \pm 0.1$ & 578 & $946 \pm 19$ \\
\hline \multirow[t]{2}{*}{ P0 F0 } & TV1 & 0.10 & $0.05 \pm 0.01$ & 0.048 & $-0.02 \pm 0.009$ & 0.0072 & $-0.16 \pm 0.07$ & -2 & $316 \pm 27$ \\
\hline & TV2 & 0.10 & $0.04 \pm 0.02$ & -0.015 & $-0.04 \pm 0.008$ & -0.01 & $-0.31 \pm 0.002$ & -1 & $312 \pm 28$ \\
\hline \multirow[t]{2}{*}{ P0 F5 } & TV1 & 0.37 & $0.3 \pm 0.02$ & 2.73 & $2.86 \pm 0.03$ & 1.91 & $2.39 \pm 0.04$ & 265 & $586 \pm 28$ \\
\hline & TV2 & 0.33 & $0.3 \pm 0.03$ & 2.92 & $3.10 \pm 0.03$ & 0.87 & $1.27 \pm 0.01$ & 276 & $604 \pm 35$ \\
\hline \multirow[t]{2}{*}{ P0 F10 } & TV1 & 0.53 & $0.5 \pm 0.05$ & 5.09 & $5.22 \pm 0.01$ & -0.61 & $-0.46 \pm 0.1$ & 572 & $915 \pm 34$ \\
\hline & TV2 & 0.62 & $0.5 \pm 0.05$ & 5.52 & $5.64 \pm 0.009$ & 0.69 & $1.23 \pm 0.08$ & 577 & $896 \pm 34$ \\
\hline \multirow[t]{2}{*}{$\overline{\text { P3 F0 }}$} & TV1 & 3.00 & $2.4 \pm 0.04$ & -0.18 & $-0.39 \pm 0.03$ & -0.21 & $-0.22 \pm 0.008$ & -49 & $279 \pm 31$ \\
\hline & TV2 & 3.08 & $2.4 \pm 0.03$ & -0.28 & $-0.30 \pm 0.009$ & 1.63 & $2.31 \pm 0.05$ & -50 & $261 \pm 29$ \\
\hline \multirow[t]{2}{*}{ P3 F5 } & TV1 & 3.64 & $3.1 \pm 0.03$ & 2.15 & $2.32 \pm 0.007$ & -0.48 & $-0.80 \pm 0.07$ & 205 & $636 \pm 31$ \\
\hline & TV2 & 3.62 & $3.1 \pm 0.03$ & 2.42 & $2.57 \pm 0.01$ & 1.56 & $1.62 \pm 0.004$ & 216 & $587 \pm 32$ \\
\hline \multirow[t]{2}{*}{ P3 F10 } & TV1 & 4.48 & $4.2 \pm 0.06$ & 4.79 & $5.02 \pm 0.03$ & 1.32 & $3.04 \pm 0.2$ & 500 & $906 \pm 23$ \\
\hline & TV2 & 4.46 & $4.2 \pm 0.05$ & 5.05 & $5.25 \pm 0.02$ & 2.09 & $2.67 \pm 0.1$ & 502 & $859 \pm 19$ \\
\hline \multirow[t]{2}{*}{$\overline{\text { P6 F0 }}$} & TV1 & 5.96 & $5.1 \pm 0.03$ & -0.96 & $-0.86 \pm 0.02$ & 0.60 & $0.81 \pm 9 e-04$ & -98 & $219 \pm 29$ \\
\hline & TV2 & 5.96 & $5.2 \pm 0.03$ & -0.56 & $-0.58 \pm 0.01$ & 1.19 & $1.16 \pm 0.03$ & -96 & $258 \pm 27$ \\
\hline \multirow[t]{2}{*}{ P6 F5 } & TV1 & 6.54 & $6.0 \pm 0.03$ & 1.96 & $2.12 \pm 0.009$ & 1.02 & $1.28 \pm 9 e-04$ & 164 & $595 \pm 30$ \\
\hline & TV2 & 6.57 & $6.0 \pm 0.03$ & 2.37 & $2.54 \pm 0.01$ & 1.41 & $1.07 \pm 0.04$ & 169 & $538 \pm 29$ \\
\hline \multirow[t]{2}{*}{ P6 F10 } & TV1 & 7.39 & $7.0 \pm 0.03$ & 4.43 & $5.04 \pm 0.2$ & 0.98 & $1.99 \pm 0.1$ & 449 & $791 \pm 21$ \\
\hline & TV2 & 7.59 & $7.2 \pm 0.04$ & 4.78 & $5.03 \pm 0.01$ & 1.97 & $2.19 \pm 0.05$ & 453 & $850 \pm 34$ \\
\hline \multirow[t]{2}{*}{ P9 F0 } & TV1 & 8.98 & $8.0 \pm 0.03$ & -1.37 & $-0.81 \pm 0.02$ & 0.95 & $2.53 \pm 0.1$ & -141 & $197 \pm 32$ \\
\hline & TV2 & 9.01 & $8.0 \pm 0.03$ & -0.94 & $-1.03 \pm 0.02$ & 2.38 & $2.37 \pm 0.1$ & -146 & $175 \pm 30$ \\
\hline \multirow[t]{2}{*}{ P9 F5 } & TV1 & 9.46 & $9.0 \pm 0.04$ & 1.56 & $1.80 \pm 0.01$ & 0.55 & $0.64 \pm 0.002$ & 113 & $497 \pm 19$ \\
\hline & TV2 & 9.52 & $9.1 \pm 0.02$ & 1.97 & $2.12 \pm 0.02$ & 1.66 & $1.22 \pm 0.04$ & 119 & $457 \pm 23$ \\
\hline \multirow[t]{2}{*}{ P9 F10 } & TV1 & 10.39 & $9.9 \pm 0.02$ & 4.15 & $4.59 \pm 0.04$ & -1.09 & $-3.61 \pm 0.2$ & 411 & $770 \pm 33$ \\
\hline & TV2 & 10.52 & $9.9 \pm 0.06$ & 4.85 & $5.22 \pm 0.05$ & 2.40 & $2.78 \pm 0.01$ & 422 & $747 \pm 32$ \\
\hline$t=0$ & Versuchsbeg & & & $\mathrm{N}_{\mathrm{p}}$ & $\ldots \mathrm{Se}$ & & & & \\
\hline \multirow[t]{3}{*}{$t>100 \ldots$} & & & (20) & $w_{z}$ & .. & hurrt & hbiegung & & \\
\hline & & & & $v_{y}$ & $\ldots$ hor & ntale D & archbiegung & & \\
\hline & & & & $\varepsilon_{\text {kon }}$ & ... komp & ensierte & Verzerrung & & \\
\hline
\end{tabular}


Temperaturlast Referenzträger unbewehrt
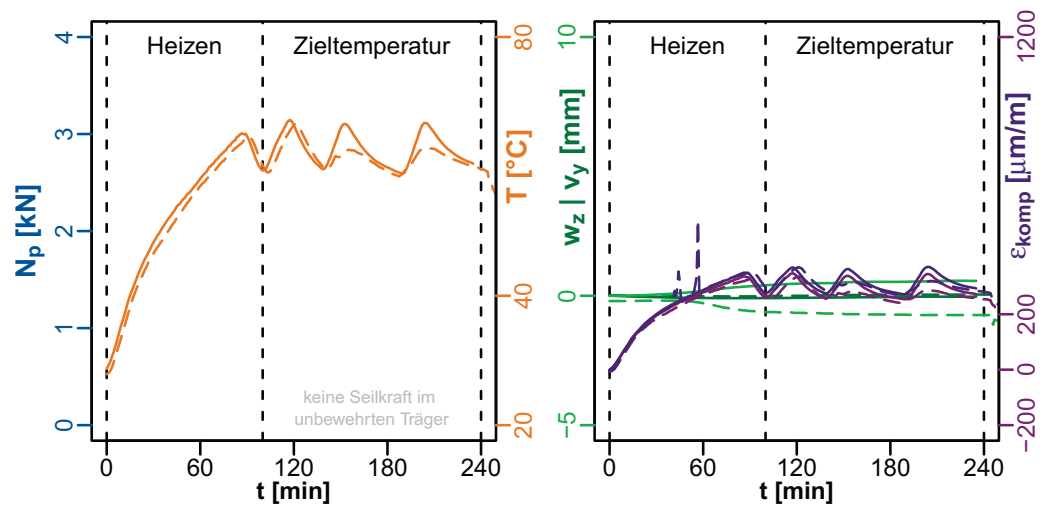

- TV01

PX F0

- - TV02

- Seilkraft

- Temperatur

- horizontale

Durchbiegung

— Verzerrung $\varepsilon_{2-4}$

- Verzerrung $\varepsilon_{\text {ref }}$
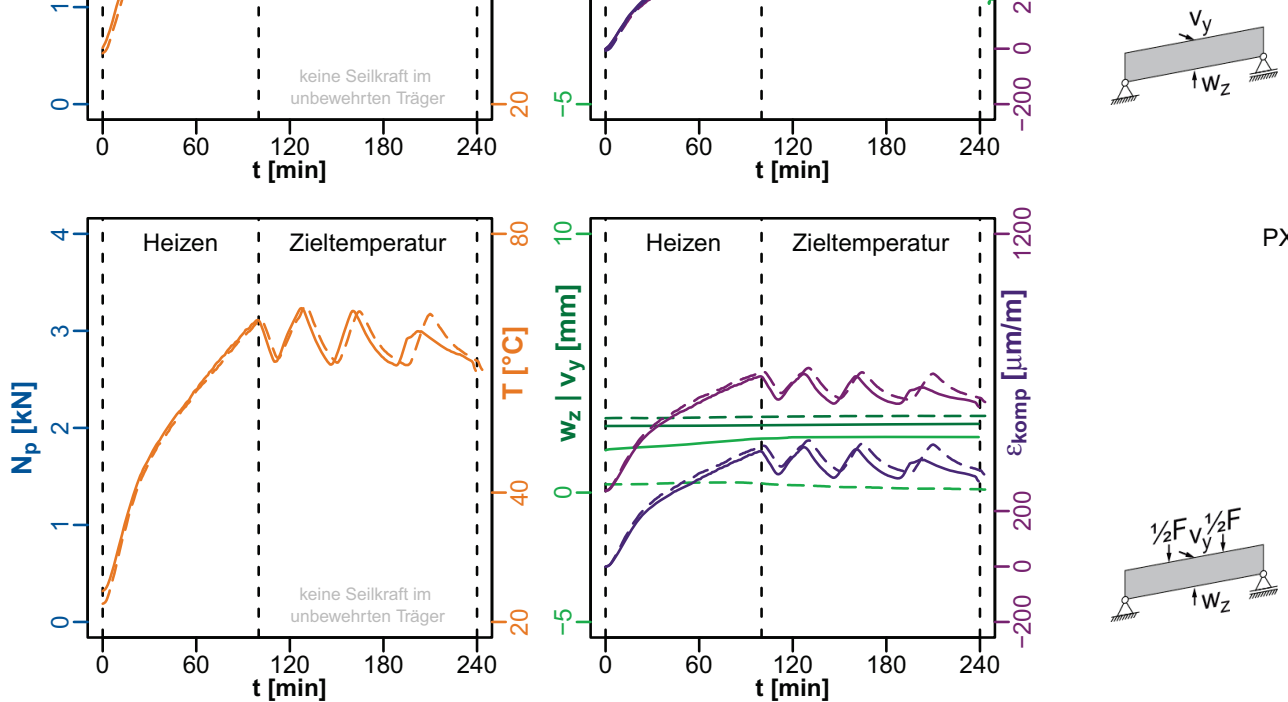

PX F5
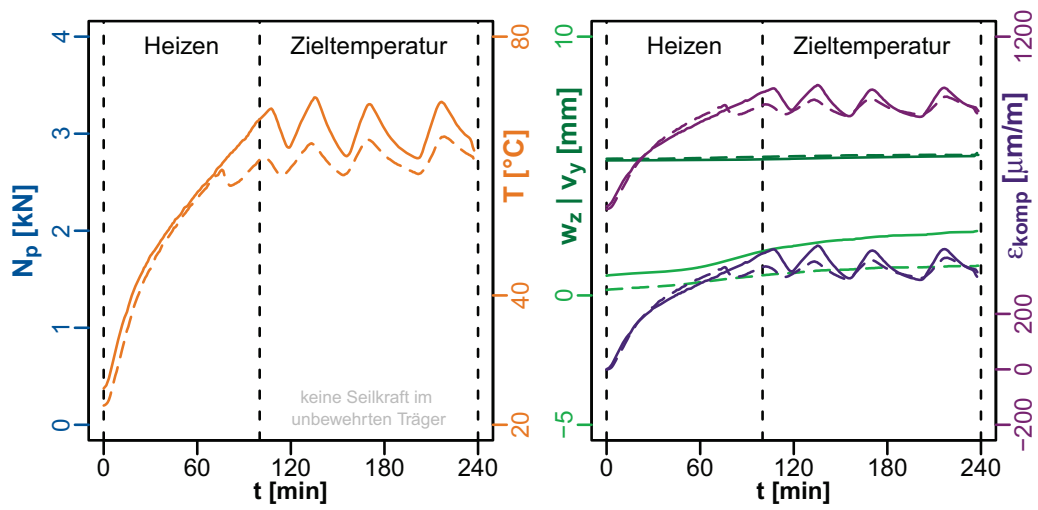

PXF10

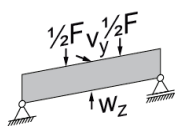

\begin{tabular}{llllllll}
\hline Versuch & Prüfkörper & $\Delta \mathbf{T}[\mathrm{K}]$ & $\Delta \mathbf{P}[\mathbf{N}]$ & $\Delta \mathbf{w}_{\mathbf{z}}[\mathrm{mm}]$ & $\Delta \mathbf{v}_{\mathbf{y}}[\mathrm{mm}]$ & $\Delta \varepsilon_{\text {komp }}[\mu \mathrm{m} / \mathbf{m}]$ & $\Delta \varepsilon_{\text {ref }}[\mu \mathrm{m} / \mathbf{m}]$ \\
\hline PX F0 & TV01 & 33.9 & - & -0.057 & 0.52 & 295.9 & 316.3 \\
& TV02 & 33.5 & - & 0.007 & -0.50 & 274.2 & 310.9 \\
PX F5 & TV01 & 38.4 & - & 0.06 & 0.51 & 356.5 & 363.6 \\
& TV02 & 40.9 & - & 0.078 & -0.11 & 376.1 & 391.0 \\
\multirow{2}{*}{ PX F10 } & TV01 & 40.1 & - & 0.12 & 1.43 & 378.4 & 382.5 \\
& TV02 & 38.3 & - & 0.13 & 0.80 & 368.0 & 361.3 \\
\hline
\end{tabular}


Temperaturlast Referenzträger schlaff bewehrt $d_{s}=\mathbf{8 , 1} \mathbf{m m}$
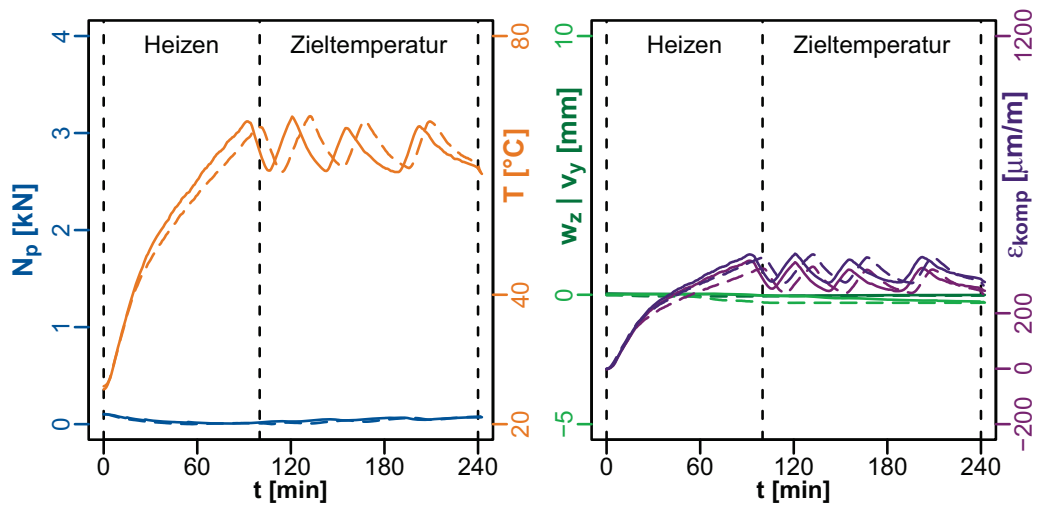

- TV01

- - TV02

- Seilkraft

- Temperatur

- vertikale und

- horizontale

Durchbiegung

- Verzerrung $\varepsilon_{2-4}$

- Verzerrung $\varepsilon_{\text {ref }}$
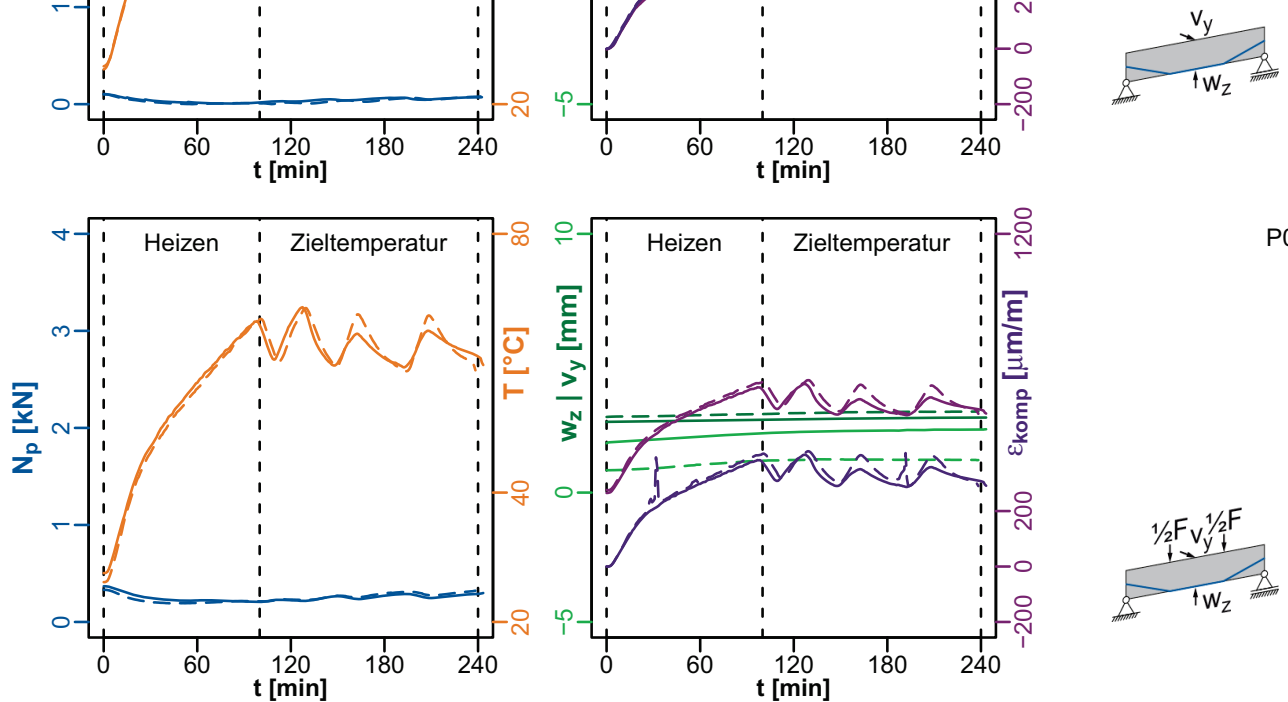

P0 F5
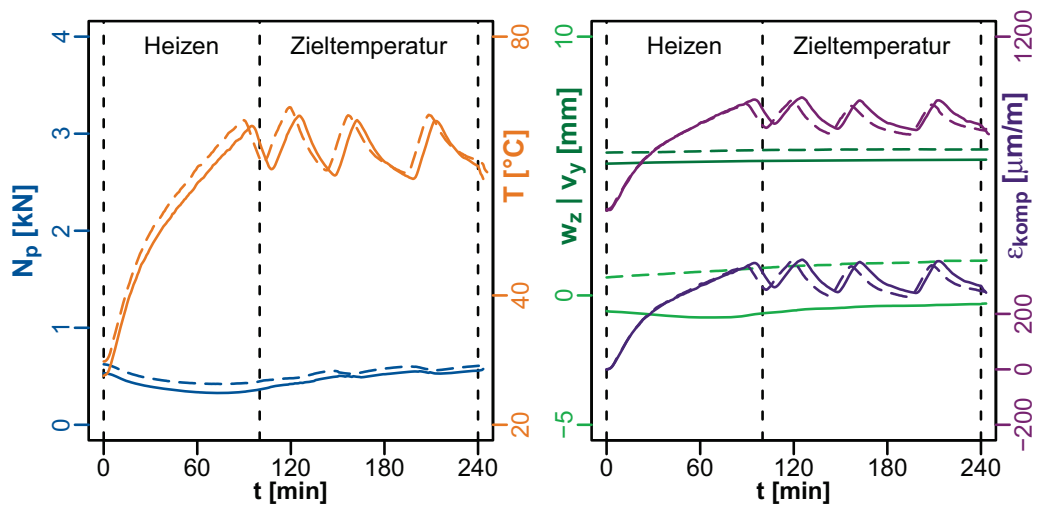

P0 F10

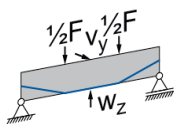

\begin{tabular}{llllllll}
\hline Versuch & Prüfkörper & $\Delta \mathbf{T}[\mathrm{K}]$ & $\Delta \mathbf{P}[\mathbf{N}]$ & $\Delta \mathbf{w}_{\mathbf{z}}[\mathrm{mm}]$ & $\Delta \mathbf{v}_{\mathbf{y}}[\mathrm{mm}]$ & $\Delta \varepsilon_{\text {komp }}[\mu \mathrm{m} / \mathbf{m}]$ & $\Delta \varepsilon_{\text {ref }}[\mu \mathrm{m} / \mathbf{m}]$ \\
\hline P0 F0 & TV01 & 36.3 & -52.9 & -0.069 & -0.16 & 318.3 & 350.5 \\
& TV02 & 37.6 & -67.3 & -0.025 & -0.30 & 313.7 & 352.5 \\
P0 F5 & TV01 & 35.2 & -116.0 & 0.14 & 0.48 & 320.4 & 330.1 \\
& TV02 & 37.0 & -63.4 & 0.18 & 0.40 & 328.1 & 349.5 \\
\multirow{2}{*}{ P0 F10 } & TV01 & 34.9 & -38.1 & 0.13 & 0.15 & 342.6 & 330.3 \\
& TV02 & 33.4 & -77.1 & 0.12 & 0.53 & 319.1 & 314.0 \\
\hline
\end{tabular}


Temperaturlast Träger mit $P_{m 0}=3 \mathrm{kN}$ und $d_{p}=8,1 \mathrm{~mm}$
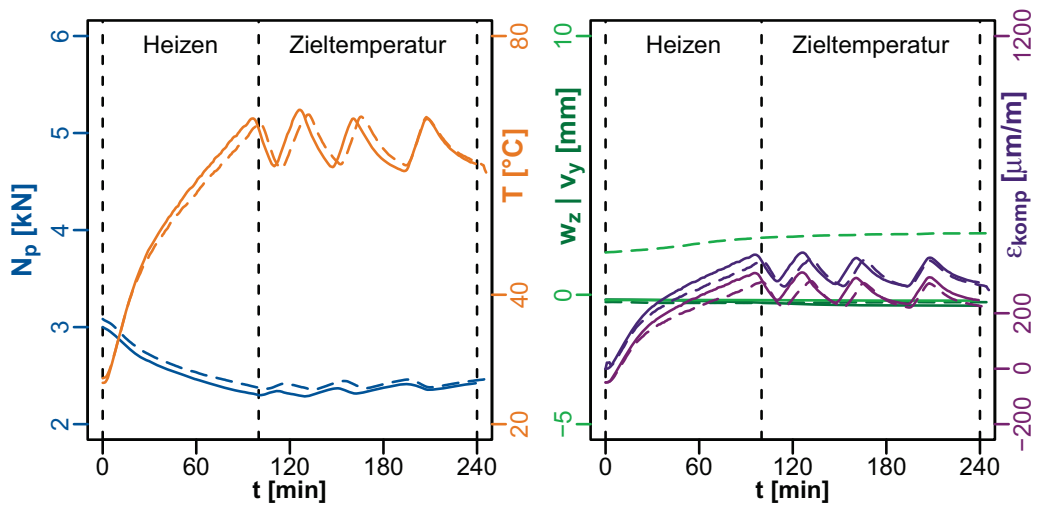

- TV01

P3 F0

- - TV02

- Seilkraft

- Temperatur

- vertikale und

- horizontale

Durchbiegung

— Verzerrung $\varepsilon_{2-4}$

- Verzerrung $\varepsilon_{\text {ref }}$
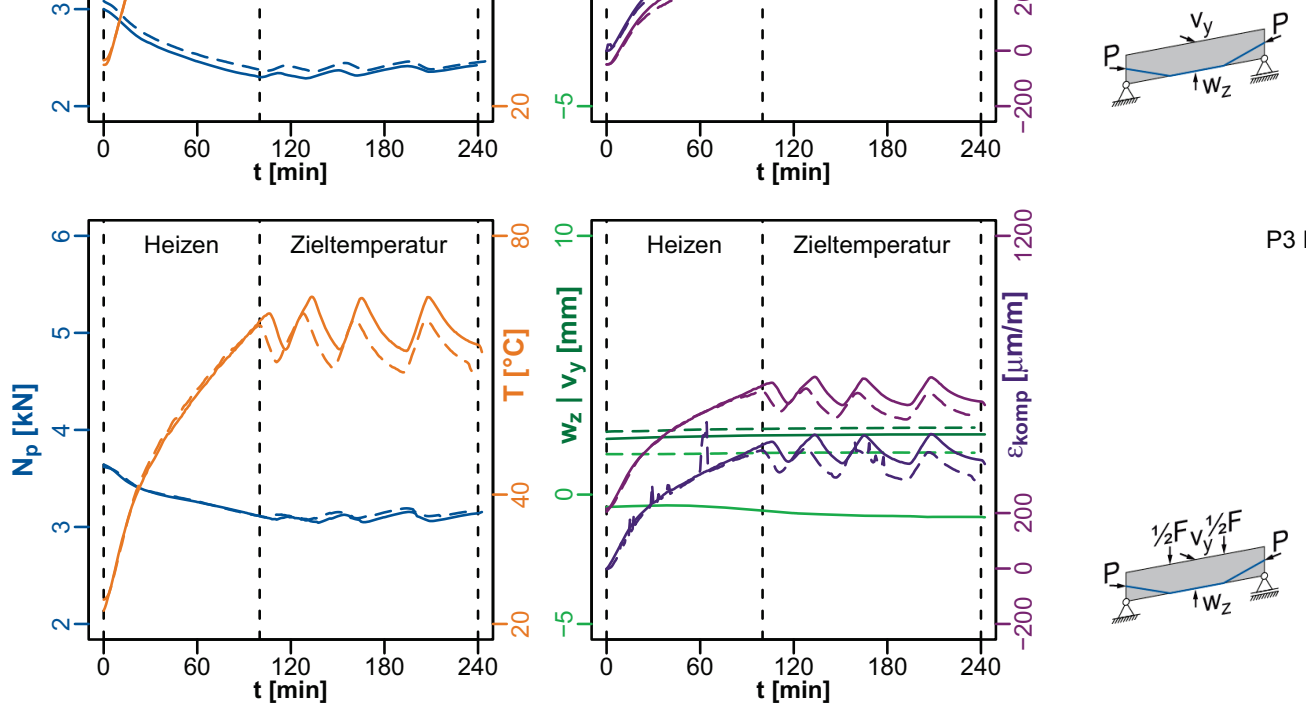

P3 F5
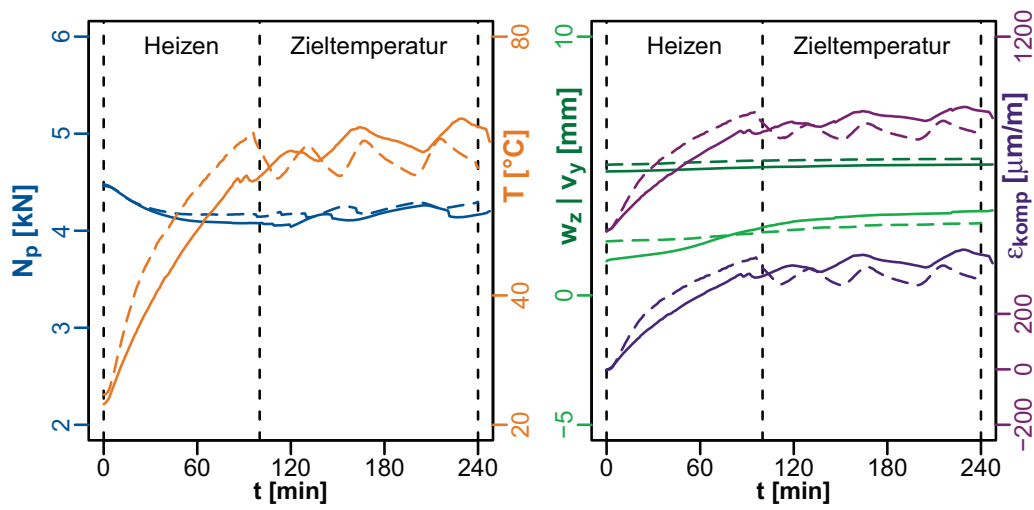

P3 F10

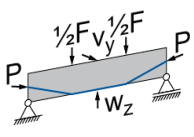

\begin{tabular}{llllllll}
\hline Versuch & Prüfkörper & $\Delta \mathbf{T}[\mathrm{K}]$ & $\Delta \mathbf{P}[\mathbf{N}]$ & $\Delta \mathbf{w}_{\mathbf{z}}[\mathrm{mm}]$ & $\Delta \mathbf{v}_{\mathbf{y}}[\mathrm{mm}]$ & $\Delta \varepsilon_{\text {komp }}[\mu \mathrm{m} / \mathbf{m}]$ & $\Delta \varepsilon_{\text {ref }}[\mu \mathrm{m} / \mathbf{m}]$ \\
\hline P3 F0 & TV01 & 36.7 & -646.5 & -0.21 & -0.012 & 328.5 & 348.5 \\
& TV02 & 36.3 & -677.7 & -0.015 & 0.68 & 311.0 & 340.0 \\
P3 F5 & TV01 & 44.0 & -548.0 & 0.16 & -0.31 & 430.7 & 427.6 \\
& TV02 & 39.3 & -492.3 & 0.14 & 0.059 & 371.5 & 377.0 \\
P3 F10 & TV01 & 40.3 & -327.5 & 0.23 & 1.72 & 406.8 & 386.6 \\
& TV02 & 36.4 & -244.2 & 0.20 & 0.58 & 357.1 & 337.4 \\
\hline
\end{tabular}


Temperaturlast Träger mit $P_{m 0}=6 \mathrm{kN}$ und $d_{p}=8,1 \mathrm{~mm}$
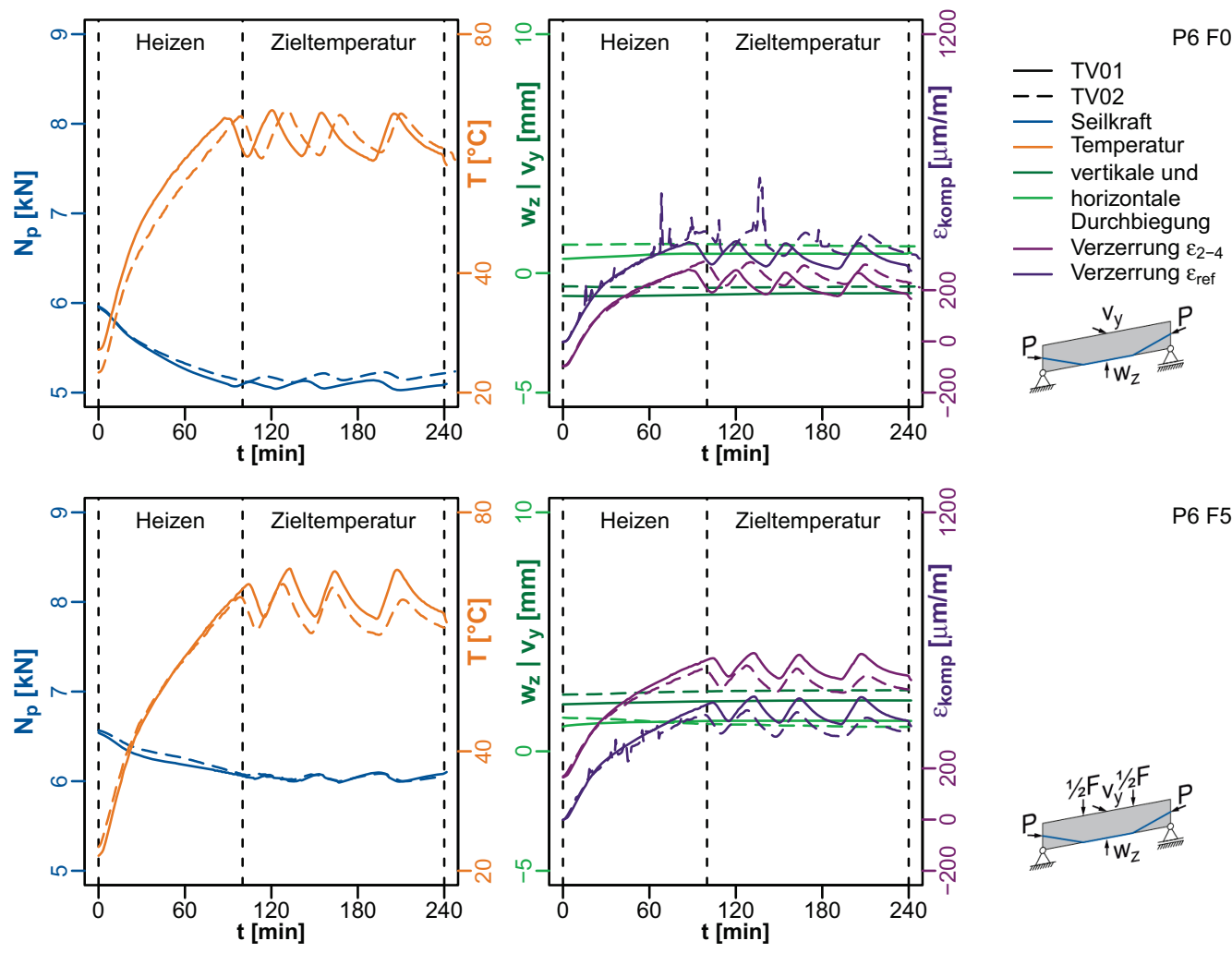

P6 F5
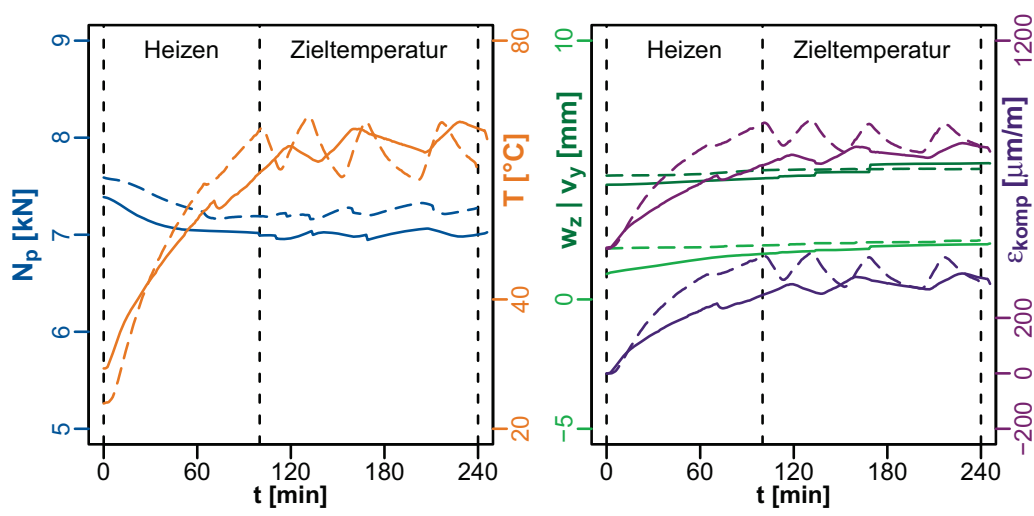

P6 F10

\begin{tabular}{llllllll}
\hline Versuch & Prüfkörper & $\Delta \mathbf{T}[\mathbf{K}]$ & $\Delta \mathbf{P}[\mathbf{N}]$ & $\Delta \mathbf{w}_{\mathbf{z}}[\mathbf{m m}]$ & $\Delta \mathbf{v}_{\mathbf{y}}[\mathbf{m m}]$ & $\Delta \varepsilon_{\text {komp }}[\mu \mathrm{m} / \mathbf{m}]$ & $\Delta \varepsilon_{\text {ref }}[\mu \mathrm{m} / \mathbf{m}]$ \\
\hline P6 F0 & TV01 & 35.4 & -874.4 & 0.098 & 0.22 & 316.4 & 331.6 \\
& TV02 & 39.7 & -792.8 & -0.027 & -0.029 & 353.6 & 390.2 \\
P6 F5 & TV01 & 43.5 & -502.2 & 0.16 & 0.26 & 430.8 & 423.8 \\
& TV02 & 39.1 & -523.6 & 0.17 & -0.34 & 369.3 & 367.6 \\
P6 F10 & TV01 & 34.7 & -384.6 & 0.61 & 1.00 & 341.9 & 320.9 \\
& TV02 & 39.2 & -354.3 & 0.25 & 0.22 & 397.0 & 367.5 \\
\hline
\end{tabular}


Temperaturlast Träger mit $P_{m 0}=9 \mathrm{kN}$ und $d_{p}=8,1 \mathrm{~mm}$
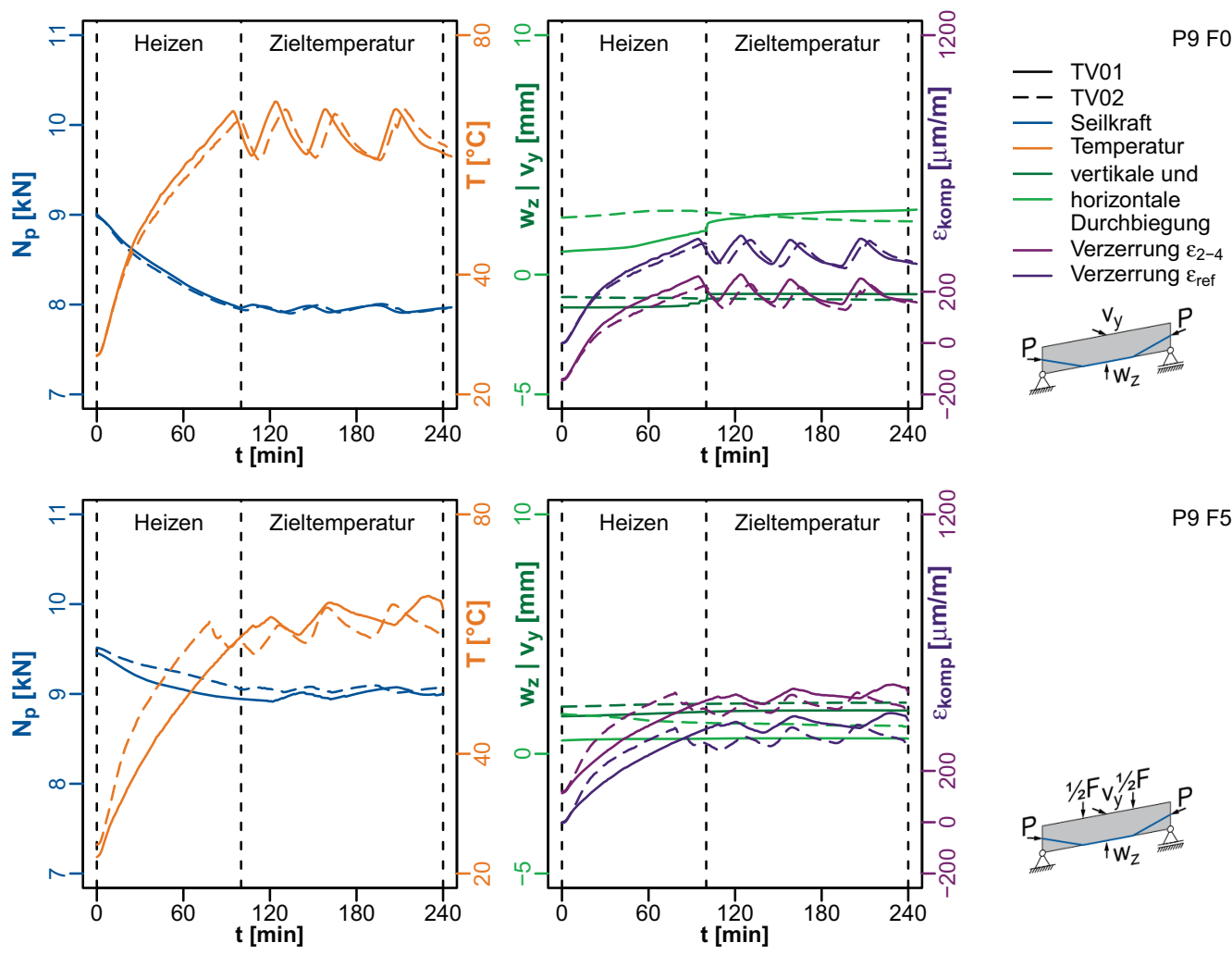

P9 F5
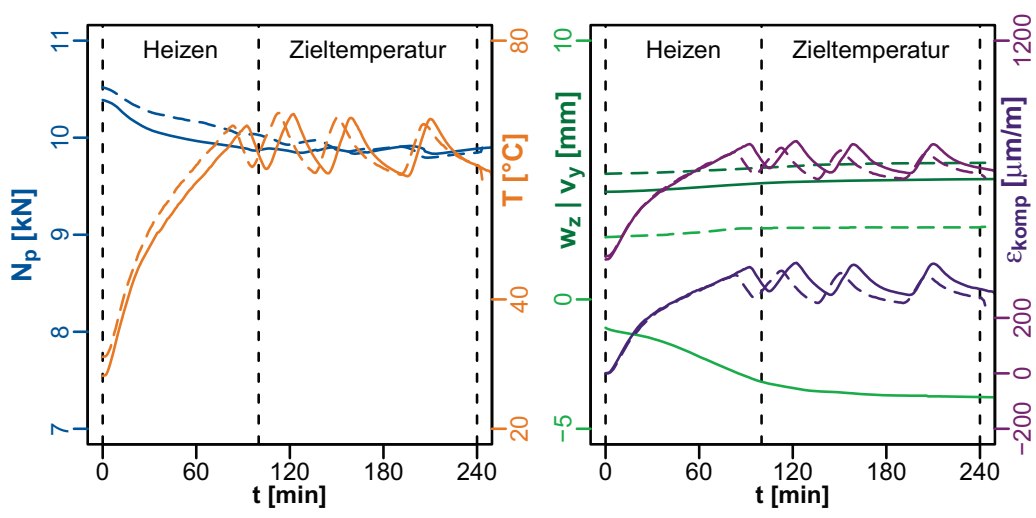

P9 F10

\begin{tabular}{llllllll}
\hline Versuch & Prüfkörper & $\Delta \mathbf{T}[\mathrm{K}]$ & $\Delta \mathbf{P}[\mathbf{N}]$ & $\Delta \mathbf{w}_{\mathbf{z}}[\mathrm{mm}]$ & $\Delta \mathbf{v}_{\mathbf{y}}[\mathrm{mm}]$ & $\Delta \varepsilon_{\text {komp }}[\mu \mathrm{m} / \mathbf{m}]$ & $\Delta \varepsilon_{\text {ref }}[\mu \mathrm{m} / \mathbf{m}]$ \\
\hline P9 F0 & TV01 & 36.7 & -1029.5 & 0.56 & 1.58 & 337.7 & 349.2 \\
& TV02 & 36.7 & -1058.1 & -0.092 & -0.014 & 321.7 & 343.2 \\
P9 F5 & TV01 & 40.2 & -466.2 & 0.24 & 0.091 & 384.0 & 386.8 \\
& TV02 & 36.1 & -463.2 & 0.15 & -0.44 & 337.8 & 330.1 \\
P9 F10 & TV01 & 35.1 & -517.6 & 0.44 & -2.52 & 359.8 & 332.8 \\
& TV02 & 32.2 & -627.4 & 0.37 & 0.38 & 325.0 & 302.3 \\
\hline
\end{tabular}




\section{F $\quad$ SOFiSTiK Quelltext}

\section{Parameter}

\begin{tabular}{|c|c|c|}
\hline sto\#LS & 2.000 & \$Spannweite $[\mathrm{m}]$ \\
\hline sto\#imp & 300 & \$Imperfektions-Divisor L/X \\
\hline sto\#H & 0.150 & \$Trägerhöhe [m] \\
\hline sto\#t & 0.006 & \$Glasdicke [m] \\
\hline sto\#teff & 0.012 & \$effektive Glasdicke [m] \\
\hline sto\#d 1 & 0.028 & \$Abstand Glaspakete [m] \\
\hline sto\#Ap & 15 & \$Seilquerschnitt $\left[\mathrm{mm}^{2}\right]$ \\
\hline sto\#Ep & 130000 & \$Seilmodul $\left[\mathrm{N} / \mathrm{mm}^{2}\right]$ \\
\hline sto\#etap & 1.000 & \$Seil-Verlustfaktor [-] \\
\hline sto\#alphap & $1.6 e-5$ & $\begin{array}{l}\text { \$Wärmedehnzahl Bewehrung } \\
{\left[\mathrm{K}^{-1}\right]}\end{array}$ \\
\hline sto\#cm & 10 & $\begin{array}{l}\text { \$Gelenksteifigkeit Klebung } \\
{[\mathrm{kNm} / \mathrm{rad}]}\end{array}$ \\
\hline sto\#P0 & 9.18 & \$Vorspannkraft $[\mathrm{kN}]$ \\
\hline sto\#F & 10 & \$Biegelast [kN] \\
\hline sto\#dT & 10.0 & \$Temperaturänderung [K] \\
\hline
\end{tabular}

\section{Materialdefinition und Querschnitte}

+ prog aqua

kopf Materialdefinition

mat $\mathrm{nr} 1$ e $7 \mathrm{e} 7$ mue 0.23 gam 25 alfa $0.9 \mathrm{e}-5$ bez 'Glas'

mat $\mathrm{nr} 2$ e $10 \mathrm{e} 10$ mue 0.3 gam 0 alfa 0 bez 'steif'

mat $\mathrm{nr} 3$ e \#Ep*1000 mue 0.3 gam 0 alfa \#alphap bez

'Spannstahl' \$Eigengewicht vernachlässigt

smat nr 30 ltyp ihng bez 'Stabgelenk'

sarb s \#cm typ my

sarb s \#cm typ mz

ende

+ prog aqua

kopf Querschnittsdefinition

quer $100 \mathrm{mnr} 1$ a $2 * \# \mathrm{t} * \# \mathrm{H}$ iy $2 * \# \mathrm{t} * \# \mathrm{H}^{\wedge} 3 / 12$ iz (\#teff)^3*\#H/12

it (\#teff) $3 * \# \mathrm{H} / 3$ ymin -\#teff/2 ymax \#teff/2 zmin

$-\# \mathrm{H} / 2$ zmax \#H/2 bez 'Glaspaket' 
quer $\mathrm{nr} 2 \mathrm{mnr} 1$ a $2 \mathrm{e}-5$ iy $8 \mathrm{e}-9$

qc $\mathrm{nr} 3 \mathrm{~d} \operatorname{sqr}(4 * \# A \mathrm{p} / 3.1415) / 1000$ t $0 \mathrm{mnr} 3 \mathrm{bez}$ 'Spannseil'

ende

\section{Geometrie}

+prog sofimsha

kopf 'Geometrie'

syst RAUM gdiv 10000

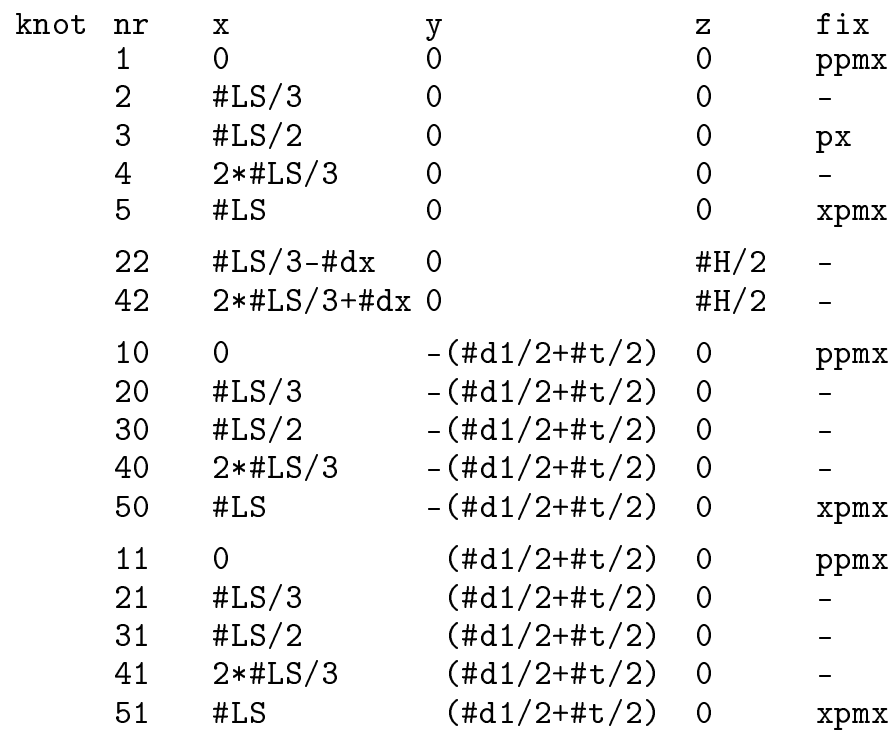

grup 10 bez 'Glaspaket 1 '

stab $\mathrm{nr}$ mesh ka (10 40 10) ke (20 10) qnr 100 teil 10

grup 11 bez 'Glaspaket 2'

stab nr mesh ka (11 41 10) ke (21 10) qnr 100 teil 10

grup 201 bez 'Umlenkung A'

stab nr 221 ka 20 ke 21 qnr 100 teil 10 anfa 30

ende 30

grup 200 bez 'Umlenkbock A'

fach nr 222 ka 22 ke 20 qnr 2

fach nr 223 ka 22 ke 21 qnr 2 


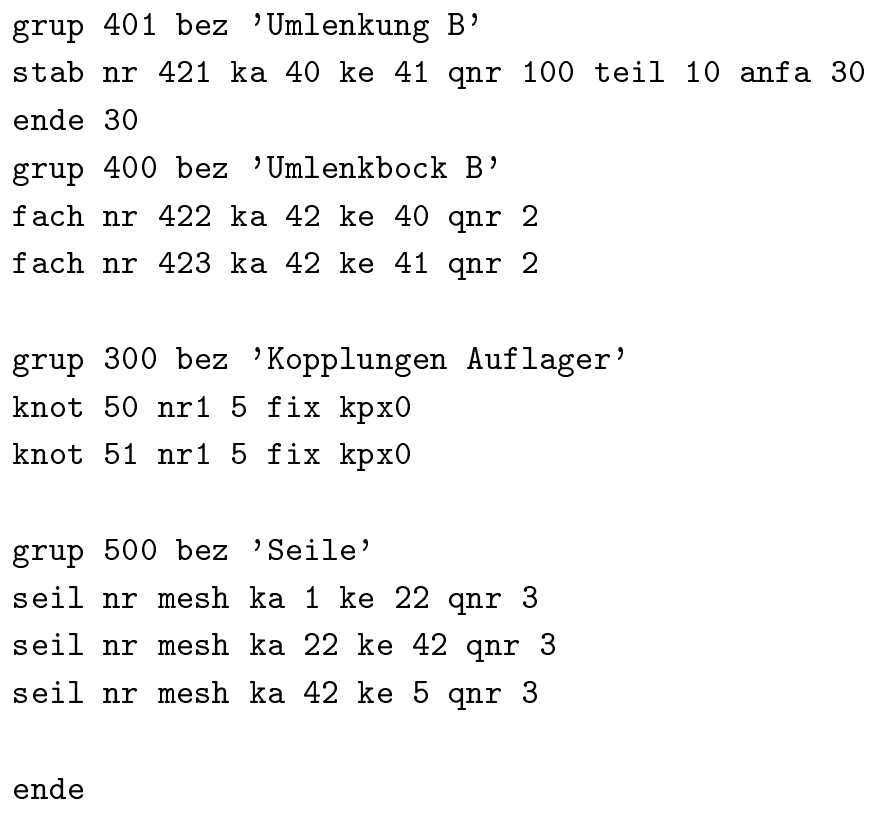

\section{Lasten und Lastkombinationen}

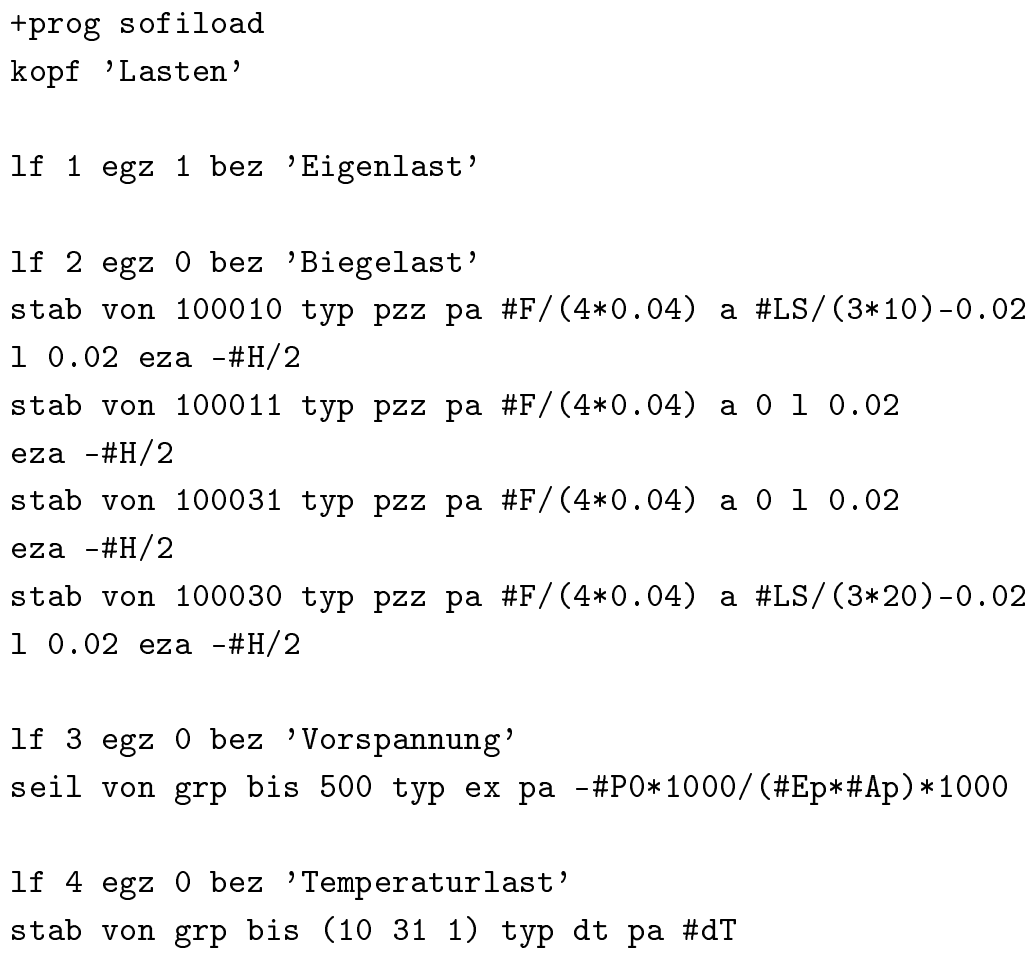




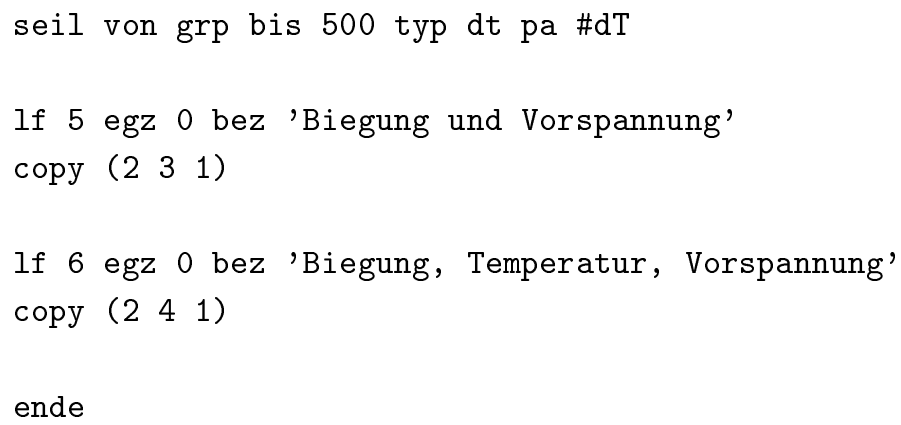

\section{Lösungen Theorie I. Ordnung}

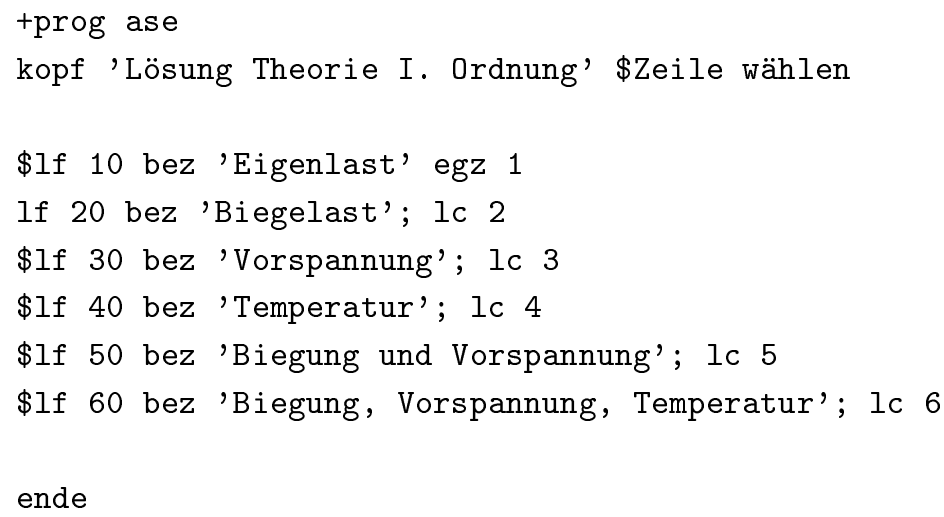

\section{Eigenwertermittlung}

+ prog ase

kopf 'Eigenwerte aus Biegelast'

syst plf 20 fakv 0.0

eige neig 4 etyp beul lf 200 lmin auto

ende 


\section{Skalierte Eigenform als Imperfektionslastfall}

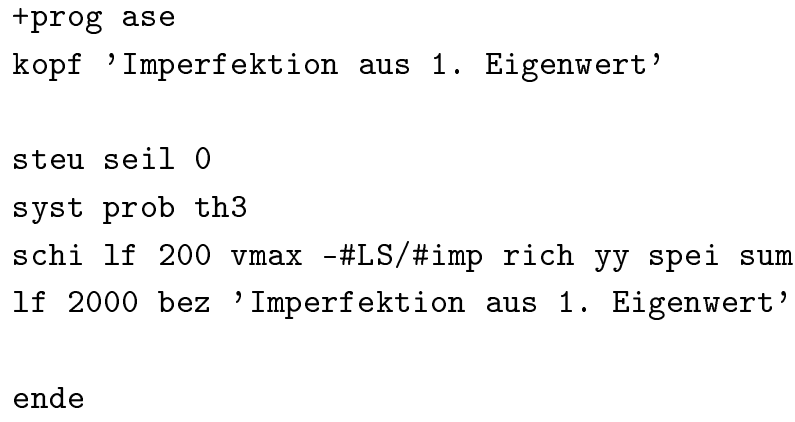

\section{Lösung des Gleichungssystems}

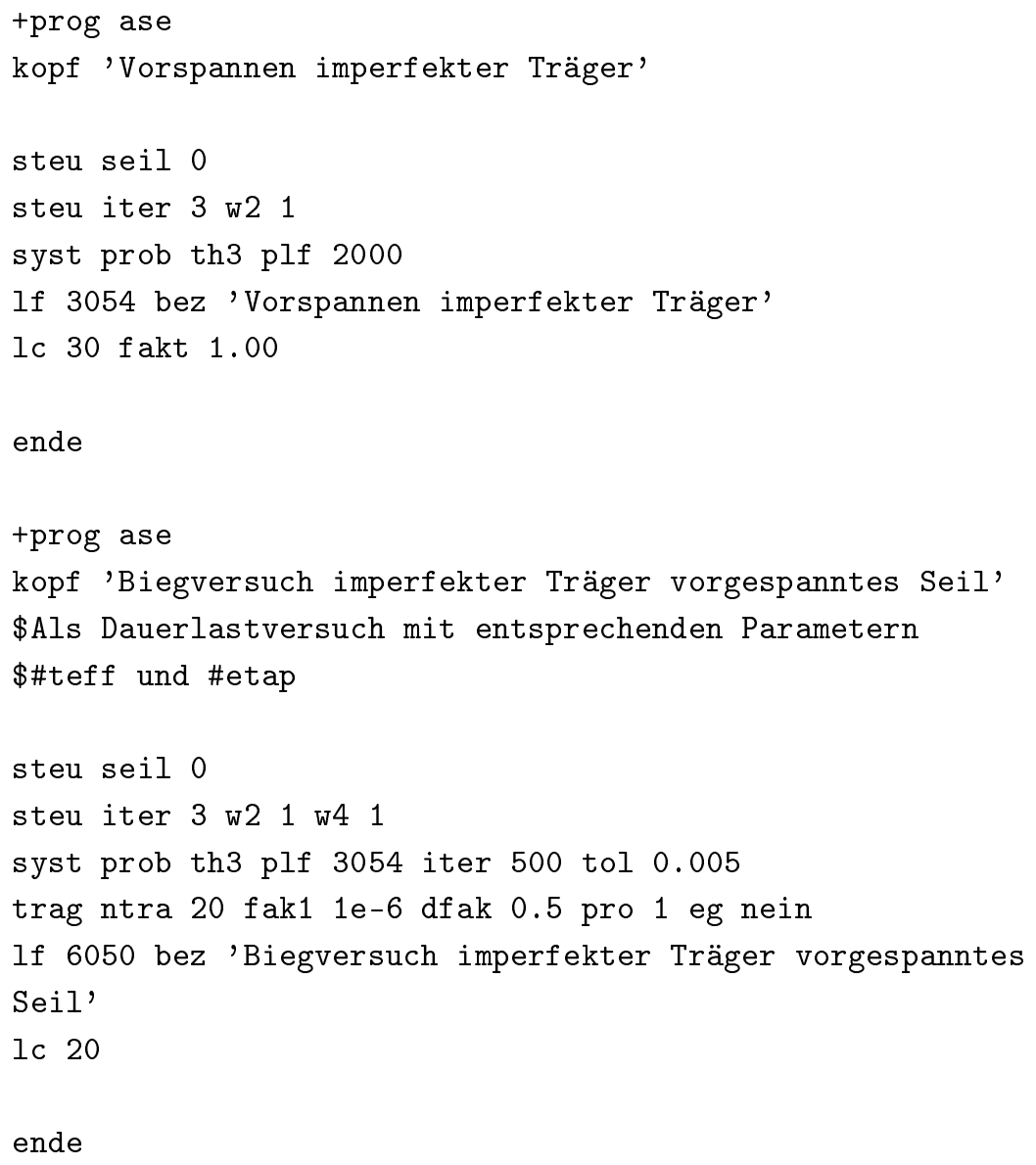

